TA7

W34

no. CHL-98-33

c. 3

US Army Corps

of Engineers

Waterways Experiment

Station

C O O PROPERTY OF THE

UNITEO STATES GOVERNMENT

\title{
Hurricane-Induced Stage-Frequency Relationships for the Territory of American Samoa
}

by Adele Militello, Norman W. Scheffner

Approved For Public Release; Distribution Is Unlimited

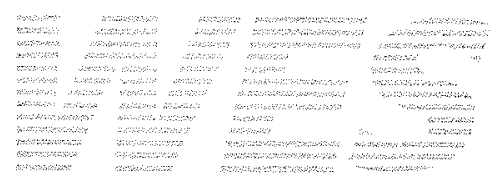

REOERACH LIERARY

USARMVENONO-TWATERNAYS

EXPERIMENT STATION

VICKSBURG, MISSISSIPPI

Prepared for U.S. Army Engineer District, Honolulu 
The contents of this report are not to be used for advertising, publication, or promotional purposes. Citation of trade names does not constitute an official endorsement or approval of the use of such commercial products.

The findings of this report are not to be construed as an official Department of the Army position, unless so designated by other authorized documents. 

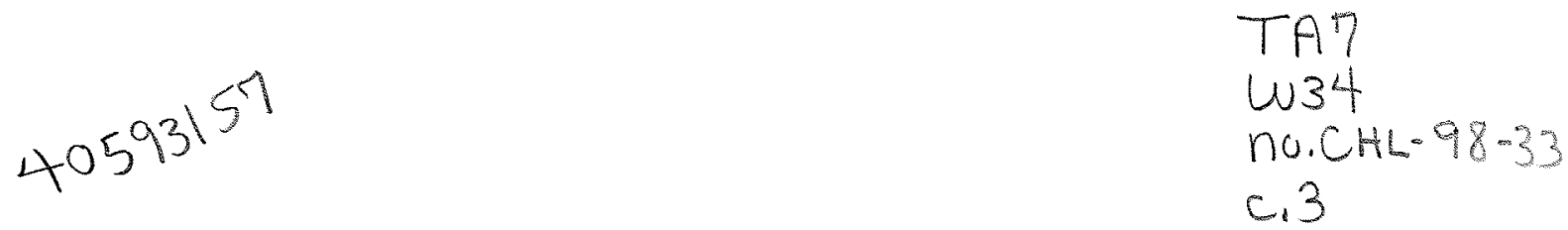

Technical Report CHL-98-33

December 1998

\section{Hurricane-Induced Stage-Frequency Relationships for the Territory of American Samoa}

by Adele Militello, Norman W. Scheffner

U.S. Army Corps of Engineers

Waterways Experiment Station

3909 Halls Ferry Road

Vicksburg, MS 39180-6199

Final report

Approved for public release; distribution is unlimited 


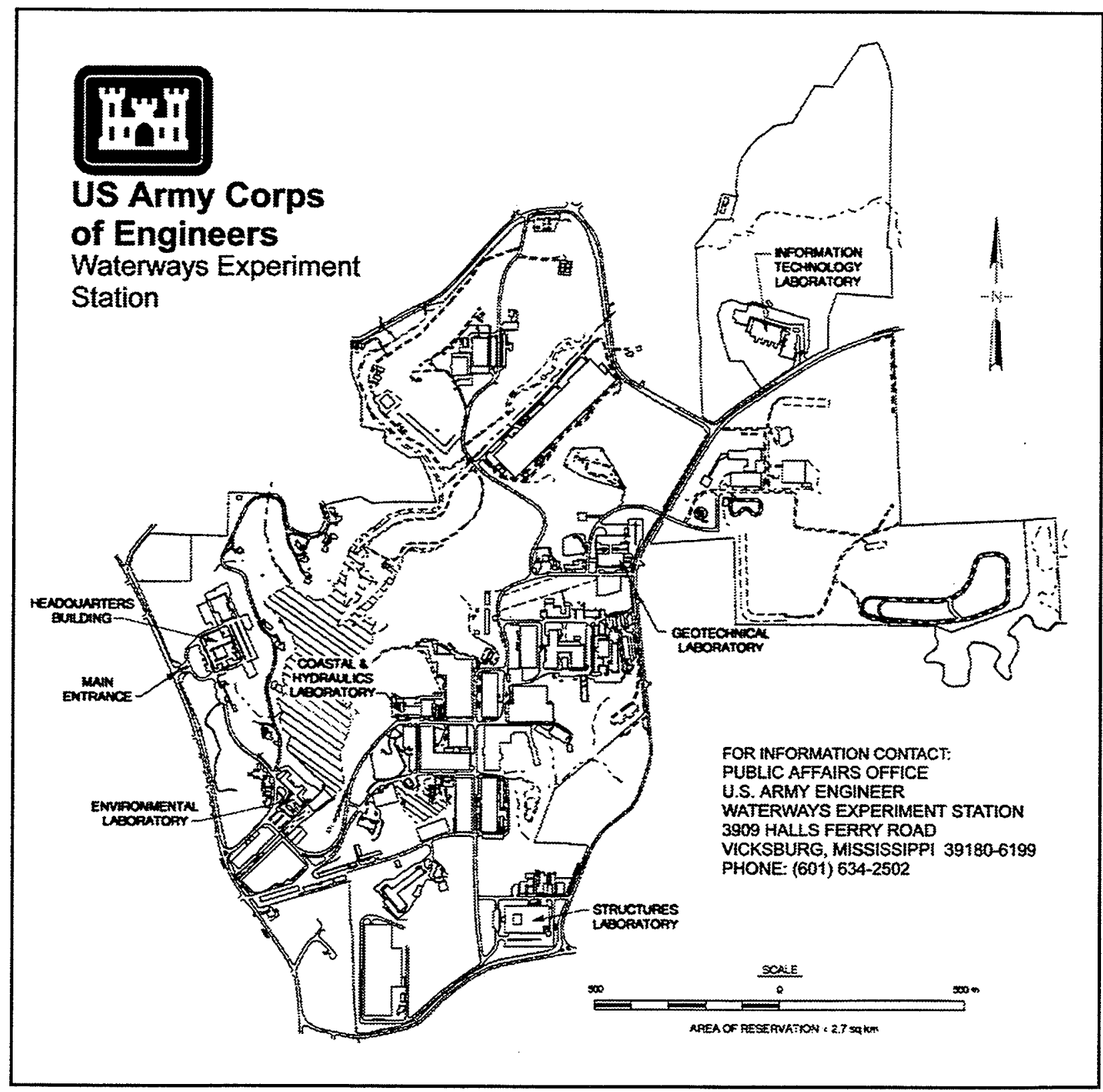

\section{Waterways Experiment Station Cataloging-in-Publication Data}

Militello, Adele.

Hurricane-induced stage-frequency relationships for the Territory of American Samoa / by Adele Militello, Norman W. Scheffner; prepared for U.S. Army Engineer District, Honolulu.

286 p. : ill. ; $28 \mathrm{~cm}$. - (Technical report ; CHL-98-33)

Includes bibliographic references.

1. Storm surges - Mathematical models. 2. Wind waves - Mathematical models. 3. Hurricanes American Samoa. I. Scheffner, Norman W. II. United States. Army. Corps of Engineers. Honolulu District. III. U.S. Army Engineer Waterways Experiment Station. IV. Coastal and Hydraulics Laboratory (U.S. Army Engineer Waterways Experiment Station) V. Title. VI. Series: Technical report (U.S. Army Engineer Waterways Experiment Station); CHL-98-33.

TA7 W34 no.CHL-98-33 


\section{Contents}

Preface vii

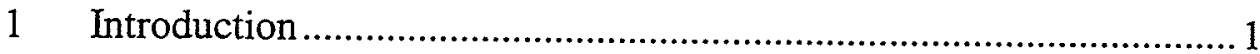

2 Empirical Simulation Technique …………….................................

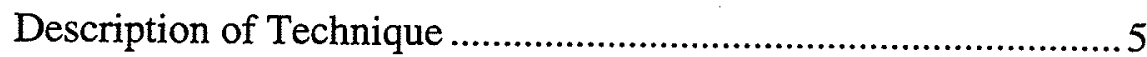

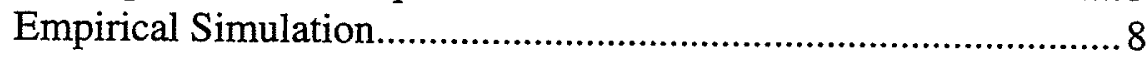

Recurrence Relationships ........................................................

3 Description of Numerical Models...................................................... 12

Wind and Atmospheric Pressure Field Model ............................... 12

Wave and Wave Transformation Models....................................... 15

Storm Surge Model ....................................................................... 17

4 Implementation of Storm Surge Model.............................................23

Calibration of the Storm Surge Model .............................................22

Validation of the Storm Surge Model ...........................................2 24

5 Development of Stage-Frequency Relationships ..............................26

Selection of Hurricanes .................................................................26

Storm Surge/Tidal Elevation Relationship...................................26

Spectral Wave Transformation....................................................2.

Wave Impoundment Correction Procedure .....................................30

Wave Setup and Runup ................................................................. 31

Typhoon Ofa - Water Level Comparison .......................................33

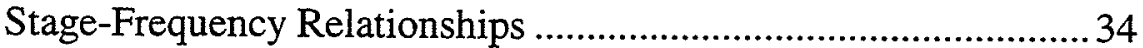

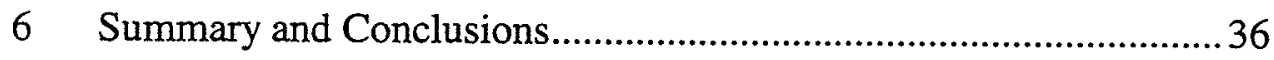

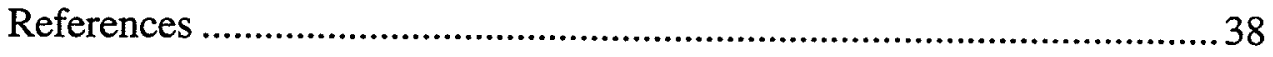

Appendix A: Station Locations ............................................................ A1

Appendix B: Stage-Frequency Relationship Tables............................... B1

Appendix C: Stage-Frequency Relationship Plots ................................... C1

Appendix D: Tables of Wave Parameters, Setup, and Ponding Level 
at Numerical Gauge Locations ....................................... D1 Appendix E: Hurricane Tracks ...........................................................E1

Appendix F: Mathematical Notation ..................................................... 


\section{List of Figures}

Figure 1. Location map of study area .................................................. 1

Figure 2. Site map showing the five islands of study ............................... 2

Figure 3. Islands of Tutuila and Aunuu ................................................. 3

Figure 4. Example of cumulative probability distribution plot ............... 11

Figure 5. Measured vs. estimated wind speed ....................................... 14

Figure 6. Computational grid for American Samoa................................ 20

Figure 7. Computational grid showing detail for Tutuila and Aunuu Islands......................................................................... 21

Figure 8. Computational grid showing detail for Pago Pago Harbor ....... 21

Figure 9. Computational grid showing detail for Ofu, Olosega, and Tau Islands................................................................................ 22

Figure 10. Tidal constituent calculated and modeled water level at the Pago Pago Harbor gauge for Jan. 4, 1996 through Jan. 29, 1996 ...... 25

Figure 11. Measured and modeled water level in Pago Pago Harbor for Hurricane Val ...................................................................... 25

Figure 12. Station locations for western Tutuila Island.............................29

Figure 13. Station locations of eastern Tutuila Island and Aunuu Island .................................................................... 29

Figure 14. Station locations for Ofu Island and Olosega Island ................. 30

Figure 15. Station locations for Tau Island ................................................ 30 


\section{List of Tables}

Table 1. Sample Distribution Function Calculation .............................. 10

Table 2. WISWAVE Grid Parameters................................................ 16

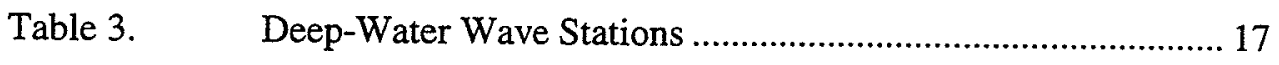

Table 4. Storm Surge Grid Parameters ................................................ 20

Table 5. Tidal Constituents in Pago Pago Harbor .................................. 24

Table 6. Historical Storms Included in the Training Set.......................22

Table 7. Ponding Level Coefficients for Irregular Waves ..................... 31

Table 8. Number of Transects for the American Samoa Islands............. 32

Table 9. Observed and Calculated Water Level for Typhoon Ofa........... 34 


\section{Preface}

This report describes the procedures and results of a hurricane stagefrequency analysis for five islands of the U.S. Territory of American Samoa. The study was performed by the U.S. Army Engineer Waterways Experiment Station (WES) Coastal and Hydraulics Laboratory (CHL) for the U.S. Army Engineer District, Honolulu (CEPOH).

The investigation reported herein was conducted by Dr. Adele Militello, Coastal Hydrodynamics Branch (CH), and Dr. Norman W. Scheffner, both of the Navigation and Harbors Division $(\mathrm{CN}), \mathrm{CHL}$. The final report was prepared by Dr. Militello. Mr. Steven H. Yamamoto, Civil Works Branch, CEPOH, was the study manager and point of contact.

This study was performed under the general supervision of Dr. James R. Houston and Mr. Charles C. Calhoun, Jr., Director and Assistant Director, respectively, $\mathrm{CHL}$. Direct supervision of this project was provided by Mr. Claude E. Chatham, Chief, CN, and by Dr. Martin C. Miller, Chief, $\mathrm{CH}, \mathrm{CN}$, CHL.

During this study, Dr. Robert W. Whalin served as the Technical Director of WES and COL Robin Cababa, EN, was the Commander of WES.

The contents of this report are not to be used for advertising, publication, or promotional purposes. Citation of trade names does not constitute an official endorsement or approval of the use of such commercial products. 



\section{Introduction}

The Territory of American Samoa consists of seven islands located in the South Pacific Ocean at approximately $170 \mathrm{~W}$ longitude and $14 \mathrm{~S}$ latitude. The Islands lie east-northeast of Australia and northeast of New Zealand as shown in Figure 1. This low-latitude location is favorable for tropical storm and hurricane formation and passage. During the period 1987 through 1991, extensive damage from three hurricanes was incurred. Storm damage included: village damage and destruction, road washout, harbor destruction, and crop damage (Sea Engineering, Inc. and Belt Collins Hawaii 1994). The present study was undertaken to calculate hurricane stage-frequency hydrographs for five of the seven islands. The development of the storm-surge hydrographs will provide information for planning and mitigation strategies to reduce the impact of future storms in the study area.

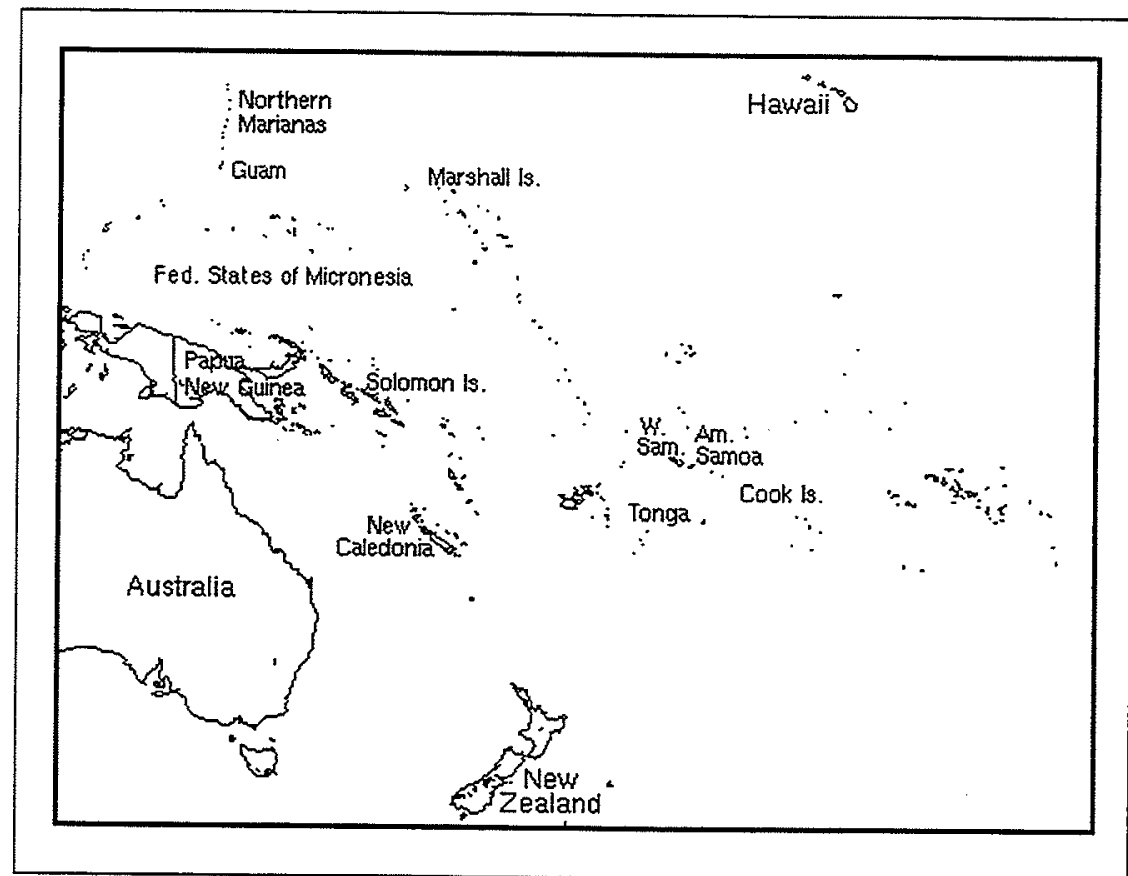

Figure 1. Location map of study area 
The islands Tutuila, Aunuu, Ofu, Olosega, and Tau, comprise the area of study (see Figure 2) and together cover an area of $77 \mathrm{sq} \mathrm{mi}(199 \mathrm{sq} \mathrm{km})$. Tutuila is the largest of the five islands. The Manu'a Group, consisting of Ofu, Olosega, and Tau, are located $60 \mathrm{mi}(160 \mathrm{~km})$ east of Tutuila and Aunuu. All five islands are volcanic, with typically narrow coastal areas and steep mountains. Fringing coral reefs are common around the islands and can extend to $2,000 \mathrm{ft}(610 \mathrm{~m})$ out from the shoreline (Sea Engineering, Inc. and Belt Collins Hawaii 1994). These reefs are typically very shallow and some are exposed at low tide.

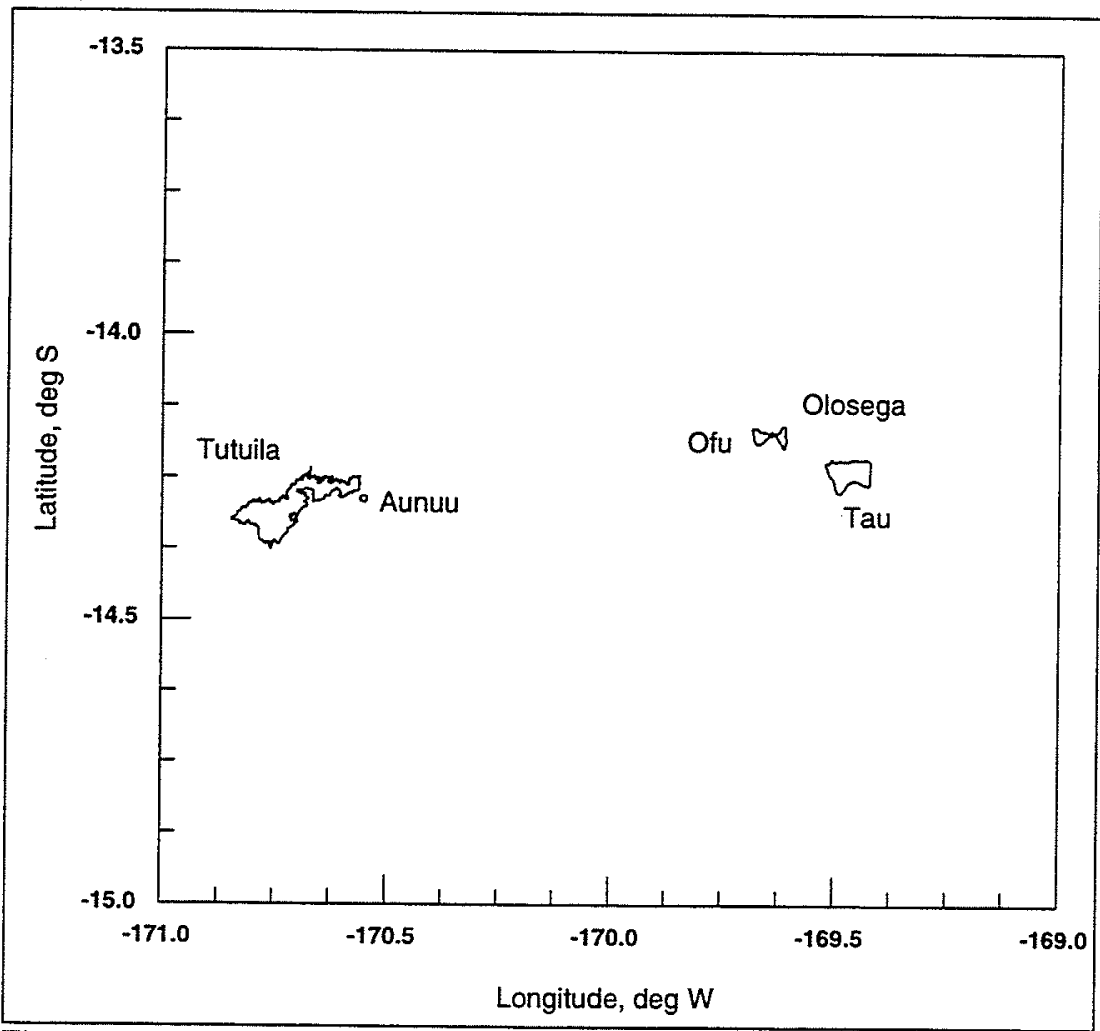

Figure 2. Site map showing the five islands of study

Pago Pago Harbor is the major embayment of the study site and is located on the southern side of Tutuila (see Figure 3). The harbor is deep, with typical depths of $200 \mathrm{ft}(60 \mathrm{~m})$ along the its main axis. Tuna canneries, a wharf, and government buildings are located on the harbor shores. Smaller embayments exist on Tutuila and most are located on the north shore of the island. Aunuu and the Manu'a Group lack natural embayments, although small harbors (Aunuu Small Boat Harbor, Ofu Harbor, and Tau Small Boat Harbor) have been constructed by the US Army Corps of Engineers.

Although most of the inhabitants of American Samoa live on Tutuila Island, villages are located in low-lying coastal areas on all five islands. The risk of inundation is greatest in these regions because of their low relief and exposure to storm waves. 


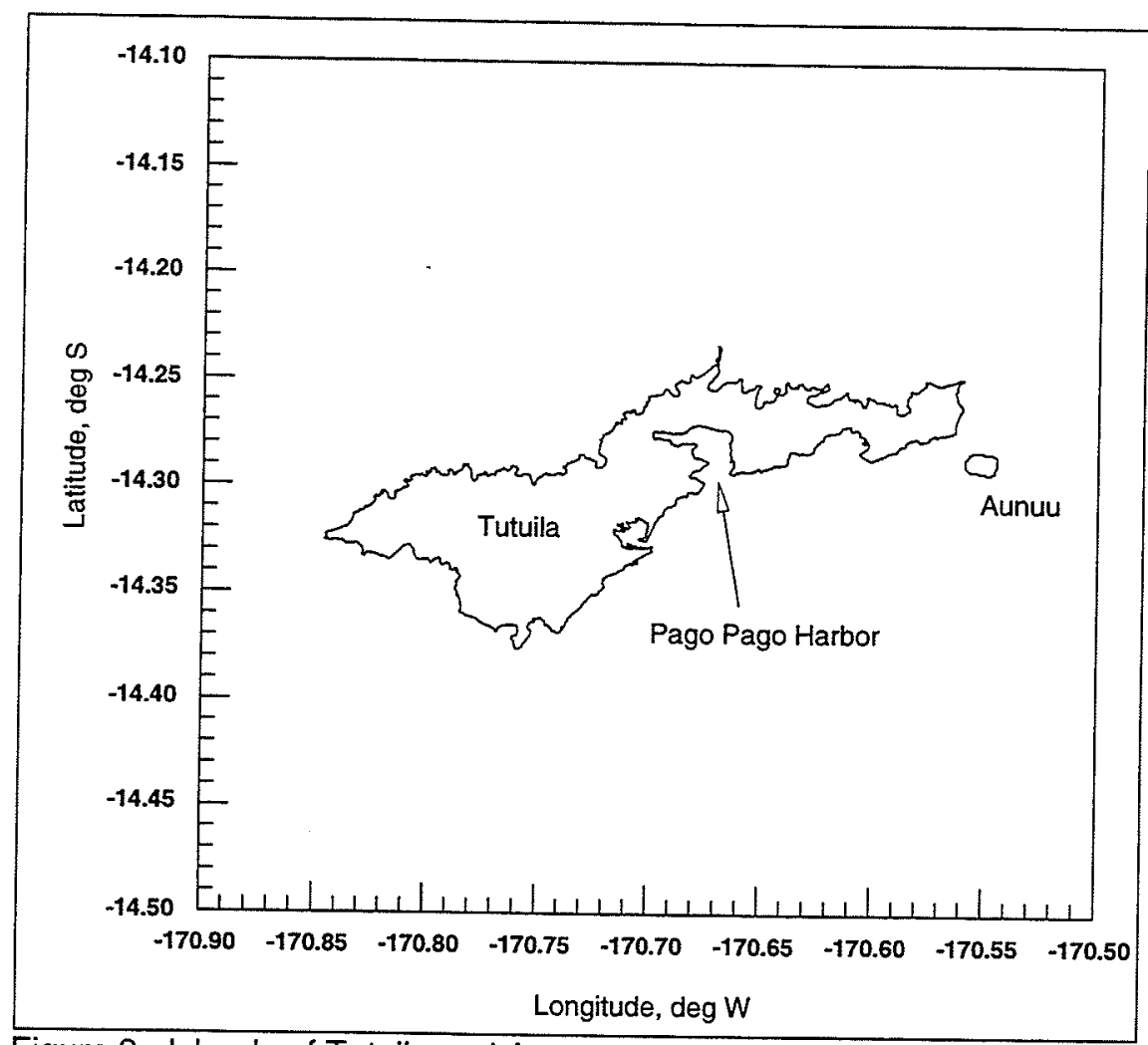

Figure 3. Islands of Tutuila and Aunuu

This report describes the procedures and results of a hurricane stagefrequency analysis for the island coastlines of the U.S. Territory of American Samoa. Many of the techniques employed in this study have been successfully applied in previous stage-frequency analyses (Mark 1996, Mark and Scheffner 1997). The analysis for this study consisted of five tasks. The first task was the development of a hurricane database for the western south Pacific Ocean and analysis of storm statistics and correlations. Storms impacting the study area were selected from the database to create a smaller, representative group of storms called the "training set." A planetary boundary layer model was applied to calculate wind and atmospheric pressure fields for each storm in the training set.

The second task consisted of simulation of storm surge by application of a long-wave, finite-element hydrodynamic model. For each storm in the training set, storm surge was calculated at specified sites in the study area. The third task consisted of wave parameter calculation for each storm by application of a wave model and a wave-transformation model. The fourth task consisted of timeseries calculation of ponding level, setup, and runup for each storm. These calculations were performed for profiles specified by $\mathrm{CEPOH}$.

The fifth task was the development of frequency-of-occurrence relationships for water level. These relationships were developed by application of the empirical simulation technique (EST) to relate hurricane parameters and the corresponding storm-surge elevations. The EST is a statistical resampling procedure that applies historical data to develop joint probability relationships 
among the various measured storm parameters (e.g., maximum wind speed). The resampling scheme generates large populations of data that are statistically similar to a much smaller database of historical events, i.e. the training set of storms. Application of the EST to the expanded storm set produces a database of peak storm-surge elevations by simulating multiple-year periods (e.g. 200-year periods) of storm activity a multiple number of times. Stage-frequency relationships are then calculated from the database of peak storm-surge elevations.

This report is divided into six chapters. Chapter 1 is the Introduction. Chapter 2 describes the EST. Chapter 3 describes the meteorological, wave, and long-wave hydrodynamic models. Chapter 4 discusses model calibration, validation, and implementation. Chapter 5 gives the methods of calculation for stage-frequency relationships. Chapter 6 provides the summary and conclusions of the study. Appendix A contains a listing of station locations for storm surge calculations. Appendix B gives stage-frequency relationship tables. Appendix C shows stage-frequency relationship plots. Appendix D contains tables of wave parameters, setup, and ponding level that correspond to peak water level for numerical gauge locations. Appendix E shows hurricane track figures for storms contained in the training set. Appendix F gives the mathematical notation used in the report. 


\section{Empirical Simulation Technique}

Storm damage reduction programs and design of coastal structures typically require a storm-surge analysis to obtain a peak water-surface elevation for design water levels. Because hurricanes occur infrequently at a given site, abundant storm-surge stages are generally not available such that standard ranking methods cannot be applied in stage-frequency analysis. Thus, numerical models are often applied for simulating a larger population of storm-surge events. Traditionally, modeled hurricanes are synthesized via a joint probability method (JPM) to describe storm attributes, such as maximum wind speeds and pressure deficits. A set of hypothetical hurricanes is built from a combination of hurricane parameter values obtained by statistical analysis of historical storms.

The JPM requires that all parameters are statistically independent. However, storm parameters are not statistically independent and the assumption that they are independent leads to errors when the JPM approach is taken. Because storm parameters are related, random grouping of parameters can cause simulation of storms that may not occur in nature. For example, one parameter may be assigned a value typical of a weak storm, whereas a second parameter may be assigned a value representative of an intense storm. Thus, a level of uncertainty is introduced into the stage-frequency computations. For this study, an alternative approach, the EST, has been taken. The EST preserves the interdependence of hurricane parameters, which is an advantage over the JPM. Details of the EST are given in Borgman et al. (1992) and Scheffner and Borgman (1993).

\section{Description of Technique}

EST is a statistical resampling technique that uses historical data to develop joint probability relationships among the various measured storm parameters. In contrast to the JPM discussed above, there are no simplifying assumptions concerning the development of the probability density functions describing historical events. Thus, the interdependence of parameters is maintained. In this manner, parameter probabilities are site-specific, do not depend on fixed parametric relationships, and do not assume parameter independence. Thus the EST is distribution-free and nonparametric. 
For this study, the EST was developed to generate numerous multi-year intervals of possible future hurricane events for the study site. The ensemble of modeled or simulated events is consistent with the statistics and correlations of past storm activity at the site. Furthermore, the EST permits random deviations in storm behavior (when compared to historic events) that could occur in the future. For example, simulated hurricanes are permitted to make landfall at locations other than those made by historical storms. These random deviations can also result in more intense storms than the historical events themselves, allowing for the possibility of a future hurricane being the storm of record.

The simulation approach requires specifying a set of parameters that describes the dynamics of some physical system, such as hurricanes. These parameters, which must be descriptive of both the physical process being modeled and the effects of that process, are defined as an $\mathrm{N}$-dimensional vector space. The parameters that describe the physical attributes of the process are referred to as input vectors. For example,

$$
\underline{v}=\left(v_{1}, v_{2}, v_{3}, \ldots, v_{N}\right)
$$

In the case of hurricanes, pertinent input vectors include: the central pressure deficit, the radius to maximum winds, minimum distance from the eye of the storm to the location of interest, forward speed of the eye, and the tidal phase during the event. These values can be defined for each specific location and correspond to each particular historical or hypothetical event of the total set of storm events used in the study.

The second class of vectors involve some selected response resulting from the $N$-dimensional parameterized storm, i.e.,

$$
\underline{r}=\left(r_{1}, r_{2}, r_{3}, \ldots, r_{M}\right)
$$

For hurricanes, response vectors can include maximum storm surge, shoreline erosion, dune recession, wind-generated wave height and period, bottom erosion, or any response that can be attributed to the passage of the storm. The maximum total water-surface elevation, resulting from the combined tide and storm surge, is the response vector of interest.

Although response vectors are related to input vectors

$$
\nu \Rightarrow r
$$

the interrelationship is highly nonlinear and involves correlation relationships which cannot be directly defined, i.e., a nonparametric relationship. For example, in addition to the storm-input parameters, storm surge is a function of local bottom topography, shoreline slope and exposure, ocean currents, etc., as well as their spatial and temporal gradients. It is assumed that these combined properties are implicit in the response vector. For the case of storm surge along the coast of the American Samoa islands, atmospheric and hydrodynamic models are applied to compute response vectors as a function of the input vectors and 
local bottom topography together with shoreline configuration. Other response vectors such as sediment transport, shoreline response, and dune recession require application of additional models.

Historical data for storms can be characterized as

$$
\left[v_{i} ; i=1, \ldots, I\right]
$$

where $I$ is the number of historical storm events. For example, let $v_{i}$ have $d_{v}$ components

$$
v_{i}=\Re^{d_{v}}
$$

where $\Re^{d_{v}}$ denotes a $d_{v}$-dimensional space. From this historical data set, a subset of storm events is selected

$$
\left[v_{j}^{*}, j=1, \ldots, J\right]
$$

where $J$ is the number of historical storms contained in the subset. The subset of storms is representative of the entire set of historical storms and is referred to as the "training set." Furthermore, those storms comprising the training set are subsequently used as input to numerical models for computing the desired response vectors. The set of $v_{j}^{*}$ usually includes historical events but may include historical storms with a deviation or perturbation, such as a hurricane with a slightly altered path. Some historical events may also be deleted from the training set if two events are nearly identical such that both would produce the same response. Because the purpose is to fill parameter space $\Re$, two similar events are redundant.

The training set of storms can be augmented with additional storms contained in the historical data set. Storm events augmenting the training set are referred to as the "statistical set" of storms. Whereas numerical models are used for calculating response vectors for those events in the training set, response vectors for the statistical set of storms are interpolated using the training set response vectors. Thus, stage-frequency relationships can be generated using the entire historical data set without need of simulating all storms in that data set.

With the augmented storm data set (i.e., training and statistical storm sets), the EST produces $\mathrm{N}$ simulations of a T-year sequence of events (hurricanes), each with their associated input vectors and response vectors. Because there are $\mathrm{N}$-repetitions of a T-year sequence of events, an error analysis of the results can be performed with respect to median, worst, least, standard deviation, etc. The following describes the procedures by which the input and response data are used to produce multiple simulations of multiple years of events. 


\section{Empirical Simulation}

Two criteria are required of the T-year sequence of events. The first criterion is that the individual events must be similar in behavior to historical events in order that the interrelationships among the input and response vectors remain realistic. For example, a hurricane with a high central pressure deficit and low maximum winds is not a reasonable event - the two parameters are not independent although their exact dependency is unknown.

Simulation of realistic events is accounted for in the nearest-neighbor interpolation resampling technique developed by Borgman et al. (1992). The basic technique can be described in two dimensions as follows. Let $X_{1}, X_{2}, X_{3}, \ldots, X_{n}$ be $n$ independent, identically distributed random vectors (storm events), each having two components $\left[X_{i}=\left\{x_{i}(1), \underline{x}_{i}(2)\right\}, i=1, n\right]$. Each event $X_{i}$ has a probability $p_{i}$ as $1 / n$; therefore, a cumulative probability relationship can be developed in which each storm event is assigned a segment of the total probability of 0.0 to 1.0 . If each event has an equal probability, then each event is assigned a segment $s_{j}$ such that $s_{j} \rightarrow X_{j}$ and has probabilities defined by

$$
\begin{gathered}
{\left[0<s_{1} \leq \frac{1}{n}\right]} \\
{\left[\frac{1}{n}<s_{2} \leq \frac{2}{n}\right]} \\
{\left[\frac{2}{n}<s_{3} \leq \frac{3}{n}\right]} \\
\cdot \\
. \\
{\left[\frac{n-1}{n}<s_{n} \leq 1\right]}
\end{gathered}
$$

A storm event is identified by random sampling from the total storm population. The procedure is equivalent to drawing and replacing random samples from the full storm event population.

The EST is not simply a resampling of historical events technique, but rather an approach intended to simulate the vector distribution contained in the training set population. The EST approach is to select a sample storm based on a random walk from the event $X_{i}$ with $x_{1}$ and $x_{2}$ response vectors to the nearest neighbor vectors. The walk is based on independent uniform random numbers with the range of $(-1,1)$ and has the effect of simulating responses that are not identical to the historical events but are similar to events that have historically occurred. 
Because simulated events correspond to a specific location, the second criterion to be satisfied is that the total number of storm events selected in the Tyears must be statistically representative of the number of historical events that have occurred at the area of study. For this study, 31 hurricane events were identified that passed within $200 \mathrm{mi}(370 \mathrm{~km})$ of the American Samoa islands during the 37-year period extending from 1958 to 1995 . Given the mean frequency of storm events for a particular region, a Poisson distribution is used to determine the average number of expected events in a given year. For example the Poisson distribution can be written as

$$
\operatorname{Pr}(s ; \lambda)=\frac{\lambda^{s} e^{-\lambda}}{s !}
$$

for $s=0,1,2,3, \ldots$ The probability $\operatorname{Pr}(s ; \lambda)$ defines the probability of having $s$ events per year where $\lambda$ is a measure of the historically based number of events per year. For this study, $\lambda$ was computed to be 0.84 (31/37).

Output from the EST program is $N$ repetitions of T-years of simulated storm event responses. It is from these responses that frequency-of-occurrence relationships are computed. The computational procedure followed is based on the generation of a probability distribution function corresponding to each of the $T$-year sequences of simulated data.

\section{Recurrence Relationships}

Estimates of frequency-of-occurrence begin with the calculation of a probability distribution function (pdf) for the response vector of interest. Let $X_{1}, X_{2}, X_{3}, \ldots, X_{n}$ be $n$ independent, identically distributed, random response variables with a cumulative pdf given by

$$
F_{x}(x)=\operatorname{Pr}[X \leq x]
$$

where $\operatorname{Pr}[X \leq x]$ represents the probability that the random variable $X$ is less than or equal to some value $x$, and $F_{x}(x)$ is the cumulative probability density function ranging from 0.0 to 1.0 . The problem is to estimate the value of $F_{x}$ without introducing some parametric relationship for probability. The following procedure is adopted because it makes use of the probability laws defined by the data and does not incorporate any prior assumptions concerning the probability relationship.

Assume a set of $n$ observations of data. The $n$ values of $x$ are first ranked in order of increasing size. In the following analysis, the parentheses surrounding

the subscript indicate that the data have been rank-ordered. The value $x_{(1)}$ is the smallest in the series and $x_{(n)}$ represents the largest value. Let $r$ denote the rank 
of the value $x_{(r)}$ such that rank $r=1$ is the smallest and rank $r=n$ is the largest.

An empirical estimate of $F_{X}\left(x_{(r)}\right)$, denoted by $\hat{F}_{X}\left(x_{(r)}\right)$, is given by Gumbel (1954) (see also Borgman and Scheffner (1991) and Scheffner and Borgman (1993)) as

$$
\hat{F}_{X}\left(x_{(r)}\right)=\frac{r}{(n+1)}
$$

for $\left\{x_{(r)}, r=1,2,3, \ldots, n\right\}$. This form of estimate allows for future values of $x$ to be less than the smallest observation $x_{(1)}$ with probability of $1 /(n+1)$, and to be larger than the largest values $x_{(n)}$. with probability $m /(n+1)$.

An example set of 10 years of observed elevations, the rank ordered set of observations, the rank, and the cumulative pdf are shown in Table 1. As can be seen in the table, this form of the cumulative distribution function allows for values of $x$ to be greater than the maximum or less than the minimum observed values in the historical database. A plot of the cumulative distribution function versus $x_{(r)}$ as computed by Equation 10 is shown in Figure 4 . In the implementation of the EST, tail functions (Borgman and Scheffner 1991) are applied to define the pdf for events larger than the largest or smaller than the smallest observed event so that there is no discontinuity in the pdf.

\section{Table 1}

Sample Distribution Function Calculation

\begin{tabular}{|l|l|l|l|l|}
\hline \hline Year & $\mathbf{X}_{1,2, \ldots, n}$ & $\mathbf{X}_{(r)}$ & Rank $\mathbf{r}$ & $\hat{\mathbf{F}}_{\boldsymbol{x}}\left(\mathbf{X}_{(r)}\right)$ \\
\hline \hline 1 & 3.2 & 10.5 & 10 & 0.91 \\
\hline 2 & 3.5 & 8.6 & 9 & 0.82 \\
\hline 3 & 8.0 & 8.0 & 8 & 0.73 \\
\hline 4 & 1.0 & 7.5 & 7 & 0.64 \\
\hline 5 & 10.5 & 5.9 & 6 & 0.55 \\
\hline 6 & 5.9 & 4.1 & 5 & 0.45 \\
\hline 7 & 8.6 & 3.5 & 4 & 0.36 \\
\hline 8 & 4.1 & 3.2 & 3 & 0.27 \\
\hline 9 & 2.3 & 2.3 & 2 & 0.18 \\
\hline 10 & 7.5 & 1.0 & 1 & 0.10 \\
\hline
\end{tabular}

The cumulative pdf as defined by Equation 10 and shown in Figure 4 is applied to develop stage-frequency relationships as follows. Consider that the cumulative probability for an $n$-year return period storm can be written as

$$
F(n)=1-\frac{1}{n}
$$


where $F(n)$ is the simulated cumulative probability of occurrence for an event with a return period of $n$ years. Frequency-of-occurrence relationships are obtained by linearly interpolating a stage from Equation 10 corresponding to the pdf associated with the return period calculated by Equation 11 .

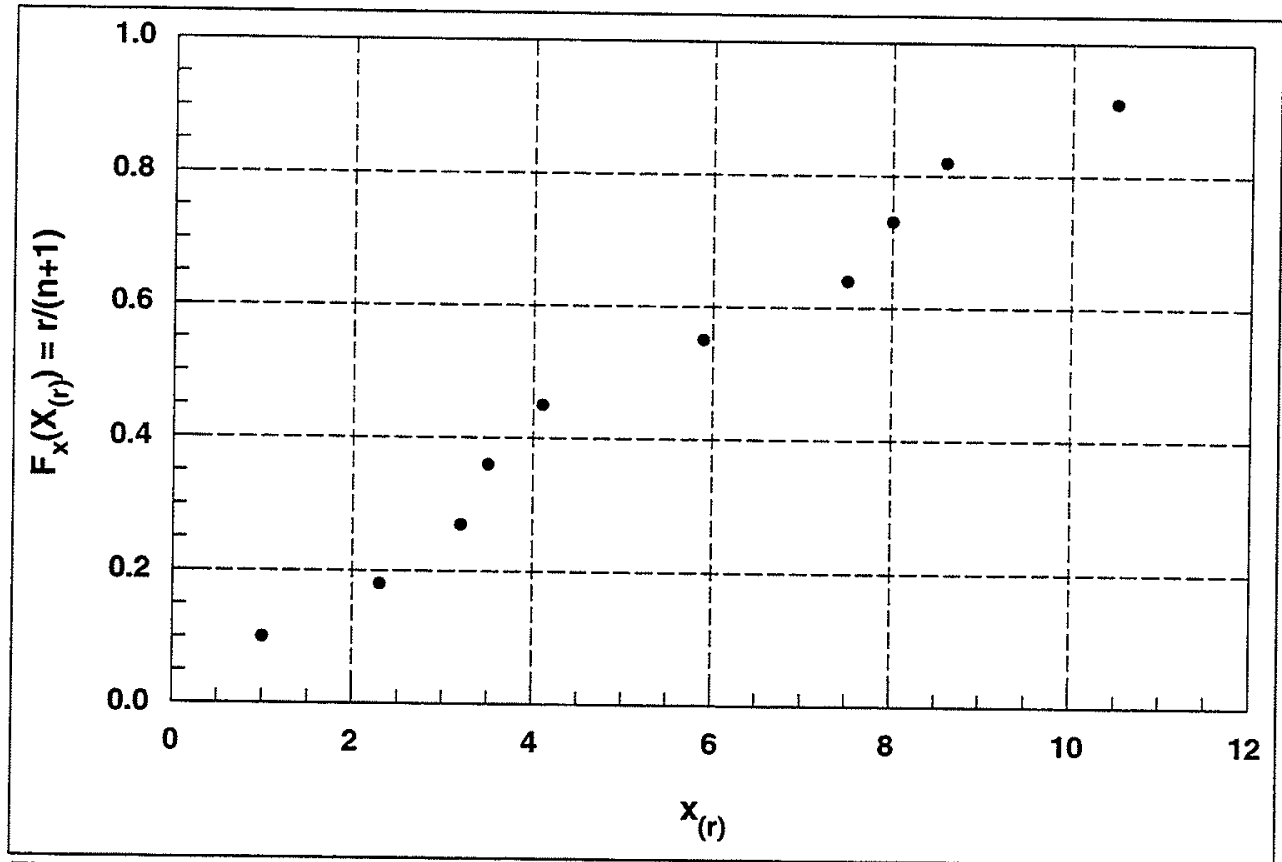

Figure 4. Example of cumulative probability distribution plot 


\section{Description of Numerical Models}

Calculation of hurricane stage-frequency relationships for the U.S. Territory of American Samoa requires application of four numerical models. The Planetary Boundary Layer (PBL) model simulates hurricane-induced wind and atmospheric pressure fields for those hurricanes that have impacted the study area. The wind-wave model WISWAVE applies the calculated wind fields to determine the deep-water wave parameters. The wave-transformation model WAVTRAN transforms the deep-water waves calculated by WISWAVE by a spectral spreading technique. These wave parameters are subsequently used to calculate wave setup, runup, and ponding level. The long-wave hydrodynamic model ADCIRC applies the calculated wind and pressure fields to obtain peak water levels for storm surge events. Descriptions of the wind and atmospheric pressure model, the wave and wave-transformation models, and the hydrodynamic model are presented below.

\section{Wind and Atmospheric Pressure Field Model}

The Planetary Boundary Layer (PBL) numerical model was selected for simulation of hurricane-generated wind and atmospheric pressure fields. The model applies the vertically averaged primitive equations of motion for predicting hurricane wind velocities. The model includes parameterization of the momentum, heat, and moisture fluxes together with surface drag and roughness formulations. Through hindcast applications, Cardone, Greenwood, and Greenwood (1992) found that the PBL model calculates accurate surface wind speeds and directions as compared to measurements collected in hurricanes over open water.

The PBL model requires a set of "snapshots" for input. The snapshots consist of meteorological storm parameters that define the storm at various stages in its development or at particular times during its life. These parameters include: latitude and longitude of the storm's eye; track direction and forward speed measured at the eye; radius to maximum winds; central and peripheral atmospheric pressures; and an estimate of the geostrophic wind speed and direction. Also, the direction and speed of steering currents can be provided for representing asymmetric hurricanes. 
For application to the American Samoa study site, the PBL model was upgraded to calculate storm wind and pressure fields for the Southern Hemisphere. Prior to the present study, the model had not been applied in the Southern Hemisphere and an upgrade was required for correct calculation of wind direction. Upon completion of the upgrade, the calculated wind fields were verified for directional accuracy.

Storm parameters were obtained from a database developed by the National Oceanic and Atmospheric Administration's (NOAA) National Hurricane Center (NHC). The present database contains hurricanes and tropical storms that occurred in the south Pacific Ocean from 1958 through 1995, a period of 37 years. Information contained in this database is provided at $0000,0600,1200$, and $1800 \mathrm{hr}$ Greenwich Mean Time (GMT), and includes: latitude and longitude of the storm, central pressure, and maximum wind speed. For some storms, data for maximum wind speed were not available. An empirical approximation was developed to estimate the maximum wind speed where these data were missing.

An empirical approximation for hurricane wind speed was developed through modification of published relationships between maximum wind speed and central pressure. The new approximation is given by

$$
W=\left(1.74+\beta \frac{P_{a}}{P_{c}}\right)\left(P_{c}-P_{a}\right)^{\alpha}
$$

where $W$ is the maximum wind speed, $P_{a}$ is the ambient pressure, $P_{c}$ is the central pressure, and $\alpha$ and $\beta$ are empirically-determined coefficients. The multiplier for the pressure deficit is variable and changes with the ratio of ambient to central pressure. The pressure ratio is effectively a scaling parameter for the multiplier and compounds the influence of decreasing central pressure on the wind speed estimate.

Tests of the approximation were performed for 6991 data points obtained from the HURDAT database for the western south Pacific Ocean. The best fit between the measured and approximated wind speed was found for $\alpha=0.671$ and $\beta=1.41$. The ambient pressure was taken to be $1013 \mathrm{mb}$. Figure 5 shows the measured and calculated wind speed. The solid line in Figure 5 plots measured = calculated wind speed. Maximum error occurs at low wind speed. For wind speeds above about $66 \mathrm{ft} / \mathrm{s}(20 \mathrm{~m} / \mathrm{s})$ the error is small, typically under $3 \mathrm{ft} / \mathrm{s}(1 \mathrm{~m} / \mathrm{s})$. For the test data set, the average absolute deviation of approximated wind speed from measured wind speed was $2.6 \mathrm{ft} / \mathrm{s}(0.8 \mathrm{~m} / \mathrm{s})$. The maximum overprediction was $9.2 \mathrm{ft} / \mathrm{s}(2.8 \mathrm{~m} / \mathrm{s})$ and corresponded to a measured wind speed of $26 \mathrm{ft} / \mathrm{s}(8 \mathrm{~m} / \mathrm{s})$. The maximum underprediction was $17.1 \mathrm{ft} / \mathrm{s}$ $(5.2 \mathrm{~m} / \mathrm{s})$ and corresponded to a measured wind speed of $39 \mathrm{ft} / \mathrm{s}(12 \mathrm{~m} / \mathrm{s})$.

Estimated wind speeds from published relationships (Kraft, 1961; Atkinson and Holliday, 1977) were compared to those calculated by the approximation given by Equation 12. The new approximation was found to give more accurate 
estimates of wind speed than the published approximations for measured wind speed greater than $65 \mathrm{ft} / \mathrm{s}(20 \mathrm{~m} / \mathrm{s})$. The ambient pressure applied in the Atkinson and Holliday (1977) approximation for the north Pacific was $1010 \mathrm{mb}$, but was modified for this analysis to be $1013 \mathrm{mb}$. Average absolute deviations were $10.2 \mathrm{ft} / \mathrm{s}(3.1 \mathrm{~m} / \mathrm{s})$ and $6.9 \mathrm{ft} / \mathrm{s}(2.1 \mathrm{~m} / \mathrm{s})$ for the Kraft (1961) and Atkinson and Holliday (1977) approximations, respectively.

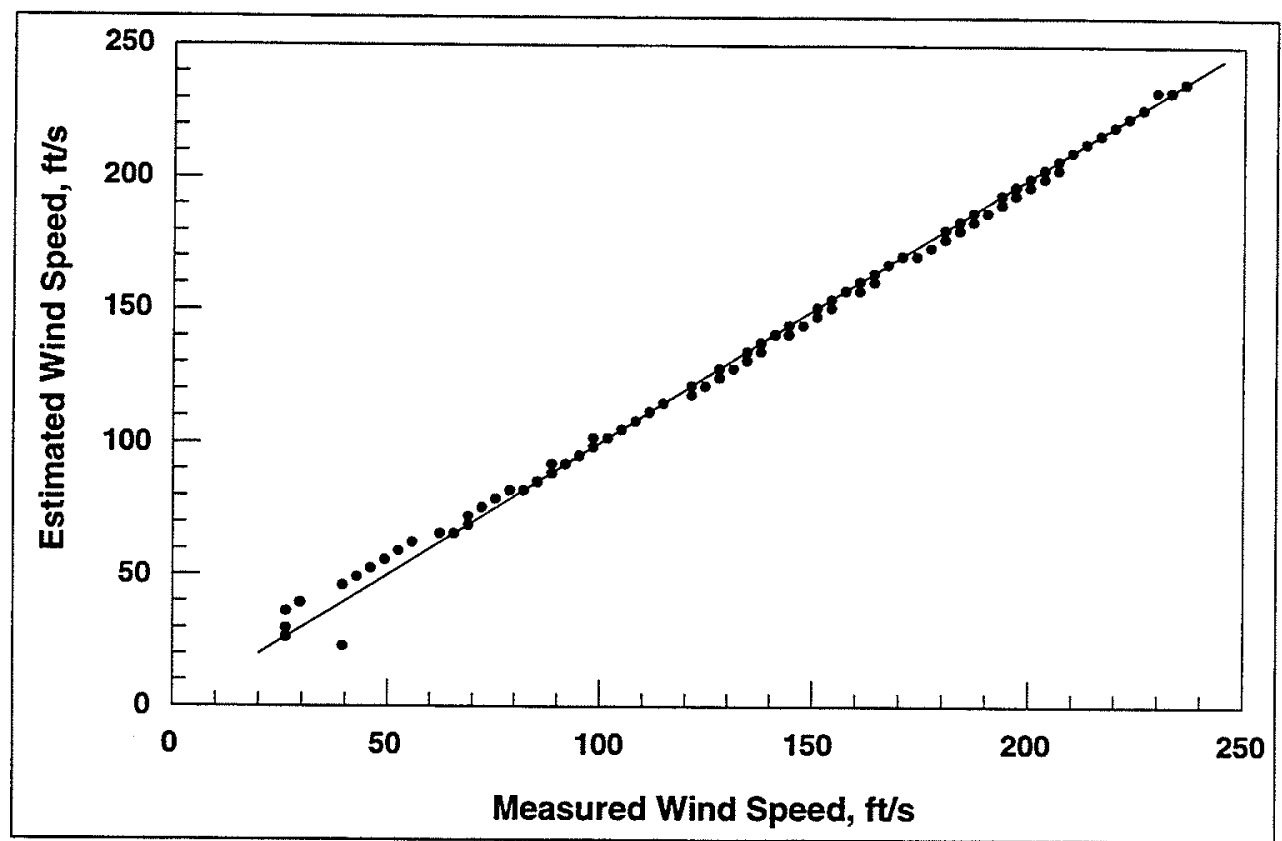

Figure 5. Measured vs. estimated wind speed

Radius to maximum winds ( $R M W$ ) is approximated by application of a function relating the maximum wind speed and the atmospheric pressure deficit (Jelesnianski and Taylor 1973). Track directions and forward speeds required by the PBL model are approximated by cubic spline interpolation at hourly intervals from the 6-hour coordinate positions provided in the database. Peripheral atmospheric pressures were assumed equal to $1013 \mathrm{mb}$, and geostrophic wind speeds were specified as 6 knots.

The spatial area covered by a hurricane at a given time is specified in the model to correspond to a set of nodes on a numerical grid. Wind velocities and atmospheric pressure values are computed at each node in the grid. Whereas some models employ a fixed grid system to simulate a hurricane (i.e., stationary grid with a moving storm), the PBL model simulates the hurricane as a stationary storm with a moving grid. A hurricane's forward motion is calculated as the vector sum of the forward and rotational velocity vector components. The numerical grid is moved under the storm at the calculated forward velocity at each time step.

The distribution of wind speed and radial change in wind speed varies spatially within a hurricane such that higher spatial resolution of the wind field is required in the central region of the storm, whereas coarser resolution is required on the outer areas. To provide spatially-graded resolution of the wind field, a 
nested gridding technique is applied consisting of five layers or subgrids. The grid nesting is applied such that all subgrids contain the same number of nodes, however, the spatial coverage and resolution differs and is successively graded. Each subgrid is composed of 21 by 21 nodes in the $x$ - and $y$-directions, respectively. The centers of all subgrids lie on node $(11,11)$, defined at the eye of the hurricane. For this study, the subgrid with the finest resolution had an incremental distance of $3.1 \mathrm{mi}(5 \mathrm{~km})$ between nodes and covered an area of $3861 \mathrm{sq} \mathrm{mi}(10,000 \mathrm{sq} \mathrm{km})$. Incremental distances for the remaining subgrids were $6.2,12.4,24.9$, and $49.7 \mathrm{mi}(10,20,40$, and $80 \mathrm{~km})$ and their areas of coverage were $15,444,61,776,247,104$, and $988,428 \mathrm{sq} \mathrm{mi}(40,000,160,000$, 640,000 , and 2,560,000 sq km), respectively.

For each snapshot, the equations of motion are first solved for the subgrid covering the greatest area. Computed wind velocities are then applied as boundary conditions on the second-largest grid, and the equations are solved again. This procedure is followed for the remaining grids where wind fields are computed on successively smaller grids. Thus, the nested grid technique provides wind field information over a wide spatial area while sufficient grid resolution is provided to accurately compute winds in the vicinity of the hurricane eye.

After all snapshots have been processed, hourly wind and atmospheric pressure fields are interpolated using a nonlinear blending algorithm which produces a smooth transition from one snapshot to the next. Hourly wind and pressure fields are then interpolated from the PBL grid onto the hydrodynamic grid and subsequently stored for use by the hydrodynamic model.

\section{Wave and Wave Transformation Models}

Deep-water wave fields were calculated by application of the Wave Information Studies Wave (WISWAVE) model (Resio and Perrie 1989). This model is a second-generation discrete directional spectral wave model in which the spectral wave computations are based on the integration of energy over the discrete frequency spectrum. Model output includes time series of significant wave height, peak (dominant) or mean wave period, and mean wave direction. Peak or dominant wave periods are not integral quantities in that they are not derived by summation over the spectrum. Peak period is defined as the period associated with the mid-band frequency, or that frequency band containing the largest spectral energy density. Mean wave period is an energy-weighted quantity integrated over all user-specified frequencies of interest. Model input includes a rectilinear computational grid, with water depths specified at each node, and wind speed and direction over the grid domain.

Application of the wave model required sufficient resolution of the grid such that calculation points could be distributed around and near to the islands so that representative wave conditions would be captured for all sides of the islands. To meet this requirement, a grid with constant spacing of $0.083 \mathrm{deg}$ was developed. Grid bathymetry was taken from that specified in the storm surge grid (described 
below) and interpolated onto the wave grid. The method of kriging was applied for the interpolation. The islands of Western Samoa and American Samoa were specified as land in the grid for accurate calculation of wave sheltering and refraction. Details of the grid are given in Table 2.

\begin{tabular}{|l|l||}
\hline $\begin{array}{l}\text { Table } 2 \\
\text { WISWAVE Grid Parameters }\end{array}$ \\
\hline \hline Parameter & Value \\
\hline \hline Longitude limits & $-173.987 \mathrm{~W},-165.961 \mathrm{~W}$ \\
\hline Latitude limits & $18.254 \mathrm{~S}, 10.238 \mathrm{~S}$ \\
\hline Cell side length & $0.083 \mathrm{deg}$ \\
\hline Total number of nodes & 9604 \\
\hline Number of nodes in North-South direction & 98 \\
\hline Number of nodes in East-West direction & 98 \\
\hline
\end{tabular}

Wind forcing for the wave model was calculated by application of the PBL model. Data for selected storms were taken from the HURDAT database for the south Pacific Ocean. Wind speed and direction were calculated for each point on the wave grid at 1 -hr intervals.

Deep-water wave parameters calculated by the wave model were stored at 32 stations surrounding the American Samoa Islands for each of the 31 storms in the training set (described in Chapter 5). A list of these stations is given in Table 3. The duration of the wave simulations corresponded to the time coverage of each storm in the HURDAT database. Wave parameters were stored at 1-hr intervals. These deep-water waves were transformed by application of the WAVTRAN model, then applied to calculations of ponding level, setup, and runup.

The WAVTRAN model calculates the spectral transformation of waves during propagation from one depth to another, taking into account shoreline orientation and wave sheltering (Jensen 1983, Gravens et al. 1991). The model assumes that sea and swell waves have an energy spectrum that follows the TMA spectral form (Bouws et al. 1985). Directional spread is calculated by $4^{\text {th }}$ and $8^{\text {th }}$ power cosine functions. The wave transformation calculation is dependent on the shoreline orientation because the bottom contours are assumed parallel to the shoreline. If wave sheltering is included, waves coming from directions specified by a sheltered angle band are deleted from the spectrum. Details of the model application for this study are given in Chapter 5. 


\begin{tabular}{|c|c|c|}
\hline \multicolumn{3}{|c|}{$\begin{array}{l}\text { Table } 3 \\
\text { Deep-Water Wave Stations }\end{array}$} \\
\hline Station Number & Latitude, deg $\mathrm{S}$ & Longitude, deg W \\
\hline 1 & 14.45 & 170.93 \\
\hline 2 & 14.37 & 170.93 \\
\hline 3 & 14.29 & 170.93 \\
\hline 4 & 14.20 & 170.93 \\
\hline 5 & 14.20 & 170.84 \\
\hline 6 & 14.20 & 170.76 \\
\hline 7 & 14.20 & 170.68 \\
\hline 8 & 14.20 & 170.60 \\
\hline 9 & 14.20 & 170.51 \\
\hline 10 & 14.20 & 170.43 \\
\hline 11 & 14.29 & 170.43 \\
\hline 12 & 14.37 & 170.43 \\
\hline 13 & 14.37 & 170.51 \\
\hline 14 & 14.37 & 170.60 \\
\hline 15 & 14.37 & 170.68 \\
\hline 16 & 14.45 & 170.68 \\
\hline 17 & 14.45 & 170.76 \\
\hline 18 & 14.45 & 170.84 \\
\hline 19 & 14.29 & 169.68 \\
\hline 20 & 14.20 & 169.68 \\
\hline 21 & 14.12 & 169.68 \\
\hline 22 & 14.12 & 169.60 \\
\hline 23 & 14.12 & 169.52 \\
\hline 24 & 14.12 & 169.44 \\
\hline 25 & 14.20 & 169.44 \\
\hline 26 & 14.29 & 169.44 \\
\hline 27 & 14.20 & 169.35 \\
\hline 28 & 14.29 & 169.35 \\
\hline 29 & 14.29 & 169.52 \\
\hline 30 & 14.29 & 169.60 \\
\hline 31 & 14.20 & 169.77 \\
\hline 32 & 14.12 & 169.77 \\
\hline
\end{tabular}

\section{Storm Surge Model}

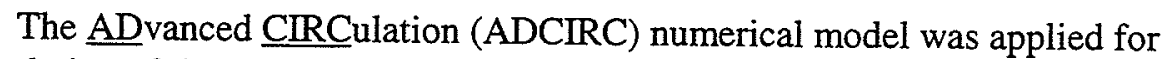
simulation of the long-wave hydrodynamic processes in the study area. The model calculates a two-dimensional, depth-integrated finite-element solution of the Generalized Wave-Continuity Equation (GWCE). The fundamental components of the GWCE are the depth-integrated continuity and Navier-Stokes 
equations for conservation of mass and momentum. The assumption of incompressibility and the Boussinesq and hydrostatic pressure approximations were applied. The primitive, non-conservative form of the governing equations, given in spherical coordinates, as applied in the model are (Flather, 1988; Kolar et al. 1993)

$$
\begin{gathered}
\frac{\partial \zeta}{\partial t}+\frac{1}{R \cos (\phi)}\left[\frac{\partial U D}{\partial \varphi}+\frac{\partial(U V \cos (\phi))}{\partial \phi}\right]=0 \\
\frac{\partial U}{\partial t}+\frac{1}{R \cos (\phi)} U \frac{\partial U}{\partial \varphi}+\frac{1}{R} V \frac{\partial U}{\partial \phi}-\left[\frac{\tan (\phi)}{R} U+f\right] V \\
=-\frac{1}{R \cos (\phi)} \frac{\partial}{\partial \varphi}\left[\frac{P_{S}}{\rho_{0}}+g(\zeta-\xi)\right]+\frac{\tau_{S \varphi}}{\rho_{0} D}-\tau_{*} U \\
\frac{\partial V}{\partial t}+\frac{1}{R \cos (\phi)} U \frac{\partial V}{\partial \varphi}+\frac{1}{R} V \frac{\partial V}{\partial \phi}-\left[\frac{\tan (\phi)}{R} U+f\right] U \\
\left.=-\frac{1}{R} \frac{\partial}{\partial \varphi\left[\frac{P_{S}}{\rho_{0}}\right.}+g(\zeta-\xi)\right]+\frac{\tau_{S \phi}}{\rho_{0} D}-\tau_{*} V
\end{gathered}
$$

where $t$ is time, $\varphi$ is degrees longitude (east of Greenwich is taken positive), $\phi$ is degrees latitude (north of the equator is taken positive), $\zeta$ is the free-surface elevation relative to the geoid, $U$ is the depth-averaged velocity component parallel to the East-West axis, $V$ is the depth-averaged velocity component parallel to the North-South axis, $R$ is the radius of the Earth, $D=\zeta+h$ is the total water-column depth, $h$ is the ambient depth relative to the geoid, $f=2 \Omega \cos (\phi)$ is the Coriolis parameter, $\Omega$ is the angular speed of the Earth's rotation, $P_{S}$ is the atmospheric pressure at the free surface, $g$ is the acceleration due to gravity, $\xi$ is the effective Newtonian equilibrium tide potential, $\rho_{0}$ is the reference density of water, $\tau_{S \varphi}$ and $\tau_{S \phi}$ are the applied free-surface stresses, and $\tau_{*}$ is the bottom stress given by $C_{f}\left(U^{2}+V^{2}\right)^{1 / 2} / D$ where $C_{f}$ is the bottomfriction coefficient.

The time-differentiated form of the conservation of mass equation is combined with a space-differentiated form of the conservation of momentum equation to develop the GWCE (Westerink et al. 1992) given by 


$$
\begin{aligned}
& \frac{\partial^{2} \zeta}{\partial t^{2}}+\tau_{0} \frac{\partial \zeta}{\partial t}-\frac{1}{R \cos (\phi)} \frac{\partial}{\partial \varphi}\left[\frac{1}{R \cos (\phi)}\left(\frac{\partial(D U U)}{\partial \varphi}+\frac{\partial(D U V \cos (\phi))}{\partial \phi}\right)-U V D \frac{\tan (\phi)}{R}\right] \\
& {\left[-2 \omega \sin (\phi) D V+\frac{D}{R \cos (\phi)} \frac{\partial}{\partial \varphi}\left(g(\zeta-\alpha \xi)+\frac{P_{S}}{\rho_{0}}\right)+\tau_{*} D U-\tau_{0} D U-\frac{\tau_{S \varphi}}{\rho_{0}}\right]} \\
& -\frac{1}{R} \frac{\partial}{\partial \phi}\left[\frac{1}{R \cos (\phi)}\left(\frac{\partial D V V}{\partial \varphi}+\frac{\partial D V V \cos (\phi)}{\partial \phi}\right)+U U H \frac{\tan (\phi)}{R}+2 \omega \sin (\phi) D U\right] \\
& -\frac{1}{R} \frac{\partial}{\partial \phi}\left[\frac{D}{R} \frac{\partial}{\partial \phi}\left(g(\zeta-\alpha \xi)+\frac{P_{S}}{\rho_{0}}\right)+\left(\tau_{*}-\tau_{0}\right) D V-\frac{\tau_{S \phi}}{\rho_{0}}\right] \\
& -\frac{\partial}{\partial t}\left[\frac{V D}{R} \tan (\phi)\right]-\tau_{0}\left[\frac{V D}{R} \tan (\phi)\right]=0
\end{aligned}
$$

The ADCIRC model solves the GWCE (Equation (16)) in conjunction with the primitive momentum equations given by Equations (14) and (15).

The GWCE-based solution scheme eliminates several problems associated with finite-element models that solve the primitive forms of the continuity and momentum equations (i.e. Navier-Stokes equations), including spurious modes of oscillation and artificial damping of the tidal signal. Forcing functions include time-varying water-surface elevation, wind stress, atmospheric pressure, and the Coriolis effect.

The computational grid developed for this study is a large-domain circular grid with a radius of $4 \mathrm{deg}(276 \mathrm{mi})$ and center at $170 \mathrm{deg} W$ longitude and $14.25 \mathrm{deg} S$ latitude. The American Samoa islands are located in the central region of the grid. The large scale of the grid has two main advantages. First, the tidal forcing boundaries are far from the region of interest such that island shorelines are free from boundary effects. Second, because hurricanes are largescale atmospheric phenomena, a large-domain grid is preferred to maximize the interaction of the horizontal storm area with the computational grid, as well as the storm track.

The grid developed for this study is shown in Figure 6. Grid resolution is coarser in the open regions with increasing resolution toward the shore. Grid parameters and range of scale of element sizes contained in the grid are given in Table 4. The two islands of Western Samoa, Savaii and Upolu, are included in the grid. The grid around the islands of Western Samoa was not specified to be as detailed as the region surrounding the islands of American Samoa. Reefs, shallow areas, and embayments are finely resolved so that the hydrodynamics can be accurately calculated in these regions. Details of the grid for Tutuila and Aunuu Islands are shown in Figure 7, and Figure 8 shows detail of Pago Pago Harbor. Detail of the grid for Ofu, Olosega, and Tau Islands are shown in Figure 9. Because of the fine grid resolution in reef areas coupled with the extreme hydrodynamic conditions (strong currents and rapid change in water level) associated with the storms, a time step of $5 \mathrm{sec}$ was required for model runs. 


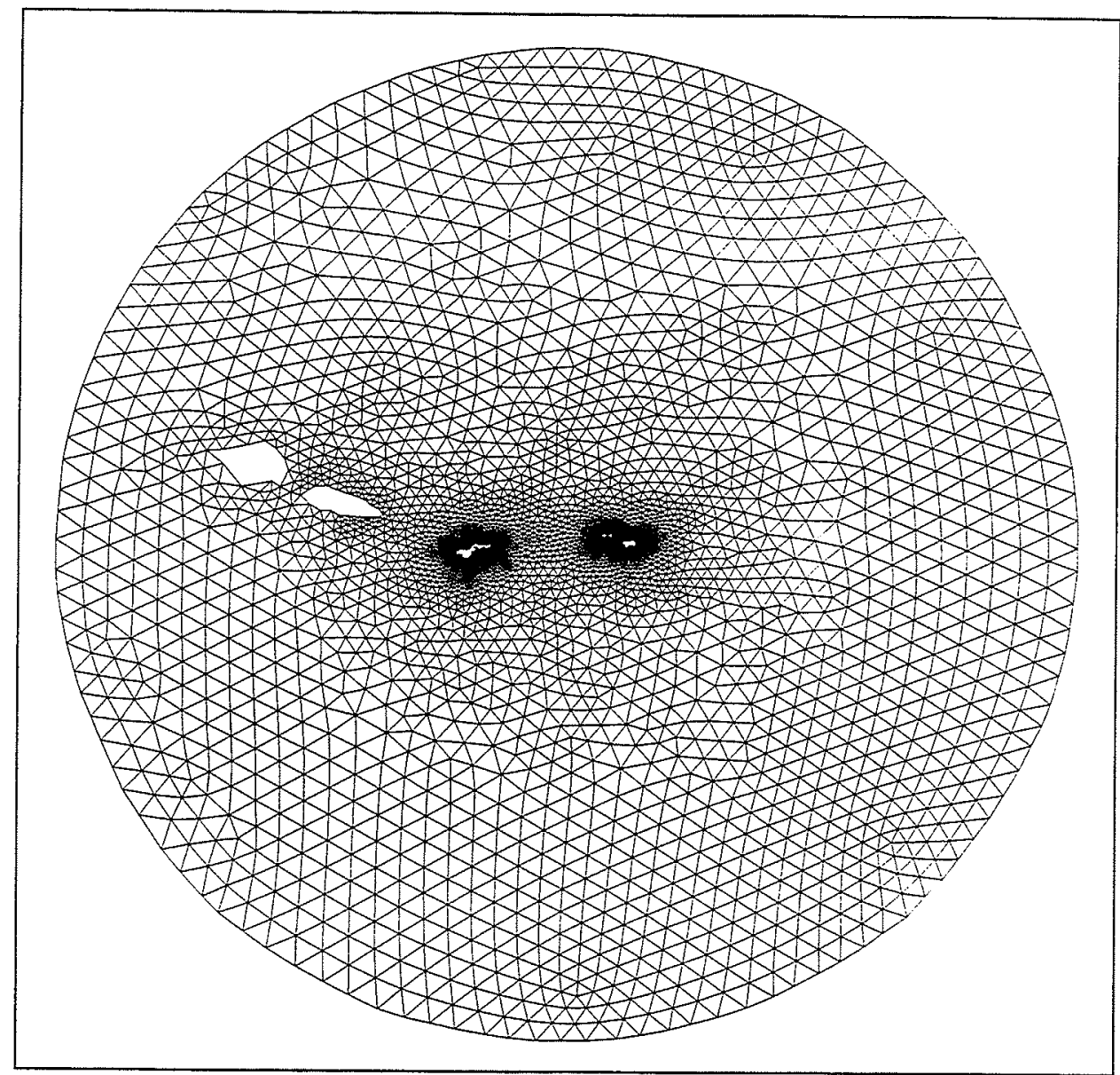

Figure 6. Computational grid for American Samoa

\begin{tabular}{|c|c|}
\hline $\begin{array}{l}\text { Table } 4 \\
\text { Storm Surge Grid Parameters }\end{array}$ & \\
\hline Parameter & Value \\
\hline Maximum element area & $81,628,823,171 \mathrm{tt}^{2}\left(7,583,565,680 \mathrm{~m}^{2}\right)$ \\
\hline Minimum element area & $14973 \mathrm{ft}^{2}\left(1391 \mathrm{~m}^{2}\right)$ \\
\hline Ratio of maximum to minimum element areas & $5,451,880$ \\
\hline Number of elements & 41,667 \\
\hline Number of nodes & 22,072 \\
\hline Center longitude and latitude & $170 \mathrm{~W}, 14.25 \mathrm{~S}$ \\
\hline Circular grid diameter & $4 \mathrm{deg}$ \\
\hline
\end{tabular}




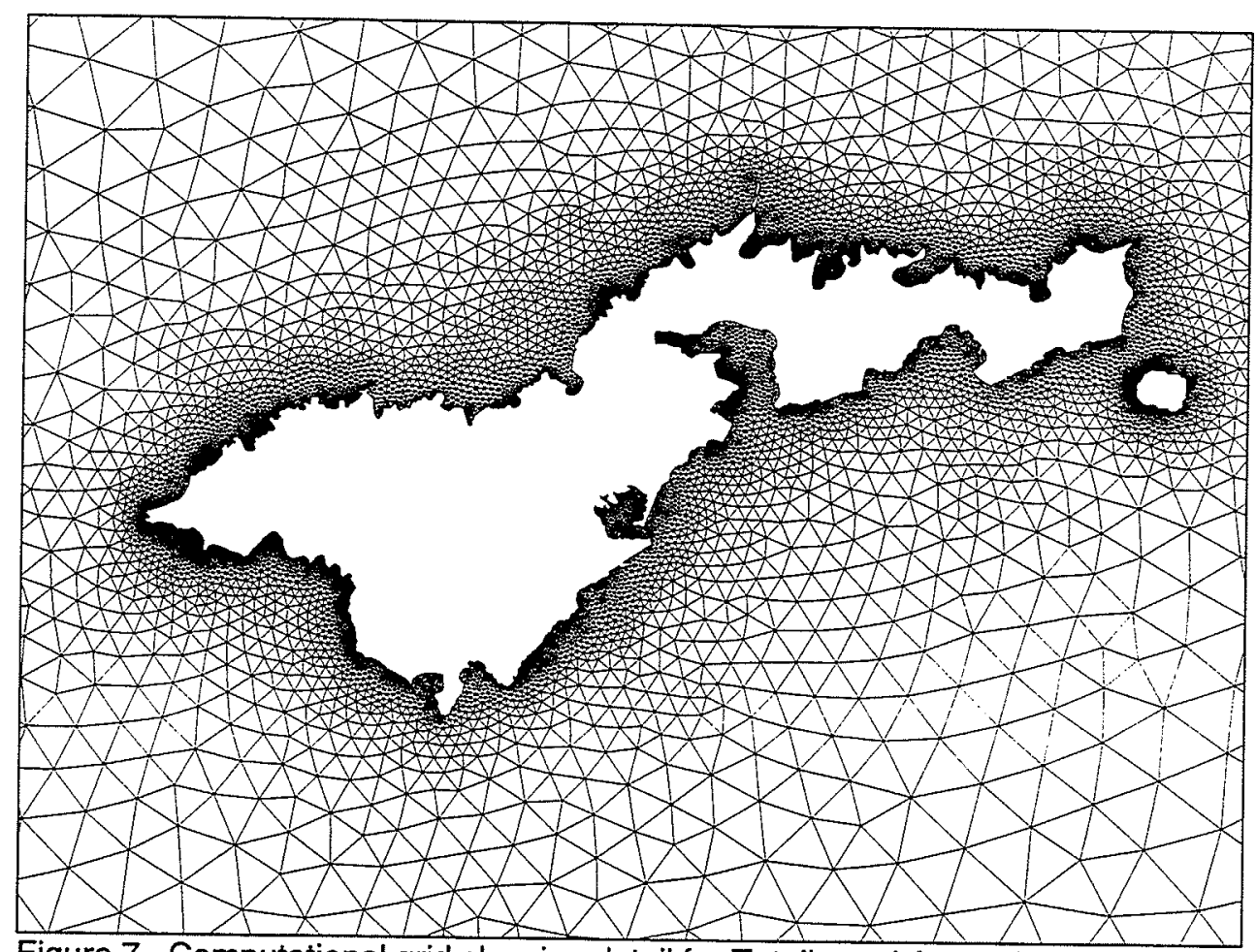

Figure 7. Computational grid showing detail for Tutuila and Aunuu Islands

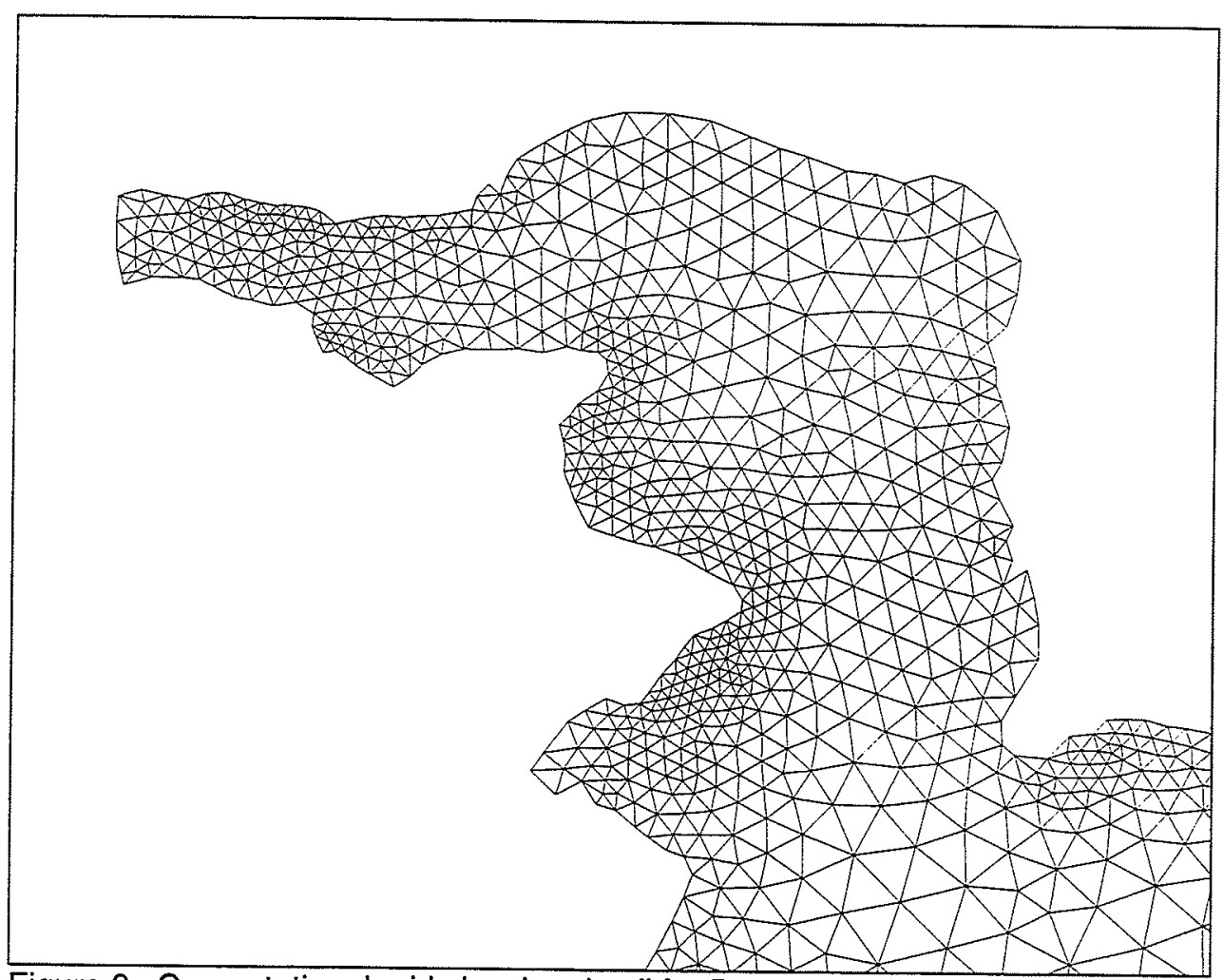

Figure 8. Computational grid showing detail for Pago Pago Harbor 


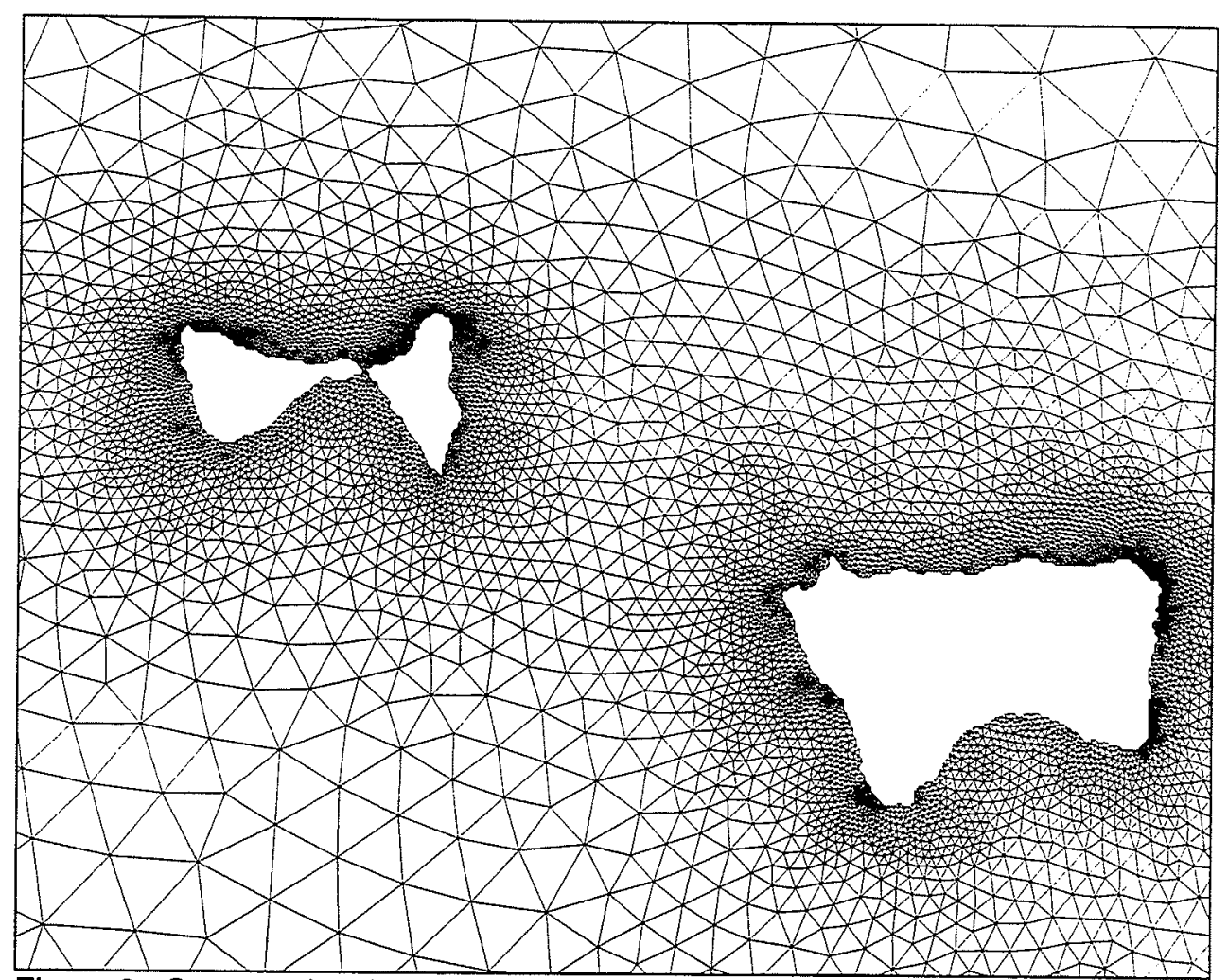

Figure 9. Computational grid showing detail for Ofu, Olosega, and Tau Islands

Several data sources were accessed for development of the computational grid. Initially, shoreline data were obtained from the World Vector Shoreline database and applied as the coastal boundary for the grid. Later, it was determined that the shoreline positions were displaced from the positions given in NOAA Chart \#83484 (Samoa Islands). The island boundaries were transposed and corrected according to the shoreline digitized from NOAA Chart \#83484. Deep-water bottom topography data were obtained from the National Center for Atmospheric Research ETOPO5 database. Depths for shallow regions surrounding the islands of interest for the study were digitized from NOAA Chart \#83484, where data were available. Grid depths are referenced to Mean Sea Level (MSL).

Tidal elevations specified at the open water boundary were calculated from tidal amplitudes and phases contained in the LeProvost World Tidal Constituent Database, which provides constituent data at 1-deg increments in latitude and longitude. A bilinear interpolation algorithm was applied to calculate tidal amplitudes and phases at 118 open boundary nodes. The four tidal constituents applied at the open boundaries were: $\mathrm{M}_{2}, \mathrm{~S}_{2}, \mathrm{~N}_{2}$, and $\mathrm{O}_{1}$. 


\section{Implementation of Storm Surge Model}

The process required for application of a long-wave hydrodynamic numerical model at a particular site includes grid generation, model calibration, model validation, and production runs. Accuracy of model results is greatly influenced by the accuracy of boundary and forcing conditions, representation of the geometry of the study area (i.e. bottom topography and land/water interface), and to a lesser degree, the values of certain "calibration" parameters. Model calibration involves adjustment of the calibration parameters, such as the bottom friction coefficient, to maximize agreement between model results and measurements.

Upon completion of calibration, the model is subject to a validation that consists of applying the model over a different segment of time from that of the calibration and where no changes have been made to the calibration parameters. The model is validated if results agree with measurements within an acceptable degree of error. The validation procedure provides confidence that the model can accurately simulate the hydrodynamic processes in the study area.

The strategy for calibrating and validating the storm surge model requires that the model accurately simulate both tidal propagation and storm surge in the study area. The model was first tested for simulation of tidal motion, then tested with the hurricane wind and pressure fields. Procedures applied in conducting model testing and the results of these tests are presented in the following sections.

\section{Calibration of the Storm Surge Model}

The model was calibrated for tidal propagation and subsequently verified for both tides and storms. Water-level measurements were available from one gauge within the study area located in the upper reach of Pago Pago Harbor, Tutuila Island. The position of the gauge is $14.2783 \mathrm{~S}, 170.6817 \mathrm{~W}$. Because measurements were only available at one location, the model could not be tested for accuracy at other locations in the study area. However, because the inner regions of bays and harbors are relatively difficult to calibrate, in comparison to deeper coastal and open ocean regions, it is expected that water-level calculations in other regions of the model domain are accurate if calculated 
water level in the harbor is accurate. Stations where accuracy may be compromised are located in Pala Lagoon, Tutuila Island (see Table A1 for positions of stations TU49 and TU50). Bathymetric data were not available for this shallow lagoon or Avatele Passage, the entrance to the lagoon. The exchange between Pala Lagoon and the ocean may be severely restricted because of the shallow depth of Avatele Passage, which is a shallow reef area according to NOAA Chart \#83484.

Calibration of the storm-surge model was conducted by driving the model with four tidal constituents and comparing the results of a harmonic analysis of the water level at the Pago Pago Harbor gauge to that calculated by NOS. Table 5 gives the tidal constituents at Pago Pago Harbor as determined by NOS and calculated by ADCRRC. Originally, the $K_{1}$ and $K_{2}$ tidal constituents were included in the calibration but were subsequently removed because their amplitude and phase calculated by the model were incorrect. It is thought that the source of error for these two constituents was an error in the tidal boundary condition specified by the tidal-constituent database. Error in tidal propagation would have been present in other constituents, which did not occur. The simulated water level constituents at the gauge location compare favorably to those calculated from measurements. The maximum error in amplitude is 0.38 in $(0.96 \mathrm{~cm})$ for the $\mathrm{M}_{2}$ and $\mathrm{N}_{2}$ constituents and the maximum error in phase is $7.4 \mathrm{deg}$ for the $\mathrm{S}_{2}$ constituent.

\begin{tabular}{|c|c|c|c|c|c|c|}
\hline \multicolumn{7}{|c|}{$\begin{array}{l}\text { Table } 5 \\
\text { Tidal Constituents in Pago Pago Harbor }\end{array}$} \\
\hline Constituent & $\begin{array}{l}\text { NOS } \\
\text { Amplitude } \\
\text { in (cm) }\end{array}$ & $\begin{array}{l}\text { Nos } \\
\text { Phase } \\
\text { Deg } \\
\end{array}$ & $\begin{array}{l}\text { ADCIRC } \\
\text { Amplitude } \\
\text { in (cm) }\end{array}$ & $\begin{array}{l}\text { ADCIRC } \\
\text { Phase } \\
\text { deg }\end{array}$ & $\begin{array}{l}\text { Amplitude } \\
\text { Error }^{1} \\
\text { in }(\mathrm{cm}) \\
\end{array}$ & $\begin{array}{l}\text { Phase } \\
\text { Error }^{2} \\
\text { Deg }\end{array}$ \\
\hline $\mathrm{M}_{2}$ & $14.80(37.58)$ & 180.0 & $14.42(36.62)$ & 178.1 & $-0.38(-0.96)$ & -1.9 \\
\hline$\overline{S_{2}}$ & $2.43(6.17)$ & 165.5 & $2.10(5.33)$ & 158.1 & $-0.33(-0.84)$ & -7.4 \\
\hline$\overline{N_{2}}$ & $4.22(10.73)$ & 161.6 & $3.85(9.77)$ & 162.4 & $-0.38(-0.96)$ & 0.8 \\
\hline$\overline{O_{1}}$ & $1.14(2.90)$ & 68.3 & $1.08(2.73)$ & 65.6 & $-0.07(-0.17)$ & -2.7 \\
\hline
\end{tabular}

Water level calculated by the model for the period Jan. 4, 1996 through Jan. 29, 1996 was compared to water level calculated from tidal constituents (Figure 10) for the Pago Pago Harbor gauge location. Twenty-three constituents were included in the calculation of water level from tidal constituents. Figure 10 shows generally good agreement between the model results and tidal-constituent calculated water level. Error in the modeled water level is probably due mostly to the $\mathrm{K}_{1}$ component missing from the model tidal forcing. The comparisons for Jan. 1996 validate the model for tidal conditions.

\section{Validation of the Storm Surge Model}

The storm surge model was validated by comparison of measured and calculated time-series water levels at the Pago Pago Harbor gauge for the period when Hurricane Val passed through the study area. Comparison of measured 
and calculated water level during the passage Hurricane Val is shown in Figure 11. The calculated water level shown does not contain wave setup, which may explain the underestimation of surge shown in the figure.

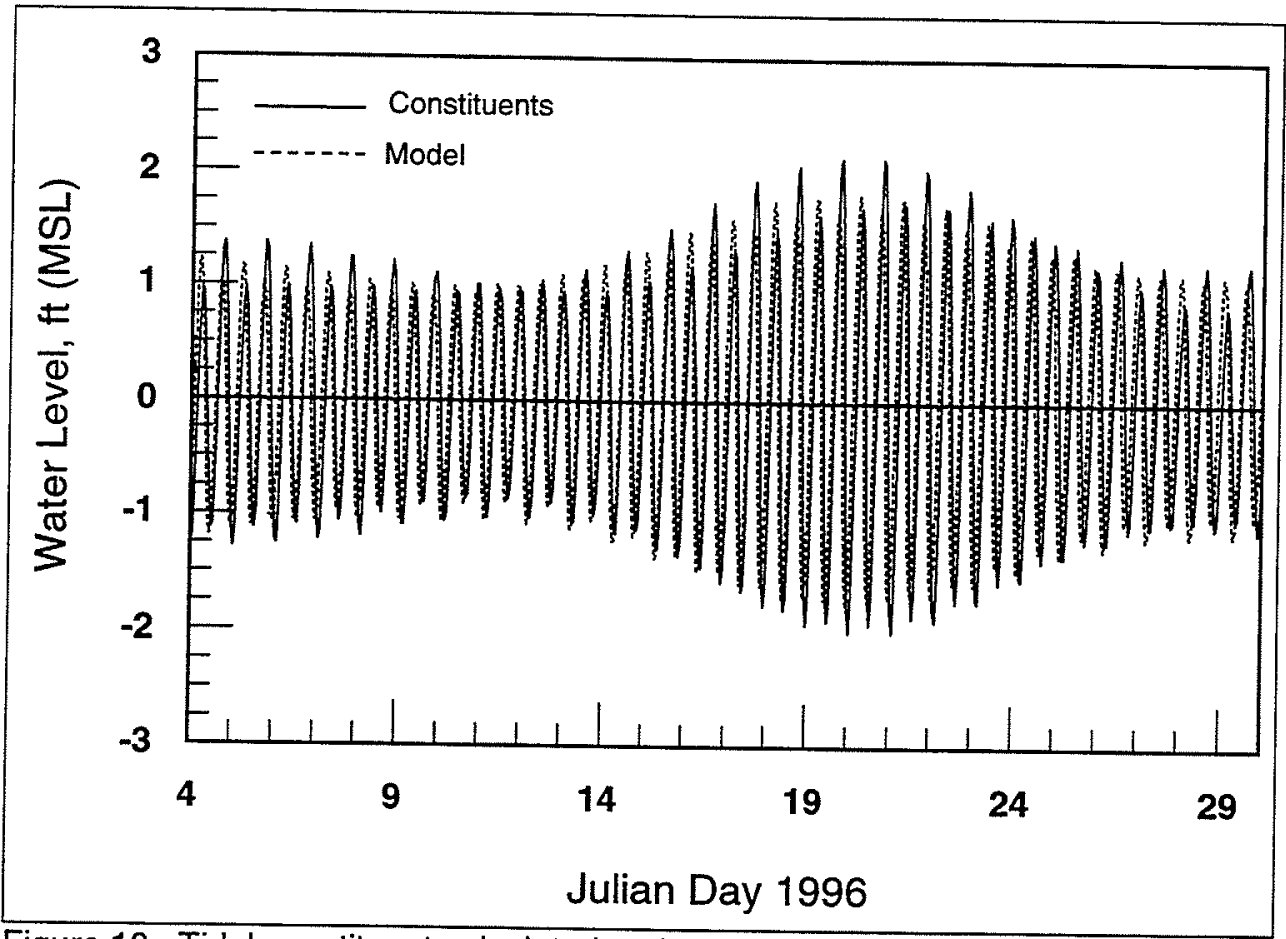

Figure 10. Tidal constituent calculated and modeled water level at the Pago Pago Harbor gauge for Jan. 4, 1996 through Jan. 29, 1996

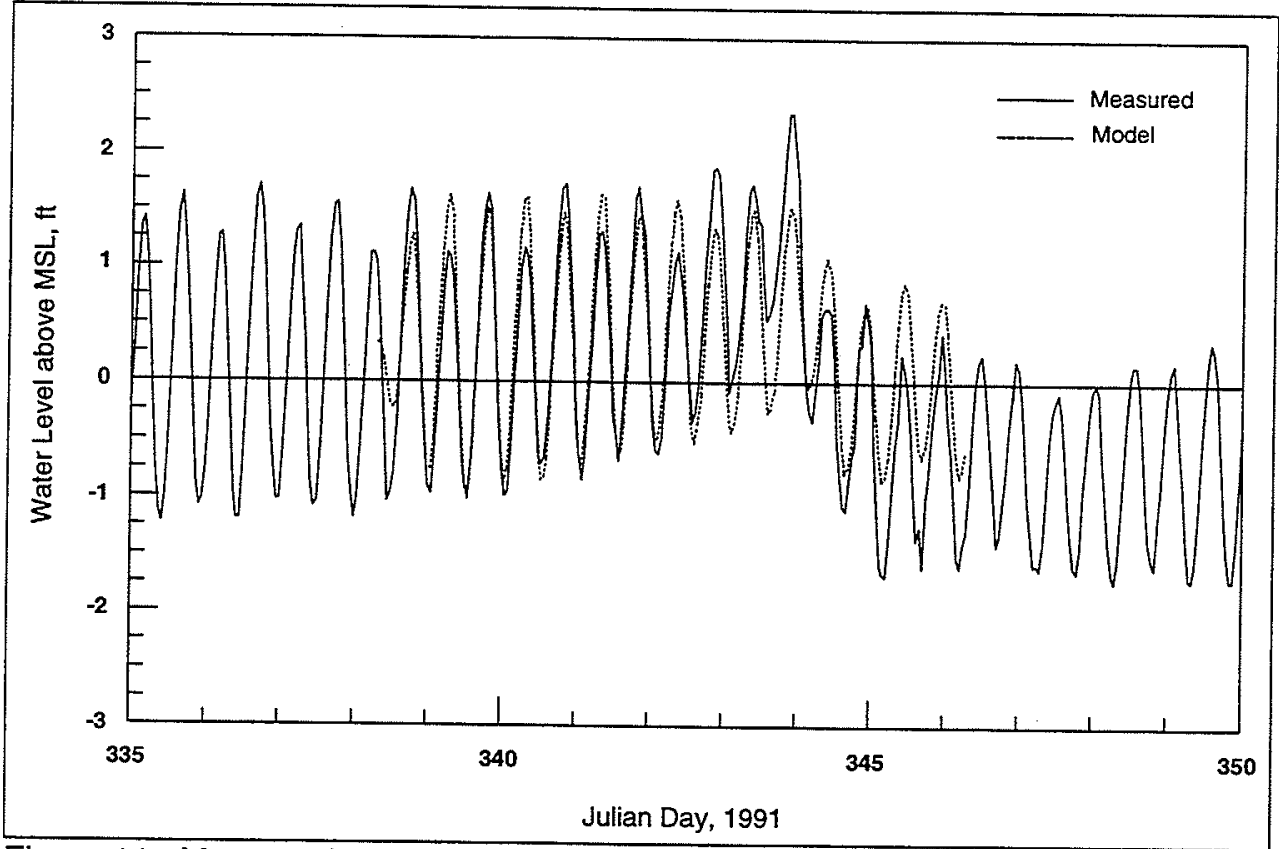

Figure 11. Measured and modeled water level in Pago Pago Harbor for Hurricane Val 


\section{Development of Stage- Frequency Relationships}

Stage-frequency relationships were developed for the Territory of American Samoa in four tasks. First, the training set of storms was developed from a storm database for the southern Pacific Ocean, and the PBL model was applied to calculate the wind fields associated with each storm in the training set. Second, the storm-surge model was applied with wind and atmospheric pressure forcing from the PBL model as time-dependent input. Time-series of storm-surge elevations associated with each storm were calculated for specified stations. Third, time series of wave parameters were calculated by application of the wave and wave-transformation models. Time series of ponding level, setup, and runup were calculated for each profile location in the study site. Fourth, the EST was applied to compute stage-frequency relationships based on the hurricane event parameters and calculated storm surge elevations.

\section{Selection of Hurricanes}

The HURDAT database for the south Pacific Ocean contains hurricane and tropical storm information covering a large area including Australia and New Zealand. A subset of storms was selected from the database to comprise the training set for American Samoa. An initial criterion for inclusion in the training set was that the storm track had to pass within a 200 mi radius of any station given in a station list. The station list consisted of 88 stations near the island coasts. Of the 622 storms contained in the HURDAT database for the south Pacific Ocean for 1958 through 1995, 31 storms met the 200 mi radius criteria. These storms were further examined to determine whether they impacted the coasts of the study site. All storms in the training set were found to produce wave-induced setup and runup on the coast, so no storms were eliminated from the training set. The set of historical storms included in the training set is given in Table 6 and storm tracks are provided in Appendix E.

\section{Storm Surge/Tidal Elevation Relationship}

Storm-surge elevations are dependent on water depth as well as intensity and angle of approach of the storm. The most accurate method for calculation of 


\begin{tabular}{|c|c|c|c|c|c|}
\hline \multicolumn{6}{|c|}{$\begin{array}{l}\text { Table } 6 \\
\text { Historical Storms Included in the Training Set }\end{array}$} \\
\hline \multicolumn{2}{|l|}{ Hurricane } & \multicolumn{2}{|c|}{ Starting Time } & \multicolumn{2}{|c|}{ Ending Time } \\
\hline Number & Name & Date & Time, GMT & Date & Time, GMT \\
\hline 18 & & $02 / 10 / 59$ & 0000 & $02 / 15 / 59$ & 0000 \\
\hline 20 & & $02 / 13 / 59$ & 0000 & $02 / 16 / 59$ & 0000 \\
\hline 21 & & $02 / 23 / 59$ & 0000 & $03 / 02 / 59$ & 0000 \\
\hline 28 & & $01 / 16 / 60$ & 0000 & $01 / 20 / 60$ & 0000 \\
\hline 33 & & $03 / 17 / 60$ & 0000 & $03 / 23 / 60$ & 0000 \\
\hline 49 & & $03 / 12 / 61$ & 0000 & $03 / 20 / 61$ & 0000 \\
\hline 60 & & $02 / 17 / 62$ & 0000 & $02 / 19 / 62$ & 0000 \\
\hline 64 & & $12 / 21 / 62$ & 0000 & $12 / 21 / 62$ & 0000 \\
\hline 82 & & $03 / 07 / 63$ & 1200 & $03 / 07 / 63$ & 0000 \\
\hline 96 & & $01 / 20 / 64$ & 0000 & $01 / 28 / 64$ & 0000 \\
\hline 97 & & $01 / 24 / 64$ & 0000 & $01 / 26 / 64$ & 0000 \\
\hline 127 & & $01 / 24 / 66$ & 0000 & $02 / 10 / 66$ & 0000 \\
\hline 146 & & $12 / 14 / 67$ & 0000 & $12 / 20 / 67$ & 0000 \\
\hline 179 & & $02 / 11 / 70$ & 1200 & $02 / 24 / 70$ & 1200 \\
\hline 231 & & $01 / 29 / 73$ & 0000 & $02 / 01 / 73$ & 1200 \\
\hline 274 & & $01 / 24 / 75$ & 0000 & $02 / 05 / 75$ & 1200 \\
\hline 335 & & $02 / 13 / 78$ & 1200 & $03 / 01 / 78$ & 0000 \\
\hline 352 & & $02 / 20 / 79$ & 0000 & $02 / 23 / 79$ & 1200 \\
\hline 390 & & $02 / 20 / 81$ & 0000 & $02 / 24 / 81$ & 0000 \\
\hline 393 & & $03 / 01 / 81$ & 0000 & $03 / 03 / 81$ & 1200 \\
\hline 414 & & $02 / 28 / 82$ & 0000 & $03 / 03 / 82$ & 1200 \\
\hline 500 & & $01 / 15 / 87$ & 0000 & $01 / 20 / 87$ & 0600 \\
\hline 504 & & $02 / 03 / 87$ & 0000 & $02 / 05 / 87$ & 1200 \\
\hline 510 & & $02 / 27 / 87$ & 0000 & $03 / 07 / 87$ & 0000 \\
\hline 513 & & $04 / 19 / 87$ & 1800 & $04 / 26 / 87$ & 1200 \\
\hline 525 & Gina & $01 / 06 / 89$ & 0600 & $01 / 10 / 89$ & 0000 \\
\hline 543 & Ofa & $01 / 28 / 90$ & 0000 & $02 / 09 / 90$ & 1200 \\
\hline 562 & Val & $12 / 04 / 91$ & 0600 & $12 / 13 / 91$ & 1200 \\
\hline 575 & Gene & $03 / 13 / 92$ & 1800 & $03 / 19 / 92$ & 0000 \\
\hline 586 & Lin & $01 / 29 / 93$ & 0000 & $02 / 04 / 93$ & 0000 \\
\hline 588 & Mick & $02 / 03 / 93$ & 0000 & $02 / 09 / 93$ & 1200 \\
\hline
\end{tabular}

surge is inclusion of tides in the storm-surge simulation. However, this approach is not practical for stage-frequency analysis because numerous tidal phases would have to be modeled for each storm in the training set to acquire a representative set of surge and tide combinations. An alternative approach was taken in this study to estimate the combined water-surface elevation of the surge and tide. Simulations were performed for each of the 31 storms in the training set, where the still-water level was taken to be MSL. Tides were not included in the computations. Because storm surges are small for the study site, the 
water-surface elevation for the combined surge and tide can be approximated as a linear superposition of the two. Thus, still-water level for stage-frequency computations was calculated by addition of the surge to a specific tidal elevation.

A total of 88 numerical gauge stations was specified as locations for surge output from the storm-surge model. The stations for Tutuila and Aunuu are shown in Figures 12 and 13. The stations for the Manua' Islands are shown in Figures 14 and 15. Appendix A gives the latitude and longitude of stations. Water-level values were stored at 15 -min intervals at each station. Combined time-series water-level and wave information were applied for the ponding level, setup, and runup calculations.

\section{Spectral Wave Transformation}

Waves calculated by the WISWAVE model were transformed by application of the spectral spreading model WAVTRAN, described in Chapter 3. Estimates were made of the general shoreline orientation closest to each of the numerical gauge locations specified in Appendix A. In addition, estimates of sheltering angle bands were made based on shoreline geometry (bays, coves), existence of land points, and islands. For waves calculated for many of the numerical gauge locations, two-sided sheltering was applied.

For numerical gauge locations located within Pago Pago Harbor, WAVTRAN was applied multiple times to calculate wave parameters within the harbor. Pago Pago Harbor is protected from waves by the presence of Taema Bank which is located approximately $1.5 \mathrm{n} . \mathrm{m} .(2.8 \mathrm{~km})$ from the harbor mouth. Typical depth over the bank is approximately $30 \mathrm{ft}(9 \mathrm{~m})$, which will cause shoaling and breaking of storm waves. Waves were transformed over the bank and propagated into the harbor.

Waves propagated into Pago Pago Harbor were assumed to travel along the main axis of the harbor. The WAVETRAN model was successively applied to three locations along the harbor axis. The first location was the harbor entrance between Niuloa Point and Breakers Point. Waves calculated at the entrance were propagated to the second point located in the center of the harbor near the village of Utulei. The third calculation point was located in the center of the harbor between Nuutotoi Point and Leasi Point, at the bend in the harbor. For each of the numerical gauge locations within Pago Pago Harbor, WAVTRAN was applied using waves calculated from the appropriate location in the harbor center.

For the most severe storms, such as Hurricane Val, some swell waves that propagate into Pago Pago Harbor are transitional waves rather than deep-water waves. Thus, some waves applied for runup calculations within the harbor were transitional waves rather than deep-water waves. Application of transitional waves for runup calculations will introduce error into the runup calculations. This error will bias the runup to be higher than if deep-water waves were applied because the transitional wave will have a larger wave height than a deep-water wave, i.e., the transitional wave will have started to shoal. Examination of 
waves propagated into Pago Pago Harbor for Hurricane Val revealed minimum depth/wavelength $(d / L)$ ratios of approximately $1 / 5$. Transitional waves, by definition, have $d / L$ ratios ranging from $1 / 25$ to $1 / 2$.

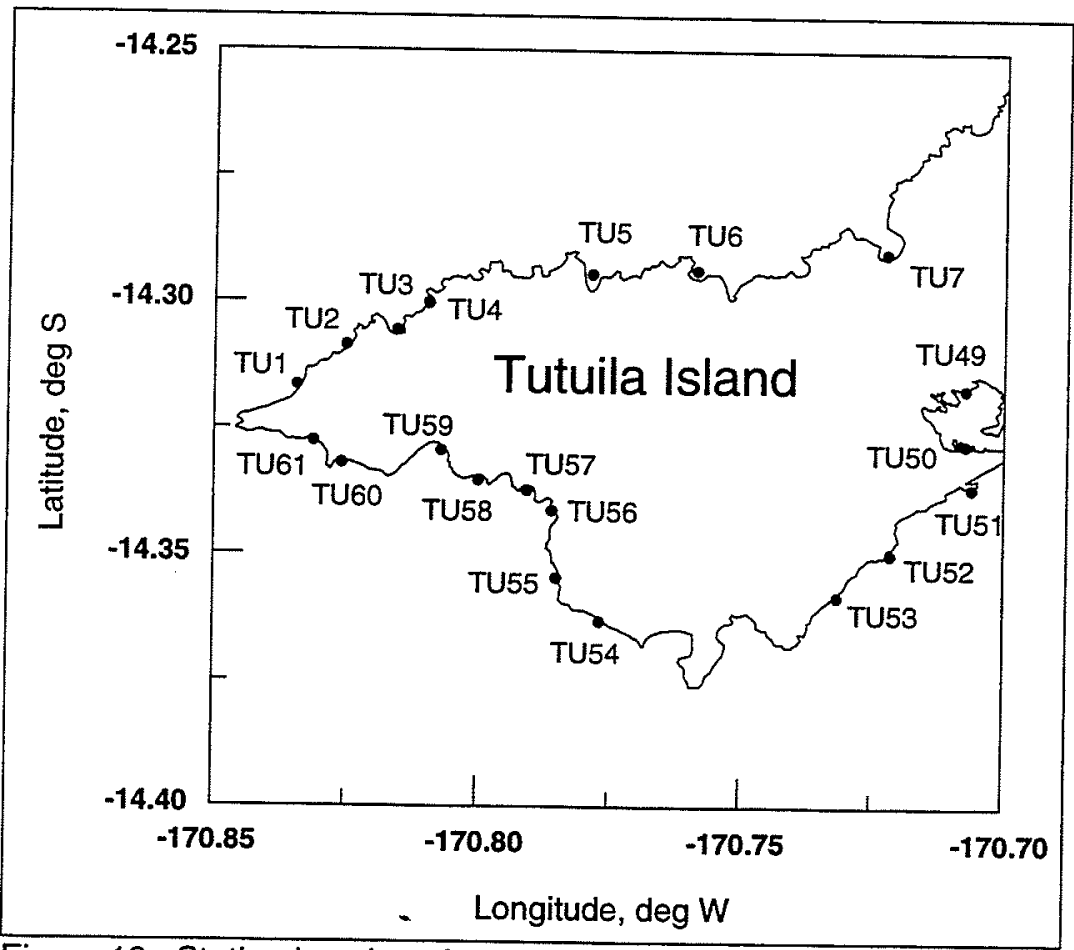

Figure 12. Station locations for western Tutuila Island

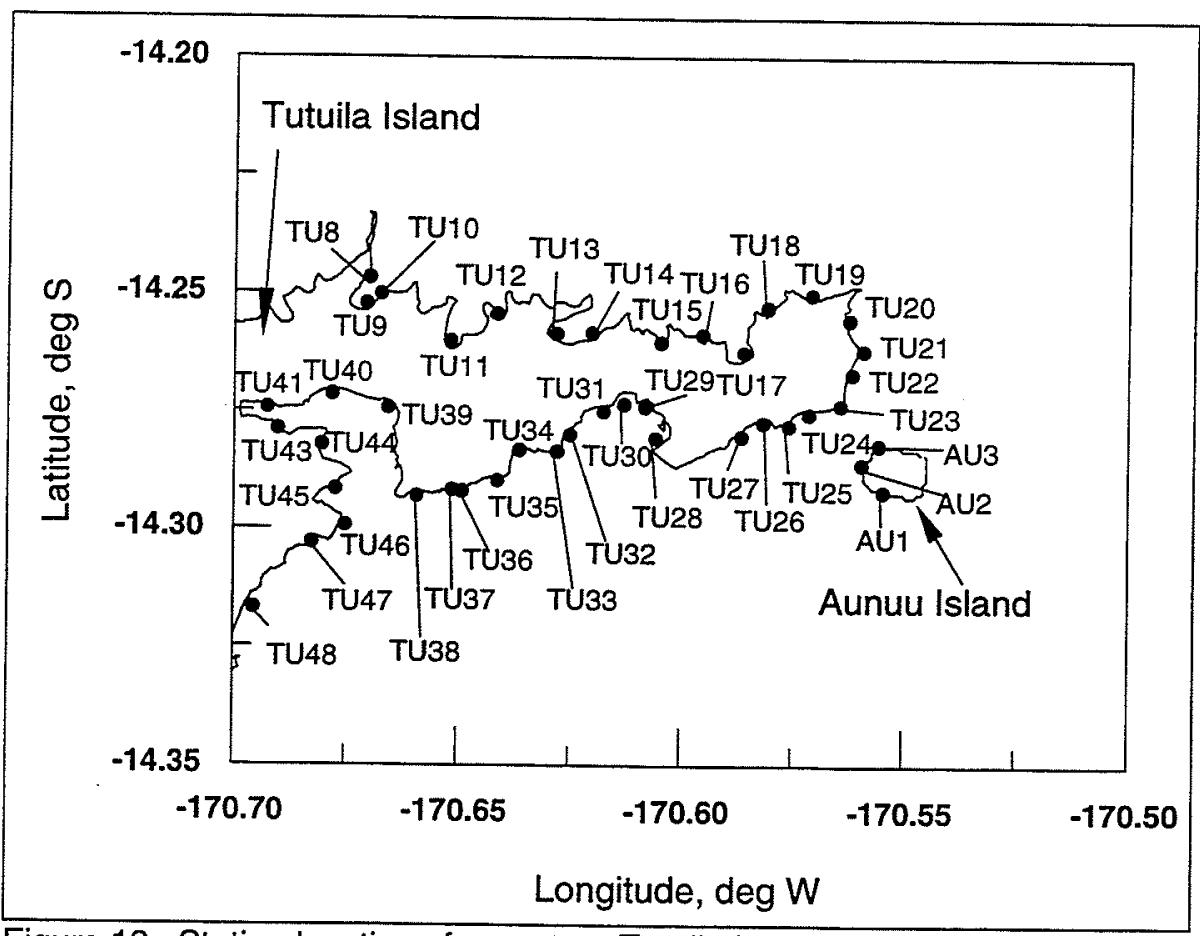

Figure 13. Station locations for eastern Tutuila Island and Aunuu Island 


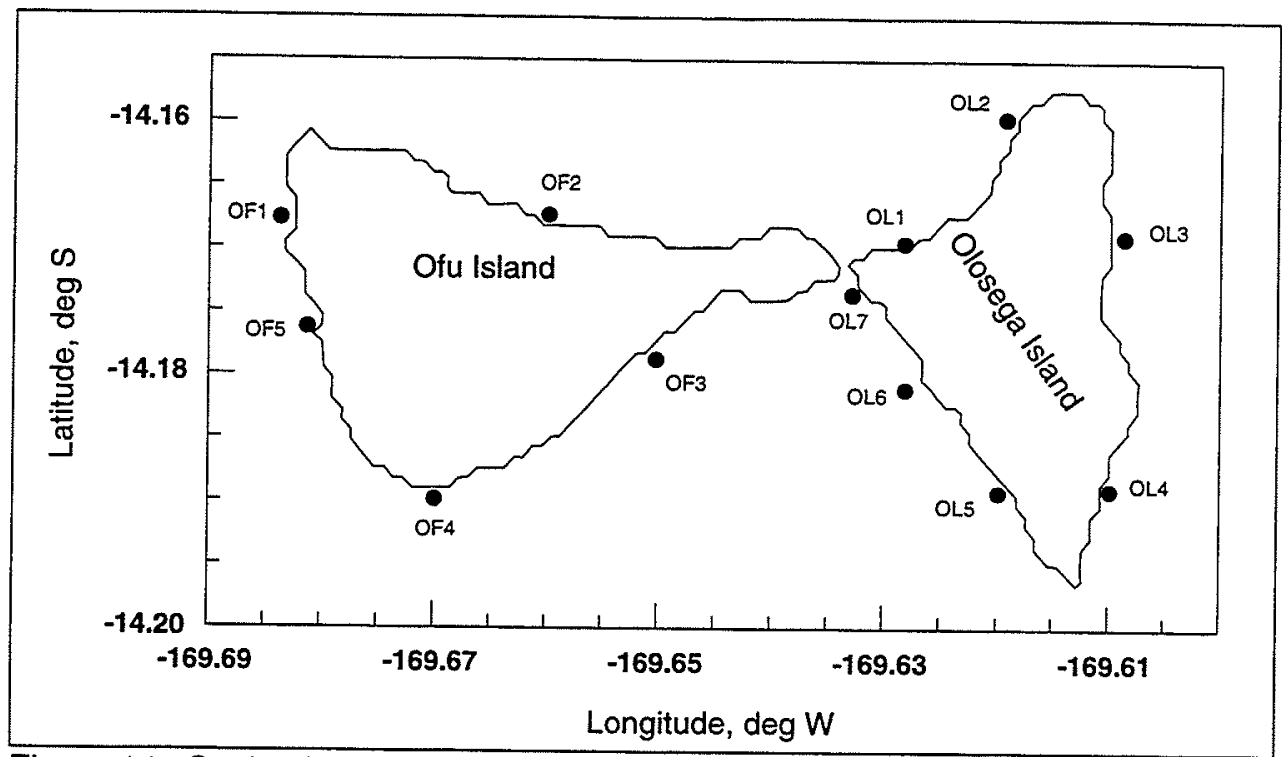

Figure 14. Station locations for Ofu Island and Olosega Island

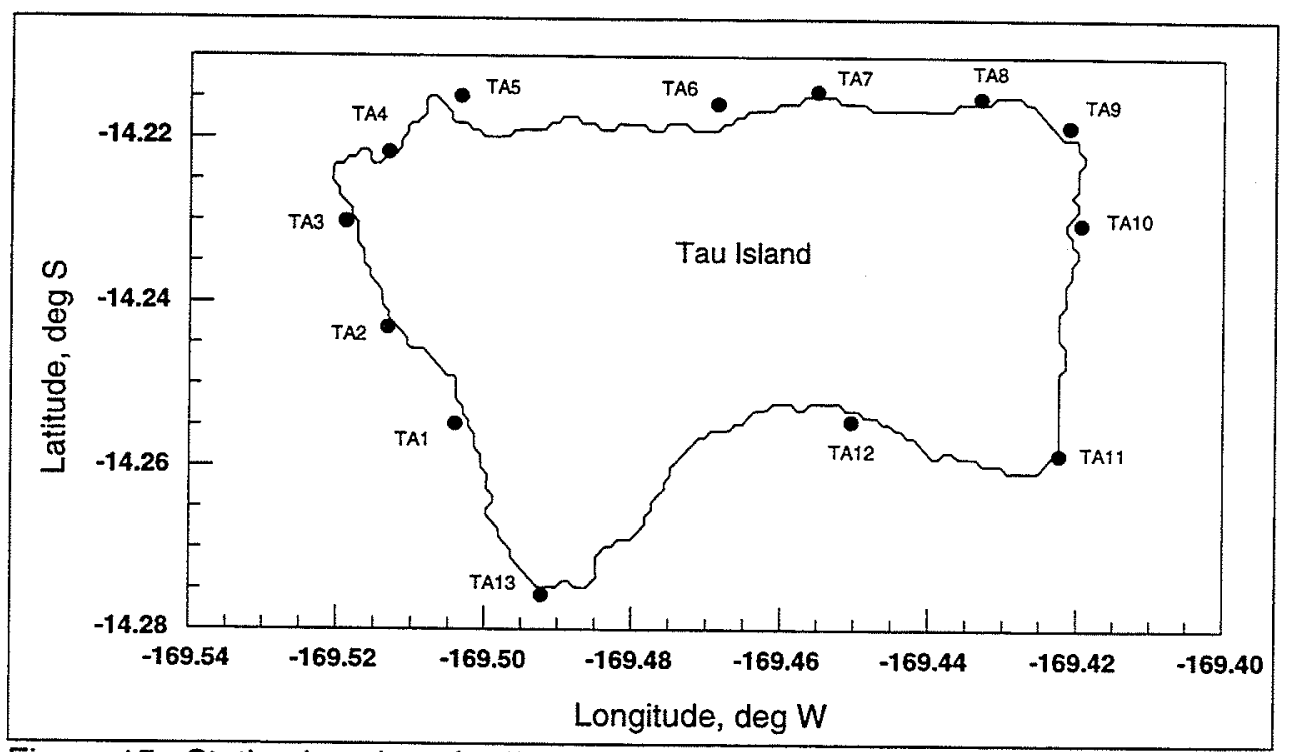

Figure 15. Station locations for Tau Island

\section{Wave Impoundment Correction Procedure}

Wave setup on reefs owes to overtopping of waves onto the reef platform. As waves break on the reef, water is deposited causing elevated water level. Seelig (1983) conducted a set of laboratory experiments for fringing reefs typical of Guam to investigate hydraulics of reef-lagoon systems. Because the reef systems of Guam are similar to those of the Territory of American Samoa, Seeling's formulations were applied in this study. Wave setup and ponding level resulting from wave overtopping were examined. Parameter ranges were varied as follows: the still-water depth at the reef crest was specified to be $0 \mathrm{ft}(0 \mathrm{~m})$ and $6.6 \mathrm{ft}(2 \mathrm{~m})$, the wave periods ranged from 8 to $16 \mathrm{sec}$, and the irregular deep-water significant wave height ranged from 8.2 to $35.1 \mathrm{ft}(2.5$ to $10.7 \mathrm{~m})$. 
Seelig found that the ponding water level is a function of the still-water level (astronomical tide and storm surge), deep-water significant wave height, and wave period. Gourlay (1996) confirmed these findings. Contributions to the ponding level do include waves breaking on the reef, but do not include setup from reformed waves. Ponding level can be estimated by (Seelig, 1983)

$$
\eta=a_{1}+a_{2} \log \left(H_{0}^{2} T\right)
$$

where $\eta$ is the ponding level, $H_{0}$ is the deep-water significant wave height, $T$ is the wave period, and $a_{1}$ and $a_{2}$ are empirical coefficients dependent on the stillwater level and wave spectrum (monochromatic or irregular). Table 7 gives values of the empirical coefficients for irregular waves.

\begin{tabular}{|c|c|c|}
\hline \multicolumn{3}{|c|}{$\begin{array}{l}\text { Table } 7 \\
\text { Ponding Level Coefficients for Irregular Waves (Seelig 1983) }\end{array}$} \\
\hline Depth, ft (m) & $a_{1}$ & $a_{2}$ \\
\hline $0(0)$ & -0.92 & 0.77 \\
\hline $6.6(2)$ & -1.25 & 0.73 \\
\hline
\end{tabular}

\section{Wave Setup and Runup}

Ponding levels include the contribution from wave setup at the reef. The setup is a super-elevation of the still-water level due to waves breaking on the reef. Another potential increase in water level is caused by breaking of waves re-formed within the lagoon system after breaking on the reef. This process is not included in the ponding level calculation.

Wave setup results when the pressure gradient of the sloping water surface (i.e. mean still-water depth) is in equilibrium with the cross-shore directed radiation stress, which represents the gradient of momentum of incoming waves in the shoreward direction:

$$
\frac{d \bar{\eta}}{d x}=-\frac{1}{\rho g d} \frac{d S_{x x}}{d x}
$$

where $\bar{\eta}$ is the mean still-water level, $\rho$ is the water density, $g$ is the acceleration due to gravity, $S_{x x}$ is the cross-shore component of the cross-shore directed radiation stress, $d$ is depth, and $x$ is the cross-shore distance. Under the assumption of linear wave theory, wave setup in the surf zone is

$$
\frac{d \bar{\eta}}{d x}=-\frac{3}{16} \frac{1}{d+\bar{\eta}} \frac{d\left(H^{2}\right)}{d x}
$$

where $H$ is the wave height. 
Wave setup was computed from Equation 19 along 375 transects within the study area. Transects were specified by elevation profiles surveyed for this study and provided by CEPOH. Elevations were specified relative to MSL. The number of transects for each of the five islands comprising the study area is given in Table 8. In addition to the measured profile data, reef width normal to the beach was estimated from topographic maps supplied by $\mathrm{CEPOH}$. The profiles were extended seaward by the estimated reef width. Depths on the reefs were assumed to vary from 1 to $3 \mathrm{ft}(0.3$ to $0.9 \mathrm{~m})$.

\begin{tabular}{|c|c|}
\hline \multicolumn{2}{|c|}{$\begin{array}{l}\text { Table } 8 \\
\text { Number of Transects for the American Samoa Islands }\end{array}$} \\
\hline Island & Number of Transects \\
\hline Tutuila & 337 \\
\hline Aunuu & 5 \\
\hline Ofu & 6 \\
\hline Olosega & 10 \\
\hline Tau & 17 \\
\hline
\end{tabular}

Wave runup is the maximum water-surface elevation caused by the uprush of water from a breaking wave. The computational method presented in the Shore Protection Manual (1984) was applied for the runup calculations for this study. The composite slope method, developed by Saville (1958), was applied to account for changes in grade along a given profile.

The deep-water wave height for the setup and runup calculations was assumed to be equal to the breaking wave height on the reef estimated by

$$
H_{b}=\gamma_{b} h_{b}
$$

where $H_{b}$ is the height of the breaking wave, $h_{b}$ is the water depth at breaking, and $\gamma_{b}$ is the breaking depth index. The breaking depth index can range from 1.1 to 0.4 across reefs (Gerritsen 1980, Hardy et al. 1990). A typical breaking depth index for beaches has a value of 0.78 , but this value is overly conservative for calculation of design wave heights landward of the reef edge (Smith 1993). An approximation for the breaking depth index is given by (Smith and Kraus 1991)

$$
\gamma_{b}=\frac{1.12}{1+e^{-60 S}}-5.0\left(1-e^{-43 S}\right)\left(\frac{H_{0}}{L_{0}}\right)
$$

where $S$ is the bottom slope. This approximation is valid for the ranges $0.0007 \leq H_{0} / L_{0} \leq 0.0921$ and $1 / 80 \leq S \leq 1 / 10$. The breaking depth index was applied as a tuning parameter for calculation of setup and runup. Adjustments to the breaking depth index were based on deep-water significant wave height and applied consistently for all profiles. The adjusted breaking depth index $\gamma_{b}^{\prime}$ was specified as 


$$
\begin{array}{ll}
\gamma_{b}^{\prime}=\gamma_{b} / 0.7, & H_{0}<3.3 \\
\gamma_{b}^{\prime}=\gamma_{b} / 0.4, & 3.3 \leq H_{0} \leq 6.6 \\
\gamma_{b}^{\prime}=\gamma_{b} / 0.3, & H_{0}>3.3
\end{array}
$$

where the significant wave heights are given in units of feet.

\section{Typhoon Ofa - Water Level Comparison}

Maximum water elevation calculations, which includes wave runup, were compared to debris-line water elevations survey by FEMA for Typhoon Ofa. The observed values were supplied for villages on Tutuila Island. Water levels were computed by linear supposition of the surge, wave setup, ponding level, and wave runup. These parameters were calculated as previously described. Time series of water levels were computed at villages for Typhoon Ofa and the minimum and maximum values of water level, referenced to MSL, were extracted from the time series. Table 9 shows the comparisons of measured and calculated minimum and maximum water level for 18 villages. Calculated water elevations under-predicted the observed water elevations at 15 villages and overpredicted at 3 villages. On average, the calculated water elevations underpredicted observed values by $5.5 \mathrm{ft}$ for the 18 villages.

The underprediction of water elevations at several of the villages stems, in part, from oversimplification of wave processes over the reef and in situations where the shoreline is curved. Presently, little is known about runup on fringing reefs. The composite-slope method for calculating runup, applied in this study, was developed for sloping beaches with structures. The accuracy of the composite-slope method for fringing reef applications has not been established and may introduce error into the runup calculations. Additionally, onedimensional models were applied to a two-dimensional problem. Waves propagating over reefs will be transformed. In bays with arcuate shorelines and reef edges, the transformation will be complex. In this application, waves were assumed to propagate straight in from the bay mouth or ocean, but in a prototype system, refraction and other processes will modify the waves.

A second source of error results from comparison of calculated water level to debris-line water levels. The debris-line water levels are the highest water level that occurred during the storm. Processes that contribute to the debris-line water levels include localized wind-driven water, spray, locally-generated waves, surge, ponding level, setup, and runup. While the surge, ponding level, setup, and runup were calculated, other processes that contribute to debris-line elevations were not calculated. In addition, all calculated wave processes were based on deep-water significant wave height, peak period, and mean wave direction. Waves having other heights, periods, and directions were not used in the computations, but would have contributed to elevated water level. 


\begin{tabular}{|l|l|l|l||}
\hline \multicolumn{4}{|l|}{$\begin{array}{l}\text { Table 9 } \\
\text { Observed and Calculated }\end{array}$} \\
\hline \hline Water Level for Typhoon Ofa \\
\hline \hline Village & $\begin{array}{l}\text { Observed Water } \\
\text { Level (FEMA), ft msl }\end{array}$ & $\begin{array}{l}\text { Minimum Calculated } \\
\text { Water Level, ft msl }\end{array}$ & $\begin{array}{l}\text { Maximum Calculated } \\
\text { Water Level, ft msl }\end{array}$ \\
\hline Masausi & 5 & 3.6 & 3.9 \\
\hline Sailele & 16 & 2.9 & 2.9 \\
\hline Aoa & 16 & 6.3 & 6.3 \\
\hline Oneona & 12.5 & 6.2 & 6.2 \\
\hline Amouli & 22 & 7.4 & 7.4 \\
\hline Aloa & 12 & 12.2 & 12.9 \\
\hline Lauagae & 22 & 9.5 & 14.3 \\
\hline Fagalii & 22 & 7.0 & 8.8 \\
\hline Poloa & 15 & 11.4 & 15.3 \\
\hline Amanaue & 13 & 13.8 & 13.8 \\
\hline Fagamalo & 21 & 13.4 & 19.1 \\
\hline Maloate & 21 & 6.9 & 8.6 \\
\hline Afao & 17 & 10.8 & 10.8 \\
\hline Utomea & 17 & 12.5 & 12.5 \\
\hline Fagasa & 8 & 12.9 & 12.9 \\
\hline Vatia & 7.5 & 5.5 & 6.1 \\
\hline Afono & 8.5 & 2.5 & 2.5 \\
\hline & 3.6 & 3.6 \\
\hline
\end{tabular}

\section{Stage-Frequency Relationships}

Stage-frequency relationships were calculated for 375 profiles along the coast of the American Samoa Islands by application of the EST. These relationships were computed for maximum water level at intervals of 5, 10,25, 50 , and 100 years. Input for the EST included the maximum water level calculated for each of the 31 storms in the training set. The maximum water level was calculated as the linear superposition of the storm surge, ponding level, setup, and runup. Tables of stage-frequency relationship values for each profile are given in Appendix B. Maximum expected water-level values and standard deviations are given in the tables. Figures showing stage-frequency relationships for profiles representative of numerical gauge locations are given in Appendix C.

Because the American Samoa Islands are located in a region of tropical storm and hurricane formation and propagation, the Islands can experience storms or waves from any direction. All coastlines of the Islands are subject to storm waves and may experience significant inundation, with the exception of the protected areas Pago Pago Harbor and Pala Lagoon. The arcuate shape of many of the coves, particularly on Tutuila, provides sheltering from waves in specific angle bands. 
In addition to the stage-frequency hydrographs for runup, values of setup and ponding level corresponding to peak water level for each storm are presented in Appendix D for each of the numerical gauges. Setup and ponding levels were computed for each profile and calculated values corresponding to profiles representative of the numerical gauge locations are given. In addition to the peak setup and ponding level for each storm, the significant wave height, wave period, and wave angle used to calculate peak water level are included in Appendix D. Setup values computed for low wave heights are overpredicted, but these values are expected to have insignificant influence on the frequency calculation for extreme water level. 


\section{Summary and Conclusions}

A set of hurricane-induced stage-frequency histograms was developed for the Territory of American Samoa. The subject study area consists of the five volcanic islands Tutuila, Aunuu, Ofu, Olosega, and Tau. Calculation of surge, wind and pressure field, and wave characteristics were performed for 31 historical storms through application of four numerical models. Wave-induced setup and runup were calculated at profile locations specified by CEPOD.

The circulation model ADCIRC was applied for calculation of storm surge in the study area. Model calculations compared well to NOAA tidal constituents calculated for Pago Pago Harbor. For storm surge calculation, ADCIRC applied wind and pressure fields calculated by the Planetary Boundary Layer (PBL) model as the atmospheric forcing.

The PBL model was applied for simulation of storms whose path brought the storm center within a $200-\mathrm{mi}(370-\mathrm{km})$ radius of the five islands constituting the study site. Historical data from the storms were input into the PBL model for calculation of wind and pressure fields. The atmospheric fields calculated by the PBL model were applied as forcing for the circulation and wave models.

Deep-water wave heights, periods, and directions for each storm were calculated by application of the wave model WISWAVE. These deep-water waves were transformed by application of the wave-transformation model WAVTRAN. For wave propagation into Pago Pago Harbor, WAVTRAN was applied multiple times for calculation of waves at specific points within the harbor.

Storm surge (wind- and atmospheric pressure-induced) was simulated for 31 historical storms and referenced to Mean Sea Level. Because the islands of American Samoa are volcanic cones with steep sides, shallow shelf areas (such as on the east coast of the United States) do not exist around the islands, so the storm surge does not shoal. Consequently, the storm surge (without consideration of waves) is small and contributes little to coastal inundation during severe storms. Wave ponding on the reefs, wave setup, and runup cause high inundation levels during storm events.

The EST was applied to calculate stage-frequency relationships based on historical storm parameters and calculated response to the storms. These relationships were calculated from the maximum water levels computed for each 36

Chapter 6 Summary and Conclusions 
storm, which included storm surge, wave setup, ponding level, and runup. Stagefrequency values and their standard deviations were calculated for $2,5,10,25$, 50,75 , and 100 -year return periods at 375 profiles. 


\section{References}

Atkinson, G. D., and Holliday, C. R., (1977): “Tropical Cyclone Minimum Sea Level Pressure/Maximum Sustained Wind Relationship for the Western North Pacific," Monthly Weather Review, 105, 421-427.

Borgman, L. E., and Scheffner, N. W., (1991). "Simulation of time sequences of wave height, period, and direction," Technical Report DRP-91-2, U.S. Army Engineer Waterways Experiment Station, Vicksburg, MS.

Borgman, L., Miller, M., Butler, L., and Reinhard, R., (1992). "Empirical simulation of future hurricane storm histories as a tool in engineering and economic analysis." Fifth International Conference on Civil Engineering in the Oceans, ASCE, College Station, TX, 2-5 November 1992.

Bouws, E., Gunther, H., Rosenthal, W., and Vincent, C. L., (1985). "Similarity of the Wind Wave Spectrum in Finite Depth Water 1 Spectral Form," Journal of Geophysical Research, 1(C1), 975-986.

Cardone, V. J., Greenwood, C. V., and Greenwood, J. A., (1992). "Unified Program for the Specification of Hurricane Boundary Layer Winds Over Surfaces of Specified Roughness," Contract Report CERC-92-1, U.S. Army Engineer Waterways Experiment Station, Vicksburg, MS.

Flather, R. A., (1988). “A Numerical Model Investigation of Tides and DiurnalPeriod Continental Shelf Waves along Vancouver Island," Journal of Physical Oceanography, 18, 115-139.

Gerritsen, F., (1980). "Wave Attenuation and Wave Set-Up on a Coastal Reef," Proceedings of the Seventeenth Coastal Engineering Conference, American Society of Civil Engineers, 444-461.

Gourlay, M. R., (1996). "Wave Set-Up on Coral Reefs. 2. Set-Up on Reefs with Various Profiles," Coastal Engineering, 28, 17-55.

Gravens, M. B., Kraus, N. C., and Hanson, H., (1991). "GENESIS: Generalized Model for Simulating Shoreline Change. Report 2, Workbook and System User's Manual," Technical Report CERC-89-18, U.S. Army Engineer Waterways Experiment Station, Vicksburg, MS. 
Gumbel, E. J., (1954). Statistical theory of extreme values and some practical applications; a series of lectures. U.S. Government Printing Office, Washington, DC.

Hardy, T. A., Young, I. R., Nelson, R. C., and Gourley, M. R., (1990). "Wave Attenuation of an Offshore Coral Reef," Proceedings of the Twenty-Second Coastal Engineering Conference, American Society of Civil Engineers, 330334.

Jelesnianski, C. P., and Taylor, A. D., (1973). "A preliminary view of storm surges before and after storm modifications," NOAA Technical Memorandum ERL WMPO-3, Weather Modification Program Office, Boulder, $\mathrm{CO}$.

Jensen, R. E., (1983). "Methodology for the Calculation of a Shallow-Water Wave Climate," WIS Report 8, U.S. Army Engineer Waterways Experiment Station, Vicksburg, MS.

Kolar, R. L., Gray, W. G., Westerink, J. J., and Luettich, R. A., (1993).

"Shallow Water Modeling in Spherical Coordinates: Equation Formulation, Numerical Implementation, and Application," Journal of Hydraulic Research.

Kraft, R. H., (1961). "The Hurricane's Central Pressure and Highest Wind," Mariner's Weather Log, 5(5), 157pp.

Mark, D. J., (1996). "Southern Guam Typhoon Stage-Frequency Analysis," Miscellaneous Paper CERC-96-7, U.S. Army Engineer Waterways Experiment Station, Vicksburg, MS.

Mark, D. J., and Scheffner, N. W. (1997). "Coast of Delaware Hurricane StageFrequency Analysis," Miscellaneous Paper CHL-97-1, U.S. Army Engineer Waterways Experiment Station, Vicksburg, MS.

Resio, D. T., and Perrie, W. (1989). "Implications of an $f^{4}$ Equilibrium Range for Wind-Generated Waves," Journal of Physical Oceanography, Vol 19, 193-204.

Saville, T., Jr., (1958). "Wave Runup on Composite Slopes," Proceedings of the Sixth Conference on Coastal Engineering, American Society of Civil Engineers, Council on Waves.

Scheffner, N. W., and Borgman, L. E. (1993). "Stochastic time-series representation of wave data," Journal of Waterway, Port, Coastal and Ocean Engineering, American Society of Civil Engineers, 118(4), 337-351.

Sea Engineering, Inc. and Belt Collins Hawaii, (1994). American Samoa Shoreline Inventory Update II. Prepared for U.S. Army Corps of Engineers, Pacific Ocean Division, Fort Shafter, HI. 
Seelig, W. N., (1983). "Laboratory Study of Reef-Lagoon System Hydraulics," Journal of Waterway, Port, Coastal and Ocean Engineering, 109(4), 380391.

Shore Protection Manual, (1984). $4^{\text {th }}$ ed., 2 Vol., U.S. Army Engineer Waterways Experiment Station, U.S. Government Printing Office, Washington, DC.

Smith, J. M., (1993). "Nearshore Wave Breaking and Decay," Technical Report CERC-93-11, US Army Engineer Waterways Experiment Station, Vicksburg, MS.

Smith, J. M., and Kraus, N. C., (1991). "Laboratory Study of Wave-Breaking over Bars and Artificial Reefs," Journal of Waterway, Port, Coastal and Ocean Engineering, 117(4), 307-325.

Westerink, J. J., Luettich, R. A., Baptista, A. M., Scheffner, N. W., and Farrar, P., (1992). "Tide and storm surge predictions using finite element model," Journal of Hydraulic Engineering, American Society of Civil Engineers, 118(10), 1373-139 


\section{Appendix A Station Locations}

\begin{tabular}{|c|c|c|c|}
\hline \multicolumn{4}{|c|}{$\begin{array}{l}\text { Table A1 } \\
\text { Station Locations for Stage-Frequency Relationships }\end{array}$} \\
\hline Station & Nearest Village or Landmark & Latitude, deg S & Longitude, deg W \\
\hline \multicolumn{4}{|c|}{ Tutuila Island } \\
\hline TU1 & Poloa & 14.3166 & 170.8344 \\
\hline TU2 & Fagalii & 14.3087 & 170.8251 \\
\hline TU3 & Maloata & 14.3057 & 170.8154 \\
\hline TU4 & Fagamalo & 14.3002 & 170.8096 \\
\hline TU5 & Aoloautua & 14.2945 & 170.7786 \\
\hline TU6 & Aasu & 14.2937 & 170.7586 \\
\hline TU7 & Fagalea, Fagasa, Fagatele & 14.2901 & 170.7224 \\
\hline TU8 & N. Vatia Bay & 14.2468 & 170.6699 \\
\hline TU9 & Vatia & 14.2524 & 170.6705 \\
\hline TU10 & E. Vatia Bay & 14.2503 & 170.6672 \\
\hline TU11 & Afono & 14.2602 & 170.6516 \\
\hline TU12 & $\mathrm{Oa}$ & 14.2544 & 170.6412 \\
\hline TU13 & Masefau & 14.2587 & 170.6280 \\
\hline TU14 & SE Masefau Bay & 14.2585 & 170.6202 \\
\hline TU15 & Masausi & 14.2604 & 170.6045 \\
\hline TU16 & Sailele & 14.2587 & 170.5954 \\
\hline TU17 & Aoa & 14.2625 & 170.5858 \\
\hline TU18 & Onenoa & 14.2531 & 170.5806 \\
\hline TU19 & Maupua & 14.2503 & 170.5708 \\
\hline TU20 & Tula & 14.2556 & 170.5623 \\
\hline TU21 & Maliuga $\mathrm{Pt}$ & 14.2620 & 170.5591 \\
\hline TU22 & Alao & 14.2671 & 170.5617 \\
\hline TU23 & Matuli Pt & 14.2737 & 170.5644 \\
\hline TU24 & Utumea & 14.2757 & 170.5714 \\
\hline TU25 & Aganoa & 14.2780 & 170.5759 \\
\hline TU26 & Amouli & 14.2774 & 170.5816 \\
\hline TU27 & Fugaau & 14.2804 & 170.5864 \\
\hline
\end{tabular}




\begin{tabular}{|c|c|c|c|}
\hline \multicolumn{4}{|c|}{ Table A1 (Continued) } \\
\hline TU28 & Alofau & 14.2808 & 170.6056 \\
\hline TU29 & Pagai & 14.2741 & 170.6081 \\
\hline TU30 & Fagaitua & 14.2736 & 170.6128 \\
\hline TU31 & Utusia, Amaua & 14.2751 & 170.6175 \\
\hline TU32 & Auto & 14.2801 & 170.6250 \\
\hline TU33 & Avaio & 14.2836 & 170.6278 \\
\hline TU34 & Alega & 14.2833 & 170.6361 \\
\hline TU35 & Siliataligau Pt & 14.2898 & 170.6411 \\
\hline TU36 & Aumi & 14.2920 & 170.6490 \\
\hline TU37 & Lauliituai & 14.2918 & 170.6512 \\
\hline TU38 & Lauliifou & 14.2931 & 170.6590 \\
\hline TU39 & Aua & 14.2744 & 170.6655 \\
\hline TU40 & Leloaloa & 14.2715 & 170.6781 \\
\hline TU41 & Lalopua, Satala & 14.2744 & 170.6926 \\
\hline TU43 & Malalua, Fagatogo & 14.2789 & 170.6902 \\
\hline TU44 & Utulei & 14.2822 & 170.6803 \\
\hline TU45 & Fagaalu & 14.2917 & 170.6773 \\
\hline TU46 & Fatumafuti & 14.2994 & 170.6749 \\
\hline TU47 & Matuu & 14.3030 & 170.6825 \\
\hline TU48 & Coconut Pt & 14.3169 & 170.6955 \\
\hline TU49 & Tualiliu Pt (Pala Lagoon) & 14.3170 & 170.7074 \\
\hline TU50 & Avatele Passage (Pala Lagoon) & 14.3279 & 170.7072 \\
\hline TU51 & Matautuotafuna $\mathrm{Pt}$ & 14.3366 & 170.7060 \\
\hline TU52 & Alatele Cove & 14.3498 & 170.7215 \\
\hline TU53 & Vaitogi & 14.3582 & 170.7316 \\
\hline TU54 & Avaloa Pt & 14.3632 & 170.7767 \\
\hline TU55 & Vailoata & 14.3546 & 170.7851 \\
\hline TU56 & Leone & 14.3413 & 170.7859 \\
\hline TU57 & Amaluia & 14.3372 & 170.7907 \\
\hline TU58 & Afao & 14.3352 & 170.7999 \\
\hline TU59 & Nua & 14.3294 & 170.8070 \\
\hline TU60 & Failolo & 14.3320 & 170.8258 \\
\hline TU61 & Amanaue & 14.3276 & 170.8312 \\
\hline \multicolumn{4}{|c|}{ Aunuu Island } \\
\hline AU1 & Fogatia Hill & 14.2920 & 170.5545 \\
\hline AU2 & Aunuu & 14.2862 & 170.5595 \\
\hline AU3 & Alofisua Pt & 14.2821 & 170.5556 \\
\hline \multicolumn{4}{|c|}{ Ofu Island } \\
\hline OF1 & Alaufau & 14.1677 & 169.6837 \\
\hline OF2 & Tuafanua & 14.1674 & 169.6598 \\
\hline OF3 & Toaga & 14.1787 & 169.6502 \\
\hline OF4 & Papaloloa Pt & 14.1899 & 169.6699 \\
\hline
\end{tabular}




\begin{tabular}{|l|l|l|l||}
\hline \multicolumn{5}{||l|}{ Table A1 (Concluded) } & \multicolumn{3}{l|}{} \\
\hline OF5 & Nuupule Rock & 14.1763 & 169.6812 \\
\hline Olosega Island & \multicolumn{5}{|l||}{} \\
\hline OL1 & Lalomoana & 14.1695 & 169.6282 \\
\hline OL2 & Faiava & 14.1596 & 169.6193 \\
\hline OL3 & Imoa Pt & 14.1689 & 169.6086 \\
\hline OL4 & Oge & 14.1890 & 169.6099 \\
\hline OL5 & Pouono Pt & 14.1892 & 169.6198 \\
\hline OL6 & Olosega & 14.1811 & 169.6281 \\
\hline OL7 & Asaga Straight & 14.1736 & 169.6328 \\
\hline Tau Island & & & \\
\hline TA1 & Afuli Cove & 14.2549 & 169.5041 \\
\hline TA2 & Fusi & 14.2431 & 169.5132 \\
\hline TA3 & Tau, Luma & 14.2302 & 169.5189 \\
\hline TA4 & Faleasao & 14.2217 & 169.5132 \\
\hline TA5 & Siulagi Pt & 14.2149 & 169.5035 \\
\hline TA6 & Avatele Cove & 14.2158 & 169.4686 \\
\hline TA7 & Faga & 14.2142 & 169.4552 \\
\hline TA8 & Lepula, Maia & 14.2150 & 169.4332 \\
\hline TA9 & Fitiuta & 14.2185 & 169.4210 \\
\hline TA10 & Papasao Pt & 14.2304 & 169.4195 \\
\hline TA11 & Tufu Pt & 14.2586 & 169.4223 \\
\hline TA12 & Laufuti Stream & 14.2545 & 169.4504 \\
\hline TA13 & Alaufau & 14.2758 & 169.4923 \\
\hline \hline
\end{tabular}


. 


\section{Appendix B Stage-Frequency Relationship Tables}

This appendix contains stage-frequency relationship values for profiles on the five subject islands of American Samoa. Maximum water level and its standard deviation are given for seven return intervals for each profile.

Postscript: During publication of this report it was found that input data for profiles Tutuila 021 through Tutuila 024 were incorrect. These errors resulted in maximum water level values that are overpredicted. Water level values from Tutuila 025 may be applied as a surrogate value for profiles Tutuila 021 through Tutuila 024 . 


\begin{tabular}{|c|c|c|}
\hline \multicolumn{3}{|c|}{$\begin{array}{l}\text { Table B1 } \\
\text { Return Period, Maximum Water Level, and Water Level Standard } \\
\text { Deviation for Profile Tutuila } 001\end{array}$} \\
\hline Return Period, yr & Maximum Water Level, $\mathrm{ft}$ & Water Level Standard Deviation, ft \\
\hline 2 & 4.6 & 1.8 \\
\hline 5 & 10.3 & 0.7 \\
\hline 10 & 12.1 & 0.7 \\
\hline 25 & 13.9 & 0.9 \\
\hline 50 & 15.2 & 1.1 \\
\hline 75 & 16.0 & 1.2 \\
\hline 100 & 16.3 & 1.4 \\
\hline
\end{tabular}

\begin{tabular}{|c|c|c|}
\hline \multicolumn{3}{|c|}{$\begin{array}{l}\text { Table B2 } \\
\text { Return Period, Maximum Water Level, and Water Level Standard } \\
\text { Deviation for Profile Tutuila 001a }\end{array}$} \\
\hline Return Period, yr & Maximum Water Level, $\mathrm{ft}$ & Water Level Standard Deviation, $t t$ \\
\hline 2 & 4.6 & 1.8 \\
\hline 5 & 10.3 & 0.7 \\
\hline 10 & 12.1 & 0.7 \\
\hline 25 & 13.9 & 0.9 \\
\hline 50 & 15.2 & 1.1 \\
\hline 75 & 16.0 & 1.2 \\
\hline 100 & 16.3 & 1.4 \\
\hline
\end{tabular}

\section{Table B3}

Return Period, Maximum Water Level, and Water Level Standard Deviation for Profile Tutuila 002

\begin{tabular}{|l|l|l|}
\hline Return Period, $\mathbf{y} r$ & Maximum Water Level, $\mathrm{ft}$ & Water Level Standard Deviation, $\mathrm{ft}$ \\
\hline 2 & 4.6 & 1.8 \\
\hline 5 & 10.3 & 0.7 \\
\hline 10 & 12.1 & 0.7 \\
\hline 25 & 13.9 & 0.9 \\
\hline 50 & 15.2 & 1.1 \\
\hline 75 & 16.0 & 1.2 \\
\hline 100 & 16.3 & 1.4 \\
\hline
\end{tabular}

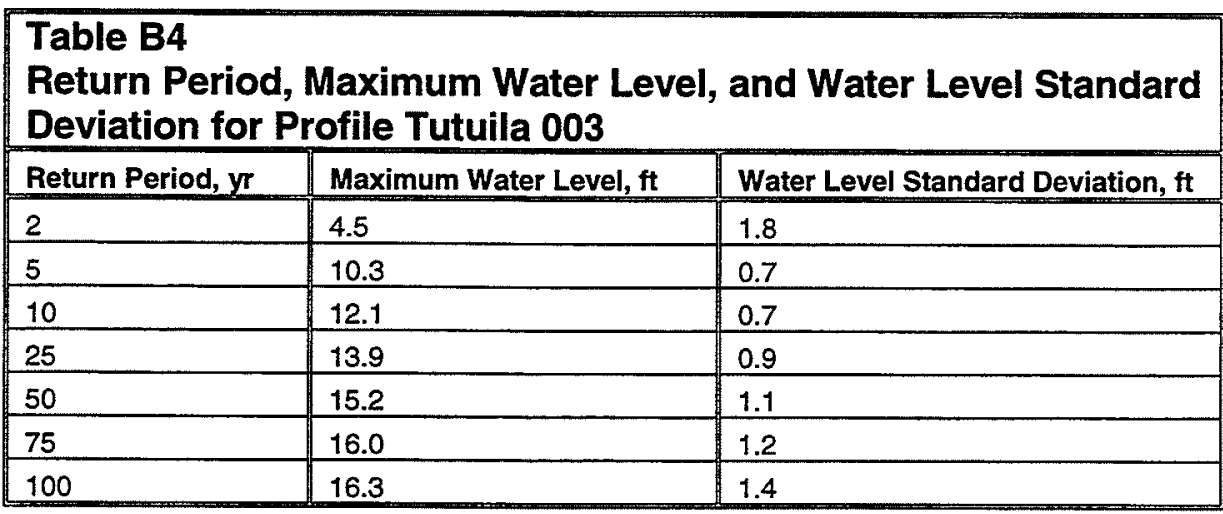




\begin{tabular}{|c|c|c|}
\hline \multicolumn{3}{|c|}{$\begin{array}{l}\text { Table B5 } \\
\text { Return Period, Maximum Water Level, and Water Level Standard } \\
\text { Deviation for Profile Tutuila } 004\end{array}$} \\
\hline Return Period, yr & Maximum Water Level, ft & Water Level Standard Deviation, $\mathrm{ft}$ \\
\hline 2 & 4.6 & 1.8 \\
\hline 5 & 10.3 & 0.7 \\
\hline 10 & 12.1 & 0.7 \\
\hline 25 & 13.9 & 0.9 \\
\hline 50 & 15.2 & 1.1 \\
\hline 75 & 16.0 & 1.2 \\
\hline 100 & 16.3 & 1.4 \\
\hline
\end{tabular}

\begin{tabular}{|c|c|c|}
\hline \multicolumn{3}{|c|}{$\begin{array}{l}\text { Table B6 } \\
\text { Return Period, Maximum Water Level, and Water Level Standard } \\
\text { Deviation for Profile Tutuila } 005\end{array}$} \\
\hline Return Period, yr & Maximum Water Level, ft & Water Level Standard Deviation, $\mathrm{ft}$ \\
\hline 2 & 4.6 & 1.8 \\
\hline 5 & 10.3 & 0.7 \\
\hline 10 & 12.1 & 0.7 \\
\hline 25 & 13.9 & 0.9 \\
\hline 50 & 15.2 & 1.1 \\
\hline 75 & 16.0 & 1.2 \\
\hline 100 & 16.3 & 1.4 \\
\hline
\end{tabular}

\section{Table B7}

Return Period, Maximum Water Level, and Water Level Standard Deviation for Profile Tutuila 006

\begin{tabular}{|l|l|l|}
\hline Return Period, yr & Maximum Water Level, $\mathrm{ft}$ & Water Level Standard Deviation, ft \\
\hline 2 & 4.6 & 1.8 \\
\hline 5 & 10.3 & 0.7 \\
\hline 10 & 12.1 & 0.7 \\
\hline 25 & 13.9 & 0.9 \\
\hline 50 & 15.2 & 1.1 \\
\hline 75 & 16.0 & 1.2 \\
\hline 100 & 16.3 & 1.4 \\
\hline
\end{tabular}

\begin{tabular}{|c|c|c|}
\hline \multicolumn{3}{|c|}{$\begin{array}{l}\text { Table B8 } \\
\text { Return Period, Maximum Water Level, and Water Level Standard } \\
\text { Deviation for Profile Tutuila } 007\end{array}$} \\
\hline Return Period, yr & Maximum Water Level, ft & Water Level Standard Deviation, $\mathrm{ft}$ \\
\hline 2 & 3.5 & 1.5 \\
\hline 5 & 10.5 & 1.0 \\
\hline 10 & 13.0 & 1.0 \\
\hline 25 & 15.4 & 1.3 \\
\hline 50 & 17.3 & 1.8 \\
\hline 75 & 18.3 & 1.8 \\
\hline 100 & 18.9 & 2.0 \\
\hline
\end{tabular}




\begin{tabular}{|l|l|l|}
\hline $\begin{array}{l}\text { Table B9 } \\
\text { Return Period, Maximum Water Level, and Water Level Standard } \\
\text { Deviation for Profile Tutuila 008 }\end{array}$ \\
\hline Return Period, yr & Maximum Water Level, ft & Water Level Standard Deviation, $\mathrm{tt}$ \\
\hline 2 & 3.5 & 1.5 \\
\hline 5 & 12.4 & 1.1 \\
\hline 10 & 15.1 & 1.1 \\
\hline 25 & 17.7 & 1.4 \\
\hline 50 & 19.4 & 1.6 \\
\hline 75 & 20.3 & 1.4 \\
\hline 100 & 20.8 & 1.5 \\
\hline
\end{tabular}

\begin{tabular}{|c|c|c|}
\hline \multicolumn{3}{|c|}{$\begin{array}{l}\text { Table B10 } \\
\text { Return Period, Maximum Water Level, and Water Level Standard } \\
\text { Deviation for Profile Tutuila } 009\end{array}$} \\
\hline Return Period, yr & Maximum Water Level, $\mathrm{ft}$ & Water Level Standard Deviation, ft \\
\hline 2 & 3.5 & 1.5 \\
\hline 5 & 9.8 & 0.8 \\
\hline 10 & 11.9 & 1.0 \\
\hline 25 & 14.0 & 1.1 \\
\hline 50 & 15.5 & 1.4 \\
\hline 75 & 16.2 & 1.5 \\
\hline 100 & 16.6 & 1.6 \\
\hline
\end{tabular}

\begin{tabular}{|c|c|c|}
\hline \multicolumn{3}{|c|}{$\begin{array}{l}\text { Table B11 } \\
\text { Return Period, Maximum Water Level, and Water Level Standard } \\
\text { Deviation for Profile Tutuila } 010\end{array}$} \\
\hline Return Period, yr & Maximum Water Level, ft & Water Level Standard Deviation, $\mathrm{ft}$ \\
\hline 2 & 3.5 & 1.5 \\
\hline 5 & 9.8 & 0.9 \\
\hline 10 & 12.0 & 0.9 \\
\hline 25 & 14.3 & 1.2 \\
\hline 50 & 16.6 & 2.4 \\
\hline 75 & 18.1 & 2.4 \\
\hline 100 & 18.8 & 2.6 \\
\hline
\end{tabular}

\begin{tabular}{|c|c|c|}
\hline \multicolumn{3}{|c|}{$\begin{array}{l}\text { Table B12 } \\
\text { Return Period, Maximum Water Level, and Water Level Standard } \\
\text { Deviation for Profile Tutuila } 011\end{array}$} \\
\hline Return Period, yr & Maximum Water Level, $\mathrm{ft}$ & Water Level Standard Deviation, $\mathrm{ft}$ \\
\hline 2 & 3.3 & 1.4 \\
\hline 5 & 9.4 & 0.7 \\
\hline 10 & 11.5 & 0.8 \\
\hline 25 & 13.8 & 1.0 \\
\hline 50 & 15.3 & 1.3 \\
\hline 75 & 16.1 & 1.3 \\
\hline 100 & 16.4 & 1.3 \\
\hline
\end{tabular}




\begin{tabular}{|c|c|c|}
\hline \multicolumn{3}{|c|}{$\begin{array}{l}\text { Table B13 } \\
\text { Return Period, Maximum Water Level, and Water Level Standard } \\
\text { Deviation for Profile Tutuila } 012\end{array}$} \\
\hline Return Period, yr & Maximum Water Level, $\mathrm{ft}$ & Water Level Standard Deviation, ft \\
\hline 2 & 3.3 & 1.4 \\
\hline 5 & 9.4 & 0.7 \\
\hline 10 & 11.5 & 0.8 \\
\hline 25 & 13.8 & 1.0 \\
\hline 50 & 15.3 & 1.3 \\
\hline 75 & 16.1 & 1.3 \\
\hline 100 & 16.4 & 1.3 \\
\hline
\end{tabular}

\begin{tabular}{|c|c|c|}
\hline \multicolumn{3}{|c|}{$\begin{array}{l}\text { Table B14 } \\
\text { Return Period, Maximum Water Level, and Water Level Standard } \\
\text { Deviation for Profile Tutuila } 013\end{array}$} \\
\hline Return Period, yr & Maximum Water Level, $\mathrm{ft}$ & Water Level Standard Deviation, $\mathrm{ft}$ \\
\hline 2 & 3.3 & 1.4 \\
\hline 5 & 9.4 & 0.7 \\
\hline 10 & 11.5 & 0.8 \\
\hline 25 & 13.8 & 1.0 \\
\hline 50 & 15.3 & 1.3 \\
\hline 75 & 16.1 & 1.3 \\
\hline 100 & 16.4 & 1.3 \\
\hline
\end{tabular}

\begin{tabular}{|l|l|}
\hline $\begin{array}{l}\text { Table B15 } \\
\text { Return Period, Maximum Water Level, and Water Level Standard } \\
\text { Deviation for Profile Tutuila 014 }\end{array}$ \\
\begin{tabular}{|l|l|l|}
\hline Return Period, $\mathrm{yr}$ & Maximum Water Level, $\mathrm{ft}$ & Water Level Standard Deviation, ft \\
\hline 2 & 2.9 & 1.1 \\
\hline 5 & 7.0 & 0.7 \\
\hline 10 & 9.3 & 1.0 \\
\hline 25 & 12.4 & 1.5 \\
\hline 50 & 14.1 & 1.4 \\
\hline 75 & 15.0 & 1.4 \\
\hline 100 & 15.3 & 1.5 \\
\hline
\end{tabular}
\end{tabular}

\begin{tabular}{|c|c|c|}
\hline \multicolumn{3}{|c|}{$\begin{array}{l}\text { Table B16 } \\
\text { Return Period, Maximum Water Level, and Water Level Standard } \\
\text { Deviation for Profile Tutuila } 015\end{array}$} \\
\hline Return Period, yr & Maximum Water Level, $\mathrm{ft}$ & Water Level Standard Deviation, $\mathrm{ft}$ \\
\hline 2 & 2.9 & 1.1 \\
\hline 5 & 7.3 & 1.0 \\
\hline 10 & 10.8 & 1.6 \\
\hline 25 & 15.1 & 1.9 \\
\hline 50 & 17.8 & 2.4 \\
\hline 75 & 19.1 & 2.3 \\
\hline 100 & 19.8 & 2.5 \\
\hline
\end{tabular}




\begin{tabular}{|c|c|c|}
\hline \multicolumn{3}{|c|}{$\begin{array}{l}\text { Table B17 } \\
\text { Return Period, Maximum Water Level, and Water Level Standard } \\
\text { Deviation for Profile Tutuila } 016\end{array}$} \\
\hline Return Period, yr & Maximum Water Level, ft & Water Level Standard Deviation, $\mathrm{ft}$ \\
\hline 2 & 2.9 & 1.1 \\
\hline 5 & 7.0 & 0.7 \\
\hline 10 & 9.3 & 1.0 \\
\hline 25 & 12.4 & 1.5 \\
\hline 50 & 14.1 & 1.4 \\
\hline 75 & 15.0 & 1.4 \\
\hline 100 & 15.3 & 1.5 \\
\hline
\end{tabular}

\begin{tabular}{|c|c|c|}
\hline \multicolumn{3}{|c|}{$\begin{array}{l}\text { Table B18 } \\
\text { Return Period, Maximum Water Level, and Water Level Standard } \\
\text { Deviation for Profile Tutuila } 017\end{array}$} \\
\hline Return Period, yr & Maximum Water Level, $\mathrm{tt}$ & Water Level Standard Deviation, $\mathrm{ft}$ \\
\hline 2 & 2.9 & 1.1 \\
\hline 5 & 7.0 & 0.7 \\
\hline 10 & 9.3 & 1.0 \\
\hline 25 & 12.4 & 1.5 \\
\hline 50 & 14.1 & 1.4 \\
\hline 75 & 15.0 & 1.4 \\
\hline 100 & 15.3 & 1.5 \\
\hline
\end{tabular}

\begin{tabular}{|c|c|c|}
\hline \multicolumn{3}{|c|}{$\begin{array}{l}\text { Table B19 } \\
\text { Return Period, Maximum Water Level, and Water Level Standard } \\
\text { Deviation for Profile Tutuila } 018\end{array}$} \\
\hline Return Period, yr & Maximum Water Level, $\mathrm{tt}$ & Water Level Standard Deviation, $\mathrm{ft}$ \\
\hline 2 & 1.9 & 0.8 \\
\hline 5 & 5.5 & 0.7 \\
\hline 10 & 7.9 & 1.0 \\
\hline 25 & 10.8 & 1.3 \\
\hline 50 & 12.6 & 1.4 \\
\hline 75 & 13.4 & 1.1 \\
\hline 100 & 13.9 & 1.2 \\
\hline
\end{tabular}

\begin{tabular}{|c|c|c|}
\hline \multicolumn{3}{|c|}{$\begin{array}{l}\text { Table B20 } \\
\text { Return Period, Maximum Water Level, and Water Level Standard } \\
\text { Deviation for Profile Tutuila } 019\end{array}$} \\
\hline Return Period, yr & Maximum Water Level, ft & Water Level Standard Deviation, $\mathrm{ft}$ \\
\hline 2 & 1.9 & 0.8 \\
\hline 5 & 5.5 & 0.7 \\
\hline 10 & 7.9 & 1.0 \\
\hline 25 & 10.8 & 1.3 \\
\hline 50 & 12.6 & 1.4 \\
\hline 75 & 13.4 & 1.1 \\
\hline 100 & 13.9 & 1.2 \\
\hline
\end{tabular}




\begin{tabular}{|c|c|c|}
\hline $\begin{array}{l}\text { Table B21 } \\
\text { Return Perio } \\
\text { Deviation for }\end{array}$ & $\begin{array}{l}\text { Maximum Water Lev } \\
\text { ofile Tutuila } 020\end{array}$ & and Water Level Standard \\
\hline Return Period, yr & Maximum Water Level, ft & Water Level Standard Deviation, $\mathrm{ft}$ \\
\hline 2 & 1.9 & 0.8 \\
\hline 5 & 5.5 & 0.7 \\
\hline 10 & 7.9 & 1.0 \\
\hline 25 & 10.8 & 1.3 \\
\hline 50 & 12.6 & 1.4 \\
\hline 75 & 13.4 & 1.1 \\
\hline 100 & 13.9 & 1.2 \\
\hline
\end{tabular}

\begin{tabular}{|c|c|c|}
\hline \multicolumn{3}{|c|}{$\begin{array}{l}\text { Table B22 } \\
\text { Return Period, Maximum Water Level, and Water Level Standard } \\
\text { Deviation for Profile Tutuila } 021\end{array}$} \\
\hline Return Period, yr & Maximum Water Level, $\mathrm{ft}$ & Water Level Standard Deviation, $\mathrm{ft}$ \\
\hline 2 & 3.0 & 1.1 \\
\hline 5 & 7.6 & 0.8 \\
\hline 10 & 10.7 & 1.4 \\
\hline 25 & 15.1 & 2.0 \\
\hline 50 & 17.7 & 2.1 \\
\hline 75 & 18.9 & 1.8 \\
\hline 100 & 19.5 & 1.9 \\
\hline
\end{tabular}

\begin{tabular}{|c|c|c|}
\hline \multicolumn{3}{|c|}{$\begin{array}{l}\text { Table B23 } \\
\text { Return Period, Maximum Water Level, and Water Level Standard } \\
\text { Deviation for Profile Tutuila } 022\end{array}$} \\
\hline Return Period, yr & Maximum Water Level, $\mathrm{ft}$ & Water Level Standard Deviation, $\mathrm{ft}$ \\
\hline 2 & 2.8 & 1.1 \\
\hline 5 & 7.3 & 0.8 \\
\hline 10 & 10.7 & 1.5 \\
\hline 25 & 15.5 & 2.1 \\
\hline 50 & 18.2 & 2.2 \\
\hline 75 & 19.5 & 1.9 \\
\hline 100 & 20.1 & 2.0 \\
\hline
\end{tabular}

\begin{tabular}{|c|c|c|}
\hline \multicolumn{3}{|c|}{$\begin{array}{l}\text { Table B24 } \\
\text { Return Period, Maximum Water Level, and Water Level Standard } \\
\text { Deviation for Profile Tutuila } 023\end{array}$} \\
\hline Return Period, yr & Maximum Water Level, ft & Water Level Standard Deviation, $\mathrm{ft}$ \\
\hline 2 & 2.9 & (1) \\
\hline 5 & 7.1 & 0.8 \\
\hline 10 & 10.1 & 1.4 \\
\hline 25 & 14.6 & 2.0 \\
\hline 50 & 17.2 & 2.1 \\
\hline 75 & 18.5 & 1.9 \\
\hline 100 & 19.1 & 2.0 \\
\hline
\end{tabular}




\begin{tabular}{|l|l|l|}
\hline $\begin{array}{l}\text { Table B25 } \\
\text { Return Period, Maximum Water Level, and Water Level Standard } \\
\text { Deviation for Profile Tutuila 024 }\end{array}$ \\
\hline Return Period, $\mathbf{y}$ & Maximum Water Level, tt & Water Level Standard Deviation, $\mathbf{t t}$ \\
\hline 2 & 2.8 & 1.1 \\
\hline 5 & 7.2 & 0.8 \\
\hline 10 & 10.6 & 1.5 \\
\hline 25 & 15.2 & 2.0 \\
\hline 50 & 17.9 & 2.1 \\
\hline 75 & 19.1 & 1.8 \\
\hline 100 & 19.8 & 1.9 \\
\hline
\end{tabular}

\begin{tabular}{|c|c|c|}
\hline \multicolumn{3}{|c|}{$\begin{array}{l}\text { Table B26 } \\
\text { Return Period, Maximum Water Level, and Water Level Standard } \\
\text { Deviation for Profile Tutuila } 025\end{array}$} \\
\hline Return Period, $y r$ & Maximum Water Level, $\mathrm{tt}$ & Water Level Standard Deviation, ft \\
\hline 2 & 1.9 & 0.8 \\
\hline 5 & 5.4 & 0.6 \\
\hline 10 & 7.8 & 1.0 \\
\hline 25 & 10.7 & 1.3 \\
\hline 50 & 12.5 & 1.4 \\
\hline 75 & 13.3 & 1.1 \\
\hline 100 & 13.7 & 1.2 \\
\hline
\end{tabular}

\begin{tabular}{|c|c|c|}
\hline \multicolumn{3}{|c|}{$\begin{array}{l}\text { Table B27 } \\
\text { Return Period, Maximum Water Level, and Water Level Standard } \\
\text { Deviation for Profile Tutuila } 026\end{array}$} \\
\hline Return Period, yr & Maximum Water Level, ft & Water Level Standard Deviation, $\mathrm{ft}$ \\
\hline 2 & 2.0 & 0.8 \\
\hline 5 & 5.6 & 0.6 \\
\hline 10 & 7.5 & 0.8 \\
\hline 25 & 9.9 & 1.2 \\
\hline 50 & 11.8 & 1.8 \\
\hline 75 & 12.8 & 1.7 \\
\hline 100 & 13.3 & 1.8 \\
\hline
\end{tabular}

\begin{tabular}{|l|l|l|}
\hline $\begin{array}{l}\text { Table B28 } \\
\text { Return Period, Maximum Water Level, and Water Level Standard } \\
\text { Deviation for Profile Tutuila 027 }\end{array}$ \\
\hline Return Period, yr & Maximum Water Level, $\mathrm{tt}$ & Water Level Standard Deviation, $\mathrm{ft}$ \\
\hline 2 & 2.0 & 0.8 \\
\hline 5 & 5.6 & 0.6 \\
\hline 10 & 7.5 & 0.8 \\
\hline 25 & 9.9 & 1.2 \\
\hline 50 & 11.8 & 1.8 \\
\hline 75 & 12.8 & 1.7 \\
\hline 100 & 13.3 & 1.8 \\
\hline
\end{tabular}




\begin{tabular}{|c|c|c|}
\hline \multicolumn{3}{|c|}{$\begin{array}{l}\text { Table B29 } \\
\text { Return Period, Maximum Water Level, and Water Level Standard } \\
\text { Deviation for Profile Tutuila } 028\end{array}$} \\
\hline Return Period, yr & Maximum Water Level, $\mathrm{ft}$ & Water Level Standard Deviation, ft \\
\hline 2 & 2.0 & 0.8 \\
\hline 5 & 5.6 & 0.6 \\
\hline 10 & 7.5 & 0.8 \\
\hline 25 & 9.9 & 1.2 \\
\hline 50 & 11.8 & 1.8 \\
\hline 75 & 12.8 & 1.7 \\
\hline 100 & 13.3 & 1.8 \\
\hline
\end{tabular}

\begin{tabular}{|c|c|c|}
\hline \multicolumn{3}{|c|}{$\begin{array}{l}\text { Table B30 } \\
\text { Return Period, Maximum Water Level, and Water Level Standard } \\
\text { Deviation for Profile Tutuila } 029\end{array}$} \\
\hline Return Period, yr & Maximum Water Level, $\mathrm{ft}$ & Water Level Standard Deviation, $\mathrm{ft}$ \\
\hline 2 & 2.0 & 0.8 \\
\hline 5 & 5.6 & 0.6 \\
\hline 10 & 7.5 & 0.8 \\
\hline 25 & 9.9 & 1.2 \\
\hline 50 & 11.8 & 1.8 \\
\hline 75 & 12.8 & 1.7 \\
\hline 100 & 13.3 & 1.8 \\
\hline
\end{tabular}

\begin{tabular}{|c|c|c|}
\hline \multicolumn{3}{|c|}{$\begin{array}{l}\text { Table B31 } \\
\text { Return Period, Maximum Water Level, and Water Level Standard } \\
\text { Deviation for Profile Tutuila 029A }\end{array}$} \\
\hline Return Period, yr & Maximum Water Level, ft & Water Level Standard Deviation, $\mathrm{ft}$ \\
\hline 2 & 1.4 & 0.7 \\
\hline 5 & 4.6 & 0.6 \\
\hline 10 & 6.4 & 0.7 \\
\hline 25 & 8.4 & 1.0 \\
\hline 50 & 9.9 & 1.4 \\
\hline 75 & 10.7 & 1.4 \\
\hline 100 & 11.2 & 1.5 \\
\hline
\end{tabular}

\begin{tabular}{|c|c|c|}
\hline \multicolumn{3}{|c|}{$\begin{array}{l}\text { Table B32 } \\
\text { Return Period, Maximum Water Level, and Water Level Standard } \\
\text { Deviation for Profile Tutuila } 030\end{array}$} \\
\hline Return Period, yr & Maximum Water Level, $\mathrm{ft}$ & Water Level Standard Deviation, $\mathrm{ft}$ \\
\hline 2 & 2.0 & 0.8 \\
\hline 5 & 5.6 & 0.6 \\
\hline 10 & 7.5 & 0.8 \\
\hline 25 & 9.9 & 1.2 \\
\hline 50 & 11.8 & 1.8 \\
\hline 75 & 12.8 & 1.7 \\
\hline 100 & 13.3 & 1.8 \\
\hline
\end{tabular}




\begin{tabular}{|l|l|l|}
\hline $\begin{array}{l}\text { Table B33 } \\
\text { Return Period, Maximum Water Level, and Water Level Standard } \\
\text { Deviation for Profile Tutuila 031 }\end{array}$ \\
\hline Return Period, yr & Maximum Water Level, ft & Water Level Standard Deviation, ft \\
\hline 2 & 2.0 & 0.8 \\
\hline 5 & 5.6 & 0.6 \\
\hline 10 & 7.5 & 0.8 \\
\hline 25 & 9.9 & 1.2 \\
\hline 50 & 11.8 & 1.8 \\
\hline 75 & 12.8 & 1.7 \\
\hline 100 & 13.3 & 1.8 \\
\hline
\end{tabular}

\begin{tabular}{|l|l|l|}
\hline $\begin{array}{l}\text { Table B34 } \\
\text { Return Period, Maximum Water Level, and Water Level Standard } \\
\text { Deviation for Profile Tutuila 032 }\end{array}$ \\
\hline Return Period, $y \boldsymbol{r}$ & Maximum Water Level, $\mathrm{ft}$ & Water Level Standard Deviation, $\mathrm{ft}$ \\
\hline 2 & 2.0 & 0.8 \\
\hline 5 & 5.6 & 0.6 \\
\hline 10 & 7.5 & 0.8 \\
\hline 25 & 9.9 & 1.2 \\
\hline 50 & 11.8 & 1.8 \\
\hline 75 & 12.8 & 1.7 \\
\hline 100 & 13.3 & 1.8 \\
\hline
\end{tabular}

\begin{tabular}{|l|l|l|}
\hline $\begin{array}{l}\text { Table B35 } \\
\text { Return Period, Maximum Water Level, and Water Level Standard } \\
\text { Deviation for Profile Tutuila 033 }\end{array}$ \\
\hline Return Period, yr & Maximum Water Level, ,t & Water Level Standard Deviation, ft \\
\hline 2 & 2.0 & 0.8 \\
\hline 5 & 5.6 & 0.6 \\
\hline 10 & 7.5 & 0.8 \\
\hline 25 & 9.9 & 1.2 \\
\hline 50 & 11.8 & 1.8 \\
\hline 75 & 12.8 & 1.7 \\
\hline 100 & 13.3 & 1.8 \\
\hline
\end{tabular}

\begin{tabular}{|c|c|c|}
\hline Return Period, yr & Maximum Water Level, ft & Water Level Standard Deviation, ft \\
\hline 2 & 1.6 & 0.6 \\
\hline 5 & 3.6 & 0.3 \\
\hline 10 & 4.4 & 0.4 \\
\hline 25 & 5.5 & 0.6 \\
\hline 50 & 6.3 & 0.7 \\
\hline 75 & 6.7 & 0.6 \\
\hline 100 & 6.9 & 0.6 \\
\hline
\end{tabular}

B10 


\begin{tabular}{|c|c|c|}
\hline \multicolumn{3}{|c|}{$\begin{array}{l}\text { Table B37 } \\
\text { Return Period, Maximum Water Level, and Water Level Standard } \\
\text { Deviation for Profile Tutuila } 035\end{array}$} \\
\hline Return Period, $y$ & Maximum Water Level, it & Water Level Standard Deviation, ft \\
\hline 2 & 1.6 & 0.6 \\
\hline 5 & 4.5 & 1.2 \\
\hline 10 & 8.8 & 1.4 \\
\hline 25 & 12.0 & 1.0 \\
\hline 50 & 13.1 & 0.9 \\
\hline 75 & 13.6 & 0.6 \\
\hline 100 & 13.8 & 0.6 \\
\hline
\end{tabular}

\begin{tabular}{|c|c|c|}
\hline \multicolumn{3}{|c|}{$\begin{array}{l}\text { Table B38 } \\
\text { Return Period, Maximum Water Level, and Water Level Standard } \\
\text { Deviation for Profile Tutuila } 036\end{array}$} \\
\hline Return Period, $\mathbf{y}$ & Maximum Water Level, $\mathrm{ft}$ & Water Level Standard Deviation, $\mathrm{ft}$ \\
\hline 2 & 1.5 & 0.6 \\
\hline 5 & 3.5 & 0.3 \\
\hline 10 & 4.3 & 0.3 \\
\hline 25 & 5.4 & 0.6 \\
\hline 50 & 6.2 & 0.7 \\
\hline 75 & 6.6 & 0.6 \\
\hline 100 & 6.8 & 0.6 \\
\hline
\end{tabular}

\begin{tabular}{|l|l|l|}
\hline $\begin{array}{l}\text { Table B39 } \\
\text { Return Period, Maximum Water Level, and Water Level Standard } \\
\text { Deviation for Profile Tutuila 037 }\end{array}$ \\
\hline Return Period, yr & Maximum Water Level, $\mathrm{ft}$ & Water Level Standard Deviation, $\mathrm{ft}$ \\
\hline 2 & 1.5 & 0.6 \\
\hline 5 & 3.5 & 0.3 \\
\hline 10 & 4.3 & 0.3 \\
\hline 25 & 5.4 & 0.6 \\
\hline 50 & 6.2 & 0.7 \\
\hline 75 & 6.6 & 0.6 \\
\hline 100 & 6.8 & 0.6 \\
\hline
\end{tabular}

\begin{tabular}{|l|l|l|}
\hline $\begin{array}{l}\text { Table B40 } \\
\text { Return Period, Maximum Water Level, and Water Level Standard } \\
\text { Deviation for Profile Tutuila } \mathbf{0 3 8}\end{array}$ \\
\hline Return Period, yr & Maximum Water Level, $\mathrm{ft}$ & Water Level Standard Deviation, $\mathrm{ft}$ \\
\hline 2 & 1.5 & 0.6 \\
\hline 5 & 3.5 & 0.3 \\
\hline 10 & 4.3 & 0.3 \\
\hline 25 & 5.4 & 0.6 \\
\hline 50 & 6.2 & 0.7 \\
\hline 75 & 6.6 & 0.6 \\
\hline 100 & 6.8 & 0.7 \\
\hline
\end{tabular}




\begin{tabular}{|l|l|l|}
\hline $\begin{array}{l}\text { Table B41 } \\
\text { Return Period, Maximum Water Level, and Water Level Standard } \\
\text { Deviation for Profile Tutuila 039 }\end{array}$ \\
\hline Return Period, yr & Maximum Water Level, ft & Water Level Standard Deviation, $\mathrm{ft}$ \\
\hline 2 & 1.5 & 0.6 \\
\hline 5 & 3.5 & 0.3 \\
\hline 10 & 4.3 & 0.3 \\
\hline 25 & 5.4 & 0.6 \\
\hline 50 & 6.2 & 0.7 \\
\hline 75 & 6.6 & 0.6 \\
\hline 100 & 6.8 & 0.7 \\
\hline
\end{tabular}

\begin{tabular}{|c|c|c|}
\hline \multicolumn{3}{|c|}{$\begin{array}{l}\text { Table B42 } \\
\text { Return Period, Maximum Water Level, and Water Level Standard } \\
\text { Deviation for Profile Tutuila } 040\end{array}$} \\
\hline Return Period, yr & Maximum Water Level, $\mathrm{ft}$ & Water Level Standard Deviation, $\mathrm{tt}$ \\
\hline 2 & 1.5 & 0.6 \\
\hline 5 & 3.5 & 0.3 \\
\hline 10 & 4.3 & 0.3 \\
\hline 25 & 5.4 & 0.6 \\
\hline 50 & 6.2 & 0.7 \\
\hline 75 & 6.6 & 0.6 \\
\hline 100 & 6.8 & 0.7 \\
\hline
\end{tabular}

\begin{tabular}{|l|l|l|}
\hline $\begin{array}{l}\text { Table B43 } \\
\text { Return Period, Maximum Water Level, and Water Level Standard } \\
\text { Deviation for Profile Tutuila 041 }\end{array}$ \\
\hline Return Period, yr & Maximum Water Level, $\mathrm{ft}$ & Water Level Standard Deviation, $\mathrm{ft}$ \\
\hline 2 & 1.5 & 0.6 \\
\hline 5 & 3.5 & 0.3 \\
\hline 10 & 4.3 & 0.3 \\
\hline 25 & 5.4 & 0.6 \\
\hline 50 & 6.2 & 0.7 \\
\hline 75 & 6.6 & 0.6 \\
\hline 100 & 6.8 & 0.7 \\
\hline
\end{tabular}

\begin{tabular}{|l|l|l|}
\hline $\begin{array}{l}\text { Table B44 } \\
\text { Return Period, Maximum Water Level, and Water Level Standard } \\
\text { Deviation for Profile Tutuila 042 }\end{array}$ \\
\hline Return Period, yr & Maximum Water Level, ft & Water Level Standard Deviation, $\mathrm{ft}$ \\
\hline 2 & 1.5 & 0.6 \\
\hline 5 & 3.5 & 0.3 \\
\hline 10 & 4.3 & 0.3 \\
\hline 25 & 5.4 & 0.6 \\
\hline 50 & 6.2 & 0.7 \\
\hline 75 & 6.6 & 0.6 \\
\hline 100 & 6.8 & 0.7 \\
\hline
\end{tabular}




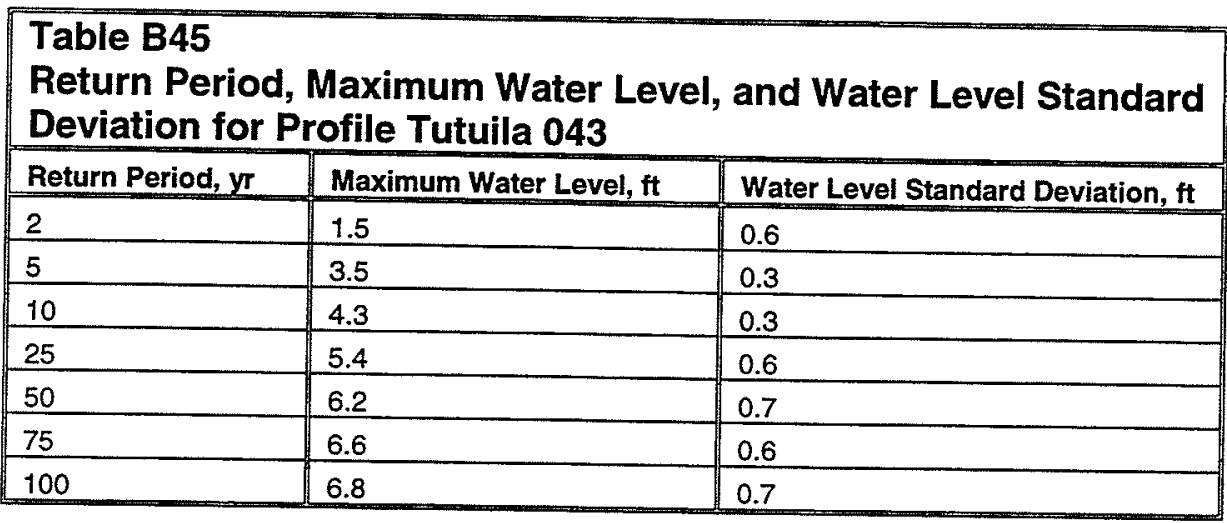

\begin{tabular}{|c|c|c|}
\hline \multicolumn{3}{|c|}{$\begin{array}{l}\text { Table B46 } \\
\text { Return Period, Maximum Water Level, and Water Level Standard } \\
\text { Deviation for Profile Tutuila } 044\end{array}$} \\
\hline Return Period, yr & Maximum Water Level, $\mathrm{ft}$ & Water Level Standard Deviation, $\mathrm{ft}$ \\
\hline 2 & 1.7 & 0.7 \\
\hline 5 & 4.3 & 0.5 \\
\hline 10 & 6.2 & 1.1 \\
\hline 25 & 9.9 & 1.5 \\
\hline 50 & 11.8 & 1.4 \\
\hline 75 & 12.6 & 1.2 \\
\hline 100 & 13.0 & 1.2 \\
\hline
\end{tabular}

\begin{tabular}{|c|c|c|}
\hline \multicolumn{3}{|c|}{$\begin{array}{l}\text { Table B47 } \\
\text { Return Period, Maximum Water Level, and Water Level Standard } \\
\text { Deviation for Profile Tutuila } 045\end{array}$} \\
\hline Return Period, yr & Maximum Water Level, $\mathrm{ft}$ & Water Level Standard Deviation, $\mathrm{ft}$ \\
\hline 2 & 1.7 & 0.7 \\
\hline 5 & 4.5 & 0.6 \\
\hline 10 & 7.1 & 1.4 \\
\hline 25 & 10.7 & 1.3 \\
\hline 50 & 12.4 & 1.0 \\
\hline 75 & 13.0 & 0.9 \\
\hline 100 & 13.4 & 0.9 \\
\hline
\end{tabular}

\begin{tabular}{|c|c|c|}
\hline \multicolumn{3}{|c|}{$\begin{array}{l}\text { Table B48 } \\
\text { Return Period, Maximum Water Level, and Water Level Standard } \\
\text { Deviation for Profile Tutuila } 046\end{array}$} \\
\hline Return Period, yr & Maximum Water Level, $\mathrm{ft}$ & Water Level Standard Deviation, $\mathrm{ft}$ \\
\hline 2 & 1.7 & 0.7 \\
\hline 5 & 4.3 & 0.5 \\
\hline 10 & 6.2 & 1.1 \\
\hline 25 & 9.9 & 1.5 \\
\hline 50 & 11.8 & 1.4 \\
\hline 75 & 12.6 & 1.2 \\
\hline 100 & 13.0 & 1.2 \\
\hline
\end{tabular}




\begin{tabular}{|c|c|c|}
\hline \multicolumn{3}{|c|}{$\begin{array}{l}\text { Table B49 } \\
\text { Return Period, Maximum Water Level, and Water Level Standard } \\
\text { Deviation for Profile Tutuila } 047\end{array}$} \\
\hline Return Period, yr & Maximum Water Level, $\mathrm{ft}$ & Water Level Standard Deviation, ft \\
\hline 2 & 1.7 & 0.7 \\
\hline 5 & 4.3 & 0.5 \\
\hline 10 & 6.2 & 1.1 \\
\hline 25 & 9.9 & 1.5 \\
\hline 50 & 11.8 & 1.4 \\
\hline 75 & 12.6 & 1.2 \\
\hline 100 & 13.0 & 1.2 \\
\hline
\end{tabular}

\begin{tabular}{|c|c|c|}
\hline \\
\hline \multicolumn{3}{|c|}{ 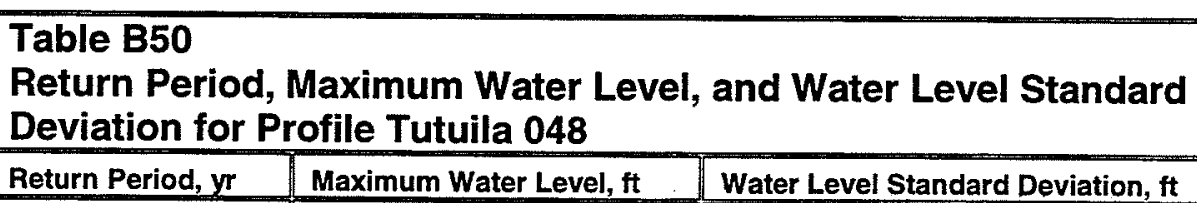 } \\
\hline \multicolumn{3}{|c|}{\begin{tabular}{|l|l|l|}
2 & 1.7 & 0.7 \\
\end{tabular}} \\
\hline 5 & 4.3 & 0.5 \\
\hline 10 & 6.2 & 1.1 \\
\hline \multirow{2}{*}{$\frac{25}{50}$} & 9.9 & 1.5 \\
\hline & 11.8 & 1.4 \\
\hline \multirow{2}{*}{$\frac{75}{100}$} & 12.6 & 1.2 \\
\hline & 13.0 & 1.2 \\
\hline
\end{tabular}

\begin{tabular}{|l|l|l|}
\hline $\begin{array}{l}\text { Table B51 } \\
\text { Return Period, Maximum Water Level, and Water Level Standard } \\
\text { Deviation for Profile Tutuila 049 }\end{array}$ \\
\hline Return Period, yr & Maximum Water Level, ft & Water Level Standard Deviation, ft \\
\hline 2 & 1.9 & 0.8 \\
\hline 5 & 5.3 & 0.6 \\
\hline 10 & 7.4 & 1.0 \\
\hline 25 & 10.5 & 1.4 \\
\hline 50 & 12.4 & 1.5 \\
\hline 75 & 13.3 & 1.3 \\
\hline 100 & 13.7 & 1.4 \\
\hline
\end{tabular}

\section{Table B52}

Return Period, Maximum Water Level, and Water Level Standard Deviation for Profile Tutuila 050

\begin{tabular}{|l|l|l|}
\hline Return Period, yr & Maximum Water Level, $\mathrm{ft}$ & Water Level Standard Deviation, $\mathrm{ft}$ \\
\hline 2 & 1.6 & 0.7 \\
\hline 5 & 4.2 & 0.5 \\
\hline 10 & 6.0 & 1.1 \\
\hline 25 & 9.6 & 1.5 \\
\hline 50 & 11.5 & 1.4 \\
\hline 75 & 12.2 & 1.1 \\
\hline 100 & 12.6 & 1.2 \\
\hline
\end{tabular}

B14 


\begin{tabular}{|c|c|c|}
\hline \multicolumn{3}{|c|}{$\begin{array}{l}\text { Table B53 } \\
\text { Return Period, Maximum Water Level, and Water Level Standard } \\
\text { Deviation for Profile Tutuila } 051\end{array}$} \\
\hline Return Period, yr & Maximum Water Level, $\mathrm{ft}$ & Water Level Standard Deviation, $\mathrm{ft}$ \\
\hline 2 & 1.6 & 0.7 \\
\hline 5 & 4.2 & 0.5 \\
\hline 10 & 6.0 & 1.1 \\
\hline 25 & 9.6 & 1.5 \\
\hline 50 & 11.5 & 1.4 \\
\hline 75 & 12.2 & 1.1 \\
\hline 100 & 12.6 & 1.2 \\
\hline
\end{tabular}

\begin{tabular}{|c|c|c|}
\hline \multicolumn{3}{|c|}{$\begin{array}{l}\text { Table B54 } \\
\text { Return Period, Maximum Water Level, and Water Level Standard } \\
\text { Deviation for Profile Tutuila } 052\end{array}$} \\
\hline Return Period, yr & Maximum Water Level, ft & Water Level Standard Deviation, $\mathrm{ft}$ \\
\hline$\underline{2}$ & 1.6 & 0.7 \\
\hline 5 & 4.2 & 0.5 \\
\hline 10 & 6.0 & 1.1 \\
\hline 25 & 9.6 & 1.5 \\
\hline 50 & 11.5 & 1.4 \\
\hline 75 & 12.2 & 1.1 \\
\hline 100 & 12.6 & 1.2 \\
\hline
\end{tabular}

\begin{tabular}{|c|c|c|}
\hline \multicolumn{3}{|c|}{$\begin{array}{l}\text { Table B55 } \\
\text { Return Period, Maximum Water Level, and Water Level Standard } \\
\text { Deviation for Profile Tutuila } 053\end{array}$} \\
\hline Return Period, yr & Maximum Water Level, ft & Water Level Standard Deviation, $\mathrm{ft}$ \\
\hline 2 & 1.6 & 0.7 \\
\hline 5 & 4.2 & 0.5 \\
\hline 10 & 6.0 & 1.1 \\
\hline 25 & 9.6 & 1.5 \\
\hline 50 & 11.5 & 1.4 \\
\hline 75 & 12.2 & 1.1 \\
\hline 100 & 12.6 & 1.2 \\
\hline
\end{tabular}

\begin{tabular}{|l|l|l|}
\hline $\begin{array}{l}\text { Table B56 } \\
\text { Return Period, Maximum Water Level, and Water Level Standard } \\
\text { Deviation for Profile Tutuila 054 }\end{array}$ \\
\hline Return Period, yr & Maximum Water Level, ft & Water Level Standard Deviation, ft \\
\hline 2 & 1.6 & 0.7 \\
\hline 5 & 4.2 & 0.5 \\
\hline 10 & 6.0 & 1.1 \\
\hline 25 & 9.6 & 1.5 \\
\hline 50 & 11.5 & 1.4 \\
\hline 75 & 12.2 & 1.1 \\
\hline 100 & 12.6 & 1.2 \\
\hline
\end{tabular}




\begin{tabular}{|l|l|l|}
\hline $\begin{array}{l}\text { Table B57 } \\
\text { Return Period, Maximum Water Level, and Water Level Standard } \\
\text { Deviation for Profile Tutuila 055 }\end{array}$ \\
\hline Return Period, yr & Maximum Water Level, ft & Water Level Standard Deviation, ft \\
\hline 2 & 1.6 & 0.7 \\
\hline 5 & 4.2 & 0.5 \\
\hline 10 & 6.0 & 1.1 \\
\hline 25 & 9.6 & 1.5 \\
\hline 50 & 11.5 & 1.4 \\
\hline 75 & 12.2 & 1.1 \\
\hline 100 & 12.6 & 1.2 \\
\hline
\end{tabular}

\begin{tabular}{|l|l|l|}
\hline $\begin{array}{l}\text { Table B58 } \\
\text { Return Period, Maximum Water Level, and Water Level Standard } \\
\text { Deviation for Profile Tutuila 056 }\end{array}$ \\
\hline Return Period, yr & Maximum Water Level, $\mathrm{ft}$ & Water Level Standard Deviation, $\mathrm{ft}$ \\
\hline 2 & 1.7 & 0.7 \\
\hline 5 & 4.3 & 0.5 \\
\hline 10 & 6.0 & 0.9 \\
\hline 25 & 9.0 & 1.3 \\
\hline 50 & 10.7 & 1.2 \\
\hline 75 & 11.4 & 0.1 \\
\hline 100 & 11.7 & 1.1 \\
\hline
\end{tabular}

\begin{tabular}{|c|c|c|}
\hline \multicolumn{3}{|c|}{$\begin{array}{l}\text { Table B59 } \\
\text { Return Period, Maximum Water Level, and Water Level Standard } \\
\text { Deviation for Profile Tutuila } 057\end{array}$} \\
\hline Return Period, yr & Maximum Water Level, ft & Water Level Standard Deviation, ft \\
\hline 2 & 1.7 & 0.7 \\
\hline 5 & 4.4 & 0.5 \\
\hline 10 & 6.1 & 0.9 \\
\hline 25 & 8.8 & 1.2 \\
\hline 50 & 10.4 & 1.2 \\
\hline 75 & 11.1 & 1.1 \\
\hline 100 & 11.5 & 1.1 \\
\hline
\end{tabular}

\begin{tabular}{|c|c|c|}
\hline \multicolumn{3}{|c|}{$\begin{array}{l}\text { Table B60 } \\
\text { Return Period, Maximum Water Level, and Water Level Standard } \\
\text { Deviation for Profile Tutuila } 058\end{array}$} \\
\hline Return Period, yr & Maximum Water Level, $\mathrm{ft}$ & Water Level Standard Deviation, $\mathrm{ft}$ \\
\hline 2 & 1.7 & 0.7 \\
\hline 5 & 4.5 & 0.5 \\
\hline 10 & 6.1 & 0.8 \\
\hline 25 & 8.7 & 1.2 \\
\hline 50 & 10.3 & 1.1 \\
\hline 75 & 11.0 & 1.0 \\
\hline 100 & 11.3 & 1.0 \\
\hline
\end{tabular}

B16 


\begin{tabular}{|c|c|c|}
\hline \multicolumn{3}{|c|}{$\begin{array}{l}\text { Table B61 } \\
\text { Return Period, Maximum Water Level, and Water Level Standard } \\
\text { Deviation for Profile Tutuila } 059\end{array}$} \\
\hline Return Period, yr & Maximum Water Level, ft & Water Level Standard Deviation, $\mathrm{ft}$ \\
\hline$\underline{2}$ & 1.8 & 0.8 \\
\hline 5 & 4.6 & 0.5 \\
\hline 10 & 6.2 & 0.8 \\
\hline 25 & 8.4 & 1.0 \\
\hline 50 & 9.8 & 1.0 \\
\hline 75 & 10.5 & 0.9 \\
\hline 100 & 10.8 & 0.9 \\
\hline
\end{tabular}

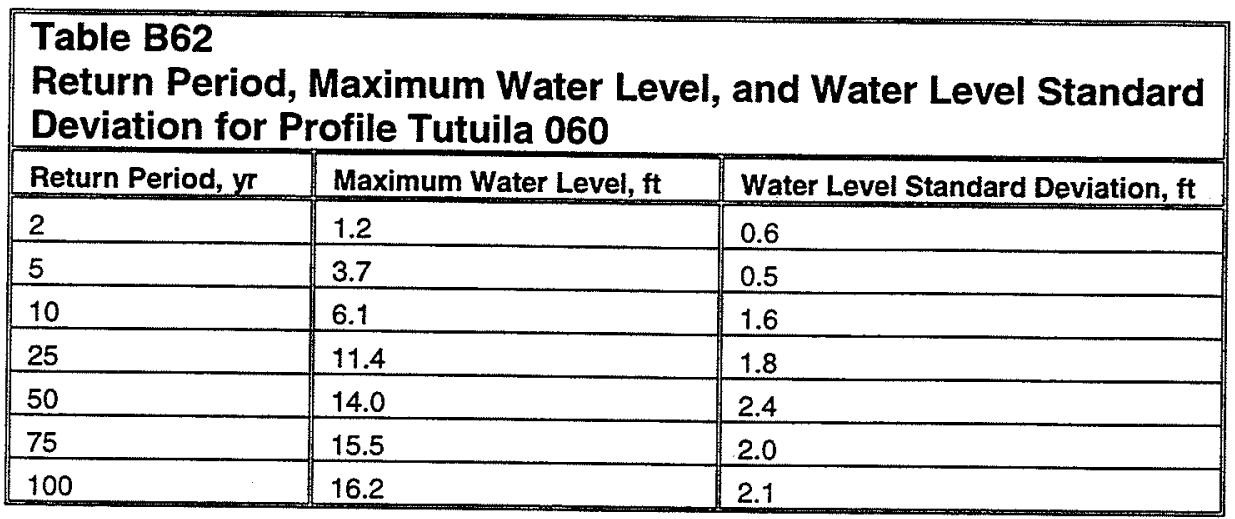

\begin{tabular}{|c|c|c|}
\hline \multicolumn{3}{|c|}{$\begin{array}{l}\text { Table B63 } \\
\text { Return Period, Maximum Water Level, and Water Level Standard } \\
\text { Deviation for Profile Tutuila } 061\end{array}$} \\
\hline Return Period, yr & Maximum Water Level, $\mathrm{ft}$ & Water Level Standard Deviation, $\mathrm{ft}$ \\
\hline 2 & 1.2 & 0.6 \\
\hline 5 & 3.7 & 0.5 \\
\hline 10 & 5.6 & 1.0 \\
\hline 25 & 8.5 & 1.1 \\
\hline 50 & 10.1 & 1.1 \\
\hline 75 & 10.8 & 1.1 \\
\hline 100 & 11.2 & 1.2 \\
\hline
\end{tabular}

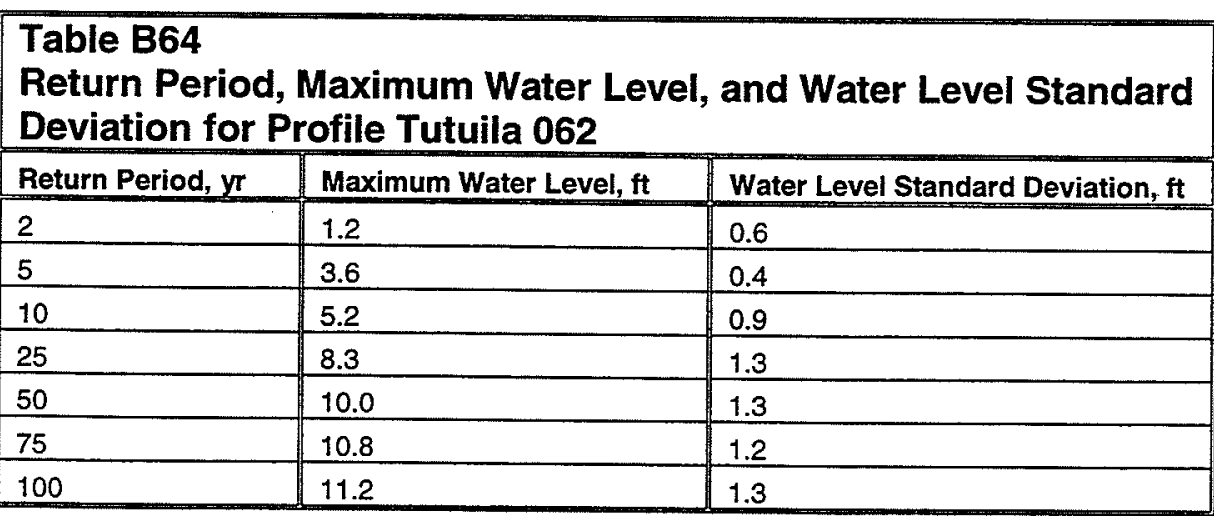




\begin{tabular}{|c|c|c|}
\hline \multicolumn{3}{|c|}{$\begin{array}{l}\text { Table B65 } \\
\text { Return Period, Maximum Water Level, and Water Level Standard } \\
\text { Deviation for Profile Tutuila } 063\end{array}$} \\
\hline Return Period, yr & Maximum Water Level, $\mathrm{ft}$ & Water Level Standard Deviation, ft \\
\hline 2 & 2.3 & 1.1 \\
\hline 5 & 9.5 & 1.2 \\
\hline 10 & 12.2 & 1.1 \\
\hline 25 & 15.1 & 1.4 \\
\hline 50 & 17.2 & 1.6 \\
\hline 75 & 18.2 & 1.6 \\
\hline 100 & 18.7 & 1.7 \\
\hline
\end{tabular}

\begin{tabular}{|c|c|c|}
\hline \multicolumn{3}{|c|}{$\begin{array}{l}\text { Table B66 } \\
\text { Return Period, Maximum Water Level, and Water Level Standard } \\
\text { Deviation for Profile Tutuila } 064\end{array}$} \\
\hline Return Period, yr & Maximum Water Level, $\mathrm{ft}$ & Water Level Standard Deviation, ft \\
\hline 2 & 2.3 & 1.1 \\
\hline 5 & 9.4 & 1.2 \\
\hline 10 & 12.1 & 1.1 \\
\hline 25 & 15.0 & 1.4 \\
\hline 50 & 17.1 & 1.7 \\
\hline 75 & 18.1 & 1.6 \\
\hline 100 & 18.6 & 1.8 \\
\hline
\end{tabular}

\begin{tabular}{|c|c|c|}
\hline \multicolumn{3}{|c|}{$\begin{array}{l}\text { Table B67 } \\
\text { Return Period, Maximum Water Level, and Water Level Standard } \\
\text { Deviation for Profile Tutuila } 065\end{array}$} \\
\hline Return Period, yr & Maximum Water Level, ft & Water Level Standard Deviation, $\mathrm{ft}$ \\
\hline 2 & 2.3 & 1.1 \\
\hline 5 & 8.0 & 0.8 \\
\hline 10 & 10.0 & 0.9 \\
\hline 25 & 12.4 & 1.2 \\
\hline 50 & 14.1 & 1.4 \\
\hline 75 & 14.8 & 1.2 \\
\hline 100 & 15.2 & 1.2 \\
\hline
\end{tabular}

\begin{tabular}{|l|l|l|}
\hline $\begin{array}{l}\text { Table B68 } \\
\text { Return Period, Maximum Water Level, and Water Level Standard } \\
\text { Deviation for Profile Tutuila 066 }\end{array}$ \\
\hline Return Period, $\mathbf{y r}$ & Maximum Water Level, $\mathrm{ft}$ & Water Level Standard Deviation, $\mathrm{ft}$ \\
\hline 2 & 2.3 & 1.1 \\
\hline 5 & 8.0 & 0.8 \\
\hline 10 & 10.0 & 0.9 \\
\hline 25 & 12.4 & 1.2 \\
\hline 50 & 14.1 & 1.4 \\
\hline 75 & 14.8 & 1.2 \\
\hline 100 & 15.2 & 1.2 \\
\hline
\end{tabular}




\section{Table B69}

Return Period, Maximum Water Level, and Water Level Standard Deviation for Profile Tutuila 067

\begin{tabular}{|l|l|l|}
\hline Return Period, yr & Maximum Water Level, $\mathrm{ft}$ & Water Level Standard Deviation, ft \\
\hline 2 & 2.3 & 1.1 \\
\hline 5 & 8.0 & 0.9 \\
\hline 10 & 10.5 & 1.1 \\
\hline 25 & 13.8 & 1.6 \\
\hline 50 & 16.3 & 2.1 \\
\hline 75 & 17.6 & 2.0 \\
\hline 100 & 18.2 & 2.1 \\
\hline
\end{tabular}

\begin{tabular}{|c|c|c|}
\hline \multicolumn{3}{|c|}{$\begin{array}{l}\text { Table B70 } \\
\text { Return Period, Maximum Water Level, and Water Level Standard } \\
\text { Deviation for Profile Tutuila } 068\end{array}$} \\
\hline Return Period, yr & Maximum Water Level, $\mathrm{ft}$ & Water Level Standard Deviation, ft \\
\hline 2 & 2.2 & 1.0 \\
\hline 5 & 7.2 & 0.7 \\
\hline 10 & 9.1 & 0.8 \\
\hline 25 & 11.2 & 1.0 \\
\hline 50 & 13.0 & 1.6 \\
\hline 75 & 13.7 & 1.5 \\
\hline 100 & 14.1 & 1.6 \\
\hline
\end{tabular}

\section{Table B71}

Return Period, Maximum Water Level, and Water Level Standard Deviation for Profile Tutuila 069

\begin{tabular}{|l|l|l|}
\hline Return Period, yr & Maximum Water Level, ft & Water Level Standard Deviation, $\mathrm{ft}$ \\
\hline 2 & 2.2 & 1.0 \\
\hline 5 & 7.2 & 0.7 \\
\hline 10 & 9.1 & 0.8 \\
\hline 25 & 11.2 & 1.0 \\
\hline 50 & 13.0 & 1.6 \\
\hline 75 & 13.7 & 1.5 \\
\hline 100 & 14.1 & 1.6 \\
\hline
\end{tabular}

\section{Table B72}

Return Period, Maximum Water Level, and Water Level Standard Deviation for Profile Tutuila 070

\begin{tabular}{|l|l|l|}
\hline Return Period, $y \mathbf{r}$ & Maximum Water Level, ft & Water Level Standard Deviation, $\mathrm{ft}$ \\
\hline 2 & 2.2 & 1.0 \\
\hline 5 & 7.2 & 0.7 \\
\hline 10 & 9.1 & 0.8 \\
\hline 25 & 11.2 & 1.0 \\
\hline 50 & 13.0 & 1.6 \\
\hline 75 & 13.7 & 1.5 \\
\hline 100 & 14.1 & 1.6 \\
\hline
\end{tabular}




\begin{tabular}{|c|c|c|}
\hline \multicolumn{3}{|c|}{$\begin{array}{l}\text { Table B73 } \\
\text { Return Period, Maximum Water Level, and Water Level Standard } \\
\text { Deviation for Profile Tutuila } 071\end{array}$} \\
\hline Return Period, yr & Maximum Water Level, $\mathrm{ft}$ & Water Level Standard Deviation, $\mathrm{tt}$ \\
\hline 2 & 2.2 & 1.0 \\
\hline 5 & 7.2 & 0.7 \\
\hline 10 & 9.1 & 0.8 \\
\hline 25 & 11.2 & 1.0 \\
\hline 50 & 13.0 & 1.6 \\
\hline 75 & 13.7 & 1.5 \\
\hline 100 & 14.1 & 1.6 \\
\hline
\end{tabular}

\begin{tabular}{|c|c|c|}
\hline \multicolumn{3}{|c|}{$\begin{array}{l}\text { Table B74 } \\
\text { Return Period, Maximum Water Level, and Water Level Standard } \\
\text { Deviation for Profile Tutuila } 072\end{array}$} \\
\hline Return Period, yr & Maximum Water Level, ft & Water Level Standard Deviation, ft \\
\hline 2 & 2.2 & 1.0 \\
\hline 5 & 7.2 & 0.7 \\
\hline 10 & 9.1 & 0.8 \\
\hline 25 & 11.2 & 1.0 \\
\hline 50 & 13.0 & 1.6 \\
\hline 75 & 13.7 & 1.5 \\
\hline 100 & 14.1 & 1.6 \\
\hline
\end{tabular}

\begin{tabular}{|c|c|c|}
\hline \multicolumn{3}{|c|}{$\begin{array}{l}\text { Table B75 } \\
\text { Return Period, Maximum Water Level, and Water Level Standard } \\
\text { Deviation for Profile Tutuila } 073\end{array}$} \\
\hline Return Period, yr & Maximum Water Level, $\mathrm{ft}$ & Water Level Standard Deviation, $\mathrm{ft}$ \\
\hline 2 & 2.2 & 1.0 \\
\hline 5 & 7.2 & 0.7 \\
\hline 10 & 9.1 & 0.8 \\
\hline 25 & 11.2 & 1.0 \\
\hline 50 & 13.0 & 1.6 \\
\hline 75 & 13.7 & 1.5 \\
\hline 100 & 14.1 & 1.6 \\
\hline
\end{tabular}

\section{Table B76}

Return Period, Maximum Water Level, and Water Level Standard Deviation for Profile Tutuila 074

\begin{tabular}{|l|l|l|}
\hline Return Period, yr & Maximum Water Level, $\mathrm{ft}$ & Water Level Standard Deviation, $\mathrm{ft}$ \\
\hline 2 & 2.2 & 1.0 \\
\hline 5 & 7.2 & 0.7 \\
\hline 10 & 9.1 & 0.8 \\
\hline 25 & 11.2 & 1.0 \\
\hline 50 & 13.0 & 1.6 \\
\hline 75 & 13.7 & 1.5 \\
\hline 100 & 14.1 & 1.6 \\
\hline
\end{tabular}




\begin{tabular}{|c|c|c|}
\hline \multicolumn{3}{|c|}{$\begin{array}{l}\text { Table B77 } \\
\text { Return Period, Maximum Water Level, and Water Level Standard } \\
\text { Deviation for Profile Tutuila } 075\end{array}$} \\
\hline Return Period, yr & Maximum Water Level, $\mathrm{tt}$ & Water Level Standard Deviation, $\mathrm{ft}$ \\
\hline 2 & 2.2 & 1.0 \\
\hline 5 & 7.2 & 0.7 \\
\hline 10 & 9.1 & 0.8 \\
\hline 25 & 11.2 & 1.0 \\
\hline 50 & 13.0 & 1.6 \\
\hline 75 & 13.7 & 1.5 \\
\hline 100 & 14.1 & 1.6 \\
\hline
\end{tabular}

\begin{tabular}{|c|c|c|}
\hline \multicolumn{3}{|c|}{$\begin{array}{l}\text { Table B78 } \\
\text { Return Period, Maximum Water Level, and Water Level Standard } \\
\text { Deviation for Profile Tutuila } 076\end{array}$} \\
\hline Return Period, yr & Maximum Water Level, ft & Water Level Standard Deviation, $\mathrm{ft}$ \\
\hline 2 & 2.2 & 1.0 \\
\hline 5 & 7.2 & 0.7 \\
\hline 10 & 9.1 & 0.8 \\
\hline 25 & 11.2 & 1.0 \\
\hline 50 & 13.0 & 1.6 \\
\hline 75 & 13.7 & 1.5 \\
\hline 100 & 14.1 & 1.6 \\
\hline
\end{tabular}

\section{Table B79}

Return Period, Maximum Water Level, and Water Level Standard Deviation for Profile Tutuila 077

\begin{tabular}{|l|l|l|}
\hline Return Period, $\mathbf{y r}$ & Maximum Water Level, $\mathrm{ft}$ & Water Level Standard Deviation, $\mathrm{ft}$ \\
\hline 2 & 2.4 & 1.1 \\
\hline 5 & 8.3 & 0.8 \\
\hline 10 & 10.2 & 0.8 \\
\hline 25 & 12.5 & 1.1 \\
\hline 50 & 14.0 & 1.4 \\
\hline 75 & 14.8 & 1.3 \\
\hline 100 & 15.1 & 1.3 \\
\hline
\end{tabular}

\begin{tabular}{|l|l|l|}
\hline $\begin{array}{l}\text { Table B80 } \\
\text { Return Period, Maximum Water Level, and Water Level Standard } \\
\text { Deviation for Profile Tutuila 078 }\end{array}$ \\
\hline Return Period, yr & Maximum Water Level, ft & Water Level Standard Deviation, ft \\
\hline 2 & 2.4 & 1.1 \\
\hline 5 & 8.3 & 0.8 \\
\hline 10 & 10.2 & 0.8 \\
\hline 25 & 12.5 & 1.1 \\
\hline 50 & 14.0 & 1.4 \\
\hline 75 & 14.8 & 1.3 \\
\hline 100 & 15.1 & 1.3 \\
\hline
\end{tabular}




\begin{tabular}{|c|c|c|}
\hline \multicolumn{3}{|c|}{$\begin{array}{l}\text { Table B81 } \\
\text { Return Period, Maximum Water Level, and Water Level Standard } \\
\text { Deviation for Profile Tutuila } 079\end{array}$} \\
\hline Return Period, yr & Maximum Water Level, $\mathrm{ft}$ & Water Level Standard Deviation, $\mathrm{ft}$ \\
\hline 2 & 2.4 & 1.1 \\
\hline 5 & 8.3 & 0.8 \\
\hline 10 & 10.2 & 0.8 \\
\hline 25 & 12.5 & 1.1 \\
\hline 50 & 14.0 & 1.4 \\
\hline 75 & 14.8 & 1.3 \\
\hline 100 & 15.1 & 1.3 \\
\hline
\end{tabular}

\section{Table B82}

Return Period, Maximum Water Level, and Water Level Standard Deviation for Profile Tutuila 080

\begin{tabular}{|l|l|l|}
\hline Return Period, yr & Maximum Water Level, ft & Water Level Standard Deviation, ft \\
\hline 2 & 2.4 & 1.1 \\
\hline 5 & 8.6 & 0.9 \\
\hline 10 & 10.9 & 1.0 \\
\hline 25 & 13.9 & 1.4 \\
\hline 50 & 16.2 & 2.1 \\
\hline 75 & 17.4 & 2.0 \\
\hline 100 & 18.1 & 2.1 \\
\hline
\end{tabular}

\begin{tabular}{|l|l|l|}
\hline $\begin{array}{l}\text { Table B83 } \\
\text { Return Period, Maximum Water Level, and Water Level Standard } \\
\text { Deviation for Profile Tutuila 081 }\end{array}$ \\
\hline Return Period, yr & Maximum Water Level, $\mathrm{ft}$ & Water Level Standard Deviation, $\mathrm{ft}$ \\
\hline 2 & 2.4 & 1.1 \\
\hline 5 & 8.3 & 0.8 \\
\hline 10 & 10.4 & 0.8 \\
\hline 25 & 12.7 & 1.1 \\
\hline 50 & 14.3 & 1.3 \\
\hline 75 & 14.9 & 1.2 \\
\hline 100 & 15.3 & 1.2 \\
\hline
\end{tabular}

\section{Table B84}

Return Period, Maximum Water Level, and Water Level Standard Deviation for Profile Tutuila 082

\begin{tabular}{|l|l|l|}
\hline Return Period, yr & Maximum Water Level, ft & Water Level Standard Deviation, ft \\
\hline 2 & 2.4 & 1.1 \\
\hline 5 & 10.7 & 1.2 \\
\hline 10 & 13.5 & 1.0 \\
\hline 25 & 16.0 & 1.2 \\
\hline 50 & 17.7 & 1.4 \\
\hline 75 & 18.5 & 1.4 \\
\hline 100 & 18.9 & 1.6 \\
\hline
\end{tabular}




\begin{tabular}{|c|c|c|}
\hline \multicolumn{3}{|c|}{$\begin{array}{l}\text { Table B85 } \\
\text { Return Period, Maximum Water Level, and Water Level Standard } \\
\text { Deviation for Profile Tutuila } 083\end{array}$} \\
\hline Return Period, yr & Maximum Water Level, ft & Water Level Standard Deviation, ft \\
\hline 2 & 2.2 & 0.9 \\
\hline 5 & 6.7 & 0.8 \\
\hline 10 & 9.4 & 1.1 \\
\hline 25 & 12.7 & 1.6 \\
\hline 50 & 15.5 & 2.5 \\
\hline 75 & 17.0 & 2.2 \\
\hline 100 & 17.8 & 2.3 \\
\hline
\end{tabular}

\begin{tabular}{|c|c|c|}
\hline \multicolumn{3}{|c|}{$\begin{array}{l}\text { Table B86 } \\
\text { Return Period, Maximum Water Level, and Water Level Standard } \\
\text { Deviation for Profile Tutuila } 084\end{array}$} \\
\hline Return Period, yr & Maximum Water Level, ft & Water Level Standard Deviation, $\mathrm{ft}$ \\
\hline 2 & 2.2 & 0.9 \\
\hline 5 & 6.7 & 0.8 \\
\hline 10 & 9.4 & 1.1 \\
\hline 25 & 12.7 & 1.6 \\
\hline 50 & 15.5 & 2.5 \\
\hline 75 & 17.0 & 2.2 \\
\hline 100 & 17.8 & 2.3 \\
\hline
\end{tabular}

\begin{tabular}{|c|c|c|}
\hline \multicolumn{3}{|c|}{$\begin{array}{l}\text { Table B87 } \\
\text { Return Period, Maximum Water Level, and Water Level Standard } \\
\text { Deviation for Profile Tutuila } 085\end{array}$} \\
\hline Return Period, yr & Maximum Water Level, $\mathrm{ft}$ & Water Level Standard Deviation, $\mathrm{ft}$ \\
\hline$x_{2}$ & 2.2 & - \\
\hline 5 & 6.7 & 0.8 \\
\hline 10 & 9.4 & 1.1 \\
\hline 25 & 12.7 & 1.6 \\
\hline 50 & 15.5 & 2.5 \\
\hline 75 & 17.0 & 2.2 \\
\hline 100 & 17.8 & 2.3 \\
\hline
\end{tabular}

\begin{tabular}{|c|c|c|}
\hline \multicolumn{3}{|c|}{$\begin{array}{l}\text { Table B88 } \\
\text { Return Period, Maximum Water Level, and Water Level Standard } \\
\text { Deviation for Profile Tutuila } 086\end{array}$} \\
\hline Return Period, yr & Maximum Water Level, $\mathrm{ft}$ & Water Level Standard Deviation, $\mathrm{ft}$ \\
\hline 2 & 2.2 & 0.9 \\
\hline 5 & 6.7 & 0.8 \\
\hline 10 & 9.4 & 1.1 \\
\hline 25 & 12.7 & 1.6 \\
\hline 50 & 15.5 & 2.5 \\
\hline 75 & 17.0 & 2.2 \\
\hline 100 & 17.8 & 2.3 \\
\hline
\end{tabular}




\begin{tabular}{|l|l|l|}
\hline $\begin{array}{l}\text { Table B89 } \\
\text { Return Period, Maximum Water Level, and Water Level Standard } \\
\text { Deviation for Profile Tutuila 087 }\end{array}$ \\
\hline Return Period, yr & Maximum Water Level, ft & Water Level Standard Deviation, ft \\
\hline 2 & 2.2 & 0.9 \\
\hline 5 & 6.7 & 0.8 \\
\hline 10 & 9.4 & 1.1 \\
\hline 25 & 12.7 & 1.6 \\
\hline 50 & 15.5 & 2.5 \\
\hline 75 & 17.0 & 2.2 \\
\hline 100 & 17.8 & 2.3 \\
\hline
\end{tabular}

\begin{tabular}{|c|c|c|}
\hline \multicolumn{3}{|c|}{$\begin{array}{l}\text { Table B90 } \\
\text { Return Period, Maximum Water Level, and Water Level Standard } \\
\text { Deviation for Profile Tutuila } 088\end{array}$} \\
\hline Return Period, yr & Maximum Water Level, $\mathrm{ft}$ & Water Level Standard Deviation, $\mathrm{ft}$ \\
\hline 2 & 3.4 & 1.4 \\
\hline 5 & 8.8 & 0.7 \\
\hline 10 & 10.8 & 0.8 \\
\hline 25 & 13.4 & 1.7 \\
\hline 50 & 16.0 & 2.8 \\
\hline 75 & 17.8 & 2.4 \\
\hline 100 & 18.6 & 2.6 \\
\hline
\end{tabular}

\begin{tabular}{|c|c|c|}
\hline \multicolumn{3}{|c|}{$\begin{array}{l}\text { Table B91 } \\
\text { Return Period, Maximum Water Level, and Water Level Standard } \\
\text { Deviation for Profile Tutuila } 089\end{array}$} \\
\hline Return Period, yr & Maximum Water Level, ft & Water Level Standard Deviation, $\mathrm{ft}$ \\
\hline 2 & 3.4 & 1.4 \\
\hline 5 & 8.8 & 0.7 \\
\hline 10 & 10.8 & 0.8 \\
\hline 25 & 13.4 & 1.7 \\
\hline 50 & 16.0 & 2.8 \\
\hline 75 & 17.8 & 2.4 \\
\hline 100 & 18.6 & 2.6 \\
\hline
\end{tabular}

\begin{tabular}{|c|c|c|}
\hline \multicolumn{3}{|c|}{$\begin{array}{l}\text { Table B92 } \\
\text { Return Period, Maximum Water Level, and Water Level Standard } \\
\text { Deviation for Profile Tutuila } 090\end{array}$} \\
\hline Return Period, yr & Maximum Water Level, $\mathrm{ft}$ & Water Level Standard Deviation, ft \\
\hline 2 & 3.2 & 1.4 \\
\hline 5 & 9.5 & 0.9 \\
\hline 10 & 12.2 & 1.0 \\
\hline 25 & 14.8 & 1.5 \\
\hline 50 & 17.3 & 2.6 \\
\hline 75 & 19.0 & 2.3 \\
\hline 100 & 19.8 & 2.5 \\
\hline
\end{tabular}




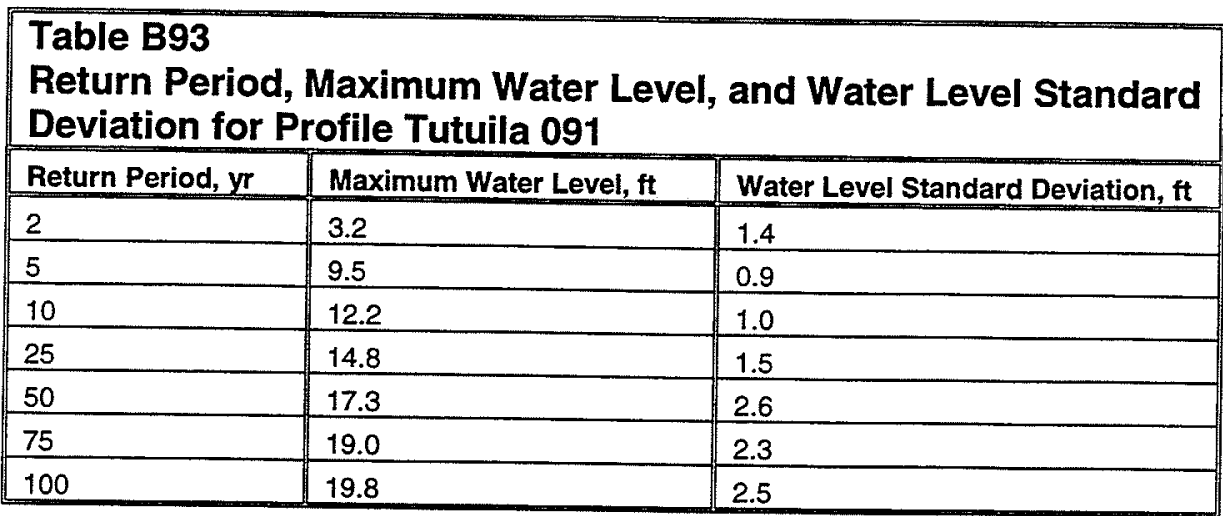

\begin{tabular}{|c|c|c|}
\hline \multicolumn{3}{|c|}{$\begin{array}{l}\text { Table B94 } \\
\text { Return Period, Maximum Water Level, and Water Level Standard } \\
\text { Deviation for Profile Tutuila } 092\end{array}$} \\
\hline Return Period, $y$ & Maximum Water Level, $\mathrm{ft}$ & Water Level Standard Deviation, it \\
\hline 2 & 4.4 & 1.8 \\
\hline 5 & 10.0 & 0.8 \\
\hline 10 & 12.4 & 0.9 \\
\hline 25 & 15.6 & 1.9 \\
\hline 50 & 18.7 & 2.7 \\
\hline 75 & 20.4 & 2.4 \\
\hline 100 & 21.2 & 2.5 \\
\hline
\end{tabular}

\begin{tabular}{|c|c|c|}
\hline \multicolumn{3}{|c|}{$\begin{array}{l}\text { Table B95 } \\
\text { Return Period, Maximum Water Level, and Water Level Standard } \\
\text { Deviation for Profile Tutuila } 093\end{array}$} \\
\hline Return Period, yr & Maximum Water Level, tt & Water Level Standard Deviation, it \\
\hline 2 & 4.7 & 2.0 \\
\hline 5 & 10.7 & 0.8 \\
\hline 10 & 13.1 & 0.9 \\
\hline 25 & 16.0 & 1.9 \\
\hline 50 & 19.3 & 3.0 \\
\hline 75 & 21.1 & 2.7 \\
\hline 100 & 22.1 & 2.8 \\
\hline
\end{tabular}

\begin{tabular}{|l|l|l|}
\hline $\begin{array}{l}\text { Table B96 } \\
\text { Return Period, Maximum Water Level, and Water Level Standard } \\
\text { Deviation for Profile Tutuila 094 }\end{array}$ \\
\hline Return Period, yr & Maximum Water Level, ft & Water Level Standard Deviation, $\mathrm{ft}$ \\
\hline 2 & 4.8 & 2.0 \\
\hline 5 & 10.9 & 0.9 \\
\hline 10 & 13.2 & 0.8 \\
\hline 25 & 16.0 & 1.9 \\
\hline 50 & 19.1 & 3.0 \\
\hline 75 & 21.0 & 2.6 \\
\hline 100 & 21.9 & 2.8 \\
\hline
\end{tabular}




\begin{tabular}{|c|c|c|}
\hline \multicolumn{3}{|c|}{$\begin{array}{l}\text { Table B97 } \\
\text { Return Period, Maximum Water Level, and Water Level Standard } \\
\text { Deviation for Profile Tutuila } 095\end{array}$} \\
\hline Return Period, $y$ & Maximum Water Level, ft & Water Level Standard Deviation, ft \\
\hline 2 & 5.7 & 2.4 \\
\hline 5 & 12.8 & 0.9 \\
\hline 10 & 15.3 & 0.8 \\
\hline 25 & 17.8 & 1.7 \\
\hline 50 & 20.7 & 3.1 \\
\hline 75 & 22.6 & 2.8 \\
\hline 100 & 23.6 & 3.0 \\
\hline
\end{tabular}

\begin{tabular}{|c|c|c|}
\hline \multicolumn{3}{|c|}{$\begin{array}{l}\text { Table B98 } \\
\text { Return Period, Maximum Water Level, and Water Level Standard } \\
\text { Deviation for Profile Tutuila } 096\end{array}$} \\
\hline Return Period, yr & Maximum Water Level, $\mathrm{ft}$ & Water Level Standard Deviation, ft \\
\hline 2 & 5.7 & 2.4 \\
\hline 5 & 12.8 & 0.9 \\
\hline 10 & 15.3 & 0.8 \\
\hline 25 & 17.8 & 1.7 \\
\hline 50 & 20.7 & 3.1 \\
\hline 75 & 22.6 & 2.8 \\
\hline 100 & 23.6 & 3.0 \\
\hline
\end{tabular}

\begin{tabular}{|c|c|c|}
\hline \multicolumn{3}{|c|}{$\begin{array}{l}\text { Table B99 } \\
\text { Return Period, Maximum Water Level, and Water Level Standard } \\
\text { Deviation for Profile Tutuila } 097\end{array}$} \\
\hline Return Period, yr & Maximum Water Level, $\mathrm{ft}$ & Water Level Standard Deviation, $\mathrm{ft}$ \\
\hline 2 & 5.1 & 2.0 \\
\hline 5 & 10.6 & 0.8 \\
\hline 10 & 12.7 & 0.8 \\
\hline 25 & 15.4 & 1.6 \\
\hline 50 & 18.0 & 2.3 \\
\hline 75 & 19.4 & 2.1 \\
\hline 100 & 20.1 & 2.1 \\
\hline
\end{tabular}

\section{Table B100}

Return Period, Maximum Water Level, and Water Level Standard Deviation for Profile Tutuila 098

\begin{tabular}{|l|l|l|}
\hline Return Period, yr & Maximum Water Level, ft & Water Level Standard Deviation, ft \\
\hline 2 & 4.8 & 1.9 \\
\hline 5 & 10.8 & 0.8 \\
\hline 10 & 13.2 & 0.9 \\
\hline 25 & 16.2 & 1.7 \\
\hline 50 & 19.1 & 2.6 \\
\hline 75 & 20.7 & 2.4 \\
\hline 100 & 21.5 & 2.6 \\
\hline
\end{tabular}




\begin{tabular}{|c|c|c|}
\hline \multicolumn{3}{|c|}{$\begin{array}{l}\text { Table B101 } \\
\text { Return Period, Maximum Water Level, and Water Level Standard } \\
\text { Deviation for Profile Tutuila } 99\end{array}$} \\
\hline Return Period, yr & Maximum Water Level, ft & Water Level Standard Deviation, $\mathrm{ft}$ \\
\hline 2 & 4.7 & 1.9 \\
\hline 5 & 11.1 & 0.9 \\
\hline 10 & 13.7 & 1.1 \\
\hline 25 & 17.0 & 1.8 \\
\hline 50 & 19.9 & 2.8 \\
\hline 75 & 21.6 & 2.6 \\
\hline 100 & 22.5 & 2.8 \\
\hline
\end{tabular}

\begin{tabular}{|c|c|c|}
\hline \multicolumn{3}{|c|}{$\begin{array}{l}\text { Table B102 } \\
\text { Return Period, Maximum Water Level, and Water Level Standard } \\
\text { Deviation for Profile Tutuila } 100\end{array}$} \\
\hline Return Period, yr & Maximum Water Level, $\mathrm{ft}$ & Water Level Standard Deviation, $\mathrm{ft}$ \\
\hline 2 & 4.2 & 1.7 \\
\hline 5 & 11.2 & 1.1 \\
\hline 10 & 15.0 & 1.5 \\
\hline 25 & 20.5 & 3.1 \\
\hline 50 & 24.3 & 2.6 \\
\hline 75 & 25.6 & 2.1 \\
\hline 100 & 26.2 & 2.0 \\
\hline
\end{tabular}

\begin{tabular}{|c|c|c|}
\hline \multicolumn{3}{|c|}{$\begin{array}{l}\text { Table B103 } \\
\text { Return Period, Maximum Water Level, and Water Level Standard } \\
\text { Deviation for Profile Tutuila } 101\end{array}$} \\
\hline Return Period, yr & Maximum Water Level, $\mathrm{ft}$ & Water Level Standard Deviation, ft \\
\hline 2 & 3.6 & 1.4 \\
\hline 5 & 9.1 & 0.9 \\
\hline 10 & 11.9 & 1.1 \\
\hline 25 & 14.7 & 1.3 \\
\hline 50 & 16.8 & 2.0 \\
\hline 75 & 18.0 & 1.9 \\
\hline 100 & 18.6 & 2.1 \\
\hline
\end{tabular}

\begin{tabular}{|c|c|c|}
\hline \multicolumn{3}{|c|}{$\begin{array}{l}\text { Table B104 } \\
\text { Return Period, Maximum Water Level, and Water Level Standard } \\
\text { Deviation for Profile Tutuila } 102\end{array}$} \\
\hline Return Period, yr & Maximum Water Level, $\mathrm{tt}$ & Water Level Standard Deviation, $\mathrm{ft}$ \\
\hline 2 & 1.5 & 0.6 \\
\hline 5 & 4.5 & 0.6 \\
\hline 10 & 7.3 & 1.4 \\
\hline 25 & 12.1 & 2.6 \\
\hline 50 & 16.0 & 3.6 \\
\hline 75 & 18.2 & 2.9 \\
\hline 100 & 19.3 & 2.9 \\
\hline
\end{tabular}




\begin{tabular}{|c|c|c|}
\hline \multicolumn{3}{|c|}{$\begin{array}{l}\text { Table B105 } \\
\text { Return Period, Maximum Water Level, and Water Level Standard } \\
\text { Deviation for Profile Tutuila } 103\end{array}$} \\
\hline Return Period, yr & Maximum Water Level, $\mathrm{ft}$ & Water Level Standard Deviation, $\mathrm{ft}$ \\
\hline 2 & 0.1 & 0.2 \\
\hline 5 & 4.3 & 1.2 \\
\hline 10 & 9.3 & 2.1 \\
\hline 25 & 14.9 & 2.6 \\
\hline 50 & 18.7 & 3.2 \\
\hline 75 & 20.7 & 2.7 \\
\hline 100 & 21.7 & 2.7 \\
\hline
\end{tabular}

\begin{tabular}{|l|l|l|}
\hline $\begin{array}{l}\text { Table B106 } \\
\text { Return Period, Maximum Water Level, and Water Level Standard } \\
\text { Deviation for Profile Tutuila 104 }\end{array}$ \\
\hline Return Period, yr & Maximum Water Level, ft & Water Level Standard Deviation, ft \\
\hline 2 & 0.1 & 0.2 \\
\hline 5 & 4.3 & 1.2 \\
\hline 10 & 9.3 & 2.1 \\
\hline 25 & 14.9 & 2.6 \\
\hline 50 & 18.7 & 3.2 \\
\hline 75 & 20.7 & 2.7 \\
\hline 100 & 21.7 & 2.7 \\
\hline
\end{tabular}

\begin{tabular}{|l|l|l|}
\hline $\begin{array}{l}\text { Table B107 } \\
\text { Return Period, Maximum Water Level, and Water Level Standard } \\
\text { Deviation for Profile Tutuila 105 }\end{array}$ \\
\hline Return Period, $y r$ & Maximum Water Level, ,t & Water Level Standard Deviation, ft \\
\hline 2 & 0.1 & 0.2 \\
\hline 5 & 4.3 & 1.2 \\
\hline 10 & 9.3 & 2.1 \\
\hline 25 & 14.9 & 2.6 \\
\hline 50 & 18.7 & 3.2 \\
\hline 75 & 20.7 & 2.7 \\
\hline 100 & 21.7 & 2.7 \\
\hline
\end{tabular}

\begin{tabular}{|l|l|l|}
\hline $\begin{array}{l}\text { Table B108 } \\
\text { Return Period, Maximum Water Level, and Water Level Standard } \\
\text { Deviation for Profile Tutuila 106 }\end{array}$ \\
\hline Return Period, yr & Maximum Water Level, $\mathrm{ft}$ & Water Level Standard Deviation, $\mathrm{ft}$ \\
\hline 2 & 1.2 & 0.7 \\
\hline 5 & 5.4 & 0.8 \\
\hline 10 & 8.8 & 1.8 \\
\hline 25 & 14.1 & 2.5 \\
\hline 50 & 17.7 & 3.0 \\
\hline 75 & 19.5 & 2.6 \\
\hline 100 & 20.4 & 2.7 \\
\hline
\end{tabular}




\begin{tabular}{|c|c|c|}
\hline \multicolumn{3}{|c|}{$\begin{array}{l}\text { Table B109 } \\
\text { Return Period, Maximum Water Level, and Water Level Standard } \\
\text { Deviation for Profile Tutuila } 107\end{array}$} \\
\hline Return Period, yr & Maximum Water Level, ft & Water Level Standard Deviation, $\mathrm{ft}$ \\
\hline 2 & 1.5 & 0.9 \\
\hline 5 & 7.2 & 1.2 \\
\hline 10 & 11.1 & 1.7 \\
\hline 25 & 16.2 & 2.3 \\
\hline 50 & 19.6 & 2.8 \\
\hline 75 & 21.3 & 2.5 \\
\hline 100 & 22.2 & 2.6 \\
\hline
\end{tabular}

\begin{tabular}{|c|c|c|}
\hline \multicolumn{3}{|c|}{$\begin{array}{l}\text { Table B110 } \\
\text { Return Period, Maximum Water Level, and Water Level Standard } \\
\text { Deviation for Profile Tutuila } 108\end{array}$} \\
\hline Return Period, yr & Maximum Water Level, $\mathrm{ft}$ & Water Level Standard Deviation, $\mathrm{H}$ \\
\hline 2 & 1.5 & 0.7 \\
\hline 5 & 6.5 & 1.2 \\
\hline 10 & 11.0 & 2.0 \\
\hline 25 & 16.4 & 2.3 \\
\hline 50 & 19.9 & 2.7 \\
\hline 75 & 21.3 & 2.3 \\
\hline 100 & 22.1 & 2.4 \\
\hline
\end{tabular}

\begin{tabular}{|c|c|c|}
\hline \multicolumn{3}{|c|}{$\begin{array}{l}\text { Table B111 } \\
\text { Return Period, Maximum Water Level, and Water Level Standard } \\
\text { Deviation for Profile Tutuila } 109\end{array}$} \\
\hline Return Period, yr & Maximum Water Level, $\mathrm{ft}$ & Water Level Standard Deviation, ft \\
\hline$\underline{2}$ & 1.5 & 0.7 \\
\hline 5 & 6.4 & 1.1 \\
\hline 10 & 9.9 & 1.3 \\
\hline 25 & 13.7 & 1.8 \\
\hline 50 & 16.4 & 2.2 \\
\hline 75 & 17.7 & 2.1 \\
\hline 100 & 18.4 & 2.2 \\
\hline
\end{tabular}

\begin{tabular}{|c|c|c|}
\hline \multicolumn{3}{|c|}{$\begin{array}{l}\text { Table B112 } \\
\text { Return Period, Maximum Water Level, and Water Level Standard } \\
\text { Deviation for Profile Tutuila 109A }\end{array}$} \\
\hline Return Period, yr & Maximum Water Level, $\mathrm{ft}$ & Water Level Standard Deviation, $\mathrm{ft}$ \\
\hline 2 & 1.5 & 0.7 \\
\hline 5 & 6.5 & 1.2 \\
\hline 10 & 10.8 & 1.9 \\
\hline 25 & 16.4 & 2.5 \\
\hline 50 & 20.1 & 3.1 \\
\hline 75 & 21.9 & 2.7 \\
\hline 100 & 22.8 & 2.7 \\
\hline
\end{tabular}




\begin{tabular}{|c|c|c|}
\hline \multicolumn{3}{|c|}{$\begin{array}{l}\text { Table B113 } \\
\text { Return Period, Maximum Water Level, and Water Level Standard } \\
\text { Deviation for Profile Tutuila } 110\end{array}$} \\
\hline Return Period, yr & Maximum Water Level, ft & Water Level Standard Deviation, $\mathrm{ft}$ \\
\hline 2 & 1.5 & 0.7 \\
\hline 5 & 6.4 & 1.1 \\
\hline 10 & 9.9 & 1.3 \\
\hline 25 & 13.7 & 1.8 \\
\hline 50 & 16.4 & 2.2 \\
\hline 75 & 17.7 & 2.1 \\
\hline 100 & 18.4 & 2.2 \\
\hline
\end{tabular}

\section{Table B114}

Return Period, Maximum Water Level, and Water Level Standard Deviation for Profile Tutuila 111

\begin{tabular}{|l|l|l|}
\hline Return Period, yr & Maximum Water Level, ft & Water Level Standard Deviation, ft \\
\hline 2 & 1.5 & 0.7 \\
\hline 5 & 6.4 & 1.1 \\
\hline 10 & 9.9 & 1.3 \\
\hline 25 & 13.7 & 1.8 \\
\hline 50 & 16.4 & 2.2 \\
\hline 75 & 17.7 & 2.1 \\
\hline 100 & 18.4 & 2.2 \\
\hline
\end{tabular}

\begin{tabular}{|c|c|c|}
\hline \multicolumn{3}{|c|}{$\begin{array}{l}\text { Table B115 } \\
\text { Return Period, Maximum Water Level, and Water Level Standard } \\
\text { Deviation for Profile Tutuila } 112\end{array}$} \\
\hline Return Period, yr & Maximum Water Level, ft & Water Level Standard Deviation, $\mathrm{ft}$ \\
\hline 2 & 1.5 & 0.7 \\
\hline 5 & 6.4 & 1.1 \\
\hline 10 & 9.9 & 1.3 \\
\hline 25 & 13.7 & 1.8 \\
\hline 50 & 16.4 & 2.2 \\
\hline 75 & 17.7 & 2.1 \\
\hline 100 & 18.4 & 2.2 \\
\hline
\end{tabular}

\section{Table B116}

Return Period, Maximum Water Level, and Water Level Standard Deviation for Profile Tutuila 113

\begin{tabular}{|l|l|l|}
\hline Return Period, yr & Maximum Water Level, ft & Water Level Standard Deviation, $\mathbf{t t}$ \\
\hline 2 & 1.5 & 0.7 \\
\hline 5 & 6.4 & 1.1 \\
\hline 10 & 9.9 & 1.3 \\
\hline 25 & 13.7 & 1.8 \\
\hline 50 & 16.4 & 2.2 \\
\hline 75 & 17.7 & 2.1 \\
\hline 100 & 18.4 & 2.2 \\
\hline
\end{tabular}




\begin{tabular}{|c|c|c|}
\hline \multicolumn{3}{|c|}{$\begin{array}{l}\text { Table B117 } \\
\text { Return Period, Maximum Water Level, and Water Level Standard } \\
\text { Deviation for Profile Tutuila } 114\end{array}$} \\
\hline Return Period, yr & Maximum Water Level, it & Water Level Standard Deviation, $\mathrm{ft}$ \\
\hline 2 & 2.4 & 1.0 \\
\hline 5 & 8.6 & 1.2 \\
\hline 10 & 12.3 & 1.5 \\
\hline 25 & 16.9 & 2.3 \\
\hline 50 & 20.2 & 2.7 \\
\hline 75 & 21.8 & 2.4 \\
\hline 100 & 22.6 & 2.5 \\
\hline
\end{tabular}

\begin{tabular}{|c|c|c|}
\hline \multicolumn{3}{|c|}{$\begin{array}{l}\text { Table B118 } \\
\text { Return Period, Maximum Water Level, and Water Level Standard } \\
\text { Deviation for Profile Tutuila } 115\end{array}$} \\
\hline Return Period, yr & Maximum Water Level, $\mathrm{ft}$ & Water Level Standard Deviation, $\mathrm{ft}$ \\
\hline 2 & 2.4 & 1.0 \\
\hline 5 & 8.6 & 1.2 \\
\hline 10 & 12.3 & 1.5 \\
\hline 25 & 16.9 & 2.3 \\
\hline 50 & 20.2 & 2.7 \\
\hline 75 & 21.8 & 2.4 \\
\hline 100 & 22.6 & 2.5 \\
\hline
\end{tabular}

\begin{tabular}{|c|c|c|}
\hline \multicolumn{3}{|c|}{$\begin{array}{l}\text { Table B119 } \\
\text { Return Period, Maximum Water Level, and Water Level Standard } \\
\text { Deviation for Profile Tutuila } 116\end{array}$} \\
\hline Return Period, yr & Maximum Water Level, ft & Water Level Standard Deviation, $\mathrm{ft}$ \\
\hline 2 & 2.4 & 1.0 \\
\hline 5 & 8.6 & 1.2 \\
\hline 10 & 12.3 & 1.5 \\
\hline 25 & 16.9 & 2.3 \\
\hline 50 & 20.2 & 2.7 \\
\hline 75 & 21.8 & 2.4 \\
\hline 100 & 22.6 & 2.5 \\
\hline
\end{tabular}

\begin{tabular}{|c|c|c|}
\hline \multicolumn{3}{|c|}{$\begin{array}{l}\text { Table B120 } \\
\text { Return Period, Maximum Water Level, and Water Level Standard } \\
\text { Deviation for Profile Tutuila } 117\end{array}$} \\
\hline Return Period, yr & Maximum Water Level, $\mathrm{ft}$ & Water Level Standard Deviation, $\mathrm{ft}$ \\
\hline 2 & 2.4 & 1.0 \\
\hline 5 & 8.6 & 1.2 \\
\hline 10 & 12.3 & 1.5 \\
\hline 25 & 16.9 & 2.3 \\
\hline 50 & 20.2 & 2.7 \\
\hline 75 & 21.8 & 2.4 \\
\hline 100 & 22.6 & 2.5 \\
\hline
\end{tabular}




\begin{tabular}{|c|c|c|}
\hline \multicolumn{3}{|c|}{$\begin{array}{l}\text { Table B121 } \\
\text { Return Period, Maximum Water Level, and Water Level Standard } \\
\text { Deviation for Profile Tutuila } 118\end{array}$} \\
\hline Return Period, yr & Maximum Water Level, $\mathrm{ft}$ & Water Level Standard Deviation, $\mathrm{ft}$ \\
\hline 2 & 2.4 & 1.0 \\
\hline 5 & 8.6 & 1.2 \\
\hline 10 & 12.3 & 1.5 \\
\hline 25 & 16.9 & 2.3 \\
\hline 50 & 20.2 & 2.7 \\
\hline 75 & 21.8 & 2.4 \\
\hline 100 & 22.6 & 2.5 \\
\hline
\end{tabular}

\begin{tabular}{|c|c|c|}
\hline \multicolumn{3}{|c|}{$\begin{array}{l}\text { Table B122 } \\
\text { Return Period, Maximum Water Level, and Water Level Standard } \\
\text { Deviation for Profile Tutuila } 119\end{array}$} \\
\hline Return Period, $y$ & Maximum Water Level, ft & Water Level Standard Deviation, $\mathrm{ft}$ \\
\hline 2 & 2.4 & 1.0 \\
\hline 5 & 8.6 & 1.2 \\
\hline 10 & 12.3 & 1.5 \\
\hline 25 & 16.9 & 2.3 \\
\hline 50 & 20.2 & 2.7 \\
\hline 75 & 21.8 & 2.4 \\
\hline 100 & 22.6 & 2.5 \\
\hline
\end{tabular}

\begin{tabular}{|c|c|c|}
\hline \multicolumn{3}{|c|}{$\begin{array}{l}\text { Table B123 } \\
\text { Return Period, Maximum Water Level, and Water Level Standard } \\
\text { Deviation for Profile Tutuila } 120\end{array}$} \\
\hline Return Period, yr & Maximum Water Level, $\mathrm{ft}$ & Water Level Standard Deviation, $\mathrm{ft}$ \\
\hline 2 & 0.7 & 0.6 \\
\hline 5 & 3.9 & 0.7 \\
\hline 10 & 8.9 & 2.5 \\
\hline 25 & 14.5 & 2.4 \\
\hline 50 & 17.8 & 2.3 \\
\hline 75 & 19.0 & 2.0 \\
\hline 100 & 19.6 & 1.9 \\
\hline
\end{tabular}

\begin{tabular}{|c|c|c|}
\hline Return Period, yr & Maximum Water Level, ft & Water Level Standard Deviation, $\mathrm{ft}$ \\
\hline 2 & 0.9 & 0.6 \\
\hline 5 & 3.8 & 0.5 \\
\hline 10 & 7.3 & 2.1 \\
\hline 25 & 12.4 & 2.0 \\
\hline 50 & 15.4 & 2.3 \\
\hline 75 & 16.7 & 2.0 \\
\hline 100 & 17.4 & 2.0 \\
\hline
\end{tabular}




\begin{tabular}{|c|c|c|}
\hline \multicolumn{3}{|c|}{$\begin{array}{l}\text { Table B125 } \\
\text { Return Period, Maximum Water Level, and Water Level Standard } \\
\text { Deviation for Profile Tutuila } 122\end{array}$} \\
\hline Return Period, yr & Maximum Water Level, $\mathrm{ft}$ & Water Level Standard Deviation, $\mathrm{ft}$ \\
\hline 2 & 0.9 & 0.6 \\
\hline 5 & 3.8 & 0.5 \\
\hline 10 & 7.3 & 2.1 \\
\hline 25 & 12.4 & 2.0 \\
\hline 50 & 15.4 & 2.3 \\
\hline 75 & 16.7 & 2.0 \\
\hline 100 & 17.4 & 2.0 \\
\hline
\end{tabular}

\begin{tabular}{|c|c|c|}
\hline \multicolumn{3}{|c|}{$\begin{array}{l}\text { Table B126 } \\
\text { Return Period, Maximum Water Level, and Water Level Standard } \\
\text { Deviation for Profile Tutuila } 123\end{array}$} \\
\hline Return Period, yr & Maximum Water Level, ft & Water Level Standard Deviation, ft \\
\hline 2 & 0.9 & 0.6 \\
\hline 5 & 3.8 & 0.5 \\
\hline 10 & 7.3 & 2.1 \\
\hline 25 & 12.4 & 2.0 \\
\hline 50 & 15.4 & 2.2 \\
\hline 75 & 16.7 & 1.9 \\
\hline 100 & 17.3 & 2.0 \\
\hline
\end{tabular}

\begin{tabular}{|c|c|c|}
\hline \multicolumn{3}{|c|}{$\begin{array}{l}\text { Table B127 } \\
\text { Return Period, Maximum Water Level, and Water Level Standard } \\
\text { Deviation for Profile Tutuila } 124\end{array}$} \\
\hline Return Period, yr & Maximum Water Level, ft & Water Level Standard Deviation, $\mathrm{ft}$ \\
\hline 2 & 0.9 & 0.6 \\
\hline 5 & 3.8 & 0.5 \\
\hline 10 & 7.6 & 2.2 \\
\hline 25 & 12.8 & 2.1 \\
\hline 50 & 15.9 & 2.2 \\
\hline 75 & 17.2 & 1.9 \\
\hline 100 & 17.8 & 2.0 \\
\hline
\end{tabular}

\begin{tabular}{|c|c|c|}
\hline \multicolumn{3}{|c|}{$\begin{array}{l}\text { Table B128 } \\
\text { Return Period, Maximum Water Level, and Water Level Standard } \\
\text { Deviation for Profile Tutuila } 125\end{array}$} \\
\hline Return Period, yr & Maximum Water Level, $\mathrm{ft}$ & Water Level Standard Deviation, $\mathrm{ft}$ \\
\hline 2 & 0.9 & 0.6 \\
\hline 5 & 3.9 & 0.6 \\
\hline 10 & 8.3 & 2.2 \\
\hline 25 & 13.4 & 2.1 \\
\hline 50 & 16.6 & 2.3 \\
\hline 75 & 17.9 & 2.0 \\
\hline 100 & 18.5 & 2.1 \\
\hline
\end{tabular}




\begin{tabular}{|c|c|c|}
\hline \multicolumn{3}{|c|}{$\begin{array}{l}\text { Table B129 } \\
\text { Return Period, Maximum Water Level, and Water Level Standard } \\
\text { Deviation for Profile Tutuila } 126\end{array}$} \\
\hline Return Period, yr & Maximum Water Level, ft & Water Level Standard Deviation, $\mathrm{ft}$ \\
\hline 2 & 0.9 & 0.6 \\
\hline 5 & 3.9 & 0.6 \\
\hline 10 & 8.6 & 2.4 \\
\hline 25 & 14.3 & 2.3 \\
\hline 50 & 17.8 & 2.9 \\
\hline 75 & 19.6 & 2.4 \\
\hline 100 & 20.5 & 2.5 \\
\hline
\end{tabular}

\begin{tabular}{|c|c|c|}
\hline \multicolumn{3}{|c|}{$\begin{array}{l}\text { Table B130 } \\
\text { Return Period, Maximum Water Level, and Water Level Standard } \\
\text { Deviation for Profile Tutuila } 127\end{array}$} \\
\hline Return Period, yr & Maximum Water Level, ft & Water Level Standard Deviation, ft \\
\hline 2 & 0.9 & 0.6 \\
\hline 5 & 3.9 & 0.6 \\
\hline 10 & 8.6 & 2.4 \\
\hline 25 & 14.3 & 2.3 \\
\hline 50 & 17.8 & 2.9 \\
\hline 75 & 19.6 & 2.4 \\
\hline 100 & 20.5 & 2.5 \\
\hline
\end{tabular}

\begin{tabular}{|c|c|c|}
\hline \multicolumn{3}{|c|}{$\begin{array}{l}\text { Table B131 } \\
\text { Return Period, Maximum Water Level, and Water Level Standard } \\
\text { Deviation for Profile Tutuila } 128\end{array}$} \\
\hline Return Period, yr & Maximum Water Level, $\mathrm{ft}$ & Water Level Standard Deviation, ft \\
\hline 2 & 0.9 & 0.6 \\
\hline 5 & 3.9 & 0.6 \\
\hline 10 & 8.6 & 2.4 \\
\hline 25 & 14.3 & 2.3 \\
\hline 50 & 17.8 & 2.9 \\
\hline 75 & 19.6 & 2.4 \\
\hline 100 & 20.5 & 2.5 \\
\hline
\end{tabular}

\begin{tabular}{|c|c|c|}
\hline \multicolumn{3}{|c|}{$\begin{array}{l}\text { Table B132 } \\
\text { Return Period, Maximum Water Level, and Water Level Standard } \\
\text { Deviation for Profile Tutuila } 129\end{array}$} \\
\hline Return Period, yr & Maximum Water Level, $\mathrm{ft}$ & Water Level Standard Deviation, $\mathrm{ft}$ \\
\hline 2 & 1.3 & 0.7 \\
\hline 5 & 4.9 & 0.9 \\
\hline 10 & 9.7 & 2.2 \\
\hline 25 & 15.1 & 2.2 \\
\hline 50 & 18.5 & 3.0 \\
\hline 75 & 20.4 & 2.6 \\
\hline 100 & 21.3 & 2.7 \\
\hline
\end{tabular}




\begin{tabular}{|c|c|c|}
\hline \multicolumn{3}{|c|}{$\begin{array}{l}\text { Table B133 } \\
\text { Return Period, Maximum Water Level, and Water Level Standard } \\
\text { Deviation for Profile Tutuila } 130\end{array}$} \\
\hline Return Period, yr & Maximum Water Level, $\mathrm{ft}$ & Water Level Standard Deviation, ft \\
\hline 2 & 1.3 & 0.7 \\
\hline 5 & 4.9 & 0.9 \\
\hline 10 & 9.7 & 2.2 \\
\hline 25 & 15.1 & 2.2 \\
\hline 50 & 18.5 & 3.0 \\
\hline 75 & 20.4 & 2.6 \\
\hline 100 & 21.3 & 2.7 \\
\hline
\end{tabular}

\begin{tabular}{|c|c|c|}
\hline $\begin{array}{l}\text { Table B134 } \\
\text { Return Perioc } \\
\text { Deviation for }\end{array}$ & $\begin{array}{l}\text { ximum Water Leve } \\
\text { le Tutuila } 131\end{array}$ & Ind Water Level Standard \\
\hline Return Period, yr & Maximum Water Level, $\mathrm{ft}$ & Water Level Standard Deviation, ft \\
\hline 2 & 1.3 & 0.7 \\
\hline 5 & 4.9 & 0.9 \\
\hline 10 & 9.7 & 2.2 \\
\hline 25 & 15.1 & 2.2 \\
\hline 50 & 18.5 & 3.0 \\
\hline 75 & 20.4 & 2.6 \\
\hline 100 & 21.3 & 2.7 \\
\hline
\end{tabular}

\begin{tabular}{|c|c|c|}
\hline \multicolumn{3}{|c|}{$\begin{array}{l}\text { Table B135 } \\
\text { Return Period, Maximum Water Level, and Water Level Standard } \\
\text { Deviation for Profile Tutuila } 132\end{array}$} \\
\hline Return Period, yr & Maximum Water Level, it & Water Level Standard Deviation, $\mathrm{ft}$ \\
\hline 2 & 1.3 & 0.8 \\
\hline 5 & 5.6 & 1.0 \\
\hline 10 & 10.1 & 2.0 \\
\hline 25 & 15.2 & 2.1 \\
\hline 50 & 18.5 & 3.1 \\
\hline 75 & 20.4 & 2.7 \\
\hline 100 & 21.4 & 2.8 \\
\hline
\end{tabular}

\begin{tabular}{|c|c|c|}
\hline \multicolumn{3}{|c|}{$\begin{array}{l}\text { Table B136 } \\
\text { Return Period, Maximum Water Level, and Water Level Standard } \\
\text { Deviation for Profile Tutuila } 133\end{array}$} \\
\hline Return Period, yr & Maximum Water Level, it & Water Level Standard Deviation, $\mathrm{ft}$ \\
\hline 2 & 1.3 & 0.8 \\
\hline 5 & 5.6 & 1.0 \\
\hline 10 & 10.1 & 2.0 \\
\hline 25 & 15.2 & 2.1 \\
\hline 50 & 18.5 & 3.1 \\
\hline 75 & 20.4 & 2.7 \\
\hline 100 & 21.4 & 2.8 \\
\hline
\end{tabular}




\begin{tabular}{|c|c|c|}
\hline \multicolumn{3}{|c|}{$\begin{array}{l}\text { Table B137 } \\
\text { Return Period, Maximum Water Level, and Water Level Standard } \\
\text { Deviation for Profile Tutuila } 134\end{array}$} \\
\hline Return Period, yr & Maximum Water Level, ft & Water Level Standard Deviation, $\mathrm{ft}$ \\
\hline 2 & 1.3 & 0.8 \\
\hline 5 & 5.6 & 1.0 \\
\hline 10 & 10.1 & 2.0 \\
\hline 25 & 15.2 & 2.1 \\
\hline 50 & 18.5 & 3.1 \\
\hline 75 & 20.4 & 2.7 \\
\hline 100 & 21.4 & 2.8 \\
\hline
\end{tabular}

\begin{tabular}{|c|c|c|}
\hline \multicolumn{3}{|c|}{$\begin{array}{l}\text { Table B138 } \\
\text { Return Period, Maximum Water Level, and Water Level Standard } \\
\text { Deviation for Profile Tutuila } 135\end{array}$} \\
\hline Return Period, yr & Maximum Water Level, $\mathrm{ft}$ & Water Level Standard Deviation, $\mathrm{ft}$ \\
\hline 2 & 1.3 & 0.8 \\
\hline 5 & 5.6 & 1.0 \\
\hline 10 & 10.1 & 2.0 \\
\hline 25 & 15.2 & 2.1 \\
\hline 50 & 18.5 & 3.1 \\
\hline 75 & 20.4 & 2.7 \\
\hline 100 & 21.4 & 2.8 \\
\hline
\end{tabular}

\begin{tabular}{|c|c|c|}
\hline \multicolumn{3}{|c|}{$\begin{array}{l}\text { Table B139 } \\
\text { Return Period, Maximum Water Level, and Water Level Standard } \\
\text { Deviation for Profile Tutuila } 136\end{array}$} \\
\hline Return Period, yr & Maximum Water Level, $\mathrm{ft}$ & Water Level Standard Deviation, $\mathrm{ft}$ \\
\hline 2 & 1.9 & 0.9 \\
\hline 5 & 8.1 & 1.3 \\
\hline 10 & 11.7 & 1.5 \\
\hline 25 & 16.2 & 2.0 \\
\hline 50 & 19.2 & 2.7 \\
\hline 75 & 20.9 & 2.5 \\
\hline 100 & 21.7 & 2.6 \\
\hline
\end{tabular}

\begin{tabular}{|c|c|c|}
\hline \multicolumn{3}{|c|}{$\begin{array}{l}\text { Table B140 } \\
\text { Return Period, Maximum Water Level, and Water Level Standard } \\
\text { Deviation for Profile Tutuila } 137\end{array}$} \\
\hline Return Period, yr & Maximum Water Level, $\mathrm{ft}$ & Water Level Standard Deviation, $\mathrm{ft}$ \\
\hline 2 & 1.9 & 0.9 \\
\hline 5 & 8.1 & 1.3 \\
\hline 10 & 11.7 & 1.5 \\
\hline 25 & 16.2 & 2.0 \\
\hline 50 & 19.2 & 2.7 \\
\hline 75 & 20.9 & 2.5 \\
\hline 100 & 21.7 & 2.6 \\
\hline
\end{tabular}




\begin{tabular}{|c|c|c|}
\hline \multicolumn{3}{|c|}{$\begin{array}{l}\text { Table B141 } \\
\text { Return Period, Maximum Water Level, and Water Level Standard } \\
\text { Deviation for Profile Tutuila } 138\end{array}$} \\
\hline Return Period, yr & Maximum Water Level, $\mathrm{ft}$ & Water Level Standard Deviation, $\mathrm{ft}$ \\
\hline 2 & 1.9 & 0.9 \\
\hline 5 & 8.1 & 1.3 \\
\hline 10 & 11.7 & 1.5 \\
\hline 25 & 16.2 & 2.0 \\
\hline 50 & 19.2 & 2.7 \\
\hline 75 & 20.9 & 2.5 \\
\hline 100 & 21.7 & 2.6 \\
\hline
\end{tabular}

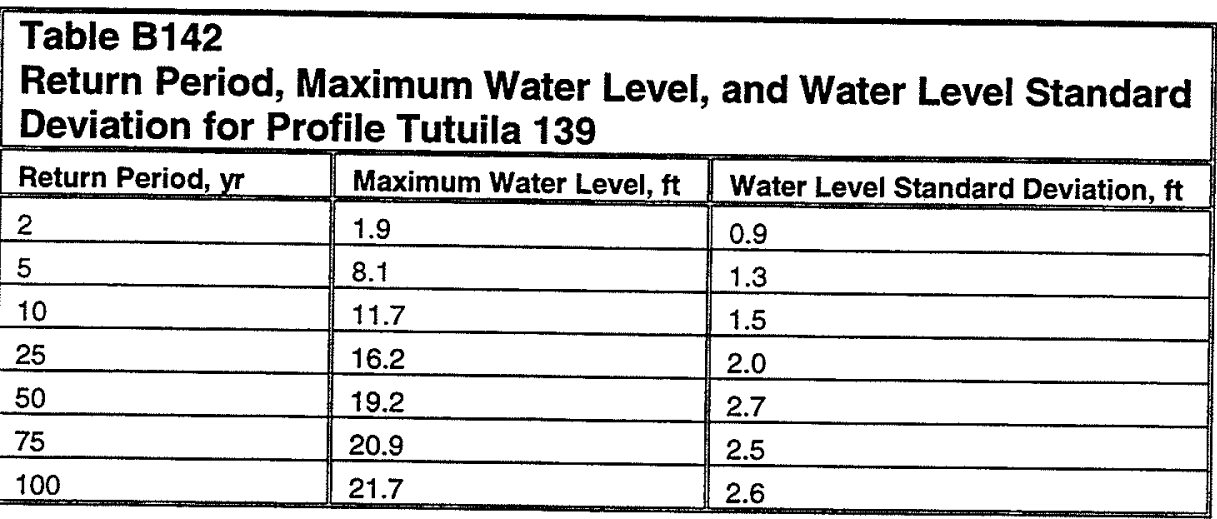

\begin{tabular}{|c|c|c|}
\hline \multicolumn{3}{|c|}{$\begin{array}{l}\text { Table B143 } \\
\text { Return Period, Maximum Water Level, and Water Level Standard } \\
\text { Deviation for Profile Tutuila } 140\end{array}$} \\
\hline Return Period, yr & Maximum Water Level, $\mathrm{ft}$ & Water Level Standard Deviation, $\mathrm{ft}$ \\
\hline 2 & 1.9 & 0.9 \\
\hline 5 & 8.1 & 1.3 \\
\hline 10 & 11.7 & 1.5 \\
\hline 25 & 16.2 & 2.0 \\
\hline 50 & 19.2 & 2.7 \\
\hline 75 & 20.9 & 2.5 \\
\hline 100 & 21.7 & 2.6 \\
\hline
\end{tabular}

\begin{tabular}{|c|c|c|}
\hline \multicolumn{3}{|c|}{$\begin{array}{l}\text { Table B144 } \\
\text { Return Period, Maximum Water Level, and Water Level Standard } \\
\text { Deviation for Profile Tutuila } 141\end{array}$} \\
\hline Return Period, yr & Maximum Water Level, $\mathrm{ft}$ & Water Level Standard Deviation, $\mathrm{ft}$ \\
\hline 2 & 1.9 & 0.9 \\
\hline 5 & 8.1 & 1.3 \\
\hline 10 & 11.7 & 1.5 \\
\hline 25 & 16.2 & 2.0 \\
\hline 50 & 19.2 & 2.7 \\
\hline 75 & 20.9 & 2.5 \\
\hline 100 & 21.7 & 2.6 \\
\hline
\end{tabular}




\begin{tabular}{|c|c|c|}
\hline \multicolumn{3}{|c|}{$\begin{array}{l}\text { Table B145 } \\
\text { Return Period, Maximum Water Level, and Water Level Standard } \\
\text { Deviation for Profile Tutuila } 142\end{array}$} \\
\hline Return Period, yr & Maximum Water Level, $\mathrm{ft}$ & Water Level Standard Deviation, $\mathrm{ft}$ \\
\hline 2 & 1.9 & 0.9 \\
\hline 5 & 8.1 & 1.3 \\
\hline 10 & 11.7 & 1.5 \\
\hline 25 & 16.2 & 2.0 \\
\hline 50 & 19.2 & 2.7 \\
\hline 75 & 20.9 & 2.5 \\
\hline 100 & 21.7 & 2.6 \\
\hline
\end{tabular}

\begin{tabular}{|c|c|c|}
\hline \multicolumn{3}{|c|}{$\begin{array}{l}\text { Table B146 } \\
\text { Return Period, Maximum Water Level, and Water Level Standard } \\
\text { Deviation for Profile Tutuila } 143\end{array}$} \\
\hline Return Period, yr & Maximum Water Level, $\mathrm{ft}$ & Water Level Standard Deviation, $\mathrm{ft}$ \\
\hline 2 & 2.3 & 1.0 \\
\hline 5 & 8.6 & 1.2 \\
\hline 10 & 12.0 & 1.4 \\
\hline 25 & 16.3 & 2.0 \\
\hline 50 & 19.4 & 2.7 \\
\hline 75 & 21.0 & 2.5 \\
\hline 100 & 21.8 & 2.6 \\
\hline
\end{tabular}

\begin{tabular}{|c|c|c|}
\hline \multicolumn{3}{|c|}{$\begin{array}{l}\text { Table B147 } \\
\text { Return Period, Maximum Water Level, and Water Level Standard } \\
\text { Deviation for Profile Tutuila } 144\end{array}$} \\
\hline Return Period, yr & Maximum Water Level, $\mathrm{ft}$ & Water Level Standard Deviation, $\mathrm{At}$ \\
\hline 2 & 2.3 & 1.0 \\
\hline 5 & 8.6 & 1.2 \\
\hline 10 & 12.0 & 1.4 \\
\hline 25 & 16.2 & 2.0 \\
\hline 50 & 19.3 & 2.7 \\
\hline 75 & 21.0 & 2.5 \\
\hline 100 & 21.8 & 2.6 \\
\hline
\end{tabular}

\begin{tabular}{|c|c|c|}
\hline \multicolumn{3}{|c|}{$\begin{array}{l}\text { Table B148 } \\
\text { Return Period, Maximum Water Level, and Water Level Standard } \\
\text { Deviation for Profile Tutuila } 145\end{array}$} \\
\hline Return Period, yr & Maximum Water Level, ft & Water Level Standard Deviation, ft \\
\hline 2 & 2.3 & 1.0 \\
\hline 5 & 8.6 & 1.2 \\
\hline 10 & 12.0 & 1.4 \\
\hline 25 & 16.2 & 2.0 \\
\hline 50 & 19.3 & 2.7 \\
\hline 75 & 21.0 & 2.5 \\
\hline 100 & 21.8 & 2.6 \\
\hline
\end{tabular}




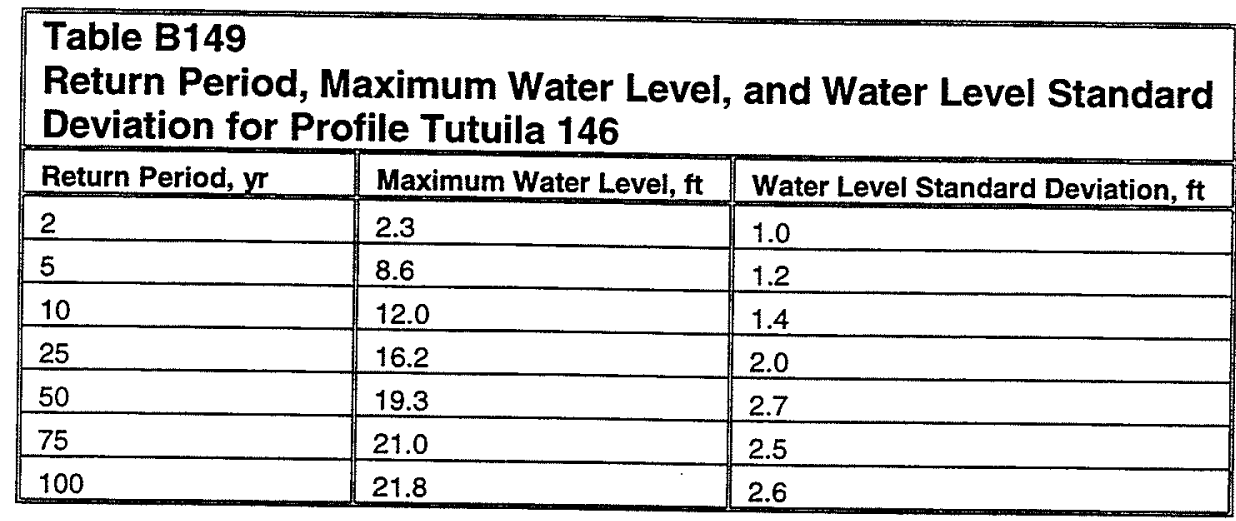

\begin{tabular}{|c|c|c|}
\hline \multicolumn{3}{|c|}{$\begin{array}{l}\text { Table B150 } \\
\text { Return Period, Maximum Water Level, and Water Level Standard } \\
\text { Deviation for Profile Tutuila } 147\end{array}$} \\
\hline Return Period, yr & Maximum Water Level, $\mathrm{ft}$ & Water Level Standard Deviation, ft \\
\hline 2 & 2.3 & 1.0 \\
\hline 5 & 8.6 & 1.2 \\
\hline 10 & 12.0 & 1.4 \\
\hline 25 & 16.2 & 2.0 \\
\hline 50 & 19.3 & 2.7 \\
\hline 75 & 21.0 & 2.5 \\
\hline 100 & 21.8 & 2.6 \\
\hline
\end{tabular}

\begin{tabular}{|c|c|c|}
\hline \multicolumn{3}{|c|}{$\begin{array}{l}\text { Table B151 } \\
\text { Return Period, Maximum Water Level, and Water Level Standard } \\
\text { Deviation for Profile Tutuila } 148\end{array}$} \\
\hline Return Period, $\mathrm{yr}$ & Maximum Water Level, $\mathrm{ft}$ & Water Level Standard Deviation, ft \\
\hline 2 & 2.3 & 1.0 \\
\hline 5 & 8.6 & 1.2 \\
\hline 10 & 12.0 & 1.4 \\
\hline 25 & 16.2 & 2.0 \\
\hline 50 & 19.3 & 2.7 \\
\hline 75 & 21.0 & 2.5 \\
\hline 100 & 21.8 & 2.6 \\
\hline
\end{tabular}

\begin{tabular}{|c|c|c|}
\hline Return Period, yr & Maximum Water Level, ft & Water Level Standard Deviation, $\mathrm{ft}$ \\
\hline 2 & 2.3 & 1.0 \\
\hline 5 & 8.6 & 1.2 \\
\hline 10 & 12.0 & 1.4 \\
\hline 25 & 16.2 & 2.0 \\
\hline 50 & 19.3 & 2.7 \\
\hline 75 & 21.0 & 2.5 \\
\hline 100 & 21.8 & 2.6 \\
\hline
\end{tabular}




\begin{tabular}{|c|c|c|}
\hline \multicolumn{3}{|c|}{$\begin{array}{l}\text { Table B153 } \\
\text { Return Period, Maximum Water Level, and Water Level Standard } \\
\text { Deviation for Profile Tutuila } 150\end{array}$} \\
\hline Return Period, yr & Maximum Water Level, ft & Water Level Standard Deviation, ft \\
\hline 2 & 2.3 & 1.0 \\
\hline 5 & 8.6 & 1.2 \\
\hline 10 & 12.0 & 1.4 \\
\hline 25 & 16.2 & 2.0 \\
\hline 50 & 19.3 & 2.7 \\
\hline 75 & 21.0 & 2.5 \\
\hline 100 & 21.8 & 2.6 \\
\hline
\end{tabular}

\begin{tabular}{|c|c|c|}
\hline $\begin{array}{l}\text { Table B154 } \\
\text { Return Perio } \\
\text { Deviation for } \\
\end{array}$ & $\begin{array}{l}\text { ximum Water Leve } \\
\text { le Tutuila } 151\end{array}$ & and Water Level Standard \\
\hline Return Period, yr & Maximum Water Level, $\mathbf{f t}$ & Water Level Standard Deviation, ft \\
\hline 2 & 2.3 & 0.9 \\
\hline 5 & 7.4 & 1.0 \\
\hline 10 & 10.5 & 1.3 \\
\hline 25 & 14.6 & 1.9 \\
\hline 50 & 17.6 & 2.7 \\
\hline 75 & 19.3 & 2.5 \\
\hline 100 & 20.2 & 2.6 \\
\hline
\end{tabular}

\begin{tabular}{|c|c|c|}
\hline \multicolumn{3}{|c|}{$\begin{array}{l}\text { Table B155 } \\
\text { Return Period, Maximum Water Level, and Water Level Standard } \\
\text { Deviation for Profile Tutuila } 152\end{array}$} \\
\hline Return Period, yr & Maximum Water Level, ft & Water Level Standard Deviation, ft \\
\hline 2 & 2.3 & 1.0 \\
\hline 5 & 8.8 & 1.2 \\
\hline 10 & 12.1 & 1.4 \\
\hline 25 & 16.4 & 2.0 \\
\hline 50 & 19.5 & 2.7 \\
\hline 75 & 21.1 & 2.5 \\
\hline 100 & 21.9 & 2.6 \\
\hline
\end{tabular}

\begin{tabular}{|c|c|c|}
\hline \multicolumn{3}{|c|}{$\begin{array}{l}\text { Table B156 } \\
\text { Return Period, Maximum Water Level, and Water Level Standard } \\
\text { Deviation for Profile Tutuila } 153\end{array}$} \\
\hline Return Period, yr & Maximum Water Level, ft & Water Level Standard Deviation, $f t$ \\
\hline$\underline{2}$ & 2.3 & 1.0 \\
\hline 5 & 8.8 & 1.2 \\
\hline 10 & 12.1 & 1.4 \\
\hline 25 & 16.4 & 2.0 \\
\hline 50 & 19.5 & 2.7 \\
\hline 75 & 21.1 & 2.5 \\
\hline 100 & 21.9 & 2.6 \\
\hline
\end{tabular}




\begin{tabular}{|c|c|c|}
\hline \multicolumn{3}{|c|}{$\begin{array}{l}\text { Table B157 } \\
\text { Return Period, Maximum Water Level, and Water Level Standard } \\
\text { Deviation for Profile Tutuila } 154\end{array}$} \\
\hline Return Period, yr & Maximum Water Level, $\mathrm{ft}$ & Water Level Standard Deviation, $\mathrm{ft}$ \\
\hline 2 & 2.3 & 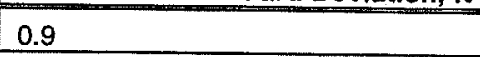 \\
\hline 5 & 7.5 & 1.0 \\
\hline 10 & 10.6 & 1.3 \\
\hline 25 & 14.7 & 1.9 \\
\hline 50 & 17.7 & 2.7 \\
\hline 75 & 19.4 & 2.5 \\
\hline 100 & 20.2 & 2.6 \\
\hline
\end{tabular}

\begin{tabular}{|c|c|c|}
\hline \multicolumn{3}{|c|}{$\begin{array}{l}\text { Table B158 } \\
\text { Return Period, Maximum Water Level, and Water Level Standard } \\
\text { Deviation for Profile Tutuila } 155\end{array}$} \\
\hline Return Period, yr & Maximum Water Level, $\mathrm{ft}$ & Water Level Standard Deviation, $\mathrm{ft}$ \\
\hline 2 & 2.3 & 1.0 \\
\hline 5 & 8.8 & 1.2 \\
\hline 10 & 12.1 & 1.4 \\
\hline 25 & 16.4 & 2.0 \\
\hline 50 & 19.5 & 2.7 \\
\hline 75 & 21.1 & 2.5 \\
\hline 100 & 21.9 & 2.6 \\
\hline
\end{tabular}

\begin{tabular}{|c|c|c|}
\hline \multicolumn{3}{|c|}{$\begin{array}{l}\text { Table B159 } \\
\text { Return Period, Maximum Water Level, and Water Level Standard } \\
\text { Deviation for Profile Tutuila } 156\end{array}$} \\
\hline Return Period, yr & Maximum Water Level, $\mathrm{ft}$ & Water Level Standard Deviation, $\mathrm{ft}$ \\
\hline 2 & 1.3 & 0.7 \\
\hline 5 & 6.2 & 1.0 \\
\hline 10 & 8.7 & 1.0 \\
\hline 25 & 11.8 & 1.4 \\
\hline 50 & 14.0 & 1.9 \\
\hline 75 & 15.1 & 1.8 \\
\hline 100 & 15.7 & 1.9 \\
\hline
\end{tabular}

\begin{tabular}{|c|c|c|}
\hline \multicolumn{3}{|c|}{$\begin{array}{l}\text { Table B160 } \\
\text { Return Period, Maximum Water Level, and Water Level Standard } \\
\text { Deviation for Profile Tutuila } 157\end{array}$} \\
\hline Return Period, yr & Maximum Water Level, ft & Water Level Standard Deviation, $\mathrm{ft}$ \\
\hline 2 & 2.3 & -1 \\
\hline 5 & 8.8 & 1.2 \\
\hline 10 & 12.1 & 1.4 \\
\hline 25 & 16.4 & 2.0 \\
\hline 50 & 19.5 & 2.7 \\
\hline 75 & 21.1 & 2.5 \\
\hline 100 & 21.9 & 2.6 \\
\hline
\end{tabular}




\begin{tabular}{|c|c|c|}
\hline \multicolumn{3}{|c|}{$\begin{array}{l}\text { Table B161 } \\
\text { Return Period, Maximum Water Level, and Water Level Standard } \\
\text { Deviation for Profile Tutuila } 158\end{array}$} \\
\hline Return Period, yr & Maximum Water Level, $\mathrm{ft}$ & Water Level Standard Deviation, $\mathrm{ft}$ \\
\hline 2 & 2.3 & 1.0 \\
\hline 5 & 8.8 & 1.2 \\
\hline 10 & 12.1 & 1.4 \\
\hline 25 & 16.4 & 2.0 \\
\hline 50 & 19.5 & 2.7 \\
\hline 75 & 21.1 & 2.5 \\
\hline 100 & 21.9 & 2.6 \\
\hline
\end{tabular}

\begin{tabular}{l}
\hline $\begin{array}{l}\text { Table B162 } \\
\text { Return Period, Maximum Water Level, and Water Level Standard } \\
\text { Deviation for Profile Tutuila 159 }\end{array}$ \\
\begin{tabular}{|l|l|l|}
\hline Return Period, yr & Maximum Water Level, $\mathrm{ft}$ & Water Level Standard Deviation, ft \\
\hline 2 & 2.4 & 1.0 \\
\hline 5 & 9.0 & 1.1 \\
\hline 10 & 12.2 & 1.3 \\
\hline 25 & 16.5 & 2.0 \\
\hline 50 & 19.6 & 2.7 \\
\hline 75 & 21.2 & 2.5 \\
\hline 100 & 22.0 & 2.6 \\
\hline
\end{tabular}
\end{tabular}

\begin{tabular}{|c|c|c|}
\hline \multicolumn{3}{|c|}{$\begin{array}{l}\text { Table B163 } \\
\text { Return Period, Maximum Water Level, and Water Level Standard } \\
\text { Deviation for Profile Tutuila } 160\end{array}$} \\
\hline Return Period, yr & Maximum Water Level, $\mathrm{ft}$ & Water Level Standard Deviation, ft \\
\hline 2 & 2.4 & 1.0 \\
\hline 5 & 9.0 & 1.1 \\
\hline 10 & 12.2 & 1.3 \\
\hline 25 & 16.5 & 2.0 \\
\hline 50 & 19.6 & 2.7 \\
\hline 75 & 21.2 & 2.5 \\
\hline 100 & 22.0 & 2.6 \\
\hline
\end{tabular}

\begin{tabular}{|c|c|c|}
\hline \multicolumn{3}{|c|}{$\begin{array}{l}\text { Table B164 } \\
\text { Return Period, Maximum Water Level, and Water Level Standard } \\
\text { Deviation for Profile Tutuila } 161\end{array}$} \\
\hline Return Period, yr & Maximum Water Level, $\mathrm{ft}$ & Water Level Standard Deviation, ft \\
\hline 2 & 2.4 & 1.0 \\
\hline 5 & 9.0 & 1.1 \\
\hline 10 & 12.2 & 1.3 \\
\hline 25 & 16.5 & 2.0 \\
\hline 50 & 19.6 & 2.7 \\
\hline 75 & 21.2 & 2.5 \\
\hline 100 & 22.0 & 2.6 \\
\hline
\end{tabular}




\begin{tabular}{|c|c|c|}
\hline \multicolumn{3}{|c|}{$\begin{array}{l}\text { Table B165 } \\
\text { Return Period, Maximum Water Level, and Water Level Standard } \\
\text { Deviation for Profile Tutuila } 162\end{array}$} \\
\hline Return Period, yr & Maximum Water Level, ft & Water Level Standard Deviation, ft \\
\hline 2 & 2.4 & 1.0 \\
\hline 5 & 9.0 & 1.1 \\
\hline 10 & 12.2 & 1.3 \\
\hline 25 & 16.5 & 2.0 \\
\hline 50 & 19.6 & 2.7 \\
\hline 75 & 21.2 & 2.5 \\
\hline 100 & 22.0 & 2.6 \\
\hline
\end{tabular}

\begin{tabular}{|c|c|c|}
\hline \multicolumn{3}{|c|}{$\begin{array}{l}\text { Table B166 } \\
\text { Return Period, Maximum Water Level, and Water Level Standard } \\
\text { Deviation for Profile Tutuila } 163\end{array}$} \\
\hline Return Period, yr & Maximum Water Level, ft & Water Level Standard Deviation, ft \\
\hline 2 & 2.2 & 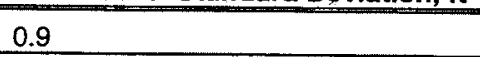 \\
\hline 5 & 7.1 & 0.8 \\
\hline 10 & 10.0 & 1.2 \\
\hline 25 & 14.2 & 2.3 \\
\hline 50 & 17.7 & 2.8 \\
\hline 75 & 19.4 & 2.5 \\
\hline 100 & 20.3 & 2.6 \\
\hline
\end{tabular}

\begin{tabular}{|c|c|c|}
\hline \multicolumn{3}{|c|}{$\begin{array}{l}\text { Table B167 } \\
\text { Return Period, Maximum Water Level, and Water Level Standard } \\
\text { Deviation for Profile Tutuila } 164\end{array}$} \\
\hline Return Period, yr & Maximum Water Level, ft & Water Level Standard Deviation, $\mathrm{ft}$ \\
\hline 2 & 2.1 & 0.9 \\
\hline 5 & 7.4 & 1.1 \\
\hline 10 & $\$ 1.0$ & 1.4 \\
\hline 25 & 15.8 & 2.5 \\
\hline 50 & 19.4 & 2.8 \\
\hline 75 & 21.1 & 2.5 \\
\hline 100 & 21.9 & 2.7 \\
\hline
\end{tabular}

\begin{tabular}{|c|c|c|}
\hline \multicolumn{3}{|c|}{$\begin{array}{l}\text { Table B168 } \\
\text { Return Period, Maximum Water Level, and Water Level Standard } \\
\text { Deviation for Profile Tutuila } 165\end{array}$} \\
\hline Return Period, yr & Maximum Water Level, ft & Water Level Standard Deviation, $\mathrm{ft}$ \\
\hline 2 & 2.1 & 0.9 \\
\hline 5 & 7.4 & 1.1 \\
\hline 10 & 11.0 & 1.4 \\
\hline 25 & 15.8 & 2.5 \\
\hline 50 & 19.4 & 2.8 \\
\hline 75 & 21.1 & 2.5 \\
\hline 100 & 21.9 & 2.7 \\
\hline
\end{tabular}




\begin{tabular}{|c|c|c|}
\hline \multicolumn{3}{|c|}{$\begin{array}{l}\text { Table B169 } \\
\text { Return Period, Maximum Water Level, and Water Level Standard } \\
\text { Deviation for Profile Tutuila } 166\end{array}$} \\
\hline Return Period, yr & Maximum Water Level, $\mathrm{ft}$ & Water Level Standard Deviation, $\mathrm{ft}$ \\
\hline 2 & 2.1 & 0.9 \\
\hline 5 & 7.4 & 1.1 \\
\hline 10 & 11.0 & 1.4 \\
\hline 25 & 15.8 & 2.5 \\
\hline 50 & 19.4 & 2.8 \\
\hline 75 & 21.1 & 2.5 \\
\hline 100 & 21.9 & 2.7 \\
\hline
\end{tabular}

\begin{tabular}{|c|c|c|}
\hline \multicolumn{3}{|c|}{$\begin{array}{l}\text { Table B170 } \\
\text { Return Period, Maximum Water Level, and Water Level Standard } \\
\text { Deviation for Profile Tutuila } 167\end{array}$} \\
\hline Return Period, yr & Maximum Water Level, $\mathrm{ft}$ & Water Level Standard Deviation, $\mathrm{ft}$ \\
\hline 2 & 0.6 & 0.5 \\
\hline 5 & 2.9 & 0.4 \\
\hline 10 & 4.4 & 0.8 \\
\hline 25 & 7.1 & 1.3 \\
\hline 50 & 9.2 & 1.5 \\
\hline 75 & 10.0 & 1.4 \\
\hline 100 & 10.4 & 1.5 \\
\hline
\end{tabular}

\begin{tabular}{|c|c|c|}
\hline \multicolumn{3}{|c|}{$\begin{array}{l}\text { Table B171 } \\
\text { Return Period, Maximum Water Level, and Water Level Standard } \\
\text { Deviation for Profile Tutuila } 168\end{array}$} \\
\hline Return Period, yr & Maximum Water Level, ft & Water Level Standard Deviation, $\mathrm{ft}$ \\
\hline 2 & 0.5 & 0.4 \\
\hline 5 & 2.7 & 0.3 \\
\hline 10 & 4.1 & 0.8 \\
\hline 25 & 6.8 & 1.3 \\
\hline 50 & 8.8 & 1.4 \\
\hline 75 & 9.6 & 1.4 \\
\hline 100 & 10.0 & 1.5 \\
\hline
\end{tabular}

\section{Table B172}

Return Period, Maximum Water Level, and Water Level Standard Deviation for Profile Tutuila 169

\begin{tabular}{|l|l|l|}
\hline Return Period, yr & Maximum Water Level, $\mathrm{ft}$ & Water Level Standard Deviation, $\mathrm{ft}$ \\
\hline 2 & 0.4 & 0.4 \\
\hline 5 & 2.5 & 0.3 \\
\hline 10 & 3.5 & 0.6 \\
\hline 25 & 6.1 & 1.3 \\
\hline 50 & 8.2 & 1.6 \\
\hline 75 & 9.2 & 1.5 \\
\hline 100 & 9.7 & 1.6 \\
\hline
\end{tabular}




\begin{tabular}{|c|c|c|}
\hline \multicolumn{3}{|c|}{$\begin{array}{l}\text { Table B173 } \\
\text { Return Period, Maximum Water Level, and Water Level Standard } \\
\text { Deviation for Profile Tutuila } 170\end{array}$} \\
\hline Return Period, yr & Maximum Water Level, ft & Water Level Standard Deviation, $\mathrm{ft}$ \\
\hline 2 & 0.5 & 0.5 \\
\hline 5 & 2.8 & 0.4 \\
\hline 10 & 4.2 & 0.8 \\
\hline 25 & 7.1 & 1.4 \\
\hline 50 & 9.2 & 1.5 \\
\hline 75 & 10.0 & 1.4 \\
\hline 100 & 10.5 & 1.5 \\
\hline
\end{tabular}

\begin{tabular}{l}
\hline $\begin{array}{l}\text { Table B174 } \\
\text { Return Period, Maximum Water Level, and Water Level Standard } \\
\text { Deviation for Profile Tutuila 171 }\end{array}$ \\
\begin{tabular}{|l|l|l|}
\hline Return Period, yr & Maximum Water Level, $\mathrm{ft}$ & Water Level Standard Deviation, ft \\
\hline 2 & 0.3 & 0.4 \\
\hline 5 & 2.2 & 0.3 \\
\hline 10 & 3.0 & 0.5 \\
\hline 25 & 5.4 & 1.4 \\
\hline 50 & 7.6 & 1.6 \\
\hline 75 & 8.6 & 1.5 \\
\hline 100 & 9.1 & 1.5 \\
\hline
\end{tabular}
\end{tabular}

\section{Table B175}

Return Period, Maximum Water Level, and Water Level Standard Deviation for Profile Tutuila 172

\begin{tabular}{|l|l|l|}
\hline Return Period, yr & Maximum Water Level, $\mathrm{ft}$ & Water Level Standard Deviation, $\mathrm{ft}$ \\
\hline 2 & 0.4 & 0.4 \\
\hline 5 & 2.3 & 0.3 \\
\hline 10 & 3.2 & 0.5 \\
\hline 25 & 5.6 & 1.3 \\
\hline 50 & 7.6 & 1.5 \\
\hline 75 & 8.5 & 1.4 \\
\hline 100 & 9.0 & 1.5 \\
\hline
\end{tabular}

\section{Table B176}

Return Period, Maximum Water Level, and Water Level Standard Deviation for Profile Tutuila 173

\begin{tabular}{|l|l|l|}
\hline Return Period, yr & Maximum Water Level, $\mathrm{ft}$ & Water Level Standard Deviation, $\mathbf{f t}$ \\
\hline 2 & 0.0 & 0.0 \\
\hline 5 & 0.9 & 0.4 \\
\hline 10 & 1.5 & 0.2 \\
\hline 25 & 2.1 & 0.4 \\
\hline 50 & 2.9 & 0.7 \\
\hline 75 & 3.4 & 0.7 \\
\hline 100 & 3.6 & 0.8 \\
\hline
\end{tabular}




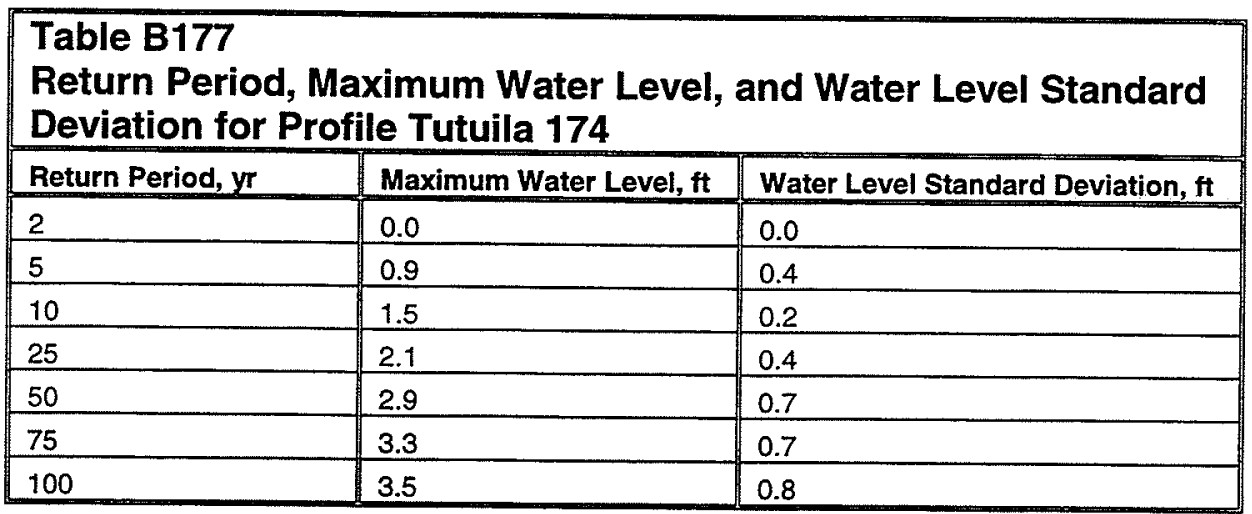

\begin{tabular}{|c|c|c|}
\hline \multicolumn{3}{|c|}{$\begin{array}{l}\text { Table B178 } \\
\text { Return Period, Maximum Water Level, and Water Level Standard } \\
\text { Deviation for Profile Tutuila } 175\end{array}$} \\
\hline Return Period, yr & Maximum Water Level, $\mathrm{ft}$ & Water Level Standard Deviation, $\mathrm{tt}$ \\
\hline 2 & 0.0 & 0.0 \\
\hline 5 & 0.9 & 0.4 \\
\hline 10 & 1.5 & 0.2 \\
\hline 25 & 2.1 & 0.4 \\
\hline 50 & 2.9 & 0.7 \\
\hline 75 & 3.3 & 0.7 \\
\hline 100 & 3.5 & 0.8 \\
\hline
\end{tabular}

\begin{tabular}{|c|c|c|}
\hline \multicolumn{3}{|c|}{$\begin{array}{l}\text { Table B179 } \\
\text { Return Period, Maximum Water Level, and Water Level Standard } \\
\text { Deviation for Profile Tutuila } 176\end{array}$} \\
\hline Return Period, yr & Maximum Water Level, $\mathrm{ft}$ & Water Level Standard Deviation, $\mathrm{ft}$ \\
\hline 2 & 0.0 & 0.0 \\
\hline 5 & 0.9 & 0.4 \\
\hline 10 & 1.5 & 0.2 \\
\hline 25 & 2.3 & 0.5 \\
\hline 50 & 3.1 & 0.7 \\
\hline 75 & 3.6 & 0.7 \\
\hline 100 & 3.8 & 0.8 \\
\hline
\end{tabular}

\begin{tabular}{|c|c|c|}
\hline \multicolumn{3}{|c|}{$\begin{array}{l}\text { Table B180 } \\
\text { Return Period, Maximum Water Level, and Water Level Standard } \\
\text { Deviation for Profile Tutuila } 177\end{array}$} \\
\hline Return Period, yr & Maximum Water Level, $\mathrm{tt}$ & Water Level Standard Deviation, $\mathrm{ft}$ \\
\hline 2 & 0.0 & 0.0 \\
\hline 5 & 1.0 & 0.4 \\
\hline 10 & 1.6 & 0.5 \\
\hline 25 & 3.9 & 1.3 \\
\hline 50 & 5.6 & 1.2 \\
\hline 75 & 6.2 & 1.1 \\
\hline 100 & 6.5 & 1.1 \\
\hline
\end{tabular}




\begin{tabular}{|c|c|c|}
\hline \multicolumn{3}{|c|}{$\begin{array}{l}\text { Table B181 } \\
\text { Return Period, Maximum Water Level, and Water Level Standard } \\
\text { Deviation for Profile Tutuila } 178\end{array}$} \\
\hline Return Period, yr & Maximum Water Level, ft & Water Level Standard Deviation, $\mathrm{ft}$ \\
\hline 2 & 0.0 & 0.0 \\
\hline 5 & 1.0 & 0.4 \\
\hline 10 & 1.6 & 0.4 \\
\hline 25 & 3.4 & 1.0 \\
\hline 50 & 4.9 & 1.0 \\
\hline 75 & 5.5 & 0.9 \\
\hline 100 & 5.8 & 0.9 \\
\hline
\end{tabular}

\begin{tabular}{|c|c|c|}
\hline \multicolumn{3}{|c|}{$\begin{array}{l}\text { Table B182 } \\
\text { Return Period, Maximum Water Level, and Water Level Standard } \\
\text { Deviation for Profile Tutuila } 179\end{array}$} \\
\hline Return Period, yr & Maximum Water Level, $\mathrm{ft}$ & Water Level Standard Deviation, $\mathrm{ft}$ \\
\hline$\underline{2}$ & 0.0 & 0.0 \\
\hline 5 & 1.0 & 0.4 \\
\hline 10 & 1.6 & 0.4 \\
\hline 25 & 3.3 & 1.0 \\
\hline 50 & 4.7 & 1.0 \\
\hline 75 & 5.2 & 0.9 \\
\hline 100 & 5.5 & 0.9 \\
\hline
\end{tabular}

\begin{tabular}{|l|l|l|}
\hline $\begin{array}{l}\text { Table B183 } \\
\text { Return Period, Maximum Water Level, and Water Level Standard } \\
\text { Deviation for Profile Tutuila 180 }\end{array}$ \\
\hline Return Period, yr & Maximum Water Level, $\mathrm{ft}$ & Water Level Standard Deviation, $\mathrm{ft}$ \\
\hline 2 & 0.0 & 0.0 \\
\hline 5 & 1.0 & 0.4 \\
\hline 10 & 1.6 & 0.4 \\
\hline 25 & 3.4 & 1.0 \\
\hline 50 & 4.9 & 1.0 \\
\hline 75 & 5.5 & 0.9 \\
\hline 100 & 5.8 & 0.9 \\
\hline
\end{tabular}

\begin{tabular}{|c|c|c|}
\hline \multicolumn{3}{|c|}{$\begin{array}{l}\text { Table B184 } \\
\text { Return Period, Maximum Water Level, and Water Level Standard } \\
\text { Deviation for Profile Tutuila } 181\end{array}$} \\
\hline Return Period, yr & Maximum Water Level, $\mathrm{ft}$ & Water Level Standard Deviation, $\mathrm{ft}$ \\
\hline 2 & 0.0 & 0.0 \\
\hline 5 & 1.0 & 0.4 \\
\hline 10 & 1.6 & 0.4 \\
\hline 25 & 3.3 & 1.0 \\
\hline 50 & 4.7 & 1.0 \\
\hline 75 & 5.2 & 0.9 \\
\hline 100 & 5.5 & 0.9 \\
\hline
\end{tabular}




\begin{tabular}{|c|c|c|}
\hline \multicolumn{3}{|c|}{$\begin{array}{l}\text { Table B185 } \\
\text { Return Period, Maximum Water Level, and Water Level Standard } \\
\text { Deviation for Profile Tutuila } 182\end{array}$} \\
\hline Return Period, yr & Maximum Water Level, ft & Water Level Standard Deviation, it \\
\hline 2 & 0.0 & 0.0 \\
\hline 5 & 0.9 & 0.4 \\
\hline 10 & 1.5 & 0.2 \\
\hline 25 & 2.2 & 0.4 \\
\hline 50 & 3.0 & 0.7 \\
\hline 75 & 3.4 & 0.7 \\
\hline 100 & 3.6 & 0.8 \\
\hline
\end{tabular}

\begin{tabular}{|c|c|c|}
\hline \multicolumn{3}{|c|}{$\begin{array}{l}\text { Table B186 } \\
\text { Return Period, Maximum Water Level, and Water Level Standard } \\
\text { Deviation for Profile Tutuila } 183\end{array}$} \\
\hline Return Period, yr & Maximum Water Level, $\mathrm{tt}$ & Water Level Standard Deviation, ft \\
\hline 2 & 0.0 & 0.1 \\
\hline 5 & 1.4 & 0.2 \\
\hline 10 & 2.1 & 0.3 \\
\hline 25 & 2.7 & 0.2 \\
\hline 50 & 3.1 & 0.4 \\
\hline 75 & 3.4 & 0.5 \\
\hline 100 & 3.6 & 0.6 \\
\hline
\end{tabular}

\begin{tabular}{|c|c|c|}
\hline \multicolumn{3}{|c|}{$\begin{array}{l}\text { Table B187 } \\
\text { Return Period, Maximum Water Level, and Water Level Standard } \\
\text { Deviation for Profile Tutuila } 184\end{array}$} \\
\hline Return Period, yr & Maximum Water Level, ft & Water Level Standard Deviation, $\mathrm{ft}$ \\
\hline 2 & 0.0 & 0.1 \\
\hline 5 & 1.5 & 0.2 \\
\hline 10 & 2.2 & 0.3 \\
\hline 25 & 2.9 & 0.2 \\
\hline 50 & 3.2 & 0.4 \\
\hline 75 & 3.5 & 0.4 \\
\hline 100 & 3.6 & 0.5 \\
\hline
\end{tabular}

\begin{tabular}{|c|c|c|}
\hline \multicolumn{3}{|c|}{$\begin{array}{l}\text { Table B188 } \\
\text { Return Period, Maximum Water Level, and Water Level Standard } \\
\text { Deviation for Profile Tutuila } 185\end{array}$} \\
\hline Return Period, yr & Maximum Water Level, ft & Water Level Standard Deviation, $\mathrm{ft}$ \\
\hline 2 & 0.1 & 0.2 \\
\hline 5 & 1.7 & 0.2 \\
\hline 10 & 2.5 & 0.3 \\
\hline 25 & 3.2 & 0.3 \\
\hline 50 & 3.6 & 0.3 \\
\hline 75 & 3.8 & 0.3 \\
\hline 100 & 3.9 & 0.4 \\
\hline
\end{tabular}




\begin{tabular}{|c|c|c|}
\hline \multicolumn{3}{|c|}{$\begin{array}{l}\text { Table B189 } \\
\text { Return Period, Maximum Water Level, and Water Level Standard } \\
\text { Deviation for Profile Tutuila } 186\end{array}$} \\
\hline Return Period, yr & Maximum Water Level, $\mathrm{ft}$ & Water Level Standard Deviation, $\mathrm{ft}$ \\
\hline 2 & 0.1 & 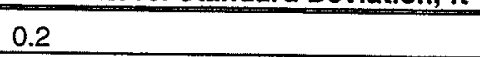 \\
\hline 5 & 1.8 & 0.2 \\
\hline 10 & 2.5 & 0.3 \\
\hline 25 & 3.2 & 0.3 \\
\hline 50 & 3.6 & 0.3 \\
\hline 75 & 3.8 & 0.3 \\
\hline 100 & 4.0 & 0.4 \\
\hline
\end{tabular}

\begin{tabular}{|l|l|l|}
\hline $\begin{array}{l}\text { Table B190 } \\
\text { Return Period, Maximum Water Level, and Water Level Standard } \\
\text { Deviation for Profile Tutuila 187 }\end{array}$ \\
\hline Return Period, yr & Maximum Water Level, ft & Water Level Standard Deviation, $\mathrm{ft}$ \\
\hline 2 & 0.0 & 0.1 \\
\hline 5 & 1.7 & 0.2 \\
\hline 10 & 2.4 & 0.3 \\
\hline 25 & 3.1 & 0.2 \\
\hline 50 & 3.4 & 0.3 \\
\hline 75 & 3.7 & 0.4 \\
\hline 100 & 3.8 & 0.4 \\
\hline
\end{tabular}

\begin{tabular}{|l|l|l|}
\hline $\begin{array}{l}\text { Table B191 } \\
\text { Return Period, Maximum Water Level, and Water Level Standard } \\
\text { Deviation for Profile Tutuila } \mathbf{1 8 8}\end{array}$ \\
\hline Return Period, yr & Maximum Water Level, $\mathrm{ft}$ & Water Level Standard Deviation, $\mathrm{ft}$ \\
\hline 2 & 0.0 & 0.1 \\
\hline 5 & 1.6 & 0.2 \\
\hline 10 & 2.3 & 0.3 \\
\hline 25 & 3.0 & 0.2 \\
\hline 50 & 3.3 & 0.3 \\
\hline 75 & 3.6 & 0.4 \\
\hline 100 & 3.7 & 0.5 \\
\hline
\end{tabular}

\begin{tabular}{|c|c|c|}
\hline \multicolumn{3}{|c|}{$\begin{array}{l}\text { Table B192 } \\
\text { Return Period, Maximum Water Level, and Water Level Standard } \\
\text { Deviation for Profile Tutuila } 189\end{array}$} \\
\hline Return Period, yr & Maximum Water Level, ft & Water Level Standard Deviation, $\mathrm{ft}$ \\
\hline 2 & 0.0 & 0.1 \\
\hline 5 & 1.6 & 0.2 \\
\hline 10 & 2.3 & 0.3 \\
\hline 25 & 3.0 & 0.2 \\
\hline 50 & 3.3 & 0.4 \\
\hline 75 & 3.6 & 0.4 \\
\hline 100 & 3.7 & 0.5 \\
\hline
\end{tabular}




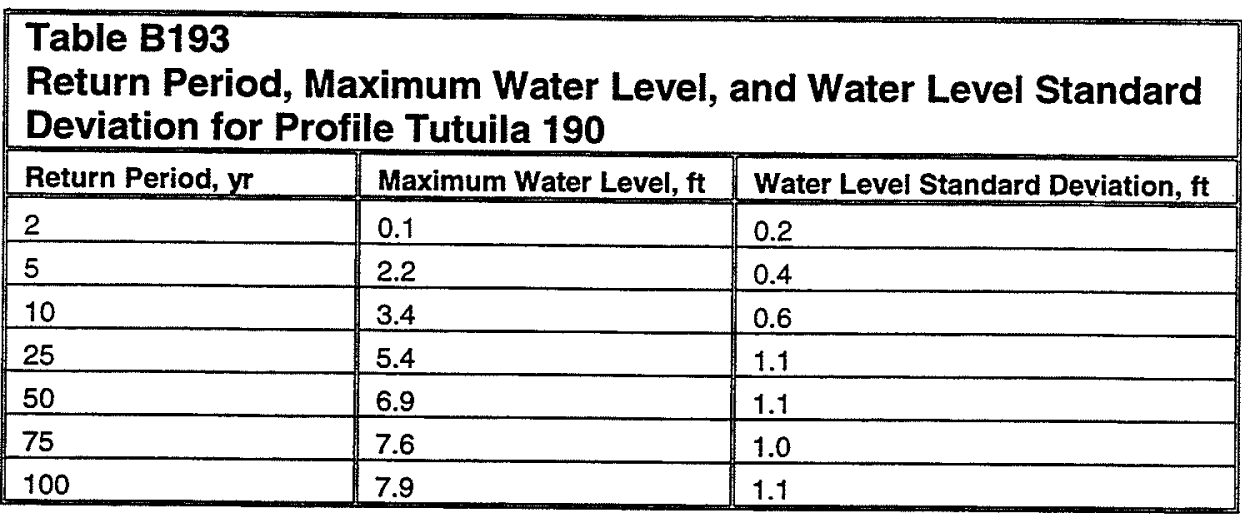

\begin{tabular}{|l|l|l|}
\hline $\begin{array}{l}\text { Table B194 } \\
\text { Return Period, Maximum Water Level, and Water Level Standard } \\
\text { Deviation for Profile Tutuila 191 }\end{array}$ \\
\hline Return Period, yr & Maximum Water Level, $\mathrm{ft}$ & Water Level Standard Deviation, $\mathrm{ft}$ \\
\hline 2 & 0.0 & 0.0 \\
\hline 5 & 1.5 & 0.4 \\
\hline 10 & 3.0 & 0.9 \\
\hline 25 & 5.3 & 1.1 \\
\hline 50 & 7.0 & 1.2 \\
\hline 75 & 7.6 & 1.0 \\
\hline 100 & 7.9 & 1.1 \\
\hline
\end{tabular}

\begin{tabular}{|c|c|c|}
\hline \multicolumn{3}{|c|}{$\begin{array}{l}\text { Table B195 } \\
\text { Return Period, Maximum Water Level, and Water Level Standard } \\
\text { Deviation for Profile Tutuila } 192\end{array}$} \\
\hline Return Period, yr & Maximum Water Level, $\mathrm{ft}$ & Water Level Standard Deviation, ft \\
\hline 2 & 0.0 & 0.0 \\
\hline 5 & 1.4 & 0.4 \\
\hline 10 & 2.6 & 0.6 \\
\hline 25 & 4.0 & 0.6 \\
\hline 50 & 4.9 & 0.8 \\
\hline 75 & 5.4 & 0.8 \\
\hline 100 & 5.7 & 0.8 \\
\hline
\end{tabular}

\begin{tabular}{|l|l|l|}
\hline $\begin{array}{l}\text { Table B196 } \\
\text { Return Period, Maximum Water Level, and Water Level Standard } \\
\text { Deviation for Profile Tutuila 193 }\end{array}$ \\
\hline Return Period, yr & Maximum Water Level, ft & Water Level Standard Deviation, ft \\
\hline 2 & 0.0 & 0.0 \\
\hline 5 & 1.5 & 0.4 \\
\hline 10 & 2.6 & 0.6 \\
\hline 25 & 4.1 & 0.6 \\
\hline 50 & 5.0 & 0.8 \\
\hline 75 & 5.5 & 0.8 \\
\hline 100 & 5.7 & 0.9 \\
\hline
\end{tabular}




\begin{tabular}{|c|c|c|}
\hline \multicolumn{3}{|c|}{$\begin{array}{l}\text { Table B197 } \\
\text { Return Period, Maximum Water Level, and Water Level Standard } \\
\text { Deviation for Profile Tutuila } 194\end{array}$} \\
\hline Return Period, yr & Maximum Water Level, $\mathrm{ft}$ & Water Level Standard Deviation, $\mathrm{ft}$ \\
\hline 2 & 0.0 & 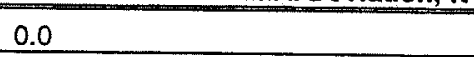 \\
\hline 5 & 1.5 & 0.4 \\
\hline 10 & 2.6 & 0.6 \\
\hline 25 & 4.1 & 0.6 \\
\hline 50 & 5.0 & 0.8 \\
\hline 75 & 5.5 & 0.8 \\
\hline 100 & 5.7 & 0.9 \\
\hline
\end{tabular}

\begin{tabular}{|c|c|c|}
\hline \multicolumn{3}{|c|}{$\begin{array}{l}\text { Table B198 } \\
\text { Return Period, Maximum Water Level, and Water Level Standard } \\
\text { Deviation for Profile Tutuila } 195\end{array}$} \\
\hline Return Period, yr & Maximum Water Level, ft & Water Level Standard Deviation, $\mathrm{ft}$ \\
\hline 2 & 0.0 & 0.0 \\
\hline 5 & 1.5 & 0.4 \\
\hline 10 & 2.6 & 0.6 \\
\hline 25 & 4.3 & 0.8 \\
\hline 50 & 5.6 & 1.0 \\
\hline 75 & 6.1 & 1.0 \\
\hline 100 & 6.4 & 1.1 \\
\hline
\end{tabular}

\begin{tabular}{|c|c|c|}
\hline \multicolumn{3}{|c|}{$\begin{array}{l}\text { Table B199 } \\
\text { Return Period, Maximum Water Level, and Water Level Standard } \\
\text { Deviation for Profile Tutuila } 196\end{array}$} \\
\hline Return Period, yr & Maximum Water Level, $\mathrm{ft}$ & Water Level Standard Deviation, $\mathrm{ft}$ \\
\hline 2 & 0.0 & 0.0 \\
\hline 5 & 1.4 & 0.3 \\
\hline 10 & 2.4 & 0.5 \\
\hline 25 & 3.8 & 0.6 \\
\hline 50 & 4.6 & 0.7 \\
\hline 75 & 5.1 & 0.7 \\
\hline 100 & 5.3 & 0.8 \\
\hline
\end{tabular}

\section{Table B200}

Return Period, Maximum Water Level, and Water Level Standard Deviation for Profile Tutuila 197

\begin{tabular}{|l|l|l|}
\hline Return Period, $\mathbf{y r}$ & Maximum Water Level, $\mathrm{tt}$ & Water Level Standard Deviation, $\mathrm{ft}$ \\
\hline 2 & 0.0 & 0.0 \\
\hline 5 & 1.3 & 0.3 \\
\hline 10 & 2.2 & 0.4 \\
\hline 25 & 3.4 & 0.6 \\
\hline 50 & 4.2 & 0.7 \\
\hline 75 & 4.6 & 0.7 \\
\hline 100 & 4.9 & 0.8 \\
\hline
\end{tabular}




\begin{tabular}{|c|c|c|}
\hline \multicolumn{3}{|c|}{$\begin{array}{l}\text { Table B201 } \\
\text { Return Period, Maximum Water Level, and Water Level Standard } \\
\text { Deviation for Profile Tutuila } 198\end{array}$} \\
\hline Return Period, yr & Maximum Water Level, $\mathrm{ft}$ & Water Level Standard Deviation, $\mathrm{ft}$ \\
\hline 2 & 0.0 & 0.0 \\
\hline 5 & 1.4 & 0.4 \\
\hline 10 & 2.6 & 0.6 \\
\hline 25 & 4.1 & 0.6 \\
\hline 50 & 5.0 & 0.8 \\
\hline 75 & 5.5 & 0.8 \\
\hline 100 & 5.7 & 0.9 \\
\hline
\end{tabular}

\begin{tabular}{|c|c|c|}
\hline $\begin{array}{l}\text { Table B202 } \\
\text { Return Perio } \\
\text { Deviation for } \\
\end{array}$ & $\begin{array}{l}\text { imum Water Level } \\
\text { Tutuila } 199\end{array}$ & nd Water Level Standard \\
\hline Return Period, yr & Maximum Water Level, $\mathrm{ft}$ & Water Level Standard Deviation, ft \\
\hline 2 & 0.0 & 0.0 \\
\hline 5 & 1.4 & 0.4 \\
\hline 10 & 2.6 & 0.6 \\
\hline 25 & 4.0 & 0.6 \\
\hline 50 & 5.0 & 0.8 \\
\hline 75 & 5.4 & 0.8 \\
\hline 100 & 5.7 & 0.8 \\
\hline
\end{tabular}

\begin{tabular}{|c|c|c|}
\hline \multicolumn{3}{|c|}{$\begin{array}{l}\text { Table B203 } \\
\text { Return Period, Maximum Water Level, and Water Level Standard } \\
\text { Deviation for Profile Tutuila } 200\end{array}$} \\
\hline Return Period, yr & Maximum Water Level, ft & Water Level Standard Deviation, it \\
\hline 2 & 0.0 & 0.0 \\
\hline 5 & 1.3 & 0.3 \\
\hline 10 & 2.1 & 0.4 \\
\hline 25 & 3.2 & 0.5 \\
\hline 50 & 3.9 & 0.6 \\
\hline 75 & 4.3 & 0.7 \\
\hline 100 & 4.5 & 0.7 \\
\hline
\end{tabular}

\begin{tabular}{|c|c|c|}
\hline \multicolumn{3}{|c|}{$\begin{array}{l}\text { Table B204 } \\
\text { Return Period, Maximum Water Level, and Water Level Standard } \\
\text { Deviation for Profile Tutuila } 201\end{array}$} \\
\hline Return Period, yr & Maximum Water Level, $\mathrm{ft}$ & Water Level Standard Deviation, $\mathrm{ft}$ \\
\hline 2 & 0.0 & 0.0 \\
\hline 5 & 1.5 & 0.4 \\
\hline 10 & 2.6 & 0.6 \\
\hline 25 & 4.1 & 0.6 \\
\hline 50 & 5.0 & 0.8 \\
\hline 75 & 5.5 & 0.8 \\
\hline 100 & 5.7 & 0.9 \\
\hline
\end{tabular}




\begin{tabular}{|c|c|c|}
\hline \multicolumn{3}{|c|}{$\begin{array}{l}\text { Table B205 } \\
\text { Return Period, Maximum Water Level, and Water Level Standard } \\
\text { Deviation for Profile Tutuila } 202\end{array}$} \\
\hline Return Period, yr & Maximum Water Level, ft & Water Level Standard Deviation, $\mathrm{ft}$ \\
\hline$\underline{2}$ & 0.0 & 0.0 \\
\hline 5 & 1.4 & 0.4 \\
\hline 10 & 2.6 & 0.6 \\
\hline 25 & 4.1 & 0.6 \\
\hline 50 & 5.0 & 0.8 \\
\hline 75 & 5.5 & 0.8 \\
\hline 100 & 5.7 & 0.9 \\
\hline
\end{tabular}

\begin{tabular}{|l|l|l|}
\hline $\begin{array}{l}\text { Table B206 } \\
\text { Return Period, Maximum Water Level, and Water Level Standard } \\
\text { Deviation for Profile Tutuila 203 }\end{array}$ \\
\hline Return Period, yr & Maximum Water Level, ft & Water Level Standard Deviation, ft \\
\hline 2 & 0.0 & 0.0 \\
\hline 5 & 1.5 & 0.4 \\
\hline 10 & 2.6 & 0.6 \\
\hline 25 & 4.3 & 0.8 \\
\hline 50 & 5.6 & 1.0 \\
\hline 75 & 6.1 & 1.0 \\
\hline 100 & 6.4 & 1.1 \\
\hline
\end{tabular}

\begin{tabular}{|c|c|c|}
\hline \multicolumn{3}{|c|}{$\begin{array}{l}\text { Table B207 } \\
\text { Return Period, Maximum Water Level, and Water Level Standard } \\
\text { Deviation for Profile Tutuila } 204\end{array}$} \\
\hline Return Period, yr & Maximum Water Level, $\mathrm{ft}$ & Water Level Standard Deviation, $\mathrm{ft}$ \\
\hline 2 & 0.0 & 0.0 \\
\hline 5 & 1.4 & 0.4 \\
\hline 10 & 2.6 & 0.6 \\
\hline 25 & 4.1 & 0.6 \\
\hline 50 & 5.0 & 0.8 \\
\hline 75 & 5.5 & 0.8 \\
\hline 100 & 5.8 & 0.9 \\
\hline
\end{tabular}

\begin{tabular}{|c|c|c|}
\hline \multicolumn{3}{|c|}{$\begin{array}{l}\text { Table B208 } \\
\text { Return Period, Maximum Water Level, and Water Level Standard } \\
\text { Deviation for Profile Tutuila } 205\end{array}$} \\
\hline Return Period, yr & Maximum Water Level, $\mathrm{ft}$ & Water Level Standard Deviation, $\mathrm{ft}$ \\
\hline 2 & 0.0 & 0.0 \\
\hline 5 & 1.4 & 0.4 \\
\hline 10 & 2.6 & 0.6 \\
\hline 25 & 4.1 & 0.6 \\
\hline 50 & 5.0 & 0.8 \\
\hline 75 & 5.5 & 0.8 \\
\hline 100 & 5.8 & 0.9 \\
\hline
\end{tabular}




\begin{tabular}{|c|c|c|}
\hline \multicolumn{3}{|c|}{$\begin{array}{l}\text { Table B209 } \\
\text { Return Period, Maximum Water Level, and Water Level Standard } \\
\text { Deviation for Profile Tutuila } 206\end{array}$} \\
\hline Return Period, yr & Maximum Water Level, $\mathrm{ft}$ & Water Level Standard Deviation, $\mathrm{ft}$ \\
\hline 2 & 0.0 & 0.0 \\
\hline 5 & 1.3 & 0.3 \\
\hline 10 & 2.0 & 0.4 \\
\hline 25 & 3.2 & 0.5 \\
\hline 50 & 3.9 & 0.6 \\
\hline 75 & 4.4 & 0.7 \\
\hline 100 & 4.6 & 0.8 \\
\hline
\end{tabular}

\section{Table B210}

Return Period, Maximum Water Level, and Water Level Standard Deviation for Profile Tutuila 207

\begin{tabular}{|l|l|l|}
\hline Return Period, yr & Maximum Water Level, ft & Water Level Standard Deviation, ft \\
\hline 2 & 0.0 & 0.0 \\
\hline 5 & 1.4 & 0.3 \\
\hline 10 & 2.3 & 0.5 \\
\hline 25 & 3.7 & 0.6 \\
\hline 50 & 4.6 & 0.7 \\
\hline 75 & 5.0 & 0.7 \\
\hline 100 & 5.3 & 0.8 \\
\hline
\end{tabular}

\begin{tabular}{|c|c|c|}
\hline \multicolumn{3}{|c|}{$\begin{array}{l}\text { Table B211 } \\
\text { Return Period, Maximum Water Level, and Water Level Standard } \\
\text { Deviation for Profile Tutuila } 208\end{array}$} \\
\hline Return Period, yr & Maximum Water Level, $\mathrm{ft}$ & Water Level Standard Deviation, $\mathrm{ft}$ \\
\hline 2 & 0.0 & 0.0 \\
\hline 5 & 1.4 & 0.4 \\
\hline 10 & 2.6 & 0.6 \\
\hline 25 & 4.1 & 0.6 \\
\hline 50 & 5.0 & 0.8 \\
\hline 75 & 5.5 & 0.8 \\
\hline 100 & 5.8 & 0.9 \\
\hline
\end{tabular}

\begin{tabular}{|l|l|l|}
\hline $\begin{array}{l}\text { Table B212 } \\
\text { Return Period, Maximum Water Level, and Water Level Standard } \\
\text { Deviation for Profile Tutuila 209 }\end{array}$ \\
\hline Return Period, $y \mathbf{r}$ & Maximum Water Level, $\mathrm{ft}$ & Water Level Standard Deviation, $\mathrm{ft}$ \\
\hline 2 & 0.0 & 0.0 \\
\hline 5 & 1.5 & 0.4 \\
\hline 10 & 2.9 & 0.9 \\
\hline 25 & 5.4 & 1.1 \\
\hline 50 & 7.1 & 1.2 \\
\hline 75 & 7.7 & 1.1 \\
\hline 100 & 8.0 & 1.1 \\
\hline
\end{tabular}




\section{Table B213}

Return Period, Maximum Water Level, and Water Level Standard Deviation for Profile Tutuila 210

\begin{tabular}{|l|l|l|}
\hline Return Period, $y r$ & Maximum Water Level, $\mathrm{ft}$ & Water Level Standard Deviation, $\mathrm{ft}$ \\
\hline 2 & 0.0 & 0.0 \\
\hline 5 & 1.4 & 0.4 \\
\hline 10 & 2.6 & 0.7 \\
\hline 25 & 4.4 & 0.8 \\
\hline 50 & 5.6 & 1.0 \\
\hline 75 & 6.2 & 1.0 \\
\hline 100 & 6.4 & 1.1 \\
\hline
\end{tabular}

\begin{tabular}{|c|c|c|}
\hline \multicolumn{3}{|c|}{$\begin{array}{l}\text { Table B214 } \\
\text { Return Period, Maximum Water Level, and Water Level Standard } \\
\text { Deviation for Profile Tutuila 211 }\end{array}$} \\
\hline Return Period, $y$ & Maximum Water Level, $\mathrm{ft}$ & Water Level Standard Deviation, $\mathrm{ft}$ \\
\hline 2 & 0.0 & 0.0 \\
\hline 5 & 1.2 & 0.3 \\
\hline 10 & 1.7 & 0.2 \\
\hline 25 & 2.5 & 0.4 \\
\hline 50 & 3.1 & 0.5 \\
\hline 75 & 3.5 & 0.5 \\
\hline 100 & 3.6 & 0.6 \\
\hline
\end{tabular}

\section{Table B215}

Return Period, Maximum Water Level, and Water Level Standard Deviation for Profile Tutuila 212

\begin{tabular}{|l|l|l|}
\hline Return Period, $y r$ & Maximum Water Level, $\mathrm{ft}$ & Water Level Standard Deviation, ft \\
\hline 2 & 0.3 & 0.3 \\
\hline 5 & 2.1 & 0.2 \\
\hline 10 & 2.8 & 0.3 \\
\hline 25 & 3.4 & 0.3 \\
\hline 50 & 3.7 & 0.2 \\
\hline 75 & 3.8 & 0.2 \\
\hline 100 & 3.9 & 0.2 \\
\hline
\end{tabular}

\begin{tabular}{|c|c|c|}
\hline \multicolumn{3}{|c|}{$\begin{array}{l}\text { Table B216 } \\
\text { Return Period, Maximum Water Level, and Water Level Standard } \\
\text { Deviation for Profile Tutuila 213 }\end{array}$} \\
\hline Return Period, yr & Maximum Water Level, ft & Water Level Standard Deviation, ft \\
\hline 2 & 0.2 & 0.3 \\
\hline 5 & 2.1 & 0.2 \\
\hline 10 & 2.7 & 0.3 \\
\hline 25 & 3.3 & 0.3 \\
\hline 50 & 3.6 & 0.2 \\
\hline 75 & 3.8 & 0.2 \\
\hline 100 & 3.8 & 0.2 \\
\hline
\end{tabular}




\begin{tabular}{|c|c|c|}
\hline \multicolumn{3}{|c|}{$\begin{array}{l}\text { Table B217 } \\
\text { Return Period, Maximum Water Level, and Water Level Standard } \\
\text { Deviation for Profile Tutuila } 214\end{array}$} \\
\hline Return Period, yr & Maximum Water Level, $\mathrm{ft}$ & Water Level Standard Deviation, $\mathrm{ft}$ \\
\hline 2 & 0.2 & 0.3 \\
\hline 5 & 2.0 & 0.2 \\
\hline 10 & 2.7 & 0.3 \\
\hline 25 & 3.3 & 0.3 \\
\hline 50 & 3.6 & 0.2 \\
\hline 75 & 3.7 & 0.2 \\
\hline 100 & 3.8 & 0.2 \\
\hline
\end{tabular}

\begin{tabular}{|l|l|l|}
\hline $\begin{array}{l}\text { Table B218 } \\
\text { Return Period, Maximum Water Level, and Water Level Standard } \\
\text { Deviation for Profile Tutuila 215 }\end{array}$ \\
\hline Return Period, yr & Maximum Water Level, $\mathrm{ft}$ & Water Level Standard Deviation, $\mathrm{ft}$ \\
\hline 2 & 0.2 & 0.3 \\
\hline 5 & 2.1 & 0.2 \\
\hline 10 & 2.7 & 0.3 \\
\hline 25 & 3.3 & 0.3 \\
\hline 50 & 3.6 & 0.2 \\
\hline 75 & 3.8 & 0.2 \\
\hline 100 & 3.8 & 0.2 \\
\hline
\end{tabular}

\begin{tabular}{|c|c|c|}
\hline $\begin{array}{l}\text { Table B219 } \\
\text { Return Perio } \\
\text { Deviation for }\end{array}$ & $\begin{array}{l}\text { ximum Water Level } \\
\text { le Tutuila } 216\end{array}$ & nd Water Level Standard \\
\hline Return Period, yr & Maximum Water Level, $\mathrm{ft}$ & Water Level Standard Deviation, $\mathrm{ft}$ \\
\hline 2 & 0.2 & 0.3 \\
\hline 5 & 2.0 & 0.2 \\
\hline 10 & 2.7 & 0.3 \\
\hline 25 & 3.3 & 0.3 \\
\hline 50 & 3.7 & 0.2 \\
\hline 75 & 3.8 & 0.3 \\
\hline 100 & 3.9 & 0.3 \\
\hline
\end{tabular}

\begin{tabular}{|l|l|l|}
\hline $\begin{array}{l}\text { Table B220 } \\
\text { Return Period, Maximum Water Level, and Water Level Standard } \\
\text { Deviation for Profile Tutuila 217 }\end{array}$ \\
\hline Return Period, yr & Maximum Water Level, $\mathrm{ft}$ & Water Level Standard Deviation, $\mathrm{ft}$ \\
\hline 2 & 0.2 & 0.3 \\
\hline 5 & 2.0 & 0.2 \\
\hline 10 & 2.7 & 0.3 \\
\hline 25 & 3.3 & 0.3 \\
\hline 50 & 3.6 & 0.2 \\
\hline 75 & 3.7 & 0.2 \\
\hline 100 & 3.8 & 0.2 \\
\hline
\end{tabular}




\begin{tabular}{|c|c|c|}
\hline \multicolumn{3}{|c|}{$\begin{array}{l}\text { Table B221 } \\
\text { Return Period, Maximum Water Level, and Water Level Standard } \\
\text { Deviation for Profile Tutuila } 218\end{array}$} \\
\hline Return Period, yr & Maximum Water Level, $\mathrm{ft}$ & Water Level Standard Deviation, $\mathrm{ft}$ \\
\hline 2 & 0.2 & 0.3 \\
\hline 5 & 2.0 & 0.2 \\
\hline 10 & 2.7 & 0.3 \\
\hline 25 & 3.3 & 0.3 \\
\hline 50 & 3.6 & 0.2 \\
\hline 75 & 3.7 & 0.2 \\
\hline 100 & 3.8 & 0.2 \\
\hline
\end{tabular}

\begin{tabular}{|c|c|c|}
\hline \multicolumn{3}{|c|}{$\begin{array}{l}\text { Table B222 } \\
\text { Return Period, Maximum Water Level, and Water Level Standard } \\
\text { Deviation for Profile Tutuila } 219\end{array}$} \\
\hline Return Period, yr & Maximum Water Level, $\mathrm{ft}$ & Water Level Standard Deviation, $\mathrm{ft}$ \\
\hline 2 & 0.2 & 0.3 \\
\hline 5 & 2.1 & 0.2 \\
\hline 10 & 2.7 & 0.3 \\
\hline 25 & 3.4 & 0.3 \\
\hline 50 & 3.7 & 0.2 \\
\hline 75 & 3.8 & 0.2 \\
\hline 100 & 3.9 & 0.2 \\
\hline
\end{tabular}

\begin{tabular}{|c|c|c|}
\hline \multicolumn{3}{|c|}{$\begin{array}{l}\text { Table B223 } \\
\text { Return Period, Maximum Water Level, and Water Level Standard } \\
\text { Deviation for Profile Tutuila } 220\end{array}$} \\
\hline Return Period, yr & Maximum Water Level, $\mathrm{ft}$ & Water Level Standard Deviation, $\mathrm{ft}$ \\
\hline 2 & 0.2 & 0.3 \\
\hline 5 & 2.0 & 0.2 \\
\hline 10 & 2.7 & 0.3 \\
\hline 25 & 3.3 & 0.3 \\
\hline 50 & 3.6 & 0.2 \\
\hline 75 & 3.7 & 0.2 \\
\hline 100 & 3.8 & 0.2 \\
\hline
\end{tabular}

\begin{tabular}{|c|c|c|}
\hline \multicolumn{3}{|c|}{$\begin{array}{l}\text { Table B224 } \\
\text { Return Period, Maximum Water Level, and Water Level Standard } \\
\text { Deviation for Profile Tutuila } 221\end{array}$} \\
\hline Return Period, yr & Maximum Water Level, ft & Water Level Standard Deviation, $t$ \\
\hline 2 & 0.2 & 0.3 \\
\hline 5 & 2.1 & 0.2 \\
\hline 10 & 2.7 & 0.3 \\
\hline 25 & 3.3 & 0.3 \\
\hline 50 & 3.6 & 0.2 \\
\hline 75 & 3.8 & 0.2 \\
\hline 100 & 3.8 & 0.2 \\
\hline
\end{tabular}




\begin{tabular}{|c|c|c|}
\hline \multicolumn{3}{|c|}{$\begin{array}{l}\text { Table B225 } \\
\text { Return Period, Maximum Water Level, and Water Level Standard } \\
\text { Deviation for Profile Tutuila } 222\end{array}$} \\
\hline Return Period, yr & Maximum Water Level, ft & Water Level Standard Deviation, tt \\
\hline 2 & 0.4 & 0.4 \\
\hline 5 & 2.1 & 0.2 \\
\hline 10 & 2.8 & 0.2 \\
\hline 25 & 3.3 & 0.2 \\
\hline 50 & 3.6 & 0.2 \\
\hline 75 & 3.7 & 0.2 \\
\hline 100 & 3.8 & 0.3 \\
\hline
\end{tabular}

\begin{tabular}{|c|c|c|}
\hline \multicolumn{3}{|c|}{$\begin{array}{l}\text { Table B226 } \\
\text { Return Period, Maximum Water Level, and Water Level Standard } \\
\text { Deviation for Profile Tutuila } 223\end{array}$} \\
\hline Return Period, yr & Maximum Water Level, $\mathrm{ft}$ & Water Level Standard Deviation, ft \\
\hline 2 & 0.6 & 0.4 \\
\hline 5 & 2.3 & 0.3 \\
\hline 10 & 3.0 & 0.2 \\
\hline 25 & 3.4 & 0.2 \\
\hline 50 & 3.7 & 0.2 \\
\hline 75 & 3.8 & 0.3 \\
\hline 100 & 3.9 & 0.3 \\
\hline
\end{tabular}

\section{Table B227}

Return Period, Maximum Water Level, and Water Level Standard Deviation for Profile Tutuila 224

\begin{tabular}{|l|l|l|}
\hline Return Period, $y \mathbf{r}$ & Maximum Water Level, ft & Water Level Standard Deviation, ft \\
\hline 2 & 0.7 & 0.4 \\
\hline 5 & 2.3 & 0.3 \\
\hline 10 & 3.0 & 0.2 \\
\hline 25 & 3.5 & 0.2 \\
\hline 50 & 3.7 & 0.2 \\
\hline 75 & 3.9 & 0.3 \\
\hline 100 & 4.0 & 0.3 \\
\hline
\end{tabular}

\begin{tabular}{|c|c|c|}
\hline \multicolumn{3}{|c|}{$\begin{array}{l}\text { Table B228 } \\
\text { Return Period, Maximum Water Level, and Water Level Standard } \\
\text { Deviation for Profile Tutuila } 225\end{array}$} \\
\hline Return Period, yr & Maximum Water Level, $\mathrm{ft}$ & Water Level Standard Deviation, $\mathrm{ft}$ \\
\hline 2 & 0.8 & 0.4 \\
\hline 5 & 2.5 & 0.2 \\
\hline 10 & 3.2 & 0.2 \\
\hline 25 & 3.7 & 0.2 \\
\hline 50 & 4.0 & 0.3 \\
\hline 75 & 4.2 & 0.3 \\
\hline 100 & 4.3 & 0.4 \\
\hline
\end{tabular}




\begin{tabular}{|c|c|c|}
\hline \multicolumn{3}{|c|}{$\begin{array}{l}\text { Table B229 } \\
\text { Return Period, Maximum Water Level, and Water Level Standard } \\
\text { Deviation for Profile Tutuila 225a }\end{array}$} \\
\hline Return Period, yr & Maximum Water Level, $\mathrm{ft}$ & Water Level Standard Deviation, $\mathrm{ft}$ \\
\hline 2 & 0.7 & 0.4 \\
\hline 5 & 2.4 & 0.3 \\
\hline 10 & 3.1 & 0.2 \\
\hline 25 & 3.6 & 0.2 \\
\hline 50 & 3.8 & 0.3 \\
\hline 75 & 3.9 & 0.3 \\
\hline 100 & 4.0 & 0.3 \\
\hline
\end{tabular}

\begin{tabular}{|l|l|l|}
\hline $\begin{array}{l}\text { Table B230 } \\
\text { Return Period, Maximum Water Level, and Water Level Standard } \\
\text { Deviation for Profile Tutuila 226 }\end{array}$ \\
\hline Return Period, yr & Maximum Water Level, ft & Water Level Standard Deviation, ft \\
\hline 2 & 0.7 & 0.4 \\
\hline 5 & 2.5 & 0.3 \\
\hline 10 & 3.1 & 0.2 \\
\hline 25 & 3.6 & 0.2 \\
\hline 50 & 3.9 & 0.3 \\
\hline 75 & 4.0 & 0.3 \\
\hline 100 & 4.1 & 0.3 \\
\hline
\end{tabular}

\begin{tabular}{|c|c|c|}
\hline \multicolumn{3}{|c|}{$\begin{array}{l}\text { Table B231 } \\
\text { Return Period, Maximum Water Level, and Water Level Standard } \\
\text { Deviation for Profile Tutuila } 227\end{array}$} \\
\hline Return Period, yr & Maximum Water Level, $\mathrm{ft}$ & Water Level Standard Deviation, ft \\
\hline$\underline{2}$ & 0.6 & 0.4 \\
\hline 5 & 2.3 & 0.3 \\
\hline 10 & 3.0 & 0.2 \\
\hline 25 & 3.4 & 0.2 \\
\hline 50 & 3.7 & 0.2 \\
\hline 75 & 3.8 & 0.3 \\
\hline 100 & 4.0 & 0.3 \\
\hline
\end{tabular}

\begin{tabular}{|c|c|c|}
\hline \multicolumn{3}{|c|}{$\begin{array}{l}\text { Table B232 } \\
\text { Return Period, Maximum Water Level, and Water Level Standard } \\
\text { Deviation for Profile Tutuila } 228\end{array}$} \\
\hline Return Period, yr & Maximum Water Level, $\mathrm{ft}$ & Water Level Standard Deviation, ft \\
\hline 2 & 1.4 & 0.6 \\
\hline 5 & 3.2 & 0.3 \\
\hline 10 & 3.9 & 0.3 \\
\hline 25 & 4.6 & 0.2 \\
\hline 50 & 4.9 & 0.4 \\
\hline 75 & 5.2 & 0.5 \\
\hline 100 & 5.3 & 0.6 \\
\hline
\end{tabular}




\begin{tabular}{|c|c|c|}
\hline \multicolumn{3}{|c|}{$\begin{array}{l}\text { Table B233 } \\
\text { Return Period, Maximum Water Level, and Water Level Standard } \\
\text { Deviation for Profile Tutuila } 229\end{array}$} \\
\hline Return Period, yr & Maximum Water Level, $\mathrm{ft}$ & Water Level Standard Deviation, $\mathrm{ft}$ \\
\hline 2 & 0.8 & 0.4 \\
\hline 5 & 2.6 & 0.2 \\
\hline 10 & 3.3 & 0.2 \\
\hline 25 & 3.8 & 0.2 \\
\hline 50 & 4.0 & 0.3 \\
\hline 75 & 4.2 & 0.4 \\
\hline 100 & 4.3 & 0.4 \\
\hline
\end{tabular}

\begin{tabular}{|c|c|c|}
\hline \multicolumn{3}{|c|}{$\begin{array}{l}\text { Table B234 } \\
\text { Return Period, Maximum Water Level, and Water Level Standard } \\
\text { Deviation for Profile Tutuila } 230\end{array}$} \\
\hline Return Period, yr & Maximum Water Level, $\mathrm{ft}$ & Water Level Standard Deviation, $\mathrm{ft}$ \\
\hline 2 & 0.7 & 0.4 \\
\hline 5 & 2.5 & 0.3 \\
\hline 10 & 3.1 & 0.2 \\
\hline 25 & 3.6 & 0.2 \\
\hline 50 & 3.9 & 0.3 \\
\hline 75 & 4.0 & 0.3 \\
\hline 100 & 4.1 & 0.3 \\
\hline
\end{tabular}

\begin{tabular}{|c|c|c|}
\hline \multicolumn{3}{|c|}{$\begin{array}{l}\text { Table B235 } \\
\text { Return Period, Maximum Water Level, and Water Level Standard } \\
\text { Deviation for Profile Tutuila } 231\end{array}$} \\
\hline Return Period, yr & Maximum Water Level, $\mathrm{ft}$ & Water Level Standard Deviation, $\mathrm{tt}$ \\
\hline 2 & 0.7 & 0.4 \\
\hline 5 & 2.5 & 0.3 \\
\hline 10 & 3.1 & 0.2 \\
\hline 25 & 3.6 & 0.2 \\
\hline 50 & 3.9 & 0.3 \\
\hline 75 & 4.1 & 0.3 \\
\hline 100 & 4.1 & 0.4 \\
\hline
\end{tabular}

\begin{tabular}{|c|c|c|}
\hline \multicolumn{3}{|c|}{$\begin{array}{l}\text { Table B236 } \\
\text { Return Period, Maximum Water Level, and Water Level Standard } \\
\text { Deviation for Profile Tutuila } 232\end{array}$} \\
\hline Return Period, yr & Maximum Water Level, ft & Water Level Standard Deviation, ft \\
\hline 2 & 0.7 & 0.4 \\
\hline 5 & 2.4 & 0.3 \\
\hline 10 & 3.1 & 0.2 \\
\hline 25 & 3.6 & 0.2 \\
\hline 50 & 3.8 & 0.3 \\
\hline 75 & 3.9 & 0.3 \\
\hline 100 & 4.0 & 0.3 \\
\hline
\end{tabular}




\begin{tabular}{|c|c|c|}
\hline \multicolumn{3}{|c|}{$\begin{array}{l}\text { Table B237 } \\
\text { Return Period, Maximum Water Level, and Water Level Standard } \\
\text { Deviation for Profile Tutuila } 233\end{array}$} \\
\hline Return Period, $y r$ & Maximum Water Level, ft & Water Level Standard Deviation, ft \\
\hline 2 & 0.6 & 0.4 \\
\hline 5 & 2.3 & 0.3 \\
\hline 10 & 3.0 & 0.2 \\
\hline 25 & 3.4 & 0.2 \\
\hline 50 & 3.7 & 0.2 \\
\hline 75 & 3.8 & 0.3 \\
\hline 100 & 3.9 & 0.3 \\
\hline
\end{tabular}

\begin{tabular}{|c|c|c|}
\hline \multicolumn{3}{|c|}{$\begin{array}{l}\text { Table B238 } \\
\text { Return Period, Maximum Water Level, and Water Level Standard } \\
\text { Deviation for Profile Tutuila } 234\end{array}$} \\
\hline Return Period, yr & Maximum Water Level, $\mathrm{ft}$ & Water Level Standard Deviation, ft \\
\hline 2 & 0.6 & 0.4 \\
\hline 5 & 2.3 & 0.3 \\
\hline 10 & 3.0 & 0.2 \\
\hline 25 & 3.4 & 0.2 \\
\hline 50 & 3.6 & 0.2 \\
\hline 75 & 3.8 & 0.3 \\
\hline 100 & 3.9 & 0.3 \\
\hline
\end{tabular}

\begin{tabular}{|c|c|c|}
\hline \multicolumn{3}{|c|}{$\begin{array}{l}\text { Table B239 } \\
\text { Return Period, Maximum Water Level, and Water Level Standard } \\
\text { Deviation for Profile Tutuila } 235\end{array}$} \\
\hline Return Period, yr & Maximum Water Level, $\mathrm{tt}$ & Water Level Standard Deviation, $\mathrm{ft}$ \\
\hline 2 & 0.8 & 0.5 \\
\hline 5 & 2.7 & 0.3 \\
\hline 10 & 3.4 & 0.3 \\
\hline 25 & 4.1 & 0.3 \\
\hline 50 & 4.6 & 0.5 \\
\hline 75 & 5.1 & 0.6 \\
\hline 100 & 5.3 & 0.7 \\
\hline
\end{tabular}

\begin{tabular}{|c|c|c|}
\hline \multicolumn{3}{|c|}{$\begin{array}{l}\text { Table B240 } \\
\text { Return Period, Maximum Water Level, and Water Level Standard } \\
\text { Deviation for Profile Tutuila } 236\end{array}$} \\
\hline Return Period, yr & Maximum Water Level, $\mathrm{ft}$ & Water Level Standard Deviation, $\mathrm{ft}$ \\
\hline 2 & 0.8 & 0.5 \\
\hline 5 & 2.7 & 0.3 \\
\hline 10 & 3.4 & 0.3 \\
\hline 25 & 4.1 & 0.3 \\
\hline 50 & 4.6 & 0.5 \\
\hline 75 & 5.1 & 0.6 \\
\hline 100 & 5.3 & 0.7 \\
\hline
\end{tabular}




\begin{tabular}{|c|c|c|}
\hline \multicolumn{3}{|c|}{$\begin{array}{l}\text { Table B241 } \\
\text { Return Period, Maximum Water Level, and Water Level Standard } \\
\text { Deviation for Profile Tutuila } 237\end{array}$} \\
\hline Return Period, yr & Maximum Water Level, ft & Water Level Standard Deviation, $\mathrm{ft}$ \\
\hline 2 & 2.1 & 0.8 \\
\hline 5 & 5.9 & 0.8 \\
\hline 10 & 8.8 & 1.2 \\
\hline 25 & 12.1 & 1.6 \\
\hline 50 & 14.4 & 2.1 \\
\hline 75 & 15.7 & 2.1 \\
\hline 100 & 16.3 & 2.2 \\
\hline
\end{tabular}

\section{Table B242}

Return Period, Maximum Water Level, and Water Level Standard Deviation for Profile Tutuila 238

\begin{tabular}{|l|l|l|}
\hline Return Period, $\mathbf{y r}$ & Maximum Water Level, $\mathbf{f t}$ & Water Level Standard Deviation, $\mathbf{f t}$ \\
\hline 2 & 2.1 & 0.8 \\
\hline 5 & 5.9 & 0.8 \\
\hline 10 & 8.8 & 1.2 \\
\hline 25 & 12.1 & 1.6 \\
\hline 50 & 14.4 & 2.1 \\
\hline 75 & 15.7 & 2.1 \\
\hline 100 & 16.3 & 2.2 \\
\hline
\end{tabular}

\begin{tabular}{|c|c|c|}
\hline \multicolumn{3}{|c|}{$\begin{array}{l}\text { Table B243 } \\
\text { Return Period, Maximum Water Level, and Water Level Standard } \\
\text { Deviation for Profile Tutuila } 239\end{array}$} \\
\hline Return Period, yr & Maximum Water Level, $\mathrm{ft}$ & Water Level Standard Deviation, it \\
\hline 2 & 2.1 & 0.8 \\
\hline 5 & 5.9 & 0.8 \\
\hline 10 & 8.8 & 1.2 \\
\hline 25 & 12.1 & 1.6 \\
\hline 50 & 14.4 & 2.1 \\
\hline 75 & 15.7 & 2.1 \\
\hline 100 & 16.3 & 2.2 \\
\hline
\end{tabular}

\begin{tabular}{|c|c|c|}
\hline \multicolumn{3}{|c|}{$\begin{array}{l}\text { Table B244 } \\
\text { Return Period, Maximum Water Level, and Water Level Standard } \\
\text { Deviation for Profile Tutuila } 240\end{array}$} \\
\hline Return Period, yr & Maximum Water Level, $\mathrm{ft}$ & Water Level Standard Deviation, $\mathrm{ft}$ \\
\hline 2 & 1.6 & 0.7 \\
\hline 5 & 5.7 & 0.9 \\
\hline 10 & 9.0 & 1.5 \\
\hline 25 & 12.9 & 1.7 \\
\hline 50 & 15.4 & 2.0 \\
\hline 75 & 16.5 & 1.8 \\
\hline 100 & 17.0 & 1.8 \\
\hline
\end{tabular}




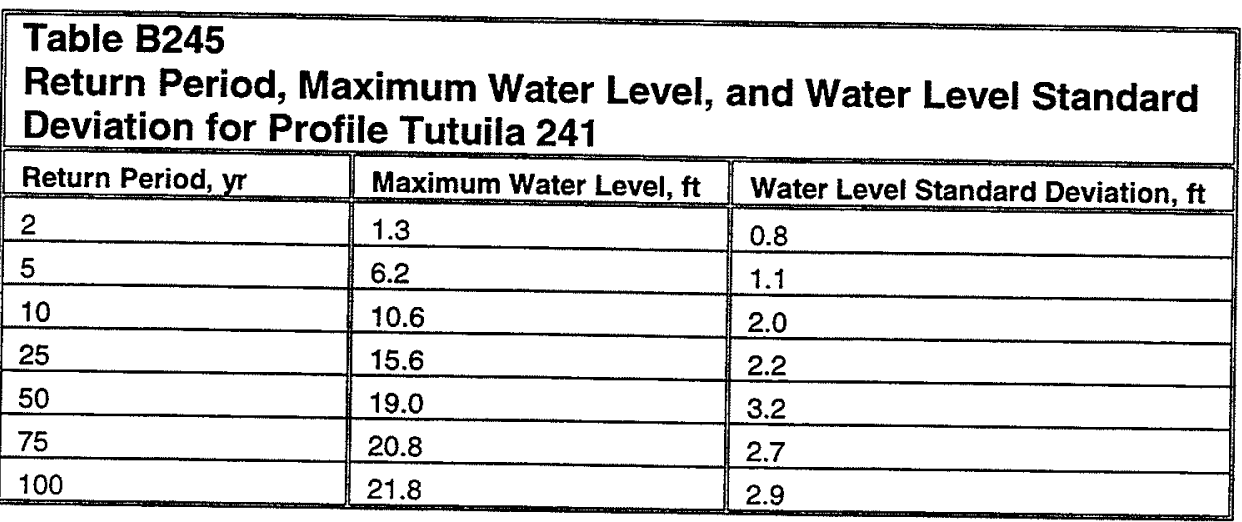

\begin{tabular}{|c|c|c|}
\hline \multicolumn{3}{|c|}{$\begin{array}{l}\text { Table B246 } \\
\text { Return Period, Maximum Water Level, and Water Level Standard } \\
\text { Deviation for Profile Tutuila } 242\end{array}$} \\
\hline Return Period, yr & Maximum Water Level, $\mathbf{f t}$ & Water Level Standard Deviation, $\mathrm{ft}$ \\
\hline 2 & 1.3 & 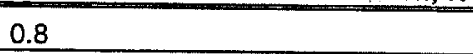 \\
\hline 5 & 6.2 & 1.1 \\
\hline 10 & 10.6 & 2.0 \\
\hline 25 & 15.6 & 2.2 \\
\hline 50 & 19.0 & 3.2 \\
\hline 75 & 20.8 & 2.7 \\
\hline 100 & 21.8 & 2.9 \\
\hline
\end{tabular}

\begin{tabular}{|c|c|c|}
\hline \multicolumn{3}{|c|}{$\begin{array}{l}\text { Table B247 } \\
\text { Return Period, Maximum Water Level, and Water Level Standard } \\
\text { Deviation for Profile Tutuila } 243\end{array}$} \\
\hline Return Period, yr & Maximum Water Level, ft & Water Level Standard Deviation, $\mathrm{ft}$ \\
\hline 2 & 1.3 & 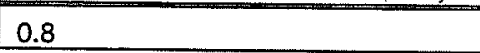 \\
\hline 5 & 6.2 & 1.2 \\
\hline 10 & 11.1 & 2.2 \\
\hline 25 & 16.5 & 2.3 \\
\hline 50 & 19.9 & 2.7 \\
\hline 75 & 21.4 & 2.3 \\
\hline 100 & 22.1 & 2.4 \\
\hline
\end{tabular}

\begin{tabular}{|c|c|c|}
\hline \multicolumn{3}{|c|}{$\begin{array}{l}\text { Table B248 } \\
\text { Return Period, Maximum Water Level, and Water Level Standard } \\
\text { Deviation for Profile Tutuila } 244\end{array}$} \\
\hline Return Period, yr & Maximum Water Level, ft & Water Level Standard Deviation, $\mathrm{ft}$ \\
\hline 2 & 1.3 & 0.8 \\
\hline 5 & 6.2 & 1.1 \\
\hline 10 & 10.6 & 1.9 \\
\hline 25 & 16.8 & 3.5 \\
\hline 50 & 22.3 & 5.1 \\
\hline 75 & 25.4 & 4.0 \\
\hline 100 & 26.9 & 4.0 \\
\hline
\end{tabular}




\begin{tabular}{|c|c|c|}
\hline \multicolumn{3}{|c|}{$\begin{array}{l}\text { Table B249 } \\
\text { Return Period, Maximum Water Level, and Water Level Standard } \\
\text { Deviation for Profile Tutuila } 245\end{array}$} \\
\hline Return Period, yr & Maximum Water Level, $\mathrm{ft}$ & Water Level Standard Deviation, ft \\
\hline 2 & 1.3 & 0.8 \\
\hline 5 & 6.2 & 1.1 \\
\hline 10 & 10.6 & 2.0 \\
\hline 25 & 15.6 & 2.2 \\
\hline 50 & 19.0 & 3.2 \\
\hline 75 & 20.8 & 2.7 \\
\hline 100 & 21.8 & 2.9 \\
\hline
\end{tabular}

\section{Table B250}

Return Period, Maximum Water Level, and Water Level Standard Deviation for Profile Tutuila 246

\begin{tabular}{|l|l|l|}
\hline Return Period, $\mathrm{yr}$ & Maximum Water Level, $\mathrm{ft}$ & Water Level Standard Deviation, $\mathrm{ft}$ \\
\hline 2 & 1.3 & 0.8 \\
\hline 5 & 6.2 & 1.1 \\
\hline 10 & 10.6 & 2.0 \\
\hline 25 & 15.6 & 2.2 \\
\hline 50 & 19.0 & 3.2 \\
\hline 75 & 20.8 & 2.7 \\
\hline 100 & 21.8 & 2.9 \\
\hline
\end{tabular}

\begin{tabular}{|c|c|c|}
\hline \multicolumn{3}{|c|}{$\begin{array}{l}\text { Table B251 } \\
\text { Return Period, Maximum Water Level, and Water Level Standard } \\
\text { Deviation for Profile Tutuila } 247\end{array}$} \\
\hline Return Period, yr & Maximum Water Level, $\mathrm{ft}$ & Water Level Standard Deviation, $\mathrm{tt}$ \\
\hline 2 & 1.3 & 0.8 \\
\hline 5 & 6.2 & 1.1 \\
\hline 10 & 10.6 & 2.0 \\
\hline 25 & 15.6 & 2.2 \\
\hline 50 & 19.0 & 3.2 \\
\hline 75 & 20.8 & 2.7 \\
\hline 100 & 21.8 & 2.9 \\
\hline
\end{tabular}

\section{Table B252}

Return Period, Maximum Water Level, and Water Level Standard Deviation for Profile Tutuila 248

\begin{tabular}{|l|l|l|}
\hline Return Period, yr & Maximum Water Level, $\mathbf{f t}$ & Water Level Standard Deviation, ft \\
\hline 2 & 1.3 & 0.8 \\
\hline 5 & 6.2 & 1.1 \\
\hline 10 & 10.6 & 2.0 \\
\hline 25 & 15.6 & 2.2 \\
\hline 50 & 19.0 & 3.2 \\
\hline 75 & 20.8 & 2.7 \\
\hline 100 & 21.8 & 2.9 \\
\hline
\end{tabular}




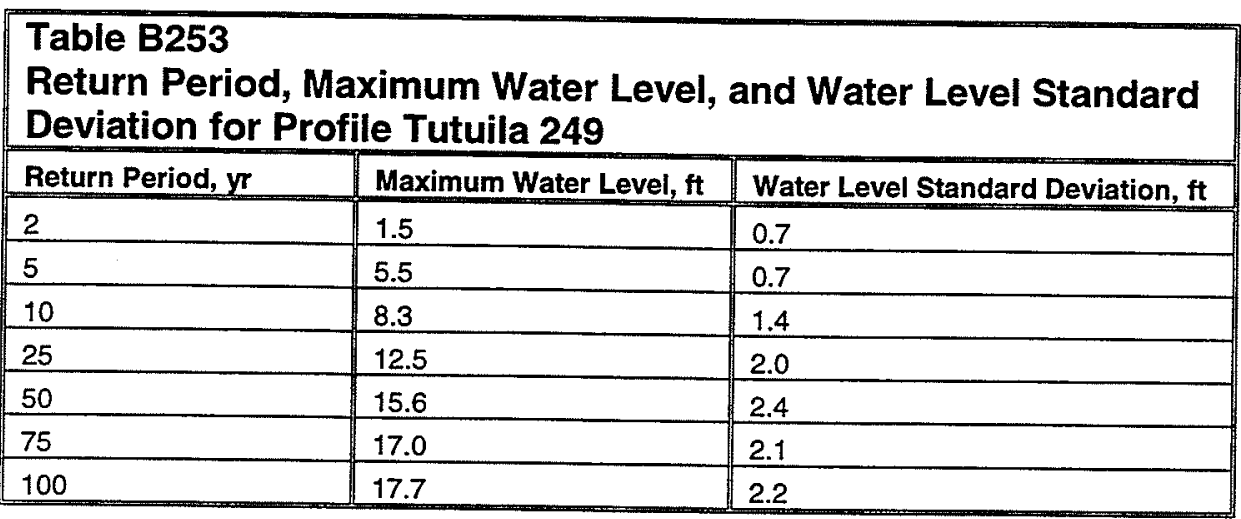

\begin{tabular}{|l|l|l|}
\hline $\begin{array}{l}\text { Table B254 } \\
\text { Return Period, Maximum Water Level, and Water Level Standard } \\
\text { Deviation for Profile Tutuila 250 }\end{array}$ \\
\hline Return Period, yr & Maximum Water Level, ft & Water Level Standard Deviation, $\mathrm{H}$ \\
\hline 2 & 1.6 & 0.8 \\
\hline 5 & 6.2 & 0.8 \\
\hline 10 & 8.8 & 1.2 \\
\hline 25 & 12.7 & 2.0 \\
\hline 50 & 15.8 & 2.7 \\
\hline 75 & 17.5 & 2.4 \\
\hline 100 & 18.4 & 2.6 \\
\hline
\end{tabular}

\begin{tabular}{|l|l|l|}
\hline $\begin{array}{l}\text { Table B255 } \\
\text { Return Period, Maximum Water Level, and Water Level Standard } \\
\text { Deviation for Profile Tutuila 251 }\end{array}$ \\
\hline Return Period, yr & Maximum Water Level, $\mathrm{ft}$ & Water Level Standard Deviation, $\mathrm{ft}$ \\
\hline 2 & 1.6 & 0.8 \\
\hline 5 & 6.2 & 0.8 \\
\hline 10 & 8.8 & 1.2 \\
\hline 25 & 12.7 & 2.0 \\
\hline 50 & 15.8 & 2.7 \\
\hline 75 & 17.5 & 2.4 \\
\hline 100 & 18.4 & 2.6 \\
\hline
\end{tabular}

\begin{tabular}{|c|c|c|}
\hline \multicolumn{3}{|c|}{$\begin{array}{l}\text { Table B256 } \\
\text { Return Period, Maximum Water Level, and Water Level Standard } \\
\text { Deviation for Profile Tutuila } 252\end{array}$} \\
\hline Return Period, yr & Maximum Water Level, ft & Water Level Standard Deviation, $\mathrm{ft}$ \\
\hline 2 & 1.6 & 0.8 \\
\hline 5 & 6.2 & 0.8 \\
\hline 10 & 8.8 & 1.2 \\
\hline 25 & 12.7 & 2.0 \\
\hline 50 & 15.8 & 2.7 \\
\hline 75 & 17.5 & 2.4 \\
\hline 100 & 18.4 & 2.6 \\
\hline
\end{tabular}




\begin{tabular}{|c|c|c|}
\hline \multicolumn{3}{|c|}{$\begin{array}{l}\text { Table B257 } \\
\text { Return Period, Maximum Water Level, and Water Level Standard } \\
\text { Deviation for Profile Tutuila } 253\end{array}$} \\
\hline Return Period, yr & Maximum Water Level, $\mathrm{ft}$ & Water Level Standard Deviation, $\mathrm{ft}$ \\
\hline 2 & 1.6 & 0.8 \\
\hline 5 & 6.2 & 0.8 \\
\hline 10 & 8.8 & 1.2 \\
\hline 25 & 12.7 & 2.0 \\
\hline 50 & 15.8 & 2.7 \\
\hline 75 & 17.5 & 2.4 \\
\hline 100 & 18.4 & 2.6 \\
\hline
\end{tabular}

\begin{tabular}{|c|c|c|}
\hline \multicolumn{3}{|c|}{$\begin{array}{l}\text { Table B258 } \\
\text { Return Period, Maximum Water Level, and Water Level Standard } \\
\text { Deviation for Profile Tutuila } 254\end{array}$} \\
\hline Return Period, yr & Maximum Water Level, tt & Water Level Standard Deviation, $\mathrm{tt}$ \\
\hline 2 & 1.7 & 0.8 \\
\hline 5 & 6.6 & 0.8 \\
\hline 10 & 9.2 & 1.1 \\
\hline 25 & 13.1 & 2.0 \\
\hline 50 & 16.2 & 2.8 \\
\hline 75 & 17.9 & 2.5 \\
\hline 100 & 18.8 & 2.6 \\
\hline
\end{tabular}

\begin{tabular}{|c|c|c|}
\hline \multicolumn{3}{|c|}{$\begin{array}{l}\text { Table B259 } \\
\text { Return Period, Maximum Water Level, and Water Level Standard } \\
\text { Deviation for Profile Tutuila } 255\end{array}$} \\
\hline Return Period, yr & Maximum Water Level, $\mathrm{ft}$ & Water Level Standard Deviation, ft \\
\hline 2 & 1.8 & 0.9 \\
\hline 5 & 6.9 & 0.9 \\
\hline 10 & 9.6 & 1.2 \\
\hline 25 & 13.5 & 2.0 \\
\hline 50 & 16.5 & 2.9 \\
\hline 75 & 18.3 & 2.5 \\
\hline 100 & 19.2 & 2.7 \\
\hline
\end{tabular}

\begin{tabular}{|c|c|c|}
\hline \multicolumn{3}{|c|}{$\begin{array}{l}\text { Table B260 } \\
\text { Return Period, Maximum Water Level, and Water Level Standard } \\
\text { Deviation for Profile Tutuila } 256\end{array}$} \\
\hline Return Period, yr & Maximum Water Level, $\mathrm{ft}$ & Water Level Standard Deviation, ft \\
\hline 2 & 2.0 & 0.9 \\
\hline 5 & 7.3 & 0.9 \\
\hline 10 & 10.1 & 1.2 \\
\hline 25 & 14.0 & 2.0 \\
\hline 50 & 17.0 & 2.9 \\
\hline 75 & 18.8 & 2.6 \\
\hline 100 & 19.8 & 2.7 \\
\hline
\end{tabular}




\begin{tabular}{|c|c|c|}
\hline \multicolumn{3}{|c|}{$\begin{array}{l}\text { Table B261 } \\
\text { Return Period, Maximum Water Level, and Water Level Standard } \\
\text { Deviation for Profile Tutuila } 257\end{array}$} \\
\hline Return Period, yr & Maximum Water Level, $\mathrm{ft}$ & Water Level Stancard Deviation, $\mathrm{ft}$ \\
\hline 2 & 2.0 & 0.9 \\
\hline 5 & 7.3 & 0.9 \\
\hline 10 & 10.1 & 1.2 \\
\hline 25 & 14.0 & 2.0 \\
\hline 50 & 17.0 & 2.9 \\
\hline 75 & 18.8 & 2.6 \\
\hline 100 & 19.8 & 2.7 \\
\hline
\end{tabular}

\begin{tabular}{|c|c|c|}
\hline \multicolumn{3}{|c|}{$\begin{array}{l}\text { Table B262 } \\
\text { Return Period, Maximum Water Level, and Water Level Standard } \\
\text { Deviation for Profile Tutuila } 258\end{array}$} \\
\hline Return Period, yr & Maximum Water Level, ft & Water Level Standard Deviation, $\mathrm{ft}$ \\
\hline 2 & 2.0 & 0.9 \\
\hline 5 & 7.3 & 0.9 \\
\hline 10 & 10.1 & 1.2 \\
\hline 25 & 14.0 & 2.0 \\
\hline 50 & 17.0 & 2.9 \\
\hline 75 & 18.8 & 2.6 \\
\hline 100 & 19.8 & 2.7 \\
\hline
\end{tabular}

\begin{tabular}{|c|c|c|}
\hline \multicolumn{3}{|c|}{$\begin{array}{l}\text { Table B263 } \\
\text { Return Period, Maximum Water Level, and Water Level Standard } \\
\text { Deviation for Profile Tutuila } 259\end{array}$} \\
\hline Return Period, yr & Maximum Water Level, ft & Water Level Standard Deviation, ft \\
\hline 2 & 2.0 & 0.9 \\
\hline 5 & 7.3 & 0.9 \\
\hline 10 & 10.1 & 1.2 \\
\hline 25 & 14.0 & 2.0 \\
\hline 50 & 17.0 & 2.9 \\
\hline 75 & 18.8 & 2.6 \\
\hline 100 & 19.8 & 2.7 \\
\hline
\end{tabular}

\begin{tabular}{|c|c|c|}
\hline \multicolumn{3}{|c|}{$\begin{array}{l}\text { Table B264 } \\
\text { Return Period, Maximum Water Level, and Water Level Standard } \\
\text { Deviation for Profile Tutuila } 260\end{array}$} \\
\hline Return Period, yz & Maximum Water Level, ft & Water Level Standard Deviation, ft \\
\hline 2 & 1.9 & 0.9 \\
\hline 5 & 7.8 & 0.9 \\
\hline 10 & 10.6 & 1.2 \\
\hline 25 & 14.6 & 2.0 \\
\hline 50 & 17.6 & 2.9 \\
\hline 75 & 19.4 & 2.6 \\
\hline 100 & 20.4 & 2.7 \\
\hline
\end{tabular}




\begin{tabular}{|c|c|c|}
\hline \multicolumn{3}{|c|}{$\begin{array}{l}\text { Table B265 } \\
\text { Return Period, Maximum Water Level, and Water Level Standard } \\
\text { Deviation for Profile Tutuila } 261\end{array}$} \\
\hline Return Period, yr & Maximum Water Level, tt & Water Level Standard Deviation, ft \\
\hline$\underline{2}$ & 1.9 & 0.9 \\
\hline 5 & 7.8 & 0.9 \\
\hline 10 & 10.6 & 1.2 \\
\hline 25 & 14.6 & 2.0 \\
\hline 50 & 17.6 & 2.9 \\
\hline 75 & 19.4 & 2.6 \\
\hline 100 & 20.4 & 2.7 \\
\hline
\end{tabular}

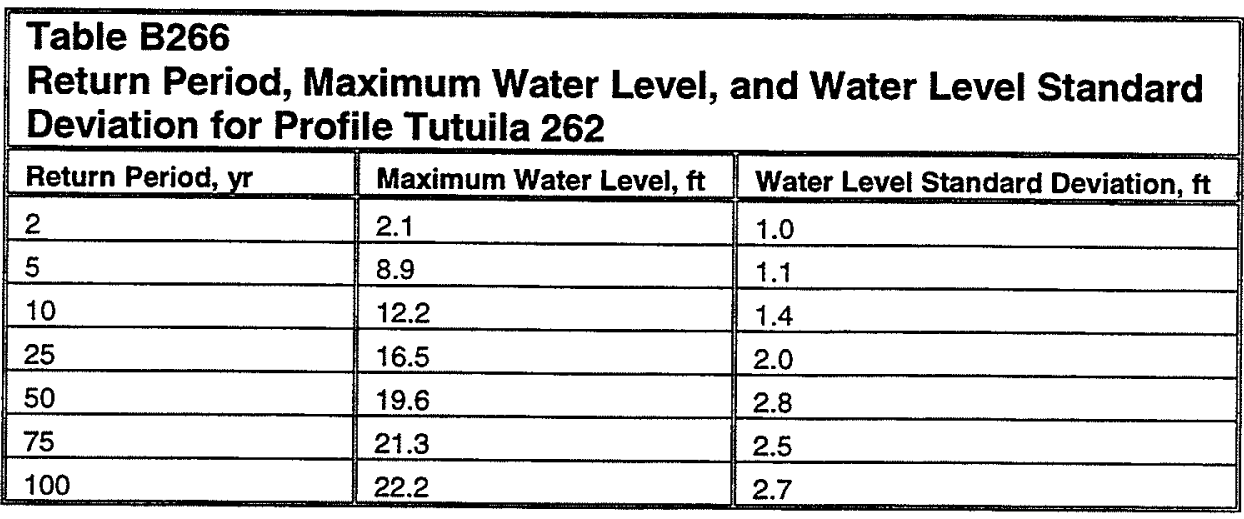

\begin{tabular}{|c|c|c|}
\hline \multicolumn{3}{|c|}{$\begin{array}{l}\text { Table B267 } \\
\text { Return Period, Maximum Water Level, and Water Level Standard } \\
\text { Deviation for Profile Tutuila } 263\end{array}$} \\
\hline Return Period, yr & Maximum Water Level, $\mathrm{ft}$ & Water Level Standard Deviation, $\mathrm{ft}$ \\
\hline 2 & 2.1 & 1.0 \\
\hline 5 & 8.9 & 1.1 \\
\hline 10 & 12.2 & 1.4 \\
\hline 25 & 16.5 & 2.0 \\
\hline 50 & 19.6 & 2.8 \\
\hline 75 & 21.3 & 2.5 \\
\hline 100 & 22.2 & 2.7 \\
\hline
\end{tabular}

\begin{tabular}{|c|c|c|}
\hline \multicolumn{3}{|c|}{$\begin{array}{l}\text { Table B268 } \\
\text { Return Period, Maximum Water Level, and Water Level Standard } \\
\text { Deviation for Profile Tutuila } 264\end{array}$} \\
\hline Return Period, yr & Maximum Water Level, $\mathrm{ft}$ & Water Level Standard Deviation, $\mathrm{ft}$ \\
\hline 2 & 0.0 & 0.0 \\
\hline 5 & 1.1 & 0.2 \\
\hline 10 & 1.6 & 0.2 \\
\hline 25 & 2.2 & 0.3 \\
\hline 50 & 2.6 & 0.4 \\
\hline 75 & 2.8 & 0.4 \\
\hline 100 & 2.9 & 0.4 \\
\hline
\end{tabular}




\begin{tabular}{|c|c|c|}
\hline \multicolumn{3}{|c|}{$\begin{array}{l}\text { Table B269 } \\
\text { Return Period, Maximum Water Level, and Water Level Standard } \\
\text { Deviation for Profile Tutuila } 265\end{array}$} \\
\hline Return Period, yr & Maximum Water Level, $\mathrm{tt}$ & Water Level Standard Deviation, $\mathrm{ft}$ \\
\hline 2 & 0.0 & 0.0 \\
\hline 5 & 1.1 & 0.2 \\
\hline 10 & 1.6 & 0.2 \\
\hline 25 & 2.2 & 0.3 \\
\hline 50 & 2.6 & 0.4 \\
\hline 75 & 2.8 & 0.4 \\
\hline 100 & 2.9 & 0.4 \\
\hline
\end{tabular}

\begin{tabular}{l}
\hline $\begin{array}{l}\text { Table B270 } \\
\text { Return Period, Maximum Water Level, and Water Level Standard } \\
\text { Deviation for Profile Tutuila 266 }\end{array}$ \\
\begin{tabular}{|l|l|l|}
\hline Return Period, yr & Maximum Water Level, $\mathrm{tt}$ & Water Level Standard Deviation, $\mathrm{tt}$ \\
\hline 2 & 0.0 & 0.0 \\
\hline 5 & 1.1 & 0.2 \\
\hline 10 & 1.6 & 0.2 \\
\hline 25 & 2.2 & 0.3 \\
\hline 50 & 2.6 & 0.4 \\
\hline 75 & 2.8 & 0.4 \\
\hline 100 & 2.9 & 0.4 \\
\hline
\end{tabular}
\end{tabular}

\begin{tabular}{|c|c|c|}
\hline \multicolumn{3}{|c|}{$\begin{array}{l}\text { Table B271 } \\
\text { Return Period, Maximum Water Level, and Water Level Standard } \\
\text { Deviation for Profile Tutuila } 267\end{array}$} \\
\hline Return Period, yr & Maximum Water Level, $\mathrm{ft}$ & Water Level Standard Deviation, ft \\
\hline 2 & 0.0 & 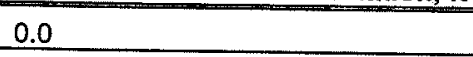 \\
\hline 5 & 1.1 & 0.2 \\
\hline 10 & 1.6 & 0.2 \\
\hline 25 & 2.2 & 0.3 \\
\hline 50 & 2.6 & 0.4 \\
\hline 75 & 2.8 & 0.4 \\
\hline 100 & 2.9 & 0.4 \\
\hline
\end{tabular}

\begin{tabular}{|c|c|c|}
\hline \multicolumn{3}{|c|}{$\begin{array}{l}\text { Table B272 } \\
\text { Return Period, Maximum Water Level, and Water Level Standard } \\
\text { Deviation for Profile Tutuila } 268\end{array}$} \\
\hline Return Period, yr & Maximum Water Level, $\mathrm{ft}$ & Water Level Standard Deviation, ft \\
\hline 2 & 0.0 & 0.0 \\
\hline 5 & 1.1 & 0.2 \\
\hline 10 & 1.6 & 0.2 \\
\hline 25 & 2.2 & 0.3 \\
\hline 50 & 2.6 & 0.4 \\
\hline 75 & 2.8 & 0.4 \\
\hline 100 & 2.9 & 0.4 \\
\hline
\end{tabular}




\begin{tabular}{|c|c|c|}
\hline \multicolumn{3}{|c|}{$\begin{array}{l}\text { Table B273 } \\
\text { Return Period, Maximum Water Level, and Water Level Standard } \\
\text { Deviation for Profile Tutuila } 269\end{array}$} \\
\hline Return Period, yr & Maximum Water Level, $\mathrm{ft}$ & Water Level Standard Deviation, $\mathrm{ft}$ \\
\hline 2 & 0.0 & 0.0 \\
\hline 5 & 1.0 & 0.2 \\
\hline 10 & 1.5 & 0.2 \\
\hline 25 & 2.1 & 0.3 \\
\hline 50 & 2.4 & 0.4 \\
\hline 75 & 2.6 & 0.4 \\
\hline 100 & 2.7 & 0.5 \\
\hline
\end{tabular}

\begin{tabular}{|c|c|c|}
\hline \multicolumn{3}{|c|}{$\begin{array}{l}\text { Table B274 } \\
\text { Return Period, Maximum Water Level, and Water Level Standard } \\
\text { Deviation for Profile Tutuila } 270\end{array}$} \\
\hline Return Period, yr & Maximum Water Level, $\mathrm{ft}$ & Water Level Standard Deviation, $\mathrm{ft}$ \\
\hline 2 & 0.0 & 0.0 \\
\hline 5 & 1.0 & 0.2 \\
\hline 10 & 1.5 & 0.2 \\
\hline 25 & 2.1 & 0.3 \\
\hline 50 & 2.4 & 0.4 \\
\hline 75 & 2.6 & 0.4 \\
\hline 100 & 2.7 & 0.5 \\
\hline
\end{tabular}

\begin{tabular}{|c|c|c|}
\hline \multicolumn{3}{|c|}{$\begin{array}{l}\text { Table B275 } \\
\text { Return Period, Maximum Water Level, and Water Level Standard } \\
\text { Deviation for Profile Tutuila } 271\end{array}$} \\
\hline Return Period, yr & Maximum Water Level, ft & Water Level Standard Deviation, ft \\
\hline 2 & 0.0 & 0.0 \\
\hline 5 & 1.0 & 0.2 \\
\hline 10 & 1.5 & 0.2 \\
\hline 25 & 2.1 & 0.3 \\
\hline 50 & 2.4 & 0.4 \\
\hline 75 & 2.6 & 0.4 \\
\hline 100 & 2.7 & 0.5 \\
\hline
\end{tabular}

\begin{tabular}{|c|c|c|}
\hline \multicolumn{3}{|c|}{$\begin{array}{l}\text { Table B276 } \\
\text { Return Period, Maximum Water Level, and Water Level Standard } \\
\text { Deviation for Profile Tutuila } 272\end{array}$} \\
\hline Return Period, yr & Maximum Water Level, ft & Water Level Standard Deviation, $\mathrm{ft}$ \\
\hline 2 & 0.0 & 0.0 \\
\hline 5 & 1.0 & 0.2 \\
\hline 10 & 1.5 & 0.2 \\
\hline 25 & 2.1 & 0.3 \\
\hline 50 & 2.4 & 0.4 \\
\hline 75 & 2.7 & 0.5 \\
\hline 100 & 2.8 & 0.6 \\
\hline
\end{tabular}

B70 


\begin{tabular}{|c|c|c|}
\hline \multicolumn{3}{|c|}{$\begin{array}{l}\text { Table B277 } \\
\text { Return Period, Maximum Water Level, and Water Level Standard } \\
\text { Deviation for Profile Tutuila } 273\end{array}$} \\
\hline Return Period, yr & Maximum Water Level, $\mathrm{ft}$ & Water Level Standard Deviation, $\mathrm{ft}$ \\
\hline 2 & 0.0 & 0.0 \\
\hline 5 & 1.0 & 0.2 \\
\hline 10 & 1.5 & 0.2 \\
\hline 25 & 2.1 & 0.3 \\
\hline 50 & 2.4 & 0.4 \\
\hline 75 & 2.7 & 0.5 \\
\hline 100 & 2.8 & 0.6 \\
\hline
\end{tabular}

\begin{tabular}{|c|c|c|}
\hline \multicolumn{3}{|c|}{$\begin{array}{l}\text { Table B278 } \\
\text { Return Period, Maximum Water Level, and Water Level Standard } \\
\text { Deviation for Profile Tutuila } 274\end{array}$} \\
\hline Return Period, yr & Maximum Water Level, $\mathrm{ft}$ & Water Level Standard Deviation, $\mathrm{ft}$ \\
\hline 2 & 0.0 & 0.0 \\
\hline 5 & 1.0 & 0.3 \\
\hline 10 & 1.5 & 0.2 \\
\hline 25 & 2.1 & 0.3 \\
\hline 50 & 2.7 & 0.6 \\
\hline 75 & 3.0 & 0.6 \\
\hline 100 & 3.2 & 0.8 \\
\hline
\end{tabular}

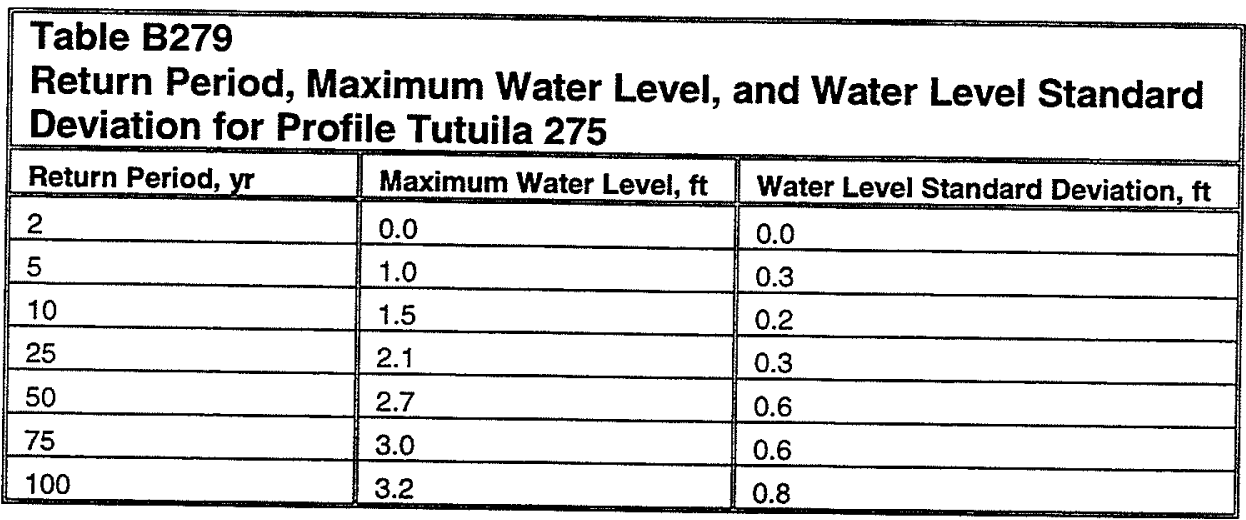

\begin{tabular}{|c|c|c|}
\hline \multicolumn{3}{|c|}{$\begin{array}{l}\text { Table B280 } \\
\text { Return Period, Maximum Water Level, and Water Level Standard } \\
\text { Deviation for Profile Tutuila } 276\end{array}$} \\
\hline Return Period, yr & Maximum Water Level, $\mathrm{ft}$ & Water Level Standard Deviation, $\mathrm{ft}$ \\
\hline 2 & 0.0 & 0.0 \\
\hline 5 & 1.0 & 0.3 \\
\hline 10 & 1.5 & 0.2 \\
\hline 25 & 2.1 & 0.3 \\
\hline 50 & 2.7 & 0.6 \\
\hline 75 & 3.0 & 0.6 \\
\hline 100 & 3.2 & 0.8 \\
\hline
\end{tabular}




\begin{tabular}{|c|c|c|}
\hline \multicolumn{3}{|c|}{$\begin{array}{l}\text { Table B281 } \\
\text { Return Period, Maximum Water Level, and Water Level Standard } \\
\text { Deviation for Profile Tutuila 276a }\end{array}$} \\
\hline Return Period, yr & Maximum Water Level, $\mathrm{ft}$ & Water Level Standard Deviation, $f t$ \\
\hline 2 & 2.4 & 1.1 \\
\hline 5 & 9.3 & 1.0 \\
\hline 10 & 12.4 & 1.3 \\
\hline 25 & 16.5 & 2.0 \\
\hline 50 & 19.5 & 2.6 \\
\hline 75 & 21.1 & 2.4 \\
\hline 100 & 21.9 & 2.5 \\
\hline
\end{tabular}

\section{Table B282}

Return Period, Maximum Water Level, and Water Level Standard Deviation for Profile Tutuila 277

\begin{tabular}{|l|l|l|}
\hline Return Period, yr & Maximum Water Level, $\mathbf{f t}$ & Water Level Standard Deviation, $\mathrm{ft}$ \\
\hline 2 & 2.6 & 1.1 \\
\hline 5 & 8.9 & 1.0 \\
\hline 10 & 11.6 & 1.1 \\
\hline 25 & 15.2 & 1.8 \\
\hline 50 & 17.9 & 2.3 \\
\hline 75 & 19.3 & 2.2 \\
\hline 100 & 20.1 & 2.3 \\
\hline
\end{tabular}

\begin{tabular}{|c|c|c|}
\hline \multicolumn{3}{|c|}{$\begin{array}{l}\text { Table B283 } \\
\text { Return Period, Maximum Water Level, and Water Level Standard } \\
\text { Deviation for Profile Tutuila } 278\end{array}$} \\
\hline Return Period, yr & Maximum Water Level, $\mathrm{ft}$ & Water Level Standard Deviation, $\mathrm{ft}$ \\
\hline 2 & 2.6 & 1.1 \\
\hline 5 & 8.0 & 0.7 \\
\hline 10 & 10.2 & 0.9 \\
\hline 25 & 13.0 & 1.4 \\
\hline 50 & 15.3 & 1.8 \\
\hline 75 & 16.4 & 1.7 \\
\hline 100 & 16.9 & 1.8 \\
\hline
\end{tabular}

\section{Table B284}

Return Period, Maximum Water Level, and Water Level Standard Deviation for Profile Tutuila 279

\begin{tabular}{|l|l|l|}
\hline Return Period, $\mathbf{y r}$ & Maximum Water Level, $\mathrm{ft}$ & Water Level Standard Deviation, $\mathrm{ft}$ \\
\hline 2 & 2.5 & 1.0 \\
\hline 5 & 7.0 & 0.6 \\
\hline 10 & 8.8 & 0.7 \\
\hline 25 & 11.1 & 1.1 \\
\hline 50 & 12.8 & 1.3 \\
\hline 75 & 13.5 & 1.3 \\
\hline 100 & 13.9 & 1.4 \\
\hline
\end{tabular}




\begin{tabular}{|c|c|c|}
\hline \multicolumn{3}{|c|}{$\begin{array}{l}\text { Table B285 } \\
\text { Return Period, Maximum Water Level, and Water Level Standard } \\
\text { Deviation for Profile Tutuila } 280\end{array}$} \\
\hline Return Period, yr & Maximum Water Level, ft & Water Level Standard Deviation, $\mathrm{ft}$ \\
\hline 2 & 2.4 & 1.0 \\
\hline 5 & 6.1 & 0.4 \\
\hline 10 & 7.5 & 0.6 \\
\hline 25 & 9.0 & 0.8 \\
\hline 50 & 10.1 & 0.8 \\
\hline 75 & 10.6 & 0.9 \\
\hline 100 & 10.9 & 0.9 \\
\hline
\end{tabular}

\begin{tabular}{|c|c|c|}
\hline \multicolumn{3}{|c|}{$\begin{array}{l}\text { Table B286 } \\
\text { Return Period, Maximum Water Level, and Water Level Standard } \\
\text { Deviation for Profile Tutuila } 281\end{array}$} \\
\hline Return Period, yr & Maximum Water Level, $\mathrm{ft}$ & Water Level Standard Deviation, $\mathrm{ft}$ \\
\hline 2 & 2.4 & 1.0 \\
\hline 5 & 6.1 & 0.4 \\
\hline 10 & 7.5 & 0.6 \\
\hline 25 & 9.0 & 0.8 \\
\hline 50 & 10.1 & 0.8 \\
\hline 75 & 10.6 & 0.9 \\
\hline 100 & 10.9 & 0.9 \\
\hline
\end{tabular}

\begin{tabular}{|c|c|c|}
\hline \multicolumn{3}{|c|}{$\begin{array}{l}\text { Table B287 } \\
\text { Return Period, Maximum Water Level, and Water Level Standard } \\
\text { Deviation for Profile Tutuila } 282\end{array}$} \\
\hline Return Period, yr & Maximum Water Level, it & Water Level Standard Deviation, ft \\
\hline 2 & 3.4 & 1.3 \\
\hline 5 & 7.8 & 0.5 \\
\hline 10 & 9.3 & 0.6 \\
\hline 25 & 11.4 & 1.3 \\
\hline 50 & 13.5 & 2.2 \\
\hline 75 & 14.9 & 2.0 \\
\hline 100 & 15.7 & 2.2 \\
\hline
\end{tabular}

\begin{tabular}{|c|c|c|}
\hline \multicolumn{3}{|c|}{$\begin{array}{l}\text { Table B288 } \\
\text { Return Period, Maximum Water Level, and Water Level Standard } \\
\text { Deviation for Profile Tutuila } 283\end{array}$} \\
\hline Return Period, yr & Maximum Water Level, ft & Water Level Standard Deviation, $\mathrm{ft}$ \\
\hline 2 & 3.4 & 1.3 \\
\hline 5 & 7.8 & 0.5 \\
\hline 10 & 9.3 & 0.6 \\
\hline 25 & 11.4 & 1.3 \\
\hline 50 & 13.5 & 2.2 \\
\hline 75 & 14.9 & 2.0 \\
\hline 100 & 15.7 & 2.2 \\
\hline
\end{tabular}




\begin{tabular}{|c|c|c|}
\hline \multicolumn{3}{|c|}{$\begin{array}{l}\text { Table B289 } \\
\text { Return Period, Maximum Water Level, and Water Level Standard } \\
\text { Deviation for Profile Tutuila } 284\end{array}$} \\
\hline Return Period, yr & Maximum Water Level, ft & Water Level Standard Deviation, ft \\
\hline 2 & 3.4 & 1.3 \\
\hline 5 & 7.8 & 0.5 \\
\hline 10 & 9.3 & 0.6 \\
\hline 25 & 11.4 & 1.3 \\
\hline 50 & 13.5 & 2.2 \\
\hline 75 & 14.9 & 2.0 \\
\hline 100 & 15.7 & 2.2 \\
\hline
\end{tabular}

\section{Table B290}

Return Period, Maximum Water Level, and Water Level Standard Deviation for Profile Tutuila 285

\begin{tabular}{|l|l|l|}
\hline Return Period, yr & Maximum Water Level, $\mathrm{ft}$ & Water Level Standard Deviation, $\mathrm{ft}$ \\
\hline 2 & 3.4 & 1.3 \\
\hline 5 & 7.8 & 0.5 \\
\hline 10 & 9.3 & 0.6 \\
\hline 25 & 11.4 & 1.3 \\
\hline 50 & 13.5 & 2.2 \\
\hline 75 & 14.9 & 2.0 \\
\hline 100 & 15.7 & 2.2 \\
\hline
\end{tabular}

\begin{tabular}{|c|c|c|}
\hline \multicolumn{3}{|c|}{$\begin{array}{l}\text { Table B291 } \\
\text { Return Period, Maximum Water Level, and Water Level Standard } \\
\text { Deviation for Profile Tutuila } 286\end{array}$} \\
\hline Return Period, yr & Maximum Water Level, ft & Water Level Standard Deviation, $\mathrm{ft}$ \\
\hline 2 & 3.4 & 1.3 \\
\hline 5 & 7.8 & 0.5 \\
\hline 10 & 9.3 & 0.6 \\
\hline 25 & 11.4 & 1.3 \\
\hline 50 & 13.5 & 2.2 \\
\hline 75 & 14.9 & 2.0 \\
\hline 100 & 15.7 & 2.2 \\
\hline
\end{tabular}

\begin{tabular}{|c|c|c|}
\hline \multicolumn{3}{|c|}{$\begin{array}{l}\text { Table B292 } \\
\text { Return Period, Maximum Water Level, and Water Level Standard } \\
\text { Deviation for Profile Tutuila } 287\end{array}$} \\
\hline Return Period, yr & Maximum Water Level, $\mathbf{f t}$ & Water Level Standard Deviation, ft \\
\hline 2 & 3.4 & 1.3 \\
\hline 5 & 7.8 & 0.5 \\
\hline 10 & 9.3 & 0.6 \\
\hline 25 & 11.4 & 1.3 \\
\hline 50 & 13.5 & 2.2 \\
\hline 75 & 14.9 & 2.0 \\
\hline 100 & 15.7 & 2.2 \\
\hline
\end{tabular}




\begin{tabular}{|c|c|c|}
\hline \multicolumn{3}{|c|}{$\begin{array}{l}\text { Table B293 } \\
\text { Return Period, Maximum Water Level, and Water Level Standard } \\
\text { Deviation for Profile Tutuila } 288\end{array}$} \\
\hline Return Period, yr & Maximum Water Level, ft & Water Level Standard Deviation, tt \\
\hline 2 & 3.4 & 1.3 \\
\hline 5 & 7.8 & 0.5 \\
\hline 10 & 9.2 & 0.6 \\
\hline 25 & 11.4 & 1.2 \\
\hline 50 & 13.6 & 2.2 \\
\hline 75 & 15.0 & 2.0 \\
\hline 100 & 15.7 & 2.1 \\
\hline
\end{tabular}

\begin{tabular}{|c|c|c|}
\hline \multicolumn{3}{|c|}{$\begin{array}{l}\text { Table B294 } \\
\text { Return Period, Maximum Water Level, and Water Level Standard } \\
\text { Deviation for Profile Tutuila } 289\end{array}$} \\
\hline Return Period, yr & Maximum Water Level, $\mathrm{ft}$ & Water Level Standard Deviation, $\mathrm{ft}$ \\
\hline 2 & 3.3 & 1.3 \\
\hline 5 & 7.8 & 0.4 \\
\hline 10 & 9.3 & 0.7 \\
\hline 25 & 11.6 & 1.4 \\
\hline 50 & 14.0 & 2.4 \\
\hline 75 & 15.6 & 2.1 \\
\hline 100 & 16.4 & 2.3 \\
\hline
\end{tabular}

\begin{tabular}{|c|c|c|}
\hline \multicolumn{3}{|c|}{$\begin{array}{l}\text { Table B295 } \\
\text { Return Period, Maximum Water Level, and Water Level Standard } \\
\text { Deviation for Profile Tutuila } 290\end{array}$} \\
\hline Return Period, yr & Maximum Water Level, ft & Water Level Standard Deviation, $\mathrm{ft}$ \\
\hline 2 & 3.3 & 1.3 \\
\hline 5 & 7.8 & 0.4 \\
\hline 10 & 9.3 & 0.7 \\
\hline 25 & 11.5 & 1.3 \\
\hline 50 & 13.7 & 2.2 \\
\hline 75 & 15.1 & 2.0 \\
\hline 100 & 16.0 & 2.1 \\
\hline
\end{tabular}

\begin{tabular}{|c|c|c|}
\hline \multicolumn{3}{|c|}{$\begin{array}{l}\text { Table B296 } \\
\text { Return Period, Maximum Water Level, and Water Level Standard } \\
\text { Deviation for Profile Tutuila } 291\end{array}$} \\
\hline Return Period, yr & Maximum Water Level, ft & Water Level Standard Deviation, $\mathrm{ft}$ \\
\hline 2 & 3.3 & 1.3 \\
\hline 5 & 7.8 & 0.4 \\
\hline 10 & 9.3 & 0.7 \\
\hline 25 & 11.5 & 1.3 \\
\hline 50 & 13.7 & 2.2 \\
\hline 75 & 15.1 & 2.0 \\
\hline 100 & 16.0 & 2.1 \\
\hline
\end{tabular}




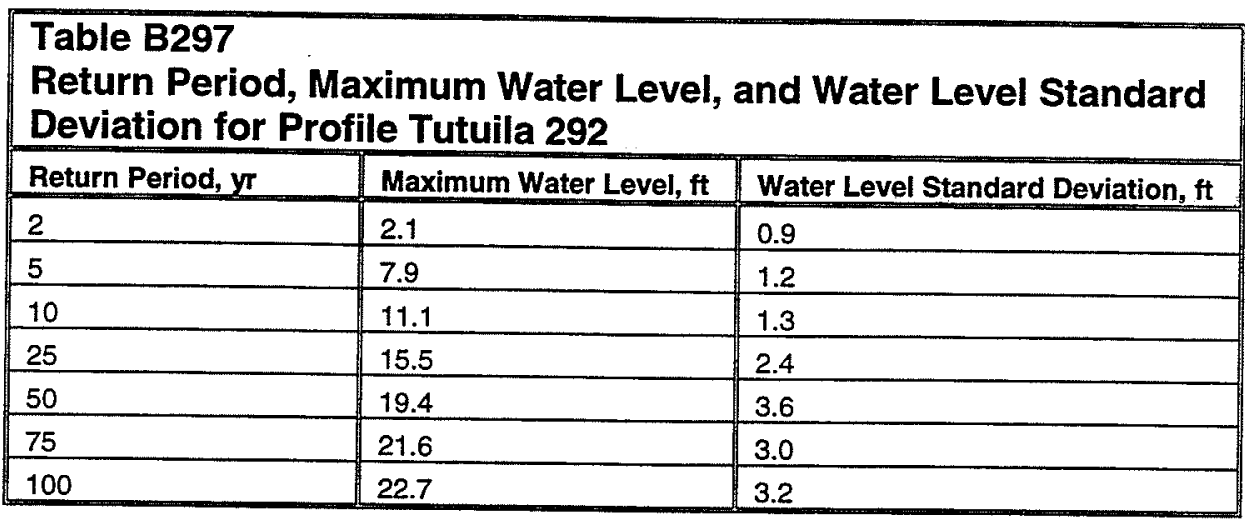

\begin{tabular}{|c|c|c|}
\hline \multicolumn{3}{|c|}{$\begin{array}{l}\text { Table B298 } \\
\text { Return Period, Maximum Water Level, and Water Level Standard } \\
\text { Deviation for Profile Tutuila } 293\end{array}$} \\
\hline Return Period, yr & Maximum Water Level, ft & Water Level Standard Deviation, $\mathrm{ft}$ \\
\hline$\underline{2}$ & 2.1 & 0.9 \\
\hline 5 & 7.9 & 1.2 \\
\hline 10 & 11.1 & 1.3 \\
\hline 25 & 15.5 & 2.4 \\
\hline 50 & 19.4 & 3.6 \\
\hline 75 & 21.6 & 3.0 \\
\hline 100 & 22.7 & 3.2 \\
\hline
\end{tabular}

\begin{tabular}{|c|c|c|}
\hline \multicolumn{3}{|c|}{$\begin{array}{l}\text { Table B299 } \\
\text { Return Period, Maximum Water Level, and Water Level Standard } \\
\text { Deviation for Profile Tutuila } 294\end{array}$} \\
\hline Return Period, yr & Maximum Water Level, $\mathrm{ft}$ & Water Level Standard Deviation, ft \\
\hline 2 & 2.1 & 0.9 \\
\hline 5 & 7.9 & 1.2 \\
\hline 10 & 11.1 & 1.3 \\
\hline 25 & 15.5 & 2.4 \\
\hline 50 & 19.4 & 3.6 \\
\hline 75 & 21.6 & 3.0 \\
\hline 100 & 22.7 & 3.2 \\
\hline
\end{tabular}

\begin{tabular}{|c|c|c|}
\hline \multicolumn{3}{|c|}{$\begin{array}{l}\text { Table B300 } \\
\text { Return Period, Maximum Water Level, and Water Level Standard } \\
\text { Deviation for Profile Tutuila } 295\end{array}$} \\
\hline Return Period, yr & Maximum Water Level, $\mathrm{ft}$ & Water Level Standard Deviation, $\mathrm{tt}$ \\
\hline 2 & 2.4 & 1.0 \\
\hline 5 & 7.0 & 0.8 \\
\hline 10 & 9.5 & 1.1 \\
\hline 25 & 13.3 & 2.1 \\
\hline 50 & 16.6 & 3.3 \\
\hline 75 & 18.7 & 2.8 \\
\hline 100 & 19.7 & 2.9 \\
\hline
\end{tabular}




\begin{tabular}{l}
\hline $\begin{array}{l}\text { Table B301 } \\
\text { Return Period, Maximum Water Level, and Water Level Standard } \\
\text { Deviation for Profile Tutuila 296 }\end{array}$ \\
\begin{tabular}{|l|l|l|}
\hline Return Period, yr & Maximum Water Level, $\mathrm{ft}$ & Water Level Standard Deviation, ft \\
\hline 2 & 2.7 & 1.2 \\
\hline 5 & 7.7 & 1.3 \\
\hline 10 & 11.8 & 1.5 \\
\hline 25 & 16.0 & 2.1 \\
\hline 50 & 19.4 & 3.4 \\
\hline 75 & 21.6 & 2.9 \\
\hline 100 & 22.6 & 3.1 \\
\hline
\end{tabular} \\
\hline
\end{tabular}

\begin{tabular}{|l|l|l|}
\hline $\begin{array}{l}\text { Table B302 } \\
\text { Return Period, Maximum Water Level, and Water Level Standard } \\
\text { Deviation for Profile Tutuila 297 }\end{array}$ \\
\hline Return Period, yr & Maximum Water Level, tt & Water Level Standard Deviation, ft \\
\hline 2 & 2.2 & 1.0 \\
\hline 5 & 6.6 & 1.1 \\
\hline 10 & 10.3 & 1.4 \\
\hline 25 & 14.2 & 1.9 \\
\hline 50 & 17.3 & 3.1 \\
\hline 75 & 19.2 & 2.7 \\
\hline 100 & 20.2 & 2.8 \\
\hline
\end{tabular}

\begin{tabular}{|l|l|l|}
\hline $\begin{array}{l}\text { Table B303 } \\
\text { Return Period, Maximum Water Level, and Water Level Standard } \\
\text { Deviation for Profile Tutuila 298 }\end{array}$ \\
\hline Return Period, yr & Maximum Water Level, ft & Water Level Standard Deviation, ft \\
\hline 2 & 1.9 & 0.9 \\
\hline 5 & 6.4 & 1.4 \\
\hline 10 & 11.9 & 2.0 \\
\hline 25 & 16.8 & 2.2 \\
\hline 50 & 20.6 & 3.1 \\
\hline 75 & 22.4 & 2.8 \\
\hline 100 & 23.4 & 3.0 \\
\hline
\end{tabular}

\begin{tabular}{|c|c|c|}
\hline \multicolumn{3}{|c|}{$\begin{array}{l}\text { Table B304 } \\
\text { Return Period, Maximum Water Level, and Water Level Standard } \\
\text { Deviation for Profile Tutuila 298a }\end{array}$} \\
\hline Return Period, yr & Maximum Water Level, $\mathrm{ft}$ & Water Level Standard Deviation, $\mathrm{ft}$ \\
\hline 2 & 2.1 & 1.0 \\
\hline 5 & 6.4 & 0.9 \\
\hline 10 & 9.6 & 1.3 \\
\hline 25 & 12.9 & 1.5 \\
\hline 50 & 15.4 & 2.2 \\
\hline 75 & 16.7 & 2.0 \\
\hline 100 & 17.4 & 2.2 \\
\hline
\end{tabular}




\begin{tabular}{|c|c|c|}
\hline \multicolumn{3}{|c|}{$\begin{array}{l}\text { Table B305 } \\
\text { Return Period, Maximum Water Level, and Water Level Standard } \\
\text { Deviation for Profile Tutuila } 299\end{array}$} \\
\hline Return Period, yr & Maximum Water Level, $\mathrm{ft}$ & Water Level Standard Deviation, ft \\
\hline 2 & 2.1 & 1.0 \\
\hline 5 & 6.3 & 0.8 \\
\hline 10 & 9.3 & 1.2 \\
\hline 25 & 12.4 & 1.4 \\
\hline 50 & 14.8 & 1.9 \\
\hline 75 & 16.0 & 1.8 \\
\hline 100 & 16.6 & 2.0 \\
\hline
\end{tabular}

\section{Table B306}

Return Period, Maximum Water Level, and Water Level Standard Deviation for Profile Tutuila 300

\begin{tabular}{|l|l|l|}
\hline Return Period, $\mathbf{y r}$ & Maximum Water Level, $\mathrm{ft}$ & Water Level Standard Deviation, $\mathrm{ft}$ \\
\hline 2 & 1.1 & 1.1 \\
\hline 5 & 6.7 & 1.3 \\
\hline 10 & 11.6 & 1.8 \\
\hline 25 & 15.4 & 1.6 \\
\hline 50 & 18.0 & 2.1 \\
\hline 75 & 19.3 & 1.9 \\
\hline 100 & 19.9 & 2.0 \\
\hline
\end{tabular}

\begin{tabular}{|c|c|c|}
\hline \multicolumn{3}{|c|}{$\begin{array}{l}\text { Table B307 } \\
\text { Return Period, Maximum Water Level, and Water Level Standard } \\
\text { Deviation for Profile Tutuila } 301\end{array}$} \\
\hline Return Period, $y r$ & Maximum Water Level, $\mathrm{ft}$ & Water Level Standard Deviation, ft \\
\hline$\underline{2}$ & 1.1 & 1.1 \\
\hline 5 & 6.7 & 1.3 \\
\hline 10 & 11.6 & 1.8 \\
\hline 25 & 15.4 & 1.6 \\
\hline 50 & 18.0 & 2.1 \\
\hline 75 & 19.3 & 1.9 \\
\hline 100 & 19.9 & 2.0 \\
\hline
\end{tabular}

\begin{tabular}{|c|c|c|}
\hline \multicolumn{3}{|c|}{$\begin{array}{l}\text { Table B308 } \\
\text { Return Period, Maximum Water Level, and Water Level Standard } \\
\text { Deviation for Profile Tutuila } 302\end{array}$} \\
\hline Return Period, yr & Maximum Water Level, ft & Water Level Standard Deviation, tt \\
\hline 2 & 1.7 & 0.9 \\
\hline 5 & 5.9 & 0.9 \\
\hline 10 & 9.2 & 1.4 \\
\hline 25 & 12.6 & 1.6 \\
\hline 50 & 15.4 & 2.2 \\
\hline 75 & 16.6 & 2.0 \\
\hline 100 & 17.3 & 2.1 \\
\hline
\end{tabular}




\begin{tabular}{|l|l|}
\hline $\begin{array}{l}\text { Table B309 } \\
\text { Return Period, Maximum Water Level, and Water Level Standard } \\
\text { Deviation for Profile Tutuila 303 }\end{array}$ \\
\begin{tabular}{|l|l|l|}
\hline Return Period, yr & Maximum Water Level, $\mathrm{ft}$ & Water Level Standard Deviation, ft \\
\hline 2 & 1.7 & 0.9 \\
\hline 5 & 6.0 & 1.2 \\
\hline 10 & 9.7 & 1.4 \\
\hline 25 & 13.2 & 1.7 \\
\hline 50 & 16.2 & 2.5 \\
\hline 75 & 17.6 & 2.2 \\
\hline 100 & 18.4 & 2.3 \\
\hline
\end{tabular}
\end{tabular}

\begin{tabular}{|c|c|c|}
\hline \multicolumn{3}{|c|}{$\begin{array}{l}\text { Table B310 } \\
\text { Return Period, Maximum Water Level, and Water Level Standard } \\
\text { Deviation for Profile Tutuila } 304\end{array}$} \\
\hline Return Period, yr & Maximum Water Level, ft & Water Level Standard Deviation, $\mathrm{ft}$ \\
\hline 2 & 1.5 & 0.8 \\
\hline 5 & 5.9 & 1.4 \\
\hline 10 & 10.1 & 1.5 \\
\hline 25 & 13.9 & 1.9 \\
\hline 50 & 17.3 & 2.9 \\
\hline 75 & 19.0 & 2.5 \\
\hline 100 & 19.9 & 2.7 \\
\hline
\end{tabular}

\begin{tabular}{|c|c|c|}
\hline \multicolumn{3}{|c|}{$\begin{array}{l}\text { Table B311 } \\
\text { Return Period, Maximum Water Level, and Water Level Standard } \\
\text { Deviation for Profile Tutuila } 305\end{array}$} \\
\hline Return Period, yr & Maximum Water Level, $\mathrm{ft}$ & Water Level Standard Deviation, $\mathrm{ft}$ \\
\hline 2 & 1.5 & 0.8 \\
\hline 5 & 5.9 & 1.4 \\
\hline 10 & 10.1 & 1.5 \\
\hline 25 & 13.9 & 1.9 \\
\hline 50 & 17.3 & 2.9 \\
\hline 75 & 19.0 & 2.5 \\
\hline 100 & 19.9 & 2.7 \\
\hline
\end{tabular}

\begin{tabular}{|c|c|c|}
\hline \multicolumn{3}{|c|}{$\begin{array}{l}\text { Table B312 } \\
\text { Return Period, Maximum Water Level, and Water Level Standard } \\
\text { Deviation for Profile Tutuila } 306\end{array}$} \\
\hline Return Period, yr & Maximum Water Level, ft & Water Level Standard Deviation, ft \\
\hline 2 & 1.5 & 0.8 \\
\hline 5 & 5.9 & 1.4 \\
\hline 10 & 10.1 & 1.5 \\
\hline 25 & 15.1 & 3.0 \\
\hline 50 & 19.9 & 4.4 \\
\hline 75 & 22.6 & 3.5 \\
\hline 100 & 24.0 & 3.7 \\
\hline
\end{tabular}




\begin{tabular}{|c|c|c|}
\hline \multicolumn{3}{|c|}{$\begin{array}{l}\text { Table B313 } \\
\text { Return Period, Maximum Water Level, and Water Level Standard } \\
\text { Deviation for Profile Tutuila } 307\end{array}$} \\
\hline Return Period, yr & Maximum Water Level, ft & Water Level Standard Deviation, ft \\
\hline 2 & 1.7 & 0.8 \\
\hline 5 & 5.6 & 0.9 \\
\hline 10 & 8.5 & 1.1 \\
\hline 25 & 12.1 & 2.0 \\
\hline 50 & 15.6 & 3.0 \\
\hline 75 & 17.4 & 2.6 \\
\hline 100 & 18.3 & 2.7 \\
\hline
\end{tabular}

\section{Table B314}

Return Period, Maximum Water Level, and Water Level Standard Deviation for Profile Tutuila 308

\begin{tabular}{|l|l|l|}
\hline Return Period, $\mathbf{y r}$ & Maximum Water Level, ft & Water Level Standard Deviation, $\mathrm{ft}$ \\
\hline 2 & 1.7 & 0.8 \\
\hline 5 & 5.6 & 0.9 \\
\hline 10 & 8.5 & 1.1 \\
\hline 25 & 12.1 & 2.0 \\
\hline 50 & 15.6 & 3.0 \\
\hline 75 & 17.4 & 2.6 \\
\hline 100 & 18.3 & 2.7 \\
\hline
\end{tabular}

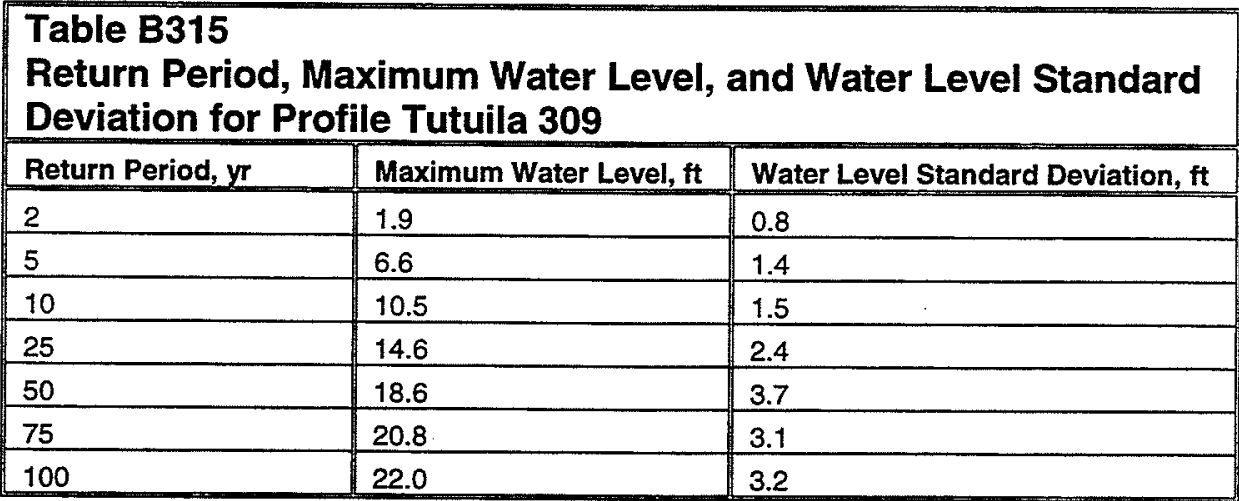

\begin{tabular}{|c|c|c|}
\hline \multicolumn{3}{|c|}{$\begin{array}{l}\text { Table B316 } \\
\text { Return Period, Maximum Water Level, and Water Level Standard } \\
\text { Deviation for Profile Tutuila } 310\end{array}$} \\
\hline Return Period, yr & Maximum Water Level, ft & Water Level Standard Deviation, it \\
\hline 2 & 1.9 & 0.8 \\
\hline 5 & 6.6 & 1.4 \\
\hline 10 & 10.5 & 1.5 \\
\hline 25 & 14.6 & 2.4 \\
\hline 50 & 18.6 & 3.7 \\
\hline 75 & 20.8 & 3.1 \\
\hline 100 & 22.0 & 3.2 \\
\hline
\end{tabular}




\begin{tabular}{|c|c|c|}
\hline \multicolumn{3}{|c|}{$\begin{array}{l}\text { Table B317 } \\
\text { Return Period, Maximum Water Level, and Water Level Standard } \\
\text { Deviation for Profile Tutuila } 311\end{array}$} \\
\hline Return Period, yr & Maximum Water Level, $\mathrm{ft}$ & Water Level Standard Deviation, $\mathrm{ft}$ \\
\hline 2 & 1.9 & 0.8 \\
\hline 5 & 6.6 & 1.4 \\
\hline 10 & 10.5 & 1.5 \\
\hline 25 & 14.6 & 2.4 \\
\hline 50 & 18.6 & 3.7 \\
\hline 75 & 20.8 & 3.1 \\
\hline 100 & 22.0 & 3.2 \\
\hline
\end{tabular}

\begin{tabular}{|c|c|c|}
\hline \multicolumn{3}{|c|}{$\begin{array}{l}\text { Table B318 } \\
\text { Return Period, Maximum Water Level, and Water Level Standard } \\
\text { Deviation for Profile Tutuila } 312\end{array}$} \\
\hline Return Period, yr & Maximum Water Level, ft & Water Level Standard Deviation, ft \\
\hline 2 & 1.9 & 0.9 \\
\hline 5 & 7.2 & 1.1 \\
\hline 10 & 10.5 & 1.5 \\
\hline 25 & 15.3 & 2.3 \\
\hline 50 & 19.2 & 3.6 \\
\hline 75 & 21.4 & 3.1 \\
\hline 100 & 22.6 & 3.2 \\
\hline
\end{tabular}

\begin{tabular}{|l|l|l|}
\hline $\begin{array}{l}\text { Table B319 } \\
\text { Return Period, Maximum Water Level, and Water Level Standard } \\
\text { Deviation for Profile Tutuila 313 }\end{array}$ \\
\hline Return Period, yr & Maximum Water Level, ft & Water Level Standard Deviation, ft \\
\hline 2 & 1.9 & 0.9 \\
\hline 5 & 7.2 & 1.1 \\
\hline 10 & 10.5 & 1.5 \\
\hline 25 & 15.3 & 2.3 \\
\hline 50 & 19.2 & 3.6 \\
\hline 75 & 21.4 & 3.1 \\
\hline 100 & 22.6 & 3.2 \\
\hline
\end{tabular}

\begin{tabular}{|l|l|l|}
\hline $\begin{array}{l}\text { Table B320 } \\
\text { Return Period, Maximum Water Level, and Water Level Standard } \\
\text { Deviation for Profile Tutuila } 314\end{array}$ \\
\hline Return Period, yr & Maximum Water Level, ft & Water Level Standard Deviation, $\mathrm{tt}$ \\
\hline 2 & 1.9 & 0.9 \\
\hline 5 & 7.2 & 1.1 \\
\hline 10 & 10.5 & 1.5 \\
\hline 25 & 15.3 & 2.3 \\
\hline 50 & 19.2 & 3.6 \\
\hline 75 & 21.4 & 3.1 \\
\hline 100 & 22.6 & 3.2 \\
\hline
\end{tabular}




\begin{tabular}{|c|c|c|}
\hline \multicolumn{3}{|c|}{$\begin{array}{l}\text { Table B321 } \\
\text { Return Period, Maximum Water Level, and Water Level Standard } \\
\text { Deviation for Profile Tutuila } 315\end{array}$} \\
\hline Return Period, yr & Maximum Water Level, $\mathrm{ft}$ & Water Level Standard Deviation, $\mathrm{ft}$ \\
\hline 2 & 1.9 & 0.9 \\
\hline 5 & 7.2 & 1.1 \\
\hline 10 & 10.5 & 1.5 \\
\hline 25 & 16.3 & 3.5 \\
\hline 50 & 22.2 & 5.2 \\
\hline 75 & 25.3 & 4.0 \\
\hline 100 & 26.9 & 4.1 \\
\hline
\end{tabular}

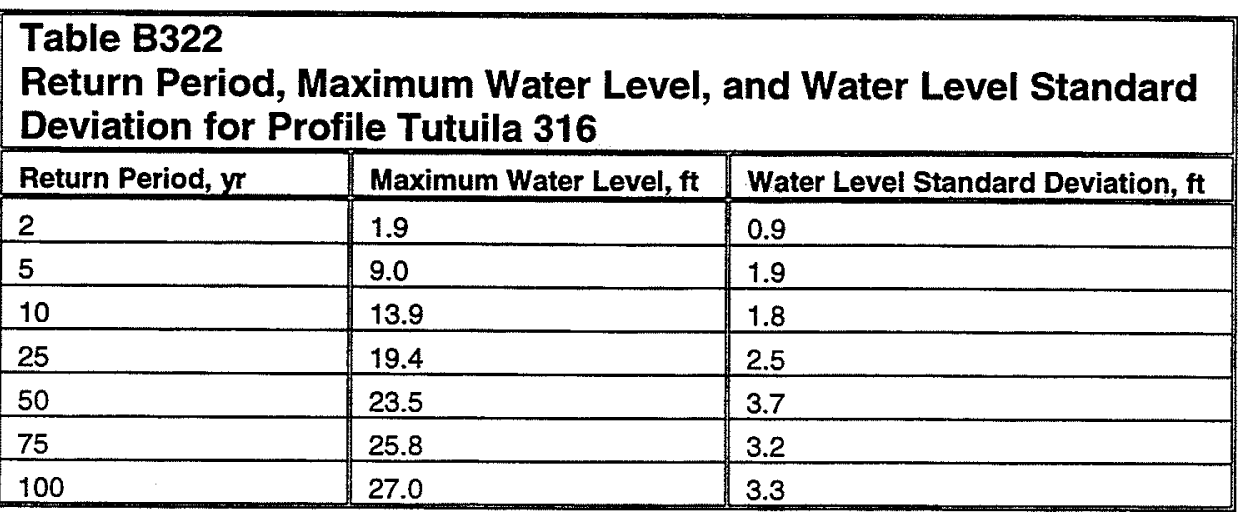

\begin{tabular}{|c|c|c|}
\hline $\begin{array}{l}\text { Table B323 } \\
\text { Return Perio } \\
\text { Deviation for }\end{array}$ & $\begin{array}{l}\text { imum Water Level } \\
\text { e Tutuila } 317\end{array}$ & and Water Level Standard \\
\hline Return Period, yr & Maximum Water Level, $\mathrm{ft}$ & Water Level Standard Deviation, ft \\
\hline 2 & 1.9 & 0.9 \\
\hline 5 & 7.2 & 1.1 \\
\hline 10 & 10.5 & 1.5 \\
\hline 25 & 15.3 & 2.3 \\
\hline 50 & 19.2 & 3.6 \\
\hline 75 & 21.4 & 3.1 \\
\hline 100 & 22.6 & 3.2 \\
\hline
\end{tabular}

\begin{tabular}{|c|c|c|}
\hline \multicolumn{3}{|c|}{$\begin{array}{l}\text { Table B324 } \\
\text { Return Period, Maximum Water Level, and Water Level Standard } \\
\text { Deviation for Profile Tutuila } 318\end{array}$} \\
\hline Return Period, yr & Maximum Water Level, ft & Water Level Standard Deviation, $\mathrm{ft}$ \\
\hline 2 & 1.9 & 0.9 \\
\hline 5 & 7.2 & 1.1 \\
\hline 10 & 10.5 & 1.5 \\
\hline 25 & 15.3 & 2.3 \\
\hline 50 & 19.2 & 3.6 \\
\hline 75 & 21.4 & 3.1 \\
\hline 100 & 22.6 & \\
\hline
\end{tabular}




\begin{tabular}{l}
\hline $\begin{array}{l}\text { Table B325 } \\
\text { Return Period, Maximum Water Level, and Water Level Standard } \\
\text { Deviation for Profile Tutuila } 319\end{array}$ \\
\begin{tabular}{|l|l|l|}
\hline Return Period, yr & Maximum Water Level, ft & Water Level Standard Deviation, ft \\
\hline 2 & 1.9 & 0.9 \\
\hline 5 & 7.2 & 1.1 \\
\hline 10 & 10.5 & 1.5 \\
\hline 25 & 15.3 & 2.3 \\
\hline 50 & 19.2 & 3.6 \\
\hline 75 & 21.4 & 3.1 \\
\hline 100 & 22.6 & 3.2 \\
\hline
\end{tabular}
\end{tabular}

\begin{tabular}{|c|c|c|}
\hline \multicolumn{3}{|c|}{$\begin{array}{l}\text { Table B326 } \\
\text { Return Period, Maximum Water Level, and Water Level Standard } \\
\text { Deviation for Profile Tutuila } 320\end{array}$} \\
\hline Return Period, yz & Maximum Water Level, ft & Water Level Standard Deviation, $\mathrm{ft}$ \\
\hline$\underline{2}$ & 1.9 & 0.9 \\
\hline 5 & 7.2 & 1.1 \\
\hline 10 & 10.5 & 1.5 \\
\hline 25 & 15.3 & 2.3 \\
\hline 50 & 19.2 & 3.6 \\
\hline 75 & 21.4 & 3.1 \\
\hline 100 & 22.6 & 3.2 \\
\hline
\end{tabular}

\begin{tabular}{|c|c|c|}
\hline \multicolumn{3}{|c|}{$\begin{array}{l}\text { Table B327 } \\
\text { Return Period, Maximum Water Level, and Water Level Standard } \\
\text { Deviation for Profile Tutuila } 321\end{array}$} \\
\hline Return Period, yr & Maximum Water Level, $\mathrm{ft}$ & Water Level Standard Deviation, $\mathrm{ft}$ \\
\hline$\underline{2}$ & 1.9 & 0.9 \\
\hline 5 & 7.2 & 1.1 \\
\hline 10 & 10.5 & 1.5 \\
\hline 25 & 15.3 & 2.3 \\
\hline 50 & 19.2 & 3.6 \\
\hline 75 & 21.4 & 3.1 \\
\hline 100 & 22.6 & 3.2 \\
\hline
\end{tabular}

\begin{tabular}{|c|c|c|}
\hline \multicolumn{3}{|c|}{$\begin{array}{l}\text { Table B328 } \\
\text { Return Period, Maximum Water Level, and Water Level Standard } \\
\text { Deviation for Profile Tutuila } 322\end{array}$} \\
\hline Return Period, yr & Maximum Water Level, tt & Water Level Standard Deviation, $\mathrm{ft}$ \\
\hline 2 & 1.9 & 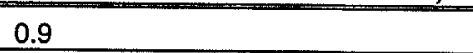 \\
\hline 5 & 7.0 & 1.0 \\
\hline 10 & 10.5 & 1.5 \\
\hline 25 & 15.4 & 2.3 \\
\hline 50 & 19.4 & 3.7 \\
\hline 75 & 21.7 & 3.1 \\
\hline 100 & 22.8 & 3.2 \\
\hline
\end{tabular}




\begin{tabular}{|c|c|c|}
\hline \multicolumn{3}{|c|}{$\begin{array}{l}\text { Table B329 } \\
\text { Return Period, Maximum Water Level, and Water Level Standard } \\
\text { Deviation for Profile Tutuila } 323\end{array}$} \\
\hline Return Period, yr & Maximum Water Level, ft & Water Level Standard Deviation, $\mathrm{ft}$ \\
\hline 2 & 1.9 & 0.9 \\
\hline 5 & 7.0 & 1.0 \\
\hline 10 & 10.5 & 1.5 \\
\hline 25 & 15.4 & 2.3 \\
\hline 50 & 19.4 & 3.7 \\
\hline 75 & 21.7 & 3.1 \\
\hline 100 & 22.8 & 3.2 \\
\hline
\end{tabular}

\begin{tabular}{|c|c|c|}
\hline \multicolumn{3}{|c|}{$\begin{array}{l}\text { Table B330 } \\
\text { Return Period, Maximum Water Level, and Water Level Standard } \\
\text { Deviation for Profile Tutuila } 324\end{array}$} \\
\hline Return Period, yr & Maximum Water Level, ft & Water Level Standard Deviation, ft \\
\hline 2 & 1.9 & 0.9 \\
\hline 5 & 7.0 & 1.0 \\
\hline 10 & 10.5 & 1.5 \\
\hline 25 & 15.4 & 2.3 \\
\hline 50 & 19.4 & 3.7 \\
\hline 75 & 21.7 & 3.1 \\
\hline 100 & 22.8 & 3.2 \\
\hline
\end{tabular}

\section{Table B331}

Return Period, Maximum Water Level, and Water Level Standard Deviation for Profile Tutuila 325

\begin{tabular}{|l|l|l|}
\hline Return Period, $\mathbf{y r}$ & Maximum Water Level, $\mathbf{f t}$ & Water Level Standard Deviation, $\mathbf{t t}$ \\
\hline 2 & 1.9 & 0.9 \\
\hline 5 & 7.0 & 1.0 \\
\hline 10 & 10.5 & 1.5 \\
\hline 25 & 15.4 & 2.3 \\
\hline 50 & 19.4 & 3.7 \\
\hline 75 & 21.7 & 3.1 \\
\hline 100 & 22.8 & 3.2 \\
\hline
\end{tabular}

\begin{tabular}{|c|c|c|}
\hline \multicolumn{3}{|c|}{$\begin{array}{l}\text { Table B332 } \\
\text { Return Period, Maximum Water Level, and Water Level Standard } \\
\text { Deviation for Profile Tutuila } 326\end{array}$} \\
\hline Return Period, yr & Maximum Water Level, ft & Water Level Standard Deviation, $\mathrm{ft}$ \\
\hline 2 & 1.9 & 0.9 \\
\hline 5 & 7.0 & 1.0 \\
\hline 10 & 10.5 & 1.5 \\
\hline 25 & 15.4 & 2.3 \\
\hline 50 & 19.4 & 3.7 \\
\hline 75 & 21.7 & 3.1 \\
\hline 100 & 22.8 & 3.2 \\
\hline
\end{tabular}




\begin{tabular}{|c|c|c|}
\hline \multicolumn{3}{|c|}{$\begin{array}{l}\text { Table B333 } \\
\text { Return Period, Maximum Water Level, and Water Level Standard } \\
\text { Deviation for Profile Tutuila } 327\end{array}$} \\
\hline Return Period, yr & Maximum Water Level, $\mathrm{ft}$ & Water Level Standard Deviation, it \\
\hline 2 & 2.2 & 1.0 \\
\hline 5 & 8.7 & 1.1 \\
\hline 10 & 11.7 & 1.1 \\
\hline 25 & 16.0 & 2.1 \\
\hline 50 & 19.5 & 3.3 \\
\hline 75 & 21.6 & 2.9 \\
\hline 100 & 22.7 & 3.1 \\
\hline
\end{tabular}

\begin{tabular}{|c|c|c|}
\hline \multicolumn{3}{|c|}{$\begin{array}{l}\text { Table B334 } \\
\text { Return Period, Maximum Water Level, and Water Level Standard } \\
\text { Deviation for Profile Tutuila } 328\end{array}$} \\
\hline Return Period, yr & Maximum Water Level, $\mathrm{ft}$ & Water Level Standard Deviation, $\mathrm{ft}$ \\
\hline 2 & 2.2 & 1.0 \\
\hline 5 & 8.7 & 1.1 \\
\hline 10 & 11.7 & 1.1 \\
\hline 25 & 16.0 & 2.1 \\
\hline 50 & 19.5 & 3.3 \\
\hline 75 & 21.6 & 2.9 \\
\hline 100 & 22.7 & 3.1 \\
\hline
\end{tabular}

\begin{tabular}{|c|c|c|}
\hline \multicolumn{3}{|c|}{$\begin{array}{l}\text { Table B335 } \\
\text { Return Period, Maximum Water Level, and Water Level Standard } \\
\text { Deviation for Profile Tutuila } 329\end{array}$} \\
\hline Return Period, yr & Maximum Water Level, ft & Water Level Standard Deviation, $\mathrm{tt}$ \\
\hline 2 & 2.4 & 1.2 \\
\hline 5 & 11.9 & 1.1 \\
\hline 10 & 15.0 & 1.3 \\
\hline 25 & 19.1 & 2.0 \\
\hline 50 & 21.9 & 2.0 \\
\hline 75 & 23.0 & 1.7 \\
\hline 100 & 23.6 & 1.7 \\
\hline
\end{tabular}

\begin{tabular}{|c|c|c|}
\hline \multicolumn{3}{|c|}{$\begin{array}{l}\text { Table B336 } \\
\text { Return Period, Maximum Water Level, and Water Level Standard } \\
\text { Deviation for Profile Tutuila } 330\end{array}$} \\
\hline Return Period, yr & Maximum Water Level, ft & Water Level Standard Deviation, $\mathrm{tt}$ \\
\hline 2 & 2.2 & 1.0 \\
\hline 5 & 10.9 & 1.4 \\
\hline 10 & 14.8 & 1.7 \\
\hline 25 & 20.3 & 2.9 \\
\hline 50 & 24.9 & 3.6 \\
\hline 75 & 27.0 & 3.1 \\
\hline 100 & 28.1 & 3.2 \\
\hline
\end{tabular}




\begin{tabular}{|c|c|c|}
\hline \multicolumn{3}{|c|}{$\begin{array}{l}\text { Table B337 } \\
\text { Return Period, Maximum Water Level, and Water Level Standard } \\
\text { Deviation for Profile Aunuu } 001\end{array}$} \\
\hline Return Period, yr & Maximum Water Level, $f t$ & Water Level Standard Deviation, $\mathrm{ft}$ \\
\hline 2 & 1.8 & 0.8 \\
\hline 5 & 6.1 & 1.0 \\
\hline 10 & 9.4 & 1.4 \\
\hline 25 & 13.2 & 1.7 \\
\hline 50 & 15.8 & 2.2 \\
\hline 75 & 17.1 & 1.9 \\
\hline 100 & 17.7 & 2.1 \\
\hline
\end{tabular}

\section{Table B338}

Return Period, Maximum Water Level, and Water Level Standard Deviation for Profile Aunuu 002

\begin{tabular}{|l|l|l|}
\hline Return Period, $\mathbf{y r}$ & Maximum Water Level, $\mathrm{ft}$ & Water Level Standard Deviation, $\mathrm{ft}$ \\
\hline 2 & 0.4 & 0.5 \\
\hline 5 & 3.5 & 0.6 \\
\hline 10 & 6.6 & 1.6 \\
\hline 25 & 10.7 & 1.6 \\
\hline 50 & 12.7 & 1.6 \\
\hline 75 & 13.5 & 1.3 \\
\hline 100 & 13.9 & 1.4 \\
\hline
\end{tabular}

\begin{tabular}{|c|c|c|}
\hline \multicolumn{3}{|c|}{$\begin{array}{l}\text { Table B339 } \\
\text { Return Period, Maximum Water Level, and Water Level Standard } \\
\text { Deviation for Profile Aunuu } 003\end{array}$} \\
\hline Return Period, yr & Maximum Water Level, ft & Water Level Standard Deviation, ft \\
\hline 2 & 1.4 & 0.7 \\
\hline 5 & 4.1 & 0.4 \\
\hline 10 & 5.3 & 0.5 \\
\hline 25 & 6.6 & 0.7 \\
\hline 50 & 7.6 & 0.9 \\
\hline 75 & 8.2 & 0.8 \\
\hline 100 & 8.5 & \\
\hline
\end{tabular}

\section{Table B340}

Return Period, Maximum Water Level, and Water Level Standard Deviation for Profile Aunuu 004

\begin{tabular}{|l|l|l|}
\hline Return Period, $\mathbf{y r}$ & Maximum Water Level, $\mathrm{ft}$ & Water Level Standard Deviation, $\mathrm{ft}$ \\
\hline 2 & 1.9 & 0.8 \\
\hline 5 & 5.6 & 0.7 \\
\hline 10 & 7.7 & 0.9 \\
\hline 25 & 9.9 & 1.1 \\
\hline 50 & 11.4 & 1.2 \\
\hline 75 & 12.1 & 1.0 \\
\hline 100 & 12.4 & 1.1 \\
\hline
\end{tabular}




\begin{tabular}{|c|c|c|}
\hline \multicolumn{3}{|c|}{$\begin{array}{l}\text { Table B341 } \\
\text { Return Period, Maximum Water Level, and Water Level Standard } \\
\text { Deviation for Profile Aunuu } 005\end{array}$} \\
\hline Return Period, yr & Maximum Water Level, ft & Water Level Standard Deviation, ft \\
\hline 2 & 2.0 & 0.8 \\
\hline 5 & 5.7 & 0.7 \\
\hline 10 & 7.8 & 0.9 \\
\hline 25 & 10.1 & 1.1 \\
\hline 50 & 11.6 & 1.2 \\
\hline 75 & 12.2 & 1.0 \\
\hline 100 & 12.6 & 1.1 \\
\hline
\end{tabular}

\begin{tabular}{|c|c|c|}
\hline \multicolumn{3}{|c|}{$\begin{array}{l}\text { Table B342 } \\
\text { Return Period, Maximum Water Level, and Water Level Standard } \\
\text { Deviation for Profile Manua } 001\end{array}$} \\
\hline Return Period, yr & Maximum Water Level, ft & Water Level Standard Deviation, $\mathrm{ft}$ \\
\hline$\underline{2}$ & 2.4 & 0.9 \\
\hline 5 & 7.2 & 1.0 \\
\hline 10 & 9.7 & 0.8 \\
\hline 25 & 11.8 & 1.0 \\
\hline 50 & 13.3 & 1.3 \\
\hline 75 & 13.9 & 1.1 \\
\hline 100 & 14.2 & 1.1 \\
\hline
\end{tabular}

\begin{tabular}{|c|c|c|}
\hline \multicolumn{3}{|c|}{$\begin{array}{l}\text { Table B343 } \\
\text { Return Period, Maximum Water Level, and Water Level Standard } \\
\text { Deviation for Profile Manua } 002\end{array}$} \\
\hline Return Period, yr & Maximum Water Level, $\mathrm{ft}$ & Water Level Standard Deviation, $\mathrm{ft}$ \\
\hline 2 & 1.9 & 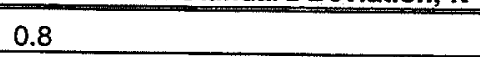 \\
\hline 5 & 6.6 & 1.0 \\
\hline 10 & 9.1 & 0.7 \\
\hline 25 & 10.9 & 0.9 \\
\hline 50 & 12.3 & 1.2 \\
\hline 75 & 12.9 & 1.1 \\
\hline 100 & 13.2 & 1.1 \\
\hline
\end{tabular}

\begin{tabular}{|c|c|c|}
\hline \multicolumn{3}{|c|}{$\begin{array}{l}\text { Table B344 } \\
\text { Return Period, Maximum Water Level, and Water Level Standard } \\
\text { Deviation for Profile Manua } 003\end{array}$} \\
\hline Return Period, yr & Maximum Water Level, it & Water Level Standard Deviation, $\mathrm{ft}$ \\
\hline 2 & 2.0 & 0.8 \\
\hline 5 & 6.8 & 1.0 \\
\hline 10 & 9.2 & 0.7 \\
\hline 25 & 11.1 & 0.9 \\
\hline 50 & 12.5 & 1.2 \\
\hline 75 & 13.2 & 1.1 \\
\hline 100 & 13.5 & 1.1 \\
\hline
\end{tabular}




\begin{tabular}{|c|c|c|}
\hline Return Period, yr & Maximum Water Level, $\mathrm{ft}$ & Water Level Standard Deviation, $\mathrm{ft}$ \\
\hline 2 & 2.2 & 0.9 \\
\hline 5 & 7.1 & 1.1 \\
\hline 10 & 10.7 & 1.6 \\
\hline 25 & 15.5 & 2.4 \\
\hline 50 & 18.7 & 2.2 \\
\hline 75 & 19.7 & 1.9 \\
\hline 100 & 20.1 & 1.9 \\
\hline
\end{tabular}

\begin{tabular}{|l|l|l|}
\hline $\begin{array}{l}\text { Table B346 } \\
\text { Return Period, Maximum Water Level, and Water Level Standard } \\
\text { Deviation for Profile Manua 005 }\end{array}$ \\
\hline Return Period, yr & Maximum Water Level, $\mathrm{tt}$ & Water Level Standard Deviation, ft \\
\hline 2 & 2.4 & 0.9 \\
\hline 5 & 7.4 & 1.0 \\
\hline 10 & 9.9 & 0.8 \\
\hline 25 & 12.1 & 1.1 \\
\hline 50 & 13.6 & 1.3 \\
\hline 75 & 14.2 & 1.1 \\
\hline 100 & 14.5 & 1.2 \\
\hline
\end{tabular}

\begin{tabular}{|c|c|c|}
\hline Return Period, yr & Maximum Water Level, $\mathrm{ft}$ & Water Level Standard Deviation, ft \\
\hline 2 & 2.0 & 0.8 \\
\hline 5 & 5.6 & 0.8 \\
\hline 10 & 8.2 & 1.2 \\
\hline 25 & 11.3 & 1.7 \\
\hline 50 & 13.8 & 2.4 \\
\hline 75 & 15.2 & 2.0 \\
\hline 100 & 15.9 & 2.2 \\
\hline
\end{tabular}

\begin{tabular}{|c|c|c|}
\hline \multicolumn{3}{|c|}{$\begin{array}{l}\text { Table B348 } \\
\text { Return Period, Maximum Water Level, and Water Level Standard } \\
\text { Deviation for Profile Manua } 007\end{array}$} \\
\hline Return Period, yr & Maximum Water Level, ft & Water Level Standard Deviation, $\mathrm{ft}$ \\
\hline 2 & 1.9 & 0.8 \\
\hline 5 & 5.4 & 0.8 \\
\hline 10 & 8.0 & 1.1 \\
\hline 25 & 11.0 & 1.6 \\
\hline 50 & 13.3 & 2.2 \\
\hline 75 & 14.7 & 2.0 \\
\hline 100 & 15.4 & 2.1 \\
\hline
\end{tabular}




\begin{tabular}{|c|c|c|}
\hline \multicolumn{3}{|c|}{$\begin{array}{l}\text { Table B349 } \\
\text { Return Period, Maximum Water Level, and Water Level Standard } \\
\text { Deviation for Profile Manua } 008\end{array}$} \\
\hline Return Period, yr & Maximum Water Level, ft & Water Level Standard Deviation, $\mathrm{ft}$ \\
\hline 2 & 1.9 & . \\
\hline 5 & 5.1 & 0.6 \\
\hline 10 & 7.4 & 1.1 \\
\hline 25 & 10.5 & 1.7 \\
\hline 50 & 13.0 & 2.4 \\
\hline 75 & 14.5 & 2.1 \\
\hline 100 & 15.2 & 2.2 \\
\hline
\end{tabular}

\begin{tabular}{|c|c|c|}
\hline \multicolumn{3}{|c|}{$\begin{array}{l}\text { Table B350 } \\
\text { Return Period, Maximum Water Level, and Water Level Standard } \\
\text { Deviation for Profile Manua } 009\end{array}$} \\
\hline Return Period, yr & Maximum Water Level, ft & Water Level Standard Deviation, tt \\
\hline 2 & 1.7 & 0.7 \\
\hline 5 & 4.9 & 0.6 \\
\hline 10 & 7.0 & 1.0 \\
\hline 25 & 9.9 & 1.7 \\
\hline 50 & 12.5 & 2.5 \\
\hline 75 & 14.1 & 2.1 \\
\hline 100 & 14.8 & 2.2 \\
\hline
\end{tabular}

\begin{tabular}{|c|c|c|}
\hline \multicolumn{3}{|c|}{$\begin{array}{l}\text { Table B351 } \\
\text { Return Period, Maximum Water Level, and Water Level Standard } \\
\text { Deviation for Profile Manua } 010\end{array}$} \\
\hline Return Period, yr & Maximum Water Level, $\mathrm{ft}$ & Water Level Standard Deviation, ft \\
\hline 2 & 1.9 & 0.8 \\
\hline 5 & 5.2 & 0.6 \\
\hline 10 & 7.2 & 1.0 \\
\hline 25 & 10.3 & 1.9 \\
\hline 50 & 13.3 & 2.8 \\
\hline 75 & 15.0 & 2.3 \\
\hline 100 & 15.9 & 2.4 \\
\hline
\end{tabular}

\begin{tabular}{|c|c|c|}
\hline \multicolumn{3}{|c|}{$\begin{array}{l}\text { Table B352 } \\
\text { Return Period, Maximum Water Level, and Water Level Standard } \\
\text { Deviation for Profile Manua } 0011\end{array}$} \\
\hline Return Period, yr & Maximum Water Level, $\mathrm{ft}$ & Water Level Standard Deviation, ft \\
\hline 2 & 1.7 & 0.7 \\
\hline 5 & 4.8 & 0.5 \\
\hline 10 & 6.6 & 0.9 \\
\hline 25 & 9.5 & 1.9 \\
\hline 50 & 12.5 & 2.8 \\
\hline 75 & 14.2 & 2.3 \\
\hline 100 & 15.1 & 2.4 \\
\hline
\end{tabular}




\begin{tabular}{|c|c|c|}
\hline \multicolumn{3}{|c|}{$\begin{array}{l}\text { Table B353 } \\
\text { Return Period, Maximum Water Level, and Water Level Standard } \\
\text { Deviation for Profile Manua } 012\end{array}$} \\
\hline Return Period, yr & Maximum Water Level, ft & Water Level Standard Deviation, $\mathrm{ft}$ \\
\hline 2 & 2.1 & 0.8 \\
\hline 5 & 5.3 & 0.5 \\
\hline 10 & 7.1 & 0.8 \\
\hline 25 & 10.3 & 2.3 \\
\hline 50 & 13.8 & 3.2 \\
\hline 75 & 15.8 & 2.6 \\
\hline 100 & 16.7 & 2.6 \\
\hline
\end{tabular}

\begin{tabular}{|c|c|c|}
\hline \multicolumn{3}{|c|}{$\begin{array}{l}\text { Table B354 } \\
\text { Return Period, Maximum Water Level, and Water Level Standard } \\
\text { Deviation for Profile Manua } 013\end{array}$} \\
\hline Return Period, yr & Maximum Water Level, $\mathrm{ft}$ & Water Level Standard Deviation, ft \\
\hline 2 & 1.7 & 0.7 \\
\hline 5 & 4.4 & 0.4 \\
\hline 10 & 5.9 & 0.7 \\
\hline 25 & 8.7 & 2.2 \\
\hline 50 & 12.3 & 3.0 \\
\hline 75 & 14.1 & 2.4 \\
\hline 100 & 15.0 & 2.4 \\
\hline
\end{tabular}

\begin{tabular}{|c|c|c|}
\hline \multicolumn{3}{|c|}{$\begin{array}{l}\text { Table B355 } \\
\text { Return Period, Maximum Water Level, and Water Level Standard } \\
\text { Deviation for Profile Manua } 014\end{array}$} \\
\hline Return Period, yr & Maximum Water Level, $\mathrm{ft}$ & Water Level Standard Deviation, $\mathrm{ft}$ \\
\hline 2 & 1.9 & 0.8 \\
\hline 5 & 4.8 & 0.4 \\
\hline 10 & 6.3 & 0.7 \\
\hline 25 & 9.3 & 2.5 \\
\hline 50 & 13.3 & 3.3 \\
\hline 75 & 15.3 & 2.6 \\
\hline 100 & 16.3 & 2.6 \\
\hline
\end{tabular}

\begin{tabular}{|c|c|c|}
\hline \multicolumn{3}{|c|}{$\begin{array}{l}\text { Table B356 } \\
\text { Return Period, Maximum Water Level, and Water Level Standard } \\
\text { Deviation for Profile Manua } 015\end{array}$} \\
\hline Return Period, yr & Maximum Water Level, ft & Water Level Standard Deviation, ft \\
\hline 2 & 1.8 & 0.7 \\
\hline 5 & 4.8 & 0.5 \\
\hline 10 & 6.9 & 1.0 \\
\hline 25 & 10.4 & 1.9 \\
\hline 50 & 13.3 & 2.6 \\
\hline 75 & 14.8 & 2.2 \\
\hline 100 & 15.6 & 2.3 \\
\hline
\end{tabular}




\begin{tabular}{|c|c|c|}
\hline \multicolumn{3}{|c|}{$\begin{array}{l}\text { Table B357 } \\
\text { Return Period, Maximum Water Level, and Water Level Standard } \\
\text { Deviation for Profile Manua } 016\end{array}$} \\
\hline Return Period, yr & Maximum Water Level, ,t & Water Level Standard Deviation, ft \\
\hline$\underline{2}$ & 1.7 & 0.7 \\
\hline 5 & 4.8 & 0.5 \\
\hline 10 & 6.9 & 1.0 \\
\hline 25 & 10.3 & 1.9 \\
\hline 50 & 13.2 & 2.6 \\
\hline 75 & 14.8 & 2.2 \\
\hline 100 & 15.6 & 2.3 \\
\hline
\end{tabular}

\begin{tabular}{|c|c|c|}
\hline \multicolumn{3}{|c|}{$\begin{array}{l}\text { Table B358 } \\
\text { Return Period, Maximum Water Level, and Water Level Standard } \\
\text { Deviation for Profile Manua } 017\end{array}$} \\
\hline Return Period, yr & Maximum Water Level, ft & Water Level Standard Deviation, $\mathrm{ft}$ \\
\hline 2 & 1.8 & 0.7 \\
\hline 5 & 4.8 & 0.5 \\
\hline 10 & 7.0 & 1.0 \\
\hline 25 & 10.5 & 1.9 \\
\hline 50 & 13.4 & 2.6 \\
\hline 75 & 15.0 & 2.3 \\
\hline 100 & 15.7 & 2.4 \\
\hline
\end{tabular}

\begin{tabular}{|c|c|c|}
\hline \multicolumn{3}{|c|}{$\begin{array}{l}\text { Table B359 } \\
\text { Return Period, Maximum Water Level, and Water Level Standard } \\
\text { Deviation for Profile Manua } 018\end{array}$} \\
\hline Return Period, yr & Maximum Water Level, it & Water Level Standard Deviation, $\mathrm{ft}$ \\
\hline 2 & 1.5 & 0.7 \\
\hline 5 & 4.9 & 0.5 \\
\hline 10 & 6.6 & 0.7 \\
\hline 25 & 9.9 & 2.3 \\
\hline 50 & 13.8 & 3.2 \\
\hline 75 & 15.8 & 2.6 \\
\hline 100 & 16.8 & 2.5 \\
\hline
\end{tabular}

\section{Table B360}

Return Period, Maximum Water Level, and Water Level Standard Deviation for Profile Manua 019

\begin{tabular}{|l|l|l|}
\hline Return Period, $\mathbf{y r}$ & Maximum Water Level, $\mathrm{ft}$ & Water Level Standard Deviation, $\mathrm{ft}$ \\
\hline 2 & 1.5 & 0.7 \\
\hline 5 & 4.7 & 0.5 \\
\hline 10 & 6.1 & 0.6 \\
\hline 25 & 9.3 & 2.3 \\
\hline 50 & 13.1 & 3.1 \\
\hline 75 & 15.0 & 2.5 \\
\hline 100 & 16.0 & 2.5 \\
\hline
\end{tabular}




\begin{tabular}{|c|c|c|}
\hline \multicolumn{3}{|c|}{$\begin{array}{l}\text { Table B361 } \\
\text { Return Period, Maximum Water Level, and Water Level Standard } \\
\text { Deviation for Profile Manua } 020\end{array}$} \\
\hline Return Period, yr & Maximum Water Level, ft & Water Level Standard Deviation, $t$ \\
\hline 2 & 1.6 & 0.7 \\
\hline 5 & 4.9 & 0.6 \\
\hline 10 & 6.9 & 1.0 \\
\hline 25 & 10.2 & 1.6 \\
\hline 50 & 13.0 & 2.4 \\
\hline 75 & 14.6 & 2.1 \\
\hline 100 & 15.4 & 2.2 \\
\hline
\end{tabular}

\begin{tabular}{|c|c|c|}
\hline \multicolumn{3}{|c|}{$\begin{array}{l}\text { Table B362 } \\
\text { Return Period, Maximum Water Level, and Water Level Standard } \\
\text { Deviation for Profile Manua } 021\end{array}$} \\
\hline Return Period, yr & Maximum Water Level, ft & Water Level Standard Deviation, ft \\
\hline 2 & 1.7 & 0.8 \\
\hline 5 & 5.2 & 0.6 \\
\hline 10 & 7.4 & 0.9 \\
\hline 25 & 10.8 & 1.7 \\
\hline 50 & 13.6 & 2.5 \\
\hline 75 & 15.2 & 2.2 \\
\hline 100 & 16.0 & 2.3 \\
\hline
\end{tabular}

\begin{tabular}{|c|c|c|}
\hline \multicolumn{3}{|c|}{$\begin{array}{l}\text { Table B363 } \\
\text { Return Period, Maximum Water Level, and Water Level Standard } \\
\text { Deviation for Profile Manua } 022\end{array}$} \\
\hline Return Period, yr & Maximum Water Level, ft & Water Level Standard Deviation, $\mathrm{ft}$ \\
\hline 2 & 1.6 & 0.7 \\
\hline 5 & 4.9 & 0.6 \\
\hline 10 & 7.0 & 1.0 \\
\hline 25 & 10.3 & 1.7 \\
\hline 50 & 13.1 & 2.4 \\
\hline 75 & 14.7 & 2.1 \\
\hline 100 & 15.5 & 2.2 \\
\hline
\end{tabular}

\begin{tabular}{|c|c|c|}
\hline \multicolumn{3}{|c|}{$\begin{array}{l}\text { Table B364 } \\
\text { Return Period, Maximum Water Level, and Water Level Standard } \\
\text { Deviation for Profile Manua } 023\end{array}$} \\
\hline Return Period, yr & Maximum Water Level, ft & Water Level Standard Deviation, $\mathrm{ft}$ \\
\hline 2 & 1.8 & 0.7 \\
\hline 5 & 4.9 & 0.5 \\
\hline 10 & 6.6 & 0.7 \\
\hline 25 & 9.6 & 1.7 \\
\hline 50 & 12.5 & 2.4 \\
\hline 75 & 14.1 & 2.1 \\
\hline 100 & 14.9 & 2.2 \\
\hline
\end{tabular}




\begin{tabular}{|c|c|c|}
\hline \multicolumn{3}{|c|}{$\begin{array}{l}\text { Table B365 } \\
\text { Return Period, Maximum Water Level, and Water Level Standard } \\
\text { Deviation for Profile Manua } 024\end{array}$} \\
\hline Return Period, $y$ & Maximum Water Level, $\mathrm{ft}$ & Water Level Standard Deviation, $\mathrm{tt}$ \\
\hline 2 & 1.8 & 0.7 \\
\hline 5 & 4.7 & 0.5 \\
\hline 10 & 6.3 & 0.7 \\
\hline 25 & 9.2 & 1.8 \\
\hline 50 & 12.2 & 2.5 \\
\hline 75 & 13.8 & 2.1 \\
\hline 100 & 14.6 & 2.2 \\
\hline
\end{tabular}

\begin{tabular}{|c|c|c|}
\hline \multicolumn{3}{|c|}{$\begin{array}{l}\text { Table B366 } \\
\text { Return Period, Maximum Water Level, and Water Level Standard } \\
\text { Deviation for Profile Manua } 025\end{array}$} \\
\hline Return Period, yr & Maximum Water Level, $\mathrm{ft}$ & Water Level Standard Deviation, ft \\
\hline 2 & 1.7 & 0.7 \\
\hline 5 & 4.6 & 0.5 \\
\hline 10 & 6.1 & 0.6 \\
\hline 25 & 9.0 & 1.8 \\
\hline 50 & 12.0 & 2.6 \\
\hline 75 & 13.6 & 2.2 \\
\hline 100 & 14.4 & 2.2 \\
\hline
\end{tabular}

\begin{tabular}{|c|c|c|}
\hline \multicolumn{3}{|c|}{$\begin{array}{l}\text { Table B367 } \\
\text { Return Period, Maximum Water Level, and Water Level Standard } \\
\text { Deviation for Profile Manua } 026\end{array}$} \\
\hline Return Period, yr & Maximum Water Level, $\mathrm{ft}$ & Water Level Standard Deviation, $\mathrm{ft}$ \\
\hline 2 & 1.9 & 0.8 \\
\hline 5 & 5.6 & 0.7 \\
\hline 10 & 7.8 & 0.8 \\
\hline 25 & 11.3 & 2.3 \\
\hline 50 & 14.9 & 3.3 \\
\hline 75 & 16.9 & 2.7 \\
\hline 100 & 18.0 & 2.7 \\
\hline
\end{tabular}

\begin{tabular}{|c|c|c|}
\hline \multicolumn{3}{|c|}{$\begin{array}{l}\text { Table B368 } \\
\text { Return Period, Maximum Water Level, and Water Level Standard } \\
\text { Deviation for Profile Manua } 027\end{array}$} \\
\hline Return Period, yr & Maximum Water Level, ft & Water Level Standard Deviation, $\mathrm{ft}$ \\
\hline 2 & 1.9 & 0.8 \\
\hline 5 & 5.8 & 0.7 \\
\hline 10 & 8.1 & 1.0 \\
\hline 25 & 11.6 & 2.3 \\
\hline 50 & 15.4 & 3.4 \\
\hline 75 & 17.5 & 2.7 \\
\hline 100 & 18.5 & 2.8 \\
\hline
\end{tabular}




\begin{tabular}{|c|c|c|}
\hline \multicolumn{3}{|c|}{$\begin{array}{l}\text { Table B369 } \\
\text { Return Period, Maximum Water Level, and Water Level Standard } \\
\text { Deviation for Profile Manua } 028\end{array}$} \\
\hline Return Period, yr & Maximum Water Level, $\mathrm{ft}$ & Water Level Standard Deviation, ft \\
\hline 2 & 2.1 & 1.0 \\
\hline 5 & 6.9 & 0.8 \\
\hline 10 & 8.9 & 0.8 \\
\hline 25 & 10.6 & 0.7 \\
\hline 50 & 11.6 & 0.9 \\
\hline 75 & 12.3 & 0.9 \\
\hline 100 & 12.6 & 1.0 \\
\hline
\end{tabular}

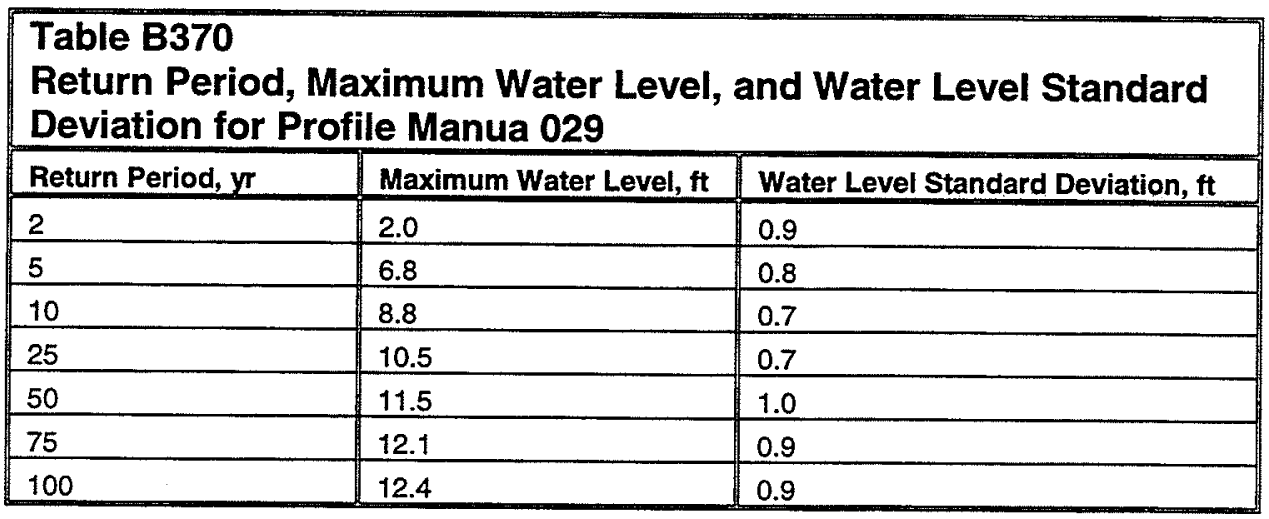

\begin{tabular}{|c|c|c|}
\hline \multicolumn{3}{|c|}{$\begin{array}{l}\text { Table B371 } \\
\text { Return Period, Maximum Water Level, and Water Level Standard } \\
\text { Deviation for Profile Manua } 030\end{array}$} \\
\hline Return Period, yr & Maximum Water Level, ft & Water Level Standard Deviation, $\mathrm{ft}$ \\
\hline 2 & 1.9 & 0.8 \\
\hline 5 & 5.3 & 0.7 \\
\hline 10 & 7.6 & 1.0 \\
\hline 25 & 10.3 & 1.4 \\
\hline 50 & 12.6 & 2.1 \\
\hline 75 & 13.9 & 1.9 \\
\hline 100 & 14.6 & 2.1 \\
\hline
\end{tabular}

\begin{tabular}{|c|c|c|}
\hline \multicolumn{3}{|c|}{$\begin{array}{l}\text { Table B372 } \\
\text { Return Period, Maximum Water Level, and Water Level Standard } \\
\text { Deviation for Profile Manua } 031\end{array}$} \\
\hline Return Period, yr & Maximum Water Level, ft & Water Level Standard Deviation, ft \\
\hline 2 & 1.9 & 0.8 \\
\hline 5 & 5.3 & 0.7 \\
\hline 10 & 7.6 & 1.0 \\
\hline 25 & 10.3 & 1.4 \\
\hline 50 & 12.6 & 2.1 \\
\hline 75 & 13.9 & 1.9 \\
\hline 100 & 14.6 & 2.1 \\
\hline
\end{tabular}




\begin{tabular}{|c|c|c|}
\hline \multicolumn{3}{|c|}{$\begin{array}{l}\text { Table B373 } \\
\text { Return Period, Maximum Water Level, and Water Level Standard } \\
\text { Deviation for Profile Manua } 032\end{array}$} \\
\hline Return Period, yr & Maximum Water Level, ft & Water Level Standard Deviation, $\mathrm{ft}$ \\
\hline 2 & 1.9 & 0.8 \\
\hline 5 & 5.3 & 0.7 \\
\hline 10 & 7.6 & 1.0 \\
\hline 25 & 10.2 & 1.4 \\
\hline 50 & 12.5 & 2.1 \\
\hline 75 & 13.8 & 1.9 \\
\hline 100 & 14.5 & 2.1 \\
\hline
\end{tabular}

\section{Table B374}

Return Period, Maximum Water Level, and Water Level Standard Deviation for Profile Manua 033

\begin{tabular}{|l|l|l|}
\hline Return Period, $\mathbf{y r}$ & Maximum Water Level, $\mathbf{f t}$ & Water Level Standard Deviation, $\mathrm{ft}$ \\
\hline 2 & 1.9 & 0.8 \\
\hline 5 & 5.4 & 0.7 \\
\hline 10 & 7.7 & 1.0 \\
\hline 25 & 10.4 & 1.4 \\
\hline 50 & 12.8 & 2.2 \\
\hline 75 & 14.1 & 2.0 \\
\hline 100 & 14.8 & 2.1 \\
\hline
\end{tabular}





\section{Appendix C Stage-Frequency Relationship Figures}

Plots of stage-frequency relationships are given for profiles representative of numerical gauge locations. Profile numbers are specified and these correspond to one of three groups of profile numbers used in the stage-frequency analysis. Each group corresponds to specific islands: Tutuila, Aunuu, and the Manua' Islands. The Manua' Islands group include Ofu, Olosega, and Tau Islands. Thus, the profile number shown will correspond to the numbering specific to the particular island group to which it belongs.

Stage-frequency relationships were developed by applying the EST to maximum water elevation values at each profile for each storm. Time-series of water elevations were computed by linear supposition of the surge, ponding level, wave setup, and wave runup. Water elevations applied within the EST were the maximum water elevations that were calculated to occur at each profile during each storm.

Each plot contains three curves. The solid line is the calculated stagefrequency curve. The upper and lower dashed lines show the calculated stagefrequency curves plus and minus one standard deviation, respectively. 


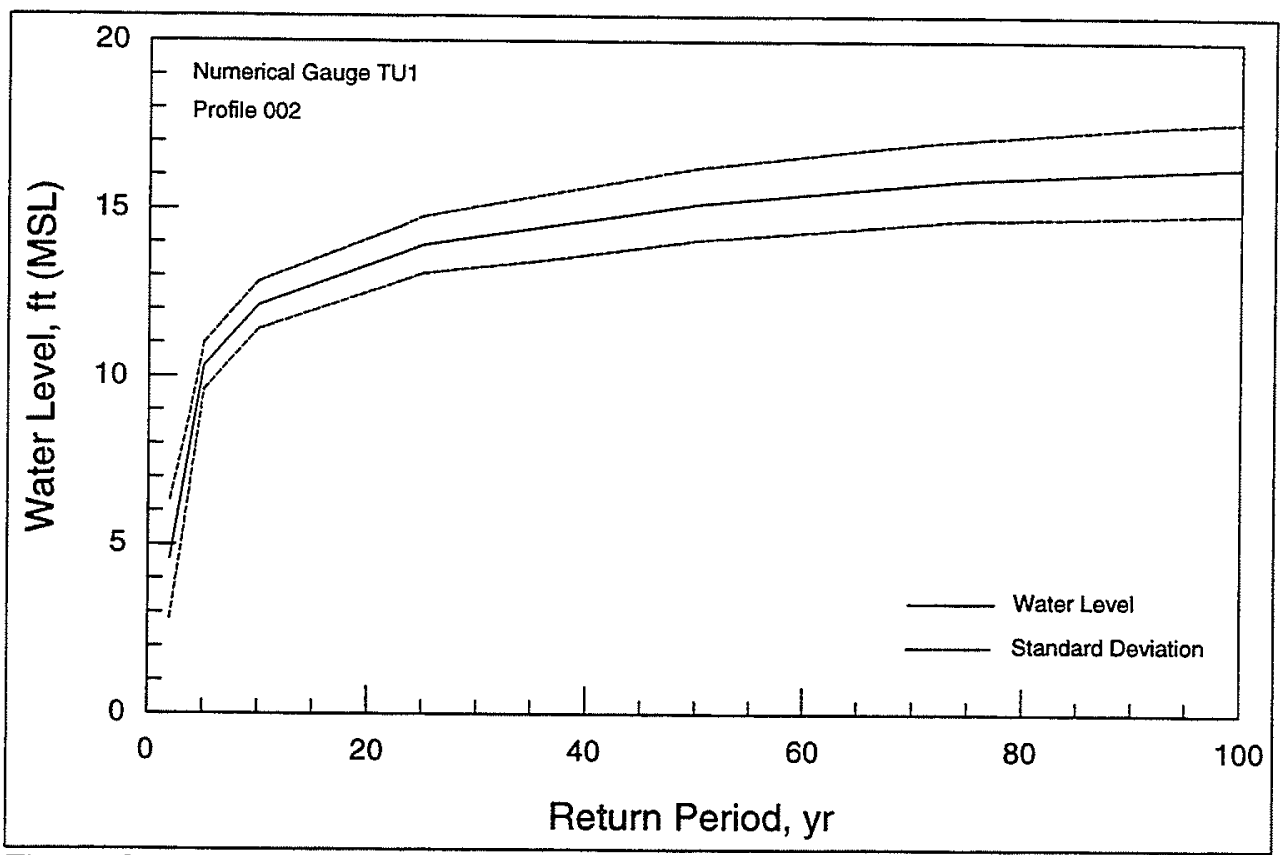

Figure C.1. Stage-frequency plot representing numerical gauge TU1 (Profile 002), Tutuila

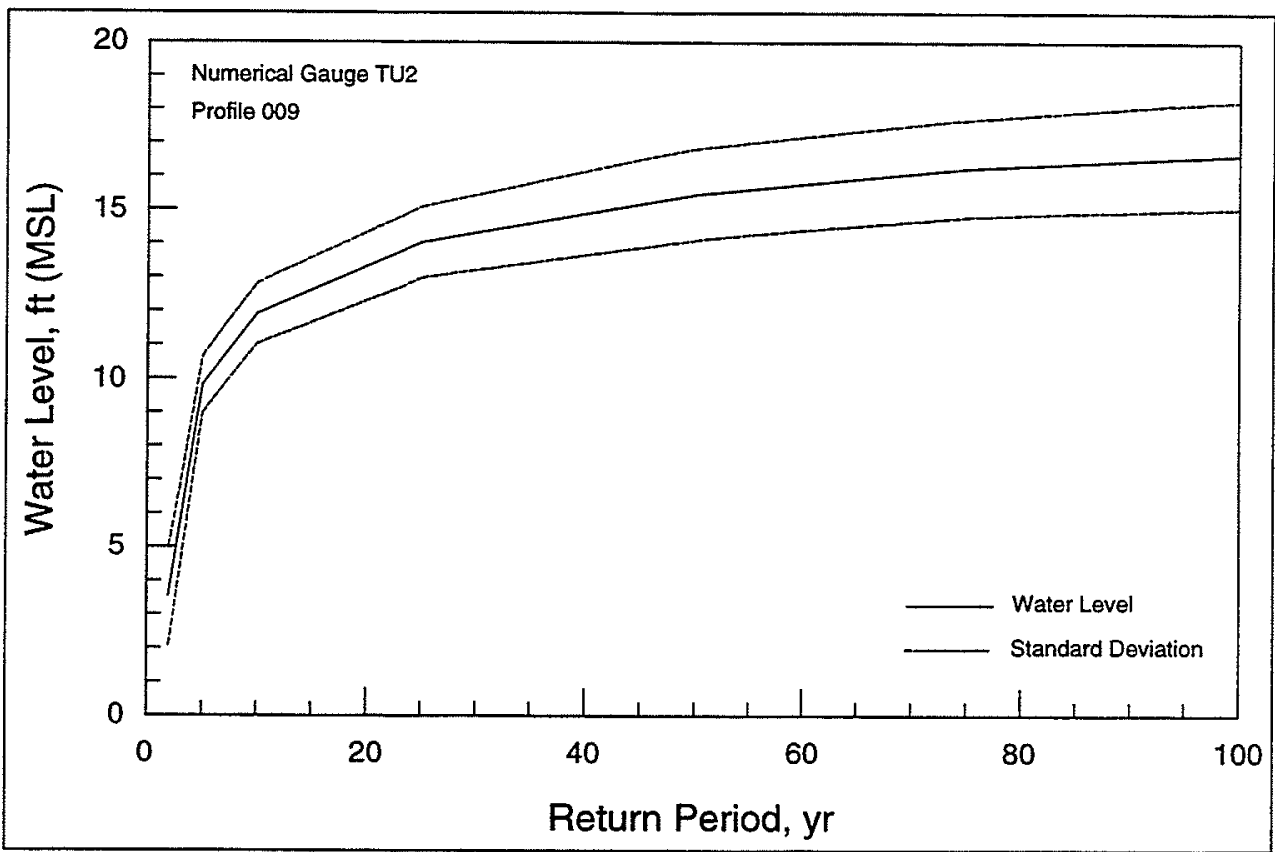

Figure C.2. Stage-frequency plot representing numerical gauge TU2 (Profile 009), Tutuila 


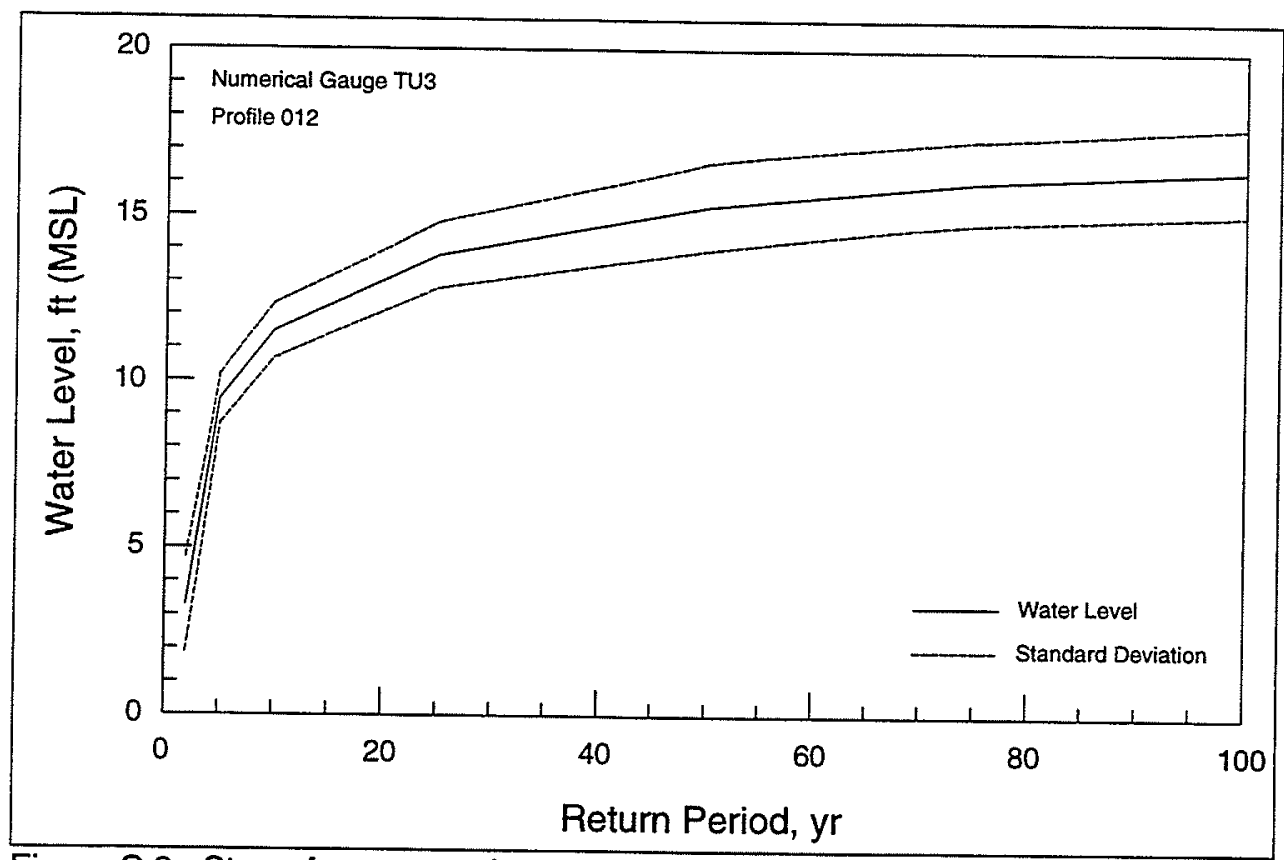

Figure C.3. Stage-frequency plot representing numerical gauge TU3 (Profile 012), Tutuila

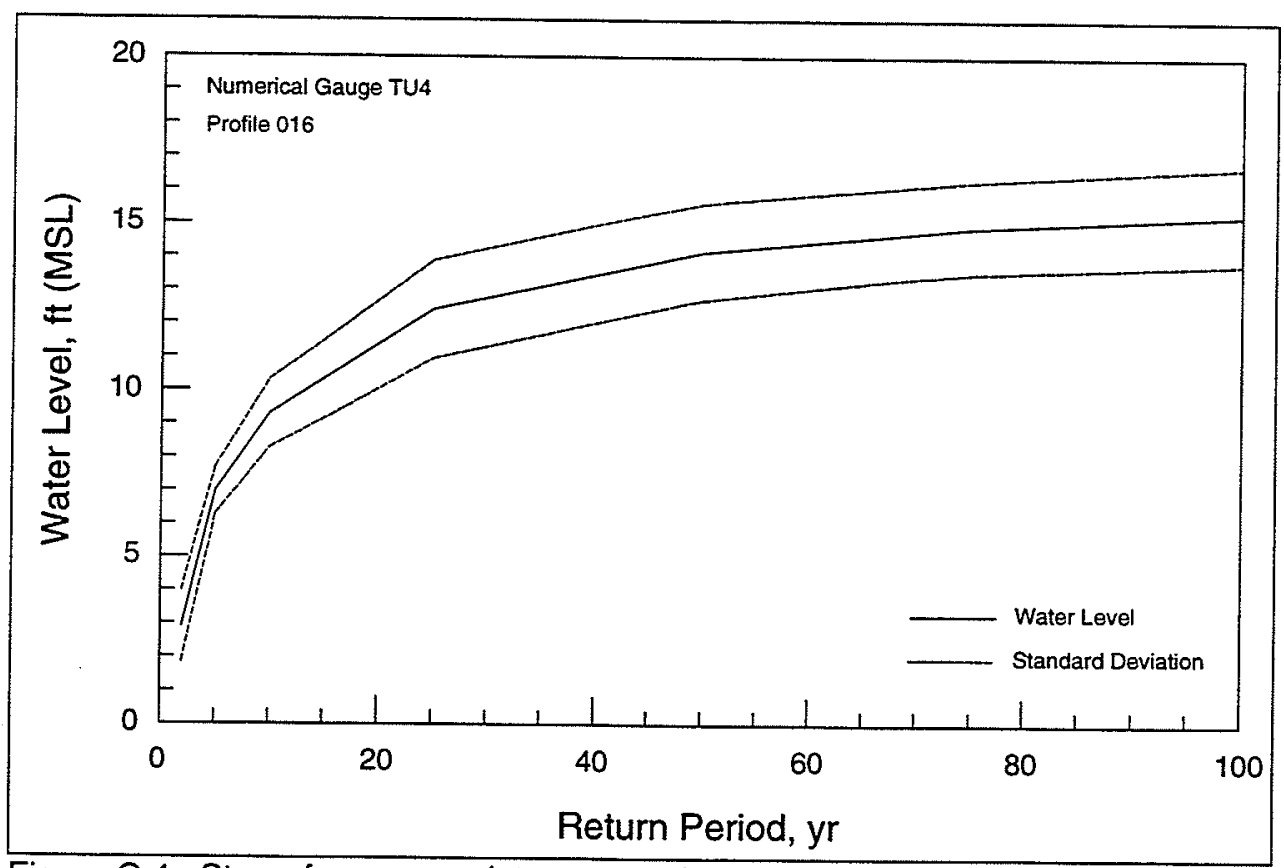

Figure C.4. Stage-frequency plot representing numerical gauge TU4 (Profile 016), Tutuila 


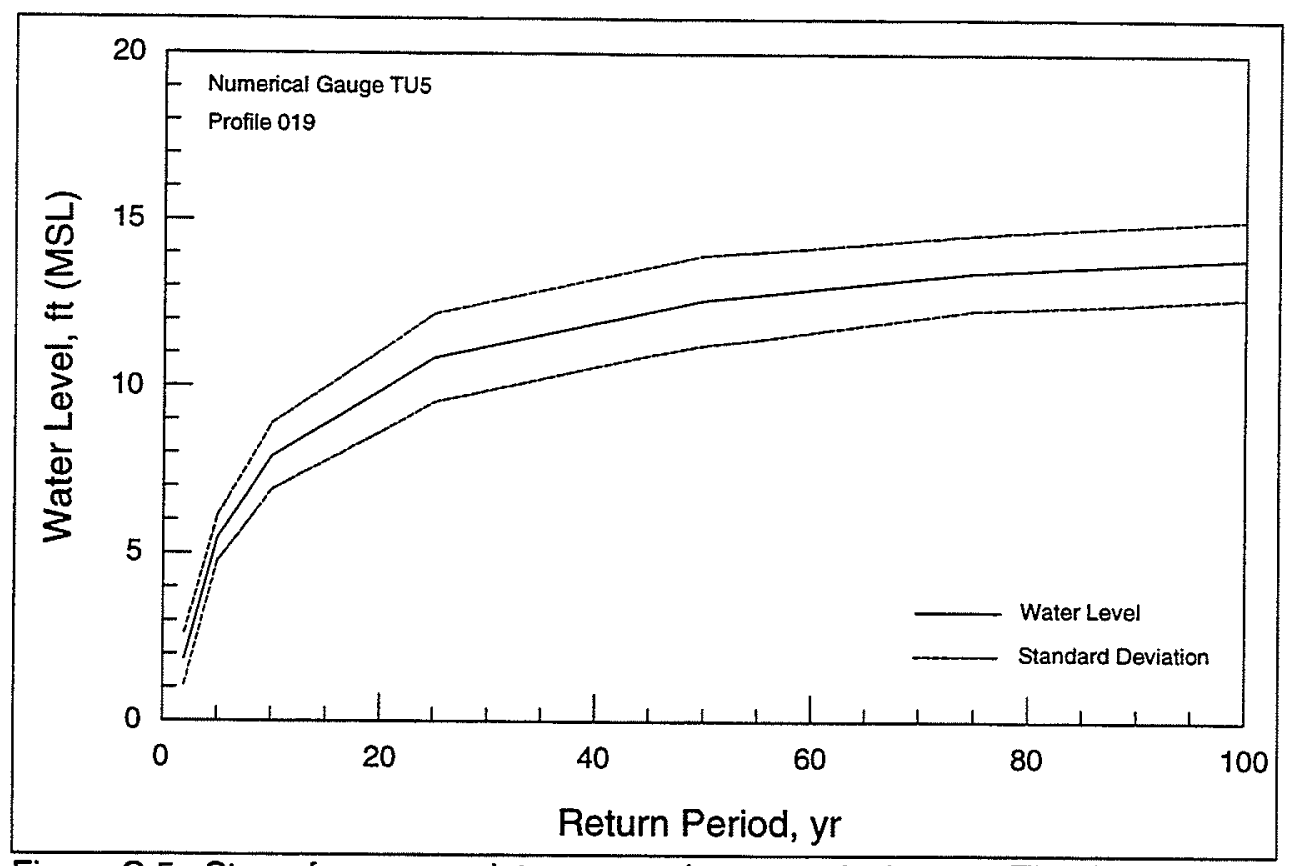

Figure C.5. Stage-frequency plot representing numerical gauge TU5 (Profile 019), Tutuila

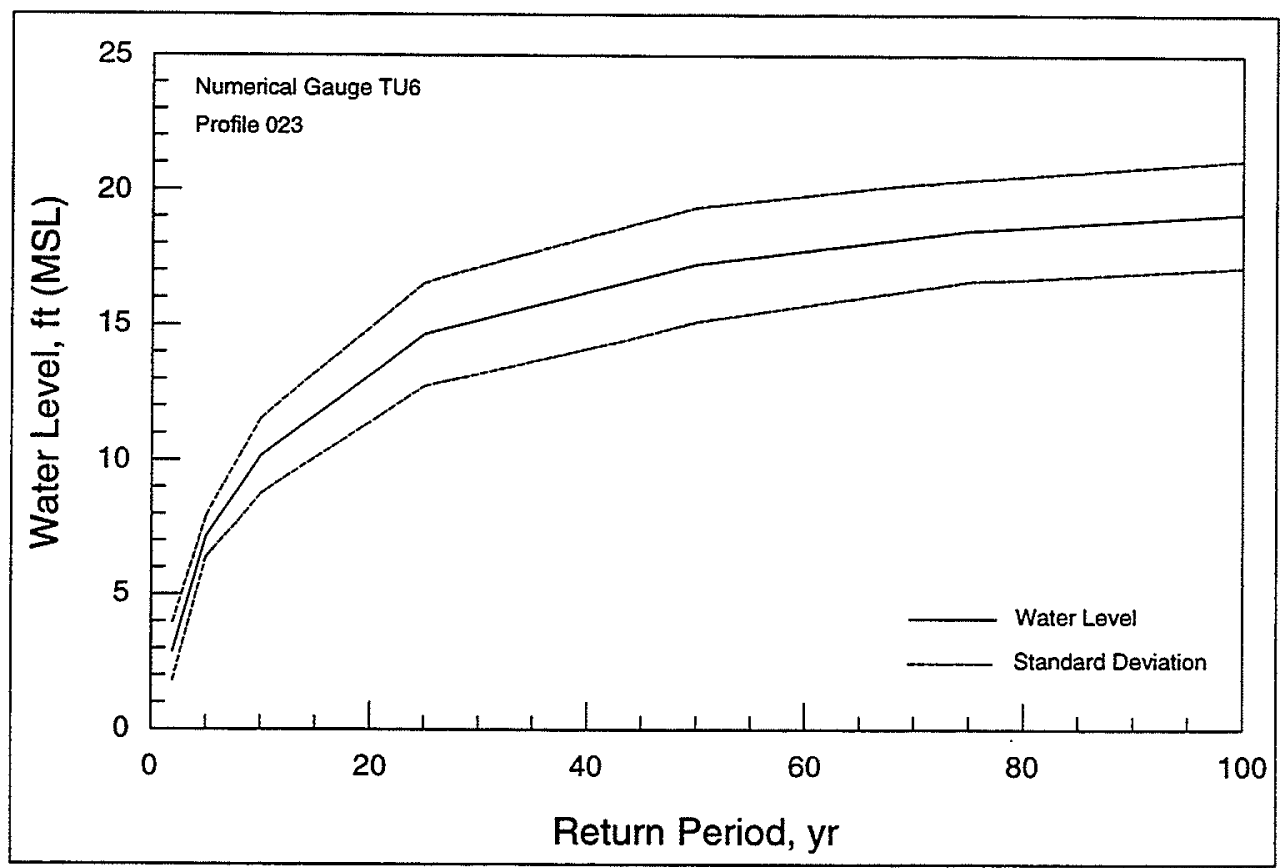

Figure C.6. Stage-frequency plot representing numerical gauge TU6 (Profile 023), Tutuila 


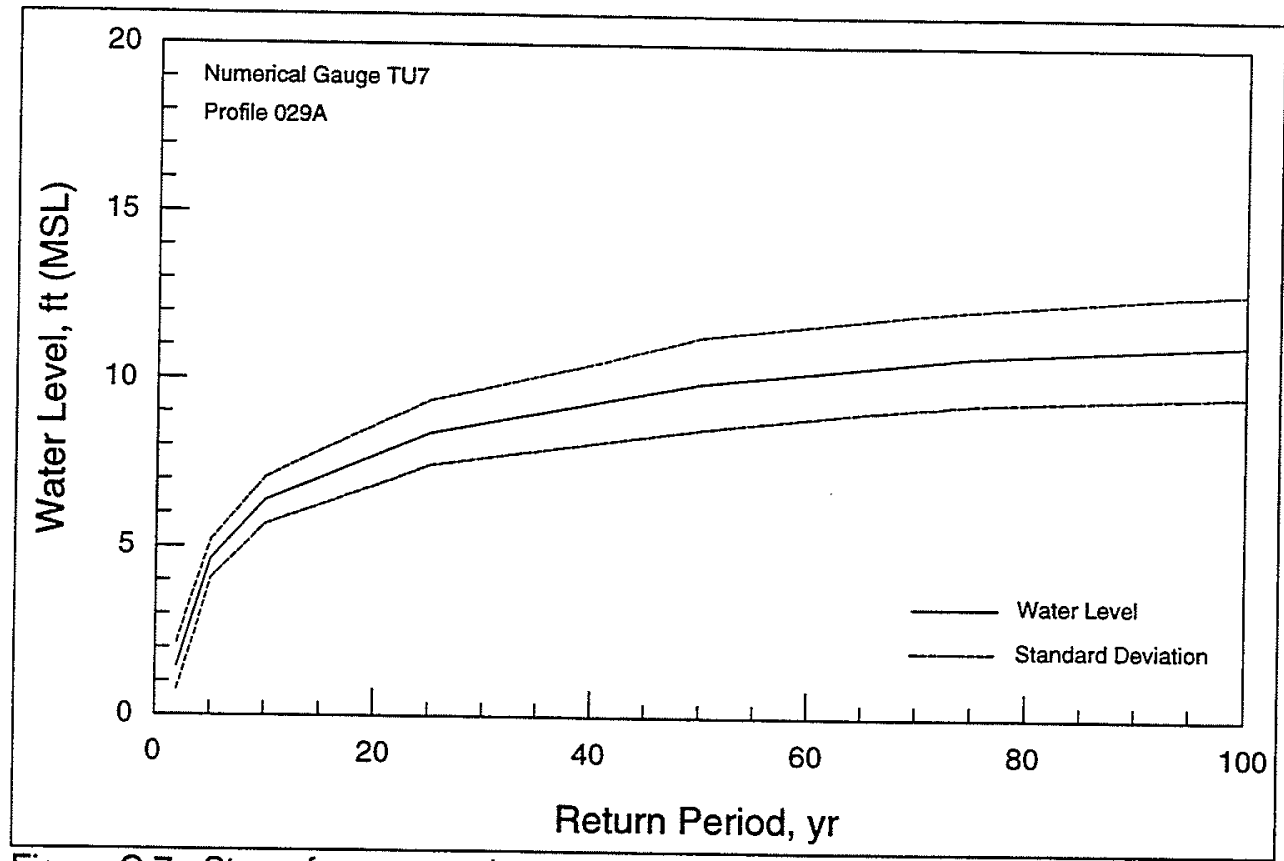

Figure C.7. Stage-frequency plot representing numerical gauge TU7 (Profile 029A), Tutuila

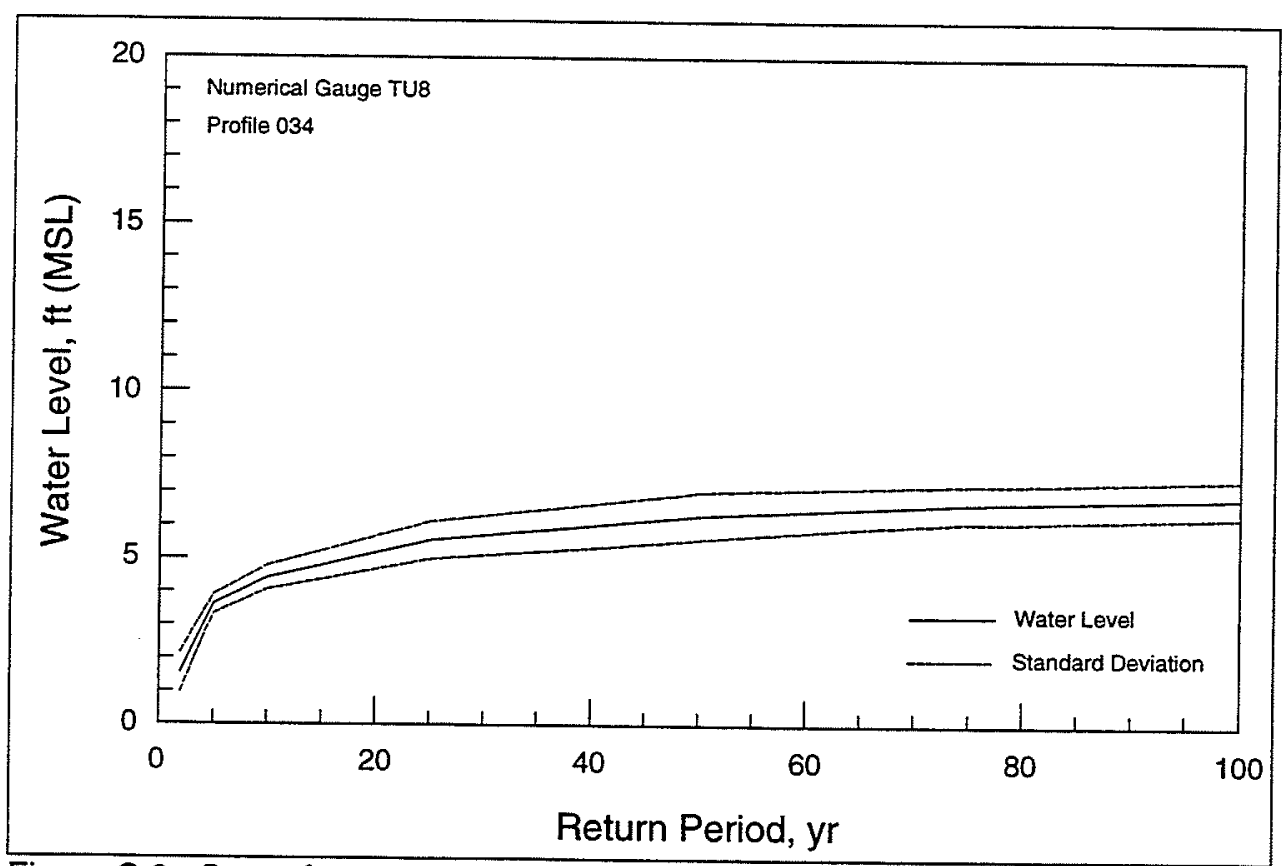

Figure C.8. Stage-frequency plot representing numerical gauge TU8 (Profile 034), Tutuila 


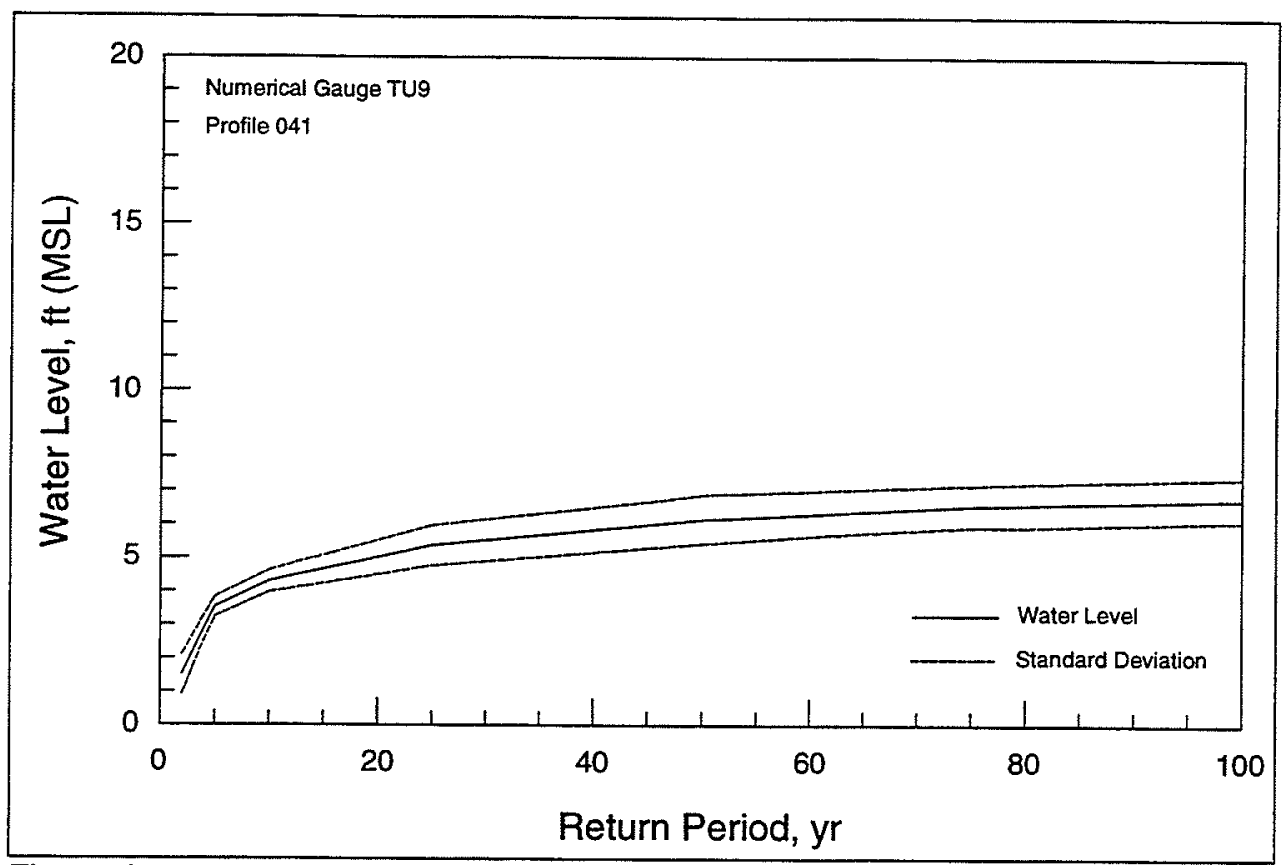

Figure C.9. Stage-frequency plot representing numerical gauge TU9 (Profile 041), Tutuila

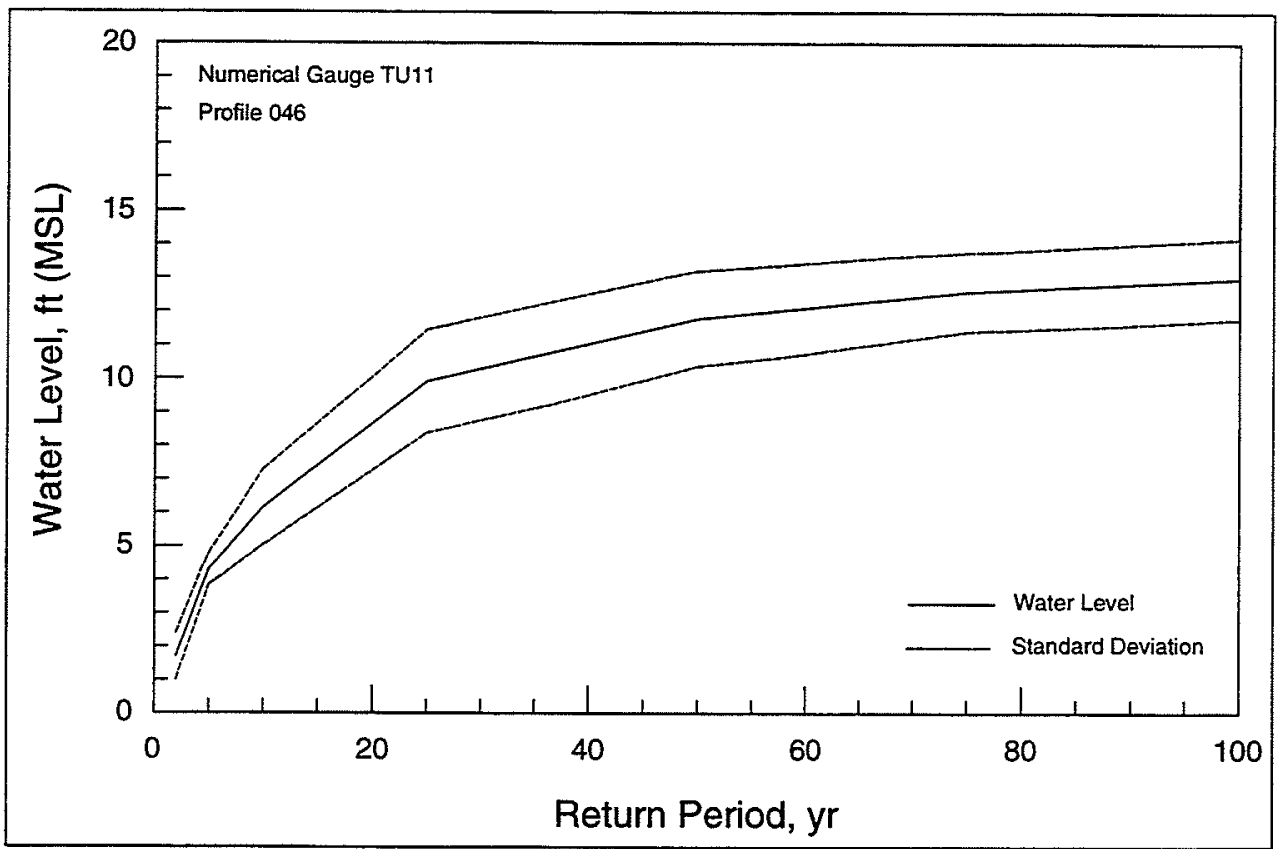

Figure C.10. Stage-frequency plot representing numerical gauge TU11 (Profile 046), Tutuila 


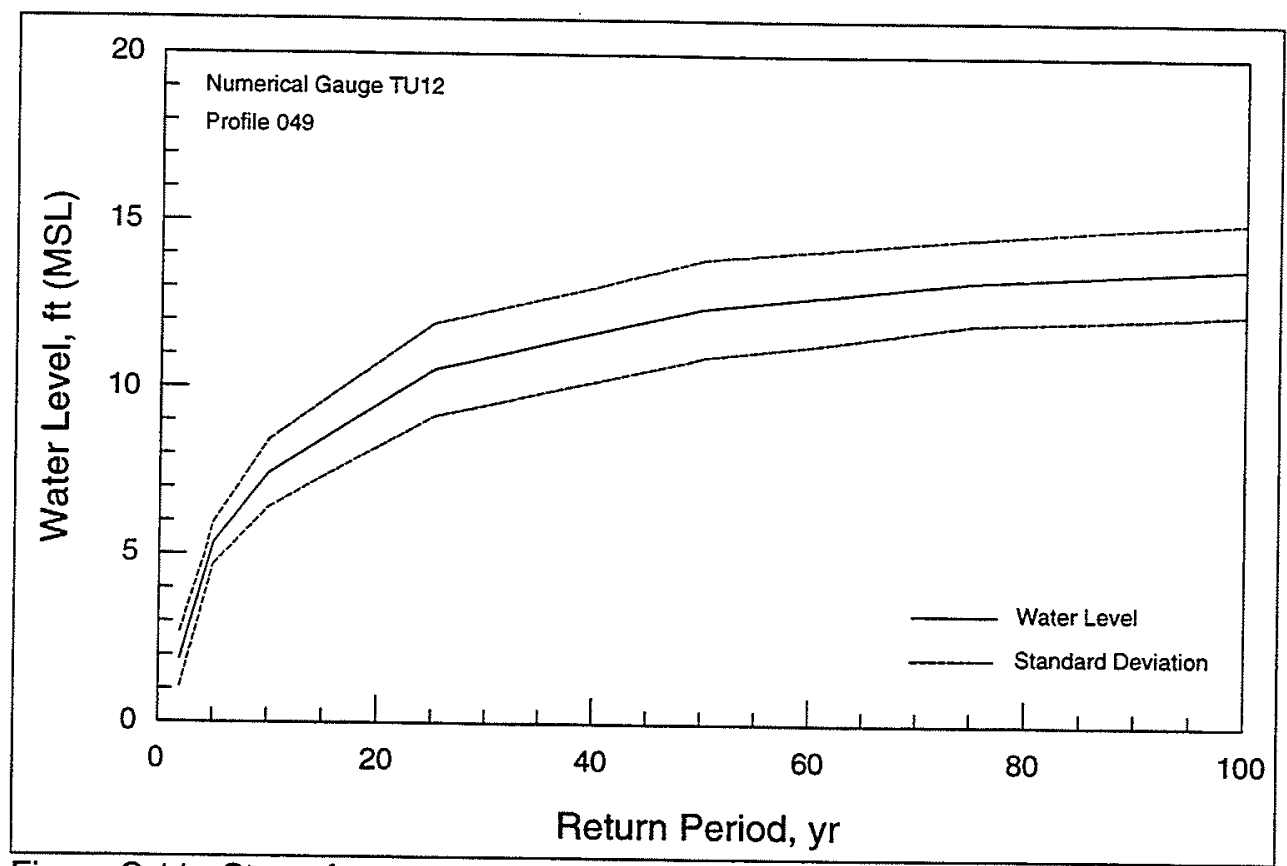

Figure C.11. Stage-frequency plot representing numerical gauge TU12 (Profile 049), Tutuila

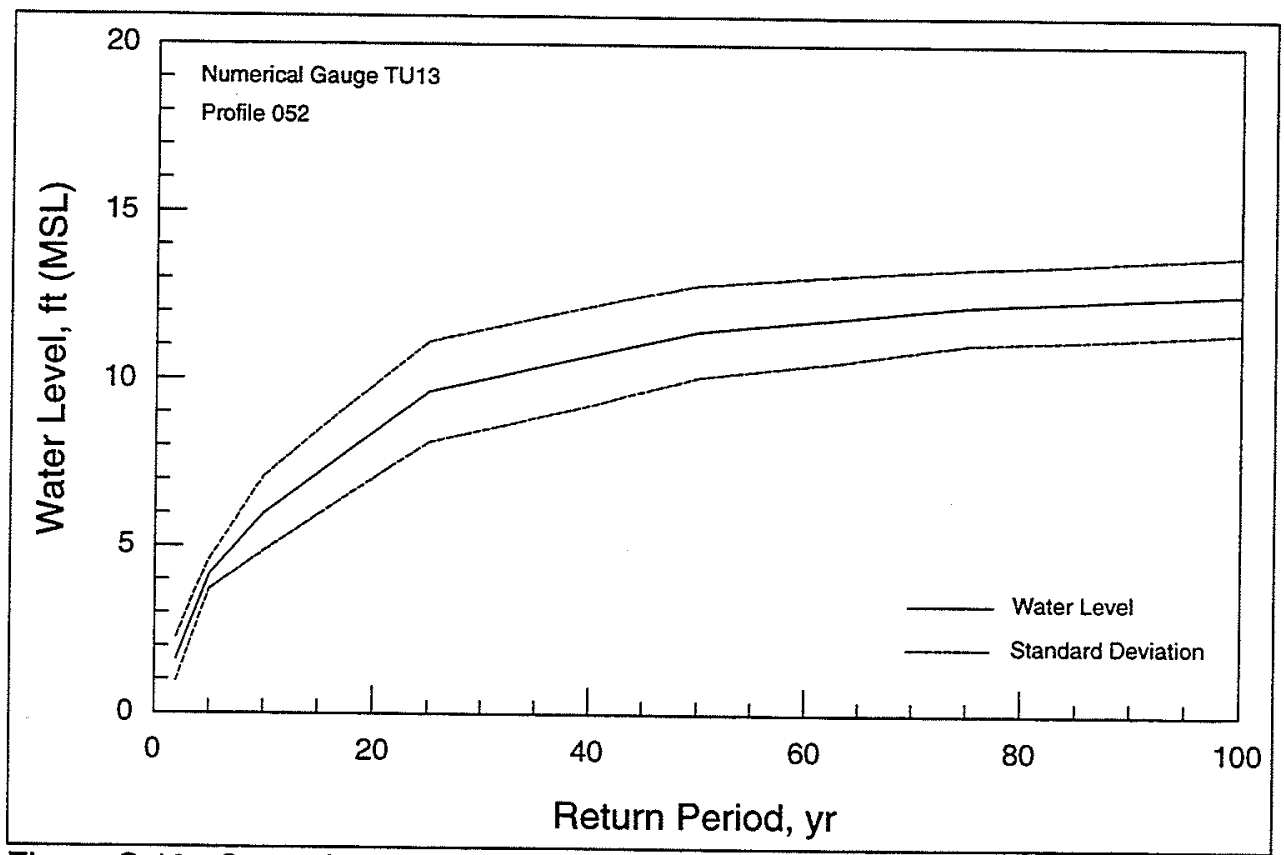

Figure C.12. Stage-frequency plot representing numerical gauge TU13 (Profile 052), Tutuila 


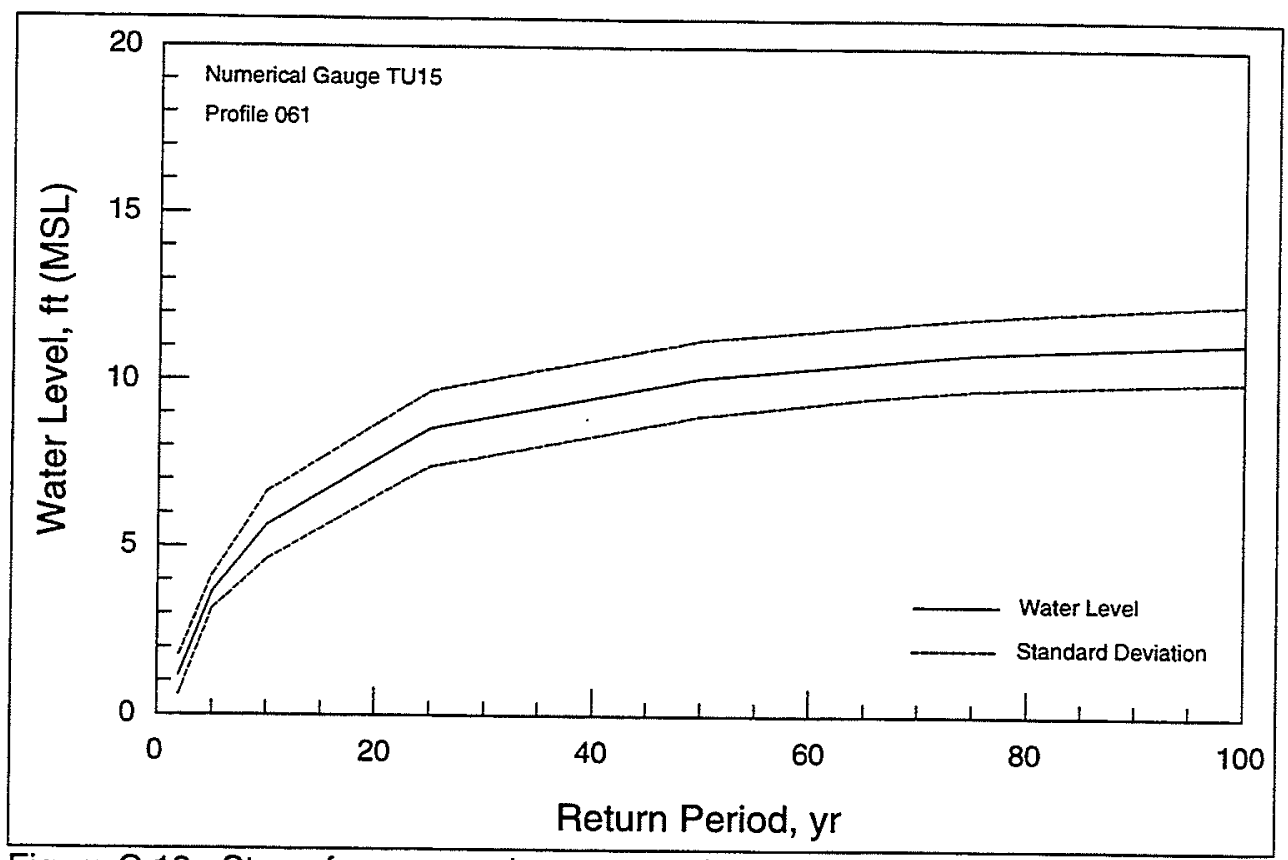

Figure C.13. Stage-frequency plot representing numerical gauge TU15 (Profile 061), Tutuila

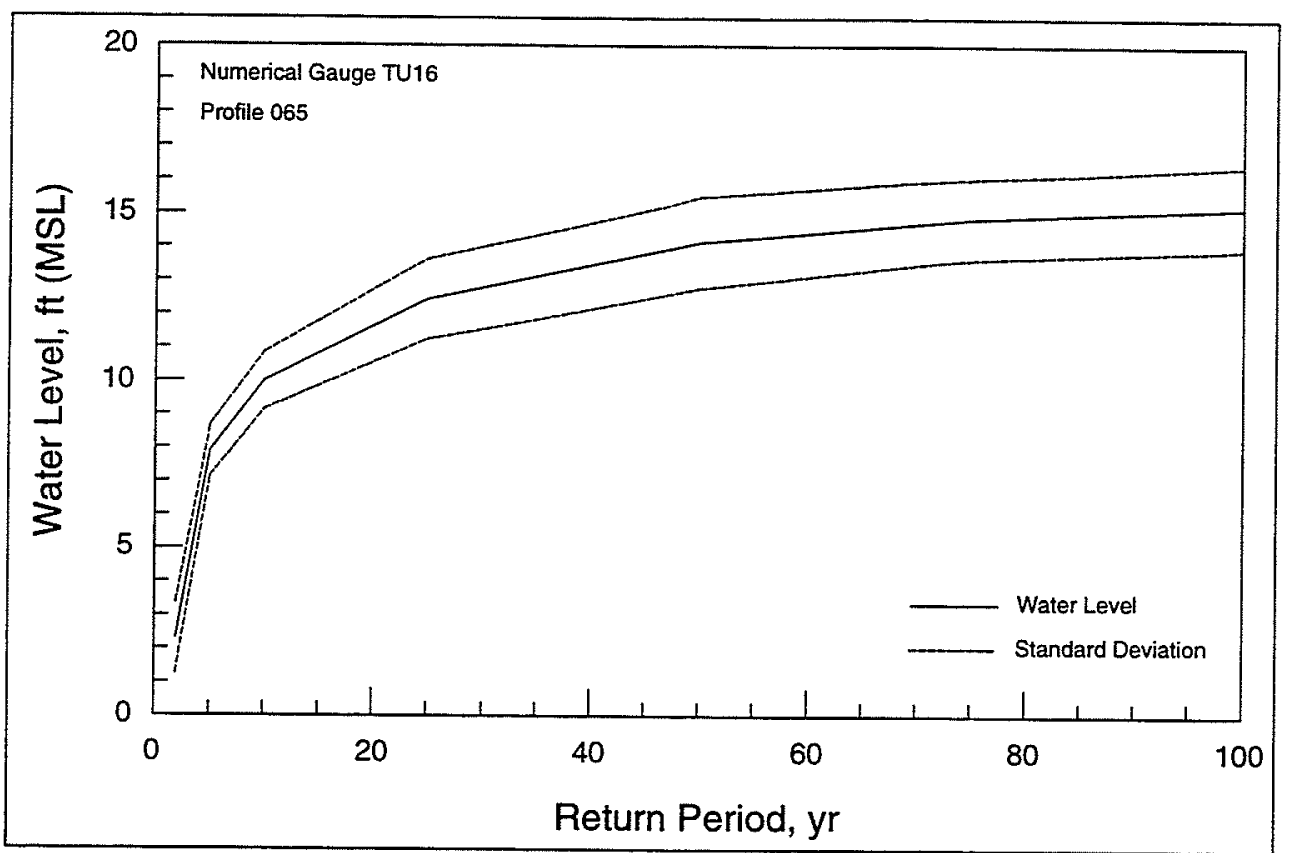

Figure C.14. Stage-frequency plot representing numerical gauge TU16 (Profile 065), Tutuila 


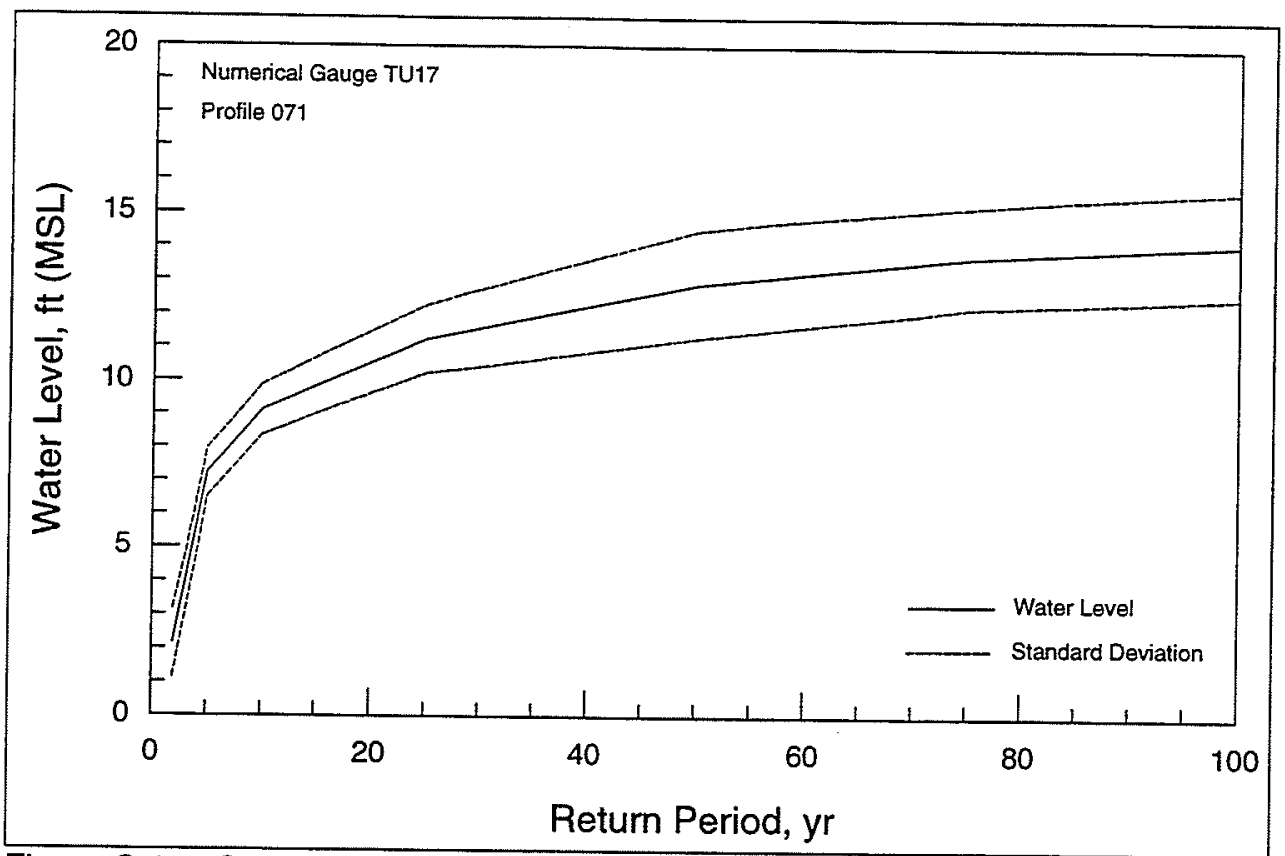

Figure C.15. Stage-frequency plot representing numerical gauge TU17 (Profile 071), Tutuila

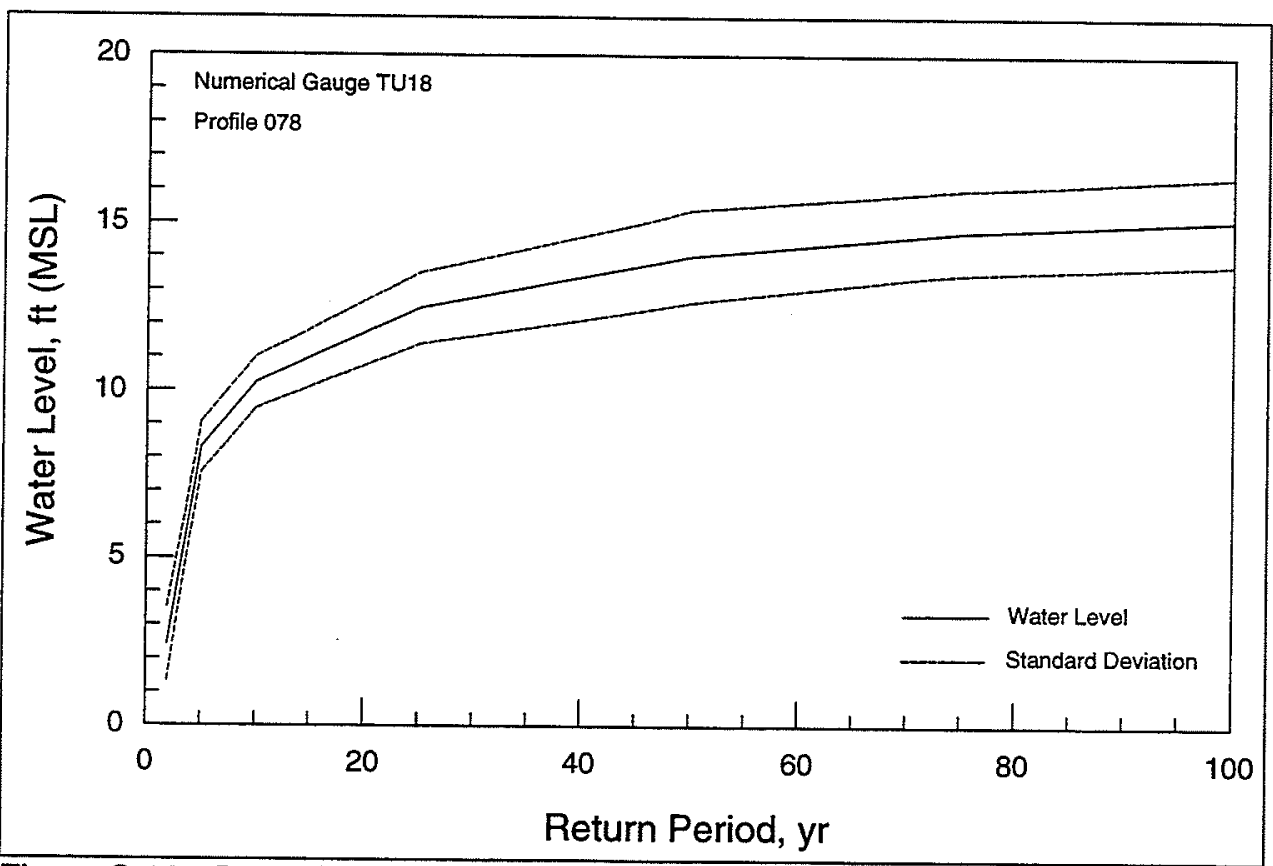

Figure C.16. Stage-frequency plot representing numerical gauge TU18 (Profile 078), Tutuila 


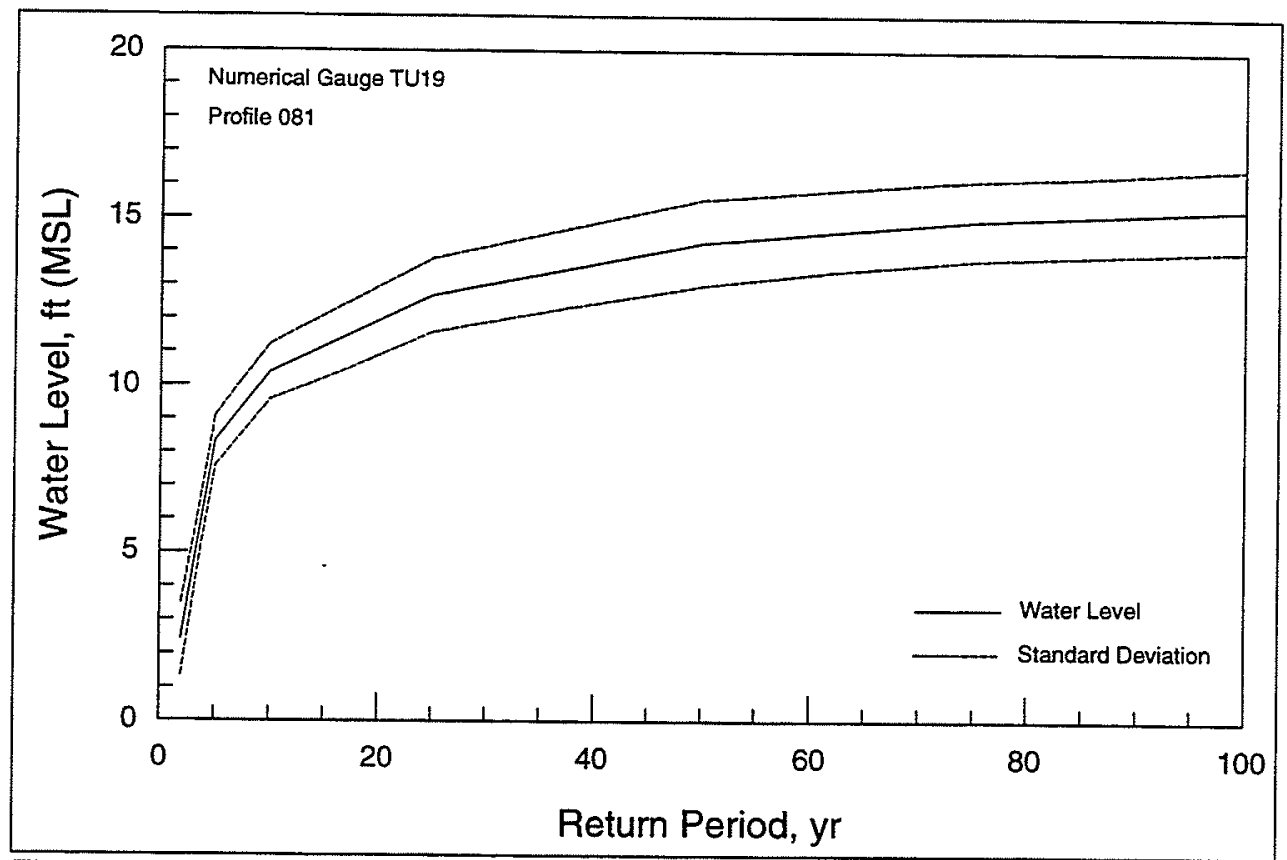

Figure C.17. Stage-frequency plot representing numerical gauge TU19 (Profile 081), Tutuila

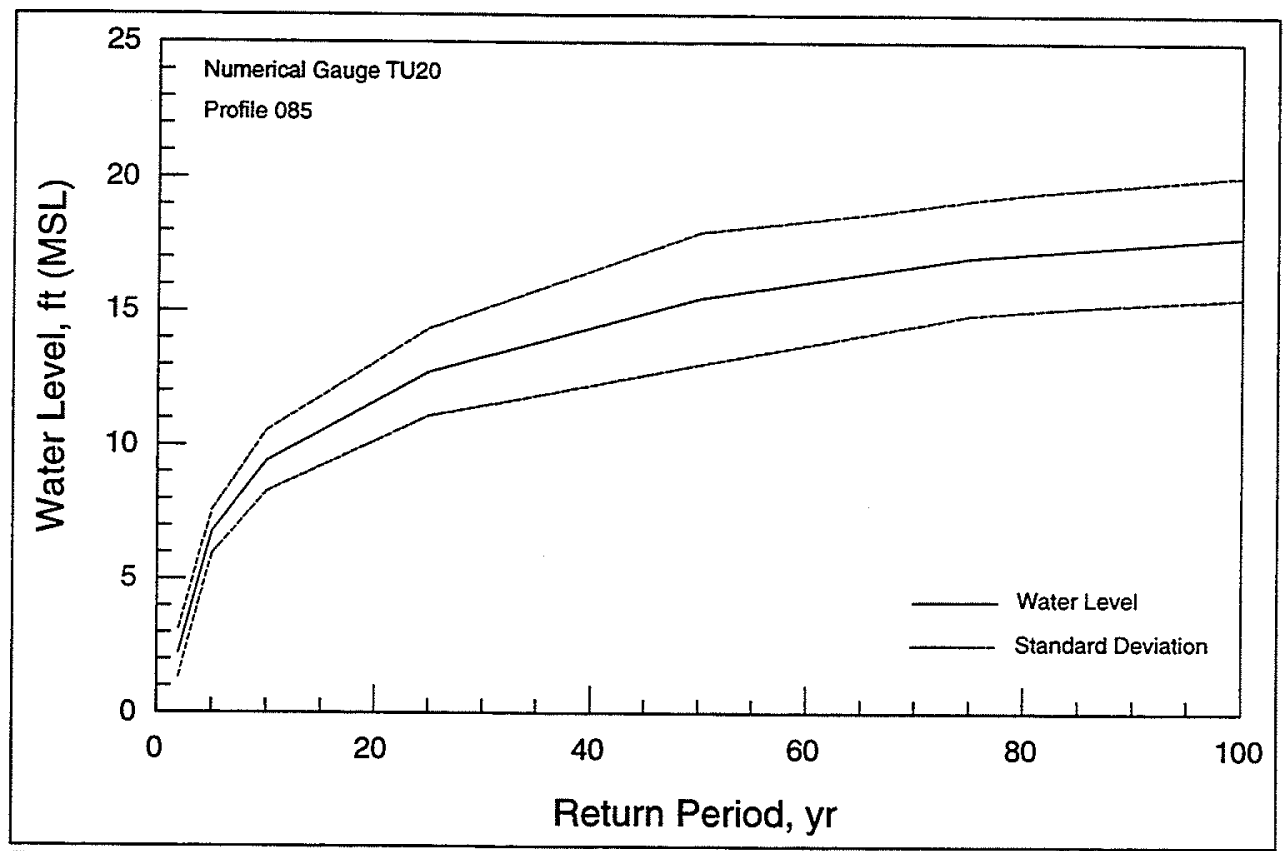

Figure C.18. Stage-frequency plot representing numerical gauge TU20 (Profile 085), Tutuila 


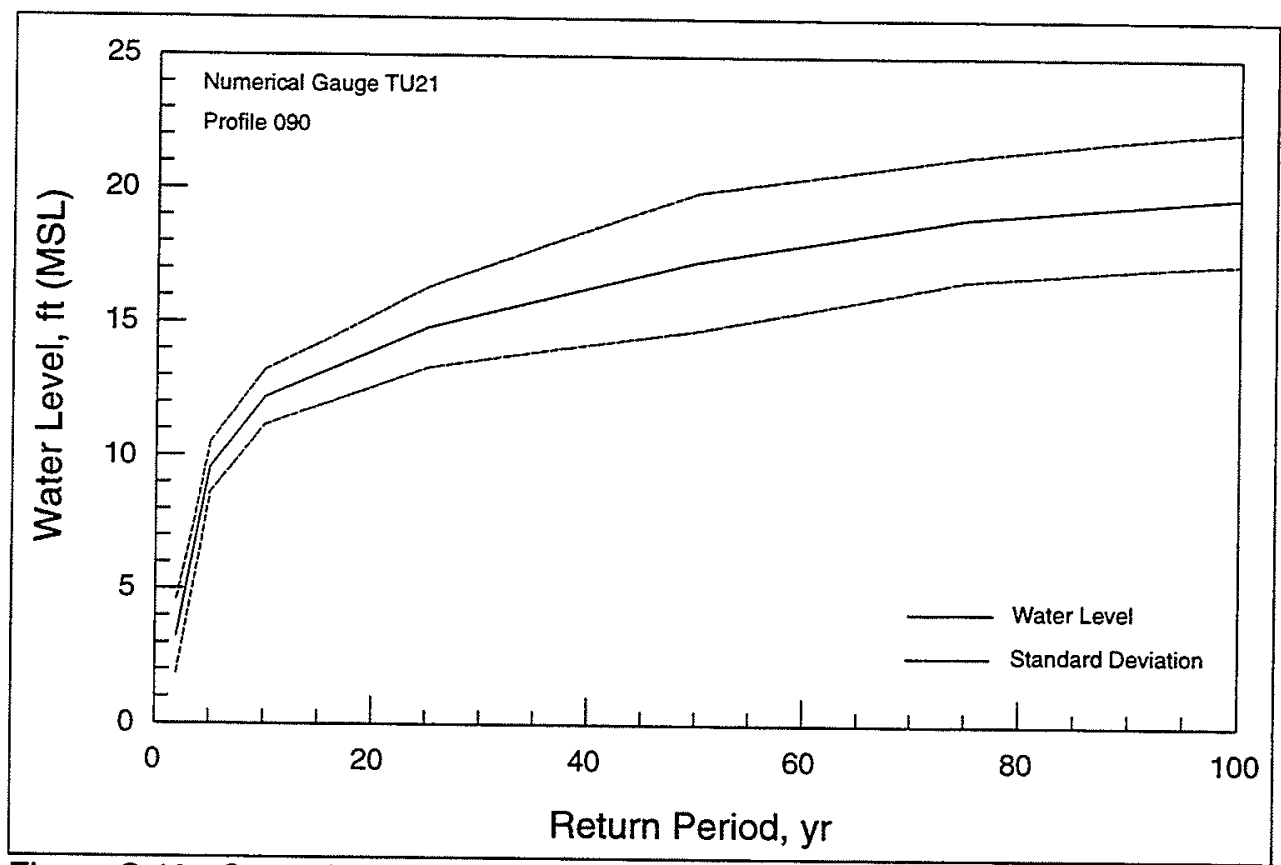

Figure C.19. Stage-frequency plot representing numerical gauge TU21 (Profile 090), Tutuila

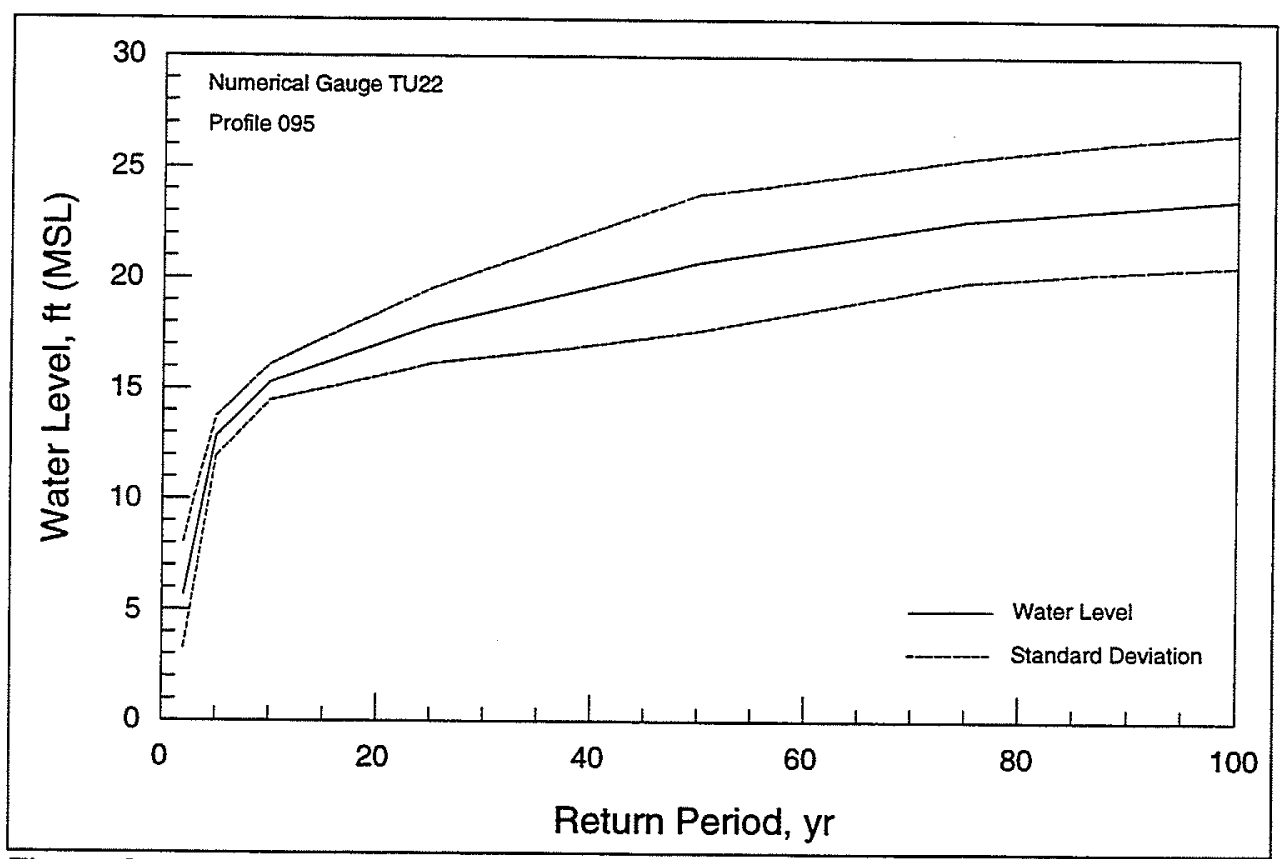

Figure C.20. Stage-frequency plot representing numerical gauge TU22 (Profile 095), Tutuila 


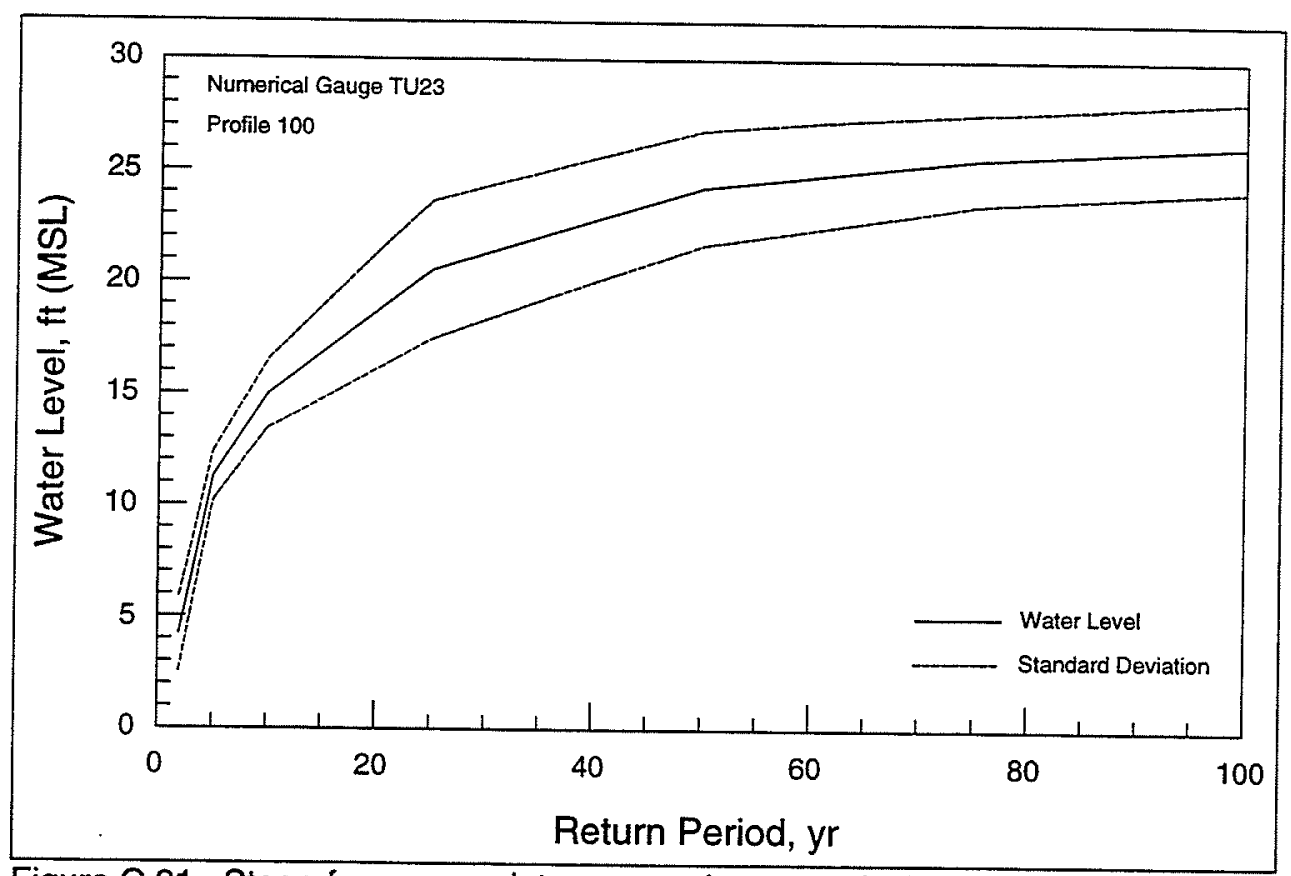

Figure C.21. Stage-frequency plot representing numerical gauge TU23 (Profile 100), Tutuila

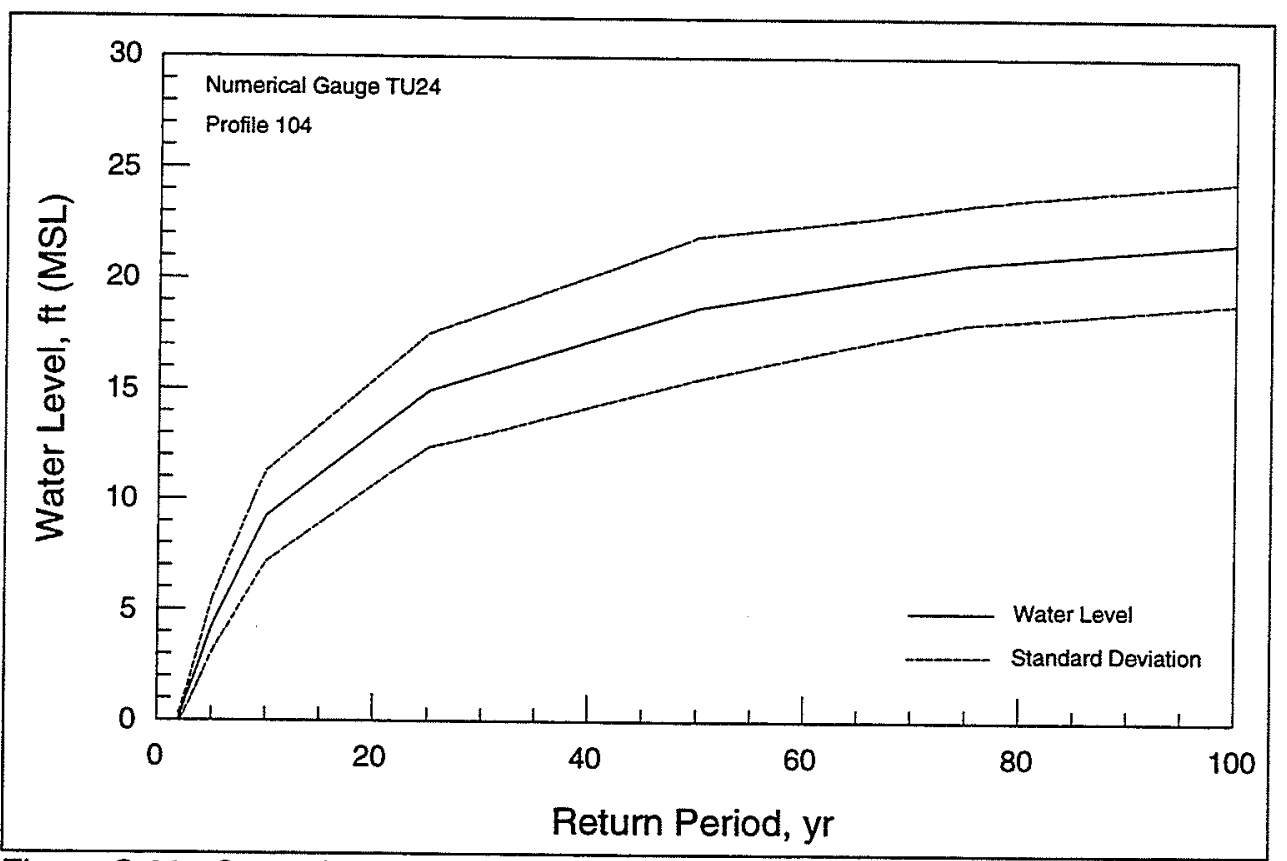

Figure C.22. Stage-frequency plot representing numerical gauge TU24 (Profile 104), Tutuila 


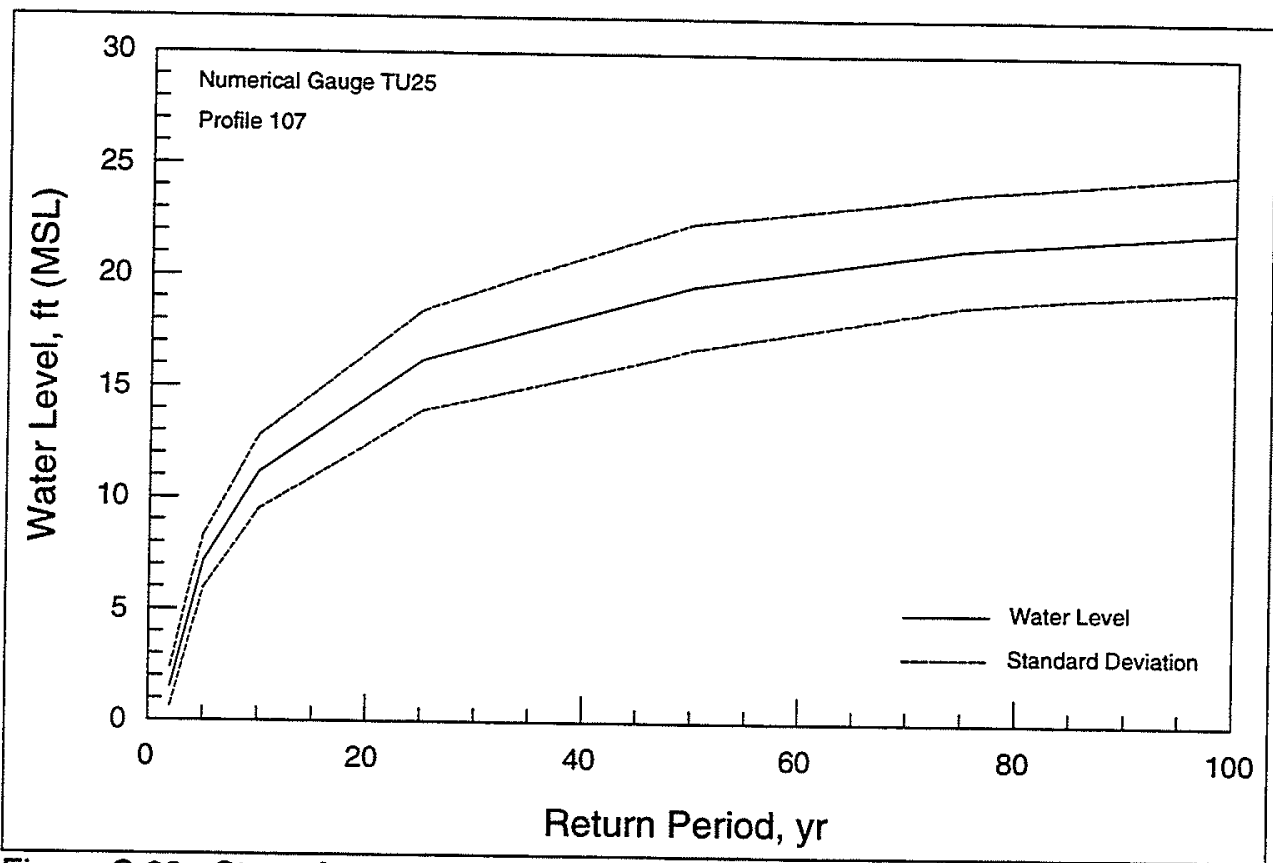

Figure C.23. Stage-frequency plot representing numerical gauge TU25 (Profile 107), Tutuila

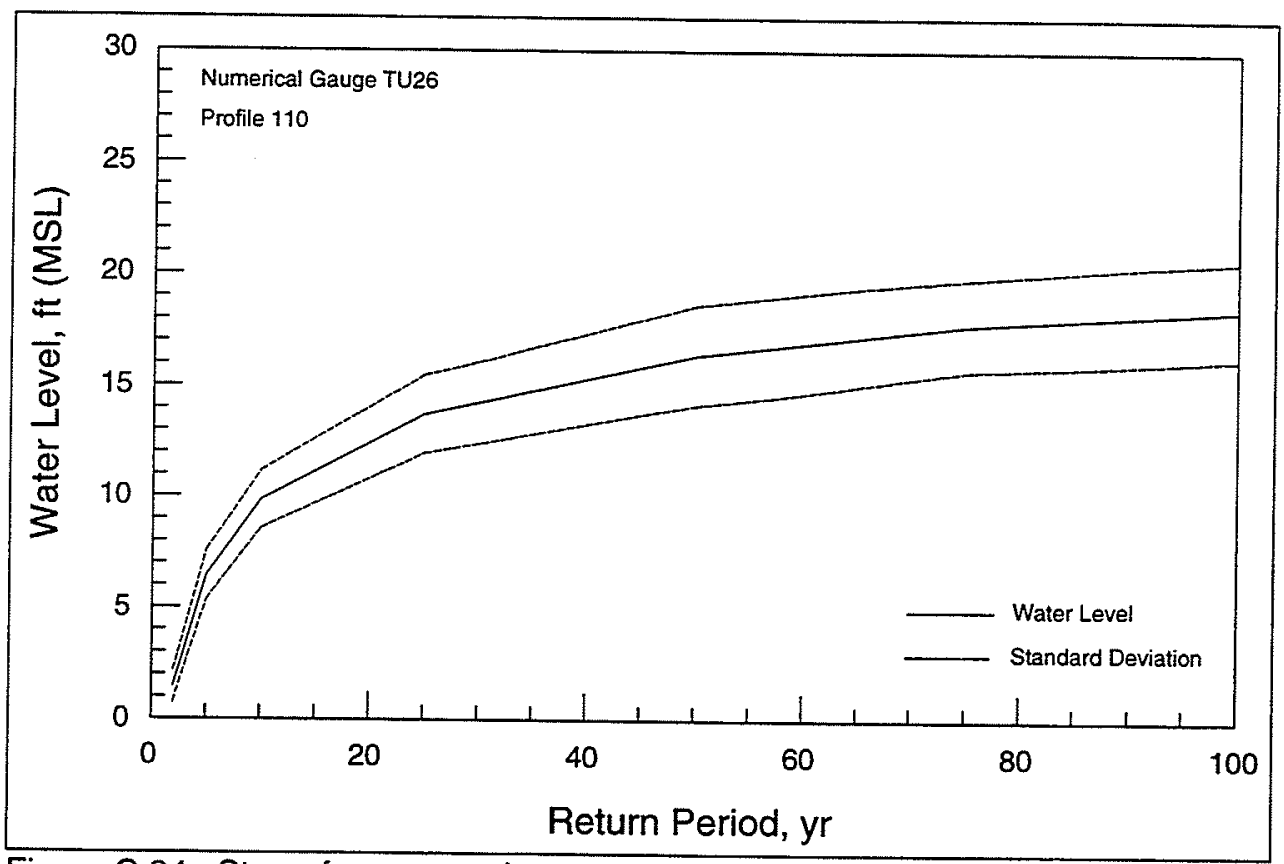

Figure C.24. Stage-frequency plot representing numerical gauge TU26 (Profile 110), Tutuila 


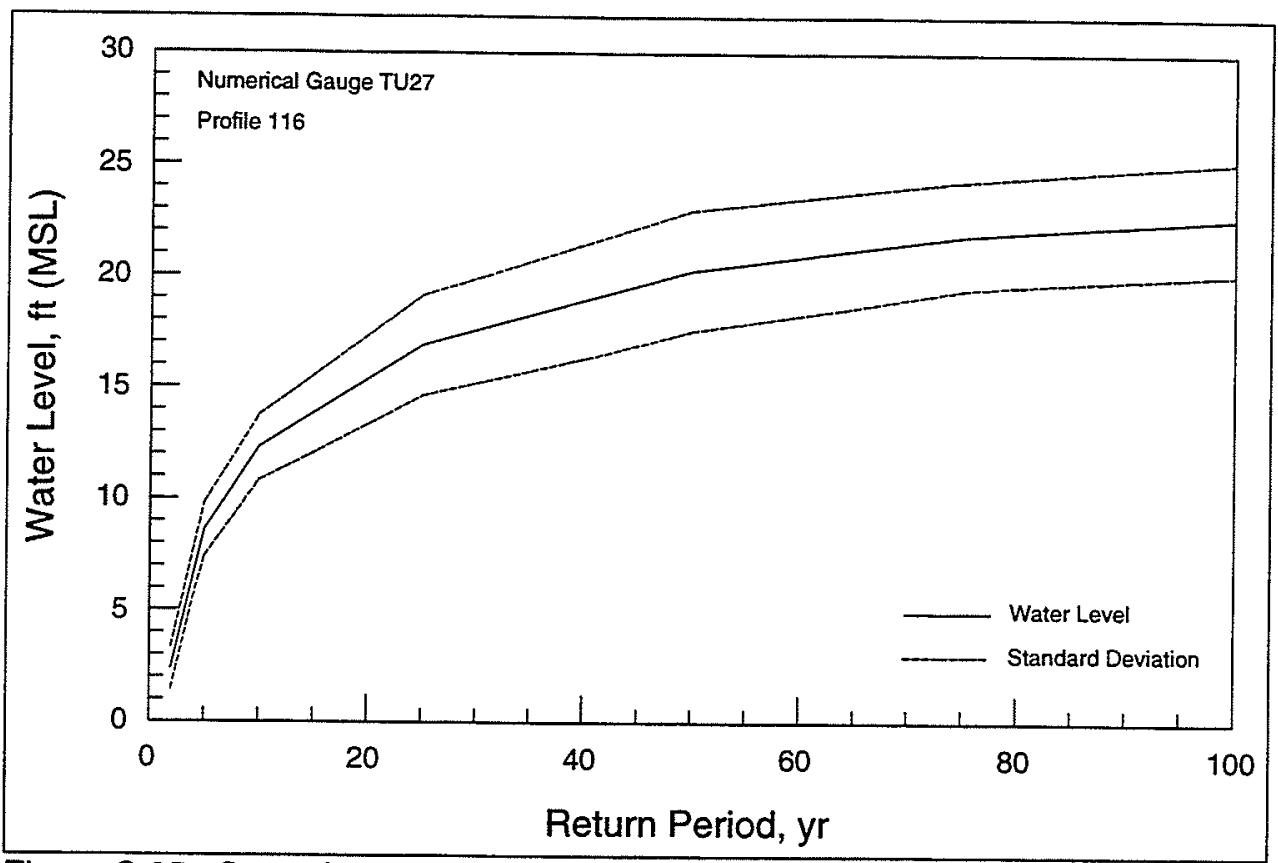

Figure C.25. Stage-frequency plot representing numerical gauge TU27 (Profile 116), Tutuila

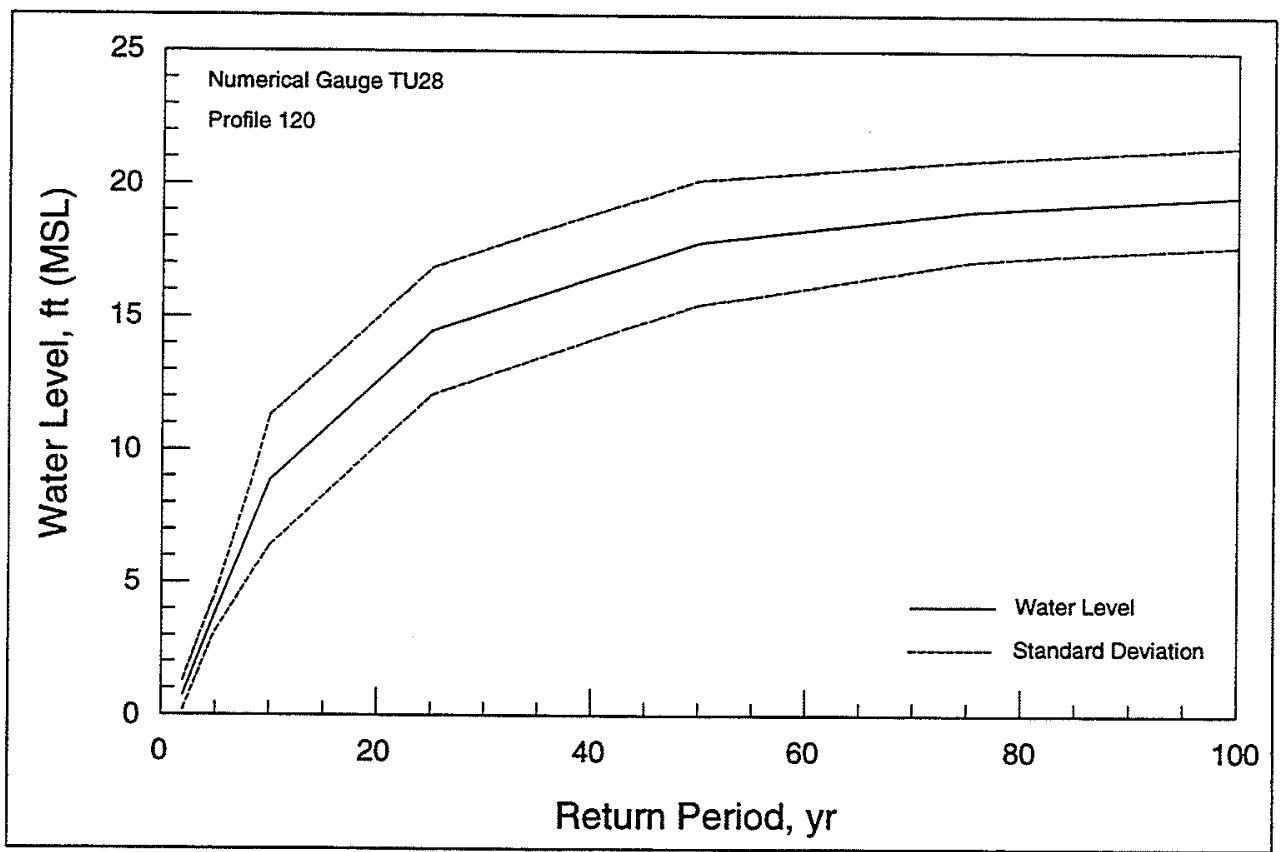

Figure C.26. Stage-frequency plot representing numerical gauge TU28 (Profile 120), Tutuila 


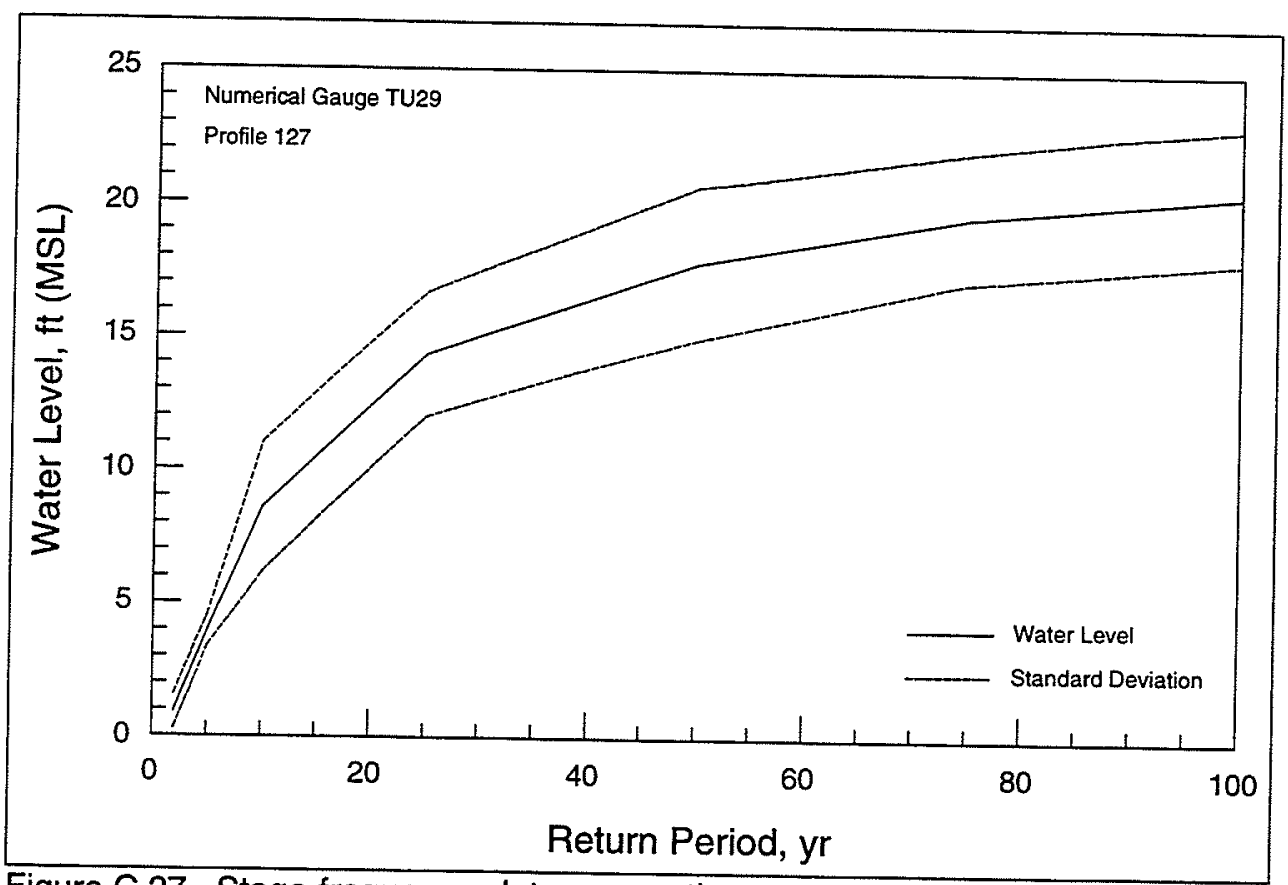

Figure C.27. Stage-frequency plot representing numerical gauge TU29 (Profile 127), Tutuila

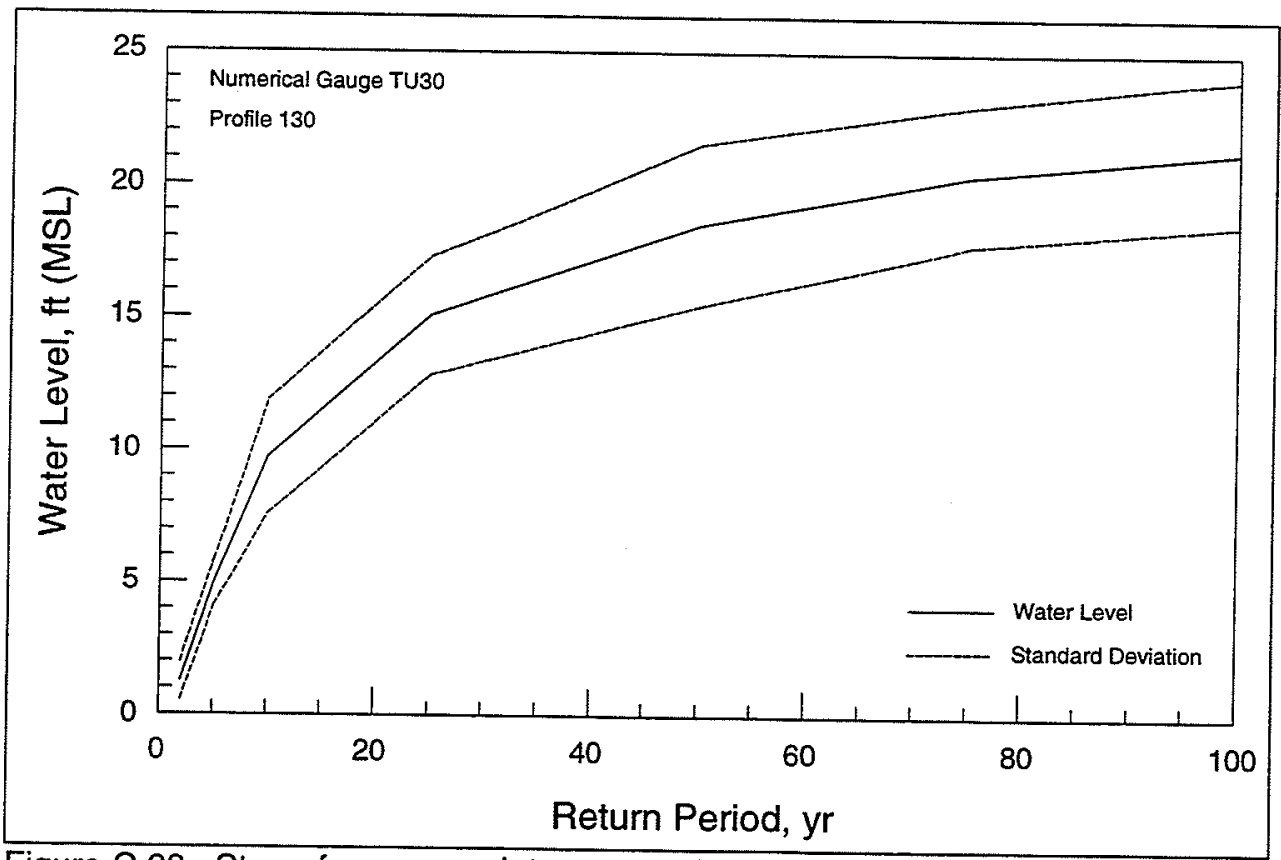

Figure C.28. Stage-frequency plot representing numerical gauge TU30 (Profile 130), Tutuila 


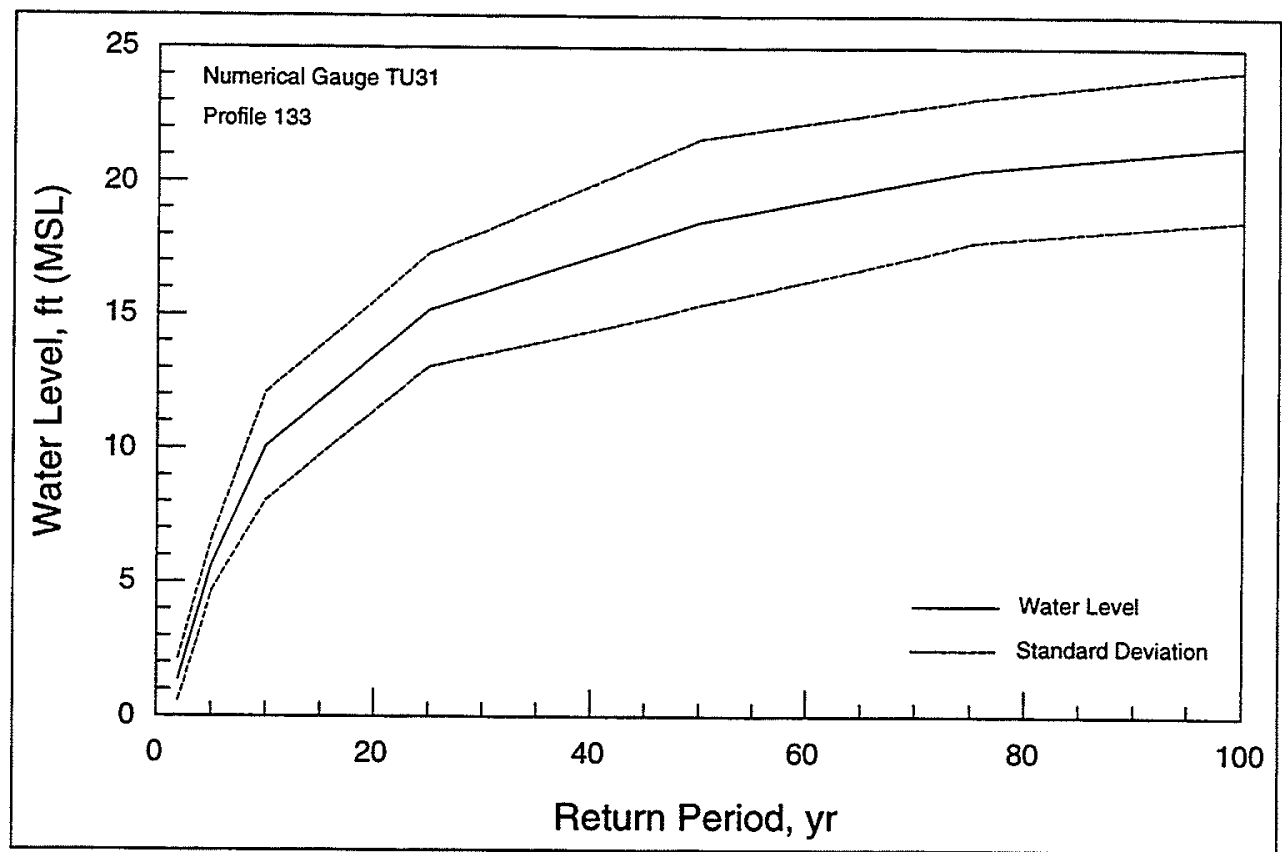

Figure C.29. Stage-frequency plot representing numerical gauge TU31 (Profile 133), Tutuila

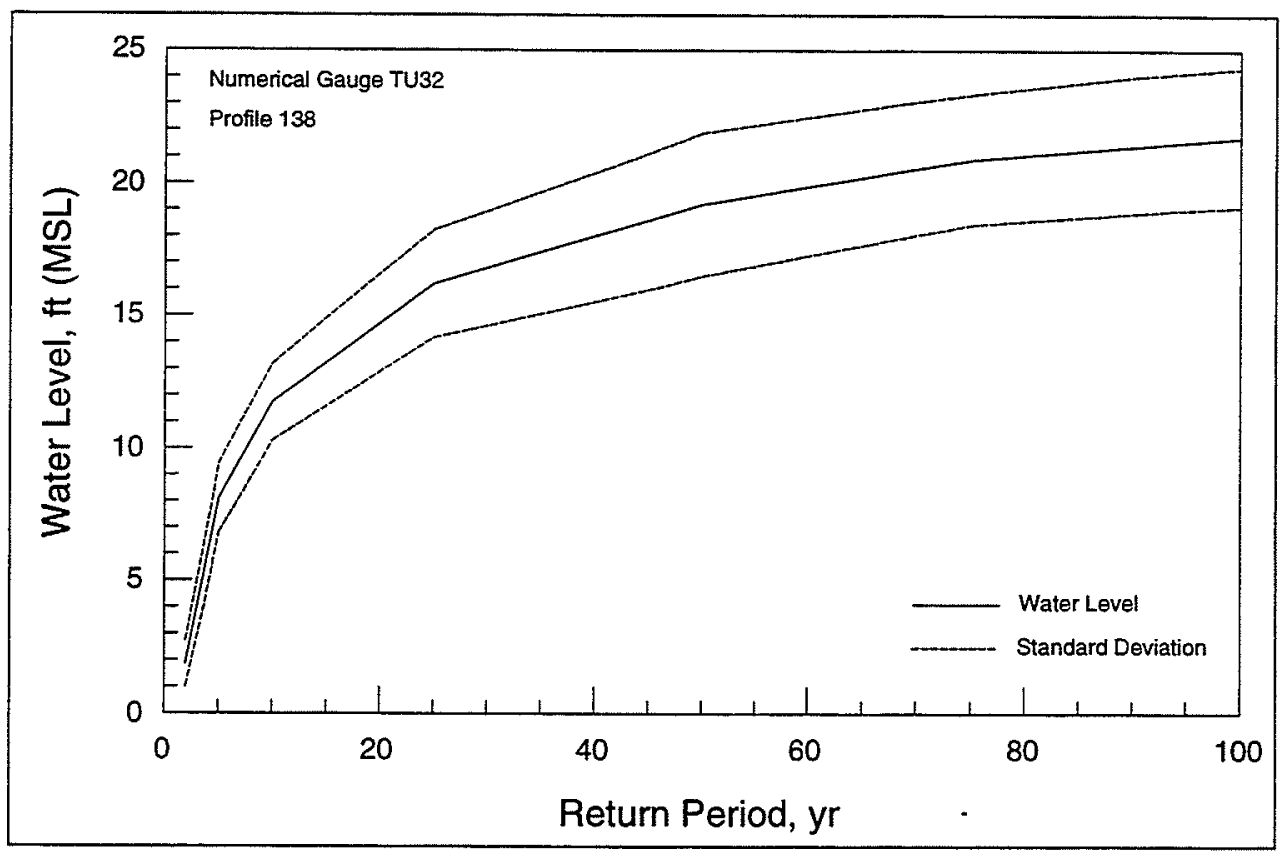

Figure C.30. Stage-frequency plot representing numerical gauge TU32 (Profile 138), Tutuila 


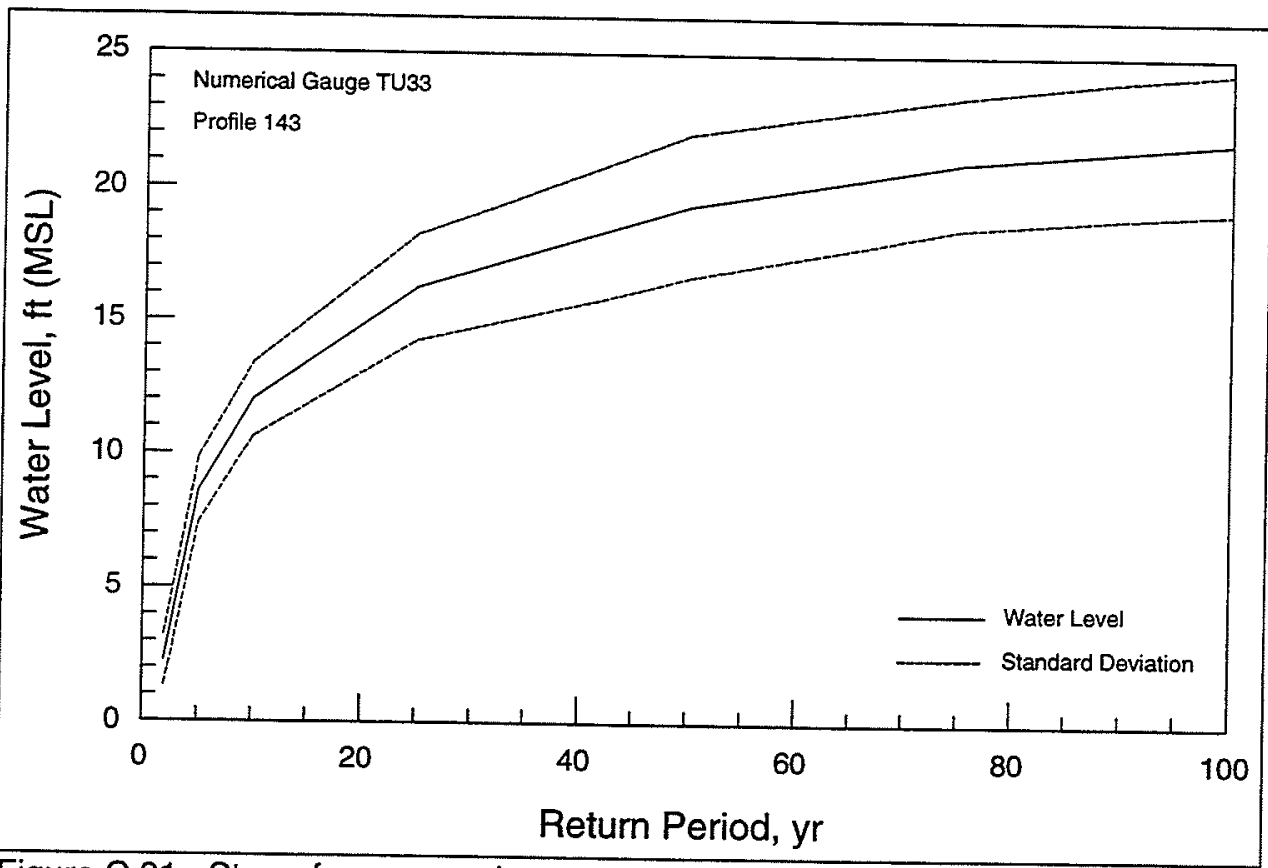

Figure C.31. Stage-frequency plot representing numerical gauge TU33 (Profile 143), Tutuila

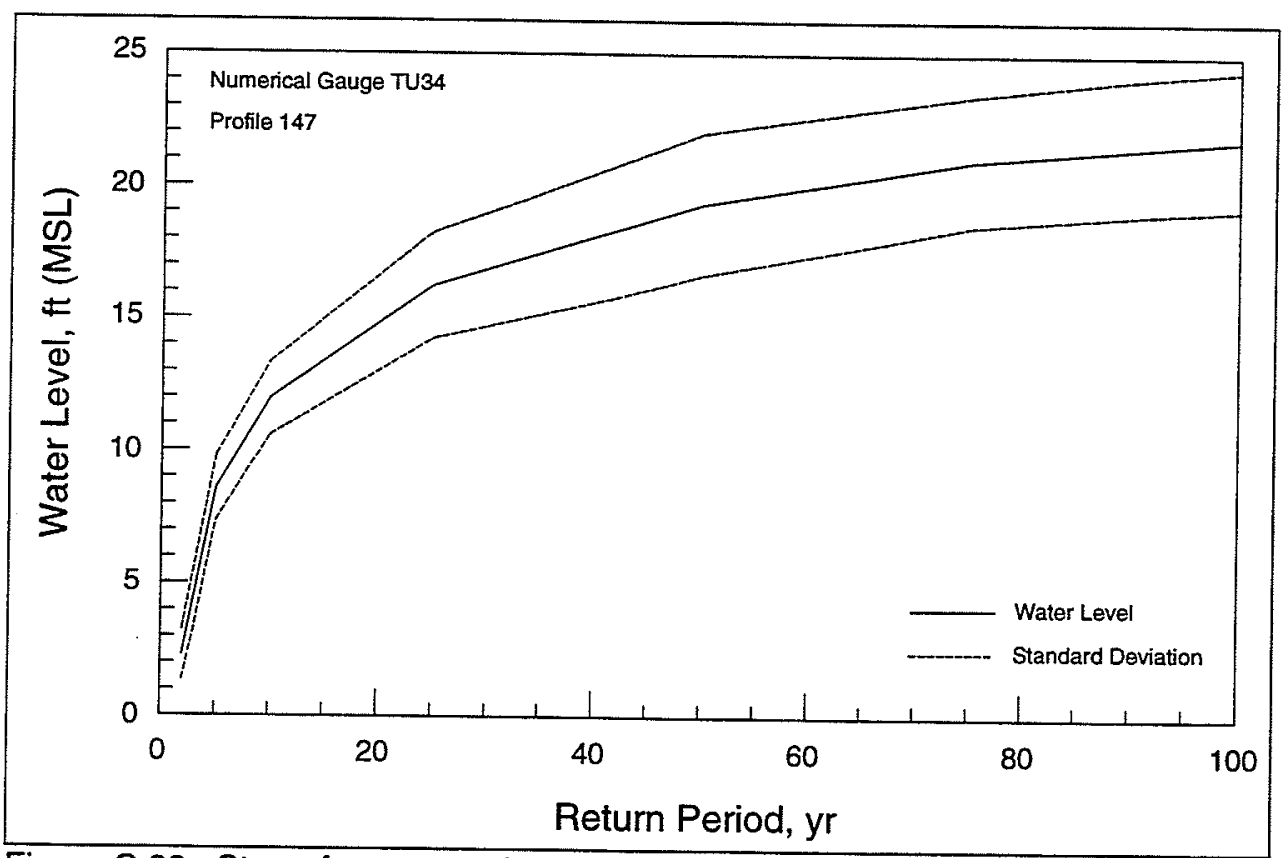

Figure C.32. Stage-frequency plot representing numerical gauge TU34 (Profile 147), Tutuila 


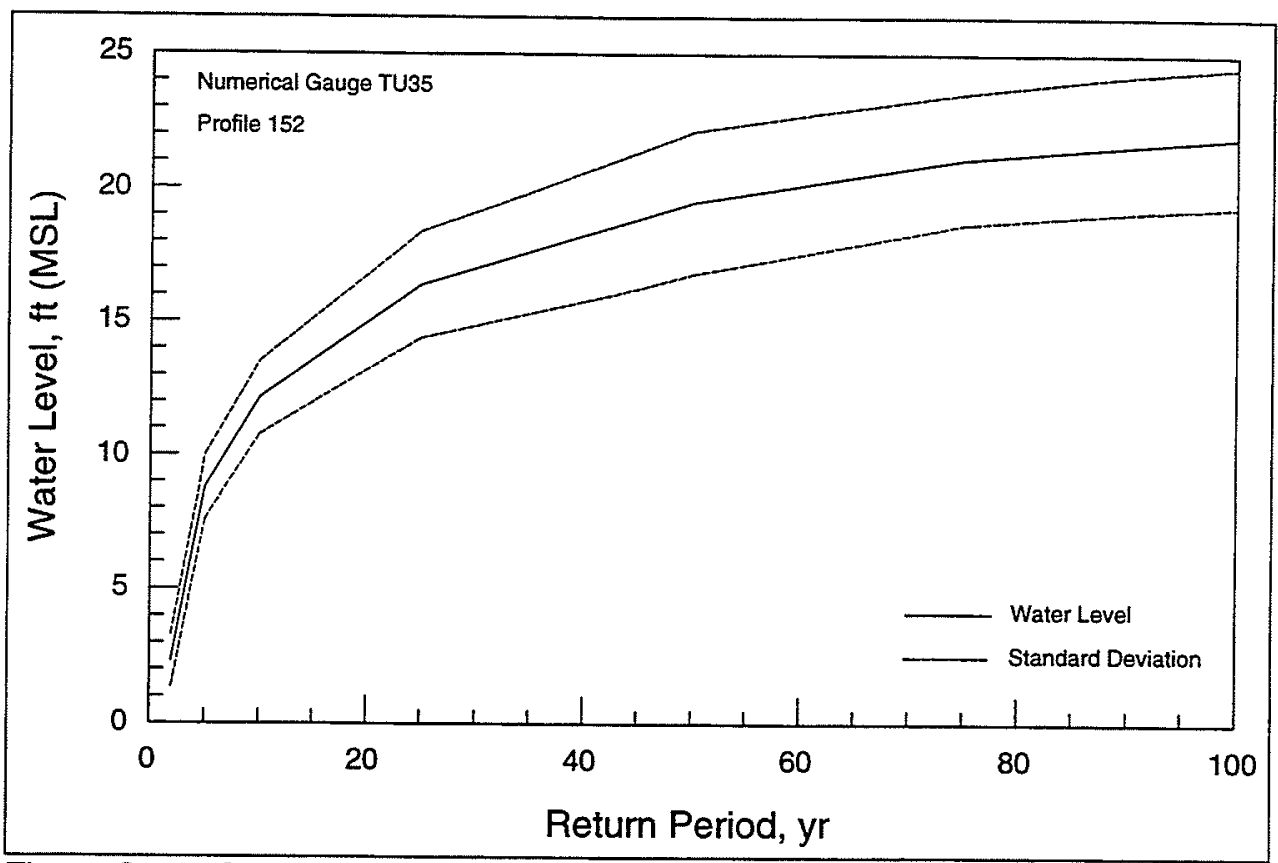

Figure C.33. Stage-frequency plot representing numerical gauge TU35 (Profile 152), Tutuila

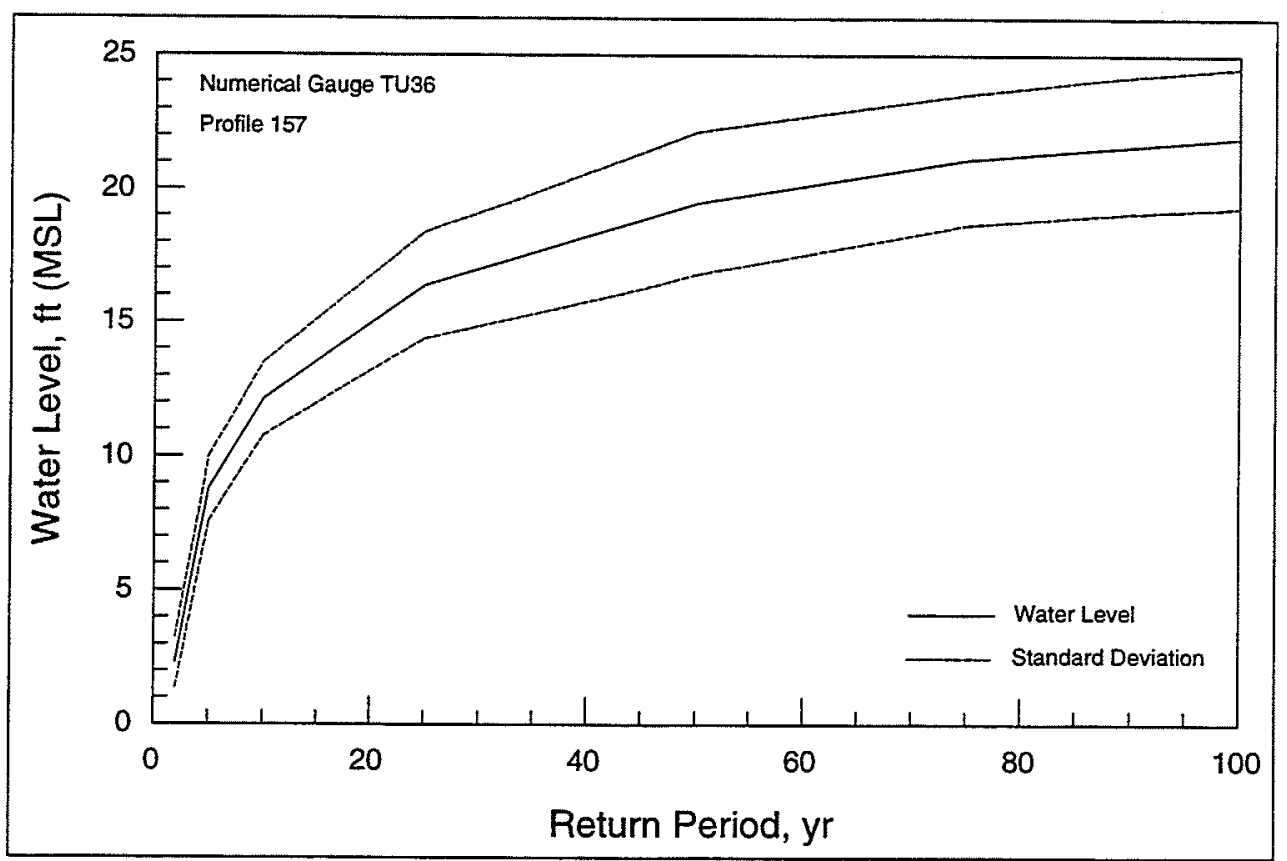

Figure C.34. Stage-frequency plot representing numerical gauge TU36 (Profile 157), Tutuila 


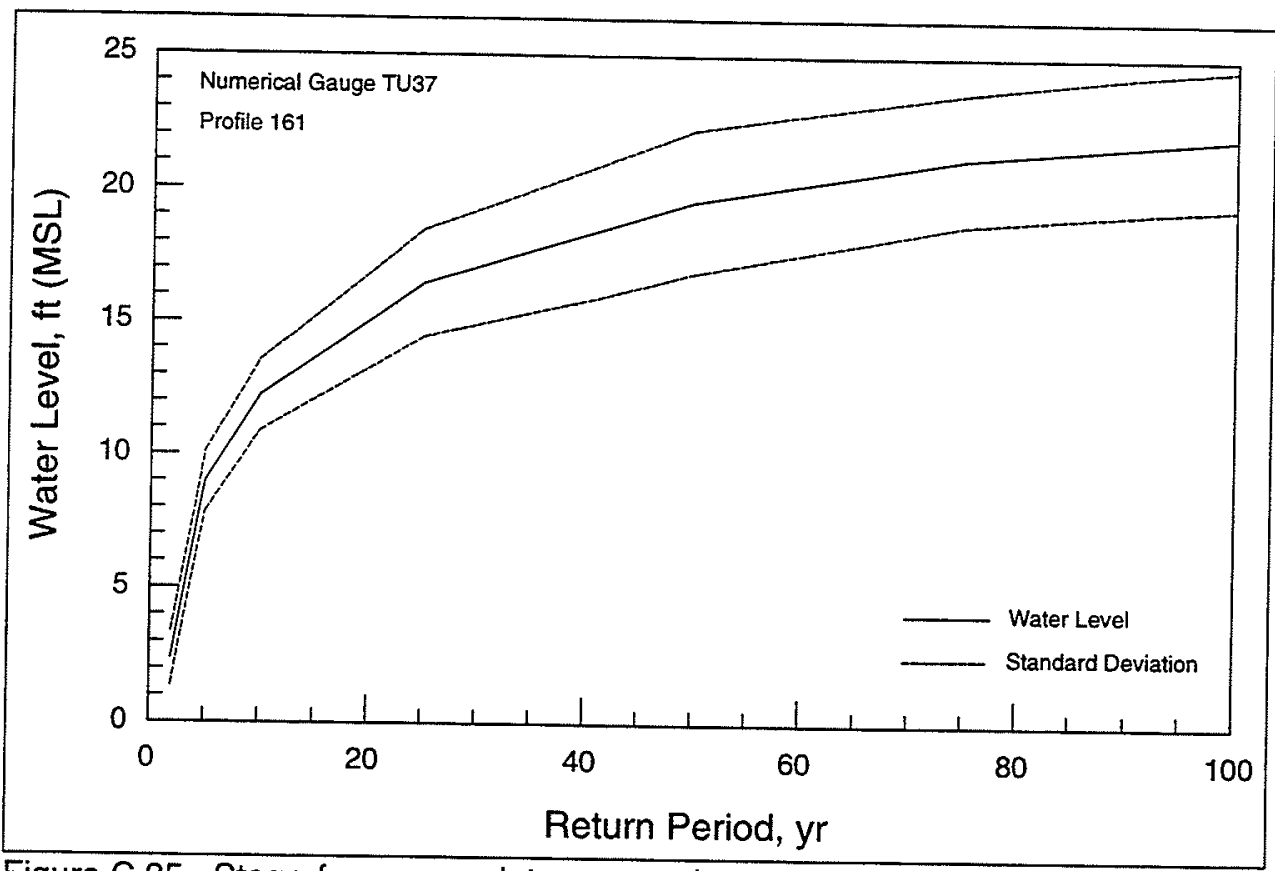

Figure C.35. Stage-frequency plot representing numerical gauge TU37 (Profile 161), Tutuila

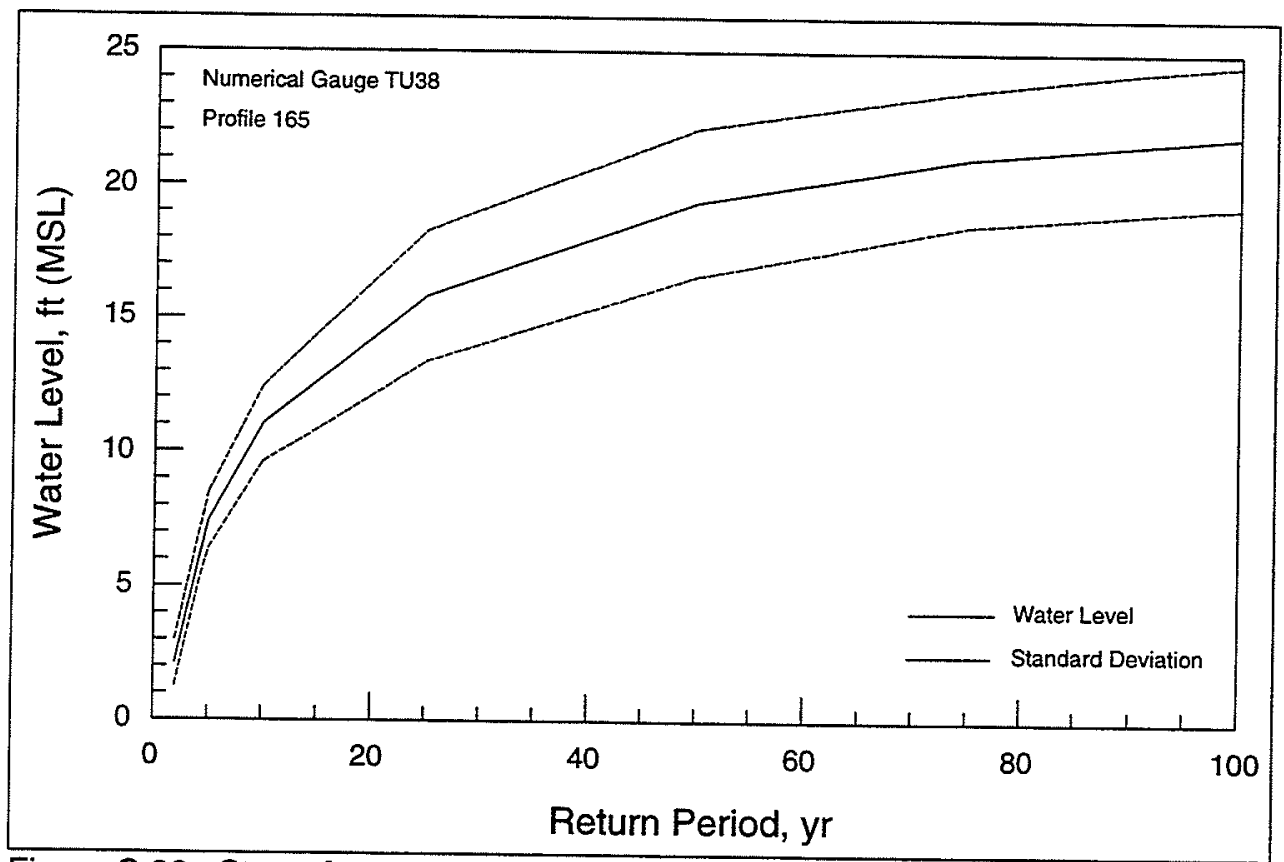

Figure C.36. Stage-frequency plot representing numerical gauge TU38 (Profile 165), Tutuila 


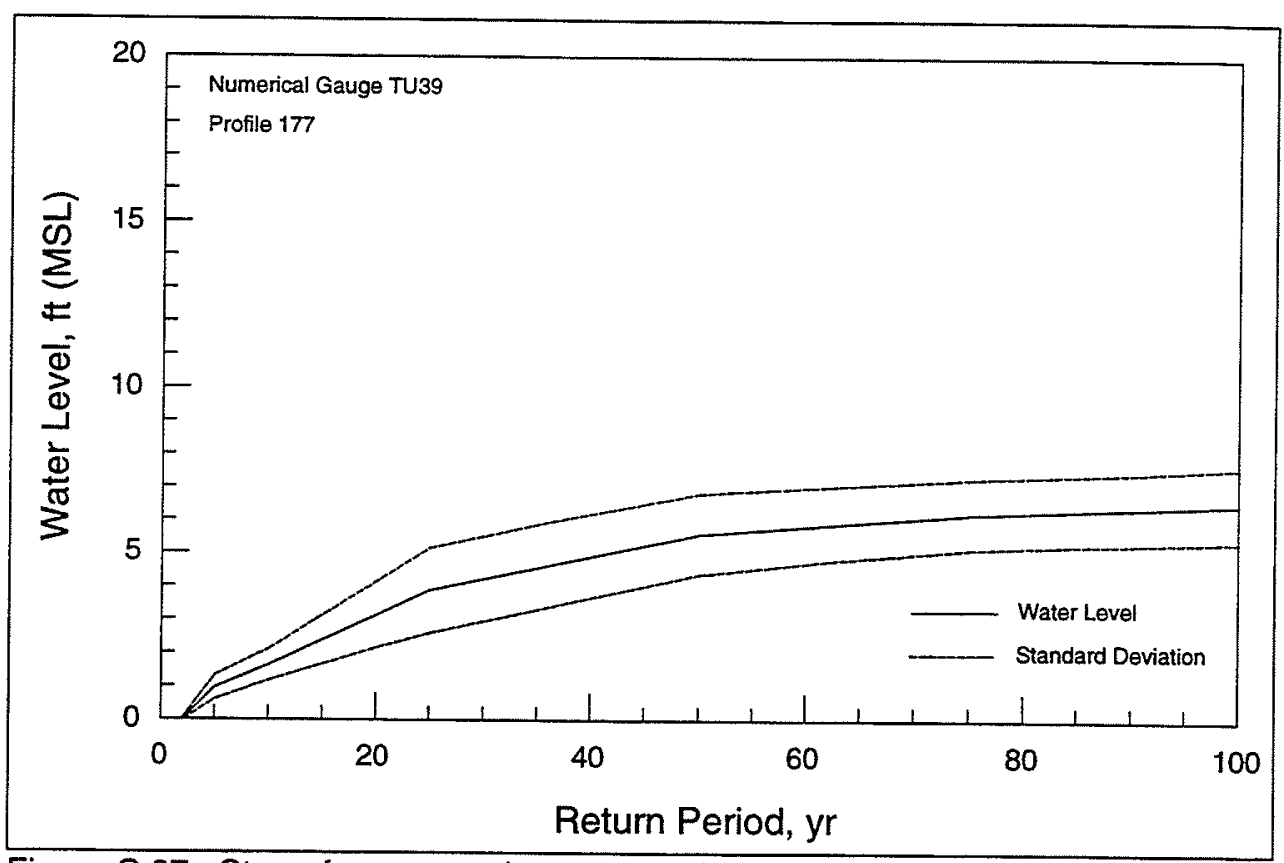

Figure C.37. Stage-frequency plot representing numerical gauge TU39 (Profile 177), Tutuila

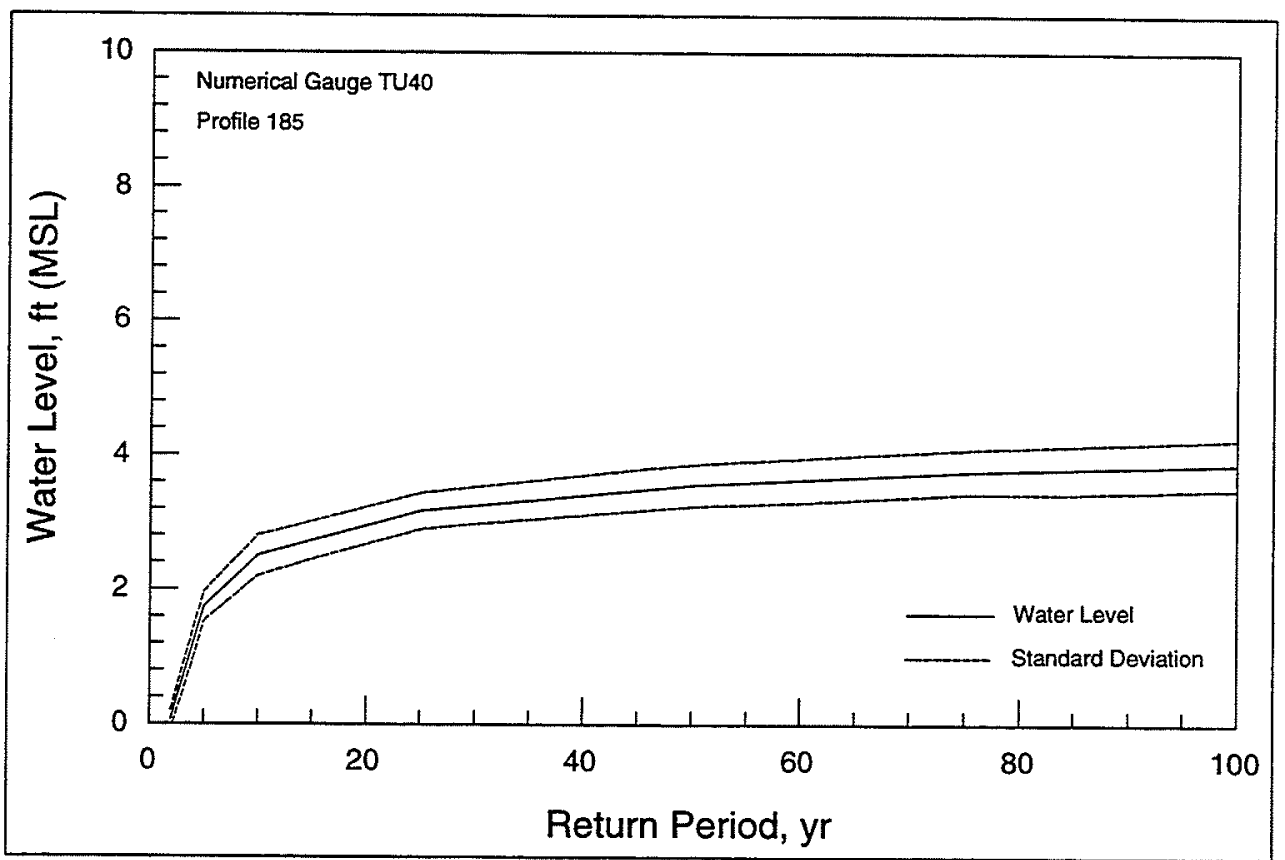

Figure C.38. Stage-frequency plot representing numerical gauge TU40 (Profile 185), Tutuila 


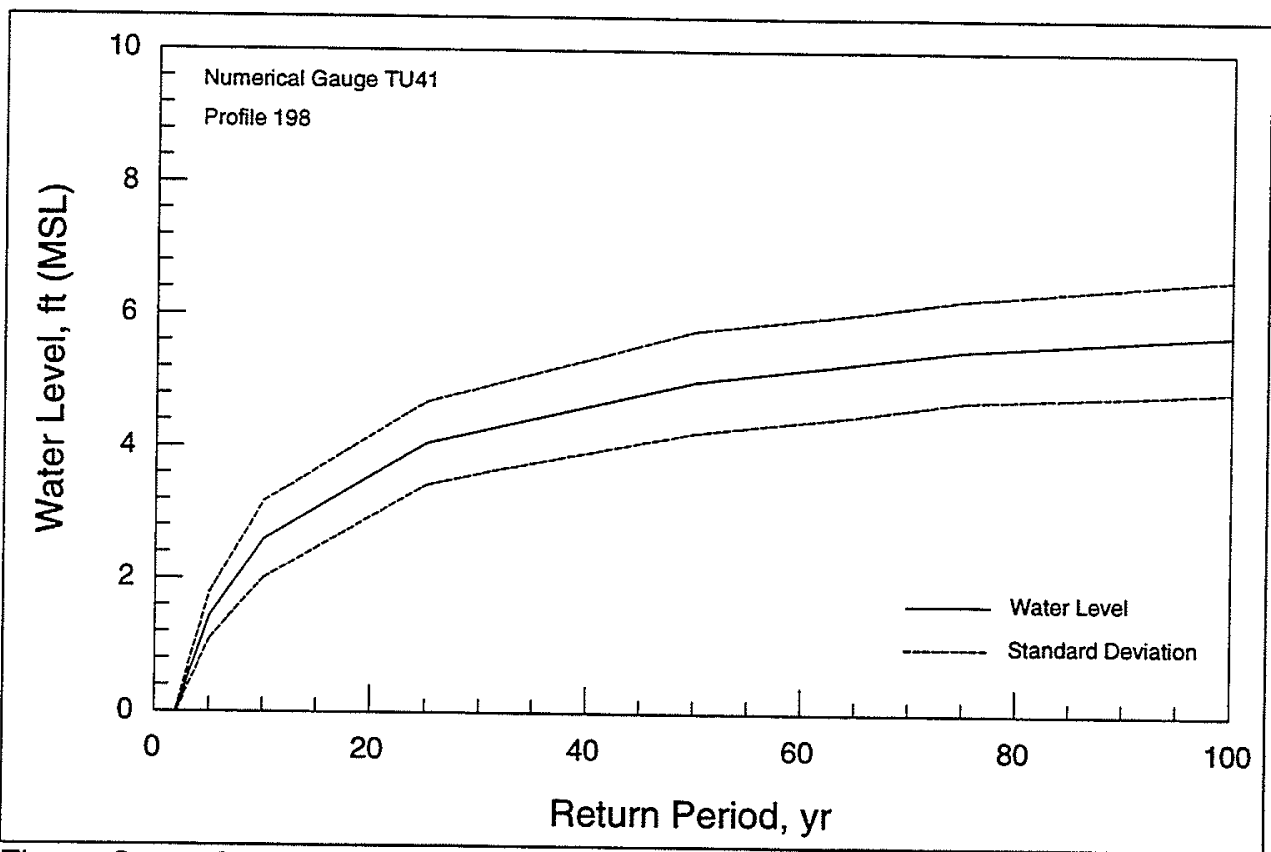

Figure C.39. Stage-frequency plot representing numerical gauge TU41 (Profile 198), Tutuila

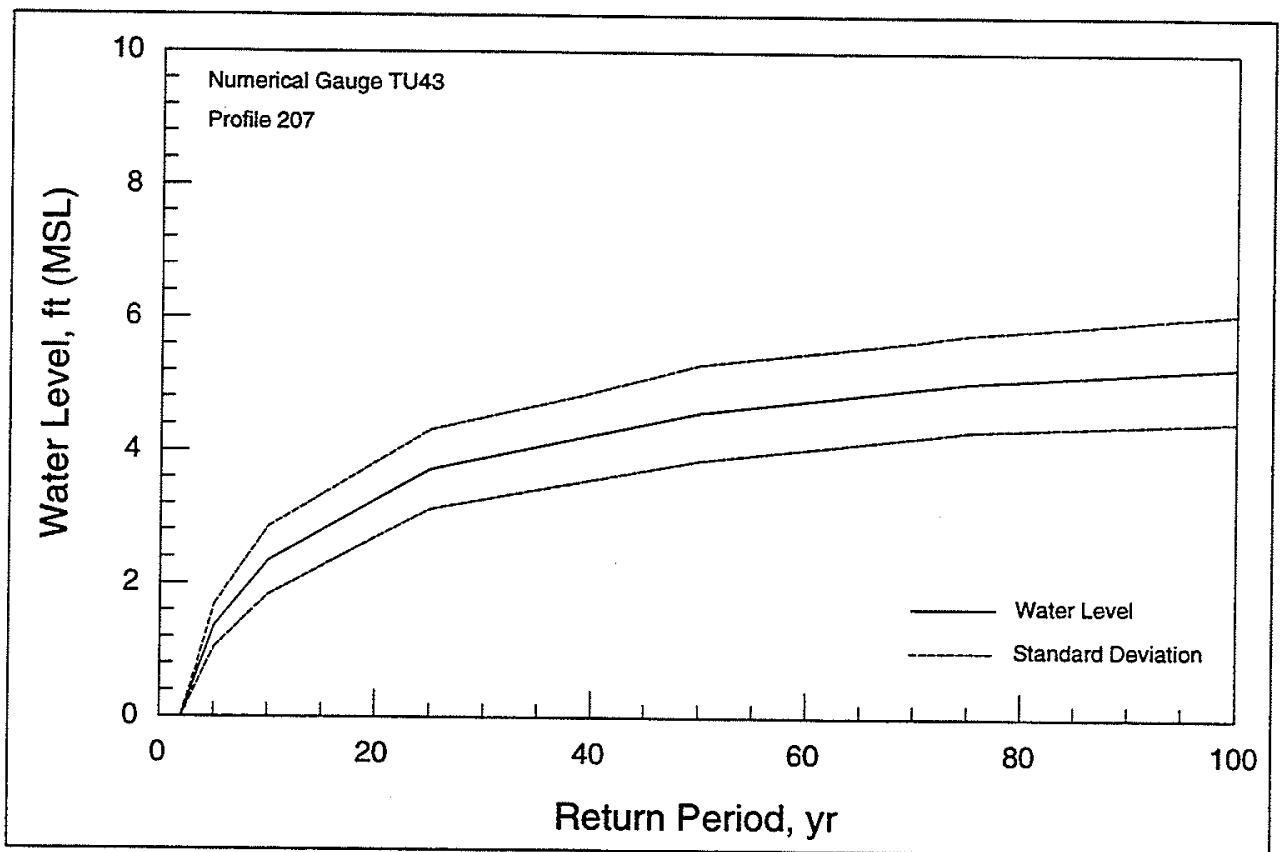

Figure C.40. Stage-frequency plot representing numerical gauge TU43 (Profile 207), Tutuila 


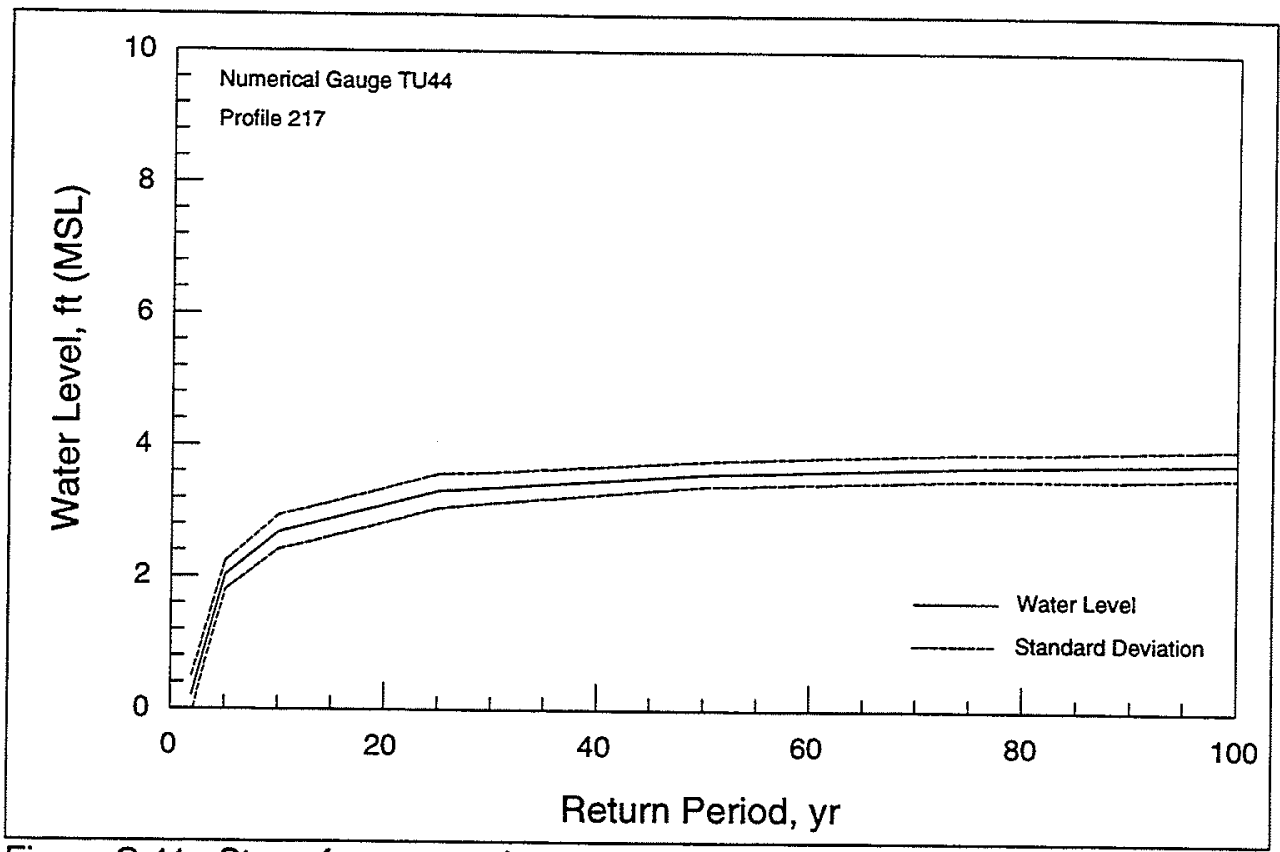

Figure C.41. Stage-frequency plot representing numerical gauge TU44 (Profile 217), Tutuila

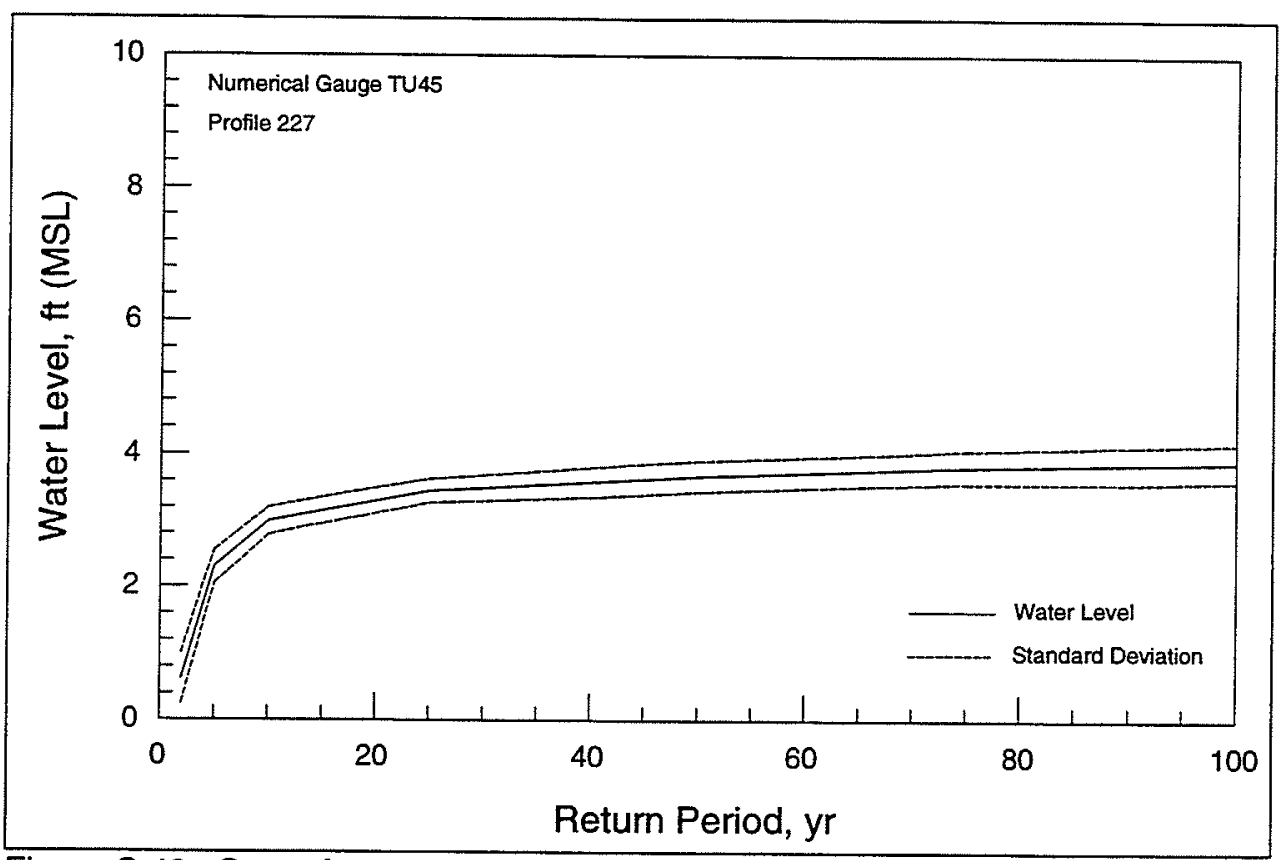

Figure C.42. Stage-frequency plot representing numerical gauge TU45 (Profile 227), Tutuila 


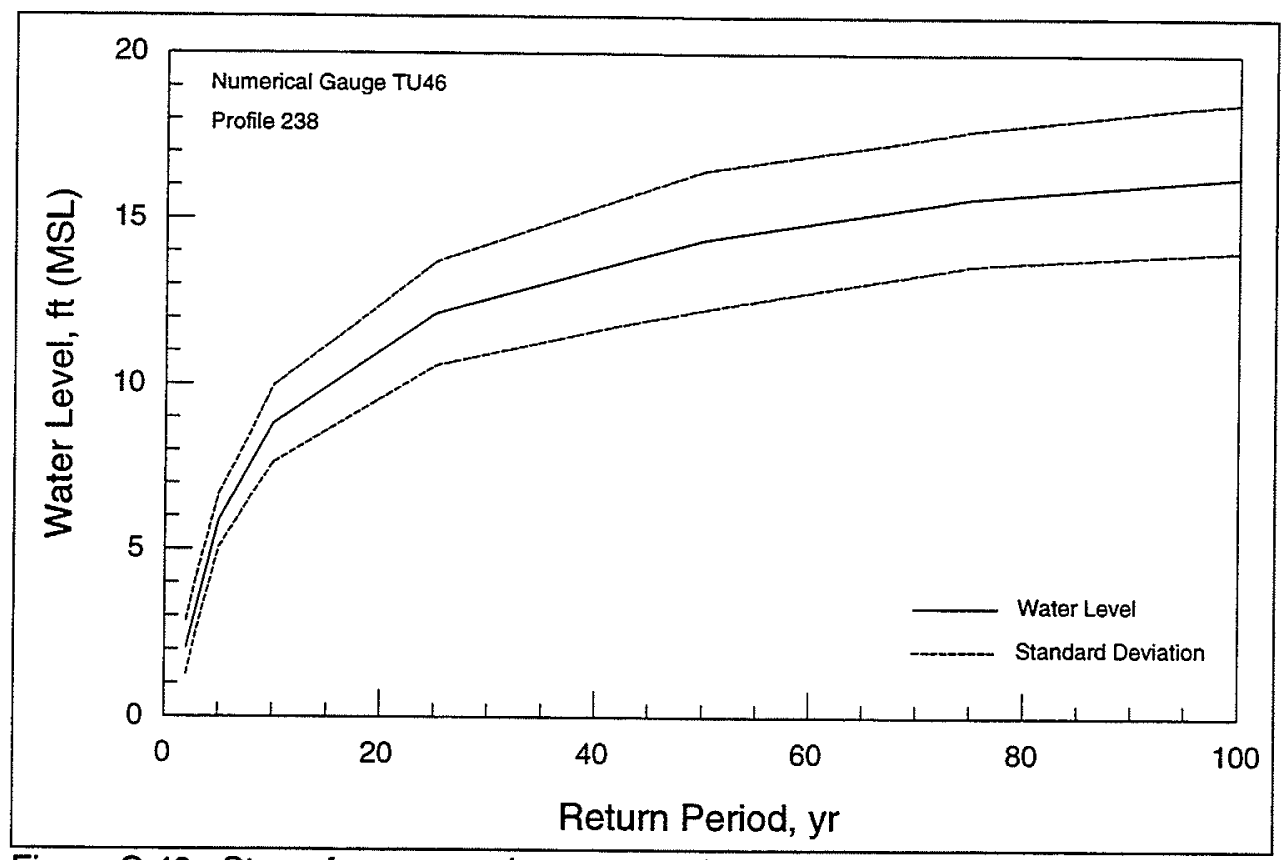

Figure C.43. Stage-frequency plot representing numerical gauge TU46 (Profile 238), Tutuila

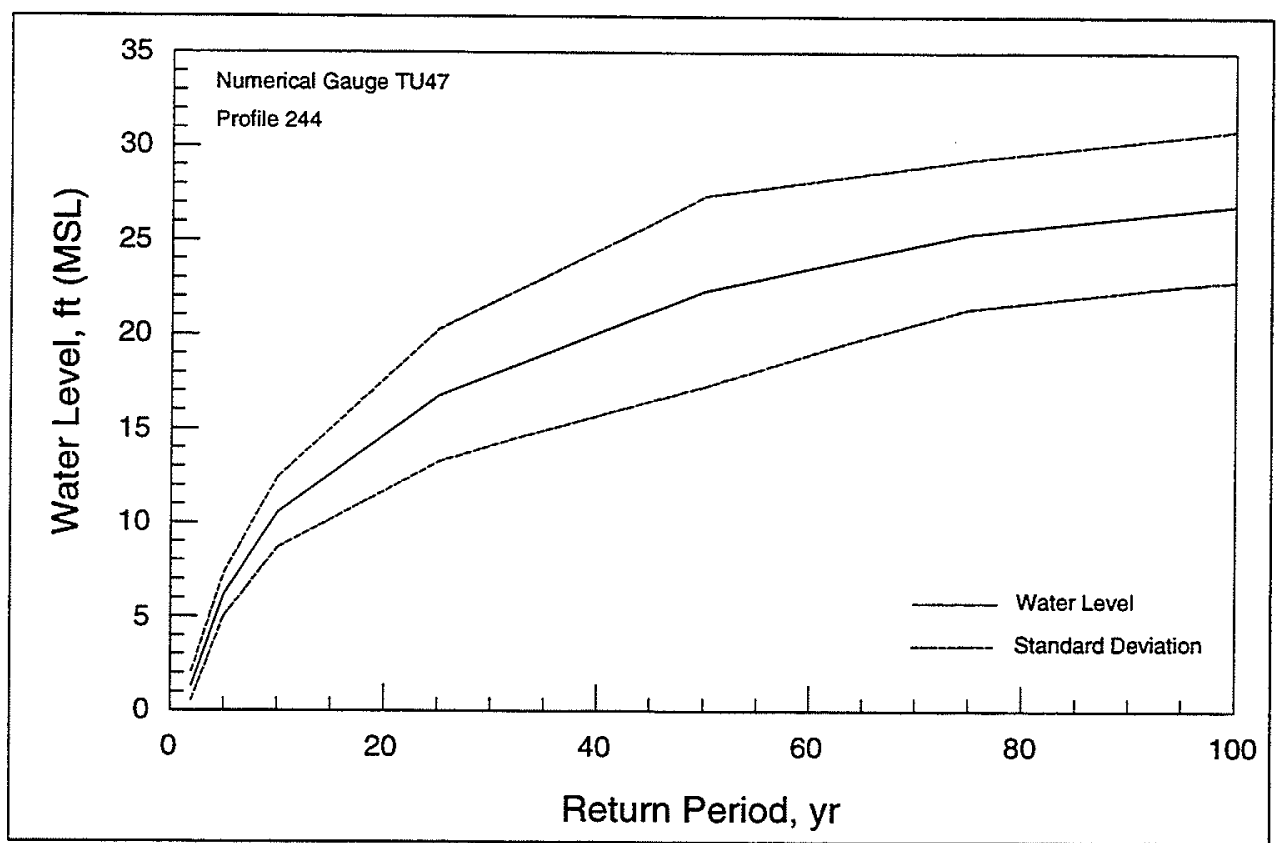

Figure C.44. Stage-frequency plot representing numerical gauge TU47 (Profile 244), Tutuila 


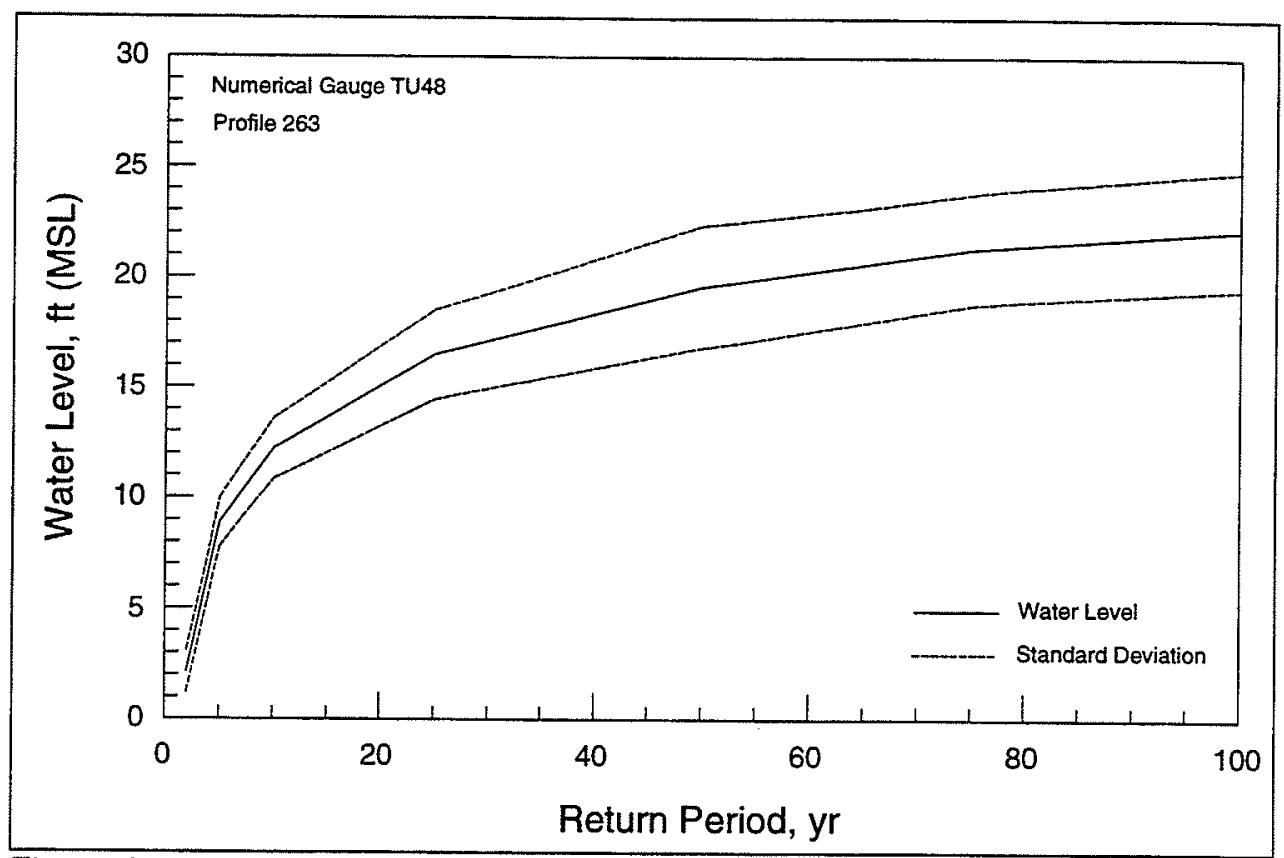

Figure C.45. Stage-frequency plot representing numerical gauge TU48 (Profile 263), Tutuila

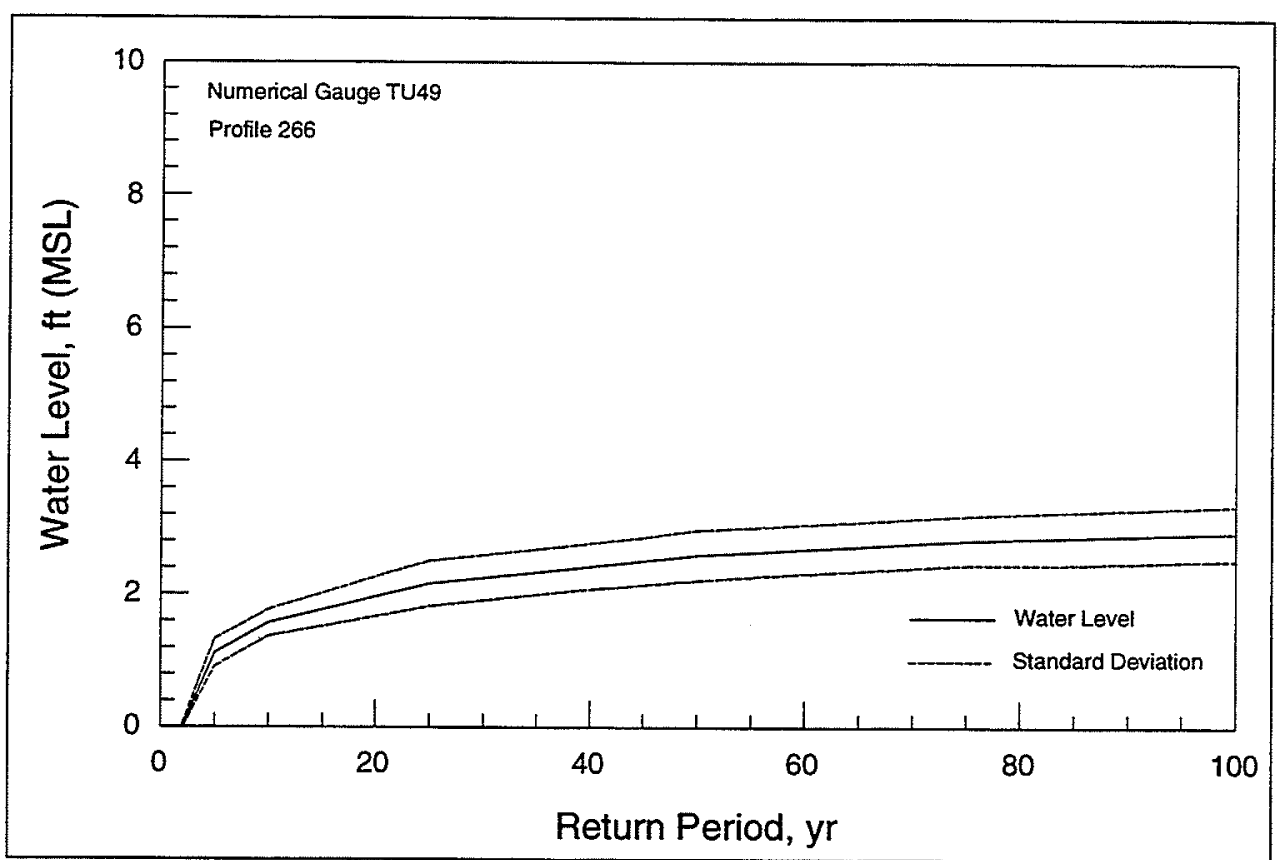

Figure C.46. Stage-frequency plot representing numerical gauge TU49 (Profile 266), Tutuila 


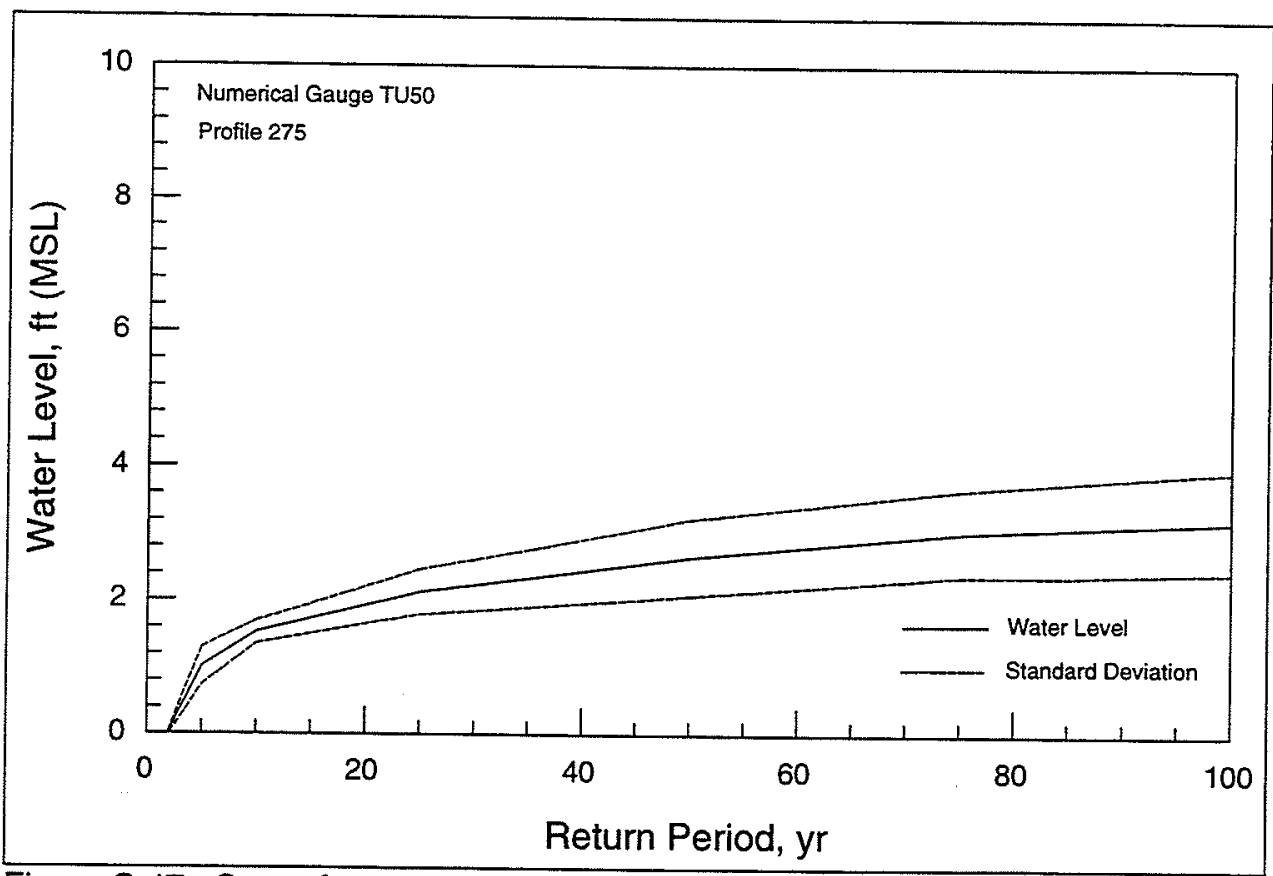

Figure C.47. Stage-frequency plot representing numerical gauge TU50 (Profile 275), Tutuila

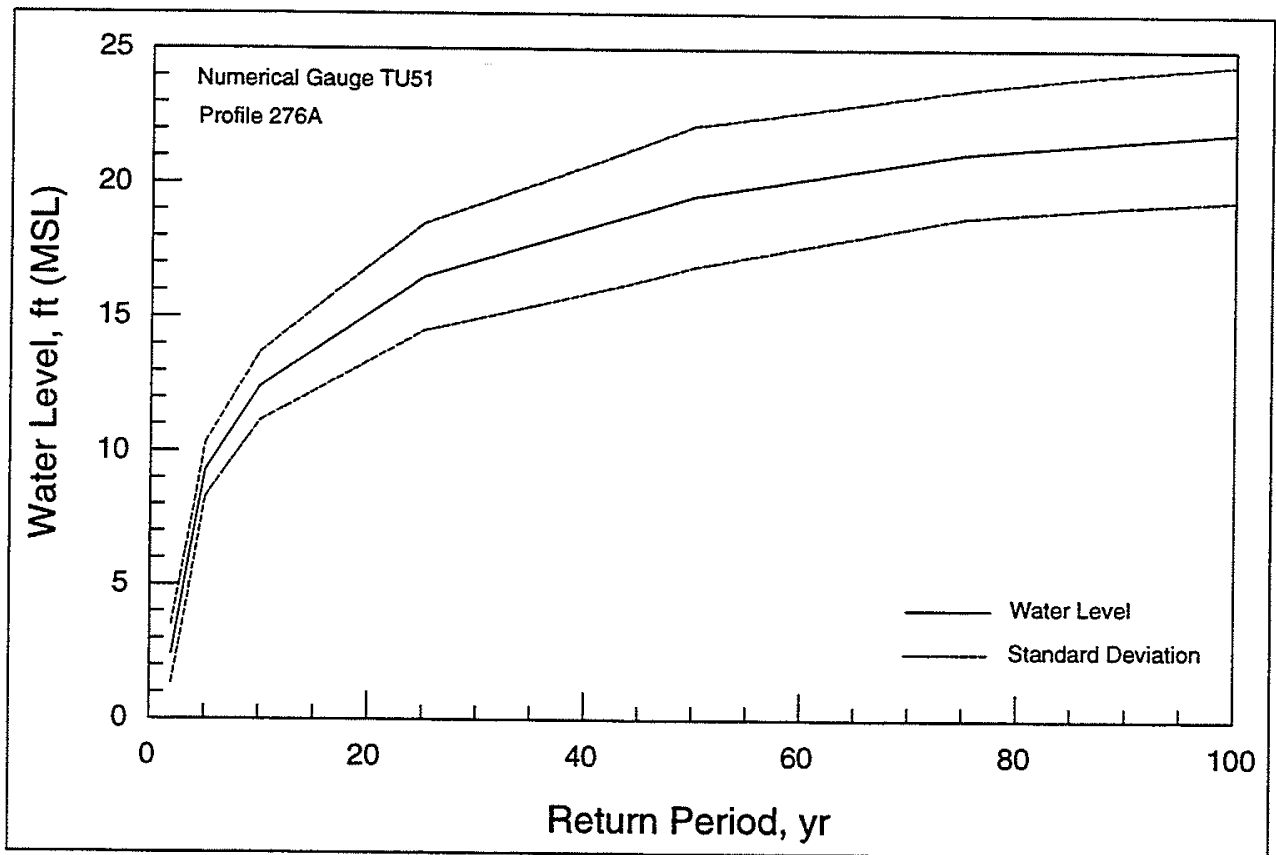

Figure C.48. Stage-frequency plot representing numerical gauge TU51 (Profile 276A), Tutuila 


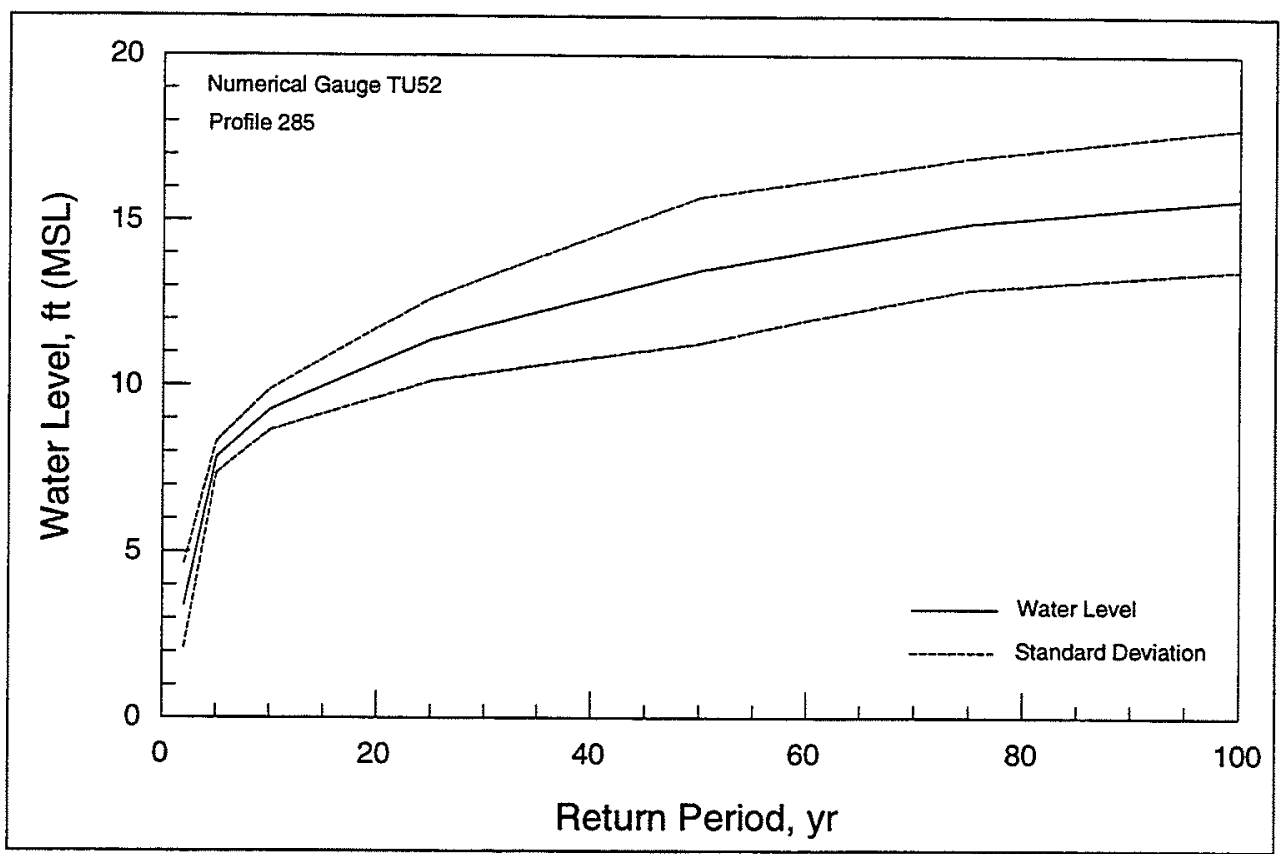

Figure C.49. Stage-frequency plot representing numerical gauge TU52 (Profile 285), Tutuila

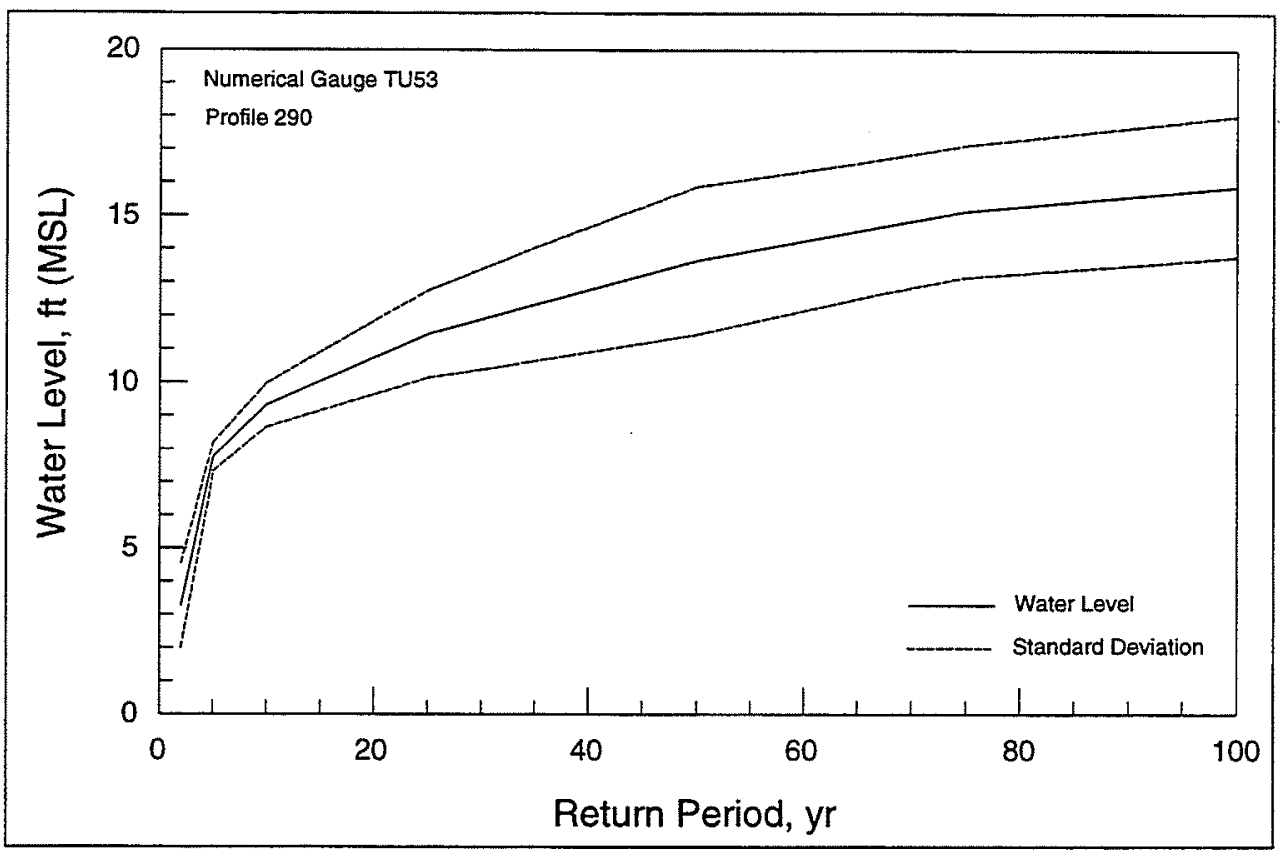

Figure C.50. Stage-frequency plot representing numerical gauge TU53 (Profile 290), Tutuila 


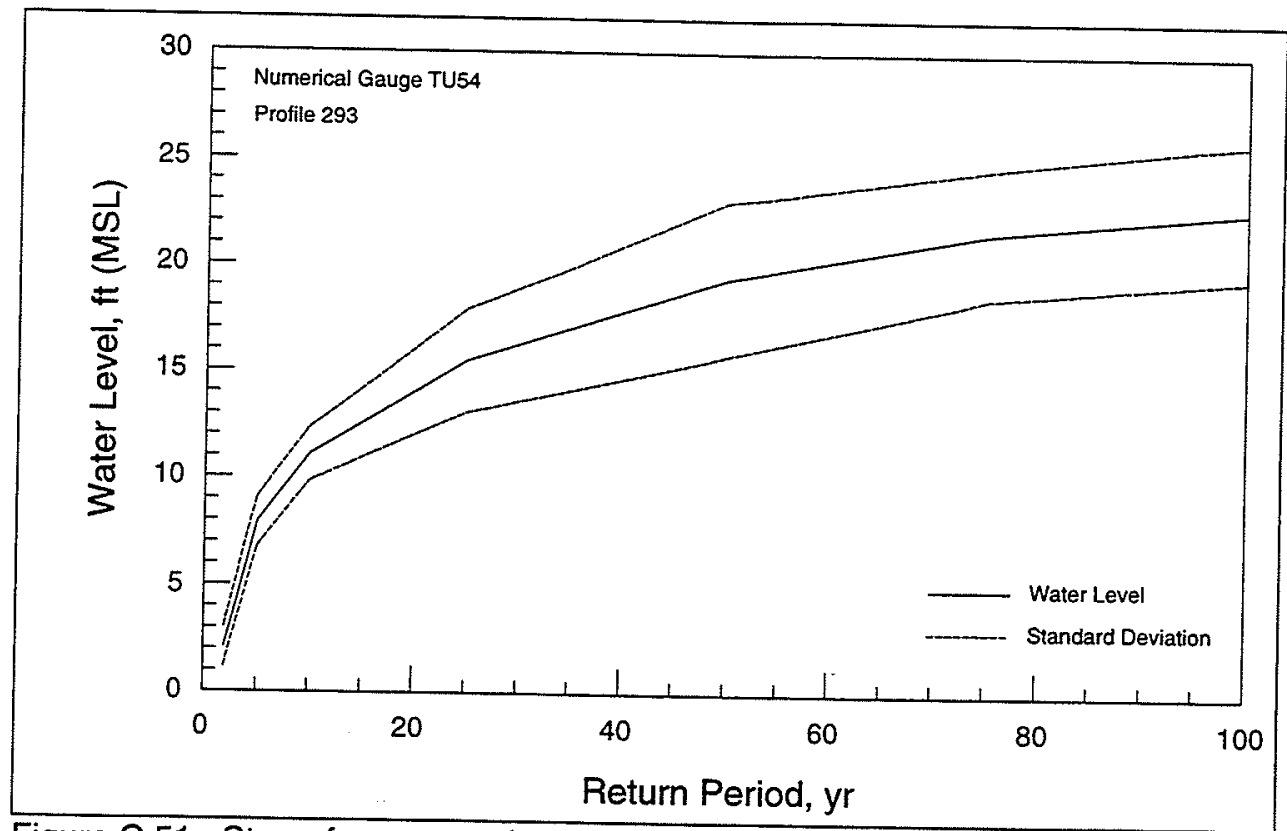

Figure C.51. Stage-frequency plot representing numerical gauge TU54 (Profile 293), Tutuila

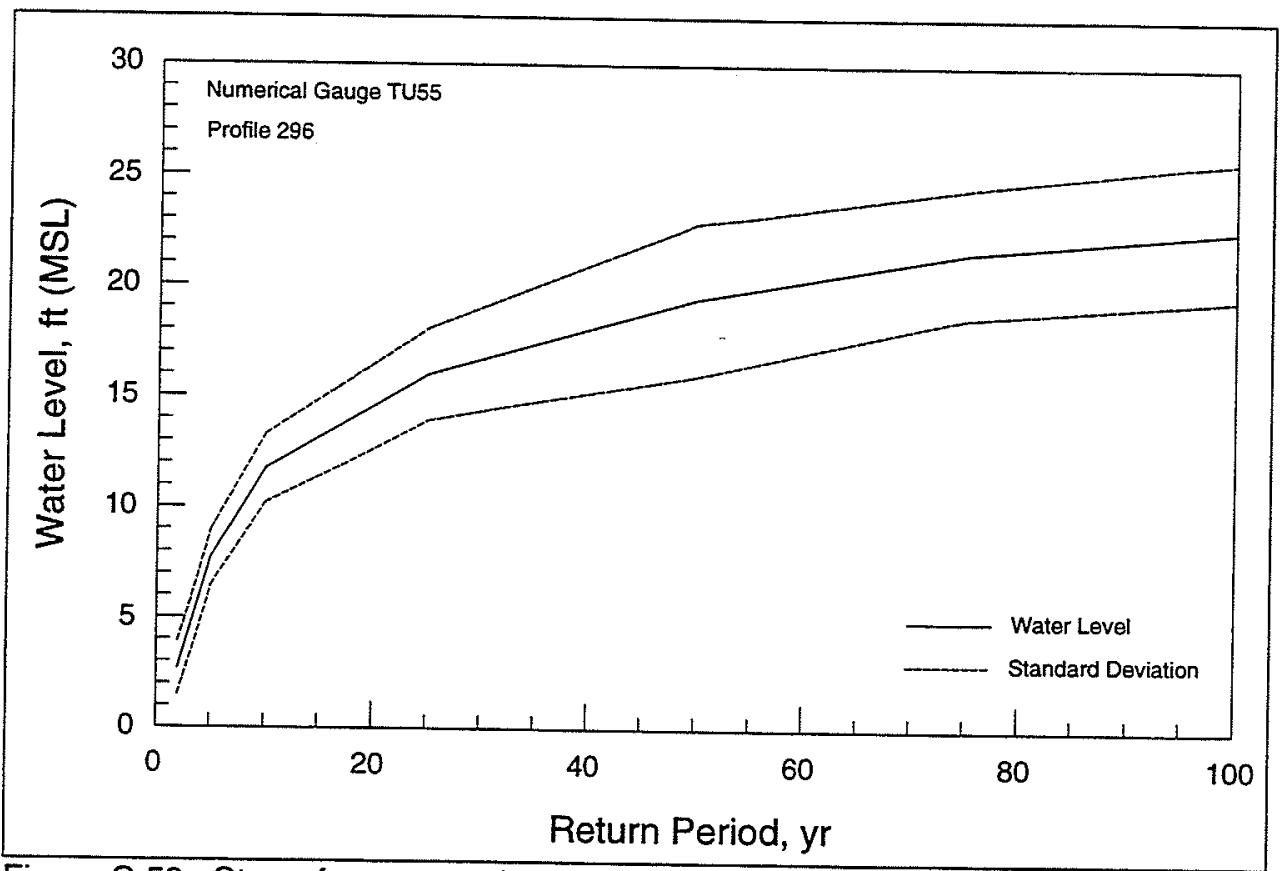

Figure C.52. Stage-frequency plot representing numerical gauge TU55 (Profile 296), Tutuila 


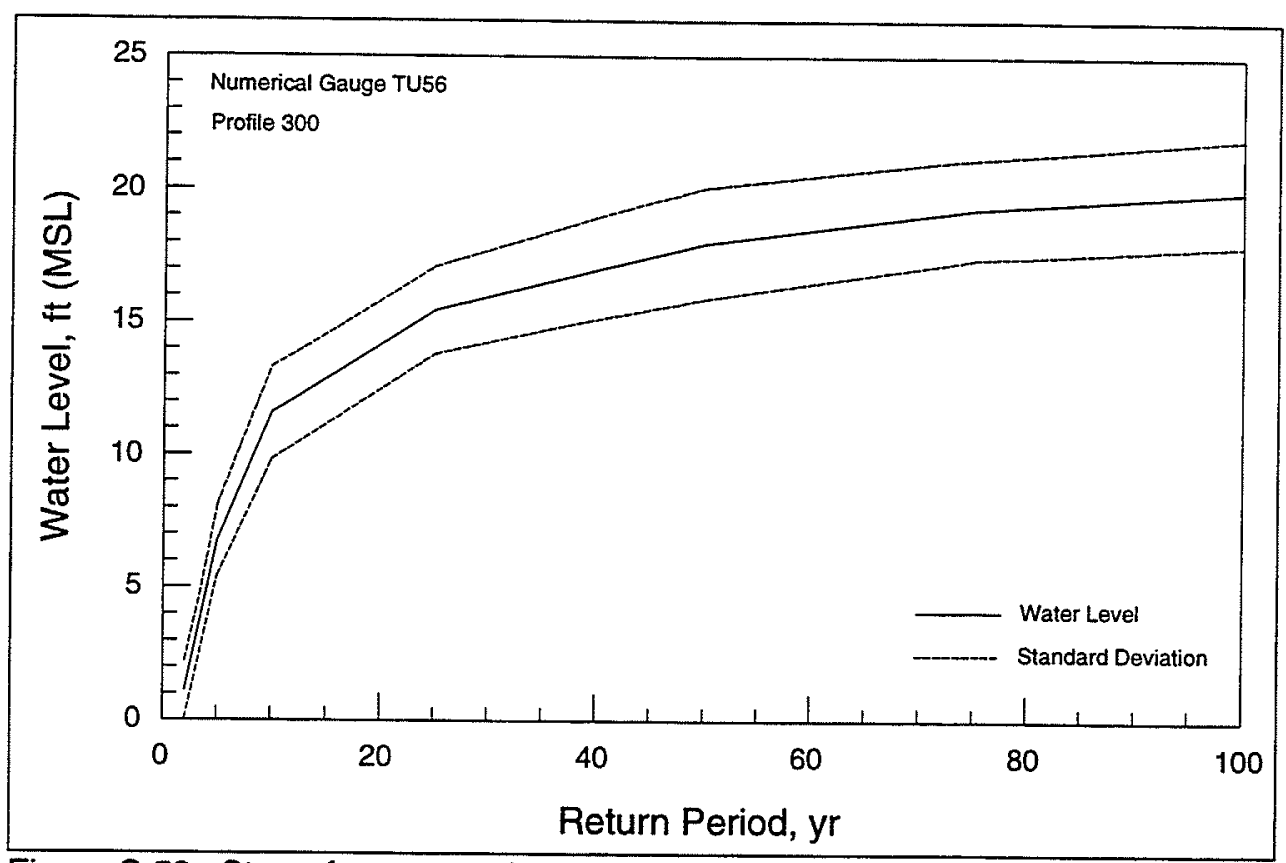

Figure C.53. Stage-frequency plot representing numerical gauge TU56 (Profile 300), Tutuila

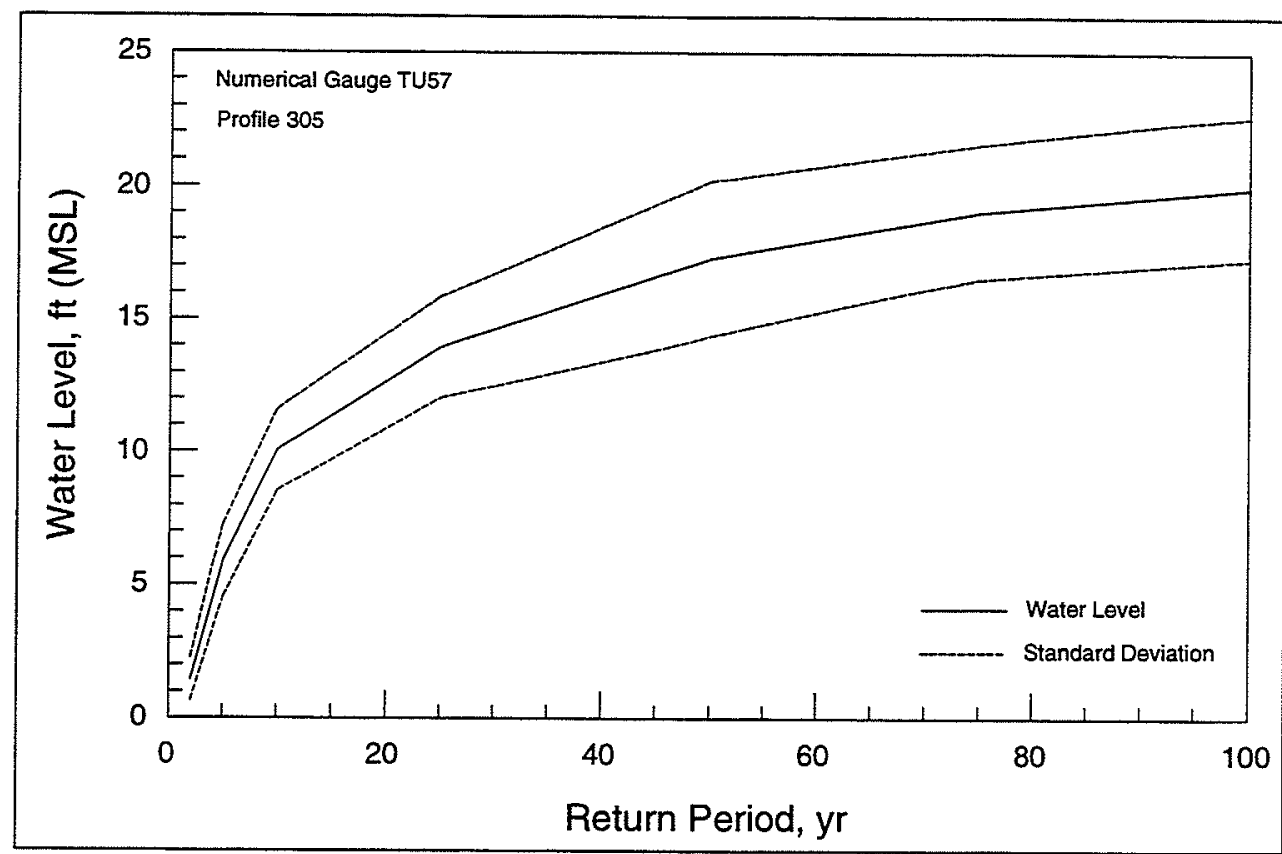

Figure C.54. Stage-frequency plot representing numerical gauge TU57 (Profile 305), Tutuila 


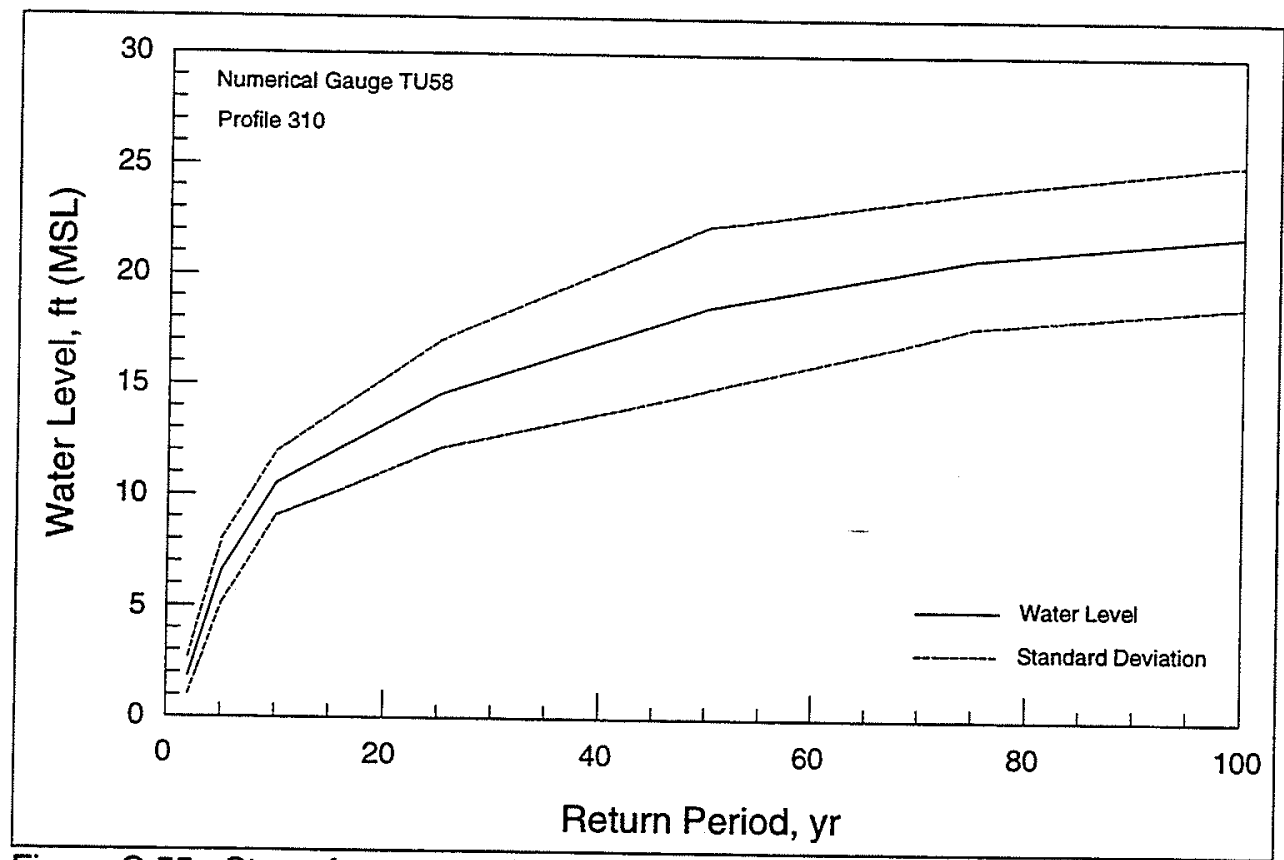

Figure C.55. Stage-frequency plot representing numerical gauge TU58 (Profile 310), Tutuila

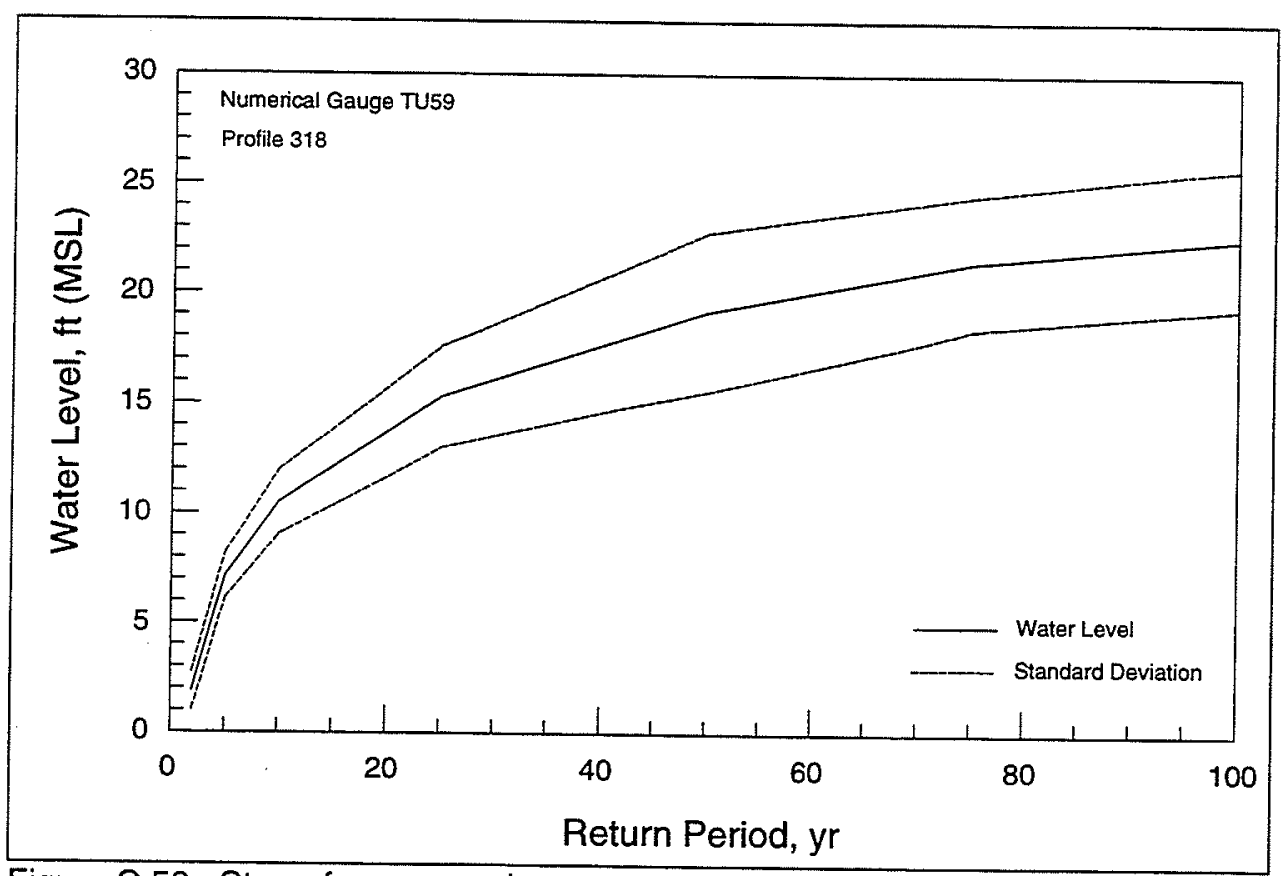

Figure C.56. Stage-frequency plot representing numerical gauge TU59 (Profile 318), Tutuila 


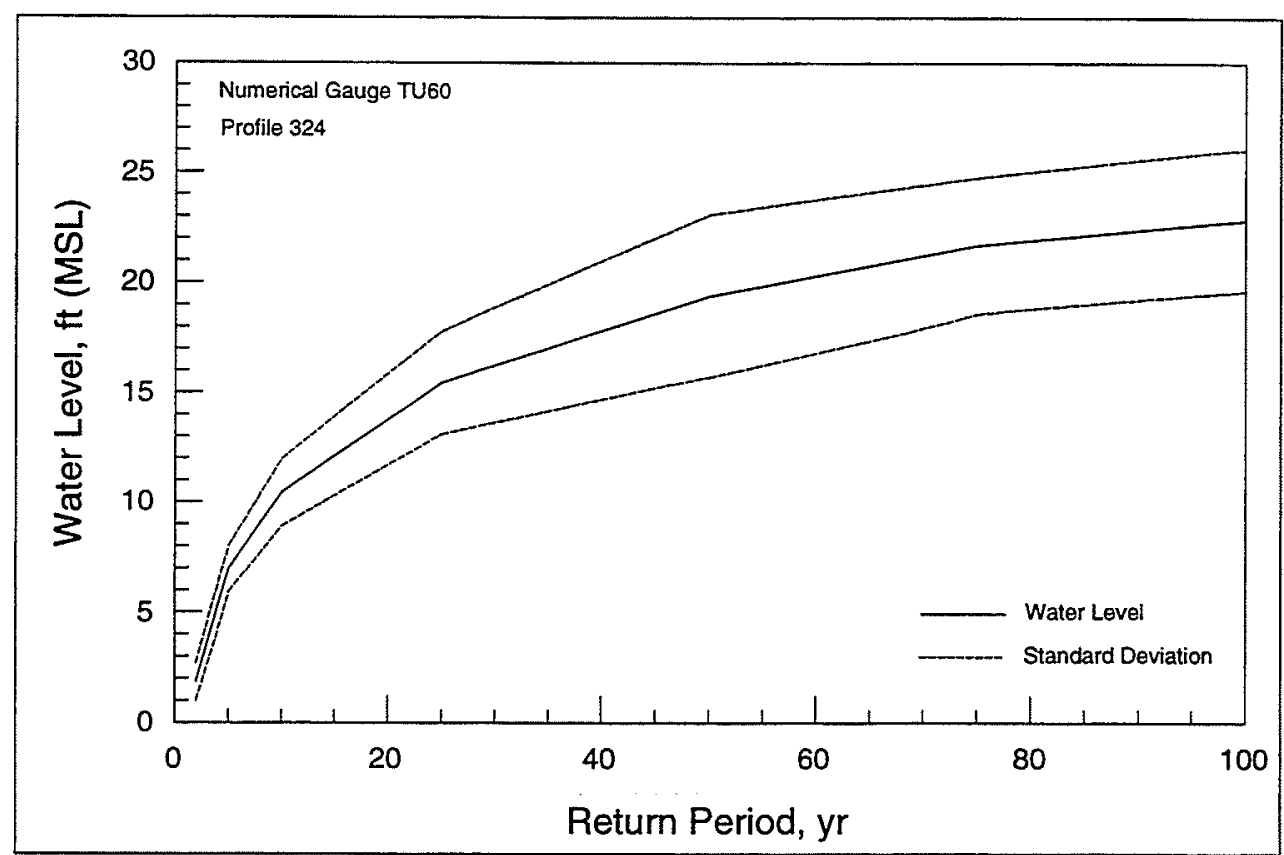

Figure C.57. Stage-frequency plot representing numerical gauge TU60 (Profile 324), Tutuila

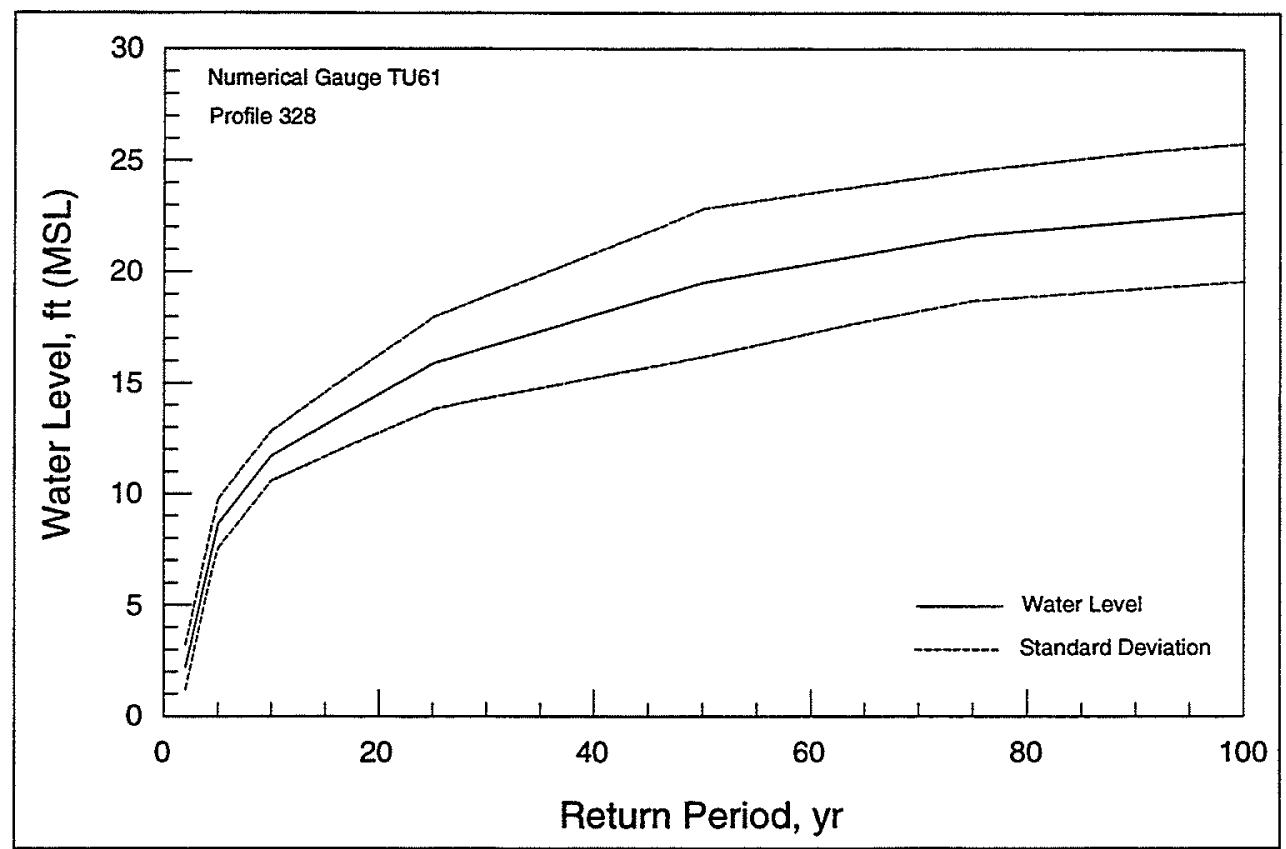

Figure C.58. Stage-frequency plot representing numerical gauge TU61 (Profile 328), Tutuila 


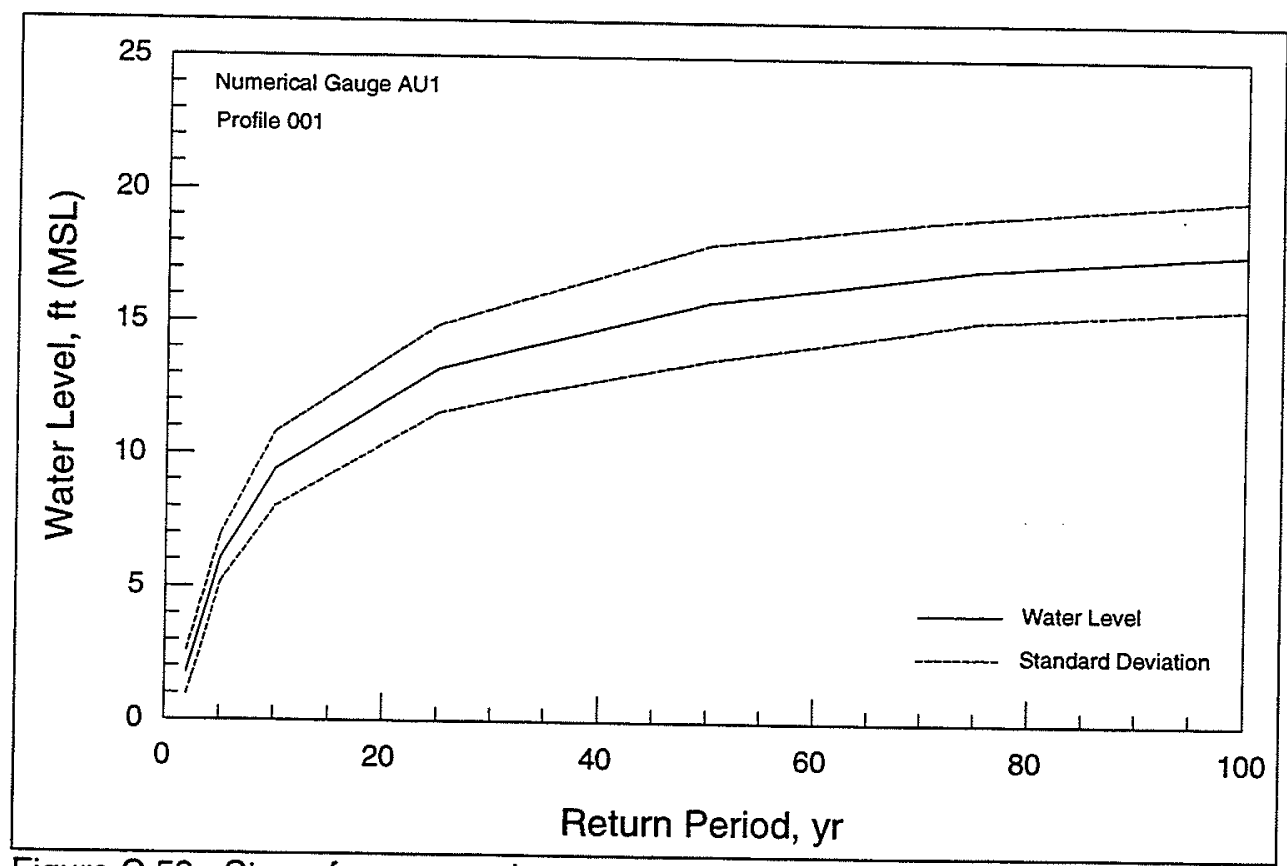

Figure C.59. Stage-frequency plot representing numerical gauge AU1 (Profile 001), Aunuu

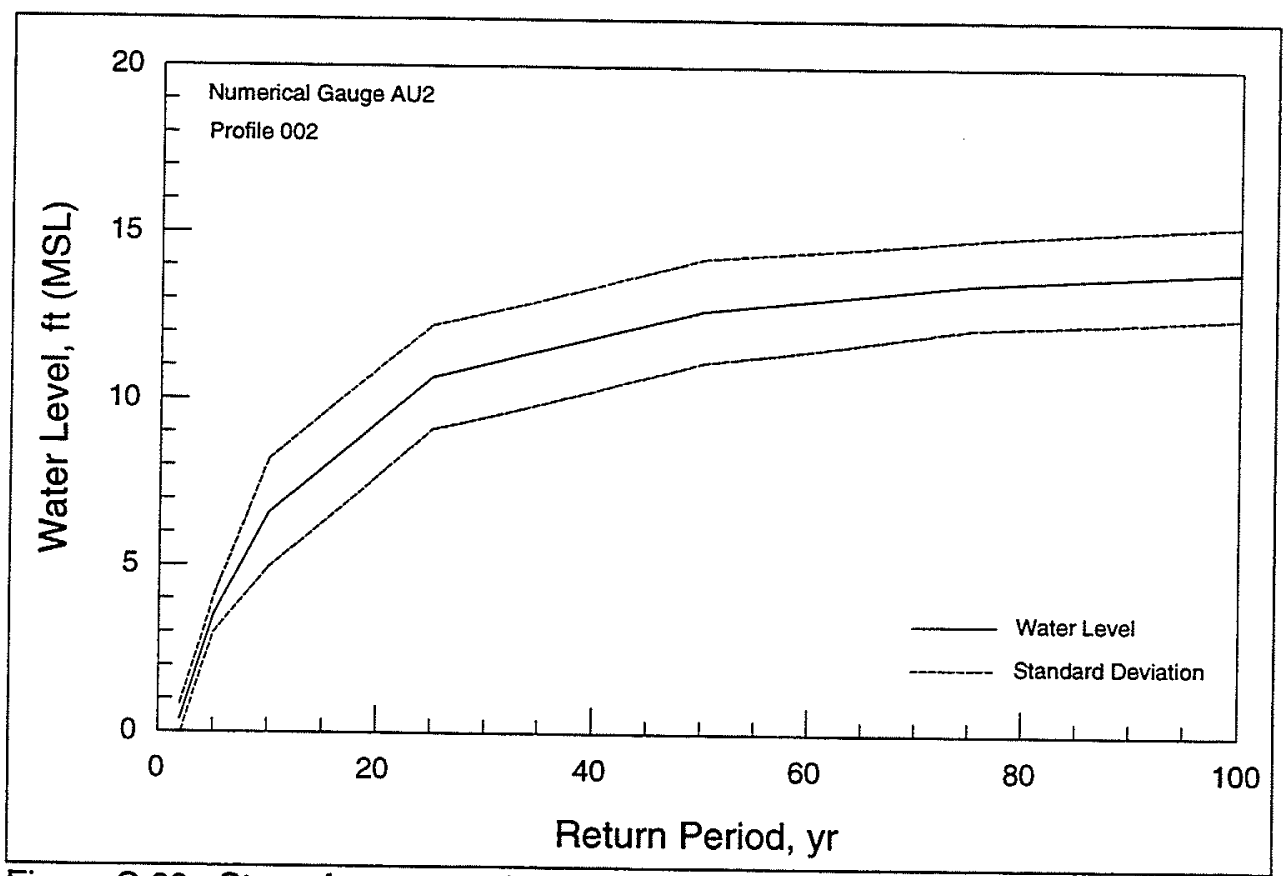

Figure C.60. Stage-frequency plot representing numerical gauge AU2 (Profile 002), Aunuu 


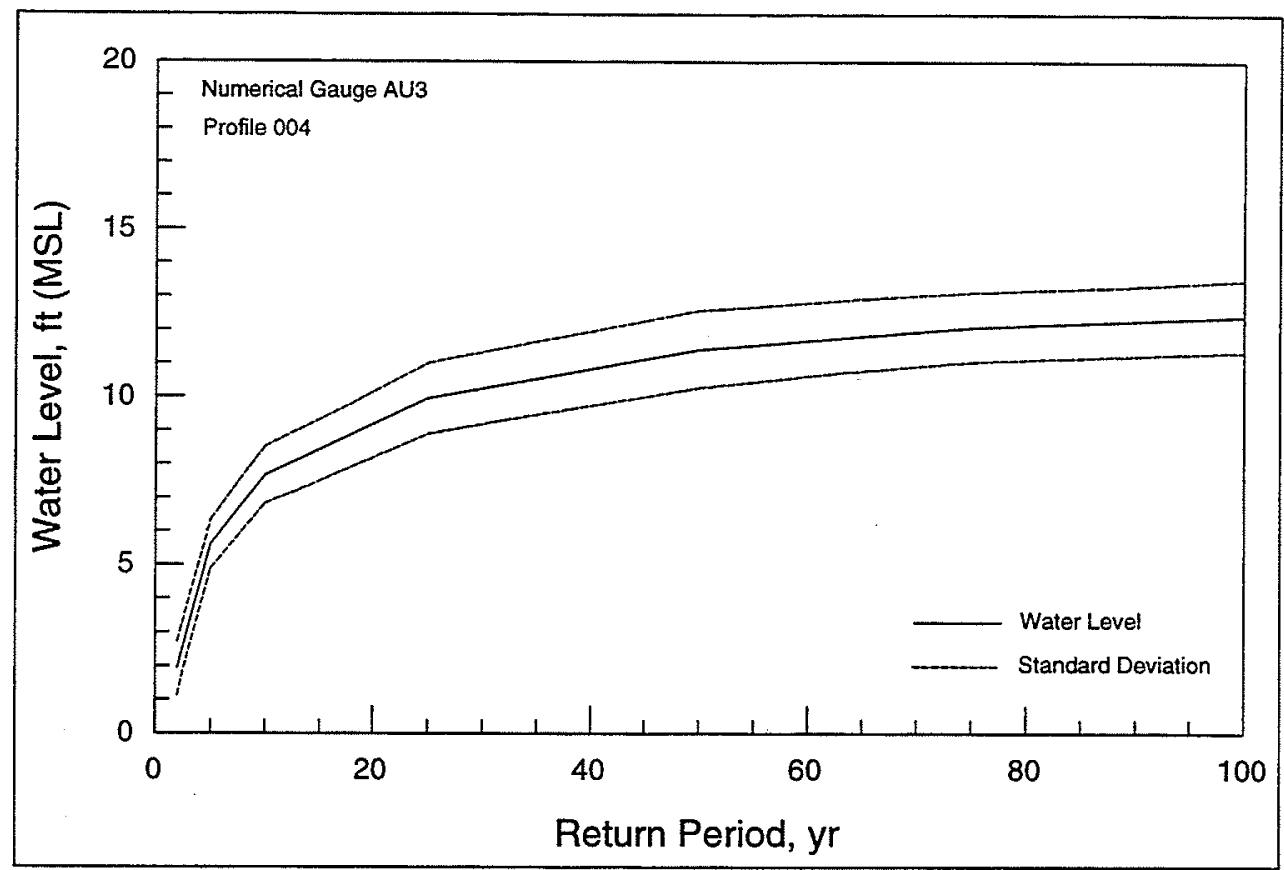

Figure C.61. Stage-frequency plot representing numerical gauge AU3 (Profile 004), Aunuu

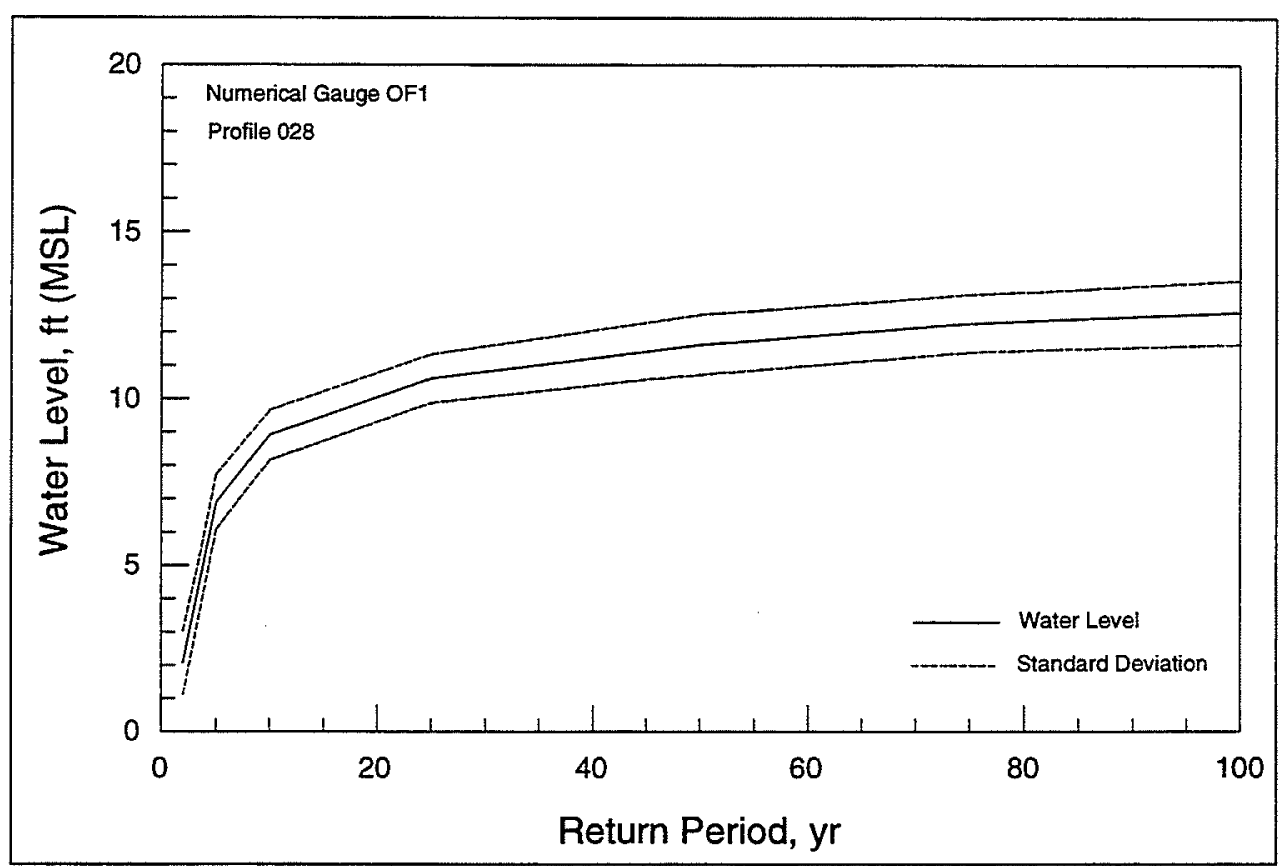

Figure C.62. Stage-frequency plot representing numerical gauge OF1 (Profile 028), Ofu 


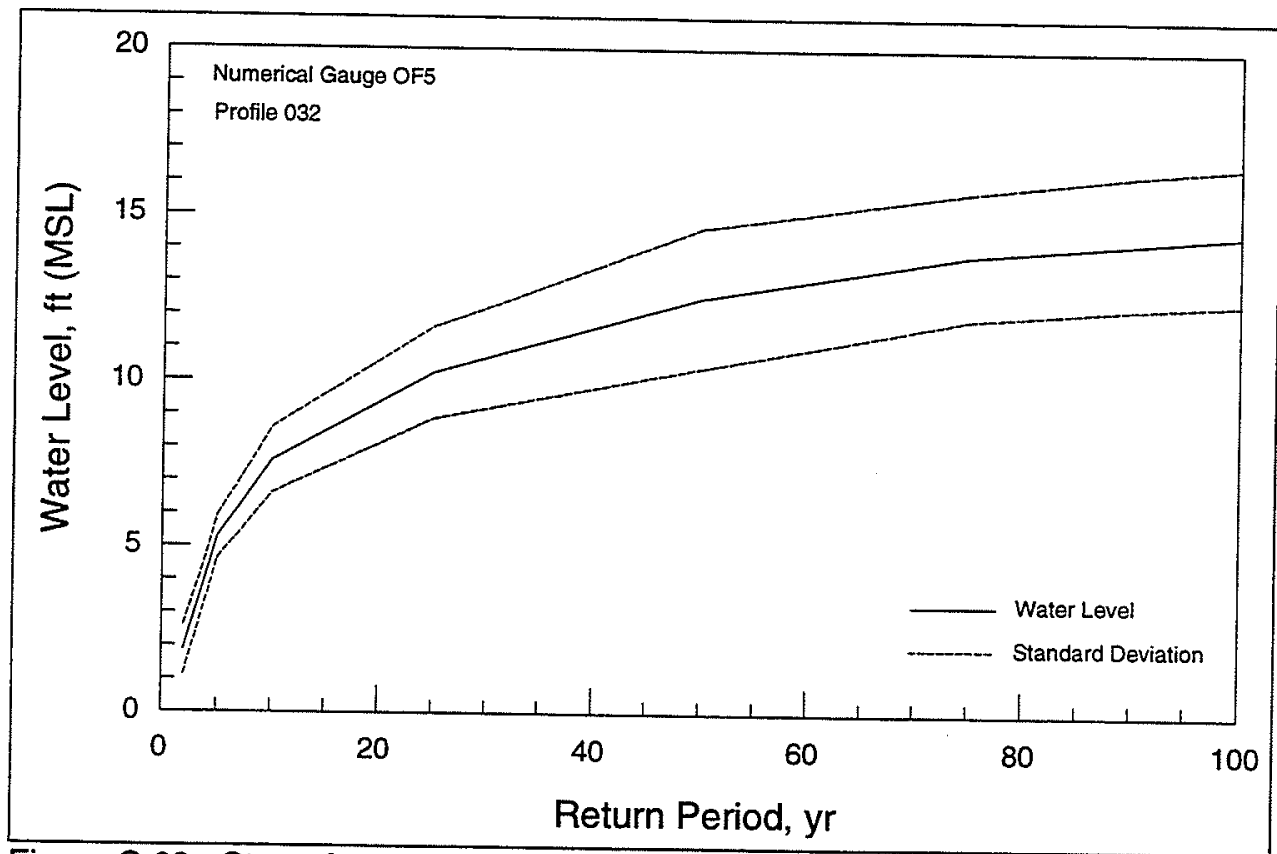

Figure C.63. Stage-frequency plot representing numerical gauge OF5 (Profile 032), Ofu

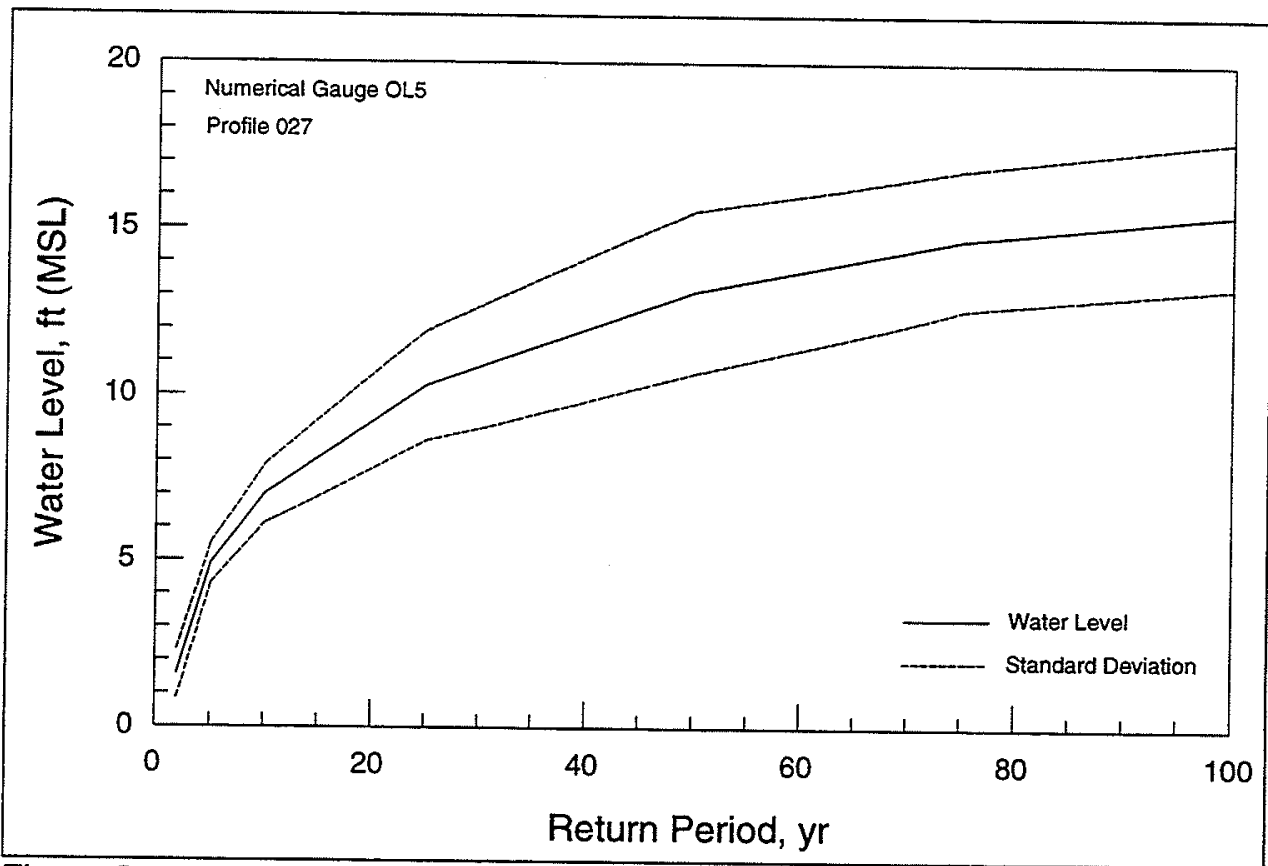

Figure C.64. Stage-frequency plot representing numerical gauge OL5 (Profile 027), Olosega 


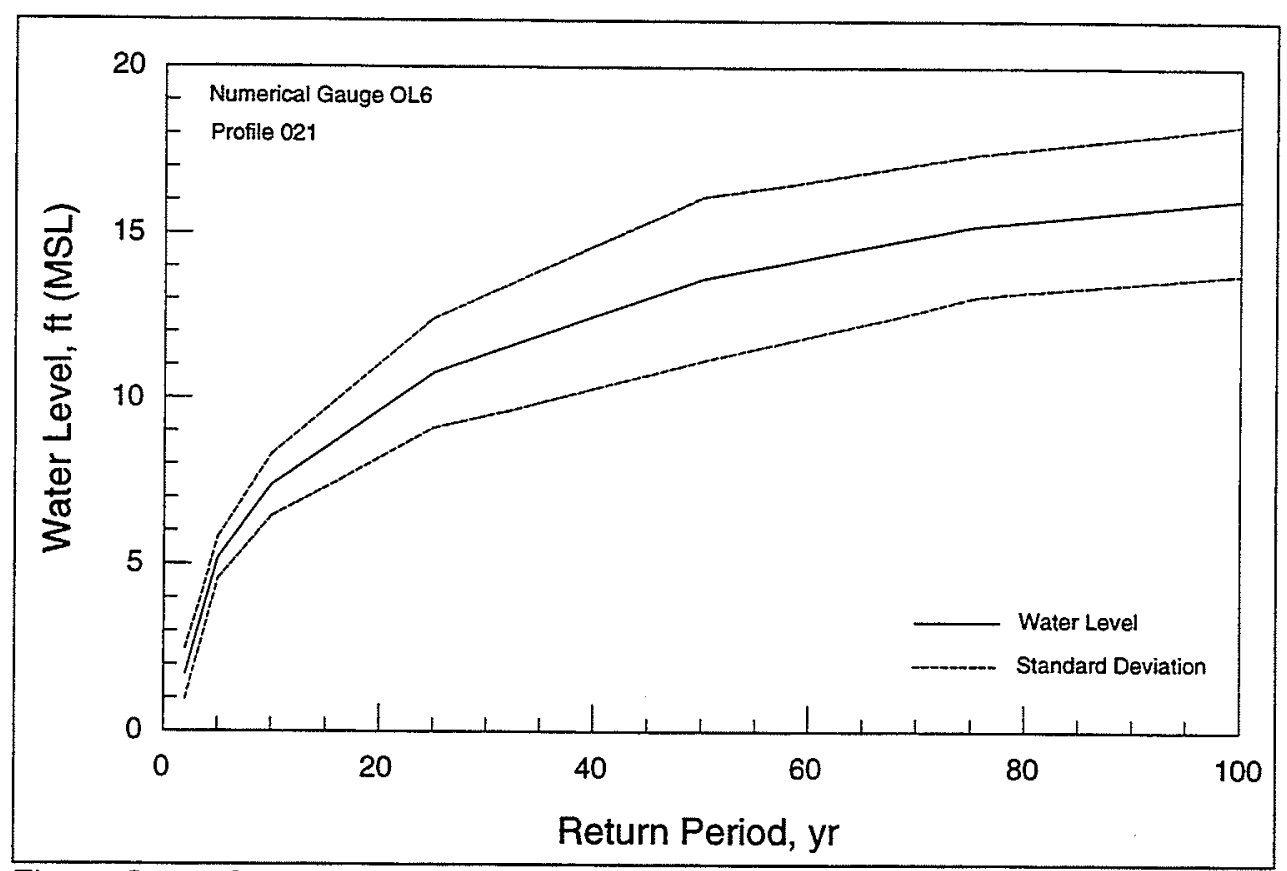

Figure C.65. Stage-frequency plot representing numerical gauge OL6 (Profile 021), Olosega

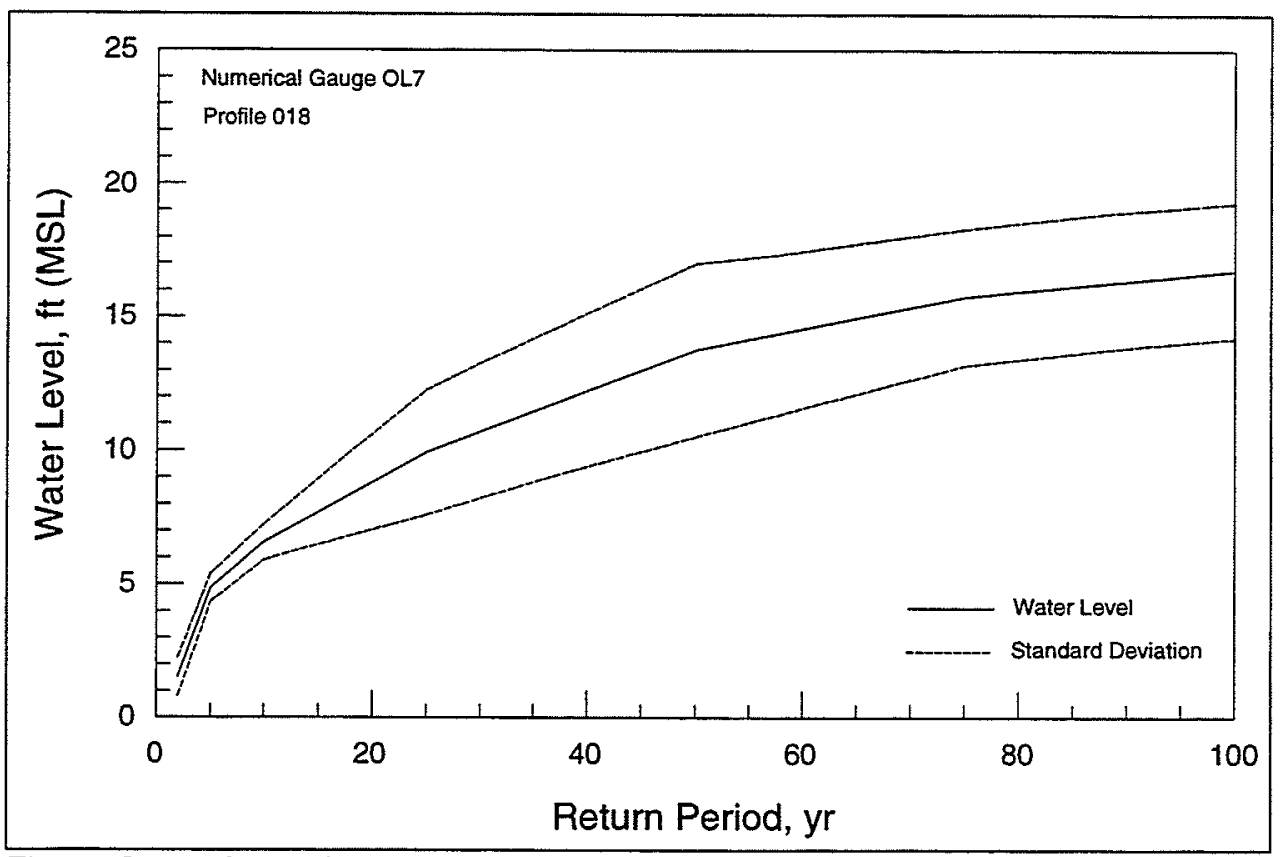

Figure C.66. Stage-frequency plot representing numerical gauge OL7 (Profile 018), Olosega 


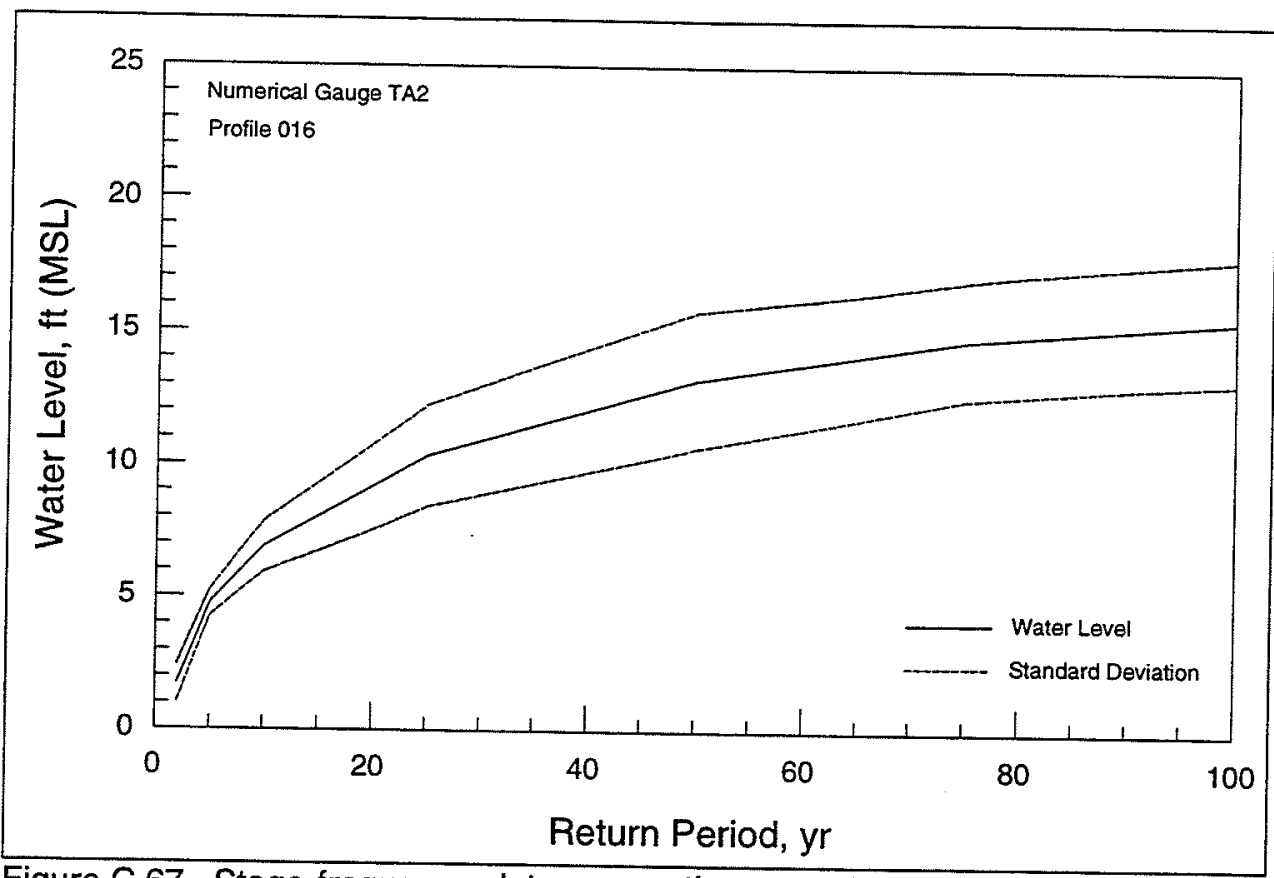

Figure C.67. Stage-frequency plot representing numerical gauge TA2 (Profile 016), Tau

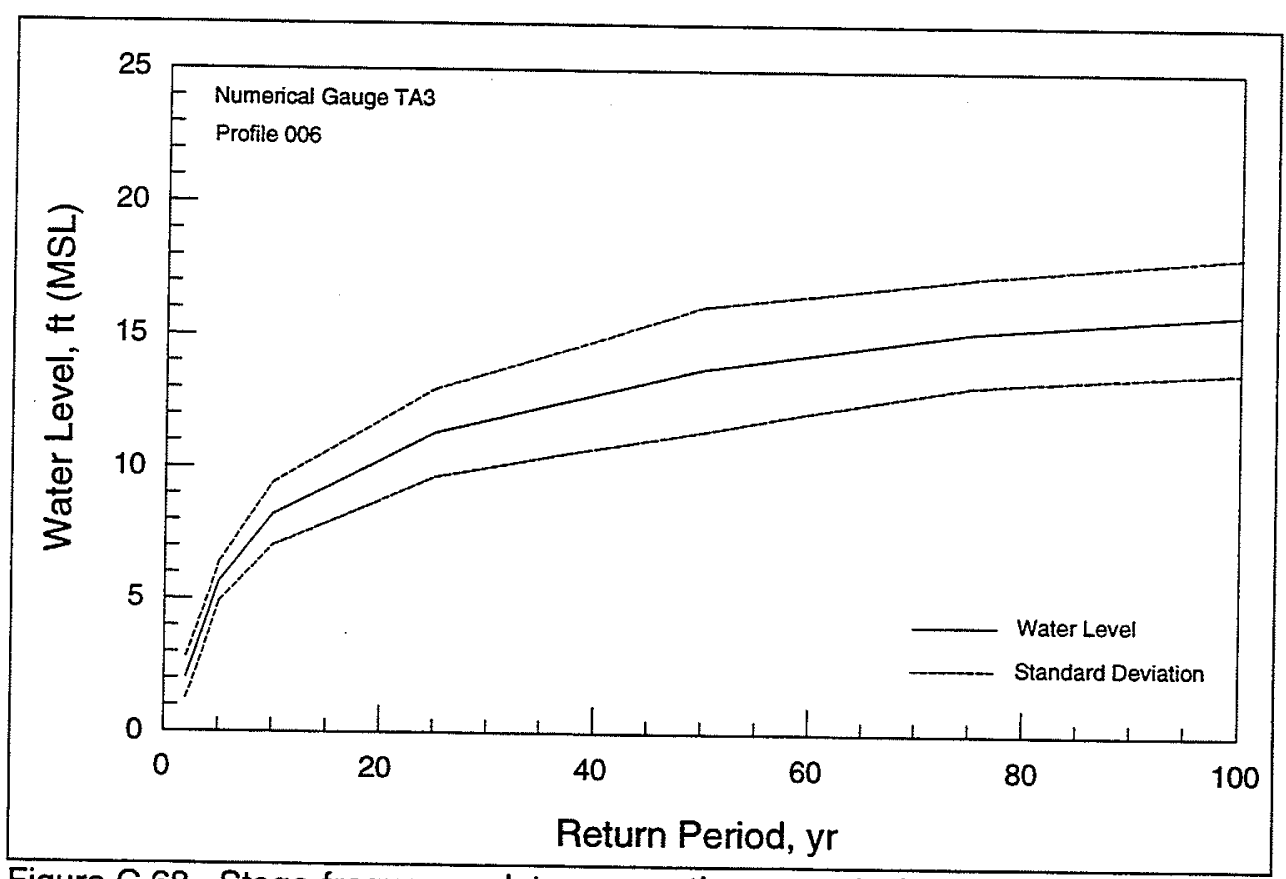

Figure C.68. Stage-frequency plot representing numerical gauge TA3 (Profile 006), Tau 


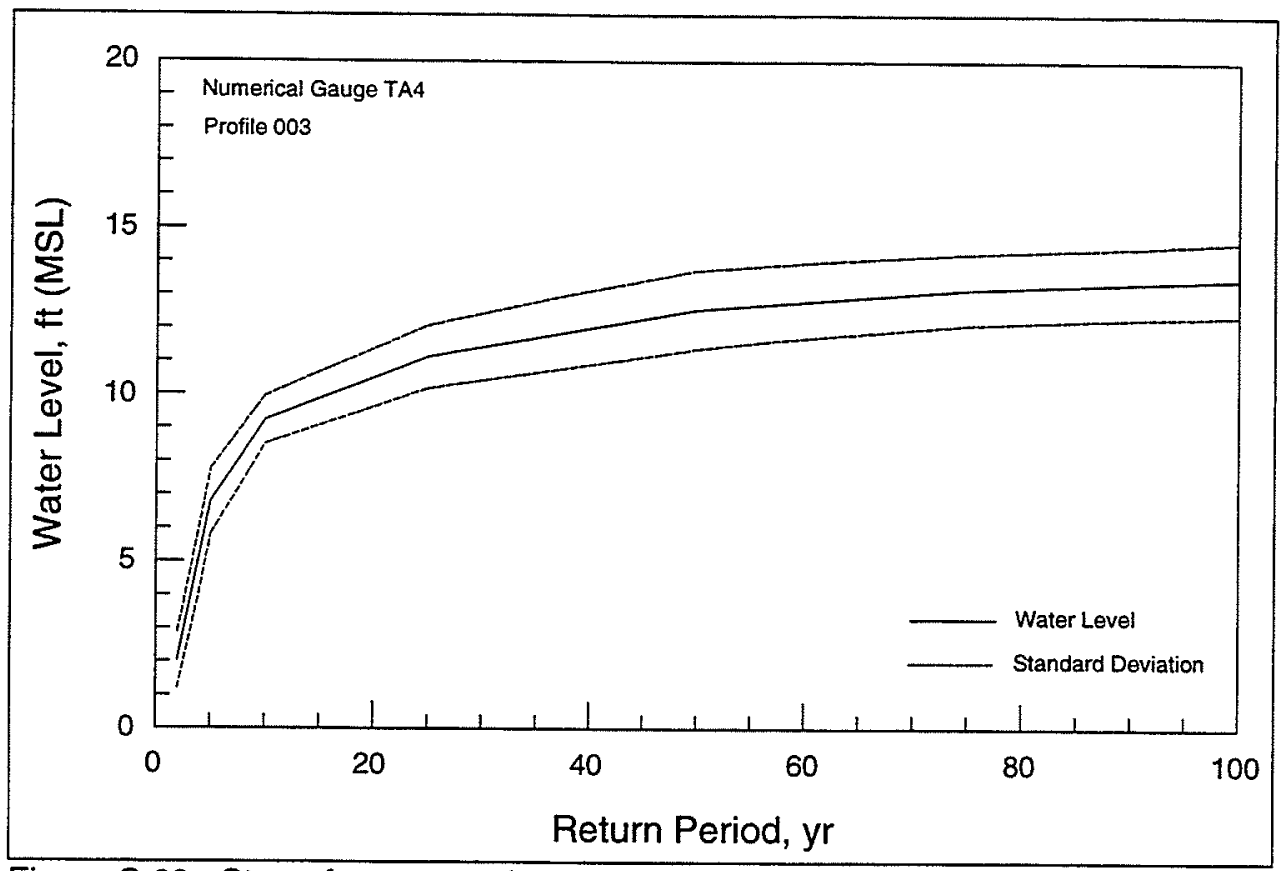

Figure C.69. Stage-frequency plot representing numerical gauge TA4 (Profile 003), Tau 


\section{Appendix D Tables of Wave Parameters, Setup, and Ponding Level at Numerical Gauge Locations}

Tables of wave parameters, setup, and ponding level that correspond to peak water level for each storm are provided for numerical gauge locations associated with profiles. Because setup and ponding level were calculated at profiles rather than for numerical gauge locations, the values given in the tables were taken from profiles representative of each numerical gauge.

Reported wave heights are the deep-water significant wave heights calculated seaward of the reef. At numerical gauge locations within Pago Pago Harbor, reported wave heights may be for transitional waves as discussed in the main text. Additionally, the reported wave heights correspond to peak water level at the representative profile and may not be the maximum wave heights experienced. 


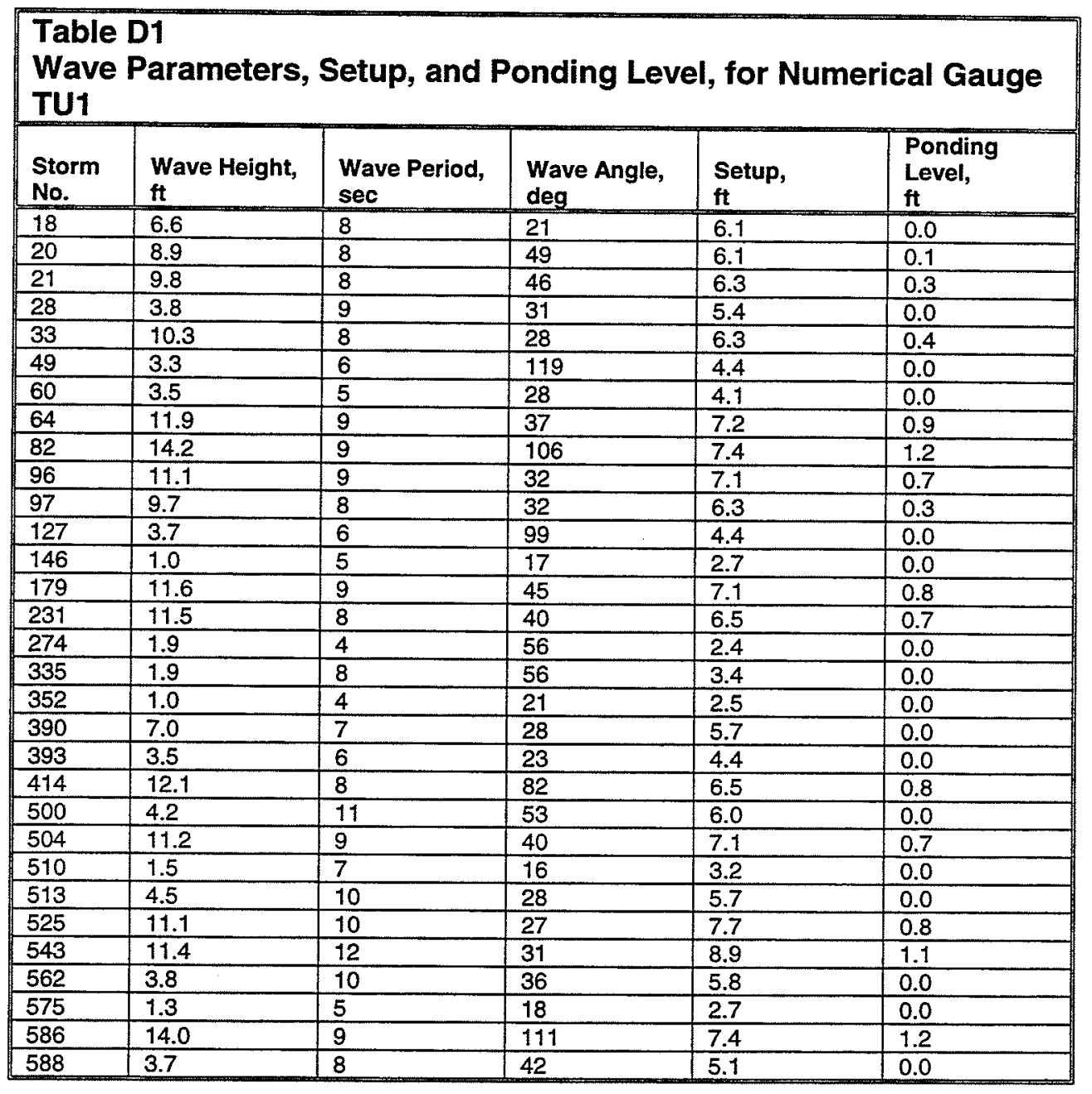




\begin{tabular}{|c|c|c|c|c|c|}
\hline $\begin{array}{l}\text { Storm } \\
\text { No. } \\
\end{array}$ & $\begin{array}{l}\text { Wave Height, } \\
\mathrm{ft}\end{array}$ & $\begin{array}{l}\text { Wave Period, } \\
\text { sec }\end{array}$ & $\begin{array}{l}\text { Wave Angle, } \\
\text { deg }\end{array}$ & $\begin{array}{l}\text { Setup, } \\
\mathrm{ft}\end{array}$ & $\begin{array}{l}\text { Ponding } \\
\text { Level, } \\
\mathrm{ft}\end{array}$ \\
\hline 18 & 6.0 & 8 & 11 & 4.4 & 0.6 \\
\hline 20 & 8.6 & 8 & 32 & 4.7 & 0.1 \\
\hline 21 & 9.4 & 8 & 29 & 6.2 & 1.6 \\
\hline 28 & 4.3 & 9 & 15 & 4.2 & 0.0 \\
\hline 33 & 8.9 & 8 & 14 & 6.1 & 1.5 \\
\hline 49 & 5.7 & 6 & 124 & 3.3 & 0.2 \\
\hline 60 & 1.7 & 5 & 24 & 2.0 & 0.0 \\
\hline 64 & 10.2 & 9 & 24 & 5.6 & 0.5 \\
\hline 82 & 12.2 & 10 & 126 & 6.5 & 1.0 \\
\hline 96 & 12.1 & 8 & 91 & 6.6 & 2.1 \\
\hline 97 & 6.6 & 9 & 24 & 5.1 & 0.0 \\
\hline 127 & 3.4 & 7 & 140 & 3.7 & 0.0 \\
\hline 146 & 0.8 & 5 & 5 & 2.1 & 0.0 \\
\hline 179 & 11.1 & 9 & 28 & 5.7 & 0.7 \\
\hline 231 & 10.8 & 8 & 24 & 6.4 & 1.9 \\
\hline 274 & 1.0 & 4 & 149 & 1.9 & 0.0 \\
\hline 335 & 1.9 & 8 & 38 & 2.6 & 0.0 \\
\hline 352 & 3.3 & 4 & 95 & 2.7 & 0.0 \\
\hline 390 & 6.8 & 7 & 16 & 5.1 & 0.7 \\
\hline 393 & 3.4 & 6 & 9 & 3.3 & 0.0 \\
\hline 414 & 12.1 & 8 & 62 & 6.6 & 2.1 \\
\hline 500 & 4.6 & 11 & 65 & 5.0 & 0.4 \\
\hline 504 & 7.7 & 8 & 15 & 5.9 & 1.2 \\
\hline 510 & 1.5 & 7 & 6 & 2.5 & 0.0 \\
\hline 513 & 6.0 & 10 & 38 & 5.2 & 0.9 \\
\hline 525 & 9.5 & 10 & 13 & 7.5 & 1.8 \\
\hline 543 & 13.2 & 11 & 20 & 7.1 & 1.3 \\
\hline 562 & 3.4 & 10 & 20 & 4.5 & 0.0 \\
\hline 575 & 1.0 & 5 & 5 & 2.1 & 0.0 \\
\hline 586 & 17.7 & 9 & 106 & 7.7 & 3.1 \\
\hline 588 & 5.4 & 8 & 13 & 4.2 & 0.4 \\
\hline
\end{tabular}




\begin{tabular}{|c|c|c|c|c|c|}
\hline \multicolumn{6}{|c|}{$\begin{array}{l}\text { Table D3 } \\
\text { Wave Parameters, Setup, and Ponding Level, for Numerical Gaug } \\
\text { TU3 }\end{array}$} \\
\hline $\begin{array}{l}\text { Storm } \\
\text { No. }\end{array}$ & $\begin{array}{l}\text { Wave Height, } \\
\text { ft }\end{array}$ & $\begin{array}{l}\text { Wave Period, } \\
\text { sec }\end{array}$ & $\begin{array}{l}\text { Wave Angle, } \\
\text { deg }\end{array}$ & $\begin{array}{l}\text { Setup, } \\
\mathrm{ft}\end{array}$ & $\begin{array}{l}\text { Ponding } \\
\text { Level, } \\
\text { ft }\end{array}$ \\
\hline 18 & 6.1 & 8 & 72 & 4.4 & 0.6 \\
\hline 20 & 8.2 & 8 & 91 & 4.7 & 0.0 \\
\hline 21 & 10.2 & 8 & 86 & 6.3 & 1.8 \\
\hline 28 & 3.8 & 9 & 73 & 4.2 & 0.0 \\
\hline 33 & 8.0 & 8 & 72 & 5.9 & 1.2 \\
\hline 49 & 0.6 & 10 & 63 & 3.0 & 0.0 \\
\hline 60 & 1.6 & 5 & 81 & 2.0 & 0.0 \\
\hline 64 & 10.1 & 9 & 79 & 5.6 & 0.5 \\
\hline 82 & 9.5 & 8 & 100 & 4.9 & 0.3 \\
\hline 96 & 9.5 & 9 & 77 & 6.8 & 1.7 \\
\hline 97 & 7.2 & 8 & 76 & 5.1 & 0.0 \\
\hline 127 & 3.9 & 8 & 90 & 3.9 & 0.0 \\
\hline 146 & 0.9 & 5 & 66 & 2.1 & 0.0 \\
\hline 179 & 10.0 & 9 & 85 & 5.6 & 0.5 \\
\hline 231 & 6.6 & 9 & 73 & 5.1 & 0.0 \\
\hline 274 & 2.4 & 4 & 90 & 1.7 & 0.0 \\
\hline 335 & 2.1 & 8 & 90 & 2.6 & 0.0 \\
\hline 352 & 1.2 & 4 & 71 & 1.8 & 0.0 \\
\hline 390 & 6.6 & 7 & 75 & 5.0 & 0.7 \\
\hline 393 & 1.6 & 6 & 66 & 2.3 & 0.0 \\
\hline 414 & 10.7 & 8 & 88 & 5.1 & 0.5 \\
\hline 500 & 8.9 & 11 & 82 & 7.9 & 1.8 \\
\hline 504 & 7.6 & 8 & 74 & 5.8 & 1.1 \\
\hline 510 & 1.0 & 7 & 64 & 2.5 & 0.0 \\
\hline 513 & 6.7 & 10 & 78 & 6.8 & 1.1 \\
\hline 525 & 7.1 & 10 & 69 & 5.4 & 0.0 \\
\hline 543 & 11.5 & 11 & 78 & 6.9 & 1.0 \\
\hline 562 & 6.0 & 11 & 84 & 5.6 & 0.9 \\
\hline 575 & 0.5 & 5 & 63 & 2.1 & 0.0 \\
\hline 586 & 14.5 & 9 & 91 & 7.5 & 2.7 \\
\hline 588 & 4.0 & 8 & 69 & 3.9 & 0.0 \\
\hline
\end{tabular}




\begin{tabular}{|c|c|c|c|c|c|}
\hline $\begin{array}{l}\text { Storm } \\
\text { No. }\end{array}$ & $\begin{array}{l}\text { Wave Height, } \\
\mathrm{ft}\end{array}$ & $\begin{array}{l}\text { Wave Period, } \\
\text { sec }\end{array}$ & $\begin{array}{l}\text { Wave Angle, } \\
\text { deg }\end{array}$ & $\begin{array}{l}\text { Setup, } \\
\mathrm{ft}\end{array}$ & $\begin{array}{l}\text { Ponding } \\
\text { Level, } \\
\mathrm{ft}\end{array}$ \\
\hline 18 & 4.7 & 8 & 64 & 4.0 & 0.1 \\
\hline 20 & 6.6 & 7 & 74 & 4.4 & 0.0 \\
\hline 21 & 5.3 & 8 & 61 & 4.2 & 0.3 \\
\hline 28 & 3.3 & 5 & 73 & 3.0 & 0.0 \\
\hline 33 & 8.5 & 9 & 100 & 5.2 & 0.2 \\
\hline 49 & 3.8 & 6 & 105 & 3.3 & 0.0 \\
\hline 60 & 0.8 & 7 & 70 & 2.5 & 0.0 \\
\hline 64 & 4.1 & 9 & 60 & 4.2 & 0.0 \\
\hline 82 & 8.7 & 9 & 78 & 6.6 & 1.5 \\
\hline 96 & 11.6 & 8 & 88 & 6.5 & 2.0 \\
\hline 97 & 7.6 & 7 & 93 & 4.3 & 0.0 \\
\hline 127 & 1.4 & 9 & 61 & 2.8 & 0.0 \\
\hline 146 & 1.3 & 5 & 62 & 2.0 & 0.0 \\
\hline 179 & 6.8 & 8 & 65 & 4.8 & 0.0 \\
\hline 231 & 4.4 & 8 & 64 & 3.9 & 0.0 \\
\hline 274 & 1.2 & 5 & 61 & 2.0 & 0.0 \\
\hline 335 & 0.9 & 8 & 61 & 2.7 & 0.0 \\
\hline 352 & 1.1 & 4 & 58 & 1.8 & 0.0 \\
\hline 390 & 2.5 & 7 & 59 & 2.4 & 0.0 \\
\hline 393 & 1.0 & 5 & 55 & 2.1 & 0.0 \\
\hline 414 & 10.1 & 8 & 81 & 6.3 & 1.7 \\
\hline 500 & 1.0 & 11 & 55 & 3.1 & 0.0 \\
\hline 504 & 7.4 & 7 & 66 & 4.3 & 0.0 \\
\hline 510 & 1.0 & 7 & 61 & 2.5 & 0.0 \\
\hline 513 & 3.9 & 10 & 61 & 4.5 & 0.0 \\
\hline 525 & 7.2 & 7 & 93 & 4.3 & 0.0 \\
\hline 543 & 10.3 & 7 & 93 & 4.4 & 0.3 \\
\hline 562 & 0.9 & 11 & 56 & 3.1 & 0.0 \\
\hline 575 & 1.5 & 5 & 69 & 2.0 & 0.0 \\
\hline 586 & 12.5 & 9 & 98 & 7.3 & 2.3 \\
\hline 588 & 5.7 & 6 & 93 & 3.3 & 0.2 \\
\hline
\end{tabular}




\begin{tabular}{|c|c|c|c|c|c|}
\hline \multicolumn{6}{|c|}{$\begin{array}{l}\text { Table D5 } \\
\text { Wave Parameters, Setup, and Ponding Level, for Numerical Gauge } \\
\text { TU5 }\end{array}$} \\
\hline $\begin{array}{l}\text { Storm } \\
\text { No. }\end{array}$ & $\begin{array}{l}\text { Wave Height, } \\
\mathrm{ft}\end{array}$ & $\begin{array}{l}\text { Wave Period, } \\
\text { sec }\end{array}$ & $\begin{array}{l}\text { Wave Angle, } \\
\text { deg }\end{array}$ & $\begin{array}{l}\text { Setup, } \\
\mathrm{ft}\end{array}$ & $\begin{array}{l}\text { Ponding } \\
\text { Level, } \\
\mathrm{ft}\end{array}$ \\
\hline 18 & 4.7 & 8 & 64 & 4.0 & 0.1 \\
\hline 20 & 6.6 & 7 & 74 & 4.3 & 0.0 \\
\hline 21 & 5.3 & 8 & 61 & 4.2 & 0.3 \\
\hline 28 & 5.0 & 5 & 84 & 2.9 & 0.0 \\
\hline 33 & 8.5 & 9 & 100 & 5.2 & 0.2 \\
\hline 49 & 3.8 & 6 & 105 & 3.3 & 0.0 \\
\hline 60 & 0.8 & 7 & 70 & 2.5 & 0.0 \\
\hline 64 & 4.1 & 9 & 60 & 4.2 & 0.0 \\
\hline 82 & 8.7 & 9 & 78 & 6.6 & 1.5 \\
\hline 96 & 11.6 & 8 & 88 & 6.5 & 2.0 \\
\hline 97 & 7.5 & 7 & 93 & 4.3 & 0.0 \\
\hline 127 & 1.4 & 9 & 61 & 2.8 & 0.0 \\
\hline 146 & 1.6 & 5 & 63 & 2.0 & 0.0 \\
\hline 179 & 6.6 & 8 & 65 & 4.8 & 0.0 \\
\hline 231 & 4.4 & 8 & 64 & 3.9 & 0.0 \\
\hline 274 & 1.2 & 5 & 61 & 2.1 & 0.0 \\
\hline 335 & 0.9 & 8 & 61 & 2.7 & 0.0 \\
\hline 352 & 1.1 & 4 & 58 & 1.8 & 0.0 \\
\hline 390 & 1.5 & 7 & 60 & 2.5 & 0.0 \\
\hline 393 & 1.0 & 5 & 54 & 2.1 & 0.0 \\
\hline 414 & 10.1 & 8 & 81 & 6.3 & 1.7 \\
\hline 500 & 1.0 & 11 & 55 & 3.1 & 0.0 \\
\hline 504 & 7.4 & 7 & 66 & 4.3 & 0.0 \\
\hline 510 & 1.0 & 7 & 61 & 2.5 & 0.0 \\
\hline 513 & 4.0 & 10 & 61 & 4.5 & 0.0 \\
\hline 525 & 7.2 & 7 & 93 & 4.3 & 0.0 \\
\hline 543 & 10.3 & 7 & 93 & 4.4 & 0.3 \\
\hline 562 & 0.9 & 11 & 54 & 3.1 & 0.0 \\
\hline 575 & 0.9 & 5 & 58 & 2.1 & 0.0 \\
\hline 586 & 12.5 & 9 & 98 & 7.3 & 2.3 \\
\hline 588 & 5.7 & 6 & 93 & 3.3 & 0.2 \\
\hline
\end{tabular}




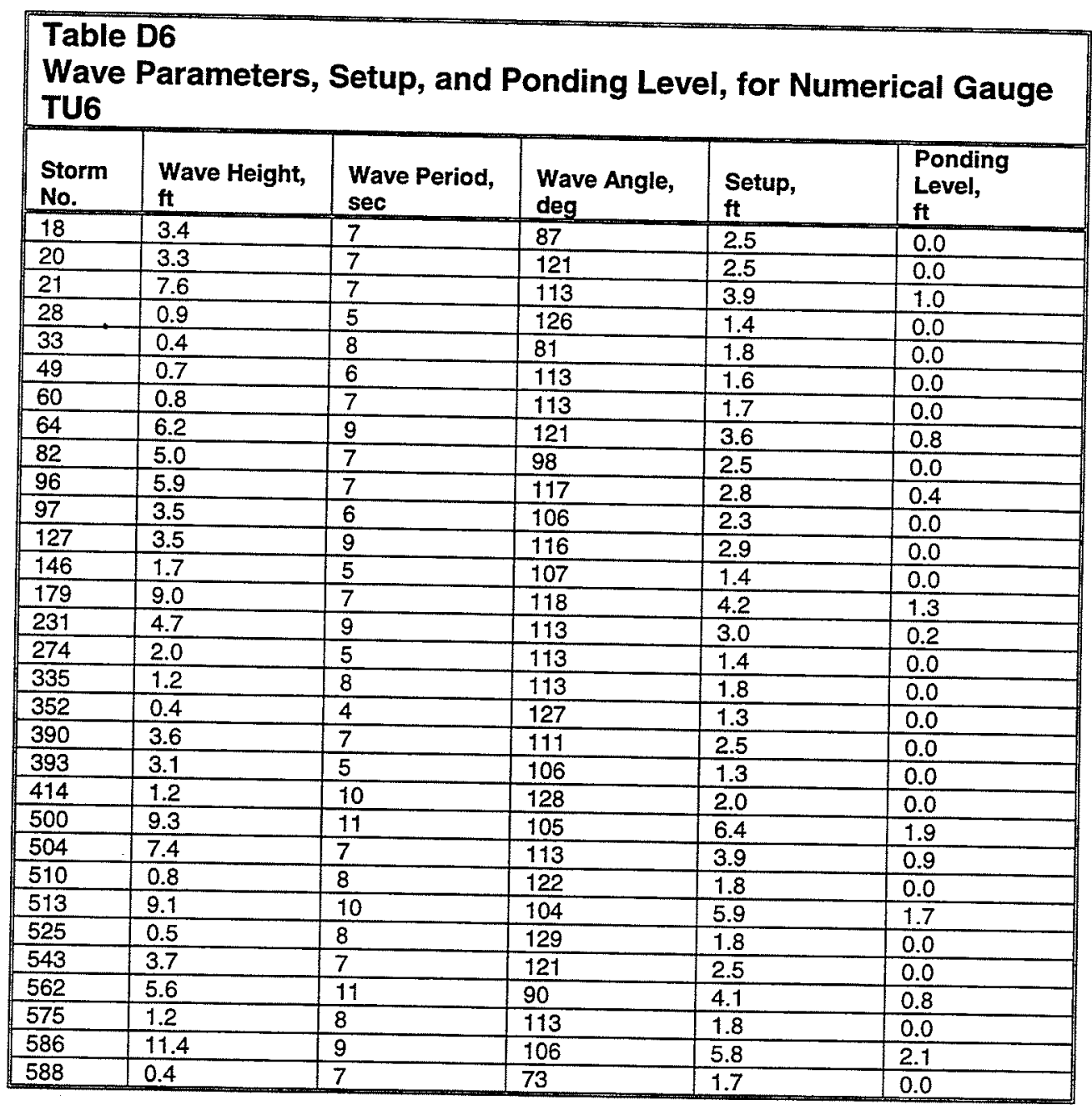




\begin{tabular}{|c|c|c|c|c|c|}
\hline \multicolumn{6}{|c|}{$\begin{array}{l}\text { Table D7 } \\
\text { Wave Parameters, Setup, and Ponding Level, for Numerical Gaug } \\
\text { TU7 }\end{array}$} \\
\hline $\begin{array}{l}\text { Storm } \\
\text { No. }\end{array}$ & $\begin{array}{l}\text { Wave Height, } \\
\mathrm{ft}\end{array}$ & $\begin{array}{l}\text { Wave Period, } \\
\text { sec }\end{array}$ & $\begin{array}{l}\text { Wave Angle, } \\
\text { deg }\end{array}$ & $\begin{array}{l}\text { Setup, } \\
\mathrm{ft}\end{array}$ & $\begin{array}{l}\text { Ponding } \\
\text { Level, } \\
\mathrm{tt}\end{array}$ \\
\hline 18 & 5.3 & 8 & 85 & 2.2 & 0.3 \\
\hline 20 & 6.9 & 6 & 89 & 2.5 & 0.6 \\
\hline 21 & 6.0 & 8 & 82 & 2.4 & 0.6 \\
\hline 28 & 3.4 & 5 & 89 & 1.5 & 0.0 \\
\hline 33 & 3.4 & 8 & 92 & 2.0 & 0.0 \\
\hline 49 & 1.6 & 6 & 95 & 1.2 & 0.0 \\
\hline 60 & 0.6 & 7 & 82 & 1.3 & 0.0 \\
\hline 64 & 6.0 & 8 & 85 & 2.4 & 0.6 \\
\hline 82 & 8.8 & 9 & 91 & 4.0 & 1.6 \\
\hline 96 & 7.9 & 8 & 89 & 3.4 & 1.2 \\
\hline 97 & 3.3 & 6 & 94 & 1.7 & 0.0 \\
\hline 127 & 1.2 & 9 & 78 & 1.5 & 0.0 \\
\hline 146 & 1.6 & 5 & 84 & 1.0 & 0.0 \\
\hline 179 & 7.9 & 8 & 87 & 3.4 & 1.2 \\
\hline 231 & 5.8 & 9 & 85 & 2.6 & 0.6 \\
\hline 274 & 1.5 & 5 & 82 & 1.0 & 0.0 \\
\hline 335 & 1.0 & 8 & 82 & 1.4 & 0.0 \\
\hline 352 & 1.5 & 4 & 91 & 0.9 & 0.0 \\
\hline 390 & 3.5 & 6 & 79 & 1.7 & 0.0 \\
\hline 393 & 1.2 & 6 & 78 & 1.2 & 0.0 \\
\hline 414 & 7.2 & 9 & 91 & 3.6 & 1.1 \\
\hline 500 & 4.4 & 10 & $\pi$ & 2.4 & 0.2 \\
\hline 504 & 6.4 & 8 & 85 & 2.5 & 0.7 \\
\hline 510 & 1.8 & 8 & 84 & 1.3 & 0.0 \\
\hline 513 & 3.6 & 10 & 77 & 2.3 & 0.0 \\
\hline 525 & 5.2 & 7 & 91 & 1.9 & 0.1 \\
\hline 543 & 7.1 & 7 & 88 & 2.9 & 0.8 \\
\hline 562 & 1.7 & 11 & 77 & 1.7 & 0.0 \\
\hline 575 & 1.0 & 8 & 82 & 1.4 & 0.0 \\
\hline 586 & 11.7 & 10 & 87 & 4.8 & 2.3 \\
\hline 588 & 3.4 & 6 & 91 & 1.7 & 0.0 \\
\hline
\end{tabular}




\begin{tabular}{|c|c|c|c|c|c|}
\hline \multicolumn{6}{|c|}{$\begin{array}{l}\text { Table D8 } \\
\text { Wave Parameters, Setup, and Ponding Level, for Numerical Gauge } \\
\text { TU8 }\end{array}$} \\
\hline $\begin{array}{l}\text { Storm } \\
\text { No. } \\
\end{array}$ & $\begin{array}{l}\text { Wave Height, } \\
\mathrm{ft}\end{array}$ & $\begin{array}{l}\text { Wave Period, } \\
\text { sec }\end{array}$ & $\begin{array}{l}\text { Wave Angle, } \\
\text { deg }\end{array}$ & $\begin{array}{l}\text { Setup, } \\
\mathrm{tt} \\
\end{array}$ & $\begin{array}{l}\text { Ponding } \\
\text { Level, } \\
\text { ft }\end{array}$ \\
\hline 18 & 1.5 & 9 & 166 & 1.9 & 0.0 \\
\hline 20 & 0.9 & 7 & 172 & 1.7 & 0.0 \\
\hline 21 & 1.2 & 8 & 167 & 1.8 & 0.0 \\
\hline 28 & 0.5 & 5 & 163 & 1.4 & 0.0 \\
\hline 33 & 0.3 & 8 & 93 & 1.8 & 0.0 \\
\hline 49 & 1.2 & 6 & 118 & 1.6 & 0.0 \\
\hline 60 & 0.8 & 7 & 168 & 1.7 & 0.0 \\
\hline 64 & 1.2 & 9 & 166 & 1.9 & 0.0 \\
\hline 82 & 0.8 & 8 & 173 & 1.8 & 0.0 \\
\hline 96 & 0.6 & 8 & 175 & 1.8 & 0.0 \\
\hline 97 & 1.1 & 7 & 168 & 1.7 & 0.0 \\
\hline 127 & 0.4 & 25 & 101 & 2.9 & 0.0 \\
\hline 146 & 1.0 & 6 & 103 & 1.6 & 0.0 \\
\hline 179 & 1.2 & 10 & 167 & 2.0 & 0.0 \\
\hline 231 & 1.2 & 8 & 167 & 1.8 & 0.0 \\
\hline 274 & 0.5 & 25 & 108 & 2.9 & 0.0 \\
\hline 335 & 0.9 & 8 & 115 & 1.8 & 0.0 \\
\hline 352 & 0.9 & 4 & 171 & 1.3 & 0.0 \\
\hline 390 & 0.7 & 7 & 169 & 1.7 & 0.0 \\
\hline 393 & 0.5 & 25 & 106 & 2.9 & 0.0 \\
\hline 414 & 1.7 & 9 & 116 & 1.9 & 0.0 \\
\hline 500 & 0.7 & 25 & 135 & 2.9 & 0.0 \\
\hline 504 & 1.0 & 9 & 170 & 1.9 & 0.0 \\
\hline 510 & 1.3 & 8 & 109 & 1.8 & 0.0 \\
\hline 513 & 1.3 & 10 & 98 & 2.0 & 0.0 \\
\hline 525 & 0.7 & 7 & 96 & 1.7 & 0.0 \\
\hline 543 & 0.8 & 7 & 172 & 1.7 & 0.0 \\
\hline 562 & 1.0 & 11 & 100 & 2.2 & 0.0 \\
\hline 575 & 1.0 & 8 & 168 & 1.8 & 0.0 \\
\hline 586 & 0.6 & 25 & 115 & 2.9 & 0.0 \\
\hline 588 & 0.9 & 8 & 99 & 1.8 & 0.0 \\
\hline
\end{tabular}




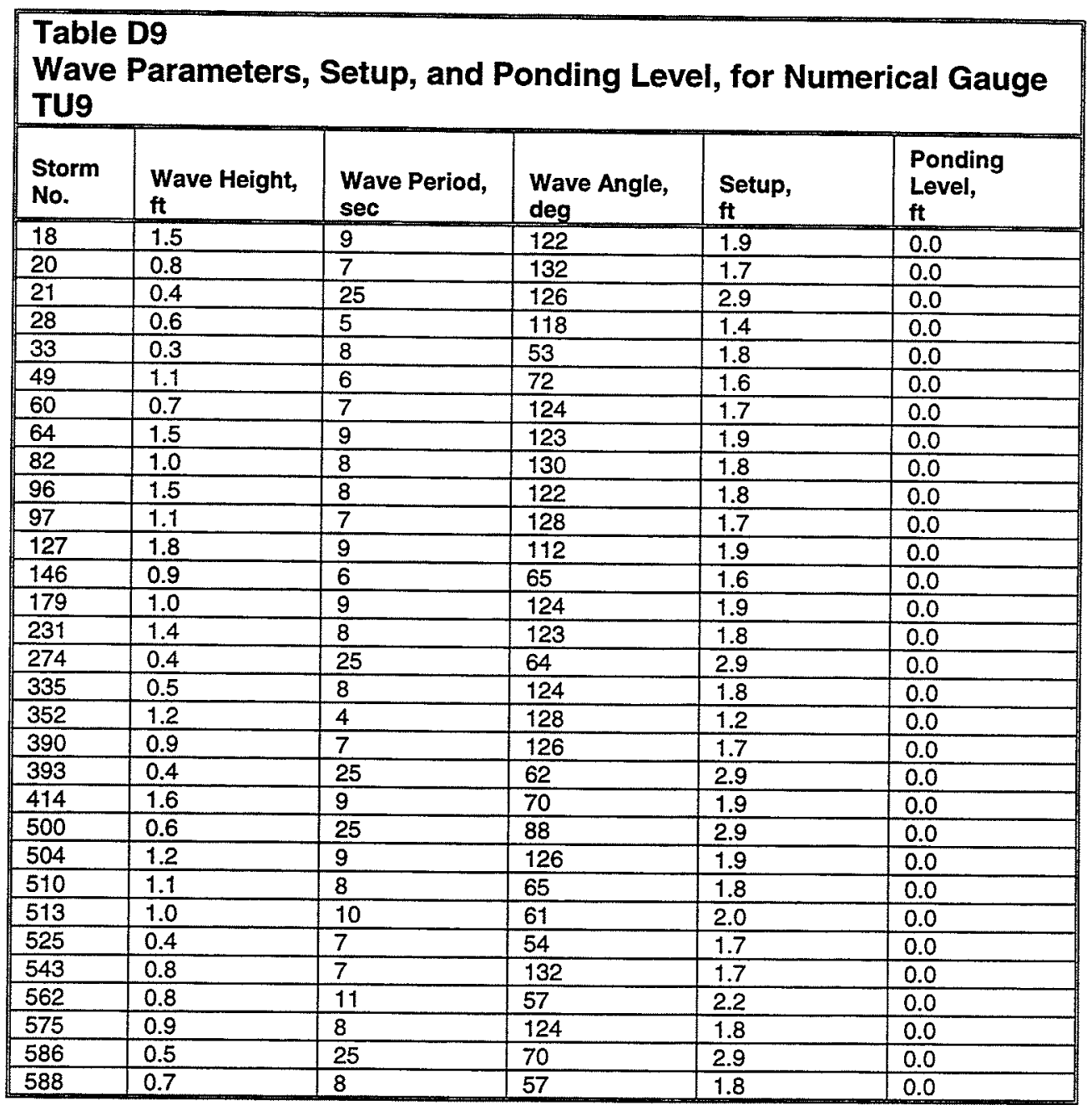




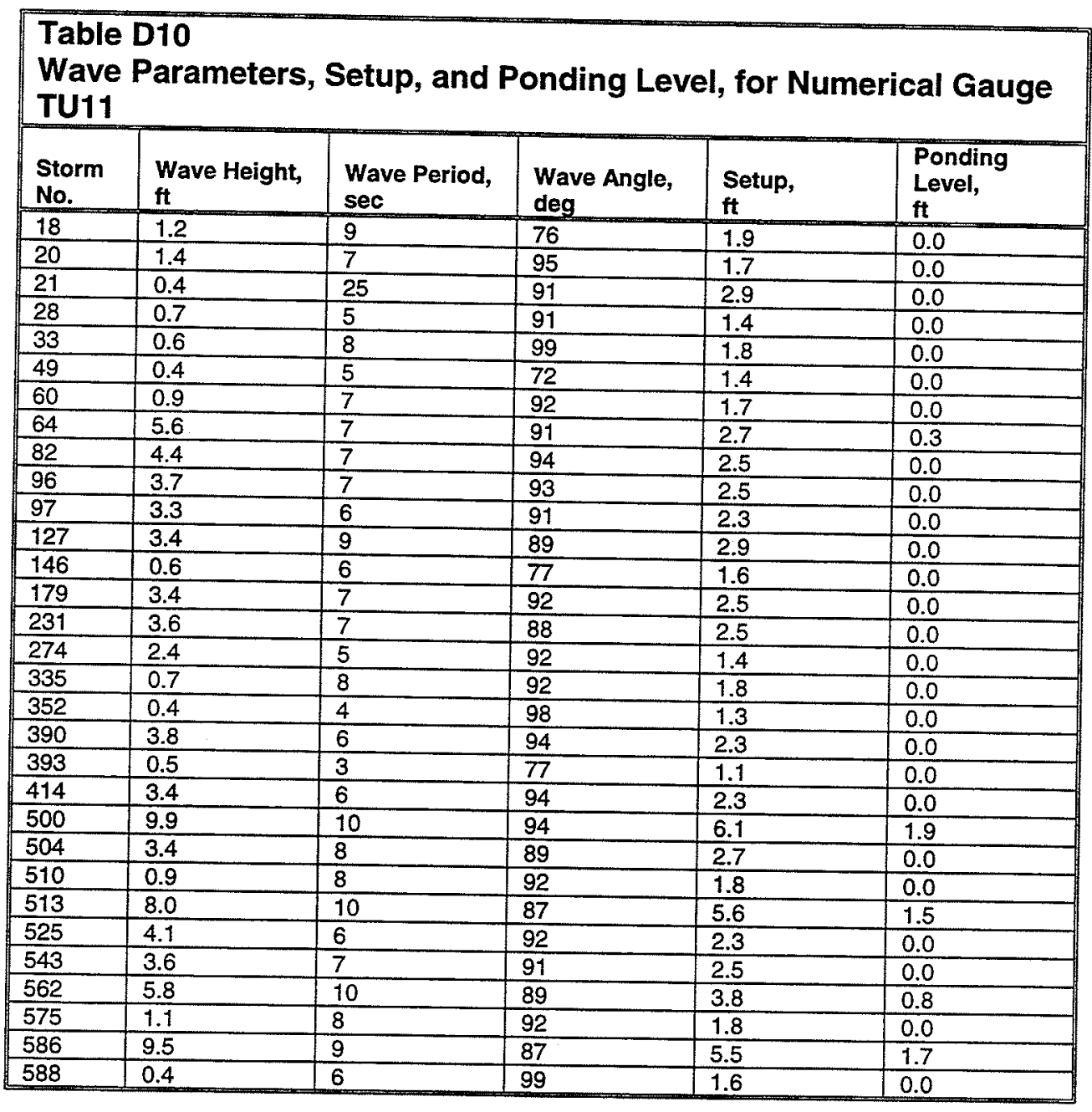




\begin{tabular}{|c|c|c|c|c|c|}
\hline \multicolumn{6}{|c|}{$\begin{array}{l}\text { Table D11 } \\
\text { Wave Parameters, Setup, and Ponding Level, for Numerical Gaug } \\
\text { TU12 }\end{array}$} \\
\hline $\begin{array}{l}\text { Storm } \\
\text { No. } \\
\end{array}$ & $\begin{array}{l}\text { Wave Height, } \\
\mathrm{ft}\end{array}$ & $\begin{array}{l}\text { Wave Period, } \\
\text { sec }\end{array}$ & $\begin{array}{l}\text { Wave Angle, } \\
\text { deg }\end{array}$ & $\begin{array}{l}\text { Setup, } \\
\mathrm{ft}\end{array}$ & $\begin{array}{l}\text { Ponding } \\
\text { Level, } \\
\mathrm{ft}\end{array}$ \\
\hline 18 & 3.8 & 8 & 115 & 2.7 & 0.0 \\
\hline 20 & 4.3 & 7 & 116 & 2.5 & 0.0 \\
\hline 21 & 7.0 & 8 & 110 & 4.3 & 0.9 \\
\hline 28 & 3.3 & 5 & 102 & 2.0 & 0.0 \\
\hline 33 & 3.7 & 4 & 97 & 1.7 & 0.0 \\
\hline 49 & 0.6 & 6 & 128 & 1.6 & 0.0 \\
\hline 60 & 0.9 & 7 & 107 & 1.7 & 0.0 \\
\hline 64 & 7.7 & 7 & 109 & 4.0 & 1.0 \\
\hline 82 & 6.6 & 7 & 117 & 3.7 & 0.7 \\
\hline 96 & 5.9 & 8 & 119 & 3.1 & 0.5 \\
\hline 97 & 3.4 & 6 & 114 & 2.3 & 0.0 \\
\hline 127 & 5.1 & 8 & 107 & 2.9 & 0.2 \\
\hline 146 & 0.5 & 6 & 86 & 1.6 & 0.0 \\
\hline 179 & 5.9 & 7 & 119 & 2.8 & 0.4 \\
\hline 231 & 3.3 & 8 & 114 & 2.7 & 0.0 \\
\hline 274 & 3.1 & 5 & 107 & 1.3 & 0.0 \\
\hline 335 & 0.9 & 8 & 107 & 1.8 & 0.0 \\
\hline 352 & 0.6 & 4 & 86 & 1.3 & 0.0 \\
\hline 390 & 4.0 & 6 & 106 & 2.3 & 0.0 \\
\hline 393 & 0.4 & 3 & 86 & 1.1 & 0.0 \\
\hline 414 & 6.0 & 7 & 109 & 2.8 & 0.5 \\
\hline 500 & 11.3 & 10 & 102 & 6.4 & 2.2 \\
\hline 504 & 5.9 & 8 & 117 & 3.1 & 0.5 \\
\hline 510 & 1.1 & 8 & 107 & 1.8 & 0.0 \\
\hline 513 & 8.1 & 10 & 100 & 5.6 & 1.5 \\
\hline 525 & 3.7 & 7 & 122 & 2.5 & 0.0 \\
\hline 543 & 6.0 & 7 & 111 & 2.8 & 0.4 \\
\hline 562 & 5.9 & 10 & 100 & 3.9 & 0.8 \\
\hline 575 & 0.9 & 8 & 115 & 1.8 & 0.0 \\
\hline 586 & 9.2 & 10 & 115 & 5.9 & 1.8 \\
\hline 588 & 1.6 & 6 & 125 & 1.5 & 0.0 \\
\hline
\end{tabular}




\begin{tabular}{|c|c|c|c|c|c|}
\hline & trameters, & Etyp, and Po & onding Leve & 1, tor Nurer & uge \\
\hline & 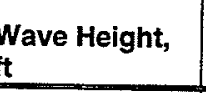 & 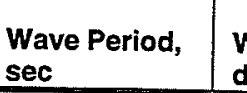 & 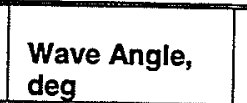 & $\begin{array}{l}\text { Saturp, } \\
\text { the }\end{array}$ & 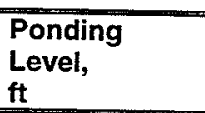 \\
\hline & & & $\pi$ & & $\bar{Z}$ \\
\hline & & & & & 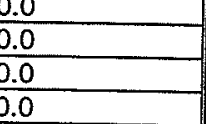 \\
\hline & & $=\frac{1}{i}$ & 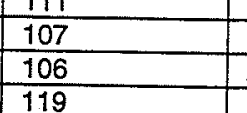 & 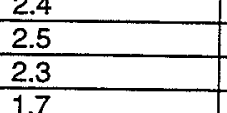 & 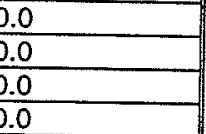 \\
\hline & 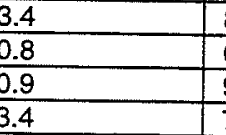 & 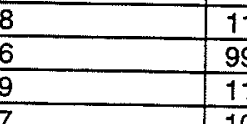 & 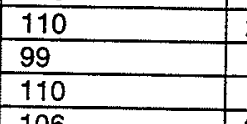 & $\bar{E}$ & $\Rightarrow$ \\
\hline 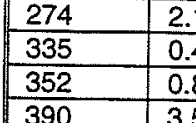 & & $\pi$ & 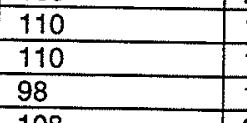 & $\bar{E}$ & 政 \\
\hline & & \pm & 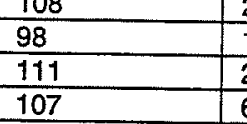 & $\bar{E}$ & 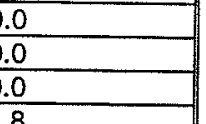 \\
\hline & & $\frac{110}{1.10}$ & 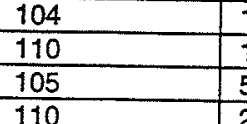 & 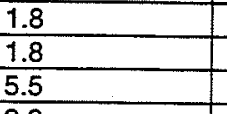 & 样 \\
\hline 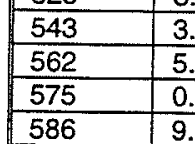 & & $\frac{110}{10}$ & & 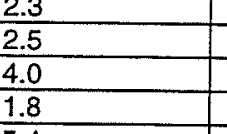 & $\theta$ \\
\hline & & & & & \\
\hline
\end{tabular}




\begin{tabular}{|c|c|c|c|c|c|}
\hline \multicolumn{6}{|c|}{$\begin{array}{l}\text { Table D13 } \\
\text { Wave Parameters, Setup, and Ponding Level, for Numerical Gauge } \\
\text { TU15 }\end{array}$} \\
\hline $\begin{array}{l}\text { Storm } \\
\text { No. }\end{array}$ & $\begin{array}{l}\text { Wave Height, } \\
\mathrm{ft}\end{array}$ & $\begin{array}{l}\text { Wave Period, } \\
\text { sec }\end{array}$ & $\begin{array}{l}\text { Wave Angle, } \\
\text { deg }\end{array}$ & $\begin{array}{l}\text { Setup, } \\
\mathrm{tt}\end{array}$ & $\begin{array}{l}\text { Ponding } \\
\text { Level, } \\
\text { ft }\end{array}$ \\
\hline 18 & 3.3 & 8 & 110 & 2.0 & 0.0 \\
\hline 20 & 3.4 & 7 & 111 & 1.8 & 0.0 \\
\hline 21 & 0.5 & 25 & 107 & 1.9 & 0.0 \\
\hline 28 & 0.5 & 5 & 104 & 1.1 & 0.0 \\
\hline 33 & 1.4 & 8 & 116 & 1.3 & 0.0 \\
\hline 49 & 0.7 & 5 & 105 & 1.1 & 0.0 \\
\hline 60 & 0.7 & 7 & 106 & 1.2 & 0.0 \\
\hline 64 & 6.6 & 7 & 107 & 3.0 & 0.6 \\
\hline 82 & 5.3 & 7 & 111 & 2.0 & 0.2 \\
\hline 96 & 5.3 & 7 & 110 & 2.0 & 0.2 \\
\hline 97 & 3.3 & 6 & 109 & 1.7 & 0.0 \\
\hline 127 & 3.6 & 8 & 106 & 2.0 & 0.0 \\
\hline 146 & 0.7 & 6 & 98 & 1.2 & 0.0 \\
\hline 179 & 3.3 & 8 & 112 & 2.0 & 0.0 \\
\hline 231 & 3.3 & 7 & 103 & 1.8 & 0.0 \\
\hline 274 & 2.7 & 5 & 106 & 1.0 & 0.0 \\
\hline 335 & 0.7 & 8 & 106 & 1.3 & 0.0 \\
\hline 352 & 0.4 & 4 & 94 & 0.9 & 0.0 \\
\hline 390 & 3.4 & 6 & 105 & 1.7 & 0.0 \\
\hline 393 & 1.4 & 4 & 99 & 0.9 & 0.0 \\
\hline 414 & 5.1 & 7 & 107 & 1.9 & 0.1 \\
\hline 500 & 9.6 & 10 & 104 & 5.2 & 1.9 \\
\hline 504 & 4.7 & 8 & 110 & 2.0 & 0.0 \\
\hline 510 & 1.0 & 8 & 106 & 1.3 & 0.0 \\
\hline 513 & 6.9 & 10 & 103 & 4.4 & 1.1 \\
\hline 525 & 4.6 & 6 & 106 & 1.6 & 0.0 \\
\hline 543 & 5.1 & 7 & 108 & 1.9 & 0.1 \\
\hline 562 & 5.0 & 10 & 103 & 2.8 & 0.4 \\
\hline 575 & 1.2 & 8 & 106 & 1.3 & 0.0 \\
\hline 586 & 7.4 & 10 & 110 & 4.6 & 1.3 \\
\hline 588 & 1.1 & 6 & 116 & 1.1 & 0.0 \\
\hline
\end{tabular}




\begin{tabular}{|c|c|c|c|c|c|}
\hline $\begin{array}{l}\text { Storm } \\
\text { No. }\end{array}$ & $\begin{array}{l}\text { Wave Height, } \\
\mathrm{ft}\end{array}$ & $\begin{array}{l}\text { Wave Period, } \\
\text { sec }\end{array}$ & $\begin{array}{l}\text { Wave Angle, } \\
\text { deg }\end{array}$ & $\begin{array}{l}\text { Setup, } \\
\mathrm{ft}\end{array}$ & $\begin{array}{l}\text { Ponding } \\
\text { Level, } \\
\mathrm{ft}\end{array}$ \\
\hline 18 & 5.7 & 8 & 143 & 3.1 & 0.5 \\
\hline 20 & 7.4 & 6 & 153 & 3.3 & 0.7 \\
\hline 21 & 8.5 & 8 & 130 & 4.7 & 1.3 \\
\hline 28 & 3.3 & 5 & 152 & 2.0 & 0.0 \\
\hline 33 & 7.7 & 8 & 166 & 4.5 & 1.2 \\
\hline 49 & 3.4 & 6 & 171 & 2.3 & 0.0 \\
\hline 60 & 1.0 & 7 & 120 & 1.7 & 0.0 \\
\hline 64 & 9.2 & 7 & 126 & 4.2 & 1.4 \\
\hline 82 & 8.3 & 9 & 167 & 5.2 & 1.4 \\
\hline 96 & 9.6 & 8 & 151 & 4.9 & 1.6 \\
\hline 97 & 6.9 & 7 & 160 & 3.8 & 0.8 \\
\hline 127 & 5.9 & 8 & 120 & 3.2 & 0.6 \\
\hline 146 & 1.2 & 6 & 68 & 1.6 & 0.0 \\
\hline 179 & 9.6 & 7 & 151 & 4.3 & 1.5 \\
\hline 231 & 7.0 & 6 & 150 & 3.2 & 0.6 \\
\hline 274 & 3.6 & 5 & 120 & 2.0 & 0.0 \\
\hline 335 & 0.5 & 8 & 61 & 1.8 & 0.0 \\
\hline 352 & 0.9 & 4 & 174 & 1.3 & 0.0 \\
\hline 390 & 3.9 & 7 & 130 & 2.5 & 0.0 \\
\hline 393 & 0.4 & 3 & 58 & 1.1 & 0.0 \\
\hline 414 & 8.6 & 9 & 164 & 5.3 & 1.5 \\
\hline 500 & 13.4 & 10 & 105 & 6.7 & 2.6 \\
\hline 504 & 9.0 & 8 & 148 & 4.8 & 1.5 \\
\hline 510 & 1.3 & 8 & 120 & 1.8 & 0.0 \\
\hline 513 & 10.3 & 10 & 92 & 6.2 & 2.0 \\
\hline 525 & 7.5 & 7 & 162 & 3.9 & 0.9 \\
\hline 543 & 7.5 & 7 & 133 & 3.9 & 0.9 \\
\hline 562 & 7.3 & 11 & 81 & 5.8 & 1.4 \\
\hline 575 & 1.3 & 8 & 142 & 1.8 & 0.0 \\
\hline 586 & 13.0 & 10 & 144 & 6.6 & 2.5 \\
\hline 588 & 4.2 & 6 & 163 & 2.2 & 0.0 \\
\hline
\end{tabular}




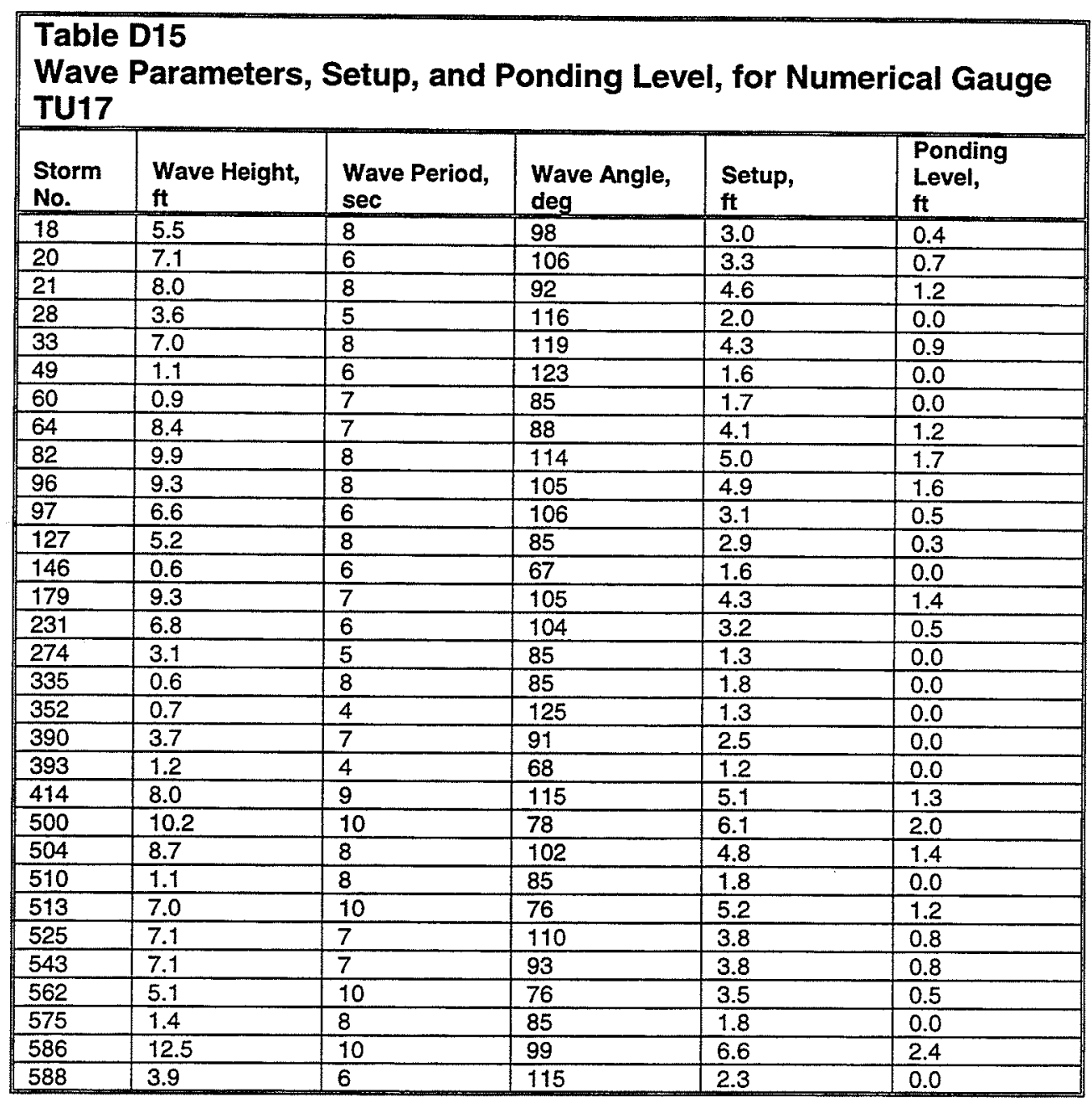




\begin{tabular}{|c|c|c|c|c|c|}
\hline $\begin{array}{l}\text { Storm } \\
\text { No. }\end{array}$ & $\begin{array}{l}\text { Wave Height, } \\
\mathrm{ft}\end{array}$ & $\begin{array}{l}\text { Wave Period, } \\
\text { sec }\end{array}$ & $\begin{array}{l}\text { Wave Angle, } \\
\text { deg }\end{array}$ & $\begin{array}{l}\text { Setup, } \\
\mathrm{ft}\end{array}$ & $\begin{array}{l}\text { Ponding } \\
\text { Level, } \\
\mathrm{ft}\end{array}$ \\
\hline 18 & 5.9 & 8 & 116 & 3.1 & 0.5 \\
\hline 20 & 8.0 & 6 & 129 & 3.4 & 0.9 \\
\hline 21 & 8.5 & 8 & 105 & 4.7 & 1.4 \\
\hline 28 & 3.6 & 5 & 103 & 2.0 & 0.0 \\
\hline 33 & 10.4 & 8 & 150 & 5.1 & 1.8 \\
\hline 49 & 3.9 & 6 & 156 & 2.3 & 0.0 \\
\hline 60 & 1.0 & 7 & 91 & \begin{tabular}{|l|}
1.7 \\
\end{tabular} & 0.0 \\
\hline 64 & 9.2 & 7 & 96 & 4.2 & 1.4 \\
\hline 82 & 10.1 & 9 & 154 & 5.6 & 1.9 \\
\hline 96 & 10.9 & 8 & 137 & 5.1 & 1.9 \\
\hline 97 & 8.0 & 7 & 138 & 4.0 & 1.1 \\
\hline 127 & 5.9 & 8 & 91 & 3.1 & 0.5 \\
\hline 146 & 3.3 & 4 & 99 & 1.7 & 0.0 \\
\hline 179 & 10.3 & 7 & 126 & 4.4 & 1.6 \\
\hline 231 & 7.4 & 6 & 125 & 3.3 & 0.8 \\
\hline 274 & 3.6 & 5 & 91 & 2.0 & 0.0 \\
\hline 335 & 1.0 & 8 & 91 & 1.8 & 0.0 \\
\hline 352 & 3.4 & 4 & 139 & 1.7 & 0.0 \\
\hline 390 & 3.3 & 7 & 101 & 2.5 & 0.0 \\
\hline 393 & 0.4 & 3 & 46 & 1.1 & 0.0 \\
\hline 414 & 10.5 & 9 & 144 & 5.7 & 2.0 \\
\hline 500 & 13.0 & 10 & 78 & 6.6 & 2.5 \\
\hline 504 & 9.4 & 8 & 122 & 4.9 & 1.6 \\
\hline 510 & 1.3 & 8 & 91 & 1.8 & 0.0 \\
\hline 513 & 9.5 & 10 & 68 & 6.0 & 1.9 \\
\hline 525 & 9.0 & 7 & 142 & 4.2 & 1.3 \\
\hline 543 & 8.9 & 7 & 145 & 4.2 & 1.3 \\
\hline 562 & 6.9 & 10 & 74 & 5.2 & 1.1 \\
\hline 575 & 1.3 & 8 & 115 & 1.8 & 0.0 \\
\hline 586 & 13.4 & 10 & 117 & 6.7 & 2.6 \\
\hline 588 & 5.6 & 6 & 143 & 2.3 & 0.1 \\
\hline
\end{tabular}




\begin{tabular}{|c|c|c|c|c|c|c|}
\hline & ters,, & Setup, and pap & Ding & for N N & ncart & \\
\hline & vave Haghth & 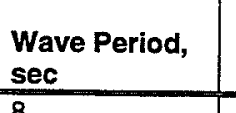 & ave Ange, & soup, & & \\
\hline & & & 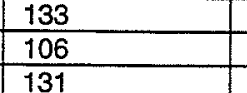 & & & \\
\hline & & & & & & \\
\hline & & & 4 & & & \\
\hline & & & $\underline{\underline{E}}$ & & & \\
\hline & & & & & & \\
\hline & 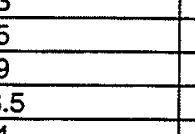 & & 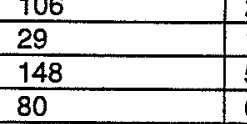 & & & \\
\hline & & & 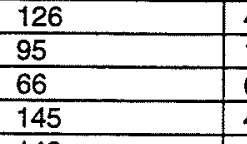 & & & \\
\hline & & & & & & \\
\hline & & & & & & \\
\hline
\end{tabular}




\begin{tabular}{|c|c|c|c|c|c|}
\hline $\begin{array}{l}\text { Storm } \\
\text { No. }\end{array}$ & $\begin{array}{l}\text { Wave Height, } \\
\mathrm{ft}\end{array}$ & $\begin{array}{l}\text { Wave Period, } \\
\text { sec }\end{array}$ & $\begin{array}{l}\text { Wave Angle, } \\
\text { deg }\end{array}$ & $\begin{array}{l}\text { Setup, } \\
\mathrm{ft}\end{array}$ & $\begin{array}{l}\text { Ponding } \\
\text { Level, } \\
\text { ft } \\
\end{array}$ \\
\hline 18 & 5.2 & 9 & 86 & 3.3 & 0.4 \\
\hline 20 & 0.6 & 7 & 138 & 1.7 & 0.0 \\
\hline 21 & 3.3 & 7 & 133 & 2.5 & 0.0 \\
\hline 28 & 0.4 & 5 & 136 & 1.4 & 0.0 \\
\hline 33 & 8.8 & 8 & 25 & 4.8 & 1.4 \\
\hline 49 & 3.7 & 7 & 35 & 2.5 & 0.0 \\
\hline 60 & 0.3 & 6 & 136 & 1.6 & 0.0 \\
\hline 64 & 0.6 & 25 & 38 & 2.9 & 0.0 \\
\hline 82 & 4.2 & 7 & 124 & 2.5 & 0.0 \\
\hline 96 & 4.5 & 7 & 100 & 2.5 & 0.0 \\
\hline 97 & 0.8 & 7 & 138 & 1.7 & 0.0 \\
\hline 127 & 9.2 & 7 & 41 & 4.3 & 1.4 \\
\hline 146 & 0.9 & 7 & 109 & 1.7 & 0.0 \\
\hline 179 & 7.0 & 8 & 102 & 4.3 & 0.9 \\
\hline 231 & 0.7 & 25 & 56 & 2.9 & 0.0 \\
\hline 274 & 0.4 & 5 & 12 & 1.4 & 0.0 \\
\hline 335 & 5.9 & 6 & 37 & 2.4 & 0.3 \\
\hline 352 & 2.0 & 4 & 91 & 1.2 & 0.0 \\
\hline 390 & 3.3 & 6 & 129 & 2.3 & 0.0 \\
\hline 393 & 0.7 & 25 & 56 & 2.9 & 0.0 \\
\hline 414 & 5.9 & 9 & 96 & 3.5 & 0.7 \\
\hline 500 & 10.8 & 10 & 121 & 6.3 & 2.1 \\
\hline 504 & 13.1 & 8 & 67 & 5.4 & 2.3 \\
\hline 510 & 5.4 & 6 & 40 & 2.2 & 0.1 \\
\hline 513 & 9.0 & 10 & 122 & 5.9 & 1.7 \\
\hline 525 & 9.8 & 7 & 35 & 4.4 & 1.5 \\
\hline 543 & 6.9 & 6 & 91 & 3.2 & 0.6 \\
\hline 562 & 18.5 & 12 & 42 & 8.4 & 3.5 \\
\hline 575 & 0.6 & 25 & 38 & 2.9 & 0.0 \\
\hline 586 & 9.2 & 8 & 121 & 4.9 & 1.5 \\
\hline 588 & 5.4 & 7 & 51 & 2.6 & 0.2 \\
\hline
\end{tabular}




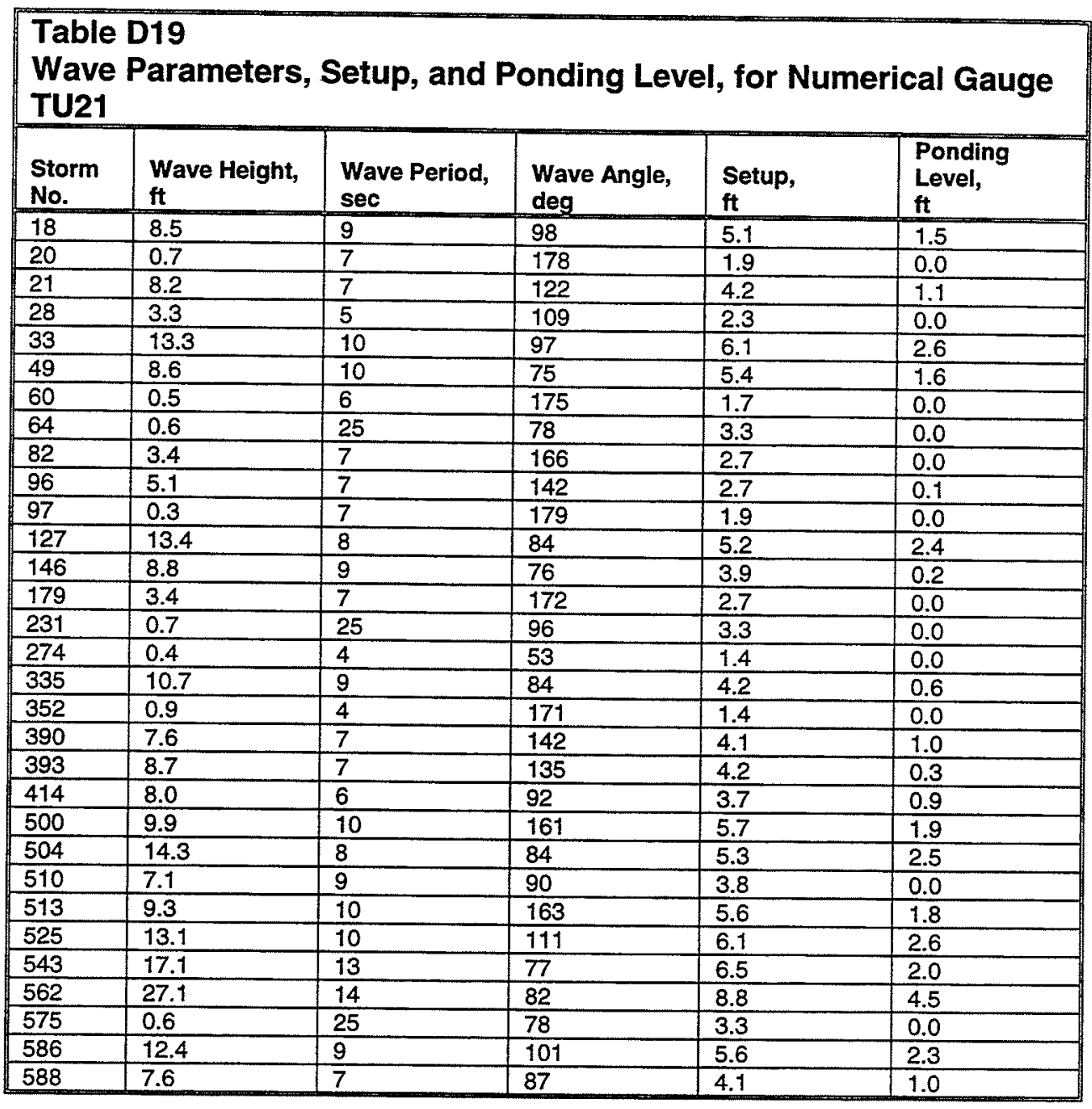




\begin{tabular}{|c|c|c|c|c|c|}
\hline $\begin{array}{l}\text { Storm } \\
\text { No. }\end{array}$ & $\begin{array}{l}\text { Wave Height, } \\
\mathrm{tt}\end{array}$ & $\begin{array}{l}\text { Wave Period, } \\
\text { sec }\end{array}$ & $\begin{array}{l}\text { Wave Angle, } \\
\text { deg }\end{array}$ & $\begin{array}{l}\text { Setup, } \\
\mathrm{ft}\end{array}$ & $\begin{array}{l}\text { Ponding } \\
\text { Level, } \\
\mathrm{ft}\end{array}$ \\
\hline 18 & 11.9 & 9 & 137 & 7.2 & 2.2 \\
\hline 20 & 3.9 & 7 & 168 & 3.7 & 0.0 \\
\hline 21 & 9.4 & 7 & 153 & 5.6 & 1.4 \\
\hline 28 & 3.3 & 5 & 115 & 3.0 & 0.0 \\
\hline 33 & 12.7 & 11 & 80 & 8.6 & 2.6 \\
\hline 49 & 11.9 & 10 & 137 & 7.9 & 2.3 \\
\hline 60 & 3.4 & 5 & 145 & 3.0 & 0.0 \\
\hline 64 & 9.9 & 8 & 154 & 6.3 & 1.7 \\
\hline 82 & 10.0 & 8 & 150 & 6.3 & 1.7 \\
\hline 96 & 8.9 & 9 & 155 & 6.7 & 1.6 \\
\hline 97 & 6.9 & 8 & 158 & 4.8 & 0.0 \\
\hline 127 & 12.4 & 9 & 97 & 7.3 & 2.3 \\
\hline 146 & 6.9 & 9 & 79 & 5.1 & 0.0 \\
\hline 179 & 12.9 & 10 & 136 & 8.0 & 2.5 \\
\hline 231 & 7.9 & 8 & 154 & 5.9 & 1.2 \\
\hline 274 & 0.6 & 5 & 63 & 2.1 & 0.0 \\
\hline 335 & 9.5 & 10 & 87 & 6.0 & 0.5 \\
\hline 352 & 1.0 & 4 & 169 & 1.9 & 0.0 \\
\hline 390 & 9.2 & 7 & 144 & 5.5 & 1.4 \\
\hline 393 & 8.4 & 7 & 136 & 5.4 & 1.2 \\
\hline 414 & 13.0 & 8 & 134 & 6.6 & 2.3 \\
\hline 500 & 11.3 & 10 & 153 & 7.8 & 2.2 \\
\hline 504 & 13.3 & 10 & 132 & 8.0 & 2.6 \\
\hline 510 & 8.8 & 9 & 94 & 5.3 & 0.2 \\
\hline 513 & 11.5 & 10 & 153 & 7.8 & 2.3 \\
\hline 525 & 17.0 & 10 & 144 & 8.3 & 3.1 \\
\hline 543 & 15.3 & 13 & 80 & 8.4 & 1.8 \\
\hline 562 & 24.9 & 14 & 83 & 11.6 & 4.3 \\
\hline 575 & 0.5 & 25 & 80 & 4.8 & 0.0 \\
\hline 586 & 16.3 & 9 & 114 & 7.6 & 2.9 \\
\hline 588 & 8.3 & 9 & 148 & 6.6 & 1.4 \\
\hline
\end{tabular}




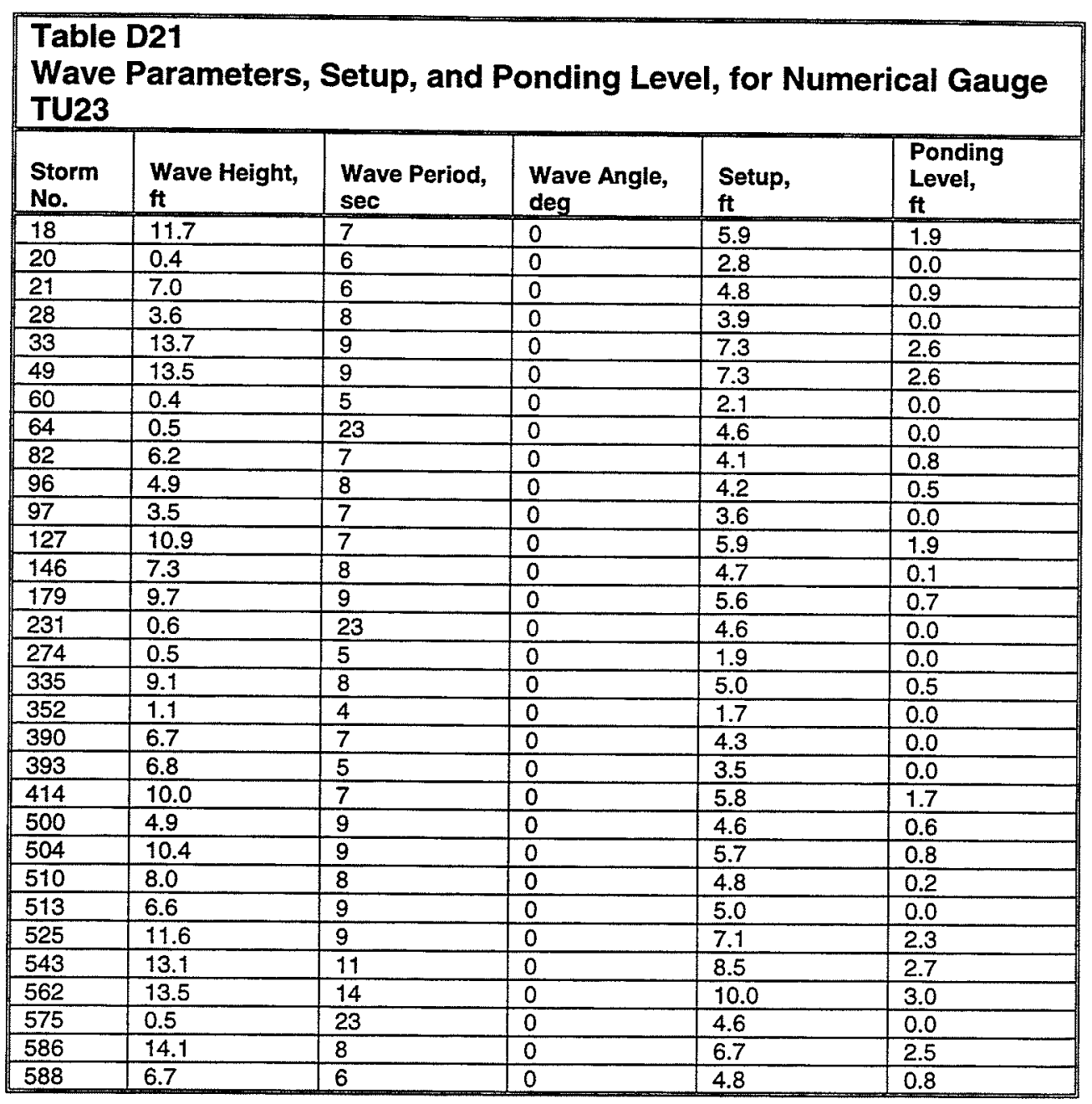




\begin{tabular}{|c|c|c|c|c|c|}
\hline $\begin{array}{l}\text { Storm } \\
\text { No. }\end{array}$ & $\begin{array}{l}\text { Wave Height, } \\
\mathrm{ft}\end{array}$ & $\begin{array}{l}\text { Wave Period, } \\
\text { sec }\end{array}$ & $\begin{array}{l}\text { Wave Angle, } \\
\text { deg }\end{array}$ & $\begin{array}{l}\text { Setup, } \\
\mathrm{ft}\end{array}$ & $\begin{array}{l}\text { Ponding } \\
\text { Level, } \\
\mathrm{ft}\end{array}$ \\
\hline 18 & 0.0 & - & -- & -- & -- \\
\hline 20 & 1.0 & 4 & 25 & 1.2 & 0.0 \\
\hline 21 & 0.0 & -- & 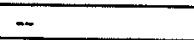 & -- & $\ldots$ \\
\hline 28 & 0.0 & $\ldots$ & -- & - & - \\
\hline 33 & 13.6 & 11 & 67 & 7.3 & 2.7 \\
\hline 49 & 7.5 & 9 & 61 & 5.0 & 1.2 \\
\hline 60 & 0.0 & - & $\cdots$ & $\cdots$ & - \\
\hline 64 & 3.6 & 5 & 40 & 2.0 & 0.0 \\
\hline 82 & 0.9 & 3 & 29 & 1.1 & 0.0 \\
\hline 96 & 0.0 & - & - & -- & -- \\
\hline 97 & 2.5 & 4 & 38 & 1.2 & 0.0 \\
\hline 127 & 9.4 & 7 & 49 & 4.3 & 1.4 \\
\hline 146 & 0.0 & - & - & -- & -- \\
\hline 179 & 7.6 & 6 & 46 & 3.4 & 0.8 \\
\hline 231 & 3.6 & 5 & 49 & 2.0 & 0.0 \\
\hline 274 & 4.3 & 5 & 51 & 2.0 & 0.0 \\
\hline 335 & 0.0 & - & - & - & -- \\
\hline 352 & 1.3 & 4 & 29 & 1.2 & 0.0 \\
\hline 390 & 5.3 & 5 & 73 & 1.9 & 0.0 \\
\hline 393 & 0.0 & -- & - & -. & - \\
\hline 414 & 5.7 & 5 & 38 & 1.9 & 0.0 \\
\hline 500 & 0.0 & -- & - & $\cdots$ & $\cdots$ \\
\hline 504 & 11.7 & 8 & 68 & 5.2 & 2.1 \\
\hline 510 & 0.0 & -- & - & $\cdots$ & - \\
\hline 513 & 0.0 & -- & -- & -- & -- \\
\hline 525 & 5.1 & 7 & 61 & 2.5 & 0.1 \\
\hline 543 & 10.6 & 12 & 36 & 7.2 & 2.3 \\
\hline 562 & 22.4 & 14 & 71 & 9.9 & 4.1 \\
\hline 575 & 0.0 & - & - & - & - \\
\hline 586 & 0.0 & - & -- & - & -- \\
\hline 588 & 4.1 & 7 & 33 & 2.5 & 0.0 \\
\hline
\end{tabular}




\begin{tabular}{|c|c|c|c|c|c|}
\hline \multicolumn{6}{|c|}{$\begin{array}{l}\text { Table D23 } \\
\text { Wave Parameters, Setup, and Ponding Level, for Numerical Gaug } \\
\text { TU25 }\end{array}$} \\
\hline $\begin{array}{l}\text { Storm } \\
\text { No. }\end{array}$ & $\begin{array}{l}\text { Wave Height, } \\
\mathrm{ft}\end{array}$ & $\begin{array}{l}\text { Wave Period, } \\
\text { sec }\end{array}$ & $\begin{array}{l}\text { Wave Angle, } \\
\text { deg }\end{array}$ & $\begin{array}{l}\text { Setup, } \\
\mathrm{ft}\end{array}$ & $\begin{array}{l}\text { Ponding } \\
\text { Level, } \\
\mathrm{ft}\end{array}$ \\
\hline 18 & 4.5 & 7 & 120 & 2.5 & 0.0 \\
\hline 20 & 0.5 & 5 & 125 & 1.4 & 0.0 \\
\hline 21 & 3.5 & 6 & 120 & 2.3 & 0.0 \\
\hline 28 & 0.6 & 5 & 127 & 1.4 & 0.0 \\
\hline 33 & 14.4 & 11 & 81 & 7.4 & 2.9 \\
\hline 49 & 13.2 & 10 & 111 & 6.7 & 2.6 \\
\hline 60 & 0.0 & -- & - & $\ldots$ & - \\
\hline 64 & 0.5 & 25 & 112 & 2.9 & 0.0 \\
\hline 82 & 0.4 & 3 & 27 & 1.1 & 0.0 \\
\hline 96 & 0.0 & 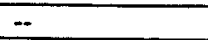 & - & -- & -- \\
\hline 97 & 0.4 & 3 & 25 & 1.1 & 0.0 \\
\hline 127 & 9.8 & 7 & 57 & 4.4 & 1.5 \\
\hline 146 & 7.6 & 9 & 111 & 5.0 & 1.3 \\
\hline 179 & 8.0 & 6 & 52 & 3.4 & 0.9 \\
\hline 231 & 0.4 & 25 & 119 & 2.9 & 0.0 \\
\hline 274 & 4.5 & 5 & 58 & 2.0 & 0.0 \\
\hline 335 & 6.9 & 9 & 117 & 4.8 & 1.0 \\
\hline 352 & 1.6 & 4 & 31 & 1.2 & 0.0 \\
\hline 390 & 3.8 & 6 & 122 & 2.3 & 0.0 \\
\hline 393 & 0.6 & 7 & 128 & 1.7 & 0.0 \\
\hline 414 & 6.4 & 5 & 42 & 2.0 & 0.2 \\
\hline 500 & 3.1 & 8 & 111 & 1.8 & 0.0 \\
\hline 504 & 12.7 & 8 & 101 & 5.3 & 2.2 \\
\hline 510 & 4.7 & 9 & 120 & 3.0 & 0.2 \\
\hline 513 & 3.4 & 8 & 118 & 2.7 & 0.0 \\
\hline 525 & 7.4 & 9 & 121 & 4.9 & 1.2 \\
\hline 543 & 12.2 & 13 & 111 & 8.0 & 2.7 \\
\hline 562 & 20.9 & 15 & 86 & 10.3 & 4.0 \\
\hline 575 & 0.5 & 25 & 112 & 2.9 & 0.0 \\
\hline 586 & 7.1 & 7 & 114 & 3.8 & 0.8 \\
\hline 588 & 4.8 & 7 & 38 & 2.4 & 0.0 \\
\hline
\end{tabular}




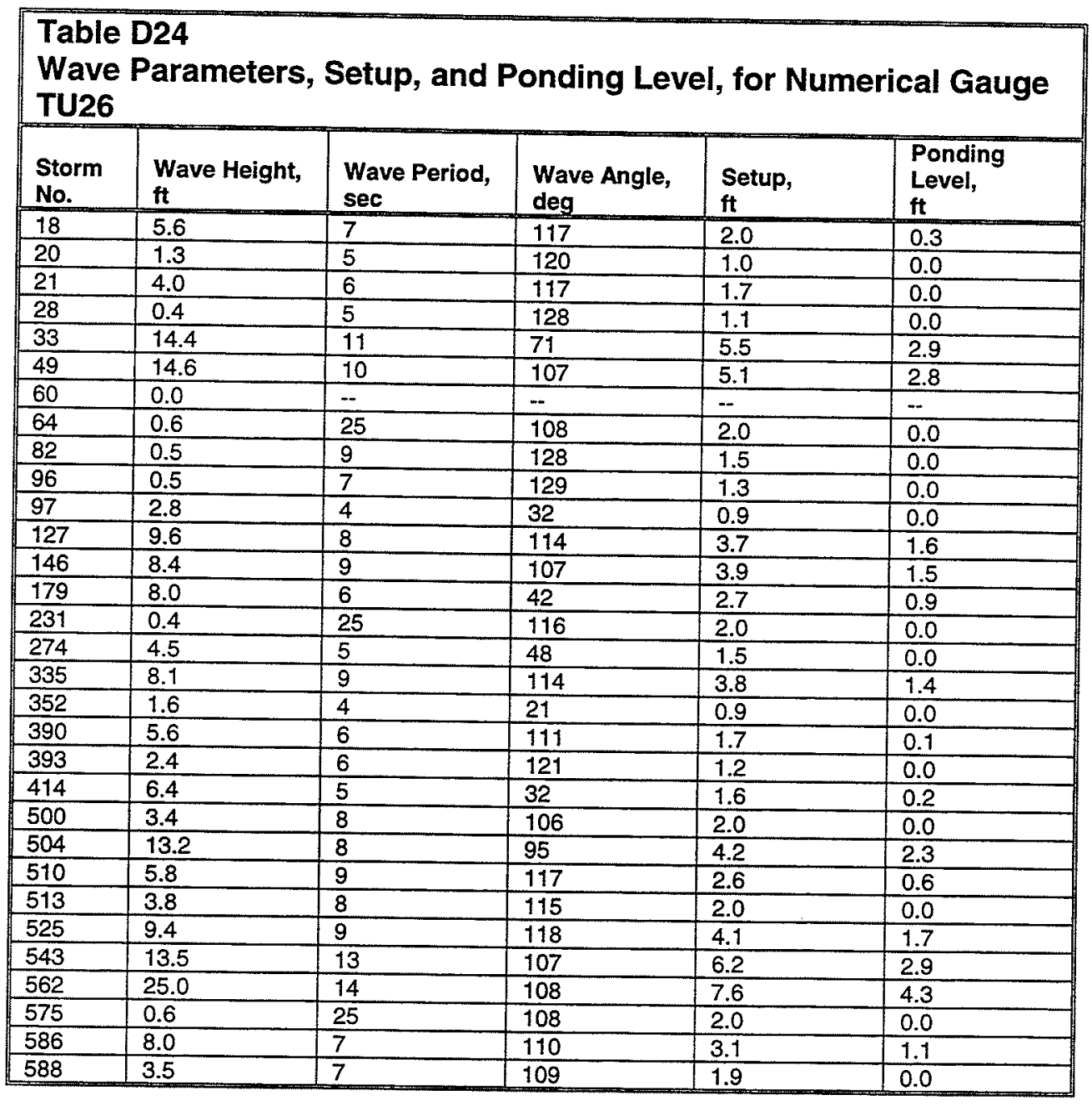




\begin{tabular}{|c|c|c|c|c|c|}
\hline \multicolumn{6}{|c|}{$\begin{array}{l}\text { Table D25 } \\
\text { Wave Parameters, Setup, and Ponding Level, for Numerical Gauge } \\
\text { TU27 }\end{array}$} \\
\hline $\begin{array}{l}\text { Storm } \\
\text { No. } \\
\end{array}$ & $\begin{array}{l}\text { Wave Height, } \\
\text { ft }\end{array}$ & $\begin{array}{l}\text { Wave Period, } \\
\text { sec }\end{array}$ & $\begin{array}{l}\text { Wave Angie, } \\
\text { deg }\end{array}$ & $\begin{array}{l}\text { Setup, } \\
\mathrm{ft}\end{array}$ & $\begin{array}{l}\text { Ponding } \\
\text { Level, } \\
\text { ft }\end{array}$ \\
\hline 18 & 7.1 & 7 & 122 & 3.8 & 0.8 \\
\hline 20 & 0.4 & 8 & 139 & 1.8 & 0.0 \\
\hline 21 & 3.8 & 7 & 126 & 2.5 & 0.0 \\
\hline 28 & 0.6 & 9 & 139 & 1.9 & 0.0 \\
\hline 33 & 14.4 & 11 & 66 & 7.4 & 2.9 \\
\hline 49 & 14.9 & 10 & 110 & 6.9 & 2.8 \\
\hline 60 & 0.4 & 5 & 138 & 1.4 & 0.0 \\
\hline 64 & 0.6 & 25 & 109 & 2.9 & 0.0 \\
\hline 82 & 0.5 & 9 & 136 & 1.9 & 0.0 \\
\hline 96 & 0.4 & 9 & 139 & 1.9 & 0.0 \\
\hline 97 & 0.5 & 8 & 139 & 1.8 & 0.0 \\
\hline 127 & 9.4 & 9 & 124 & 5.5 & 1.7 \\
\hline 146 & 9.3 & 9 & 108 & 5.4 & 1.7 \\
\hline 179 & 7.9 & 7 & 120 & 4.0 & 1.0 \\
\hline 231 & 0.5 & 25 & 120 & 2.9 & 0.0 \\
\hline 274 & 4.5 & 5 & 42 & 2.0 & 0.0 \\
\hline 335 & 9.6 & 9 & 117 & 5.5 & 1.7 \\
\hline 352 & 1.8 & 4 & 13 & 1.2 & 0.0 \\
\hline 390 & 6.6 & 6 & 125 & 3.1 & 0.5 \\
\hline 393 & 3.4 & 6 & 126 & 2.3 & 0.0 \\
\hline 414 & 6.6 & 5 & 25 & 2.5 & 0.3 \\
\hline 500 & 3.7 & 8 & 107 & 2.7 & 0.0 \\
\hline 504 & 13.6 & 8 & 92 & 5.4 & 2.4 \\
\hline 510 & 7.2 & 9 & 121 & 4.9 & 1.1 \\
\hline 513 & 5.4 & 8 & 116 & 3.0 & 0.4 \\
\hline 525 & 12.0 & 9 & 123 & 5.9 & 2.2 \\
\hline 543 & 14.8 & 13 & 108 & 8.5 & 3.1 \\
\hline 562 & 22.3 & 15 & 101 & 10.4 & 4.2 \\
\hline 575 & 0.6 & 25 & 109 & 2.9 & 0.0 \\
\hline 586 & 8.1 & 8 & 130 & 4.6 & 1.2 \\
\hline 588 & 3.4 & 8 & 107 & 2.7 & 0.0 \\
\hline
\end{tabular}




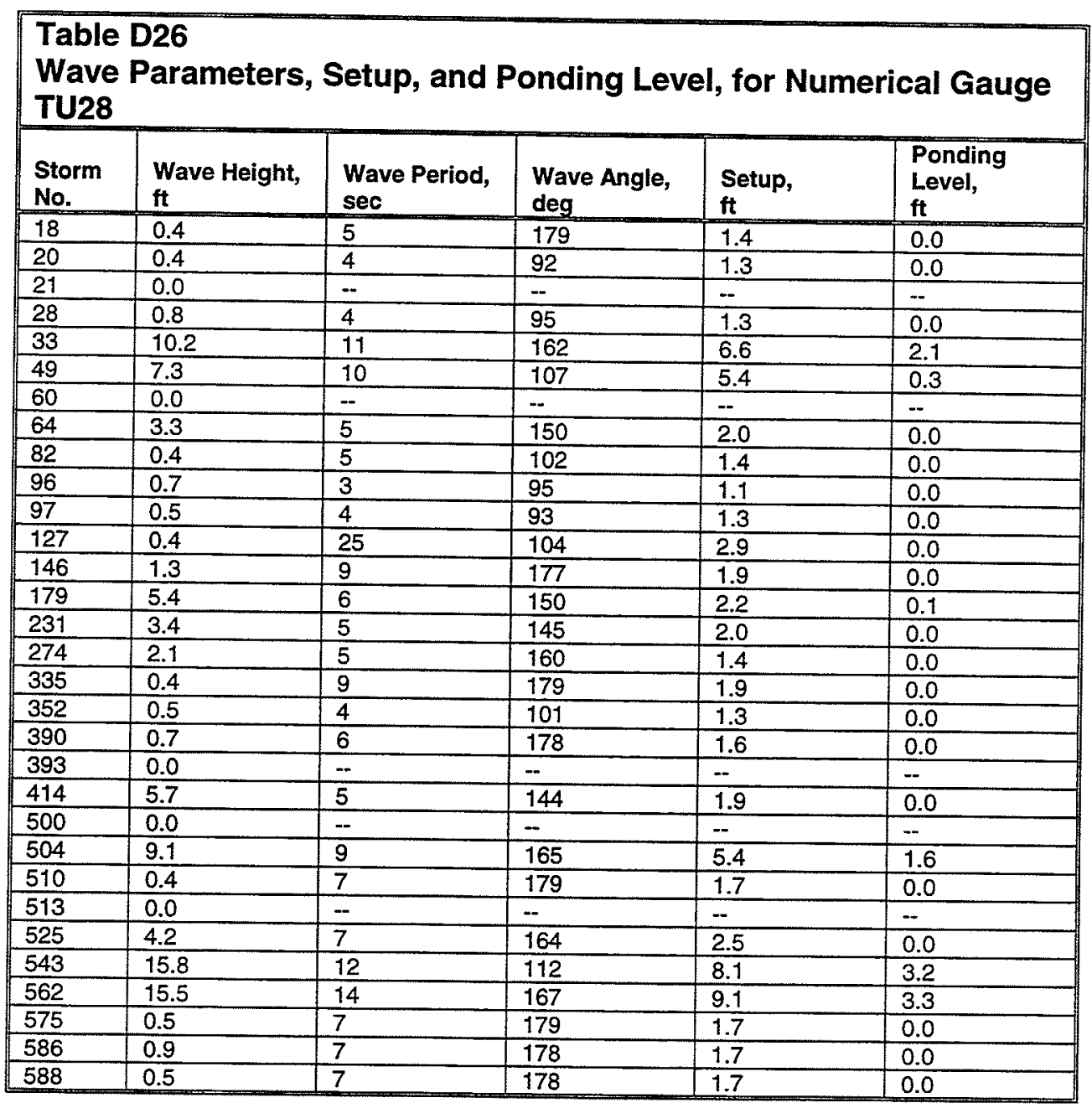




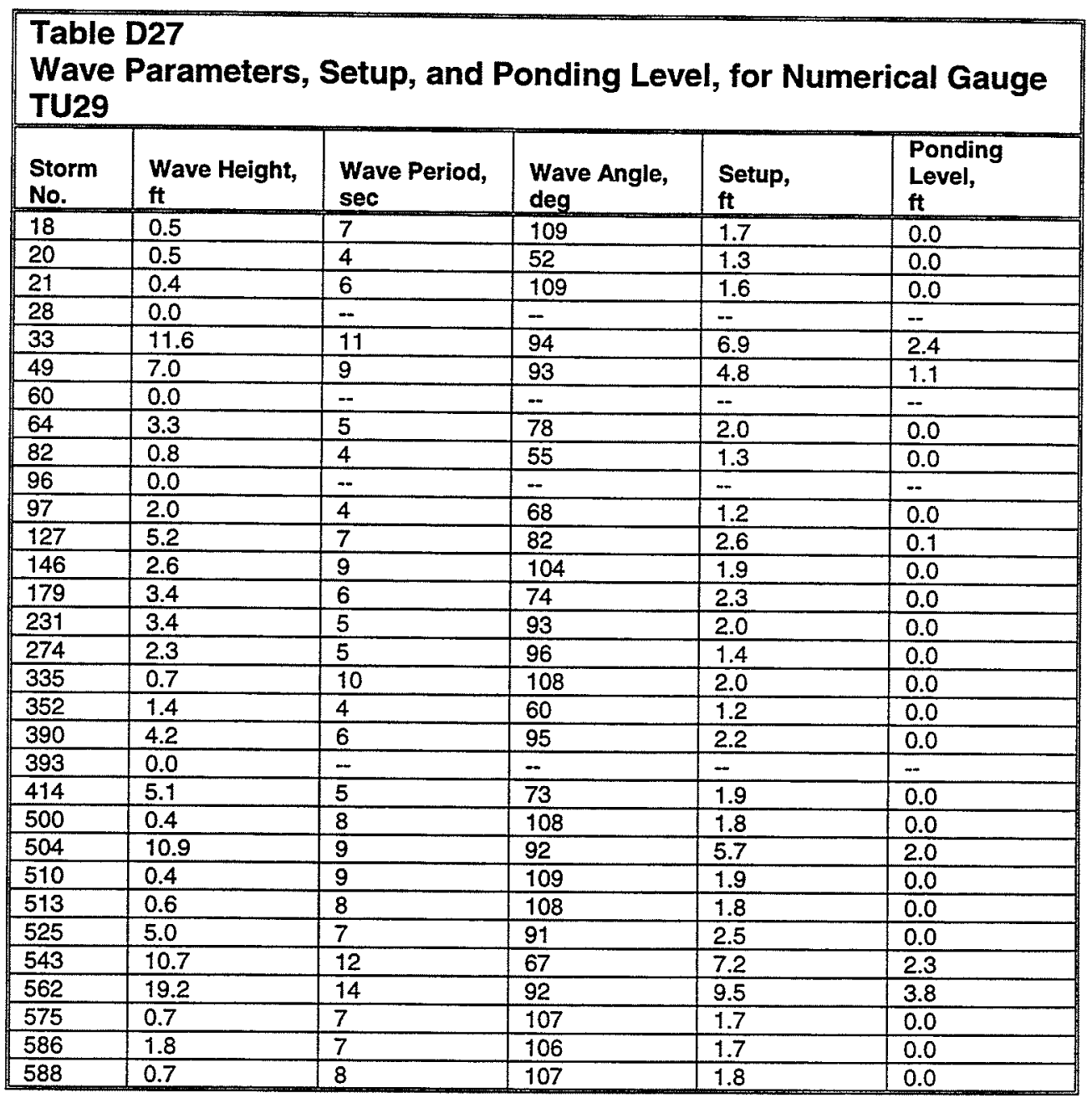




\begin{tabular}{|c|c|c|c|c|c|}
\hline \multicolumn{6}{|c|}{$\begin{array}{l}\text { Table D28 } \\
\text { Wave Parameters, Setup, and Ponding Level, for Numerical Gaug } \\
\text { TU30 }\end{array}$} \\
\hline $\begin{array}{l}\text { Storm } \\
\text { No. } \\
\end{array}$ & $\begin{array}{l}\text { Wave Height, } \\
\mathrm{ft}\end{array}$ & $\begin{array}{l}\text { Wave Period, } \\
\text { sec }\end{array}$ & $\begin{array}{l}\text { Wave Angle, } \\
\text { deg }\end{array}$ & $\begin{array}{l}\text { Setup, } \\
\mathrm{ft}\end{array}$ & $\begin{array}{l}\text { Ponding } \\
\text { Level, } \\
\mathrm{ft}\end{array}$ \\
\hline 18 & 1.5 & 7 & 97 & 1.7 & 0.0 \\
\hline 20 & 0.4 & 5 & 97 & 1.4 & 0.0 \\
\hline 21 & 0.8 & 6 & 97 & 1.6 & 0.0 \\
\hline 28 & 0.3 & 5 & 98 & 1.4 & 0.0 \\
\hline 33 & 13.1 & 11 & 65 & 7.2 & 2.7 \\
\hline 49 & 9.9 & 10 & 92 & 6.1 & 1.9 \\
\hline 60 & 0.0 & - & -- & $\cdots$ & -- \\
\hline 64 & 0.4 & 25 & 91 & 2.9 & 0.0 \\
\hline 82 & 0.8 & \begin{tabular}{|l}
4 \\
\end{tabular} & 25 & 1.3 & 0.0 \\
\hline 96 & 0.0 & - & - & -- & $-\infty$ \\
\hline 97 & 2.0 & 4 & 38 & 1.2 & 0.0 \\
\hline 127 & 6.7 & 7 & 98 & 3.7 & 0.7 \\
\hline 146 & 5.4 & 8 & 92 & 3.0 & 0.4 \\
\hline 179 & 3.5 & \begin{tabular}{|l}
6 \\
\end{tabular} & 46 & 2.3 & 0.0 \\
\hline 231 & 3.7 & 5 & 52 & 2.0 & 0.0 \\
\hline 274 & 2.6 & 5 & 61 & 1.4 & 0.0 \\
\hline 335 & 3.6 & 9 & 93 & 2.9 & 0.0 \\
\hline 352 & 1.4 & 4 & 30 & 1.2 & 0.0 \\
\hline 390 & 5.7 & 6 & 81 & 2.3 & 0.2 \\
\hline 393 & 0.7 & 6 & 98 & 1.6 & 0.0 \\
\hline 414 & 5.1 & 5 & 43 & 1.9 & 0.0 \\
\hline 500 & 1.2 & 8 & 94 & 1.8 & 0.0 \\
\hline 504 & 12.8 & 9 & 70 & 6.0 & 2.4 \\
\hline 510 & 1.8 & 9 & 95 & 1.9 & 0.0 \\
\hline 513 & 2.0 & 8 & 93 & 1.8 & 0.0 \\
\hline 525 & 5.8 & 7 & 69 & 2.7 & 0.4 \\
\hline 543 & 10.7 & 12 & 37 & 7.2 & 2.3 \\
\hline 562 & 19.3 & 15 & 73 & 10.1 & 3.8 \\
\hline 575 & 0.4 & 25 & 91 & 2.9 & 0.0 \\
\hline 586 & 4.1 & 7 & 92 & 1.7 & 0.0 \\
\hline 588 & 3.7 & 4 & 77 & 1.7 & 0.0 \\
\hline
\end{tabular}




\begin{tabular}{|c|c|c|c|c|c|}
\hline \multicolumn{6}{|c|}{$\begin{array}{l}\text { Table D29 } \\
\text { Wave Parameters, Setup, and Ponding Level, for Numerical Gauge } \\
\text { TU31 }\end{array}$} \\
\hline $\begin{array}{l}\text { Storm } \\
\text { No. }\end{array}$ & $\begin{array}{l}\text { Wave Height, } \\
\mathrm{ft}\end{array}$ & $\begin{array}{l}\text { Wave Period, } \\
\text { sec }\end{array}$ & $\begin{array}{l}\text { Wave Angle, } \\
\text { deg }\end{array}$ & $\begin{array}{l}\text { Setup, } \\
\mathrm{ft}\end{array}$ & $\begin{array}{l}\text { Ponding } \\
\text { Level, } \\
\mathrm{ft}\end{array}$ \\
\hline 18 & 3.4 & 7 & 123 & 2.5 & 0.0 \\
\hline 20 & 0.8 & 5 & 125 & 1.4 & 0.0 \\
\hline 21 & 0.6 & 6 & 128 & 1.6 & 0.0 \\
\hline 28 & 0.4 & 5 & 128 & 1.4 & 0.0 \\
\hline 33 & 13.0 & 11 & 88 & 7.2 & 2.6 \\
\hline 49 & 11.8 & 10 & 114 & 6.5 & 2.3 \\
\hline 60 & 0.0 & -- & -- & -- & - \\
\hline 64 & 0.4 & 25 & 116 & 2.9 & 0.0 \\
\hline 82 & 0.5 & 4 & 53 & 1.3 & 0.0 \\
\hline 96 & 0.0 & - & -- & -- & $\ldots$ \\
\hline 97 & 2.0 & 4 & 68 & 1.2 & 0.0 \\
\hline 127 & 7.5 & 7 & 108 & 3.9 & 0.9 \\
\hline 146 & 5.6 & 9 & 116 & 3.4 & 0.6 \\
\hline 179 & 3.7 & 6 & 73 & 2.3 & 0.0 \\
\hline 231 & 3.5 & 5 & 76 & 2.0 & 0.0 \\
\hline 274 & 2.4 & 5 & 78 & 1.4 & 0.0 \\
\hline 335 & 5.5 & 9 & 120 & 3.4 & 0.5 \\
\hline 352 & 1.1 & 4 & 57 & 1.2 & 0.0 \\
\hline 390 & 5.7 & 6 & 101 & 2.3 & 0.2 \\
\hline 393 & 1.1 & 6 & 126 & 1.6 & 0.0 \\
\hline 414 & 4.6 & 5 & 69 & 2.0 & 0.0 \\
\hline 500 & 1.6 & 8 & 122 & 1.8 & 0.0 \\
\hline 504 & 12.6 & 9 & 91 & 6.0 & 2.4 \\
\hline 510 & 3.5 & 9 & 122 & 2.9 & 0.0 \\
\hline 513 & 2.3 & 8 & 121 & 1.8 & 0.0 \\
\hline 525 & 5.5 & 9 & 123 & 3.4 & \begin{tabular}{|l}
0.5 \\
\end{tabular} \\
\hline 543 & 9.9 & 12 & 115 & 7.0 & 2.1 \\
\hline 562 & 19.1 & 15 & 94 & 10.1 & 3.8 \\
\hline 575 & 3.4 & 7 & 118 & 2.5 & 0.0 \\
\hline 586 & 5.2 & 7 & 118 & 2.5 & 0.1 \\
\hline 588 & 3.3 & 7 & 117 & 2.5 & 0.0 \\
\hline
\end{tabular}




\begin{tabular}{|c|c|c|c|c|c|}
\hline \multicolumn{6}{|c|}{$\begin{array}{l}\text { Table D30 } \\
\text { Wave Parameters, Setup, and Ponding Level, for Numerical Gaug } \\
\text { TU32 }\end{array}$} \\
\hline $\begin{array}{l}\text { Storm } \\
\text { No. } \\
\end{array}$ & $\begin{array}{l}\text { Wave Height, } \\
\mathrm{ft}\end{array}$ & $\begin{array}{l}\text { Wave Period, } \\
\text { sec }\end{array}$ & $\begin{array}{l}\text { Wave Angle, } \\
\text { deg }\end{array}$ & $\begin{array}{l}\text { Setup, } \\
\mathrm{ft}\end{array}$ & $\begin{array}{l}\text { Ponding } \\
\text { Level, } \\
\mathrm{ft}\end{array}$ \\
\hline 18 & 6.6 & 7 & 74 & 3.7 & 0.6 \\
\hline 20 & 1.6 & 5 & 78 & 1.4 & 0.0 \\
\hline 21 & 3.4 & 6 & 78 & 2.3 & 0.0 \\
\hline 28 & 0.4 & 9 & 89 & 1.9 & 0.0 \\
\hline 33 & 14.7 & 11 & 62 & 7.4 & 2.9 \\
\hline 49 & 15.4 & 10 & 62 & 6.9 & 2.8 \\
\hline 60 & 0.0 & $\ldots$ & -- & $\cdots$ & -- \\
\hline 64 & 0.6 & 25 & 62 & 2.9 & 0.0 \\
\hline 82 & 0.7 & 8 & 87 & 1.8 & 0.0 \\
\hline 96 & 0.5 & 8 & 88 & 1.8 & 0.0 \\
\hline 97 & 1.1 & 4 & 8 & 1.2 & 0.0 \\
\hline 127 & 10.6 & 8 & 69 & 5.1 & 1.8 \\
\hline 146 & 7.8 & 9 & 62 & 5.1 & 1.3 \\
\hline 179 & 4.0 & 6 & 15 & 2.3 & 0.0 \\
\hline 231 & 0.5 & 25 & 74 & 2.9 & 0.0 \\
\hline 274 & 1.7 & 5 & 14 & 1.4 & 0.0 \\
\hline 335 & 9.0 & 9 & 69 & 5.4 & 1.6 \\
\hline 352 & 0.4 & 3 & 85 & 1.1 & 0.0 \\
\hline 390 & 6.2 & 6 & 66 & 2.5 & 0.4 \\
\hline 393 & 3.4 & 6 & 82 & 2.3 & 0.0 \\
\hline 414 & 3.3 & 5 & 11 & 2.0 & 0.0 \\
\hline 500 & 3.4 & 7 & 82 & 2.5 & 0.0 \\
\hline 504 & 12.0 & 9 & 28 & 5.9 & 2.2 \\
\hline 510 & 7.0 & 8 & 73 & 4.3 & 0.9 \\
\hline 513 & 4.7 & 8 & 71 & 2.2 & 0.1 \\
\hline 525 & 10.8 & 9 & 74 & 5.7 & 2.0 \\
\hline 543 & 13.6 & 12 & 61 & 7.8 & 2.8 \\
\hline 562 & 22.1 & 14 & 32 & 9.8 & 4.1 \\
\hline 575 & 0.6 & 25 & 62 & 2.9 & 0.0 \\
\hline 586 & 7.5 & 8 & 80 & 4.5 & 1.1 \\
\hline 588 & 4.5 & 7 & 62 & 2.5 & 0.0 \\
\hline
\end{tabular}




\begin{tabular}{|c|c|c|c|c|c|}
\hline \multicolumn{6}{|c|}{$\begin{array}{l}\text { Table D31 } \\
\text { Wave Parameters, Setup, and Ponding Level, for Numerical Gauge } \\
\text { TU33 }\end{array}$} \\
\hline $\begin{array}{l}\text { Storm } \\
\text { No. } \\
\end{array}$ & $\begin{array}{l}\text { Wave Height, } \\
\text { ft }\end{array}$ & $\begin{array}{l}\text { Wave Period, } \\
\text { sec }\end{array}$ & $\begin{array}{l}\text { Wave Angle, } \\
\text { deg }\end{array}$ & $\begin{array}{l}\text { Setup, } \\
\mathrm{ft}\end{array}$ & $\begin{array}{l}\text { Ponding } \\
\text { Level, } \\
\mathrm{ft}\end{array}$ \\
\hline 18 & 7.4 & 7 & 150 & 3.9 & 0.9 \\
\hline 20 & 0.7 & 8 & 168 & 1.8 & 0.0 \\
\hline 21 & 3.4 & 6 & 160 & 2.3 & 0.0 \\
\hline 28 & 0.8 & 9 & 168 & 1.9 & 0.0 \\
\hline 33 & 15.4 & 11 & 135 & 7.5 & 3.0 \\
\hline 49 & 16.2 & 10 & 132 & 7.0 & 3.0 \\
\hline 60 & 0.4 & 5 & 168 & 1.4 & 0.0 \\
\hline 64 & 0.6 & 25 & 136 & 2.9 & 0.0 \\
\hline 82 & 0.4 & 9 & 164 & 1.9 & 0.0 \\
\hline 96 & 0.4 & 9 & 169 & 1.9 & 0.0 \\
\hline 97 & 0.7 & 8 & 169 & 1.8 & 0.0 \\
\hline 127 & 11.5 & 8 & 143 & 5.2 & 2.0 \\
\hline 146 & 8.2 & 9 & 135 & 5.2 & 1.4 \\
\hline 179 & 3.3 & 10 & 164 & 3.0 & 0.0 \\
\hline 231 & 0.5 & 25 & 150 & 2.9 & 0.0 \\
\hline 274 & 2.6 & 5 & 82 & 1.4 & 0.0 \\
\hline 335 & 9.7 & 9 & 143 & 5.5 & 1.8 \\
\hline 352 & 1.4 & 4 & 50 & 1.2 & 0.0 \\
\hline 390 & 7.1 & 6 & 151 & 3.3 & 0.7 \\
\hline 393 & 3.6 & 6 & 155 & 2.3 & 0.0 \\
\hline 414 & 3.7 & 8 & 164 & 2.7 & 0.0 \\
\hline 500 & 3.3 & 8 & 148 & 2.7 & 0.0 \\
\hline 504 & 13.1 & 9 & 92 & 6.1 & 2.4 \\
\hline 510 & 7.2 & 9 & 148 & 4.9 & 1.1 \\
\hline 513 & 5.2 & 8 & 146 & 2.9 & 0.3 \\
\hline 525 & 12.2 & 9 & 150 & 5.9 & 2.3 \\
\hline 543 & 14.2 & 12 & 134 & 7.9 & 2.9 \\
\hline 562 & 27.3 & 14 & 136 & 10.2 & 4.5 \\
\hline 575 & 0.6 & 25 & 136 & 2.9 & 0.0 \\
\hline 586 & 9.3 & 8 & 157 & 4.9 & 1.6 \\
\hline 588 & 3.5 & 8 & 143 & 2.7 & 0.0 \\
\hline
\end{tabular}




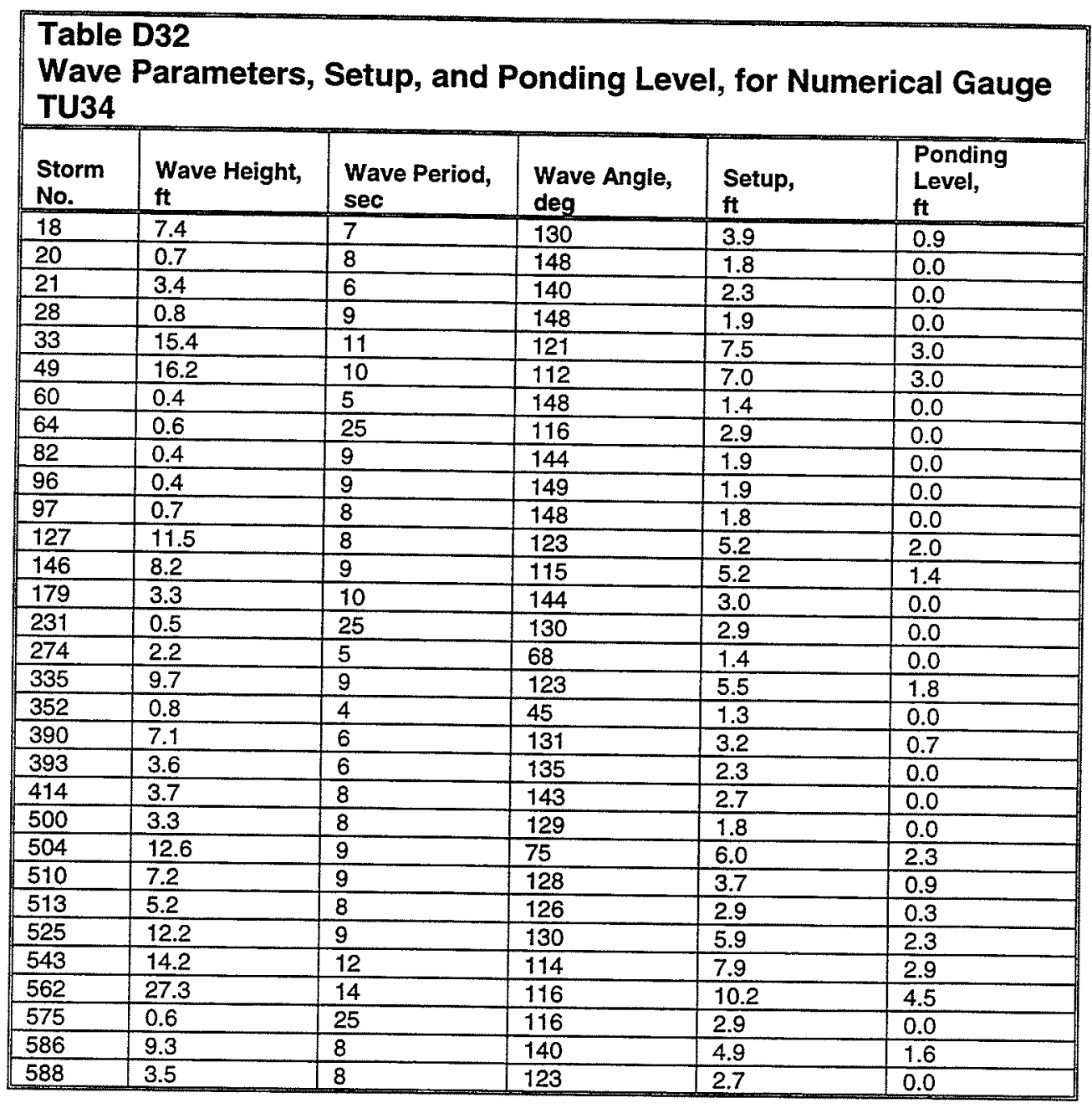




\begin{tabular}{|c|c|c|c|c|c|}
\hline \multicolumn{6}{|c|}{$\begin{array}{l}\text { Table D33 } \\
\text { Wave Parameters, Setup, and Ponding Level, for Numerical Gauge } \\
\text { TU35 }\end{array}$} \\
\hline $\begin{array}{l}\text { Storm } \\
\text { No. }\end{array}$ & $\begin{array}{l}\text { Wave Height, } \\
\mathrm{ft}\end{array}$ & $\begin{array}{l}\text { Wave Period, } \\
\text { sec }\end{array}$ & $\begin{array}{l}\text { Wave Angle, } \\
\text { deg }\end{array}$ & $\begin{array}{l}\text { Setup, } \\
\mathrm{ft}\end{array}$ & $\begin{array}{l}\text { Ponding } \\
\text { Level, } \\
\text { ft }\end{array}$ \\
\hline 18 & 7.8 & 7 & 137 & 4.0 & 1.0 \\
\hline 20 & 0.8 & 8 & 158 & 1.8 & 0.0 \\
\hline 21 & 3.8 & 6 & 149 & 2.3 & 0.0 \\
\hline 28 & 1.0 & 9 & 157 & 1.9 & 0.0 \\
\hline 33 & 15.7 & 11 & 128 & 7.5 & 3.1 \\
\hline 49 & 16.4 & 10 & 118 & 7.0 & 3.0 \\
\hline 60 & 0.6 & 5 & 155 & 1.4 & 0.0 \\
\hline 64 & 0.6 & 25 & 122 & 2.9 & 0.0 \\
\hline 82 & 0.7 & 9 & 153 & 1.9 & 0.0 \\
\hline 96 & 0.5 & 9 & 158 & 1.9 & 0.0 \\
\hline 97 & 0.5 & 9 & 159 & 1.9 & 0.0 \\
\hline 127 & 11.8 & 8 & 130 & 5.2 & 2.1 \\
\hline 146 & 8.3 & 9 & 121 & 5.2 & 1.4 \\
\hline 179 & 3.4 & 10 & 153 & 3.0 & 0.0 \\
\hline 231 & 0.6 & 25 & 138 & 2.9 & 0.0 \\
\hline 274 & 2.5 & 5 & 68 & 1.4 & 0.0 \\
\hline 335 & 10.0 & 9 & 130 & 5.6 & 1.8 \\
\hline 352 & 0.4 & 4 & 153 & 1.3 & 0.0 \\
\hline 390 & 7.5 & 6 & 139 & 3.3 & 0.8 \\
\hline 393 & 3.9 & 6 & 143 & 2.3 & 0.0 \\
\hline 414 & 5.3 & 8 & 151 & 3.0 & 0.3 \\
\hline 500 & 5.6 & 7 & 147 & 2.7 & 0.3 \\
\hline 504 & 13.0 & 9 & 77 & 6.0 & 2.4 \\
\hline 510 & 7.5 & 9 & 135 & 4.9 & 1.2 \\
\hline 513 & 5.3 & 8 & 133 & 3.0 & 0.3 \\
\hline 525 & 12.9 & 9 & 137 & 6.0 & 2.4 \\
\hline 543 & 14.4 & 12 & 120 & 7.9 & 3.0 \\
\hline 562 & 27.6 & 14 & 122 & 10.3 & 4.6 \\
\hline 575 & 0.6 & 25 & 122 & 2.9 & 0.0 \\
\hline 586 & 10.1 & 8 & 145 & 5.0 & 1.7 \\
\hline 588 & 3.6 & 8 & 130 & 2.7 & 0.0 \\
\hline
\end{tabular}




\begin{tabular}{|c|c|c|c|c|c|}
\hline $\begin{array}{l}\text { Storm } \\
\text { No. } \\
\end{array}$ & $\begin{array}{l}\text { Wave Height, } \\
\mathrm{ft}\end{array}$ & $\begin{array}{l}\text { Wave Period, } \\
\text { sec }\end{array}$ & $\begin{array}{l}\text { Wave Angle, } \\
\text { deg }\end{array}$ & $\begin{array}{l}\text { Setup, } \\
\mathrm{tt}\end{array}$ & $\begin{array}{l}\text { Ponding } \\
\text { Level, } \\
\mathrm{ft}\end{array}$ \\
\hline 18 & 7.8 & 7 & 137 & 4.0 & 1.0 \\
\hline 20 & 0.8 & 8 & 158 & 1.8 & 0.0 \\
\hline 21 & 0.0 & $\cdots$ & - & - & -- \\
\hline 28 & 1.0 & 9 & 157 & 1.9 & 0.0 \\
\hline 33 & 15.7 & 11 & 128 & 7.5 & 3.1 \\
\hline 49 & 16.4 & 10 & 118 & 7.0 & 3.0 \\
\hline 60 & 0.5 & 5 & 157 & 1.4 & 0.0 \\
\hline 64 & 0.6 & 25 & 122 & 2.9 & 0.0 \\
\hline 82 & 0.7 & 9 & 153 & 1.9 & 0.0 \\
\hline 96 & 0.5 & 9 & 158 & 1.9 & 0.0 \\
\hline 97 & 0.5 & 9 & 159 & 1.9 & 0.0 \\
\hline 127 & 11.8 & 8 & 133 & 5.2 & 2.0 \\
\hline 146 & 8.3 & 9 & 121 & 5.2 & 1.4 \\
\hline 179 & 3.4 & 10 & 153 & 3.0 & 0.0 \\
\hline 231 & 0.6 & 25 & 138 & 2.9 & 0.0 \\
\hline 274 & 2.3 & 5 & 68 & 1.4 & 0.0 \\
\hline 335 & 10.0 & 9 & 130 & 5.6 & 1.8 \\
\hline 352 & 1.2 & 4 & 38 & 1.2 & 0.0 \\
\hline 390 & 7.5 & 6 & 139 & 3.3 & 0.8 \\
\hline 393 & 3.9 & 6 & 143 & 2.3 & 0.0 \\
\hline 414 & 5.3 & 8 & 151 & 3.0 & 0.3 \\
\hline 500 & 5.6 & 7 & 147 & 2.7 & 0.3 \\
\hline 504 & 13.0 & 9 & 77 & 6.0 & 2.4 \\
\hline 510 & 7.5 & 9 & 135 & 4.9 & 1.2 \\
\hline 513 & 5.3 & 8 & 133 & 3.0 & 0.3 \\
\hline 525 & 12.9 & 9 & 137 & 6.0 & 2.4 \\
\hline 543 & 14.4 & 12 & 120 & 7.9 & 3.0 \\
\hline 562 & 27.6 & 14 & 122 & 10.3 & 4.6 \\
\hline 575 & 0.6 & 25 & 122 & 2.9 & 0.0 \\
\hline 586 & 10.1 & 8 & 145 & 5.0 & 1.7 \\
\hline 588 & 3.6 & 8 & 130 & 2.7 & 0.0 \\
\hline
\end{tabular}




\begin{tabular}{|c|c|c|c|c|c|}
\hline \multicolumn{6}{|c|}{$\begin{array}{l}\text { Table D35 } \\
\text { Wave Parameters, Setup, and Ponding Level, for Numerical Gauge } \\
\text { TU37 }\end{array}$} \\
\hline $\begin{array}{l}\text { Storm } \\
\text { No. } \\
\end{array}$ & $\begin{array}{l}\text { Wave Height, } \\
\mathrm{ft}\end{array}$ & $\begin{array}{l}\text { Wave Period, } \\
\text { sec }\end{array}$ & $\begin{array}{l}\text { Wave Angle, } \\
\text { deg }\end{array}$ & $\begin{array}{l}\text { Setup, } \\
\mathrm{ft}\end{array}$ & $\begin{array}{l}\text { Ponding } \\
\text { Level, } \\
\text { ft }\end{array}$ \\
\hline 18 & 7.2 & 8 & 119 & 4.4 & 1.0 \\
\hline 20 & 1.2 & 8 & 126 & 1.8 & 0.0 \\
\hline 21 & 3.7 & 7 & 121 & 2.5 & 0.0 \\
\hline 28 & 1.4 & 9 & 126 & 1.9 & 0.0 \\
\hline 33 & 16.1 & 11 & 94 & 7.6 & 3.1 \\
\hline 49 & 16.5 & 10 & 84 & 7.0 & 6.1 \\
\hline 60 & 0.6 & 5 & 126 & 1.4 & 0.0 \\
\hline 64 & 0.7 & 25 & 88 & 2.9 & 0.0 \\
\hline 82 & 0.9 & 9 & 122 & 1.9 & 0.0 \\
\hline 96 & 0.7 & 9 & 127 & 1.9 & 0.0 \\
\hline 97 & 0.6 & 9 & 128 & 1.9 & 0.0 \\
\hline 127 & 12.1 & 8 & 97 & 5.3 & 2.1 \\
\hline 146 & 8.4 & 9 & 87 & 5.2 & 1.5 \\
\hline 179 & 4.6 & 10 & 122 & 3.3 & 0.3 \\
\hline 231 & 0.6 & 25 & 105 & 2.9 & 0.0 \\
\hline 274 & 2.4 & 5 & 28 & 1.4 & 0.0 \\
\hline 335 & 10.0 & 9 & 96 & 5.6 & 1.8 \\
\hline 352 & 0.4 & 4 & 122 & 1.3 & 0.0 \\
\hline 390 & 7.9 & 6 & 107 & 3.4 & 0.9 \\
\hline 393 & 3.6 & 7 & 118 & 2.5 & 0.0 \\
\hline 414 & 6.1 & 8 & 120 & 3.2 & 0.6 \\
\hline 500 & 6.1 & 7 & 115 & 2.8 & 0.5 \\
\hline 504 & 12.9 & 9 & 43 & 6.0 & 2.4 \\
\hline 510 & 7.8 & 9 & 102 & 5.0 & 1.3 \\
\hline 513 & 5.5 & 8 & 100 & 3.0 & 0.4 \\
\hline 525 & 13.4 & 9 & 105 & 6.1 & 2.5 \\
\hline 543 & 14.5 & 12 & 86 & 7.9 & 3.0 \\
\hline 562 & 28.0 & 14 & 88 & 10.3 & 4.6 \\
\hline 575 & 6.0 & 7 & 94 & 2.8 & 0.4 \\
\hline 586 & 10.9 & 8 & 113 & 5.1 & 1.9 \\
\hline 588 & 3.7 & 8 & 97 & 2.7 & 0.0 \\
\hline
\end{tabular}




\begin{tabular}{|c|c|c|c|c|c|}
\hline \multicolumn{6}{|c|}{$\begin{array}{l}\text { Table D36 } \\
\text { Wave Parameters, Setup, and Ponding Level, for Numerical Gaug } \\
\text { TU38 }\end{array}$} \\
\hline $\begin{array}{l}\text { Storm } \\
\text { No. }\end{array}$ & $\begin{array}{l}\text { Wave Height, } \\
\mathrm{tt}\end{array}$ & $\begin{array}{l}\text { Wave Period, } \\
\text { sec }\end{array}$ & $\begin{array}{l}\text { Wave Angle, } \\
\text { deg }\end{array}$ & $\begin{array}{l}\text { Setup, } \\
\mathrm{ft}\end{array}$ & $\begin{array}{l}\text { Ponding } \\
\text { Level, } \\
\mathrm{ft}\end{array}$ \\
\hline 18 & 7.4 & 7 & 160 & 3.9 & 0.9 \\
\hline 20 & 0.7 & 8 & 178 & 1.8 & 0.0 \\
\hline 21 & 3.4 & 6 & 170 & 2.3 & 0.0 \\
\hline 28 & 0.8 & 9 & 178 & 1.9 & 0.0 \\
\hline 33 & 15.4 & 11 & 151 & 7.5 & 3.0 \\
\hline 49 & 8.3 & 10 & 160 & 5.7 & 1.6 \\
\hline 60 & 0.9 & 5 & 118 & 1.4 & 0.0 \\
\hline 64 & 1.9 & 10 & 144 & 2.0 & 0.0 \\
\hline 82 & 0.4 & 9 & 144 & 1.9 & 0.0 \\
\hline 96 & 0.9 & 9 & 140 & 1.9 & 0.0 \\
\hline 97 & 0.5 & 9 & 140 & 1.9 & 0.0 \\
\hline 127 & 11.5 & 8 & 153 & 5.2 & 2.0 \\
\hline 146 & 8.2 & 9 & 145 & 5.2 & 1.4 \\
\hline 179 & 3.3 & 10 & 174 & 3.0 & 0.0 \\
\hline 231 & 0.5 & 25 & 160 & 2.9 & 0.0 \\
\hline 274 & 2.4 & 5 & 88 & 1.4 & 0.0 \\
\hline 335 & 9.7 & 9 & 153 & 5.5 & 1.8 \\
\hline 352 & 1.1 & 4 & 67 & 1.2 & 0.0 \\
\hline 390 & 7.1 & 6 & 161 & 3.3 & 0.7 \\
\hline 393 & 4.1 & 6 & 137 & 2.3 & 0.0 \\
\hline 414 & 3.7 & 8 & 141 & 2.7 & 0.0 \\
\hline 500 & 4.4 & 7 & 142 & 2.5 & 0.0 \\
\hline 504 & 7.2 & 8 & 159 & 4.4 & 1.0 \\
\hline 510 & 5.1 & 8 & 153 & 2.9 & 0.2 \\
\hline 513 & 3.3 & 7 & 150 & 2.5 & 0.0 \\
\hline 525 & 7.5 & 9 & 154 & 5.0 & 1.2 \\
\hline 543 & 14.2 & 12 & 144 & 7.9 & 2.9 \\
\hline 562 & 27.3 & 14 & 146 & 10.2 & 4.5 \\
\hline 575 & 3.4 & 7 & 154 & 2.5 & 0.0 \\
\hline 586 & 6.8 & 8 & 147 & 4.3 & 0.9 \\
\hline 588 & 3.3 & 6 & 153 & 2.3 & 0.0 \\
\hline
\end{tabular}




\begin{tabular}{|c|c|c|c|c|c|}
\hline \multicolumn{6}{|c|}{$\begin{array}{l}\text { Table D37 } \\
\text { Wave Parameters, Setup, and Ponding Level, for Numerical Gaug } \\
\text { TU39 }\end{array}$} \\
\hline $\begin{array}{l}\text { Storm } \\
\text { No. } \\
\end{array}$ & $\begin{array}{l}\text { Wave Height, } \\
\mathrm{ft}\end{array}$ & $\begin{array}{l}\text { Wave Period, } \\
\text { sec }\end{array}$ & $\begin{array}{l}\text { Wave Angle, } \\
\text { deg }\end{array}$ & $\begin{array}{l}\text { Setup, } \\
\mathrm{ft}\end{array}$ & $\begin{array}{l}\text { Ponding } \\
\text { Level, } \\
\mathrm{tt}\end{array}$ \\
\hline 18 & 0.0 & -- & -- & -. & -- \\
\hline 20 & 0.0 & - & - & $\cdots$ & - \\
\hline 21 & 0.0 & - & - & -- & -- \\
\hline 28 & 0.0 & 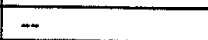 & - & ... & - \\
\hline 33 & 0.0 & -- & -- & -- & -- \\
\hline 49 & 0.0 & - & -- & -- & -- \\
\hline 60 & 0.0 & - & -- & - & $\cdots$ \\
\hline 64 & 0.0 & -- & -- & -- & -- \\
\hline 82 & 0.0 & - & -. & -- & -- \\
\hline 96 & 0.0 & - & - & -- & 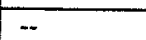 \\
\hline 97 & 0.0 & $\ldots$ & -- & -- & $\ldots$ \\
\hline 127 & 0.0 & $\cdots$ & \begin{tabular}{|l|}
- \\
\end{tabular} & - & -- \\
\hline 146 & 0.0 & -- & - & - & - \\
\hline 179 & 0.0 & -- & - & -- & -- \\
\hline 231 & 0.0 & -- & -- & -- & - \\
\hline 274 & 0.0 & -- & - & - & - \\
\hline 335 & 0.0 & -- & -. & -- & - \\
\hline 352 & 0.0 & - & - & -- & - \\
\hline 390 & 0.0 & - & -- & -- & - \\
\hline 393 & 0.0 & -- & -- & - & $\ldots$ \\
\hline 414 & 0.0 & -- & - & - & -- \\
\hline 500 & 0.0 & - & - & - & - \\
\hline 504 & 0.0 & -- & - & $=$ & $\ldots$ \\
\hline 510 & 0.0 & - & -- & -- & -- \\
\hline 513 & 0.0 & -- & - & - & $\ldots$ \\
\hline 525 & 0.0 & $\cdots$ & -- & $\cdots$ & -- \\
\hline 543 & 0.3 & 13 & 105 & 2.7 & 0.0 \\
\hline 562 & 0.7 & 12 & 105 & 2.6 & 0.0 \\
\hline 575 & 0.0 & -- & - & -0 & - \\
\hline 586 & 0.0 & - & - & -. & - \\
\hline 588 & 0.0 & - & -- & -- & - \\
\hline
\end{tabular}




\begin{tabular}{|c|c|c|c|c|c|}
\hline \multicolumn{6}{|c|}{$\begin{array}{l}\text { Table D38 } \\
\text { Wave Parameters, Setup, and Ponding Level, for Numerical Gauge } \\
\text { TU40 }\end{array}$} \\
\hline $\begin{array}{l}\text { Storm } \\
\text { No. }\end{array}$ & $\begin{array}{l}\text { Wave Height, } \\
\text { ft }\end{array}$ & $\begin{array}{l}\text { Wave Period, } \\
\text { sec }\end{array}$ & $\begin{array}{l}\text { Wave Angle, } \\
\text { deg }\end{array}$ & $\begin{array}{l}\text { Setup, } \\
\mathrm{ft}\end{array}$ & $\begin{array}{l}\text { Ponding } \\
\text { Level, } \\
\mathrm{ft}\end{array}$ \\
\hline 18 & 0.3 & 7 & 98 & 1.0 & 0.0 \\
\hline 20 & 0.3 & 4 & 97 & 0.7 & 0.0 \\
\hline 21 & 0.3 & 5 & 97 & 0.9 & 0.0 \\
\hline 28 & 0.0 & -- & -- & -- & - \\
\hline 33 & 0.3 & 10 & 97 & 1.3 & 0.0 \\
\hline 49 & 0.4 & 9 & 97 & 1.2 & 0.0 \\
\hline 60 & 0.0 & - & $\cdots$ & -- & - \\
\hline 64 & 0.0 & -- & - & -- & -- \\
\hline 82 & 0.0 & $=$ & - & -- & 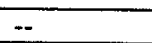 \\
\hline 96 & 0.3 & 5 & 97 & 0.9 & 0.0 \\
\hline 97 & 0.0 & - & - & - & -- \\
\hline 127 & 0.3 & 7 & 98 & 1.0 & 0.0 \\
\hline 146 & 0.0 & $\ldots$ & -- & - & - \\
\hline 179 & 0.0 & -- & - & - & $\ldots$ \\
\hline 231 & 0.0 & $\ldots$ & -- & $-\infty$ & - \\
\hline 274 & 0.0 & $\cdots$ & $=$ & -- & - \\
\hline 335 & 0.0 & $\cdots$ & -- & -- & $\ldots$ \\
\hline 352 & 0.0 & -- & $\cdots$ & $\ldots$ & -- \\
\hline 390 & 0.4 & 5 & 98 & 0.9 & 0.0 \\
\hline 393 & 0.4 & 5 & 97 & 0.8 & 0.0 \\
\hline 414 & 0.3 & 5 & 97 & 0.9 & 0.0 \\
\hline 500 & 0.4 & 5 & 97 & 0.8 & 0.0 \\
\hline 504 & 0.3 & 8 & 98 & 1.1 & 0.0 \\
\hline 510 & 0.4 & 5 & 98 & 0.9 & 0.0 \\
\hline 513 & 0.3 & 5 & 97 & 0.9 & 0.0 \\
\hline 525 & 0.4 & 8 & 98 & 1.1 & 0.0 \\
\hline 543 & 0.3 & 13 & 105 & 1.5 & 0.0 \\
\hline 562 & 0.8 & 14 & 105 & 1.5 & 0.0 \\
\hline 575 & 0.3 & 4 & 97 & 0.7 & 0.0 \\
\hline 586 & 0.3 & 7 & 98 & 1.0 & 0.0 \\
\hline 588 & 0.3 & 5 & 97 & 0.8 & 0.0 \\
\hline
\end{tabular}




\begin{tabular}{|c|c|c|c|c|c|}
\hline \multicolumn{6}{|c|}{$\begin{array}{l}\text { Table D39 } \\
\text { Wave Parameters, Setup, and Ponding Level, for Numerical Gaug } \\
\text { TU41 }\end{array}$} \\
\hline $\begin{array}{l}\text { Storm } \\
\text { No. }\end{array}$ & $\begin{array}{l}\text { Wave Height, } \\
\mathrm{ft}\end{array}$ & $\begin{array}{l}\text { Wave Period, } \\
\text { sec }\end{array}$ & $\begin{array}{l}\text { Wave Angle, } \\
\text { deg }\end{array}$ & $\begin{array}{l}\text { Setup, } \\
\mathrm{ft}\end{array}$ & $\begin{array}{l}\text { Ponding } \\
\text { Level, } \\
\text { ft }\end{array}$ \\
\hline 18 & 0.0 & - & - & -- & -- \\
\hline 20 & 0.0 & - & -- & $\ldots$ & - \\
\hline 21 & 0.0 & -- & -- & -- & -- \\
\hline 28 & 0.0 & - & - & -- & -- \\
\hline 33 & 0.0 & - & - & -- & -- \\
\hline 49 & 0.0 & - & $\cdots$ & -- & -- \\
\hline 60 & 0.0 & -- & - & - & $\cdots$ \\
\hline 64 & 0.0 & -- & -- & - & -- \\
\hline 82 & 0.0 & 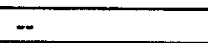 & -- & -- & -- \\
\hline 96 & 0.0 & -- & -- & - &.- \\
\hline 97 & 0.0 & -- & -- & - & -- \\
\hline 127 & 0.0 & -- & $\cdots$ & - & - \\
\hline 146 & 0.0 & - & - & -- & -- \\
\hline 179 & 0.0 & $\cdots$ & +- & $\cdots$ & -- \\
\hline 231 & 0.0 & -- & - & -- & - \\
\hline 274 & 0.0 & $\cdots$ & - & $\ldots$ & -- \\
\hline 335 & 0.0 & $-\cdots$ & - & -- & -- \\
\hline 352 & 0.0 & -- & - & -- & - \\
\hline 390 & 0.0 & -- & - & $\ldots$ & -- \\
\hline 393 & 0.0 & $\ldots$ & - & $m$ & - \\
\hline 414 & 0.0 & - & - & $=$ & $=$ \\
\hline 500 & 0.3 & 6 & 96 & 1.7 & 0.0 \\
\hline 504 & 0.0 & - & -- & -- & - \\
\hline 510 & 0.0 & - & -- & $m$ & - \\
\hline 513 & 0.4 & 5 & 96 & 1.6 & 0.0 \\
\hline 525 & 0.3 & 7 & 96 & 1.9 & 0.0 \\
\hline 543 & 0.3 & 13 & 105 & 2.7 & 0.0 \\
\hline 562 & 0.3 & 13 & 105 & 2.7 & 0.0 \\
\hline 575 & 0.0 & - & $\ldots$ &..- & 0.0 \\
\hline 586 & 0.4 & 2 & 96 & 1.9 & 0.0 \\
\hline 588 & 0.4 & 5 & 96 & 1.6 & 0.0 \\
\hline
\end{tabular}




\begin{tabular}{|c|c|c|c|c|c|}
\hline $\begin{array}{l}\text { Storm } \\
\text { No. }\end{array}$ & $\begin{array}{l}\text { Wave Height, } \\
\mathrm{ft}\end{array}$ & $\begin{array}{l}\text { Wave Period, } \\
\text { sec }\end{array}$ & $\begin{array}{l}\text { Wave Angle, } \\
\text { deg }\end{array}$ & $\begin{array}{l}\text { Setup, } \\
\mathrm{ft}\end{array}$ & $\begin{array}{l}\text { Ponding } \\
\text { Level, } \\
\mathrm{ft}\end{array}$ \\
\hline 18 & 0.0 & - & $\cdots$ & - & -- \\
\hline 20 & 0.0 & -- & -- & -- & -- \\
\hline 21 & 0.0 & $\cdots$ & - & - & - \\
\hline 28 & 0.0 & -- & - & -- & - \\
\hline 33 & 0.0 & -- & - & - & - \\
\hline 49 & 0.0 & -- & -- & $\cdots$ & -- \\
\hline 60 & 0.0 & -- & $\cdots$ & -- & -- \\
\hline 64 & 0.0 & - & -- & -- & -- \\
\hline 82 & 0.0 & -- & $\cdots$ & -- & -- \\
\hline 96 & 0.0 & -- & 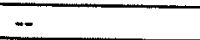 & $\cdots$ & -- \\
\hline 97 & 0.0 & $\rightarrow$ & - & -- & -- \\
\hline 127 & 0.0 & - & - & -- & $\because$ \\
\hline 146 & 0.0 & -- & $\cdots$ & - & -- \\
\hline 179 & 0.0 & $\cdots$ & -- & - & -- \\
\hline 231 & 0.0 & -- & -- & $\cdots$ & -- \\
\hline 274 & 0.0 & - & -- & $\cdots$ & -- \\
\hline 335 & 0.0 & $\cdots$ & $\cdots$ & $\cdots$ & -- \\
\hline 352 & 0.0 & -- & -- & -- & -- \\
\hline 390 & 0.0 & -- & -- & -- & - \\
\hline 393 & 0.0 & -- & $\because$ & - & - \\
\hline 414 & 0.0 & - & $\cdots$ & - & -- \\
\hline 500 & 0.3 & 6 & 96 & 1.5 & 0.0 \\
\hline 504 & 0.0 & -- & $\cdots$ & -- & - \\
\hline 510 & 0. & -- & - & $\cdots$ & -- \\
\hline 513 & 0.4 & 5 & 96 & 1.4 & 0.0 \\
\hline 525 & 0.3 & 7 & 96 & 1.6 & 0.0 \\
\hline 543 & 0.3 & 13 & 105 & 2.4 & 0.0 \\
\hline 562 & 0.6 & 13 & 105 & 2.3 & 0.0 \\
\hline 575 & 0.0 & -- & -- & $\because$ & -- \\
\hline 586 & 0.4 & 7 & 96 & 1.6 & 0.0 \\
\hline 588 & 0.4 & 5 & 96 & 1.4 & 0.0 \\
\hline
\end{tabular}




\begin{tabular}{|c|c|c|c|c|c|}
\hline \multicolumn{6}{|c|}{$\begin{array}{l}\text { Table D41 } \\
\text { Wave Parameters, Setup, and Ponding Level, for Numerical Gaug } \\
\text { TU44 }\end{array}$} \\
\hline $\begin{array}{l}\text { Storm } \\
\text { No. }\end{array}$ & $\begin{array}{l}\text { Wave Height, } \\
\mathrm{ft}\end{array}$ & $\begin{array}{l}\text { Wave Period, } \\
\text { sec }\end{array}$ & $\begin{array}{l}\text { Wave Angle, } \\
\text { deg }\end{array}$ & $\begin{array}{l}\text { Setup, } \\
\mathrm{ft}\end{array}$ & $\begin{array}{l}\text { Ponding } \\
\text { Level, } \\
\mathrm{ft}\end{array}$ \\
\hline 18 & 0.5 & 8 & 74 & 1.1 & 0.0 \\
\hline 20 & 0.3 & 5 & 75 & 0.8 & 0.0 \\
\hline 21 & 0.4 & 7 & 75 & 0.9 & 0.0 \\
\hline 28 & 0.4 & 5 & 75 & 0.8 & 0.0 \\
\hline 33 & 0.5 & 11 & 75 & 1.3 & 0.0 \\
\hline 49 & 0.6 & 10 & 75 & 1.2 & 0.0 \\
\hline 60 & 0.0 & - & - & $\cdots$ & -- \\
\hline 64 & 0.0 & - & - & -- & $\cdots$ \\
\hline 82 & 0.0 & - & -- & 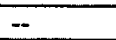 & -- \\
\hline 96 & 0.4 & 7 & 75 & 0.9 & 0.0 \\
\hline 97 & 0.0 & -- & -- & - & - \\
\hline 127 & 0.5 & 8 & 74 & 1.1 & 0.0 \\
\hline 146 & 0.3 & 8 & 75 & 1.1 & 0.0 \\
\hline 179 & 0.3 & 9 & 75 & 1.1 & 0.0 \\
\hline 231 & 0.4 & 8 & 75 & 1.1 & 0.0 \\
\hline 274 & 0.0 & $\cdots$ & - & - & - \\
\hline 335 & 0.3 & 8 & 75 & 1.1 & 0.0 \\
\hline 352 & 0.0 & -- & - & - & -- \\
\hline 390 & 0.4 & 7 & 75 & 0.9 & 0.0 \\
\hline 393 & 0.4 & 6 & 75 & 0.9 & 0.0 \\
\hline 414 & 0.4 & 8 & 75 & 1.1 & 0.0 \\
\hline 500 & 0.4 & 5 & 74 & 0.8 & 0.0 \\
\hline 504 & 0.4 & 10 & 74 & 1.2 & 0.0 \\
\hline 510 & 0.3 & 9 & 75 & 1.1 & 0.0 \\
\hline 513 & 0.5 & 6 & 75 & 0.9 & 0.0 \\
\hline 525 & 0.3 & 10 & 75 & 1.2 & 0.0 \\
\hline 543 & 0.3 & 13 & 105 & 1.4 & 0.0 \\
\hline 562 & 0.8 & 14 & 105 & 1.3 & 0.0 \\
\hline 575 & 0.4 & 5 & 74 & 0.8 & 0.0 \\
\hline 586 & 0.4 & 8 & 75 & 1.1 & 0.0 \\
\hline 588 & 0.4 & 6 & 75 & 0.9 & 0.0 \\
\hline
\end{tabular}




\begin{tabular}{|c|c|c|c|c|c|}
\hline $\begin{array}{l}\text { Storm } \\
\text { No. }\end{array}$ & $\begin{array}{l}\text { Wave Height, } \\
\mathrm{ft}\end{array}$ & $\begin{array}{l}\text { Wave Period, } \\
\text { sec }\end{array}$ & $\begin{array}{l}\text { Wave Angle, } \\
\text { deg }\end{array}$ & $\begin{array}{l}\text { Setup, } \\
\mathrm{ft}\end{array}$ & $\begin{array}{l}\text { Ponding } \\
\text { Level, } \\
\mathrm{ft}\end{array}$ \\
\hline 18 & 1.6 & 8 & 79 & 1.1 & 0.0 \\
\hline 20 & 0.6 & 8 & 79 & 1.1 & 0.0 \\
\hline 21 & 0.5 & 8 & 79 & 1.1 & 0.0 \\
\hline 28 & 0.6 & 9 & 79 & 1.2 & 0.0 \\
\hline 33 & 2.1 & 11 & 78 & 1.3 & 0.0 \\
\hline 49 & 2.5 & 10 & 79 & 1.2 & 0.0 \\
\hline 60 & 0.0 & -- & $\ldots$ & -- & - \\
\hline 64 & 1.0 & 10 & 80 & 1.2 & 0.0 \\
\hline 82 & 1.1 & 8 & 79 & 1.1 & 0.0 \\
\hline 96 & 0.7 & 9 & 79 & 1.2 & 0.0 \\
\hline 97 & 0.5 & 8 & 79 & 1.1 & 0.0 \\
\hline 127 & 0.5 & 8 & 80 & 1.1 & 0.0 \\
\hline 146 & 1.1 & 9 & 78 & 1.2 & 0.0 \\
\hline 179 & 1.0 & 10 & 80 & 1.2 & 0.0 \\
\hline 231 & 1.2 & 9 & 79 & 1.2 & 0.0 \\
\hline 274 & 0.5 & 4 & 79 & 0.7 & 0.0 \\
\hline 335 & 1.2 & 10 & 79 & 1.2 & 0.0 \\
\hline 352 & 0.6 & 3 & 77 & 0.6 & 0.0 \\
\hline 390 & 1.5 & 8 & 80 & 1.1 & 0.0 \\
\hline 393 & 1.6 & 6 & 79 & 0.9 & 0.0 \\
\hline 414 & 0.4 & 8 & 79 & 1.1 & 0.0 \\
\hline 500 & 0.7 & 8 & 79 & 1.1 & 0.0 \\
\hline 504 & 2.3 & 9 & 80 & 1.2 & 0.0 \\
\hline 510 & 1.5 & 9 & 79 & 1.2 & 0.0 \\
\hline 513 & 1.1 & 8 & 79 & 1.1 & 0.0 \\
\hline 525 & 0.4 & 10 & 79 & 1.2 & 0.0 \\
\hline 543 & 1.4 & 13 & 95 & 1.4 & 0.0 \\
\hline 562 & 1.0 & 14 & 98 & 1.4 & 0.0 \\
\hline 575 & 1.3 & 7 & 79 & 1.0 & 0.0 \\
\hline 586 & 1.2 & 9 & 79 & 1.2 & 0.0 \\
\hline 588 & 0.8 & 9 & 79 & 1.2 & 0.0 \\
\hline
\end{tabular}




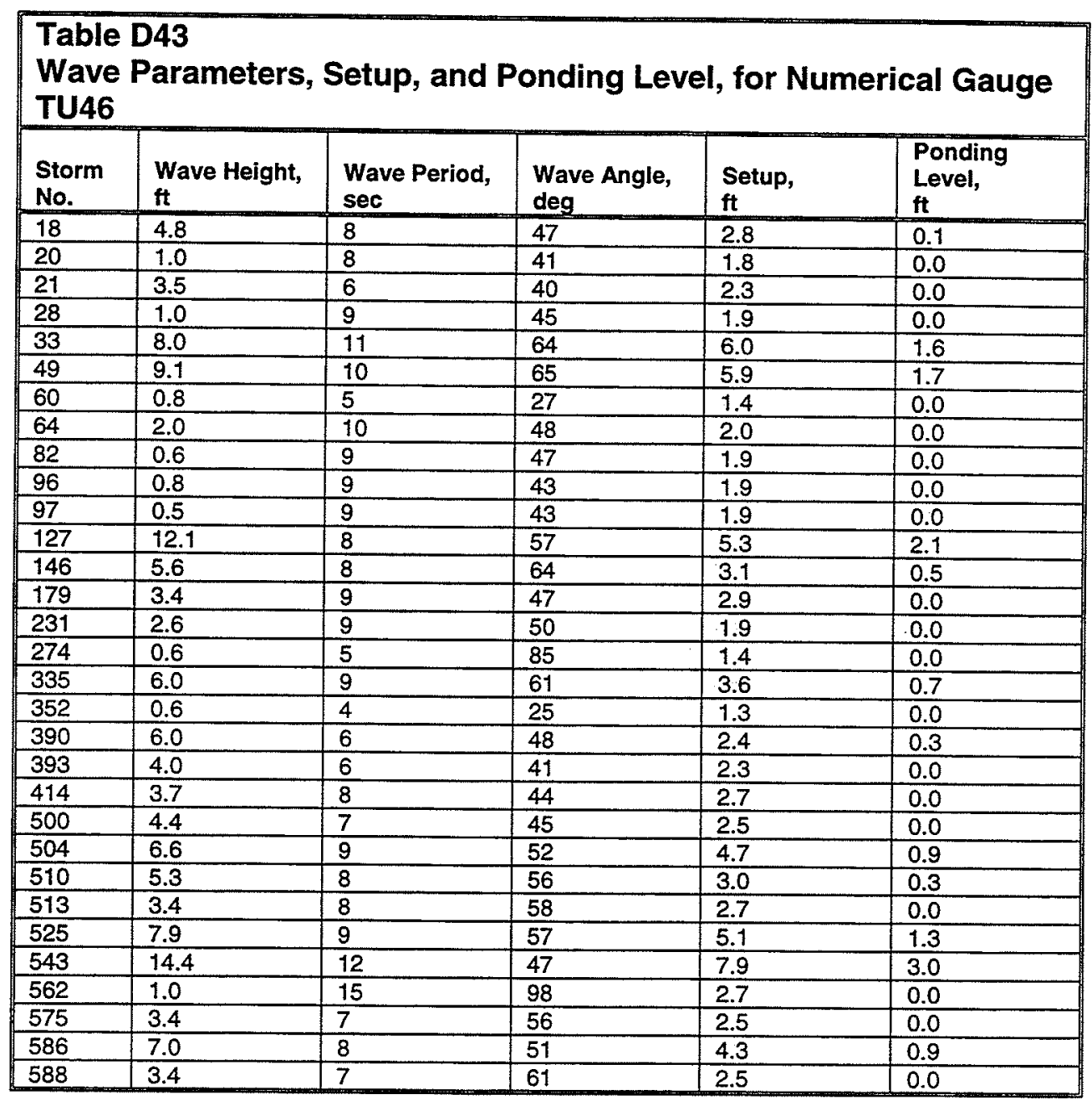




\begin{tabular}{|c|c|c|c|c|c|}
\hline $\begin{array}{l}\text { Storm } \\
\text { No. } \\
\end{array}$ & $\begin{array}{l}\text { Wave Height, } \\
\mathrm{ft}\end{array}$ & $\begin{array}{l}\text { Wave Period, } \\
\text { sec }\end{array}$ & $\begin{array}{l}\text { Wave Angle, } \\
\text { deg }\end{array}$ & $\begin{array}{l}\text { Setup, } \\
\mathrm{ft}\end{array}$ & $\begin{array}{l}\text { Ponding } \\
\text { Level, } \\
\mathrm{ft}\end{array}$ \\
\hline 18 & 3.6 & 7 & 102 & 2.5 & 0.0 \\
\hline 20 & 0.8 & 5 & 104 & 1.4 & 0.0 \\
\hline 21 & 0.4 & 6 & 109 & 1.6 & 0.0 \\
\hline 28 & 0.4 & 5 & 108 & 1.4 & 0.0 \\
\hline 33 & 13.2 & 11 & 62 & 7.2 & \begin{tabular}{|l|}
2.7 \\
\end{tabular} \\
\hline 49 & 12.7 & 10 & 92 & 6.6 & 2.5 \\
\hline 60 & 0.0 & $\cdots$ & - & -- & -- \\
\hline 64 & 0.5 & 25 & 94 & 2.9 & 0.0 \\
\hline 82 & 0.6 & \begin{tabular}{|l|}
4 \\
\end{tabular} & 24 & 1.3 & 0.0 \\
\hline 96 & 0.0 & -- & - & $\cdots$ & -- \\
\hline 97 & 2.1 & 4 & 40 & 1.2 & 0.0 \\
\hline 127 & 7.3 & 8 & 98 & 4.4 & 1.0 \\
\hline 146 & 7.0 & 8 & 93 & 4.3 & 0.9 \\
\hline 179 & 3.4 & 6 & 43 & 2.3 & 0.0 \\
\hline 231 & 3.6 & 5 & 48 & 2.0 & 0.0 \\
\hline 274 & 2.3 & 5 & 45 & 1.4 & 0.0 \\
\hline 335 & 6.2 & 9 & 98 & 3.6 & 0.8 \\
\hline 352 & 1.2 & 4 & 28 & 1.2 & 0.0 \\
\hline 390 & 6.1 & 6 & 81 & 2.4 & 0.3 \\
\hline 393 & 1.4 & 6 & 105 & 1.6 & 0.0 \\
\hline 414 & 4.8 & 5 & 41 & 1.9 & 0.0 \\
\hline 500 & 1.9 & 8 & 101 & 1.8 & 0.0 \\
\hline 504 & 12.9 & 9 & 67 & 6.0 & 2.4 \\
\hline 510 & 3.4 & 9 & 101 & 2.9 & 0.0 \\
\hline 513 & 2.7 & 8 & 101 & 1.8 & 0.0 \\
\hline 525 & 6.4 & 9 & 102 & 3.7 & 0.9 \\
\hline 543 & 10.7 & 12 & 94 & 7.2 & 2.3 \\
\hline 562 & 24.3 & 14 & 72 & 10.0 & 4.3 \\
\hline 575 & 0.5 & 25 & 94 & 2.9 & 0.0 \\
\hline 586 & 5.7 & 7 & 96 & 2.7 & 0.3 \\
\hline 588 & 3.5 & 7 & 94 & 2.5 & 0.0 \\
\hline
\end{tabular}




\begin{tabular}{|c|c|c|c|c|c|}
\hline \multicolumn{6}{|c|}{$\begin{array}{l}\text { Table D45 } \\
\text { Wave Parameters, Setup, and Ponding Level, for Numerical Gauge } \\
\text { TU48 }\end{array}$} \\
\hline $\begin{array}{l}\text { Storm } \\
\text { No. }\end{array}$ & $\begin{array}{l}\text { Wave Height, } \\
\mathrm{ft}\end{array}$ & $\begin{array}{l}\text { Wave Period, } \\
\text { sec }\end{array}$ & $\begin{array}{l}\text { Wave Angle, } \\
\text { deg }\end{array}$ & $\begin{array}{l}\text { Setup, } \\
\mathrm{ft}\end{array}$ & $\begin{array}{l}\text { Ponding } \\
\text { Level, } \\
\text { ft }\end{array}$ \\
\hline 18 & 9.0 & 8 & 94 & 4.5 & 1.5 \\
\hline 20 & 1.9 & 8 & 105 & 1.6 & 0.0 \\
\hline 21 & 4.4 & 7 & 98 & 2.2 & 0.0 \\
\hline 28 & 1.9 & 9 & 104 & 1.7 & 0.0 \\
\hline 33 & 16.2 & 11 & 65 & 7.2 & 3.1 \\
\hline 49 & 16.6 & 10 & 57 & 6.7 & 3.1 \\
\hline 60 & 1.0 & 5 & 103 & 1.2 & 0.0 \\
\hline 64 & 4.3 & 10 & 101 & 2.8 & 0.1 \\
\hline 82 & 0.3 & 9 & 107 & 1.7 & 0.0 \\
\hline 96 & 3.5 & 6 & 101 & 2.0 & 0.0 \\
\hline 97 & 1.2 & 9 & 106 & 1.7 & 0.0 \\
\hline 127 & 12.1 & 9 & 77 & 5.6 & 2.3 \\
\hline 146 & 8.5 & 8 & 56 & 4.4 & 1.3 \\
\hline 179 & 6.1 & 10 & 99 & 3.6 & 0.9 \\
\hline 231 & 4.7 & 9 & 96 & 2.7 & 0.2 \\
\hline 274 & 1.4 & 5 & 11 & 1.2 & 0.0 \\
\hline 335 & 10.6 & 9 & 69 & 5.3 & 2.0 \\
\hline 352 & 0.5 & 3 & 99 & 1.0 & 0.0 \\
\hline 390 & 7.2 & 7 & 85 & 3.5 & 0.8 \\
\hline 393 & 5.8 & 6 & 92 & 2.1 & 0.2 \\
\hline 414 & 7.7 & 8 & 96 & 4.2 & 1.1 \\
\hline 500 & 6.8 & 6 & 91 & 2.9 & 0.5 \\
\hline 504 & 11.8 & 9 & 91 & 5.5 & 2.2 \\
\hline 510 & 8.2 & 9 & 74 & 4.8 & 1.4 \\
\hline 513 & 5.4 & 8 & 73 & 2.7 & 0.4 \\
\hline 525 & 13.7 & 9 & 76 & 5.8 & 2.5 \\
\hline 543 & 13.2 & 13 & 74 & 7.8 & 2.9 \\
\hline 562 & 26.4 & 14 & 42 & 9.8 & 4.5 \\
\hline 575 & 5.8 & 7 & 69 & 2.4 & 1.4 \\
\hline 586 & 12.7 & 8 & 85 & 5.0 & 2.2 \\
\hline 588 & 3.4 & 7 & 32 & 2.2 & 0.0 \\
\hline
\end{tabular}




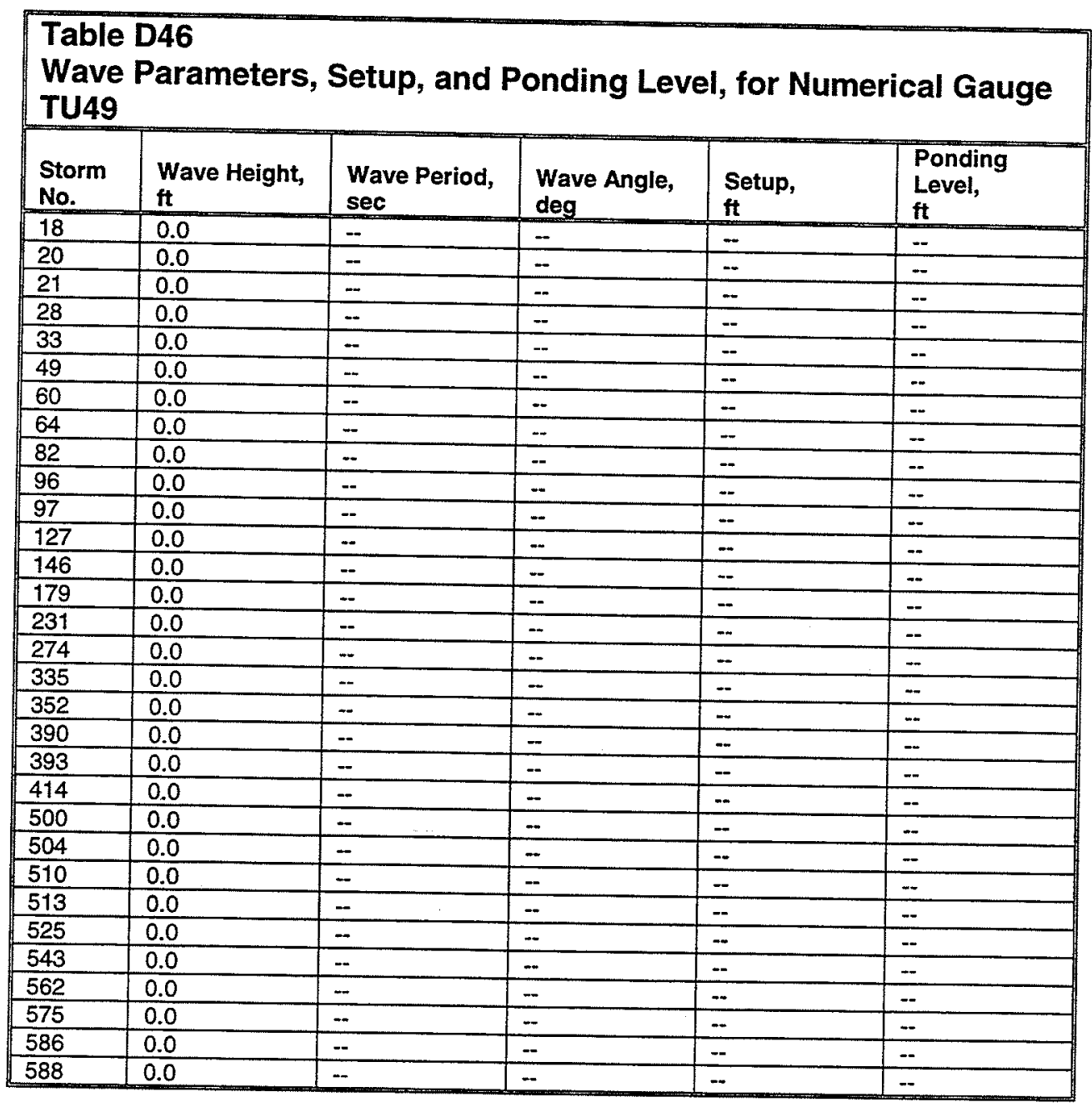




\begin{tabular}{|c|c|c|c|c|c|}
\hline $\begin{array}{l}\text { Storm } \\
\text { No. }\end{array}$ & $\begin{array}{l}\text { Wave Height, } \\
\text { ft }\end{array}$ & $\begin{array}{l}\text { Wave Period, } \\
\text { sec }\end{array}$ & $\begin{array}{l}\text { Wave Angle, } \\
\text { deg }\end{array}$ & $\begin{array}{l}\text { Setup, } \\
\text { ft }\end{array}$ & $\begin{array}{l}\text { Ponding } \\
\text { Level, } \\
\text { ft }\end{array}$ \\
\hline 18 & 0.0 & - & - & $-\cdots$ & -- \\
\hline 20 & 0.0 & $=$ & - & $=$ & $\because$ \\
\hline 21 & 0.0 & $=$ & - & $\cdots$ & $=$ \\
\hline 28 & 0.0 & -- & - & - & $=$ \\
\hline 33 & 0.0 & - & $=$ & $\cdots$ & -- \\
\hline 49 & 0.0 & - & $=$ & $=$ & -- \\
\hline 60 & 0.0 & $\cdots$ & -- & -- & - \\
\hline 64 & 0.0 & - & $\cdots$ & - & - \\
\hline 82 & 0.0 & $\cdots$ & $=$ & - & -- \\
\hline 96 & 0.0 & -- & -- & $=$ & -- \\
\hline 97 & 0.0 & $=$ & - & -- & -- \\
\hline 127 & 0.0 & -- & - & $\approx$ & $=$ \\
\hline 146 & 0.0 & -- & -- & $=$ & - \\
\hline 179 & 0.0 & - & -- & - & -- \\
\hline 231 & 0.0 & - & $m$ & -- & - \\
\hline 274 & 0.0 & $=$ & 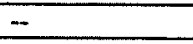 & - & - \\
\hline 335 & 0.0 & -- & -- & $=$ & - \\
\hline 352 & 0.0 & $m$ & + & - & - \\
\hline 390 & 0.0 & $=$ & - & -- & - \\
\hline 393 & 0.0 & $=$ & - & $=$ & - \\
\hline 414 & 0.0 & - & - & -- & $=$ \\
\hline 500 & 0.0 & $\cdots$ & - & - & $\cdots$ \\
\hline 504 & 0.0 & - & - & - & - \\
\hline 510 & 0.0 & $\cdots$ & $\cdots$ & - & $\cdots$ \\
\hline 513 & 0.0 & - & -- & $=$ & $\cdots$ \\
\hline 525 & 0.0 & - & - & - & -- \\
\hline 543 & 0.0 & - & $\cdots$ & $=$ & $=$ \\
\hline 562 & 0.0 & $=$ & - & $=$ & - \\
\hline 575 & 0.0 & -- & $=$ & - & - \\
\hline 586 & 0.0 & -- & -- & -- & $=$ \\
\hline 588 & 0.0 & -- & - & -- & -- \\
\hline
\end{tabular}




\begin{tabular}{|c|c|c|c|c|c|}
\hline \multicolumn{6}{|c|}{$\begin{array}{l}\text { Table D48 } \\
\text { Wave Parameters, Setup, and Ponding Level, for Numerical Gauge } \\
\text { TU51 }\end{array}$} \\
\hline $\begin{array}{l}\text { Storm } \\
\text { No. } \\
\end{array}$ & $\begin{array}{l}\text { Wave Height, } \\
\mathrm{ft}\end{array}$ & $\begin{array}{l}\text { Wave Period, } \\
\text { sec }\end{array}$ & $\begin{array}{l}\text { Wave Angle, } \\
\text { deg }\end{array}$ & $\begin{array}{l}\text { Setup, } \\
\mathrm{ft}\end{array}$ & $\begin{array}{l}\text { Ponding } \\
\text { Level, } \\
\mathrm{ft}\end{array}$ \\
\hline 18 & 10.2 & 8 & 160 & 4.7 & 1.8 \\
\hline 20 & 2.4 & 8 & 172 & 1.5 & 0.0 \\
\hline 21 & 5.2 & 7 & 165 & 2.3 & 0.2 \\
\hline 28 & 2.6 & 9 & 172 & 1.7 & 0.0 \\
\hline 33 & 16.4 & 11 & 121 & 7.2 & 3.1 \\
\hline 49 & 16.7 & 10 & 117 & 6.7 & 3.1 \\
\hline 60 & 1.4 & 5 & 171 & 1.2 & 0.0 \\
\hline 64 & 5.5 & 10 & 169 & 3.4 & 0.7 \\
\hline 82 & 3.3 & 8 & 165 & 2.3 & 0.0 \\
\hline 96 & 3.4 & 9 & 173 & 2.5 & 0.0 \\
\hline 97 & 3.4 & 8 & 172 & 2.3 & 0.0 \\
\hline 127 & 12.0 & 9 & 144 & 5.6 & 2.2 \\
\hline 146 & 8.5 & 8 & 115 & 4.4 & 1.4 \\
\hline 179 & 7.5 & 10 & 166 & 5.0 & 1.3 \\
\hline 231 & 6.4 & 8 & 168 & 3.0 & 0.7 \\
\hline 274 & 2.2 & 5 & 52 & 1.2 & 0.0 \\
\hline 335 & 10.8 & 9 & 130 & 5.4 & 2.0 \\
\hline 352 & 0.4 & 4 & 168 & 1.1 & 0.0 \\
\hline 390 & 7.6 & 7 & 149 & 3.6 & 1.0 \\
\hline 393 & 6.7 & 6 & 158 & 2.8 & 0.5 \\
\hline 414 & 8.4 & 8 & 161 & 4.3 & 1.3 \\
\hline 500 & 7.4 & 6 & 156 & 3.0 & 0.7 \\
\hline 504 & 10.4 & 10 & 161 & 5.8 & 2.1 \\
\hline 510 & 8.4 & 9 & 136 & 4.8 & 1.5 \\
\hline 513 & 5.5 & 8 & 135 & 2.7 & 0.4 \\
\hline 525 & 14.1 & 9 & 139 & 5.8 & 2.6 \\
\hline 543 & 13.5 & 13 & 136 & 7.9 & 2.9 \\
\hline 562 & 26.9 & 14 & 100 & 9.8 & 4.5 \\
\hline 575 & 5.9 & 7 & 130 & 2.5 & 0.4 \\
\hline 586 & 13.5 & 8 & 149 & 5.1 & 2.4 \\
\hline 588 & 3.3 & 8 & 170 & 1.6 & 0.0 \\
\hline
\end{tabular}




\begin{tabular}{|c|c|c|c|c|c|}
\hline \multicolumn{6}{|c|}{$\begin{array}{l}\text { Table D49 } \\
\text { Wave Parameters, Setup, and Ponding Level, for Numerical Gauge } \\
\text { TU52 }\end{array}$} \\
\hline $\begin{array}{l}\text { Storm } \\
\text { No. } \\
\end{array}$ & $\begin{array}{l}\text { Wave Height, } \\
\text { ft }\end{array}$ & $\begin{array}{l}\text { Wave Period, } \\
\text { sec }\end{array}$ & $\begin{array}{l}\text { Wave Angle, } \\
\text { deg }\end{array}$ & $\begin{array}{l}\text { Setup, } \\
\mathrm{tt}\end{array}$ & $\begin{array}{l}\text { Ponding } \\
\text { Level, } \\
\mathrm{ft}\end{array}$ \\
\hline 18 & 0.4 & 25 & 118 & 0.4 & 0.0 \\
\hline 20 & 0.4 & 6 & 128 & 0.4 & 0.0 \\
\hline 21 & 0.7 & 8 & 128 & 0.7 & 0.0 \\
\hline 28 & 0.5 & 8 & 127 & 0.5 & 0.0 \\
\hline 33 & 16.0 & 11 & 92 & 7.5 & 0.0 \\
\hline 49 & 16.5 & 10 & 88 & 7.4 & 0.0 \\
\hline 60 & 0.3 & 4 & 125 & 0.3 & 0.0 \\
\hline 64 & 0.7 & 25 & 86 & 0.6 & 0.0 \\
\hline 82 & 0.4 & 8 & 121 & 0.4 & 0.0 \\
\hline 96 & 0.7 & 9 & 128 & 0.7 & 0.0 \\
\hline 97 & 0.3 & 8 & 128 & 0.3 & 0.0 \\
\hline 127 & 10.8 & 9 & 106 & 5.1 & 0.0 \\
\hline 146 & 9.1 & 8 & 88 & 4.2 & 0.0 \\
\hline 179 & 4.8 & 10 & 122 & 2.9 & 0.0 \\
\hline 231 & 0.6 & 25 & 105 & 0.5 & 0.0 \\
\hline 274 & 0.6 & 4 & 99 & 0.4 & 0.0 \\
\hline 335 & 10.3 & 9 & 97 & 4.9 & 0.0 \\
\hline 352 & 0.4 & 3 & 122 & 0.2 & 0.0 \\
\hline 390 & 2.1 & 7 & 125 & 1.3 & 0.0 \\
\hline 393 & 3.0 & 6 & 121 & 1.6 & 0.0 \\
\hline 414 & 0.4 & 9 & 127 & 0.3 & 0.0 \\
\hline 500 & 0.4 & 4 & 126 & 0.3 & 0.0 \\
\hline 504 & 0.6 & 25 & 71 & 0.6 & 0.0 \\
\hline 510 & 7.8 & 9 & 101 & 4.0 & 0.0 \\
\hline 513 & 5.2 & 8 & 100 & 2.8 & 0.0 \\
\hline 525 & 0.9 & 10 & 126 & 0.9 & 0.0 \\
\hline 543 & 0.8 & 11 & 41 & 0.8 & 0.0 \\
\hline 562 & 28.5 & 14 & 88 & 13.2 & 0.0 \\
\hline 575 & 0.7 & 25 & 91 & 0.6 & 0.0 \\
\hline 586 & 11.6 & 8 & 110 & 5.0 & 0.0 \\
\hline 588 & 0.8 & 9 & 125 & 0.7 & 0.0 \\
\hline
\end{tabular}




\begin{tabular}{|c|c|c|c|c|c|}
\hline \multicolumn{6}{|c|}{$\begin{array}{l}\text { Table D50 } \\
\text { Wave Parameters, Setup, and Ponding Level, for Numerical Gauge } \\
\text { TU53 }\end{array}$} \\
\hline $\begin{array}{l}\text { Storm } \\
\text { No. }\end{array}$ & $\begin{array}{l}\text { Wave Height, } \\
\mathrm{tt}\end{array}$ & $\begin{array}{l}\text { Wave Period, } \\
\text { sec }\end{array}$ & $\begin{array}{l}\text { Wave Angle, } \\
\text { deg }\end{array}$ & $\begin{array}{l}\text { Setup, } \\
\mathrm{ft}\end{array}$ & $\begin{array}{l}\text { Ponding } \\
\text { Level, } \\
\mathrm{ft}\end{array}$ \\
\hline 18 & 0.5 & 25 & 132 & 0.5 & 0.0 \\
\hline 20 & 0.9 & 6 & 145 & 0.6 & 0.0 \\
\hline 21 & 0.3 & 5 & 146 & 0.3 & 0.0 \\
\hline 28 & 1.0 & 7 & 142 & 0.8 & 0.0 \\
\hline 33 & 16.3 & 11 & 96 & 7.7 & 0.0 \\
\hline 49 & 16.7 & 10 & 92 & 7.4 & 0.0 \\
\hline 60 & 1.1 & 5 & 141 & 0.7 & 0.0 \\
\hline 64 & 0.7 & 25 & 90 & 0.6 & 0.0 \\
\hline 82 & 0.3 & 6 & 148 & 0.3 & 0.0 \\
\hline 96 & 1.3 & 8 & 144 & 1.0 & 0.0 \\
\hline 97 & 1.0 & 9 & 145 & 0.9 & 0.0 \\
\hline 127 & 0.6 & 8 & 15 & 0.5 & 0.0 \\
\hline 146 & 0.3 & 7 & 147 & 0.3 & 0.0 \\
\hline 179 & 6.8 & 10 & 138 & 3.8 & 0.0 \\
\hline 231 & 0.6 & 25 & 116 & 0.6 & 0.0 \\
\hline 274 & 0.7 & 4 & 101 & 0.4 & 0.0 \\
\hline 335 & 10.7 & 9 & 105 & 5.0 & 0.0 \\
\hline 352 & 0.4 & 4 & 139 & 0.3 & 0.0 \\
\hline 390 & 6.1 & 8 & 130 & 3.1 & 0.0 \\
\hline 393 & 0.6 & 3 & 116 & 0.3 & 0.0 \\
\hline 414 & 0.7 & 9 & 144 & 0.7 & 0.0 \\
\hline 500 & 0.4 & 25 & 139 & 0.3 & 0.0 \\
\hline 504 & 0.7 & 25 & 70 & 0.6 & 0.0 \\
\hline 510 & 8.3 & 9 & 110 & 4.2 & 0.0 \\
\hline 513 & 5.4 & 8 & 109 & 2.9 & 0.0 \\
\hline 525 & 13.9 & 9 & 113 & 6.2 & 0.0 \\
\hline 543 & 13.4 & 13 & 110 & 7.2 & 0.0 \\
\hline 562 & 28.8 & 14 & 93 & 13.3 & 0.0 \\
\hline 575 & 0.7 & 25 & 95 & 0.6 & 0.0 \\
\hline 586 & 13.2 & 8 & 122 & 5.5 & 0.0 \\
\hline 588 & 0.4 & 7 & 12 & 0.3 & 0.0 \\
\hline
\end{tabular}




\begin{tabular}{|c|c|c|c|c|c|}
\hline \multicolumn{6}{|c|}{$\begin{array}{l}\text { Table D51 } \\
\text { Wave Parameters, Setup, and Ponding Level, for Numerical Gauge } \\
\text { TU54 }\end{array}$} \\
\hline $\begin{array}{l}\text { Storm } \\
\text { No. }\end{array}$ & $\begin{array}{l}\text { Wave Height, } \\
\mathrm{ft}\end{array}$ & $\begin{array}{l}\text { Wave Period, } \\
\text { sec }\end{array}$ & $\begin{array}{l}\text { Wave Angle, } \\
\text { deg }\end{array}$ & $\begin{array}{l}\text { Setup, } \\
\mathrm{ft}\end{array}$ & $\begin{array}{l}\text { Ponding } \\
\text { Level, } \\
\mathrm{ft}\end{array}$ \\
\hline 18 & 3.4 & 6 & 161 & 2.3 & 0.0 \\
\hline 20 & 4.4 & 5 & 36 & 2.0 & 0.0 \\
\hline 21 & 0.4 & 5 & 2 & 1.4 & 0.0 \\
\hline 28 & 5.2 & 5 & 26 & 1.9 & 0.0 \\
\hline 33 & 10.6 & 11 & 154 & 6.7 & 2.2 \\
\hline 49 & 12.6 & 10 & 151 & 6.6 & 2.5 \\
\hline 60 & 0.0 & -- & - & - & - \\
\hline 64 & 0.5 & 25 & 150 & 2.9 & 0.0 \\
\hline 82 & 8.8 & 9 & 30 & 5.3 & 1.6 \\
\hline 96 & 9.6 & 7 & 32 & 4.3 & 1.5 \\
\hline 97 & 3.7 & 6 & 12 & 2.3 & 0.0 \\
\hline 127 & 6.7 & 9 & 159 & 4.7 & 1.0 \\
\hline 146 & 6.7 & 8 & 151 & 4.2 & 0.8 \\
\hline 179 & 3.6 & 6 & 96 & 2.3 & 0.0 \\
\hline 231 & 0.4 & 25 & 159 & 2.9 & 0.0 \\
\hline 274 & 0.4 & 5 & 3 & 1.4 & 0.0 \\
\hline 335 & 6.3 & 9 & 156 & 3.7 & 0.8 \\
\hline 352 & 0.4 & 5 & 11 & 1.4 & 0.0 \\
\hline 390 & 5.8 & 6 & 140 & 2.3 & 0.2 \\
\hline 393 & 0.8 & 7 & 167 & 1.7 & 0.0 \\
\hline 414 & 13.3 & 8 & 45 & 5.4 & 2.3 \\
\hline 500 & 3.4 & 5 & 155 & 2.0 & 0.0 \\
\hline 504 & 10.7 & 9 & 134 & 5.7 & 2.0 \\
\hline 510 & 5.2 & 9 & 158 & 3.3 & 0.4 \\
\hline 513 & 2.0 & 7 & 162 & 1.7 & 0.0 \\
\hline 525 & 7.4 & 9 & 159 & 4.9 & 1.2 \\
\hline 543 & 15.1 & 11 & 79 & 7.5 & 3.0 \\
\hline 562 & 24.9 & 15 & 130 & 10.6 & 4.4 \\
\hline 575 & 0.5 & 25 & 154 & 2.9 & 0.0 \\
\hline 586 & 4.5 & 9 & 5 & 2.9 & 0.1 \\
\hline 588 & 4.3 & 7 & 71 & 2.5 & 0.0 \\
\hline
\end{tabular}




\begin{tabular}{|c|c|c|c|c|c|}
\hline $\begin{array}{l}\text { Storm } \\
\text { No. }\end{array}$ & $\begin{array}{l}\text { Wave Height, } \\
\mathrm{ft}\end{array}$ & $\begin{array}{l}\text { Wave Period, } \\
\text { sec }\end{array}$ & $\begin{array}{l}\text { Wave Angle, } \\
\text { deg }\end{array}$ & $\begin{array}{l}\text { Setup, } \\
\mathrm{tt}\end{array}$ & $\begin{array}{l}\text { Ponding } \\
\text { Level, } \\
\mathrm{ft}\end{array}$ \\
\hline 18 & 1.0 & 10 & 176 & 3.0 & 0.0 \\
\hline 20 & 3.3 & 6 & 58 & 3.4 & 0.0 \\
\hline 21 & 0.4 & 8 & 51 & 2.7 & 0.0 \\
\hline 28 & 3.3 & 6 & 62 & 3.4 & 0.0 \\
\hline 33 & 8.5 & 9 & 60 & 5.2 & 0.2 \\
\hline 49 & 9.9 & 11 & 91 & 8.2 & 2.0 \\
\hline 60 & 0.0 & $\cdots$ & -- & $\cdots$ & -- \\
\hline 64 & 3.9 & 5 & 131 & 2.9 & 0.0 \\
\hline 82 & 11.1 & 10 & 98 & 7.7 & 2.2 \\
\hline 96 & 9.0 & 8 & 65 & 6.1 & 1.5 \\
\hline 97 & 7.7 & 7 & 70 & 4.3 & 0.0 \\
\hline 127 & 6.2 & 7 & 133 & 3.9 & 0.5 \\
\hline 146 & 0.5 & 8 & 53 & 2.7 & 0.0 \\
\hline 179 & 3.4 & 7 & 75 & 3.7 & 0.0 \\
\hline 231 & 6.9 & 6 & 104 & 3.9 & 0.0 \\
\hline 274 & 0.4 & 5 & 53 & 2.1 & 0.0 \\
\hline 335 & 1.0 & 9 & 177 & 2.8 & 0.0 \\
\hline 352 & 3.3 & 4 & 110 & 2.7 & 0.0 \\
\hline 390 & 1.3 & 6 & 177 & 2.3 & 0.0 \\
\hline 393 & 0.0 & -- & - & - & -- \\
\hline 414 & 14.1 & 8 & 121 & 6.7 & 2.5 \\
\hline 500 & 1.1 & 8 & 176 & 2.7 & 0.0 \\
\hline 504 & 8.5 & 9 & 164 & 5.2 & 0.2 \\
\hline 510 & 0.4 & 9 & 179 & 2.8 & 0.0 \\
\hline 513 & 0.8 & 10 & 53 & 3.0 & 0.0 \\
\hline 525 & 4.9 & 7 & 161 & 3.6 & 0.0 \\
\hline 543 & 10.9 & 12 & 70 & 7.2 & 1.0 \\
\hline 562 & 17.6 & 15 & 164 & 11.6 & 3.6 \\
\hline 575 & 0.3 & 9 & 53 & 2.8 & 0.0 \\
\hline 586 & 8.8 & 9 & 60 & 5.3 & 0.2 \\
\hline 588 & 6.0 & 6 & 74 & 3.4 & 0.3 \\
\hline
\end{tabular}




\begin{tabular}{|c|c|c|c|c|c|}
\hline \multicolumn{6}{|c|}{$\begin{array}{l}\text { Table D53 } \\
\text { Wave Parameters, Setup, and Ponding Level, for Numerical Gaug } \\
\text { TU56 }\end{array}$} \\
\hline $\begin{array}{l}\text { Storm } \\
\text { No. } \\
\end{array}$ & $\begin{array}{l}\text { Wave Height, } \\
\mathrm{ft}\end{array}$ & $\begin{array}{l}\text { Wave Period, } \\
\text { sec }\end{array}$ & $\begin{array}{l}\text { Wave Angle, } \\
\text { deg }\end{array}$ & $\begin{array}{l}\text { Setup, } \\
\mathrm{ft}\end{array}$ & $\begin{array}{l}\text { Ponding } \\
\text { Level, } \\
\mathrm{ft}\end{array}$ \\
\hline 18 & 0.0 & - & -- & - & -- \\
\hline 20 & 5.1 & 5 & 91 & 2.9 & 0.0 \\
\hline 21 & 0.0 & -- & - & - & - \\
\hline 28 & 4.1 & 5 & 64 & 3.0 & 0.0 \\
\hline 33 & 6.1 & 8 & 67 & 3.9 & 0.0 \\
\hline 49 & 8.9 & 11 & 74 & 7.9 & 1.8 \\
\hline 60 & 0.0 & - & - & -- & - \\
\hline 64 & 4.5 & 5 & 101 & 2.9 & 0.0 \\
\hline 82 & 10.2 & 10 & 79 & 7.6 & 2.0 \\
\hline 96 & 7.9 & 8 & 80 & 5.9 & 1.2 \\
\hline 97 & 5.2 & 7 & 61 & 3.6 & 0.0 \\
\hline 127 & 5.7 & 7 & 102 & 3.8 & 0.3 \\
\hline 146 & 0.8 & 8 & 128 & 2.7 & 0.0 \\
\hline 179 & 3.3 & 7 & 108 & 3.7 & 0.0 \\
\hline 231 & 3.9 & 6 & 63 & 3.3 & 0.0 \\
\hline 274 & 1.6 & 5 & 114 & 2.0 & 0.0 \\
\hline 335 & 0.4 & 6 & 129 & 2.3 & 0.0 \\
\hline 352 & 0.5 & 4 & 56 & 1.9 & 0.0 \\
\hline 390 & 0.5 & 6 & 129 & 2.3 & 0.0 \\
\hline 393 & 0.0 & - & - & -- & -- \\
\hline 414 & 13.5 & 8 & 94 & 6.7 & 2.4 \\
\hline 500 & 0.0 & $\cdots$ & - & -- & -- \\
\hline 504 & 5.0 & 9 & 122 & 4.2 & 0.0 \\
\hline 510 & 0.0 & -- & $\sim$ & $\cdots$ & - \\
\hline 513 & 0.7 & 9 & 55 & 2.8 & 0.0 \\
\hline 525 & 3.5 & 6 & 118 & 3.3 & 0.0 \\
\hline 543 & 12.7 & 11 & 83 & 8.6 & 2.6 \\
\hline 562 & 10.3 & 15 & 122 & 10.3 & 2.5 \\
\hline 575 & 0.0 & -- & - & - & -- \\
\hline 586 & 3.9 & 9 & 55 & 4.2 & 0.0 \\
\hline 588 & 5.1 & 7 & 90 & 3.7 & 0.1 \\
\hline
\end{tabular}




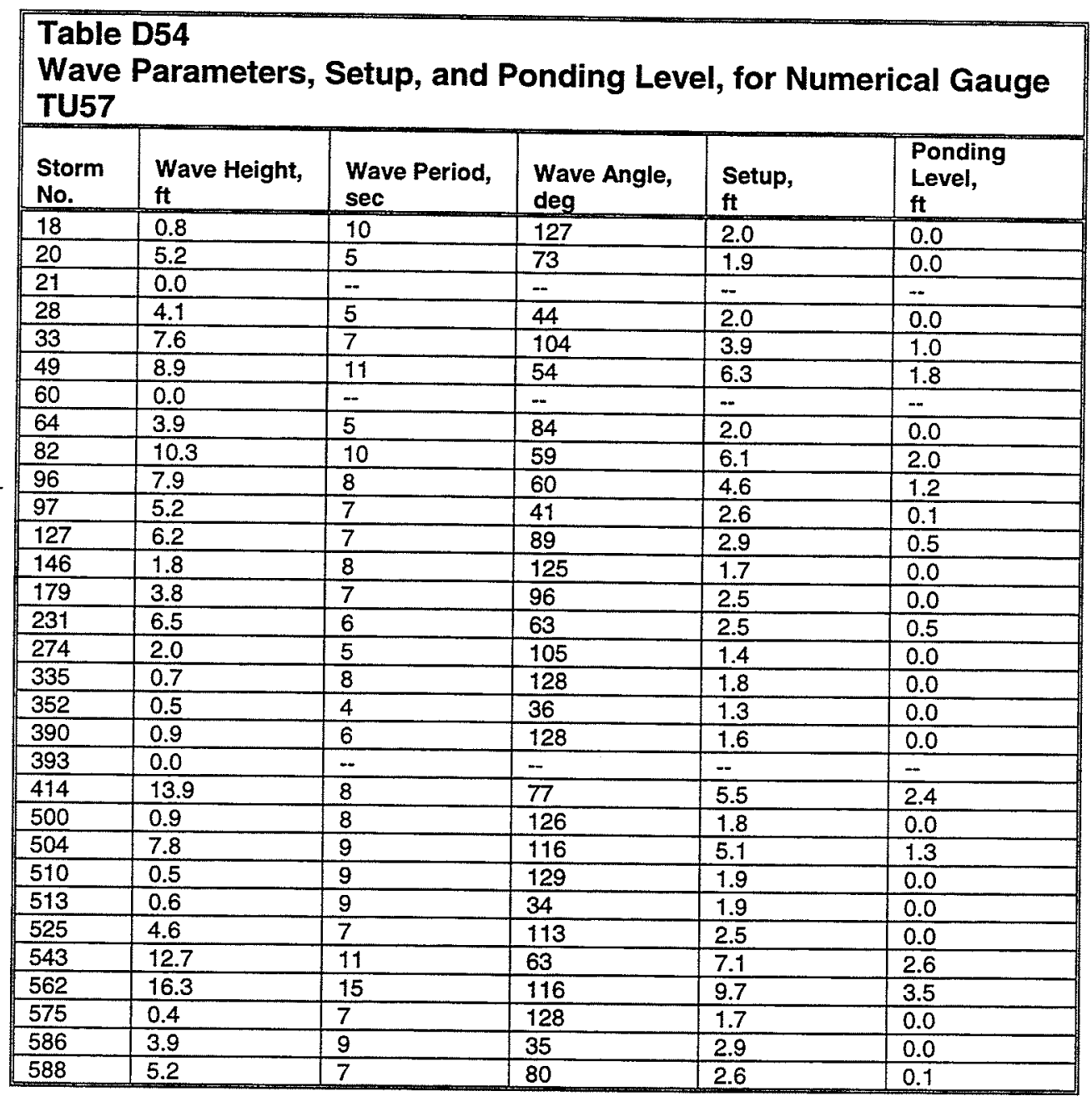




\begin{tabular}{|c|c|c|c|c|c|}
\hline \multicolumn{6}{|c|}{$\begin{array}{l}\text { Table D55 } \\
\text { Wave Parameters, Setup, and Ponding Level, for Numerical Gaug } \\
\text { TU58 }\end{array}$} \\
\hline $\begin{array}{l}\text { Storm } \\
\text { No. } \\
\end{array}$ & $\begin{array}{l}\text { Wave Height, } \\
\mathrm{ft}\end{array}$ & $\begin{array}{l}\text { Wave Period, } \\
\text { sec }\end{array}$ & $\begin{array}{l}\text { Wave Angle, } \\
\text { deg }\end{array}$ & $\begin{array}{l}\text { Setup, } \\
\mathrm{ft}\end{array}$ & $\begin{array}{l}\text { Ponding } \\
\text { Level, } \\
\mathrm{ft}\end{array}$ \\
\hline 18 & 1.0 & 10 & 136 & 2.0 & 0.0 \\
\hline 20 & 5.1 & 5 & 59 & 1.9 & 0.0 \\
\hline 21 & 0.6 & 6 & 138 & 1.6 & 0.0 \\
\hline 28 & 3.7 & 5 & 32 & 2.0 & 0.0 \\
\hline 33 & 7.9 & 10 & 130 & 5.6 & 1.4 \\
\hline 49 & 8.5 & 11 & 42 & 6.2 & 1.7 \\
\hline 60 & 0.0 & -- & $\cdots$ & -- & -- \\
\hline 64 & 0.4 & 25 & 128 & 2.9 & 0.0 \\
\hline 82 & 9.9 & 10 & 46 & 6.1 & 1.9 \\
\hline 96 & 7.3 & 8 & 60 & 4.4 & 1.0 \\
\hline 97 & 4.6 & 7 & 30 & 2.5 & 0.0 \\
\hline 127 & 6.2 & 7 & 74 & 2.9 & 0.5 \\
\hline 146 & 4.7 & 8 & 128 & 2.7 & 0.1 \\
\hline 179 & 3.9 & 7 & 85 & 2.5 & 0.0 \\
\hline 231 & 6.4 & 6 & 50 & 2.5 & 0.4 \\
\hline 274 & 2.3 & 5 & 98 & 1.4 & 0.0 \\
\hline 335 & 3.7 & 9 & 132 & 2.9 & 0.0 \\
\hline 352 & 0.4 & 4 & 25 & 1.3 & 0.0 \\
\hline 390 & 3.9 & 6 & 130 & 2.3 & 0.0 \\
\hline 393 & 0.4 & 5 & 139 & 1.4 & 0.0 \\
\hline 414 & 13.9 & 8 & 63 & 5.5 & 2.4 \\
\hline 500 & 2.3 & 8 & 131 & 1.8 & 0.0 \\
\hline 504 & 10.4 & 9 & 115 & 5.7 & 1.9 \\
\hline 510 & 3.3 & 8 & 133 & 2.7 & 0.0 \\
\hline 513 & 0.5 & 9 & 23 & 1.9 & 0.0 \\
\hline 525 & 5.8 & 7 & 110 & 2.7 & 0.4 \\
\hline 543 & 14.7 & 10 & 71 & 6.9 & 2.8 \\
\hline 562 & 21.9 & 15 & 115 & 10.4 & 4.1 \\
\hline 575 & 0.7 & 9 & 134 & 1.9 & 0.0 \\
\hline 586 & 1.5 & 9 & 22 & 1.9 & 0.0 \\
\hline 588 & 5.2 & 7 & 65 & 2.6 & 0.1 \\
\hline
\end{tabular}




\begin{tabular}{|c|c|c|c|c|c|}
\hline $\begin{array}{l}\text { Storm } \\
\text { No. } \\
\end{array}$ & $\begin{array}{l}\text { Wave Height, } \\
\mathrm{ft}\end{array}$ & $\begin{array}{l}\text { Wave Period, } \\
\text { sec }\end{array}$ & $\begin{array}{l}\text { Wave Angle, } \\
\text { deg }\end{array}$ & $\begin{array}{l}\text { Setup, } \\
\mathrm{ft}\end{array}$ & $\begin{array}{l}\text { Ponding } \\
\text { Level, } \\
\mathrm{ft} \\
\end{array}$ \\
\hline 18 & 1.0 & 10 & 136 & 2.0 & 0.0 \\
\hline 20 & 5.1 & 5 & 32 & 1.4 & 0.0 \\
\hline 21 & 0.6 & 6 & 149 & 1.6 & 0.0 \\
\hline 28 & 10.6 & 5 & 146 & 1.4 & 0.0 \\
\hline 33 & 12.6 & 11 & 134 & \begin{tabular}{|l|}
6.7 \\
\end{tabular} & 2.2 \\
\hline 49 & 0.0 & 10 & 131 & 6.6 & 2.5 \\
\hline 60 & 0.5 & - & -- & -- & -- \\
\hline 64 & 3.3 & 25 & 130 & 2.9 & 0.0 \\
\hline 82 & 3.8 & 9 & 47 & 2.9 & 0.0 \\
\hline 96 & 0.5 & 7 & 47 & 2.5 & 0.0 \\
\hline 97 & 6.7 & 6 & 41 & 1.6 & 0.0 \\
\hline 127 & 6.7 & 9 & 139 & 4.7 & 1.0 \\
\hline 146 & 3.5 & 8 & 131 & 4.2 & 0.8 \\
\hline 179 & 0.4 & 6 & 79 & 2.3 & 0.0 \\
\hline 231 & 2.2 & 25 & 139 & 2.9 & 0.0 \\
\hline 274 & 6.4 & 5 & 74 & 1.4 & 0.0 \\
\hline 335 & 1.3 & 9 & 136 & 3.7 & 0.9 \\
\hline 352 & 5.7 & 3 & 82 & 1.0 & 0.0 \\
\hline 390 & 1.1 & 5 & 122 & 1.9 & 0.0 \\
\hline 393 & 7.8 & 6 & 145 & 1.6 & 0.0 \\
\hline 414 & 3.4 & 8 & 52 & 4.6 & 1.2 \\
\hline 500 & 10.7 & 5 & 135 & 2.0 & 0.0 \\
\hline 504 & 5.2 & 9 & 114 & 5.7 & 2.0 \\
\hline 510 & 1.8 & 9 & 138 & 3.3 & 0.4 \\
\hline 513 & 7.4 & 7 & 142 & 1.7 & 0.0 \\
\hline 525 & 13.6 & 9 & 139 & 4.9 & 1.2 \\
\hline 543 & 24.9 & 11 & 67 & 7.3 & 2.7 \\
\hline 562 & 0.5 & 15 & 110 & 10.6 & 4.4 \\
\hline 575 & 8.6 & 25 & 134 & 2.9 & 0.0 \\
\hline 586 & 3.3 & 8 & 154 & 4.7 & 1.4 \\
\hline 588 & & 7 & 58 & 2.5 & 0.0 \\
\hline
\end{tabular}




\begin{tabular}{|c|c|c|c|c|c|}
\hline \multicolumn{6}{|c|}{$\begin{array}{l}\text { Table D57 } \\
\text { Wave Parameters, Setup, and Ponding Level, for Numerical Gaug } \\
\text { TU60 }\end{array}$} \\
\hline $\begin{array}{l}\text { Storm } \\
\text { No. } \\
\end{array}$ & $\begin{array}{l}\text { Wave Height, } \\
\text { ft }\end{array}$ & $\begin{array}{l}\text { Wave Period, } \\
\text { sec }\end{array}$ & $\begin{array}{l}\text { Wave Angle, } \\
\text { deg }\end{array}$ & $\begin{array}{l}\text { Setup, } \\
\mathrm{ft}\end{array}$ & $\begin{array}{l}\text { Ponding } \\
\text { Level, } \\
\mathrm{ft}\end{array}$ \\
\hline 18 & 3.4 & 6 & 142 & 1.5 & 0.0 \\
\hline 20 & 4.0 & 4 & 70 & 1.7 & 0.0 \\
\hline 21 & 1.3 & 6 & 145 & 1.6 & 0.0 \\
\hline 28 & 0.6 & 5 & 146 & 1.4 & 0.0 \\
\hline 33 & 10.6 & 11 & 134 & 6.7 & 2.2 \\
\hline 49 & 12.6 & 10 & 131 & 6.6 & 2.5 \\
\hline 60 & 0.0 & - & - & - & - \\
\hline 64 & 0.5 & 25 & 130 & 2.9 & 0.0 \\
\hline 82 & 3.3 & 9 & 47 & 2.9 & 0.0 \\
\hline 96 & 3.8 & 7 & 47 & 2.5 & 0.0 \\
\hline 97 & 0.5 & 6 & 41 & 1.6 & 0.0 \\
\hline 127 & 6.7 & 9 & 139 & 4.7 & 1.0 \\
\hline 146 & 6.7 & 8 & 131 & 4.2 & 0.8 \\
\hline 179 & 3.5 & 6 & 79 & 2.3 & 0.0 \\
\hline 231 & 0.4 & 25 & 139 & 2.9 & 0.0 \\
\hline 274 & 2.2 & 5 & 74 & 1.4 & 0.0 \\
\hline 335 & 6.4 & 9 & 136 & 3.7 & 0.9 \\
\hline 352 & 1.3 & 3 & 82 & 1.0 & 0.0 \\
\hline 390 & 5.8 & 6 & 120 & 2.3 & 0.2 \\
\hline 393 & 1.1 & 6 & 145 & 1.6 & 0.0 \\
\hline 414 & 7.8 & 8 & 52 & 4.6 & 1.2 \\
\hline 500 & 3.4 & 5 & 135 & 2.0 & 0.0 \\
\hline 504 & 10.7 & 9 & 114 & 5.7 & 2.0 \\
\hline 510 & 5.2 & 9 & 138 & 3.3 & 0.4 \\
\hline 513 & 1.8 & 7 & 142 & 1.7 & 0.0 \\
\hline 525 & 7.4 & 9 & 139 & 4.9 & 1.2 \\
\hline 543 & 13.6 & 11 & 67 & 7.3 & 2.7 \\
\hline 562 & 24.9 & 15 & 110 & 10.6 & 4.4 \\
\hline 575 & 3.3 & 7 & 135 & 2.5 & 0.0 \\
\hline 586 & 3.8 & 8 & 144 & 2.7 & 0.0 \\
\hline 588 & 3.3 & 7 & 58 & 2.5 & 0.0 \\
\hline
\end{tabular}




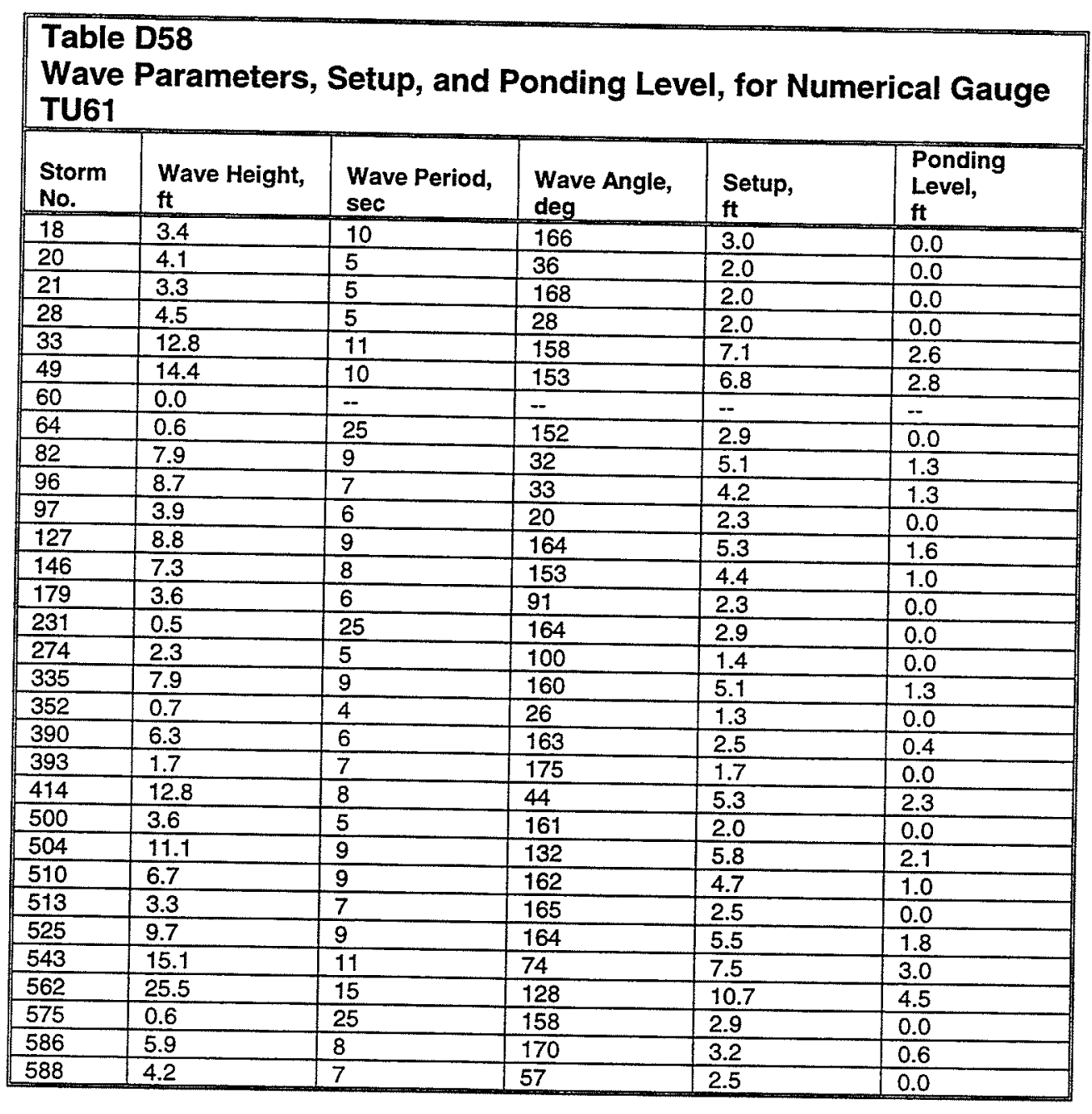




\begin{tabular}{|c|c|c|c|c|c|}
\hline \multicolumn{6}{|c|}{$\begin{array}{l}\text { Table D59 } \\
\text { Wave Parameters, Setup, and Ponding Level, for Numerical Gauge } \\
\text { AU1 }\end{array}$} \\
\hline $\begin{array}{l}\text { Storm } \\
\text { No. }\end{array}$ & $\begin{array}{l}\text { Wave Height, } \\
\mathrm{ft}\end{array}$ & $\begin{array}{l}\text { Wave Period, } \\
\text { sec }\end{array}$ & $\begin{array}{l}\text { Wave Angle, } \\
\text { deg }\end{array}$ & $\begin{array}{l}\text { Setup, } \\
\mathrm{ft}\end{array}$ & $\begin{array}{l}\text { Ponding } \\
\text { Level, } \\
\text { ft }\end{array}$ \\
\hline 18 & 5.0 & 7 & 139 & 2.2 & 0.1 \\
\hline 20 & 0.6 & 5 & 144 & 1.2 & 0.0 \\
\hline 21 & 3.6 & 6 & 139 & 2.0 & 0.0 \\
\hline 28 & 1.0 & 5 & 144 & 1.2 & 0.0 \\
\hline 33 & 14.4 & 11 & 96 & 5.4 & 2.9 \\
\hline 49 & 14.0 & 10 & 129 & 5.1 & 2.7 \\
\hline 60 & 0.0 & - & - & $\cdots$ & -- \\
\hline 64 & 0.5 & 25 & 130 & 2.4 & 0.0 \\
\hline 82 & 0.5 & 8 & 149 & 1.6 & 0.0 \\
\hline 96 & 0.4 & 7 & 150 & 1.5 & 0.0 \\
\hline 97 & 3.1 & 4 & 51 & 1.1 & 0.0 \\
\hline 127 & 8.9 & 8 & 136 & 3.9 & 1.5 \\
\hline 146 & 8.1 & 9 & 129 & 3.1 & 0.1 \\
\hline 179 & 6.7 & 5 & 59 & 2.3 & 0.0 \\
\hline 231 & 0.4 & 25 & 138 & 2.4 & 0.0 \\
\hline 274 & 3.3 & 5 & 74 & 1.9 & 0.0 \\
\hline 335 & 7.4 & 9 & 135 & 3.1 & 0.0 \\
\hline 352 & 0.4 & 4 & 26 & 1.1 & 0.0 \\
\hline 390 & 4.4 & 6 & 141 & 2.0 & 0.0 \\
\hline 393 & 2.1 & 6 & 142 & 1.4 & 0.0 \\
\hline 414 & 7.1 & 5 & 51 & 2.6 & 0.4 \\
\hline 500 & 3.4 & 5 & 133 & 1.9 & 0.0 \\
\hline 504 & 13.0 & 8 & 118 & 4.3 & 2.3 \\
\hline 510 & 4.5 & 9 & 139 & 2.6 & 0.1 \\
\hline 513 & 3.7 & 8 & 137 & 2.4 & 0.0 \\
\hline 525 & 8.4 & 9 & 140 & 4.1 & 1.5 \\
\hline 543 & 12.9 & 13 & 129 & 6.0 & 2.8 \\
\hline 562 & 24.1 & 14 & 101 & 7.2 & 4.3 \\
\hline 575 & 0.5 & 25 & 130 & 2.4 & 0.0 \\
\hline 586 & 7.5 & 7 & 132 & 3.3 & 0.9 \\
\hline 588 & 5.7 & 7 & 44 & 2.3 & 0.3 \\
\hline
\end{tabular}




\begin{tabular}{|c|c|c|c|c|c|}
\hline \multicolumn{6}{|c|}{$\begin{array}{l}\text { Table D60 } \\
\text { Wave Parameters, Setup, and Ponding Level, for Numerical Gaug } \\
\text { AU2 }\end{array}$} \\
\hline $\begin{array}{l}\text { Storm } \\
\text { No. }\end{array}$ & $\begin{array}{l}\text { Wave Height, } \\
\mathrm{ft}\end{array}$ & $\begin{array}{l}\text { Wave Period, } \\
\text { sec }\end{array}$ & $\begin{array}{l}\text { Wave Angle, } \\
\text { deg }\end{array}$ & $\begin{array}{l}\text { Setup, } \\
\mathrm{ft}\end{array}$ & $\begin{array}{l}\text { Ponding } \\
\text { Level, } \\
\mathrm{ft}\end{array}$ \\
\hline 18 & 0.0 & - & -- & $=$ & -- \\
\hline 20 & 1.0 & 4 & 95 & 1.1 & 0.0 \\
\hline 21 & 0.0 & -- & -- & - & -- \\
\hline 28 & 0.3 & 4 & 98 & 1.1 & 0.0 \\
\hline 33 & 8.0 & 11 & 168 & 4.7 & 1.6 \\
\hline 49 & 8.3 & 10 & 115 & 4.4 & 1.6 \\
\hline 60 & 0.0 & -- & -- & -- & - \\
\hline 64 & 3.8 & 5 & 126 & 1.9 & 0.0 \\
\hline 82 & 0.5 & 5 & 108 & 1.3 & 0.0 \\
\hline 96 & 0.7 & 7 & 91 & 1.5 & 0.0 \\
\hline 97 & 2.4 & 4 & 153 & 1.0 & 0.0 \\
\hline 127 & 0.5 & 25 & 110 & 2.4 & 0.0 \\
\hline 146 & 0.5 & 9 & 179 & 1.7 & 0.0 \\
\hline 179 & 3.4 & 6 & 156 & 2.1 & 0.0 \\
\hline 231 & 3.1 & 6 & 128 & 1.4 & 0.0 \\
\hline 274 & 2.0 & 5 & 162 & 1.2 & 0.0 \\
\hline 335 & 0.5 & 6 & 178 & 1.4 & 0.0 \\
\hline 352 & 0.7 & 4 & 106 & 1.1 & 0.0 \\
\hline 390 & 2.0 & 6 & 174 & 1.4 & 0.0 \\
\hline 393 & 0.0 & - & -- & -- & -- \\
\hline 414 & 5.3 & 5 & 153 & 1.9 & 0.0 \\
\hline 500 & 0.0 & $\cdots$ & - & - & -- \\
\hline 504 & 6.9 & 9 & 170 & 3.1 & 0.0 \\
\hline 510 & 0.0 & - & $\cdots$ & - & -- \\
\hline 513 & 0.0 & $\cdots$ & - & - & - \\
\hline 525 & 3.3 & 6 & 169 & 2.1 & 0.0 \\
\hline 543 & 12.8 & 11 & 130 & 5.3 & 2.6 \\
\hline 562 & 11.1 & 14 & 171 & 6.1 & 2.6 \\
\hline 575 & 0.0 & - & - & -- & -- \\
\hline 586 & 0.7 & 6 & 178 & 1.4 & 0.0 \\
\hline 588 & 3.8 & 5 & 131 & 1.9 & 0.0 \\
\hline
\end{tabular}




\begin{tabular}{|c|c|c|c|c|c|}
\hline \multicolumn{6}{|c|}{$\begin{array}{l}\text { Table D61 } \\
\text { Wave Parameters, Setup, and Ponding Level, for Numerical Gauge } \\
\text { AU3 }\end{array}$} \\
\hline $\begin{array}{l}\text { Storm } \\
\text { No. }\end{array}$ & $\begin{array}{l}\text { Wave Height, } \\
\mathrm{ft}\end{array}$ & $\begin{array}{l}\text { Wave Period, } \\
\text { sec }\end{array}$ & $\begin{array}{l}\text { Wave Angle, } \\
\text { deg }\end{array}$ & $\begin{array}{l}\text { Setup, } \\
\mathrm{ft}\end{array}$ & $\begin{array}{l}\text { Ponding } \\
\text { Level, } \\
\mathrm{tt}\end{array}$ \\
\hline 18 & 5.9 & 8 & 63 & 2.6 & 0.6 \\
\hline 20 & 6.7 & 6 & 69 & 2.5 & 0.0 \\
\hline 21 & 8.4 & 8 & 57 & 3.7 & 1.3 \\
\hline 28 & 4.0 & 5 & 66 & 1.9 & 0.0 \\
\hline 33 & 5.7 & 8 & 79 & 2.6 & \begin{tabular}{|l|}
0.5 \\
\end{tabular} \\
\hline 49 & 0.4 & 7 & 4 & 1.5 & 0.0 \\
\hline 60 & 0.8 & 7 & 68 & 1.5 & 0.0 \\
\hline 64 & 9.2 & 7 & 54 & 2.7 & 0.1 \\
\hline 82 & 6.6 & 8 & 72 & 2.6 & 0.6 \\
\hline 96 & 8.2 & 8 & 70 & 3.7 & 1.3 \\
\hline 97 & 5.8 & 7 & 70 & 2.6 & 0.0 \\
\hline 127 & 5.4 & 8 & 49 & 2.5 & 0.4 \\
\hline 146 & 1.4 & 7 & 25 & 1.5 & 0.0 \\
\hline 179 & 8.2 & 7 & 53 & 2.7 & 0.0 \\
\hline 231 & 7.6 & 6 & 36 & 2.5 & 0.0 \\
\hline 274 & 3.5 & 5 & 49 & 1.9 & 0.0 \\
\hline 335 & 0.9 & 8 & 16 & 1.5 & 0.0 \\
\hline 352 & 0.9 & 4 & 9 & 1.1 & 0.0 \\
\hline 390 & 3.7 & 7 & 57 & 2.2 & 0.0 \\
\hline 393 & 1.6 & 4 & 9 & 1.1 & 0.0 \\
\hline 414 & 6.7 & 9 & 79 & 3.7 & 1.0 \\
\hline 500 & 12.7 & 10 & 29 & 4.9 & 2.5 \\
\hline 504 & 5.8 & 8 & 65 & 2.6 & 0.5 \\
\hline 510 & 1.0 & 8 & 20 & 1.5 & 0.0 \\
\hline 513 & 10.1 & 10 & 27 & 4.5 & 2.0 \\
\hline 525 & 5.4 & 8 & 80 & 2.5 & $\begin{array}{l}0.4 \\
\end{array}$ \\
\hline 543 & 6.7 & 6 & 60 & 2.5 & 0.0 \\
\hline 562 & 7.8 & 10 & 34 & 4.2 & 1.4 \\
\hline 575 & 1.6 & 8 & 49 & 1.5 & 0.0 \\
\hline 586 & 11.9 & 10 & 66 & 4.8 & 2.3 \\
\hline 588 & 3.4 & 6 & 79 & 2.0 & 0.0 \\
\hline
\end{tabular}




\begin{tabular}{|c|c|c|c|c|c|}
\hline $\begin{array}{l}\text { Storm } \\
\text { No. } \\
\end{array}$ & $\begin{array}{l}\text { Wave Height, } \\
\mathrm{ft}\end{array}$ & $\begin{array}{l}\text { Wave Period, } \\
\text { sec }\end{array}$ & $\begin{array}{l}\text { Wave Angle, } \\
\text { deg }\end{array}$ & $\begin{array}{l}\text { Setup, } \\
\text { ft }\end{array}$ & $\begin{array}{l}\text { Ponding } \\
\text { Level, } \\
\text { ft }\end{array}$ \\
\hline 18 & 6.3 & 9 & 62 & 3.0 & 0.8 \\
\hline 20 & 5.1 & 6 & 67 & 2.1 & 0.0 \\
\hline 21 & 11.6 & 10 & 76 & 4.9 & 2.3 \\
\hline 28 & 3.4 & 4 & 71 & 1.7 & 0.0 \\
\hline 33 & 8.8 & 7 & 104 & 3.5 & 1.3 \\
\hline 49 & 5.8 & 9 & 100 & 2.9 & 0.7 \\
\hline 60 & 1.7 & 8 & 65 & 1.6 & 0.0 \\
\hline 64 & 3.8 & 8 & 49 & 2.4 & 0.0 \\
\hline 82 & 11.3 & 10 & 101 & 4.8 & 2.2 \\
\hline 96 & 11.4 & 8 & 72 & 4.2 & 2.0 \\
\hline 97 & 3.4 & 7 & 52 & 2.2 & 0.0 \\
\hline 127 & 6.7 & 6 & 79 & 2.5 & 0.0 \\
\hline 146 & 2.0 & 8 & 55 & 1.6 & 0.0 \\
\hline 179 & 6.8 & 8 & 67 & 2.9 & 0.0 \\
\hline 231 & 6.9 & 6 & 69 & 2.5 & 0.0 \\
\hline 274 & 3.1 & 5 & 60 & 1.2 & 0.0 \\
\hline 335 & 0.7 & 7 & 117 & 1.5 & 0.0 \\
\hline 352 & 0.7 & 4 & 47 & 1.1 & 0.0 \\
\hline 390 & 3.5 & 7 & 50 & 2.2 & 0.0 \\
\hline 393 & 0.8 & 7 & 41 & 1.5 & 0.0 \\
\hline 414 & 12.7 & 9 & 97 & 4.7 & 2.4 \\
\hline 500 & 6.7 & 11 & 53 & 4.4 & 1.2 \\
\hline 504 & 8.1 & 8 & 73 & 3.8 & 1.3 \\
\hline 510 & 0.4 & 7 & 41 & 1.5 & 0.0 \\
\hline 513 & 8.2 & 9 & 76 & 4.1 & 1.4 \\
\hline 525 & 7.6 & 10 & 53 & 3.4 & 0.1 \\
\hline 543 & 6.7 & 10 & 93 & 3.4 & 0.0 \\
\hline 562 & 13.5 & 9 & 68 & 4.7 & 2.5 \\
\hline 575 & 0.5 & 5 & 41 & 1.3 & 0.0 \\
\hline 586 & 9.8 & 8 & 59 & 4.0 & 1.7 \\
\hline 588 & 8.8 & 7 & 93 & 3.5 & 1.3 \\
\hline
\end{tabular}




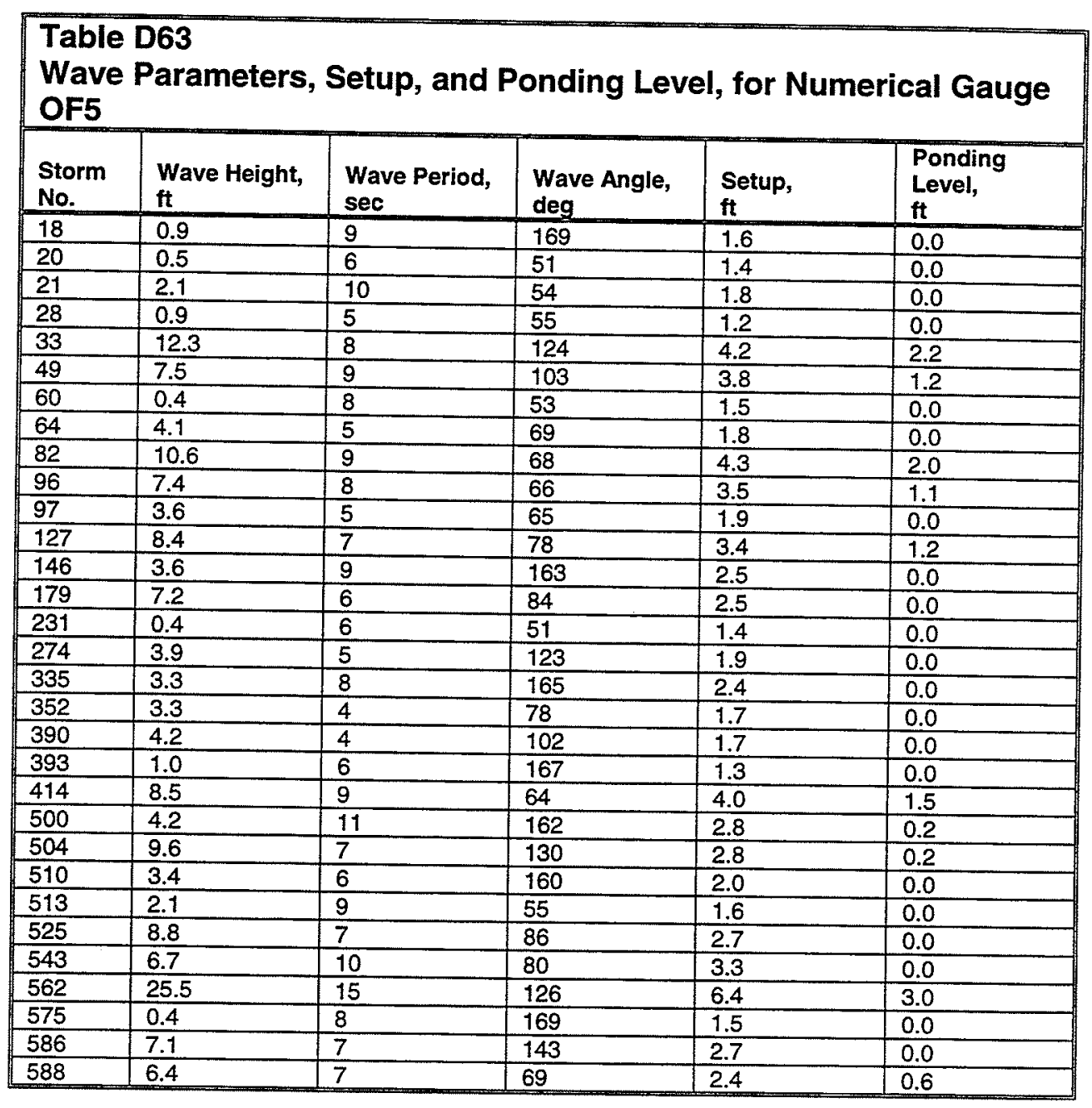




\begin{tabular}{|c|c|c|c|c|c|}
\hline \multicolumn{6}{|c|}{$\begin{array}{l}\text { Table D64 } \\
\text { Wave Parameters, Setup, and Ponding Level, for Numerical Gauge } \\
\text { OL5 }\end{array}$} \\
\hline $\begin{array}{l}\text { Storm } \\
\text { No. } \\
\end{array}$ & $\begin{array}{l}\text { Wave Height, } \\
\mathrm{ft}\end{array}$ & $\begin{array}{l}\text { Wave Period, } \\
\text { sec }\end{array}$ & $\begin{array}{l}\text { Wave Angle, } \\
\text { deg }\end{array}$ & $\begin{array}{l}\text { Setup, } \\
\mathrm{ft}\end{array}$ & $\begin{array}{l}\text { Ponding } \\
\text { Level, } \\
\mathrm{ft}\end{array}$ \\
\hline 18 & 4.6 & 8 & 158 & 2.4 & 0.0 \\
\hline 20 & 0.6 & 6 & 42 & 1.4 & 0.0 \\
\hline 21 & 1.8 & 10 & 43 & 1.8 & 0.0 \\
\hline 28 & 0.7 & 5 & 166 & 1.2 & 0.0 \\
\hline 33 & 11.1 & 9 & 138 & 4.4 & 2.1 \\
\hline 49 & 8.2 & 10 & 147 & 4.3 & 1.5 \\
\hline 60 & 0.4 & 7 & 43 & 1.5 & 0.0 \\
\hline 64 & 0.9 & 6 & 43 & 1.4 & 0.0 \\
\hline 82 & 9.3 & 9 & 55 & 4.2 & 1.7 \\
\hline 96 & 6.2 & 7 & 50 & 2.4 & 0.5 \\
\hline 97 & 0.9 & 6 & 43 & 1.4 & 0.0 \\
\hline 127 & 8.4 & 6 & 126 & 3.1 & 1.0 \\
\hline 146 & 6.9 & 8 & 156 & 2.9 & 0.0 \\
\hline 179 & 4.1 & 6 & 57 & 2.0 & 0.0 \\
\hline 231 & 0.6 & 6 & 42 & 1.4 & 0.0 \\
\hline 274 & 3.6 & 5 & 112 & 1.9 & 0.0 \\
\hline 335 & 7.5 & 7 & 151 & 3.3 & 0.9 \\
\hline 352 & 3.3 & 5 & 62 & 1.9 & 0.0 \\
\hline 390 & 3.8 & 4 & 77 & 1.7 & 0.0 \\
\hline 393 & 0.4 & 6 & 169 & 1.4 & 0.0 \\
\hline 414 & 6.3 & 9 & 51 & 3.0 & 0.8 \\
\hline 500 & 7.9 & 11 & 154 & 4.6 & 1.5 \\
\hline 504 & 7.2 & 6 & 121 & 2.5 & 0.0 \\
\hline 510 & 3.9 & 8 & 161 & 2.4 & 0.0 \\
\hline 513 & 3.3 & 8 & 162 & 2.4 & 0.0 \\
\hline 525 & 6.4 & 6 & 81 & 2.2 & 0.4 \\
\hline 543 & 7.2 & 9 & 87 & 3.8 & 1.1 \\
\hline 562 & 27.2 & 15 & 125 & 7.7 & 4.6 \\
\hline 575 & 3.6 & 8 & 161 & 2.4 & 0.0 \\
\hline 586 & 9.2 & 7 & 132 & 3.5 & 1.4 \\
\hline 588 & 6.0 & 7 & 148 & 2.4 & 0.5 \\
\hline
\end{tabular}




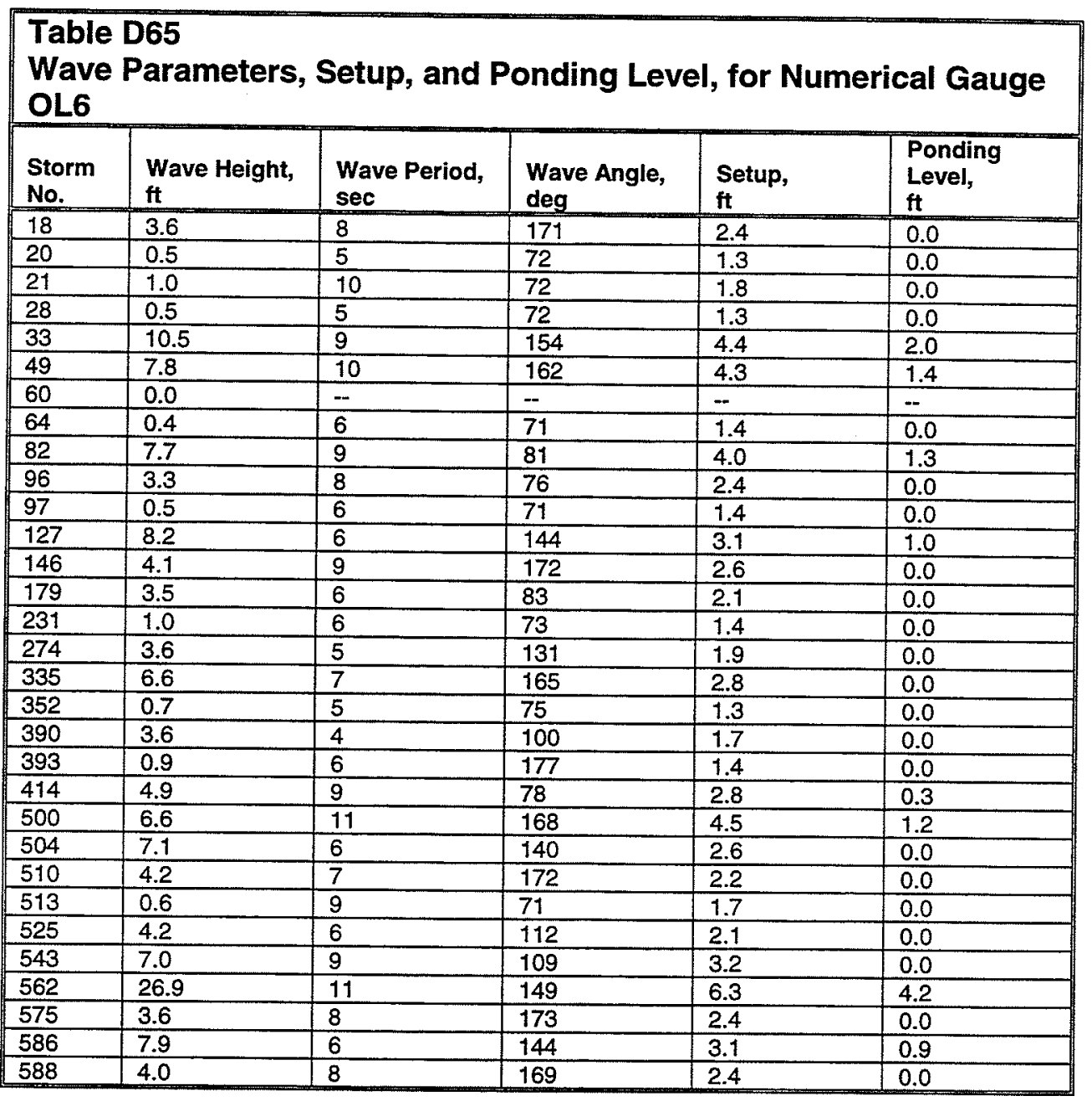




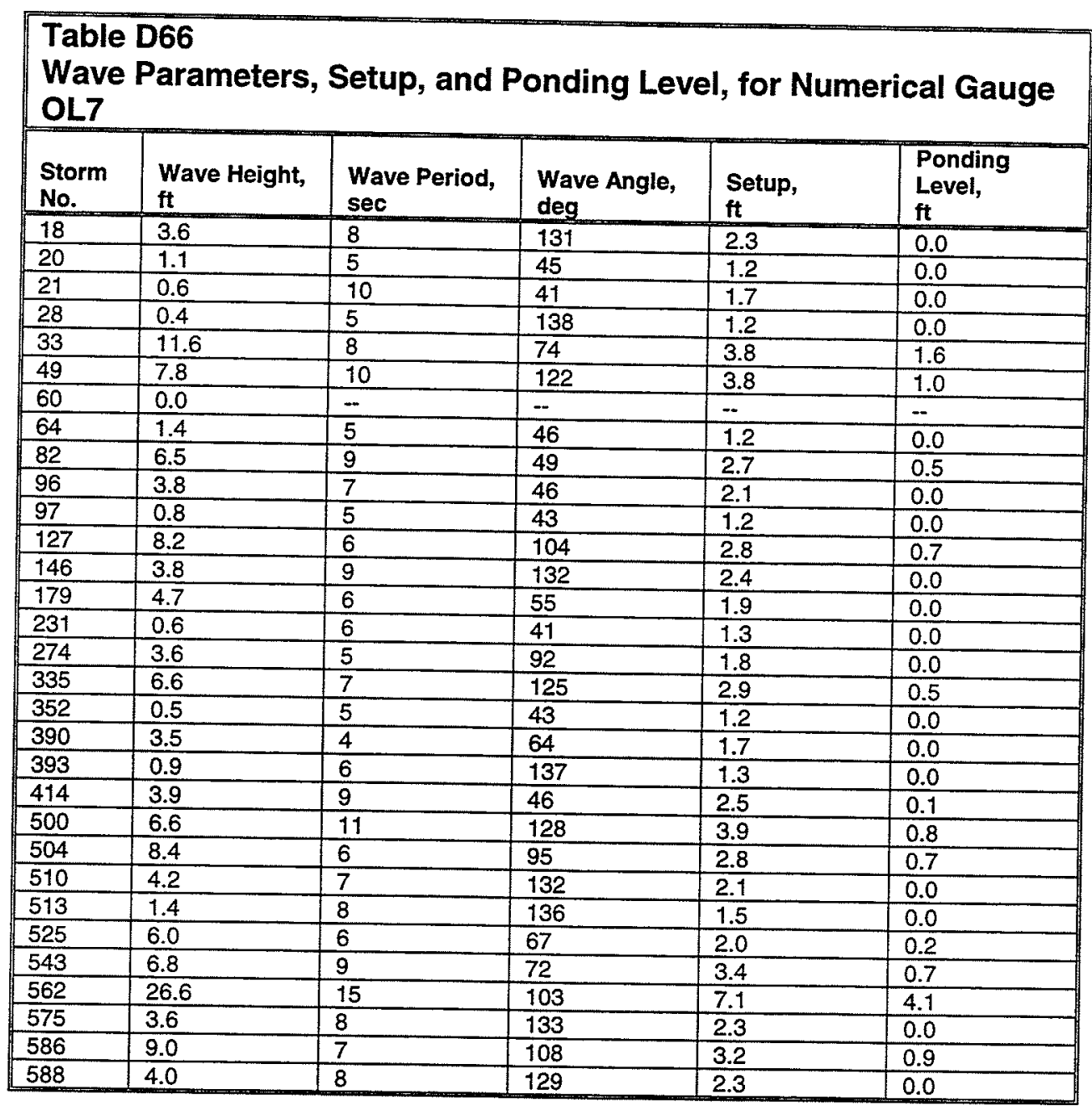




\begin{tabular}{|c|c|c|c|c|c|}
\hline \multicolumn{6}{|c|}{$\begin{array}{l}\text { Table D67 } \\
\text { Wave Parameters, Setup, and Ponding Level, for Numerical Gaug } \\
\text { TA2 }\end{array}$} \\
\hline $\begin{array}{l}\text { Storm } \\
\text { No. }\end{array}$ & $\begin{array}{l}\text { Wave Height, } \\
\mathrm{ft}\end{array}$ & $\begin{array}{l}\text { Wave Period, } \\
\text { sec }\end{array}$ & $\begin{array}{l}\text { Wave Angle, } \\
\text { deg }\end{array}$ & $\begin{array}{l}\text { Setup, } \\
\mathrm{ft}\end{array}$ & $\begin{array}{l}\text { Ponding } \\
\text { Level, } \\
\text { ft }\end{array}$ \\
\hline 18 & 3.8 & 6 & 142 & 2.2 & 0.0 \\
\hline 20 & 3.4 & 4 & 24 & 1.8 & 0.0 \\
\hline 21 & 1.2 & 8 & 3 & 1.7 & 0.0 \\
\hline 28 & 1.0 & 4 & 5 & 1.2 & 0.0 \\
\hline 33 & 9.8 & 9 & 127 & 4.5 & 1.8 \\
\hline 49 & 6.6 & 9 & 70 & 3.9 & 0.9 \\
\hline 60 & 0.4 & 3 & 6 & 1.1 & 0.0 \\
\hline 64 & 3.6 & 5 & 30 & 2.0 & 0.0 \\
\hline 82 & 5.3 & 7 & 36 & 2.4 & 0.2 \\
\hline 96 & 6.7 & 7 & 15 & 3.3 & 0.7 \\
\hline 97 & 4.5 & 5 & 23 & 2.0 & 0.0 \\
\hline 127 & 7.2 & 6 & 116 & 3.1 & 0.7 \\
\hline 146 & 3.5 & 8 & 143 & 2.5 & 0.0 \\
\hline 179 & 0.5 & 6 & 2 & 1.4 & 0.0 \\
\hline 231 & 0.4 & 7 & 1 & 1.6 & 0.0 \\
\hline 274 & 3.8 & 5 & 111 & 2.0 & 0.0 \\
\hline 335 & 3.4 & 8 & 145 & 2.5 & 0.0 \\
\hline 352 & 0.5 & 3 & 18 & 1.1 & 0.0 \\
\hline 390 & 0.4 & 6 & 1 & 1.4 & 0.0 \\
\hline 393 & 0.5 & 6 & 149 & 1.4 & 0.0 \\
\hline 414 & 8.4 & 9 & 11 & 4.3 & 1.5 \\
\hline 500 & 1.1 & 6 & 143 & 1.4 & 0.0 \\
\hline 504 & 6.7 & 5 & 112 & 2.4 & 0.0 \\
\hline 510 & 3.7 & 7 & 142 & 2.3 & 0.0 \\
\hline 513 & 0.4 & 8 & 149 & 1.7 & 0.0 \\
\hline 525 & 4.2 & 7 & 41 & 2.3 & 0.0 \\
\hline 543 & 6.9 & 9 & 87 & 3.3 & 0.0 \\
\hline 562 & 27.0 & 15 & 117 & 6.9 & 3.1 \\
\hline 575 & 0.9 & 9 & 145 & 1.8 & 0.0 \\
\hline 586 & 8.5 & 7 & 124 & 2.8 & 0.0 \\
\hline 588 & 5.1 & 7 & 135 & 2.3 & 0.1 \\
\hline
\end{tabular}




\begin{tabular}{|c|c|c|c|c|c|}
\hline \multicolumn{6}{|c|}{$\begin{array}{l}\text { Table D68 } \\
\text { Wave Parameters, Setup, and Ponding Level, for Numerical Gaug } \\
\text { TA3 }\end{array}$} \\
\hline $\begin{array}{l}\text { Storm } \\
\text { No. } \\
\end{array}$ & $\begin{array}{l}\text { Wave Height, } \\
\mathrm{tt}\end{array}$ & $\begin{array}{l}\text { Wave Period, } \\
\text { sec }\end{array}$ & $\begin{array}{l}\text { Wave Angle, } \\
\text { deg }\end{array}$ & $\begin{array}{l}\text { Setup, } \\
\mathrm{ft}\end{array}$ & $\begin{array}{l}\text { Ponding } \\
\text { Level, } \\
\text { ft }\end{array}$ \\
\hline 18 & 4.1 & 9 & 6 & 2.8 & 0.0 \\
\hline 20 & 3.4 & 5 & 20 & 2.1 & 0.0 \\
\hline 21 & 5.2 & 8 & 12 & 2.7 & 0.3 \\
\hline 28 & 1.1 & 8 & 4 & 1.7 & 0.0 \\
\hline 33 & 9.2 & 9 & 89 & 4.6 & 1.7 \\
\hline 49 & 6.6 & 10 & 95 & 4.4 & 1.0 \\
\hline 60 & 1.3 & 5 & 9 & 1.4 & 0.0 \\
\hline 64 & 3.3 & 6 & 11 & 2.3 & 0.0 \\
\hline 82 & 7.5 & 7 & 48 & 3.6 & 0.9 \\
\hline 96 & 9.6 & 8 & 33 & 4.3 & 1.6 \\
\hline 97 & 3.5 & 5 & 21 & 2.1 & 0.0 \\
\hline 127 & 6.9 & 6 & 152 & 3.1 & 0.6 \\
\hline 146 & 1.7 & 9 & 176 & 1.8 & 0.0 \\
\hline 179 & 1.2 & 9 & 2 & 1.8 & 0.0 \\
\hline 231 & 3.7 & 5 & 13 & 2.1 & 0.0 \\
\hline 274 & 3.7 & 5 & 148 & 2.1 & 0.0 \\
\hline 335 & 3.6 & 7 & 172 & 2.4 & 0.0 \\
\hline 352 & 2.6 & 4 & 80 & 1.2 & 0.0 \\
\hline 390 & 1.4 & 7 & 4 & 1.6 & 0.0 \\
\hline 393 & 0.4 & 8 & 1 & 1.7 & 0.0 \\
\hline 414 & 14.3 & 9 & 28 & 5.2 & 2.6 \\
\hline 500 & 1.2 & 8 & 2 & 1.7 & 0.0 \\
\hline 504 & 6.9 & 6 & 146 & 2.7 & 0.0 \\
\hline 510 & 0.6 & 8 & 179 & 1.7 & 0.0 \\
\hline 513 & 0.5 & 8 & 179 & 1.7 & 0.0 \\
\hline 525 & 3.7 & 10 & 6 & 3.0 & 0.0 \\
\hline 543 & 6.6 & 10 & 72 & 3.6 & 0.0 \\
\hline 562 & 25.6 & 15 & 153 & 7.1 & 3.0 \\
\hline 575 & 0.6 & 9 & 177 & 1.8 & 0.0 \\
\hline 586 & 12.0 & 9 & 14 & 3.9 & 0.9 \\
\hline 588 & 6.4 & 7 & 15 & 2.7 & 0.6 \\
\hline
\end{tabular}




\begin{tabular}{|c|c|c|c|c|c|}
\hline \multicolumn{6}{|c|}{$\begin{array}{l}\text { Table D69 } \\
\text { Wave Parameters, Setup, and Ponding Level, for Numerical Gauge } \\
\text { TA4 }\end{array}$} \\
\hline $\begin{array}{l}\text { Storm } \\
\text { No. }\end{array}$ & $\begin{array}{l}\text { Wave Height, } \\
\mathrm{ft}\end{array}$ & $\begin{array}{l}\text { Wave Period, } \\
\text { sec }\end{array}$ & $\begin{array}{l}\text { Wave Angle, } \\
\text { deg }\end{array}$ & $\begin{array}{l}\text { Setup, } \\
\mathrm{ft}\end{array}$ & $\begin{array}{l}\text { Ponding } \\
\text { Level, } \\
\text { ft }\end{array}$ \\
\hline 18 & 6.7 & 9 & 99 & 3.8 & 1.0 \\
\hline 20 & 4.7 & 6 & 96 & 2.1 & 0.0 \\
\hline 21 & 13.4 & 10 & 107 & 5.1 & 2.6 \\
\hline 28 & 0.4 & 5 & 43 & 1.3 & 0.0 \\
\hline 33 & 9.3 & 8 & 110 & 4.0 & 1.6 \\
\hline 49 & 3.5 & 5 & 116 & 1.9 & 0.0 \\
\hline 60 & 1.9 & 8 & 94 & 1.6 & 0.0 \\
\hline 64 & 5.5 & 7 & 81 & 2.2 & 0.0 \\
\hline 82 & 7.9 & 10 & 120 & 4.3 & 1.4 \\
\hline 96 & 12.5 & 8 & 96 & 4.3 & 2.2 \\
\hline 97 & 4.1 & 6 & 96 & 2.1 & 0.0 \\
\hline 127 & 8.1 & 6 & 63 & 3.1 & 0.9 \\
\hline 146 & 1.6 & 9 & 42 & 1.7 & 0.0 \\
\hline 179 & 4.9 & 8 & 106 & 2.4 & 0.0 \\
\hline 231 & 5.6 & 8 & 96 & 2.4 & 0.0 \\
\hline 274 & 3.9 & 5 & 81 & 1.9 & 0.0 \\
\hline 335 & 2.0 & 9 & 81 & 1.7 & 0.0 \\
\hline 352 & 0.6 & 4 & 37 & 1.2 & 0.0 \\
\hline 390 & 3.5 & 7 & 95 & 2.2 & 0.0 \\
\hline 393 & 5.8 & 7 & 79 & 2.4 & 0.4 \\
\hline 414 & 14.2 & 9 & 107 & 4.8 & 2.6 \\
\hline 500 & 13.8 & 11 & 65 & 5.4 & 2.8 \\
\hline 504 & 5.8 & 8 & 104 & 2.7 & 0.5 \\
\hline 510 & 3.5 & 7 & 70 & 2.2 & 0.0 \\
\hline 513 & 7.4 & 10 & 71 & 4.2 & 1.3 \\
\hline 525 & 6.7 & 7 & 109 & 2.8 & 0.0 \\
\hline 543 & 5.0 & 6 & 111 & 2.1 & 0.0 \\
\hline 562 & 12.9 & 11 & 61 & 5.3 & 2.6 \\
\hline 575 & 2.6 & 9 & 92 & 1.7 & 0.0 \\
\hline 586 & 9.6 & 8 & 97 & 4.0 & 1.6 \\
\hline 588 & 7.6 & 7 & 110 & 3.4 & 1.0 \\
\hline
\end{tabular}




\section{Appendix E Hurricane Tracks}

This appendix shows hurricane tracks for each storm contained in the EST training set. Each figure consists of an upper and lower panel. The upper panel shows storm tracks through the immediate vicinity of the five islands of interest for the study. Some figures do not show a storm track in the upper panel because the storm did not pass within the bounds of the graphical limits. The lower panel shows storm tracks for the region covered by the numerical grid developed for the study. The outer boundary of the numerical grid is shown as the large circle. Storm tracks can also be seen outside of the grid region.

The date and time when each storm was first recorded in the HURDAT database is given under the label "Storm Track Start" in the lower panel. Dots in the upper and lower panels show the 6-hr HURDAT locations for the storms, lines show the 1-hr interpolated storm track. On the lower panels, the 6-hr data points are associated with a data and time given in the format $\mathrm{mm} / \mathrm{dd} / \mathrm{tt}$ where $\mathrm{mm}$ is the month, dd is the day, and tt is the hour referenced to GMT. 


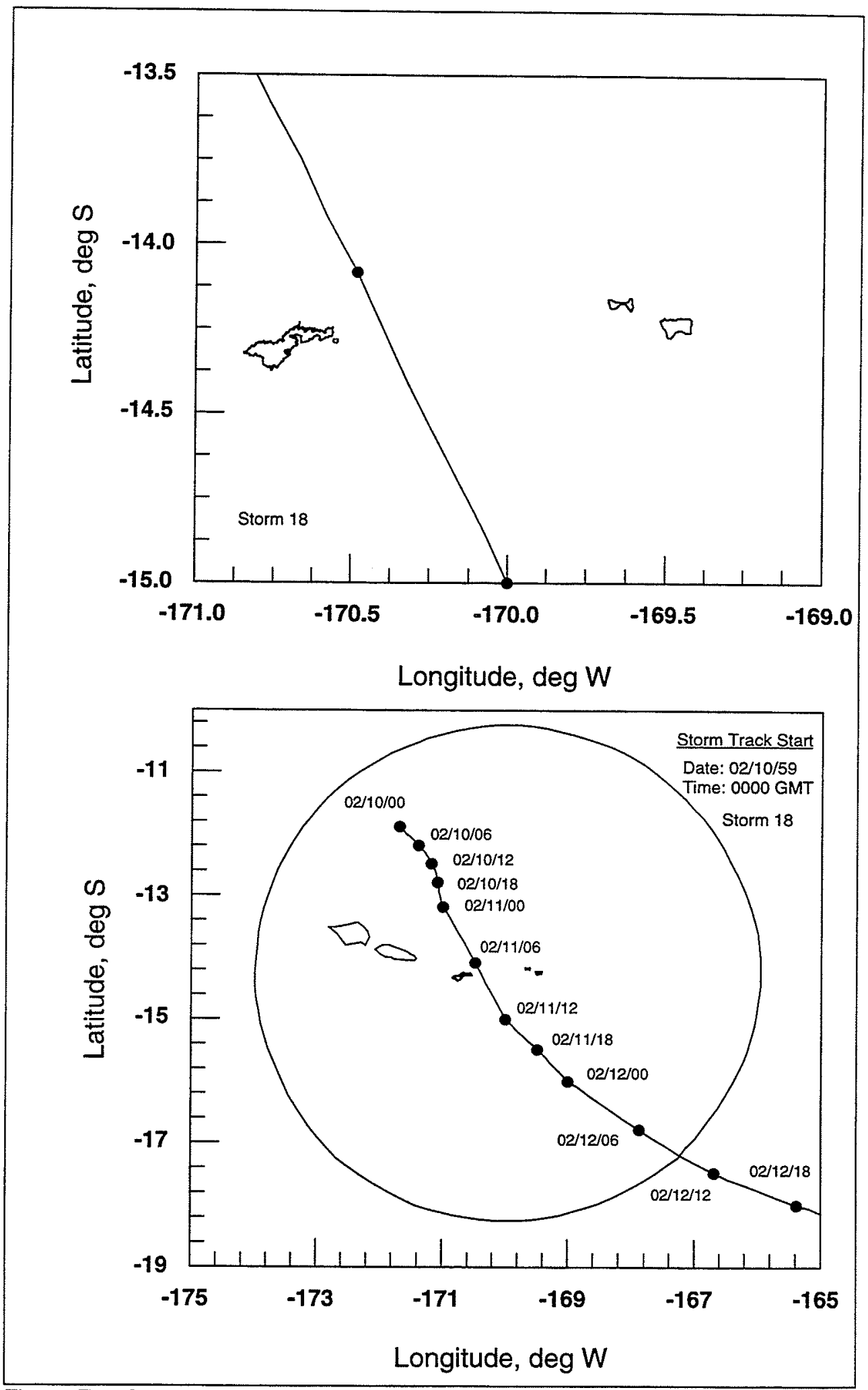

Figure E.1 Storm track for storm number 18 


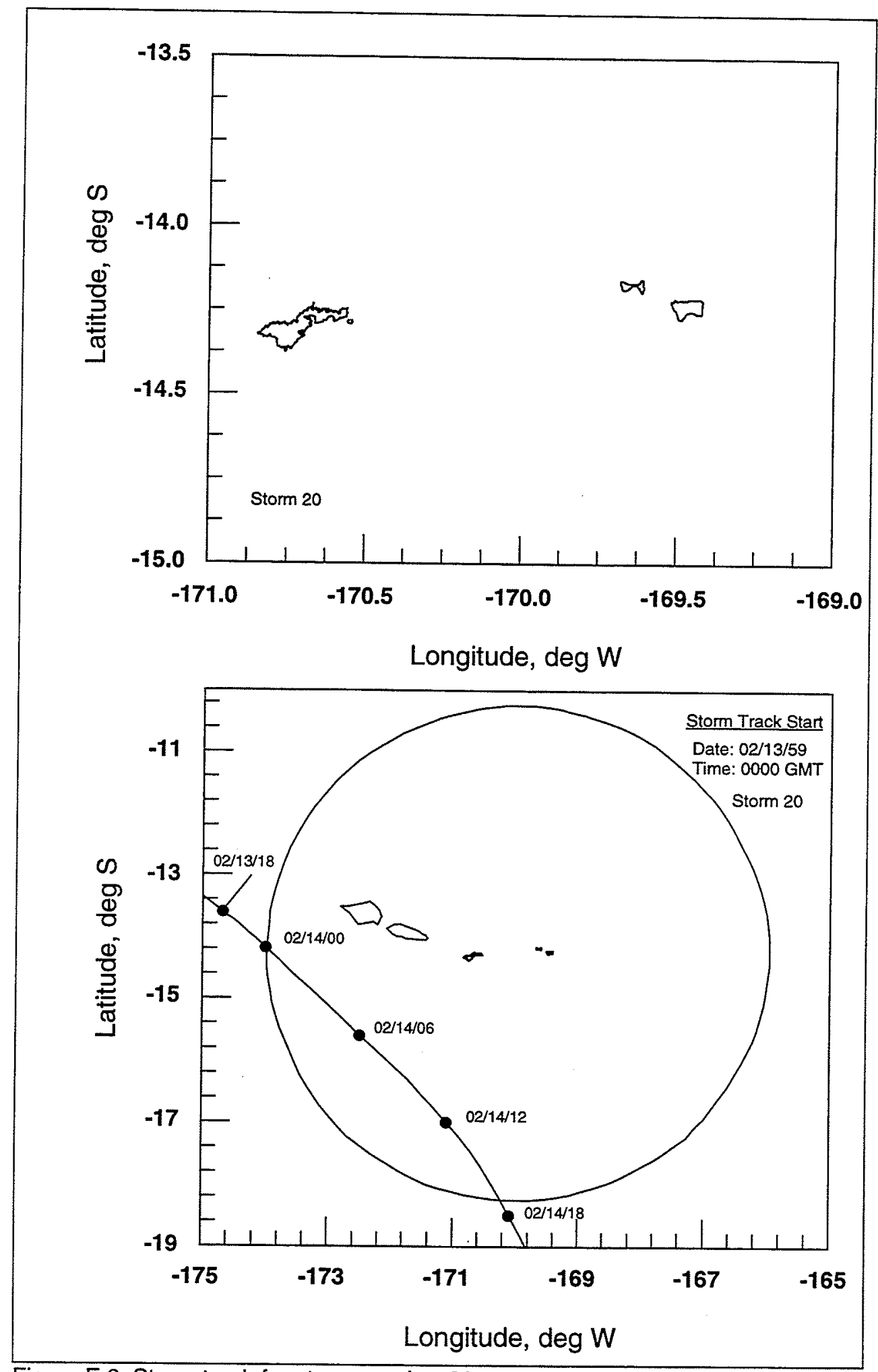

Figure E.2 Storm track for storm number 20 

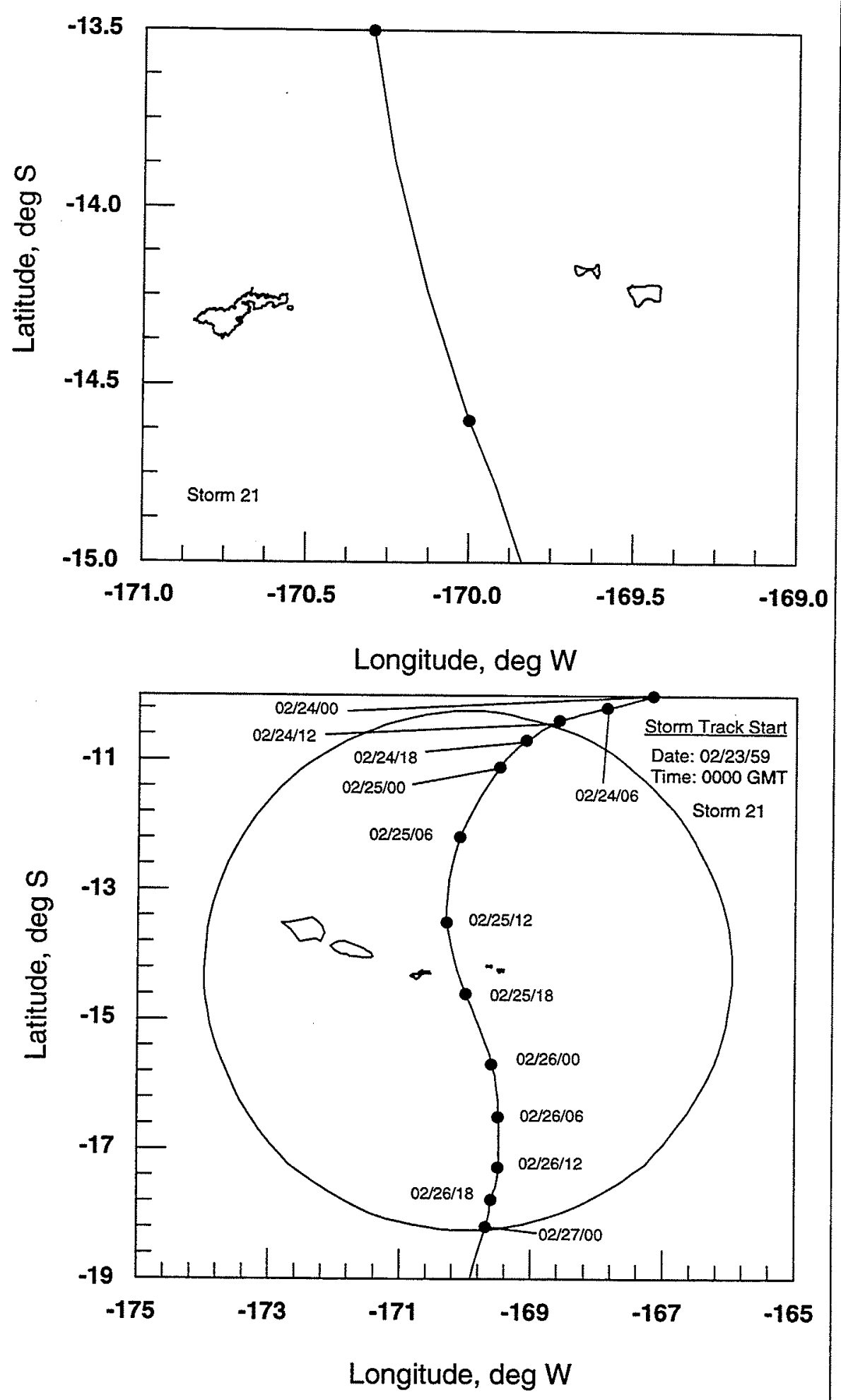

Figure E.3 Storm track for storm number 21 


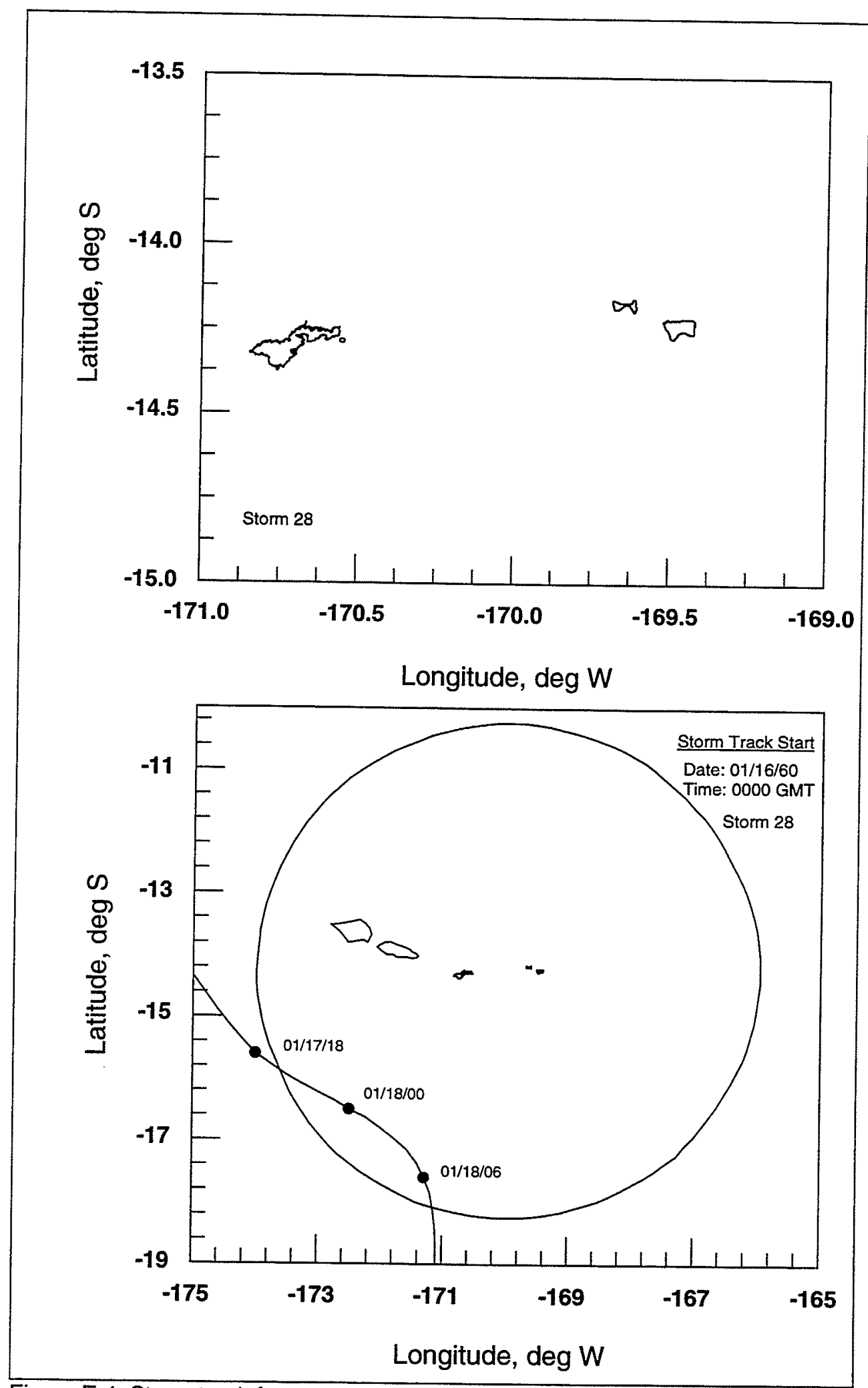

Figure E.4 Storm track for storm number 28 


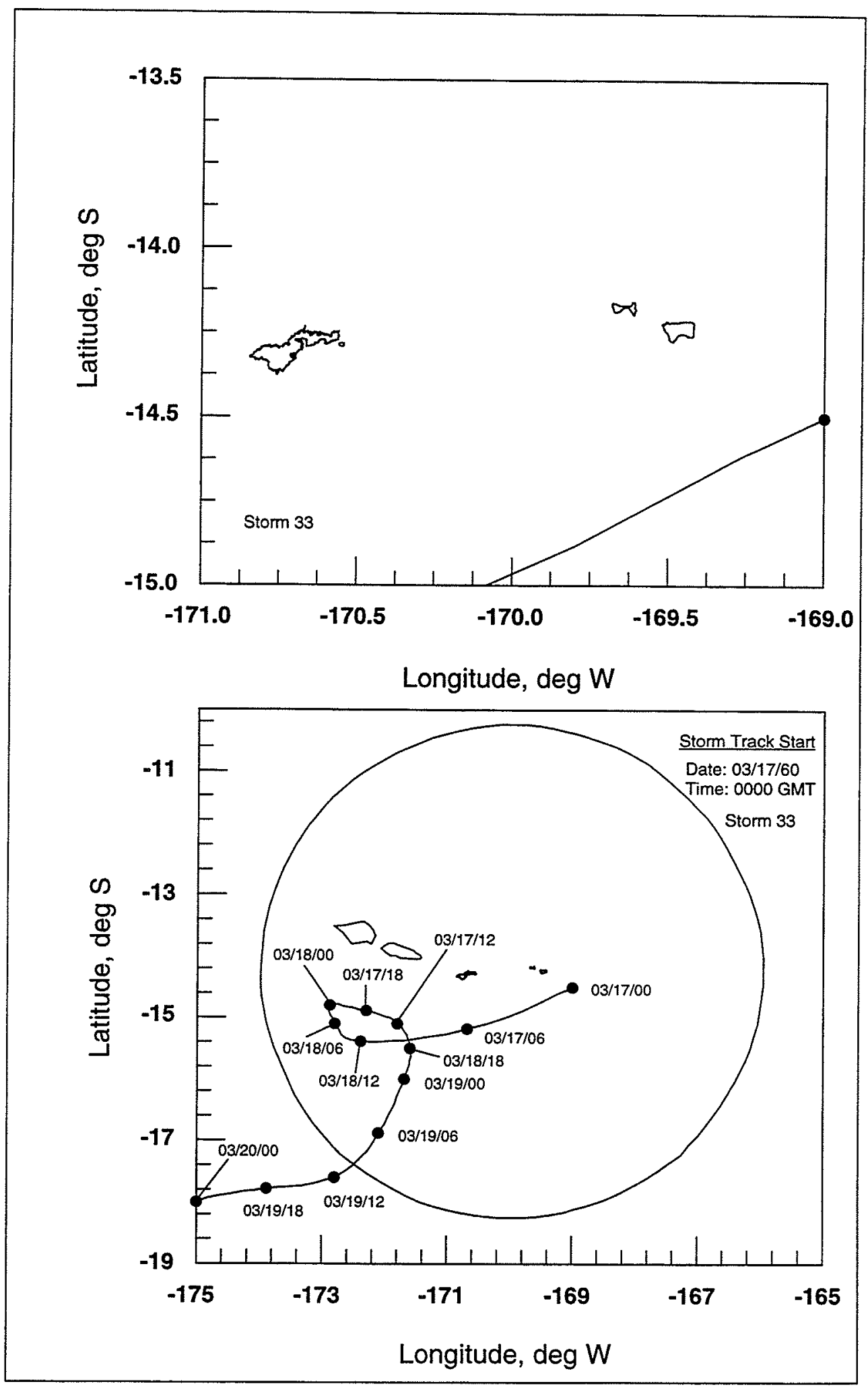

Figure E.5 Storm track for storm number 33 


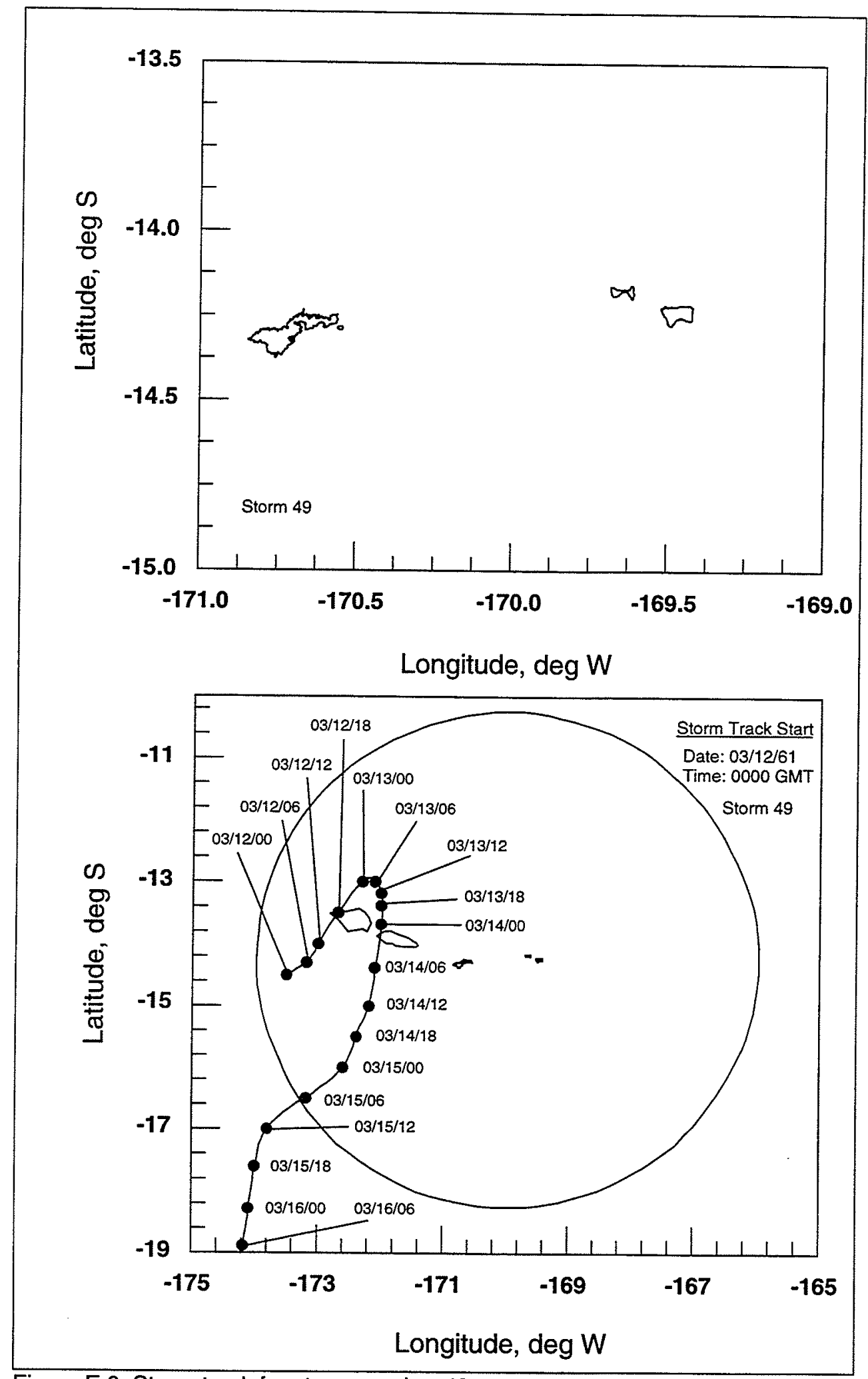

Figure E.6 Storm track for storm number 49 


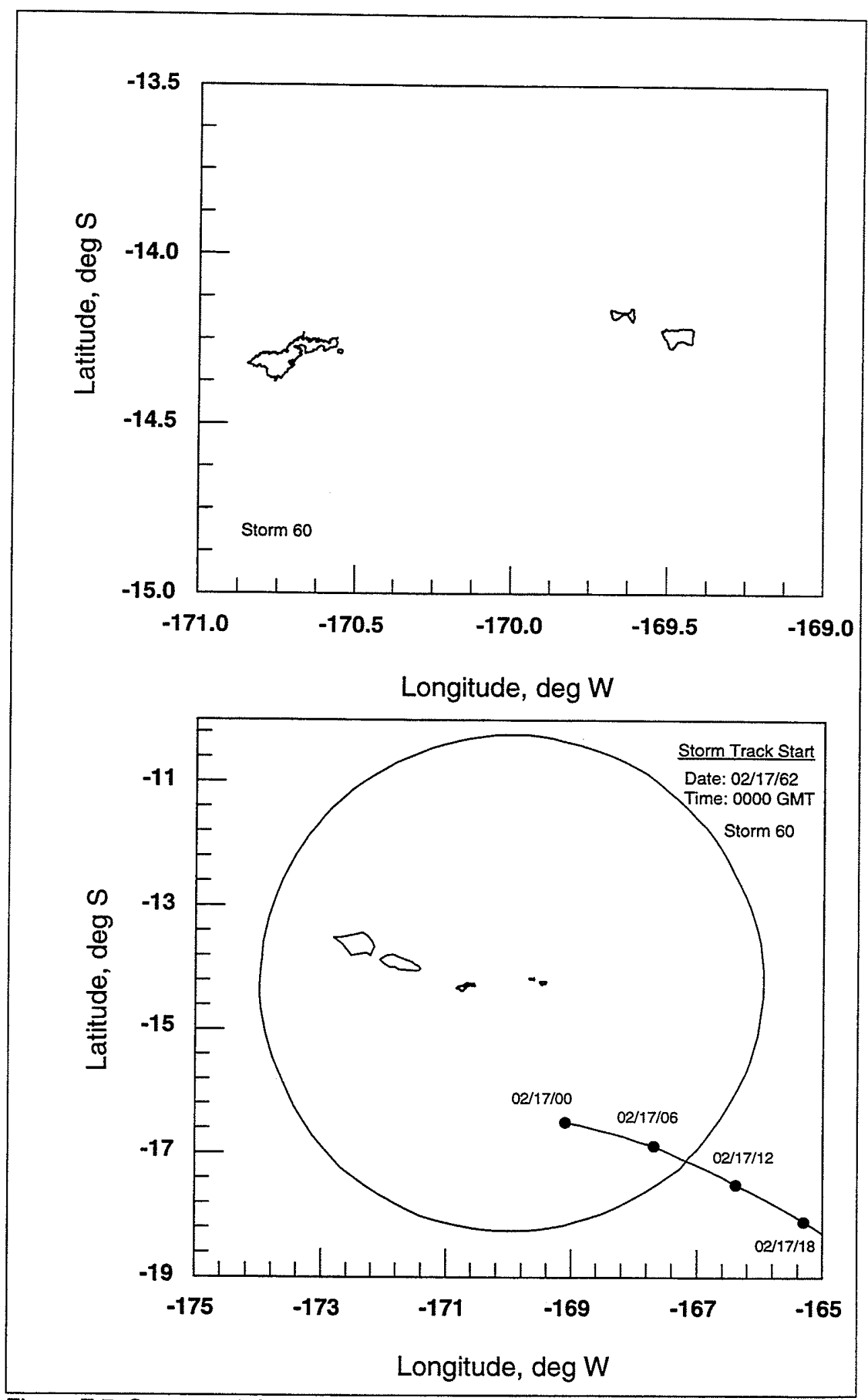

Figure E.7 Storm track for storm number 60 


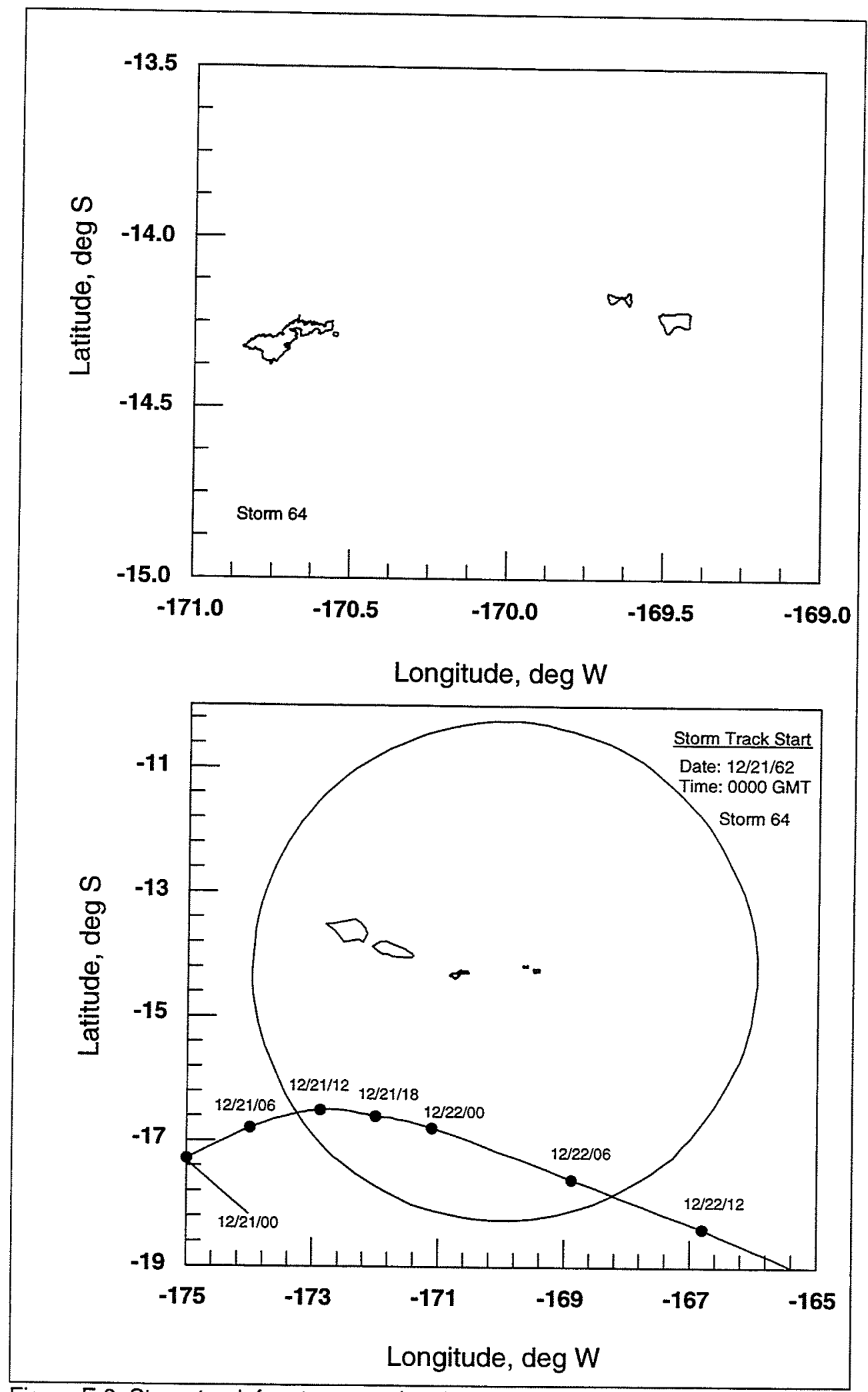

Figure E.8 Storm track for storm number 64 


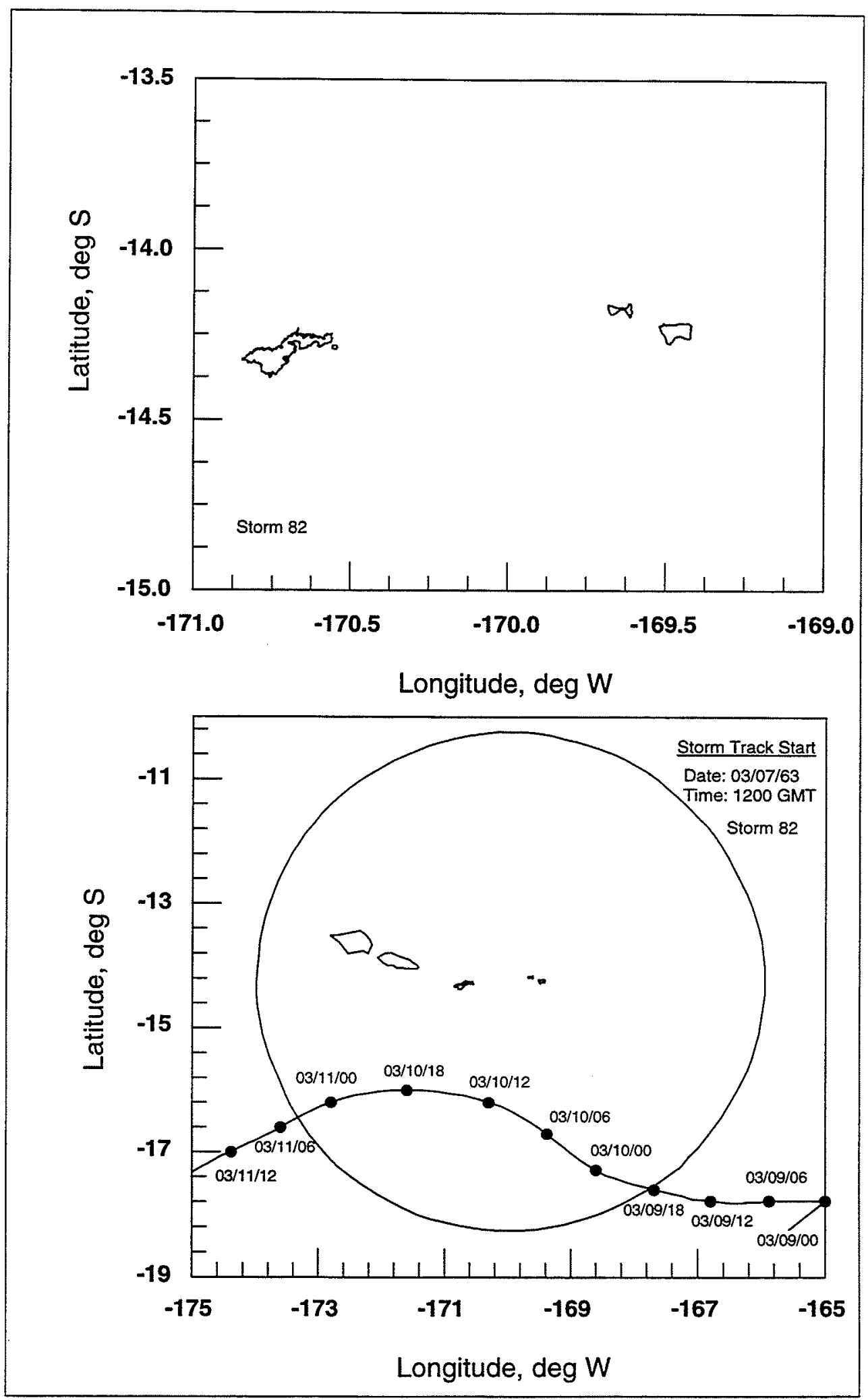

Figure E.9. Storm track for storm number 82 


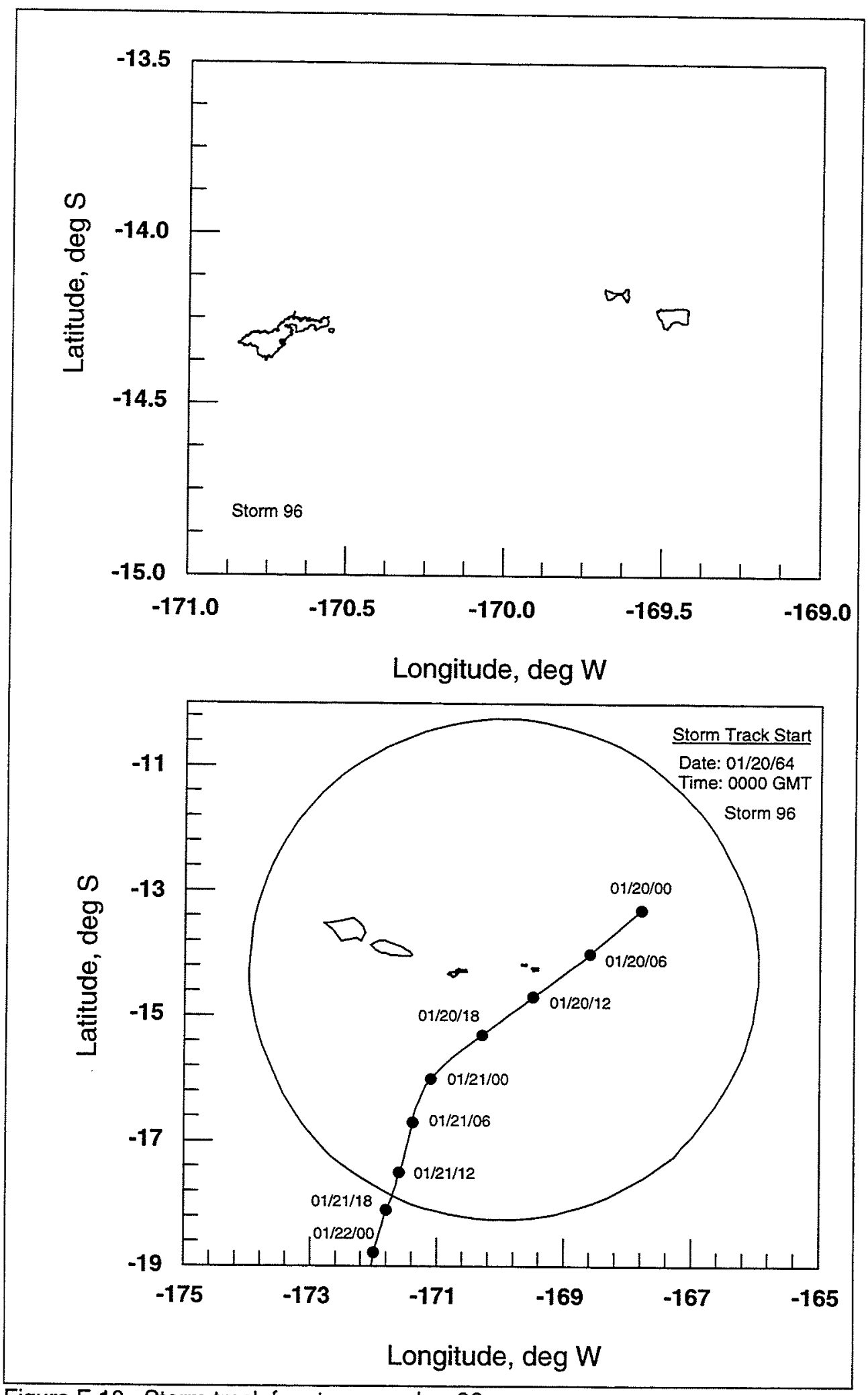

Figure E.10. Storm track for storm number 96 


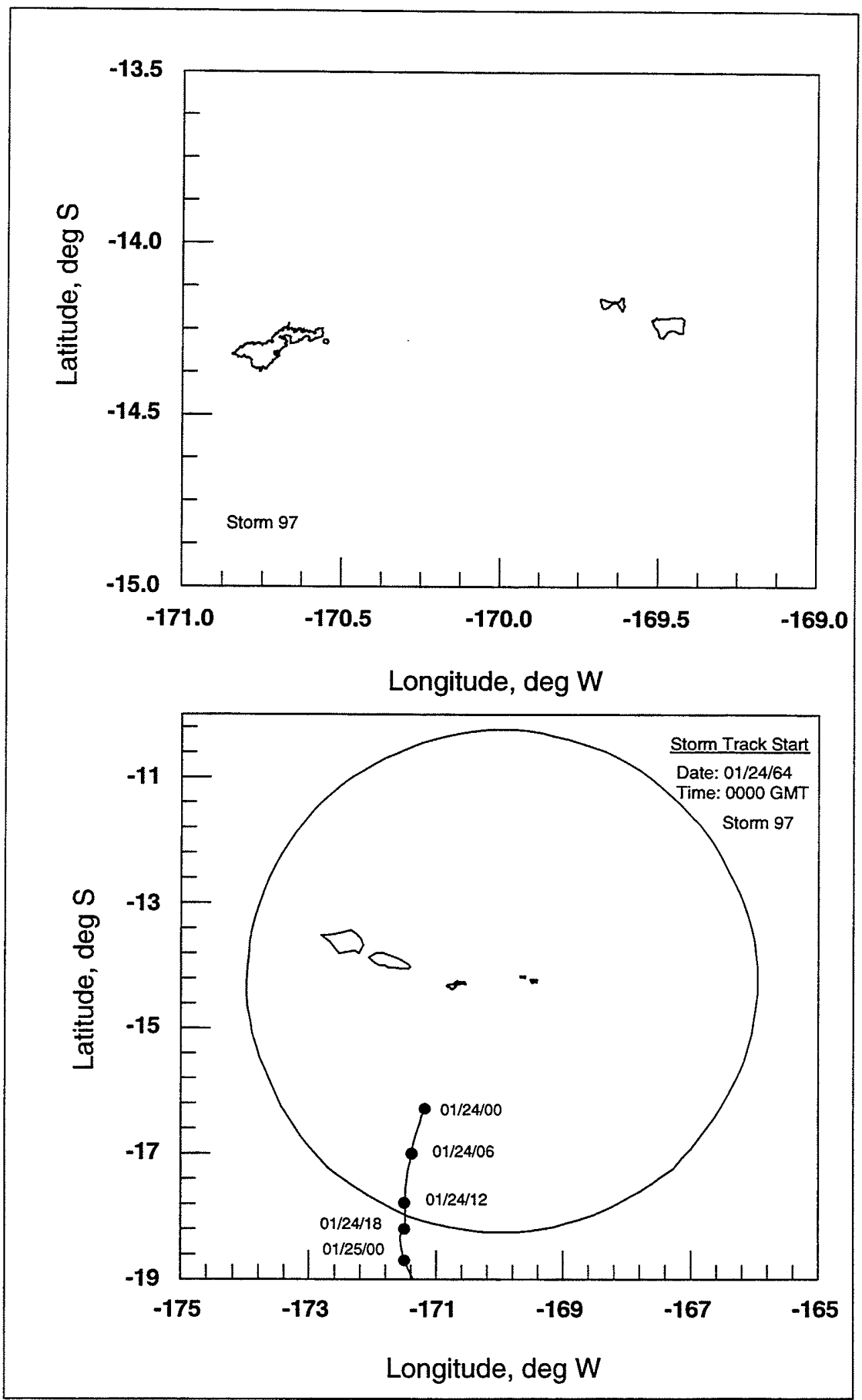

Figure E.11. Storm track for storm number 97 


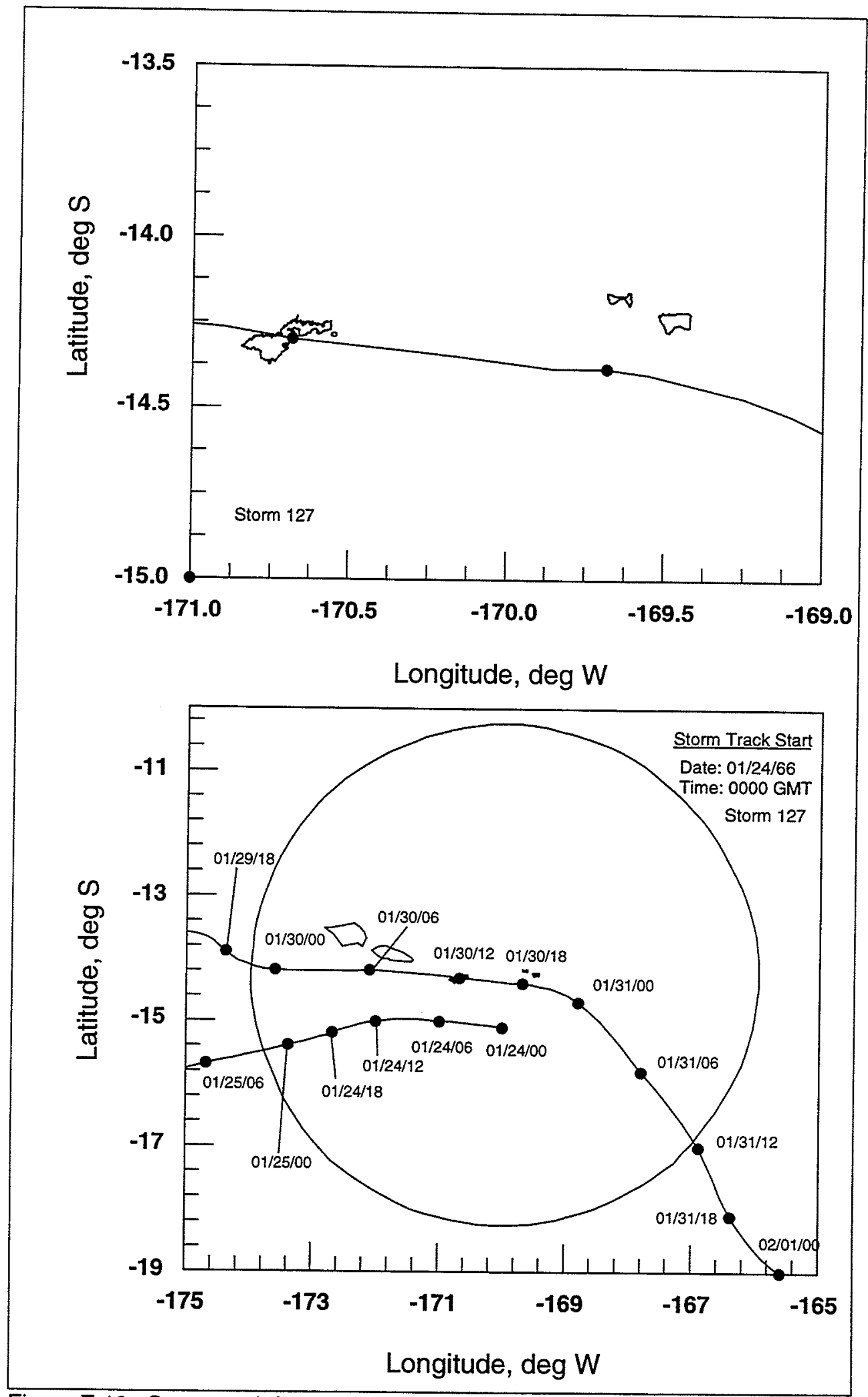

Figure E.12. Storm track for storm number 127 


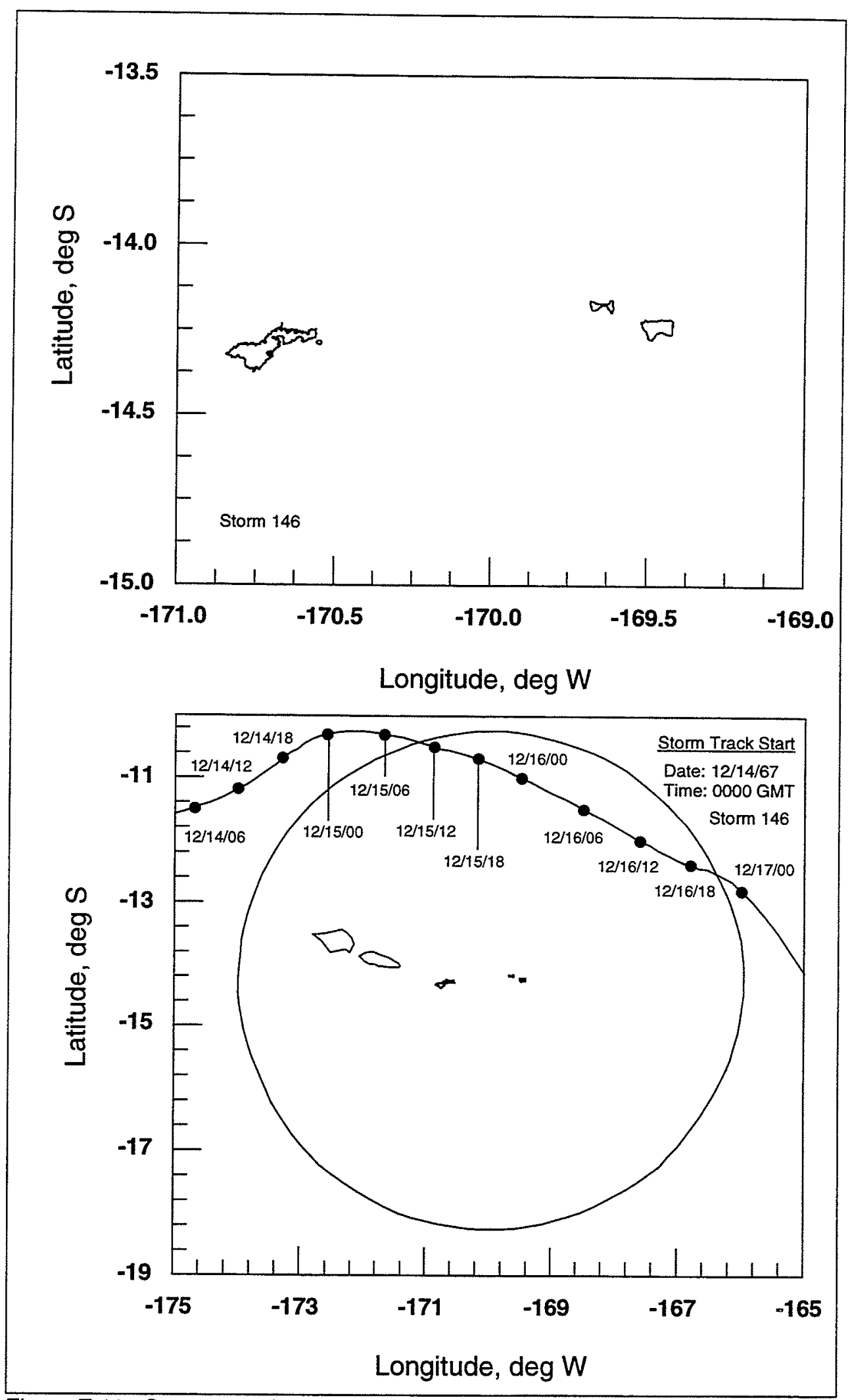

Figure E.13. Storm track for storm number 146 


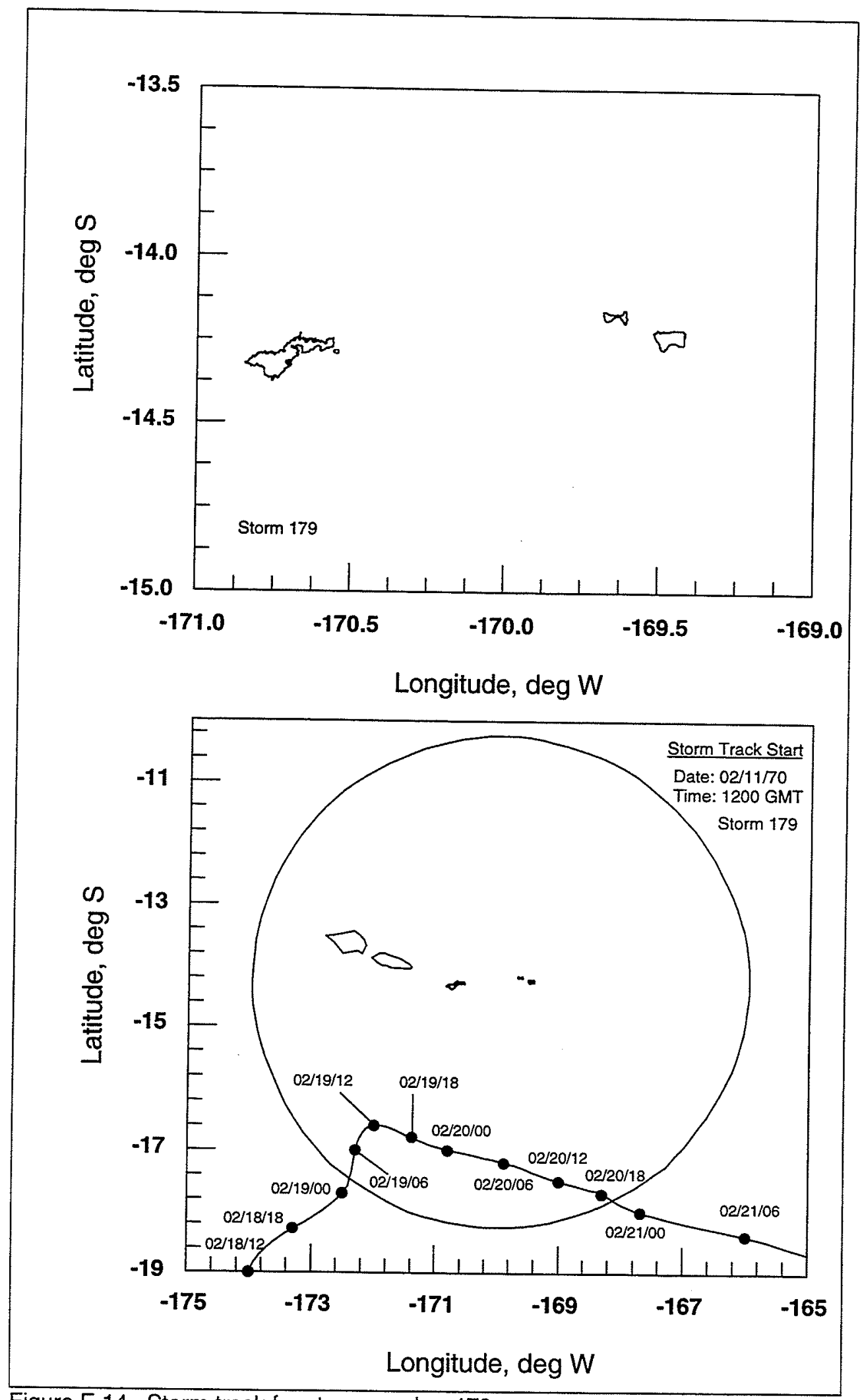

Figure E.14. Storm track for storm number 179 


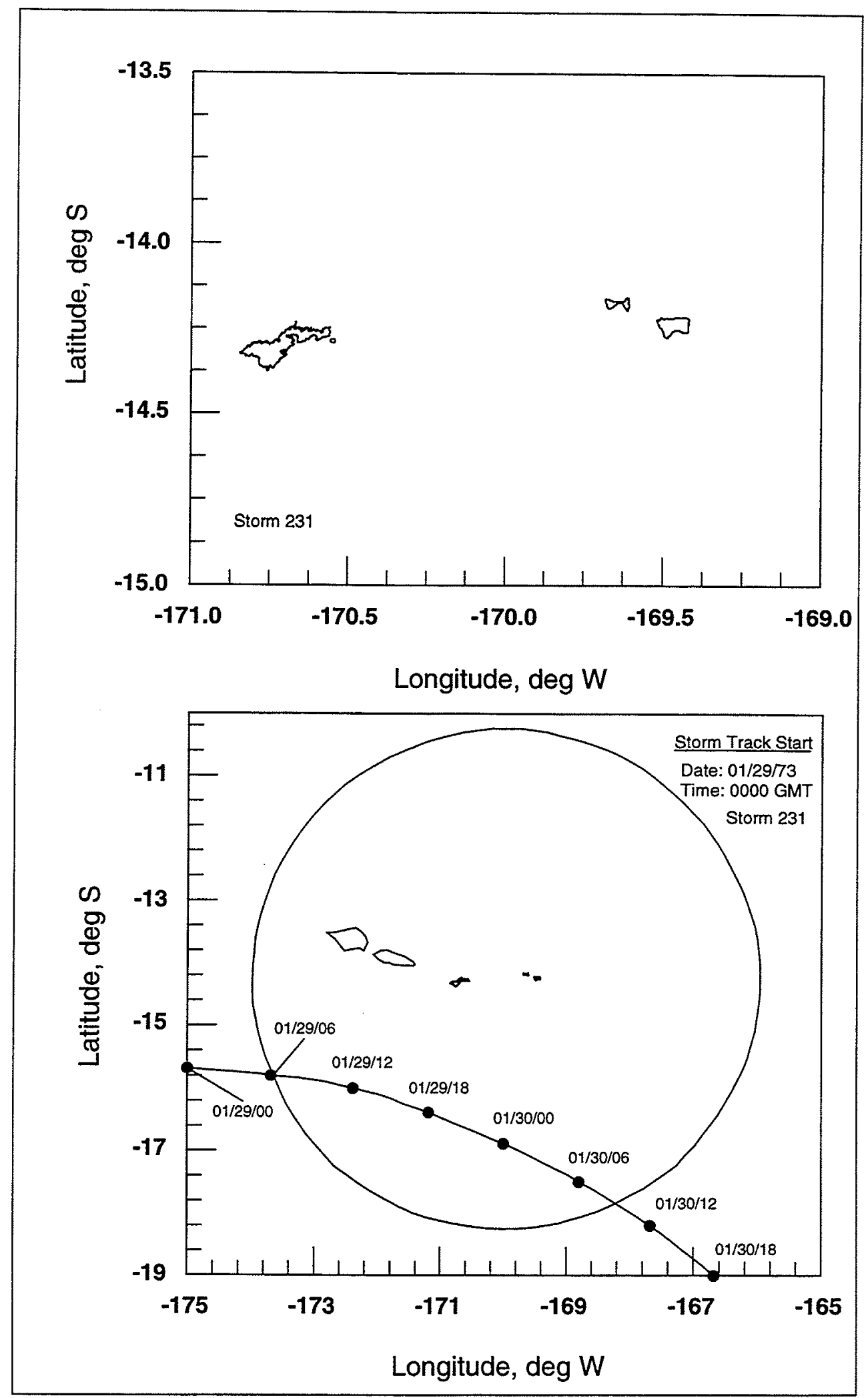

Figure E.15. Storm track for storm number 231 


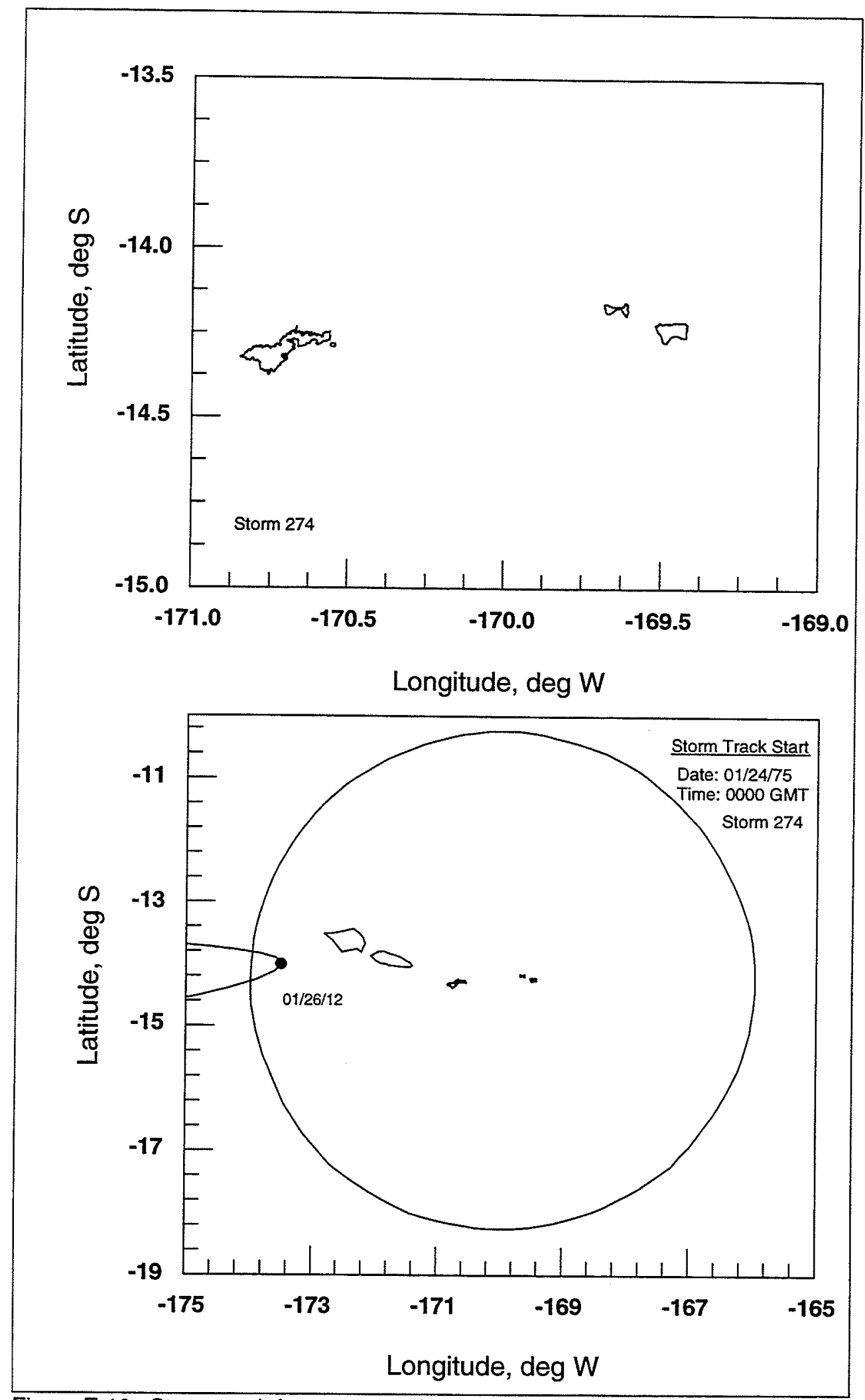

Figure E.16. Storm track for storm number 274 


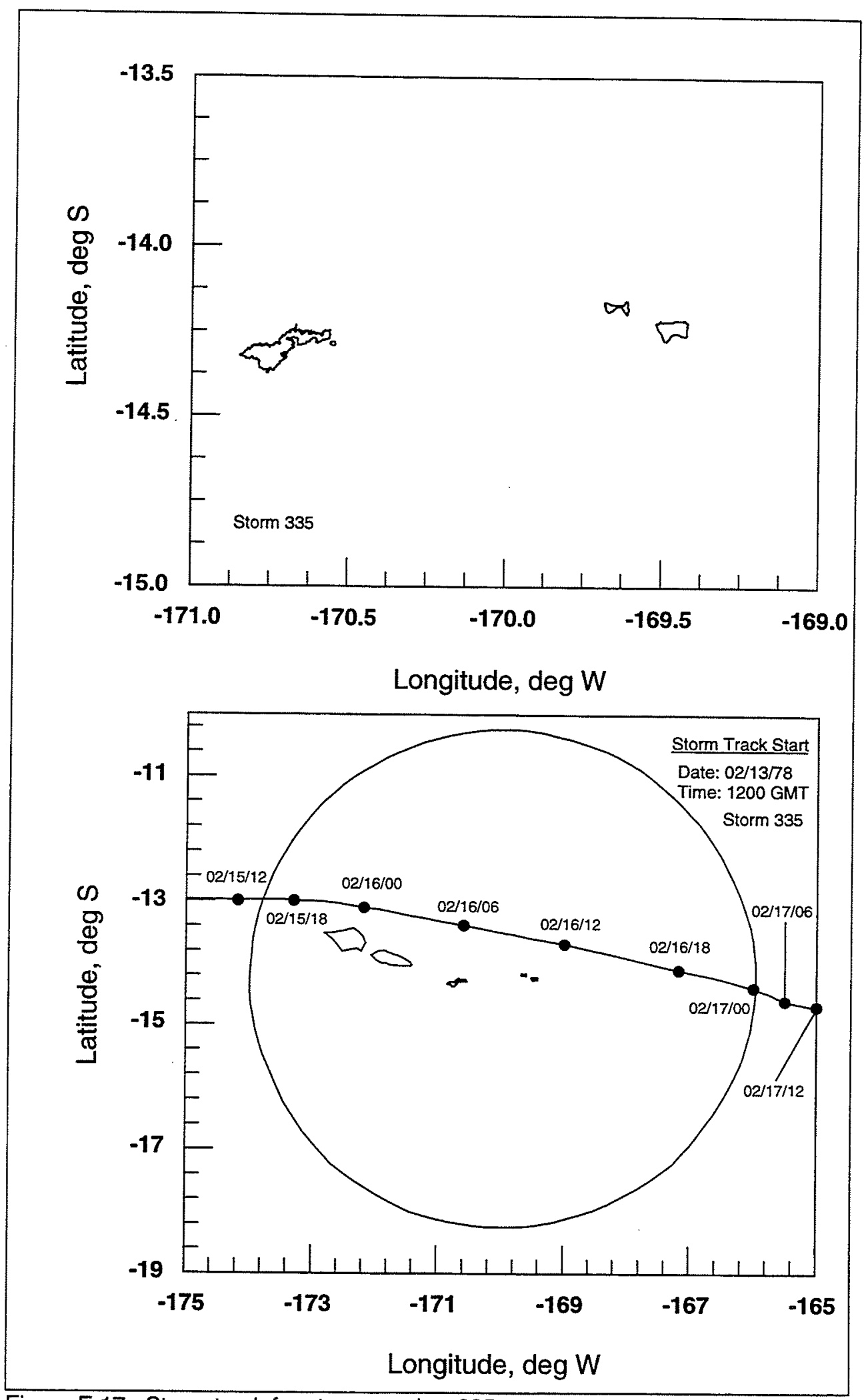

Figure E.17. Storm track for storm number 335 


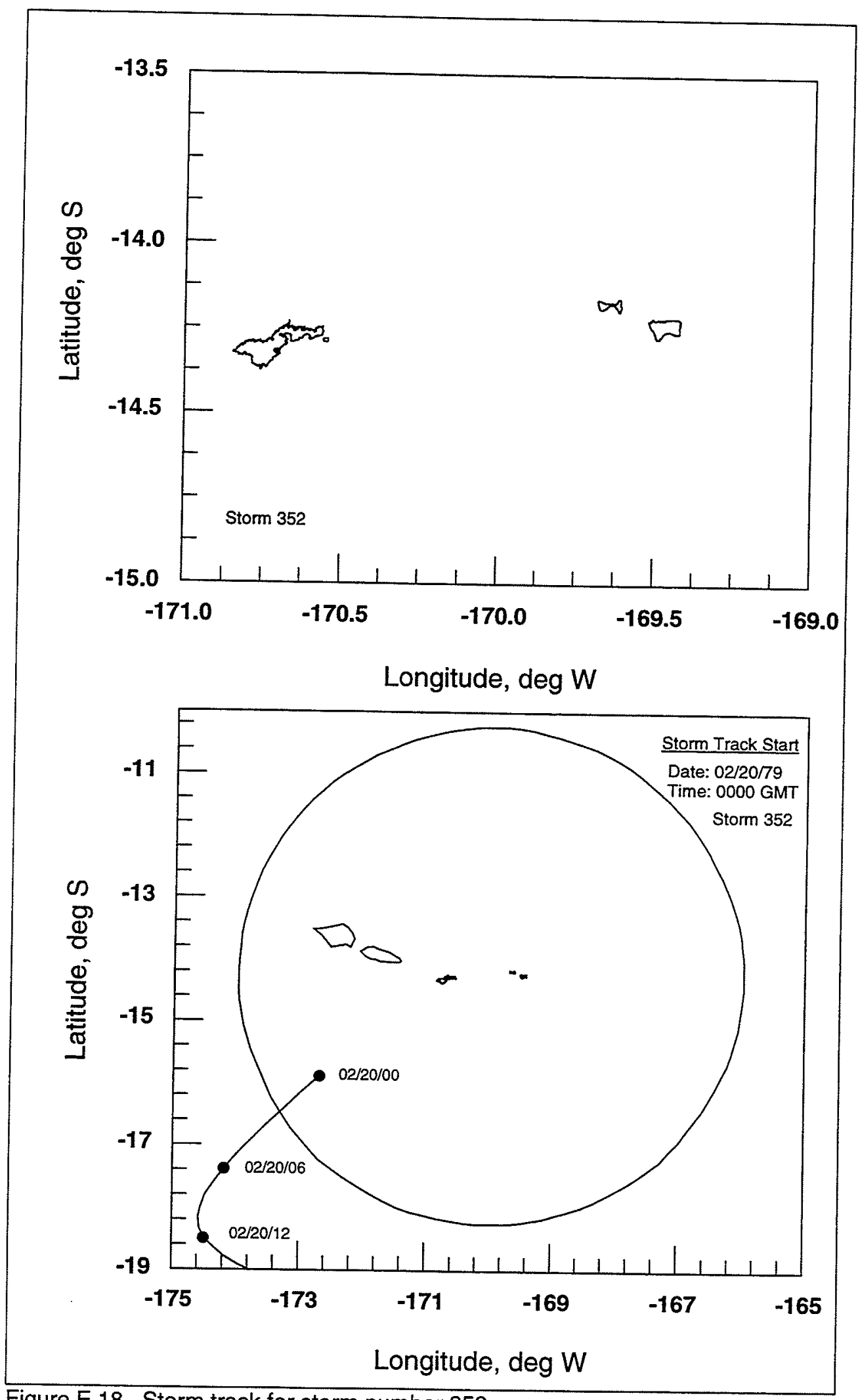

Figure E.18. Storm track for storm number 352 


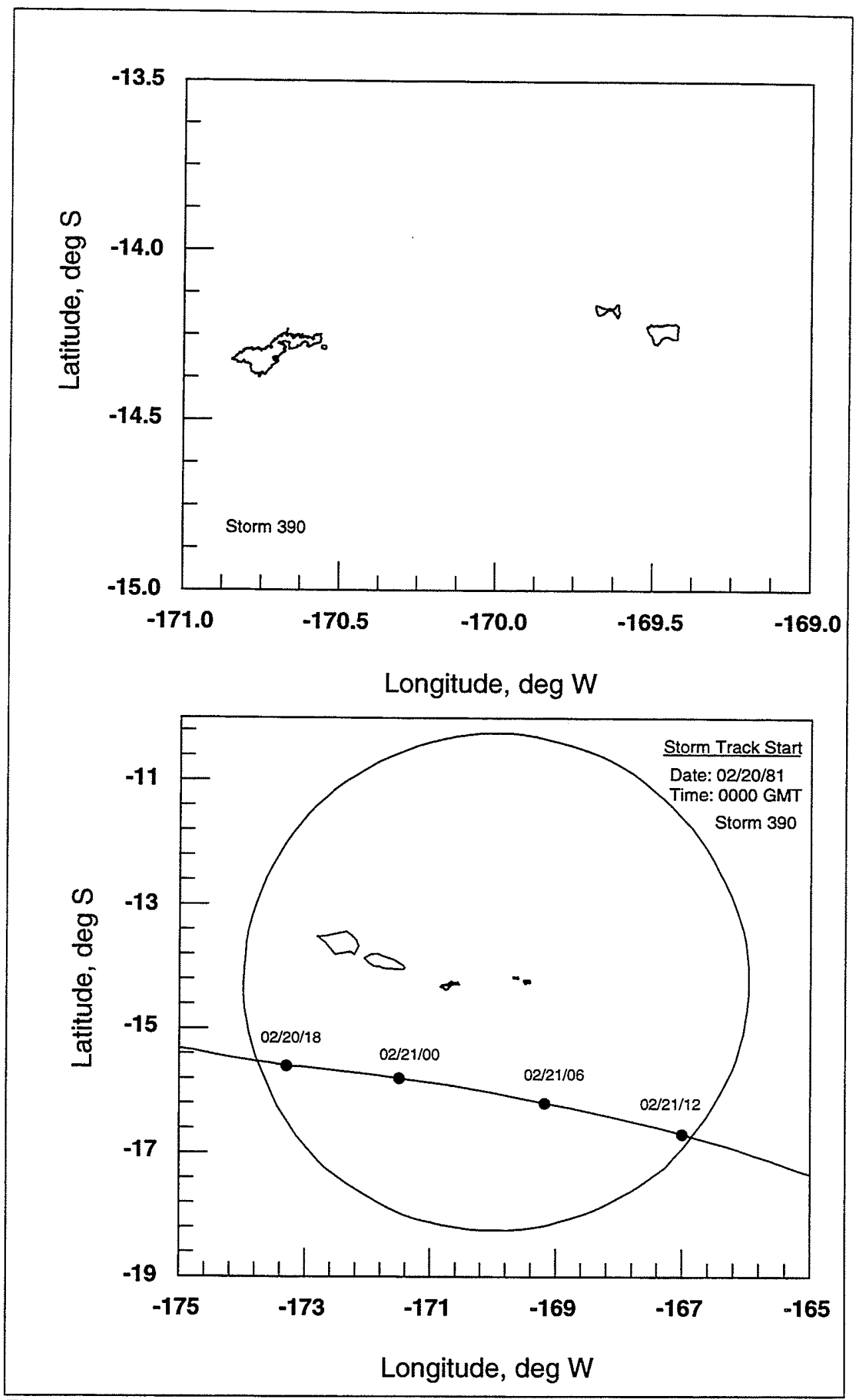

Figure E.19. Storm track for storm number 390 

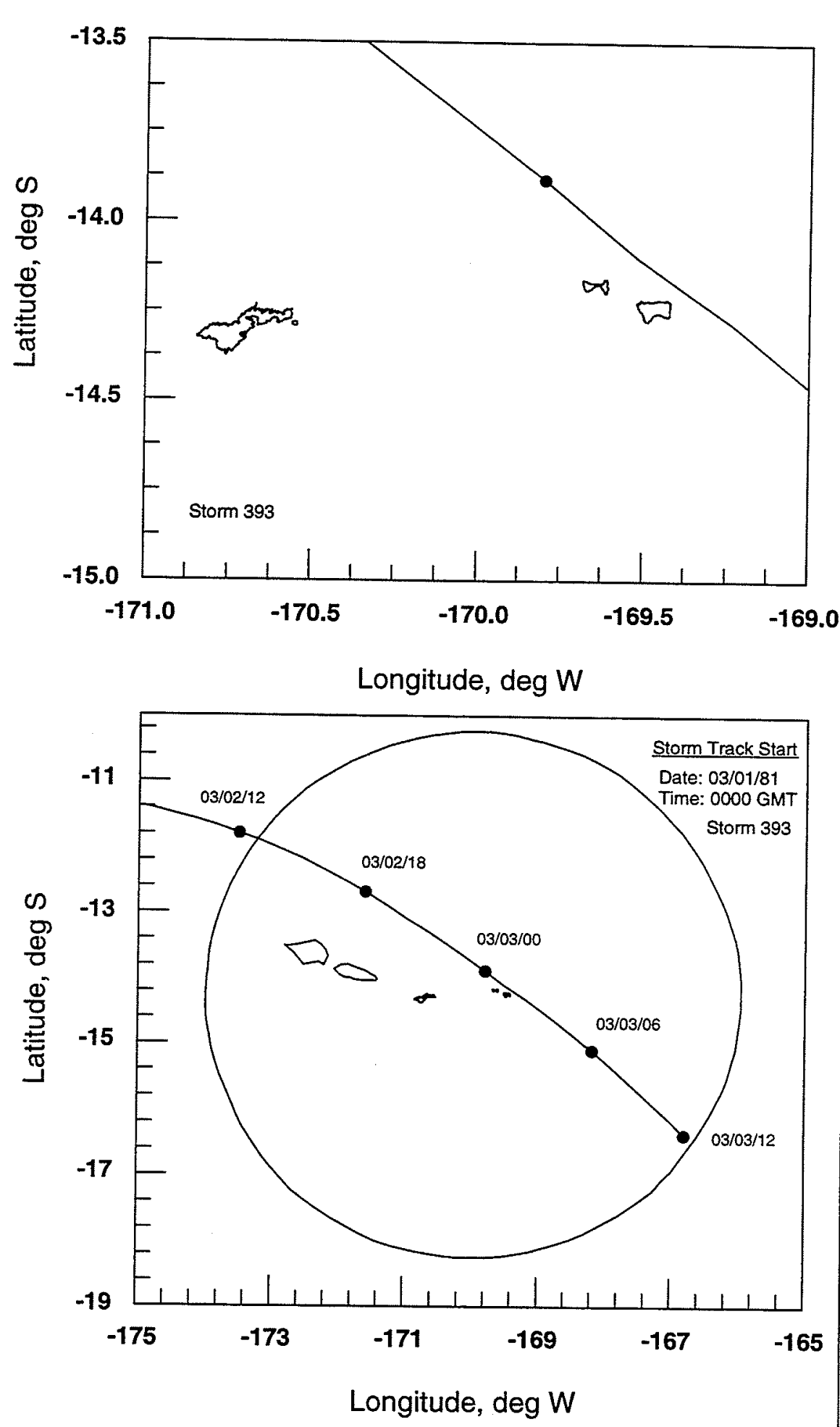

Figure E.20. Storm track for storm number 393 


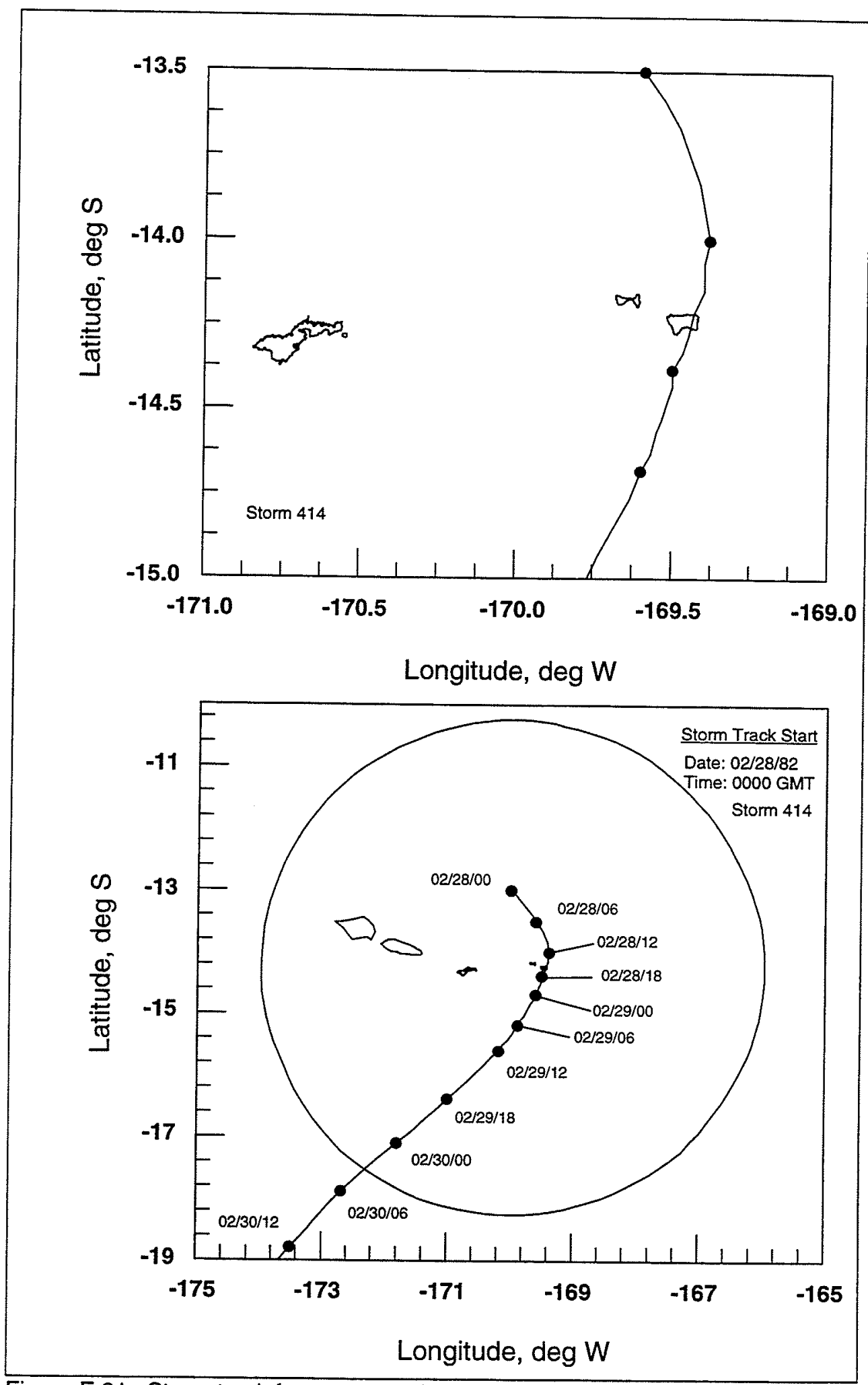

Figure E.21. Storm track for storm number 414 


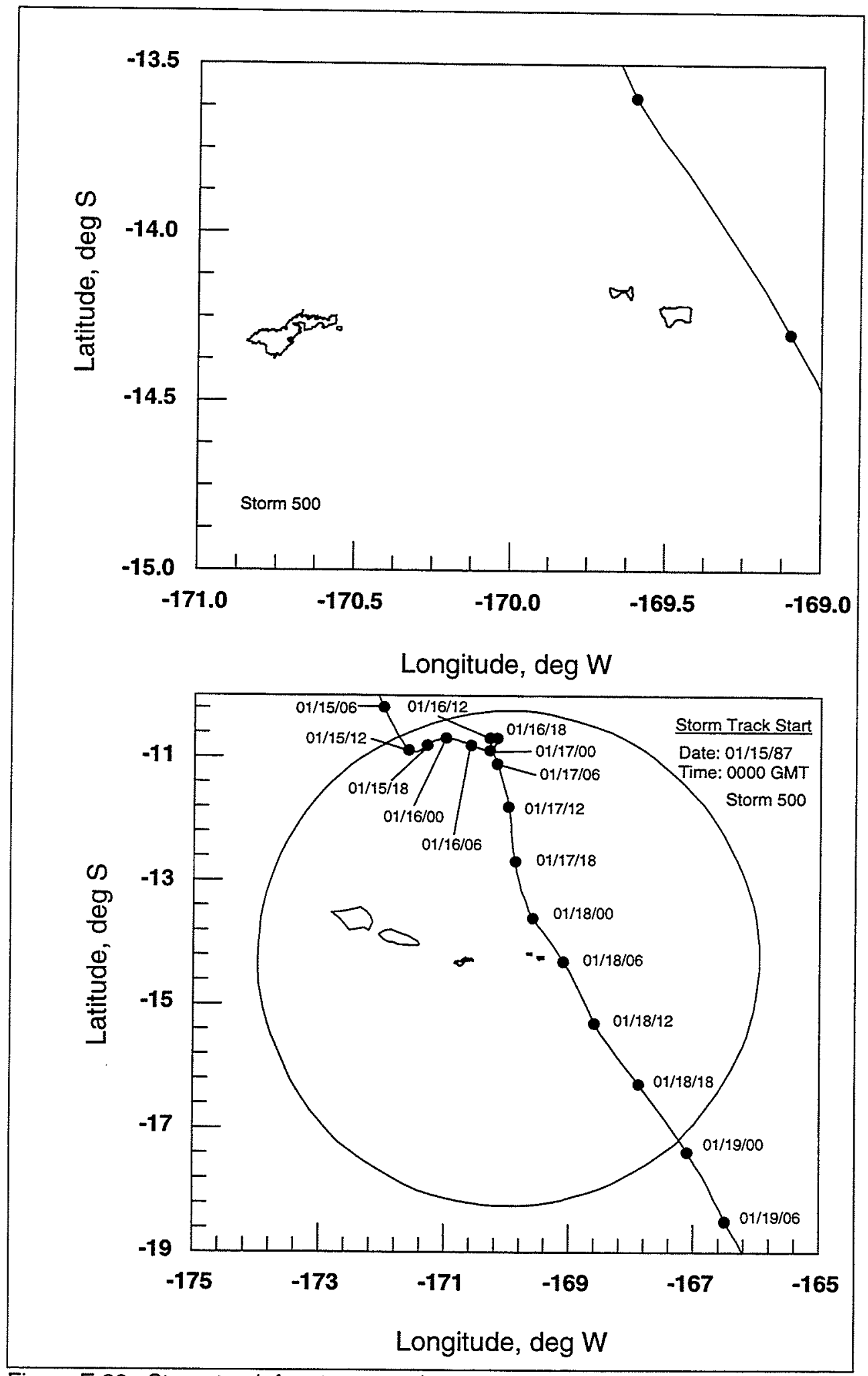

Figure E.22. Storm track for storm number 500 


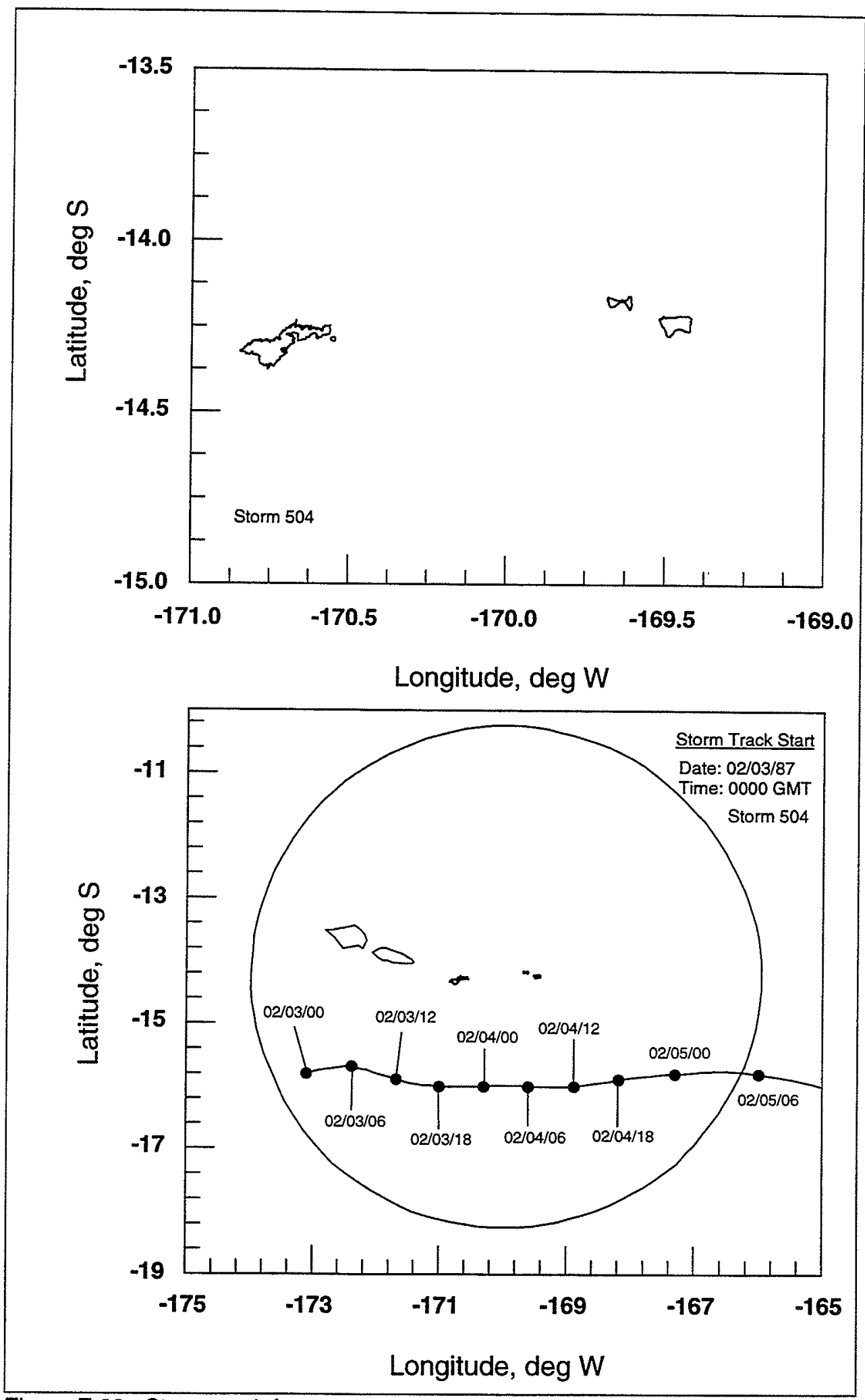

Figure E.23. Storm track for storm number 504 


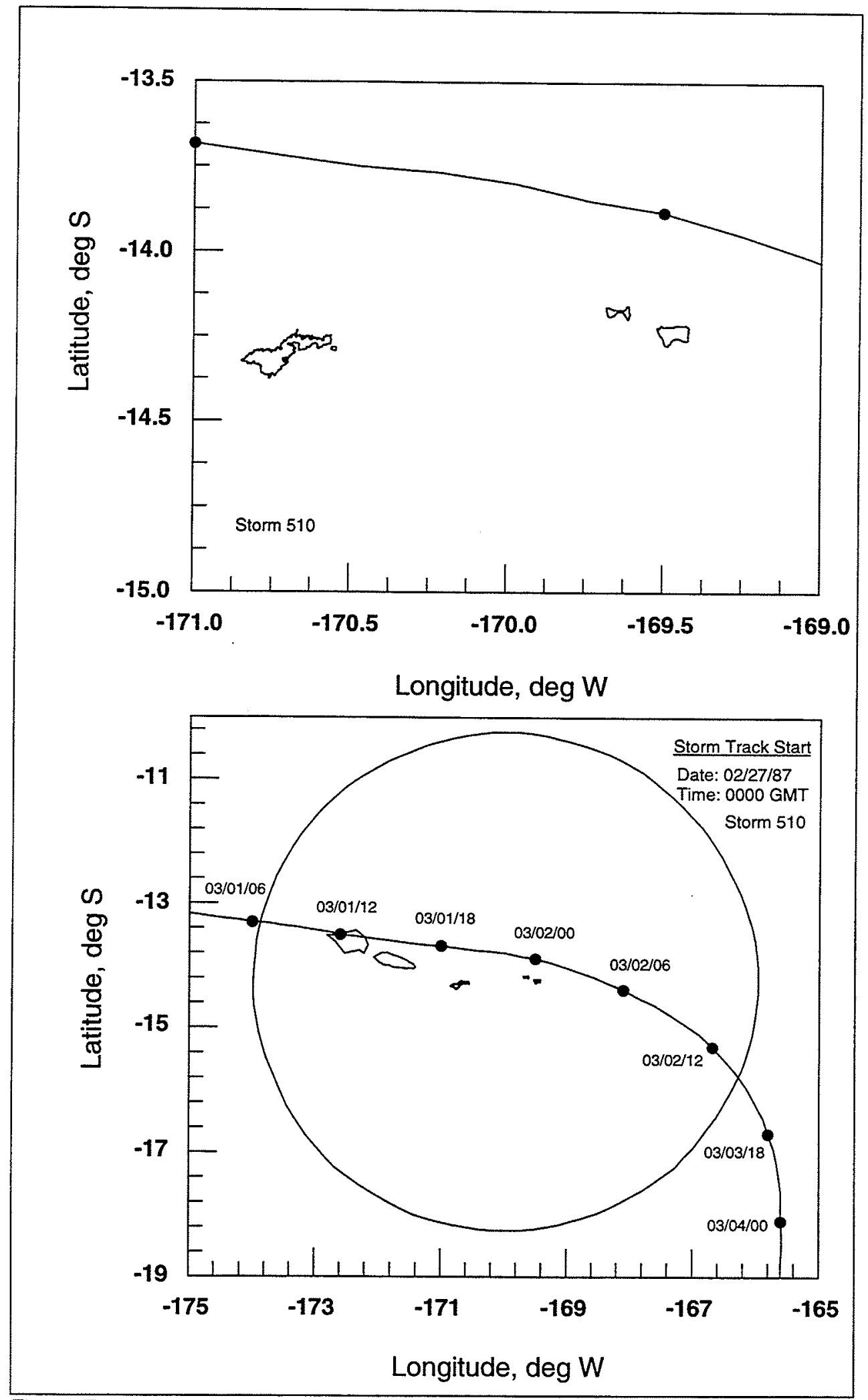

Figure E.24. Storm track for storm number $\mathbf{5 1 0}$ 


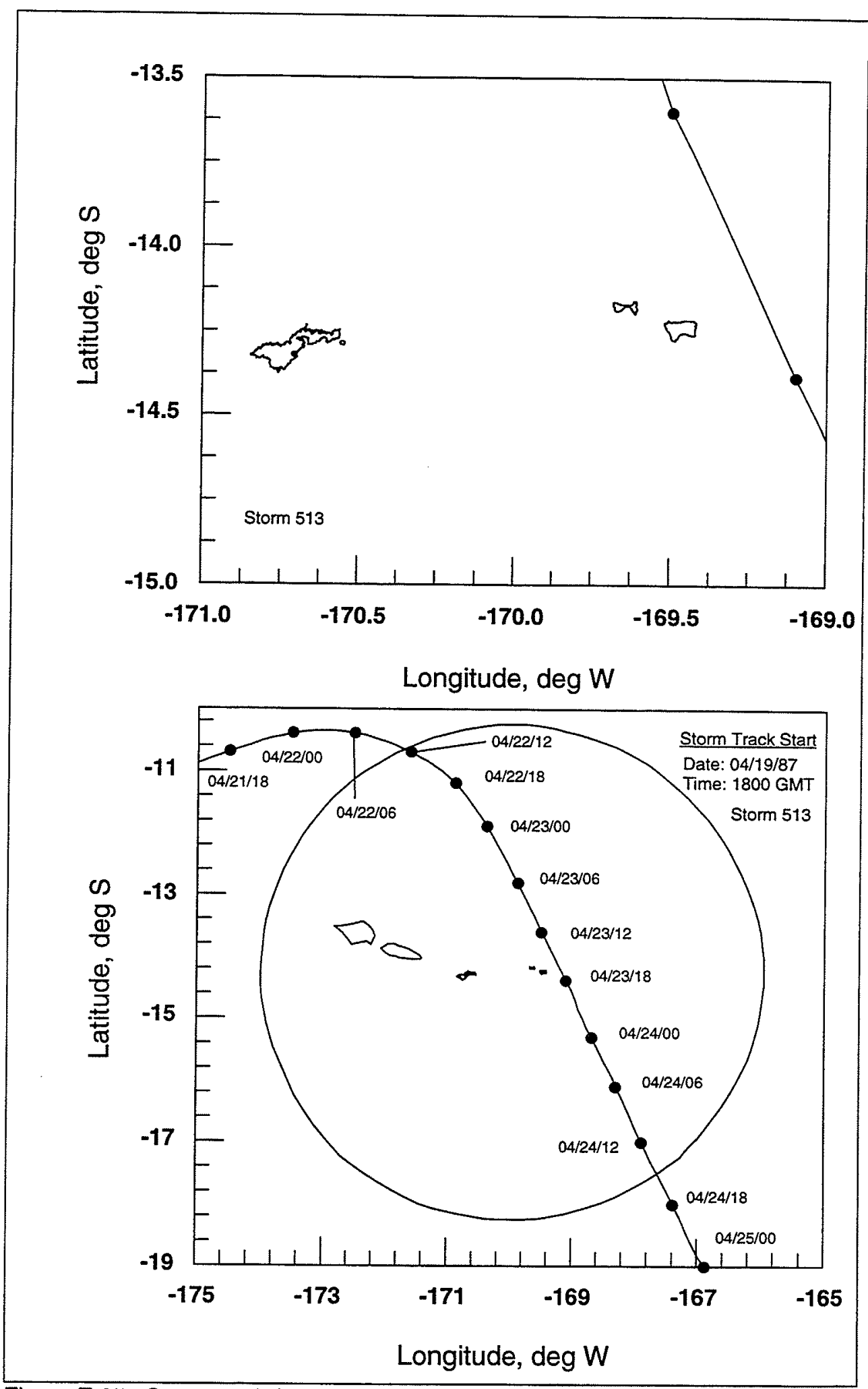

Figure E.25. Storm track for storm number 513 


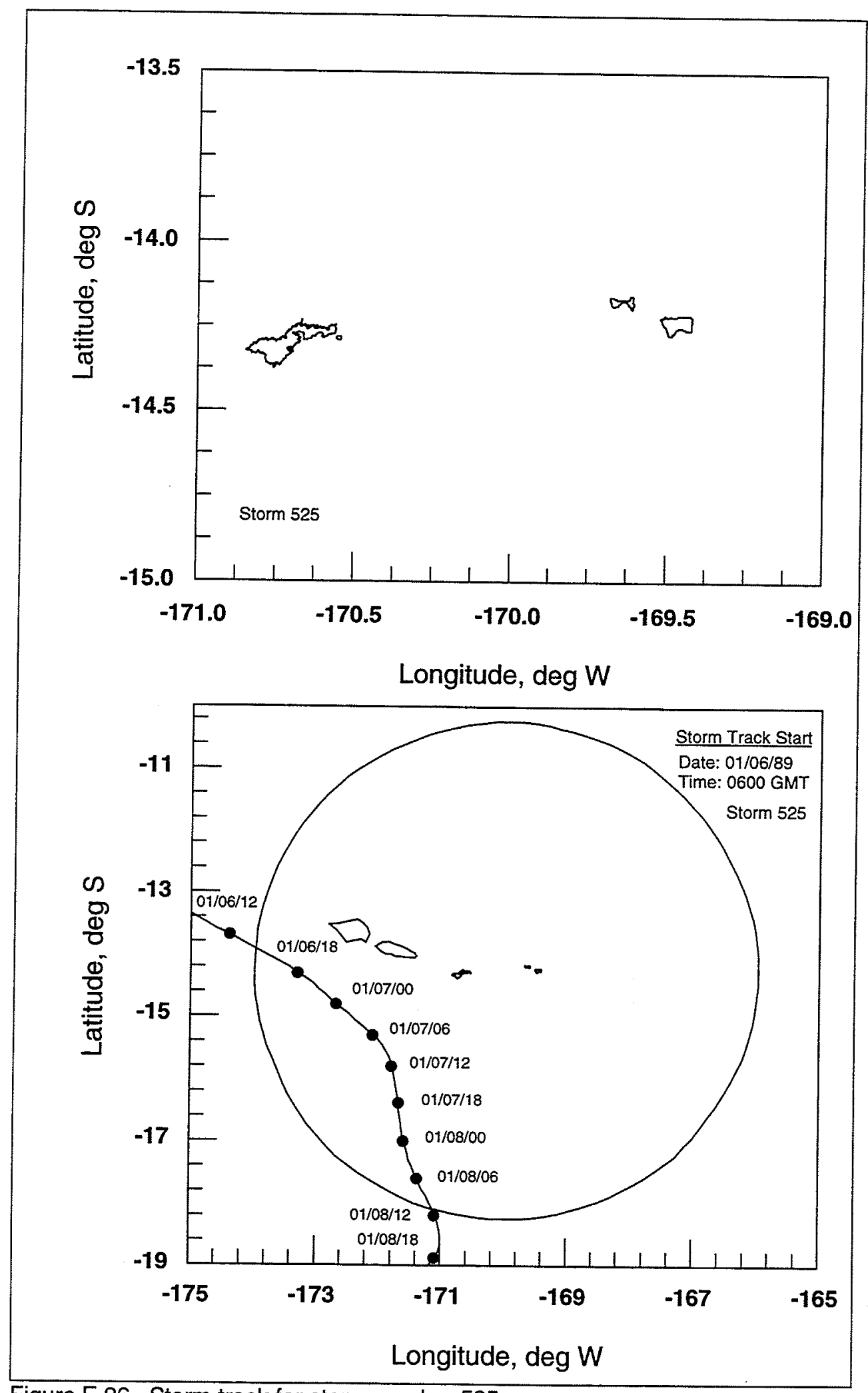

Figure E.26. Storm track for storm number 525 


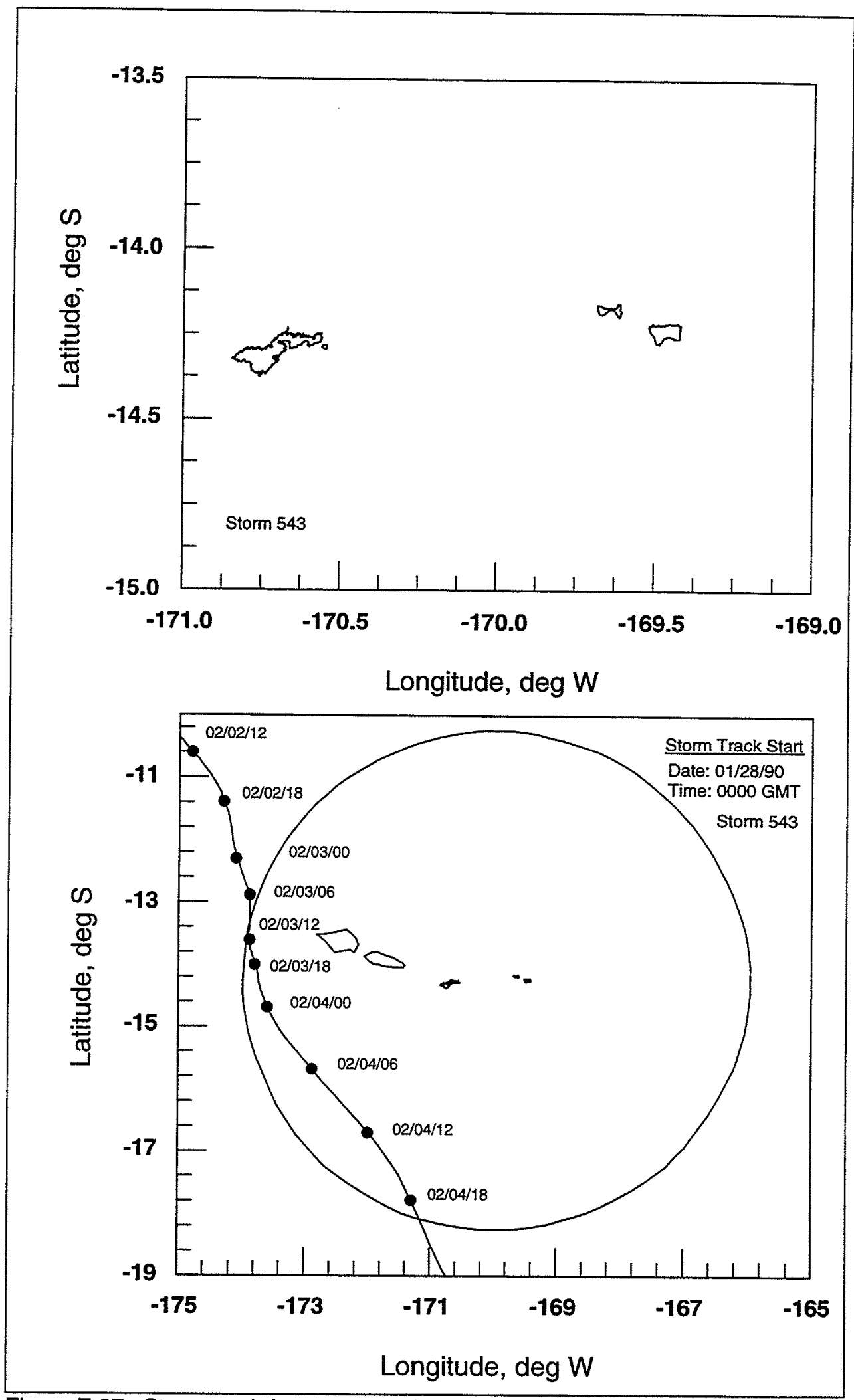

Figure E.27. Storm track for storm number 543 


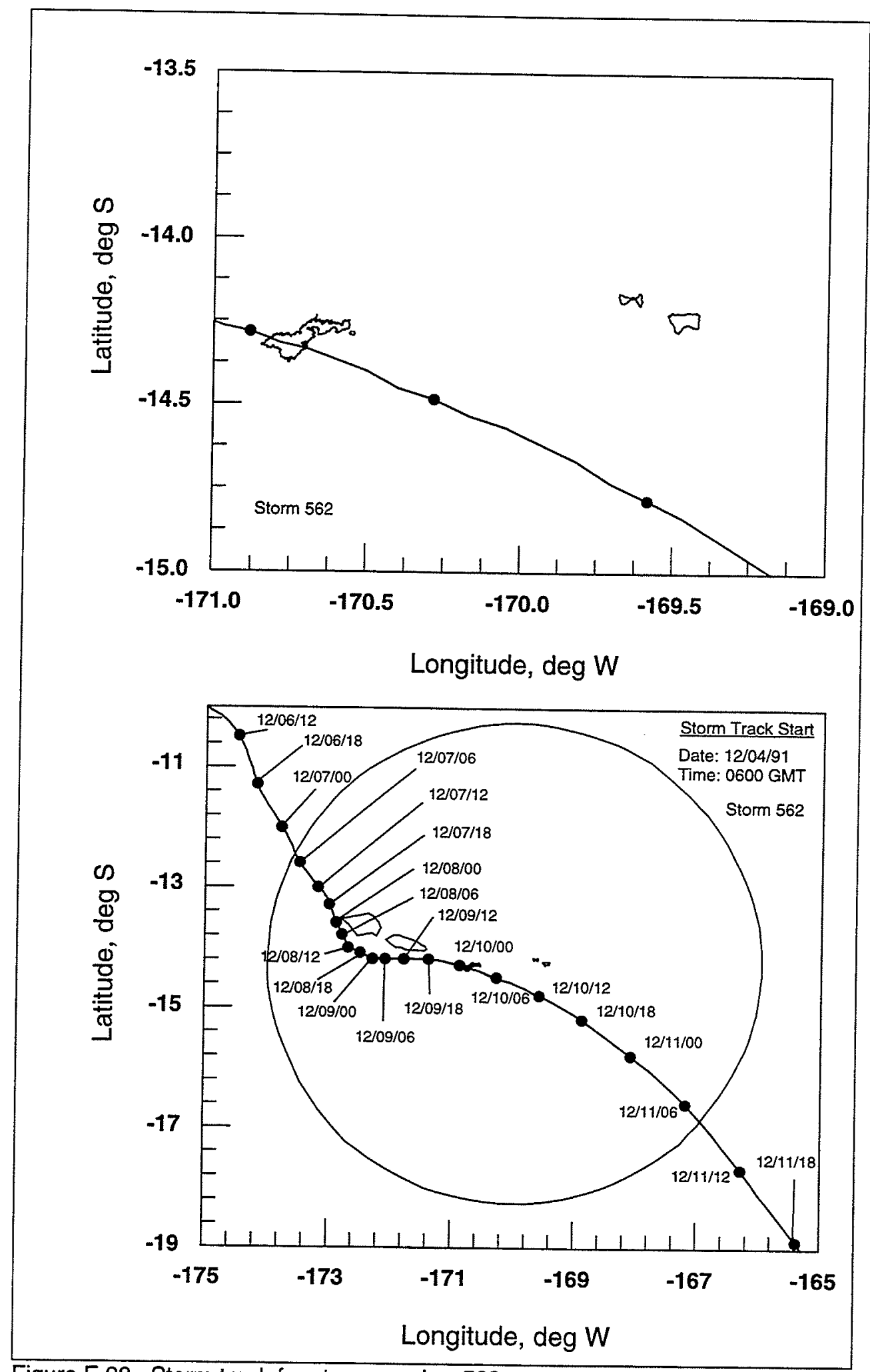

Figure E.28. Storm track for storm number 562 


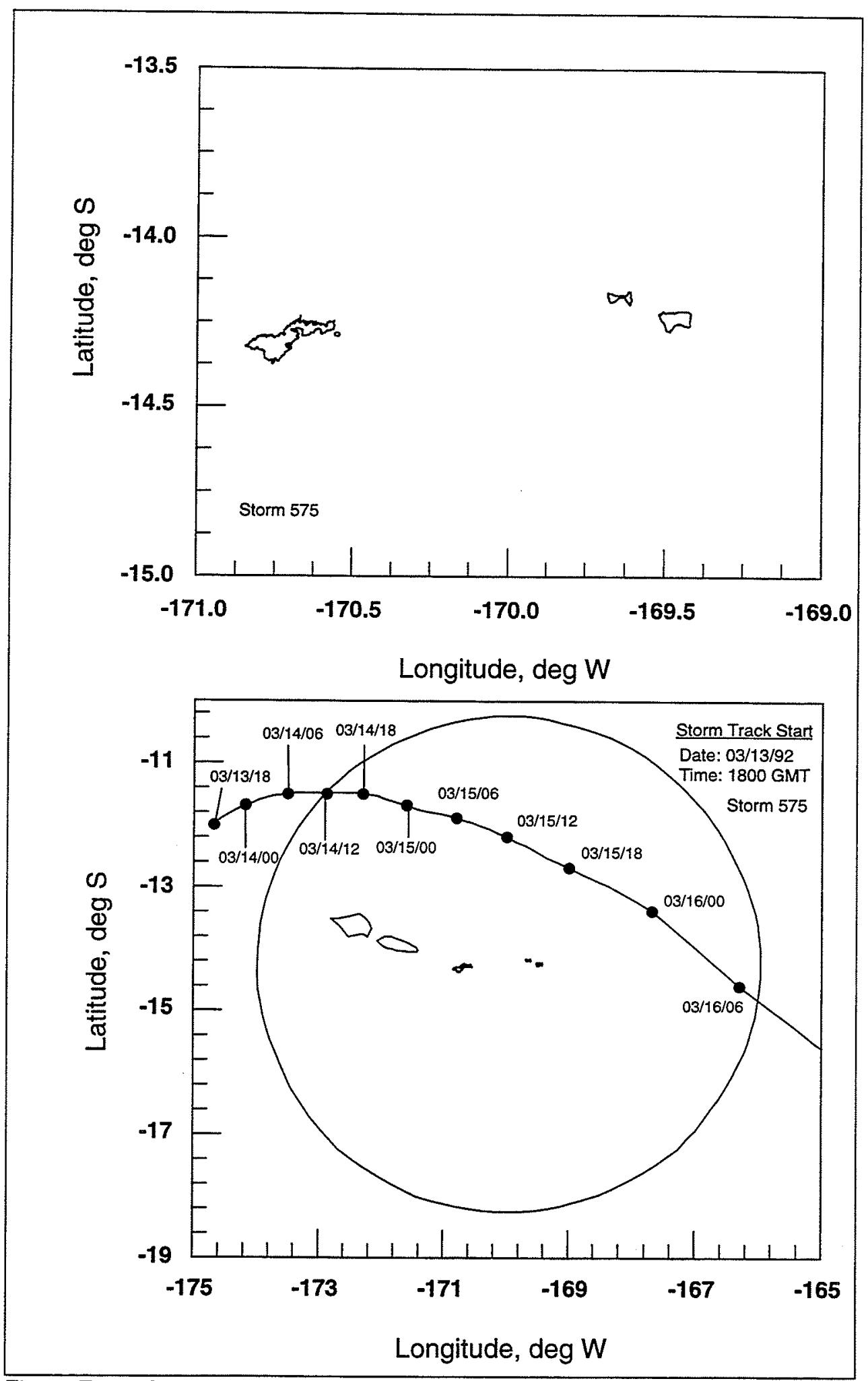

Figure E.29. Storm track for storm number 575 


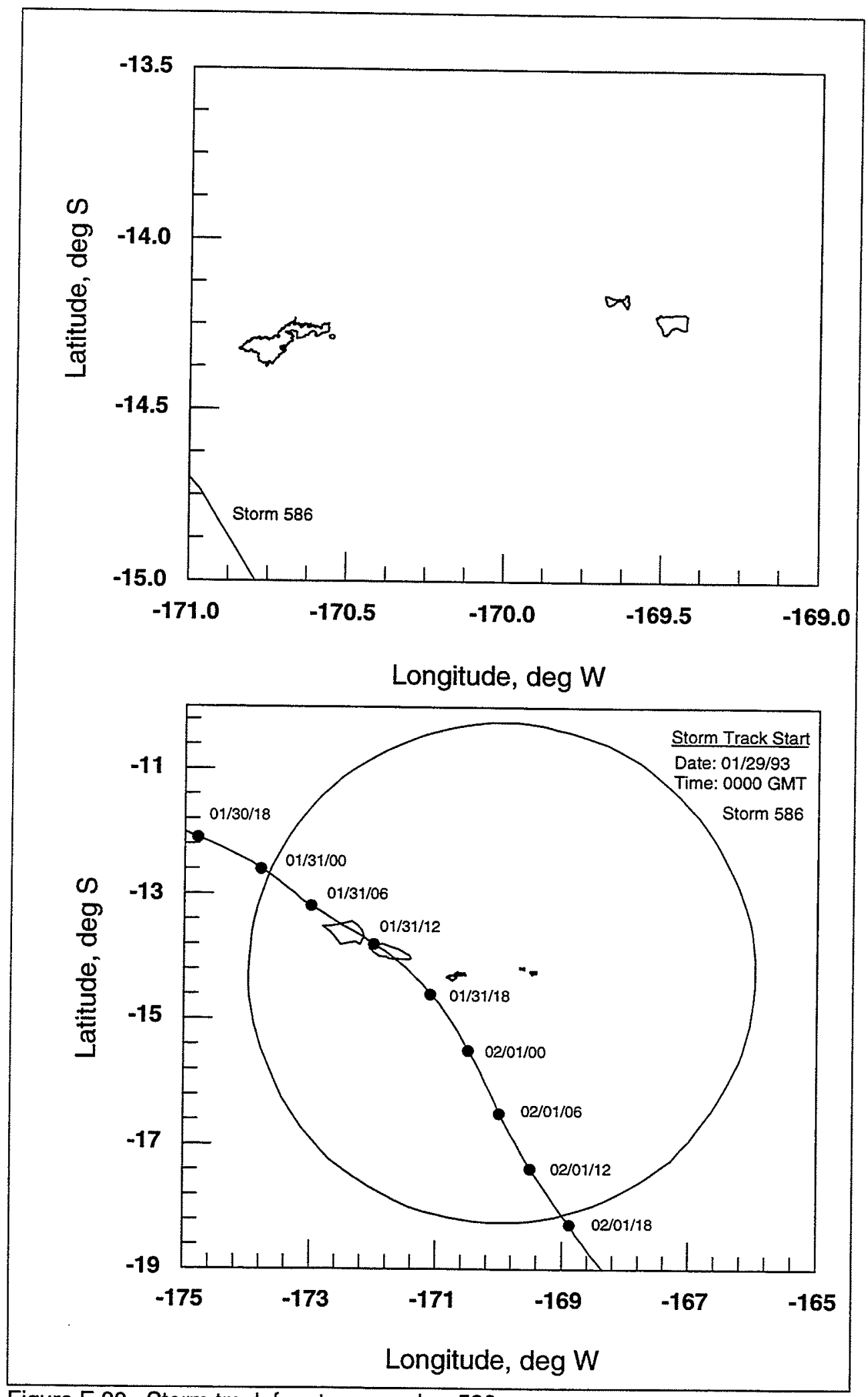

Figure E.30. Storm track for storm number 586 


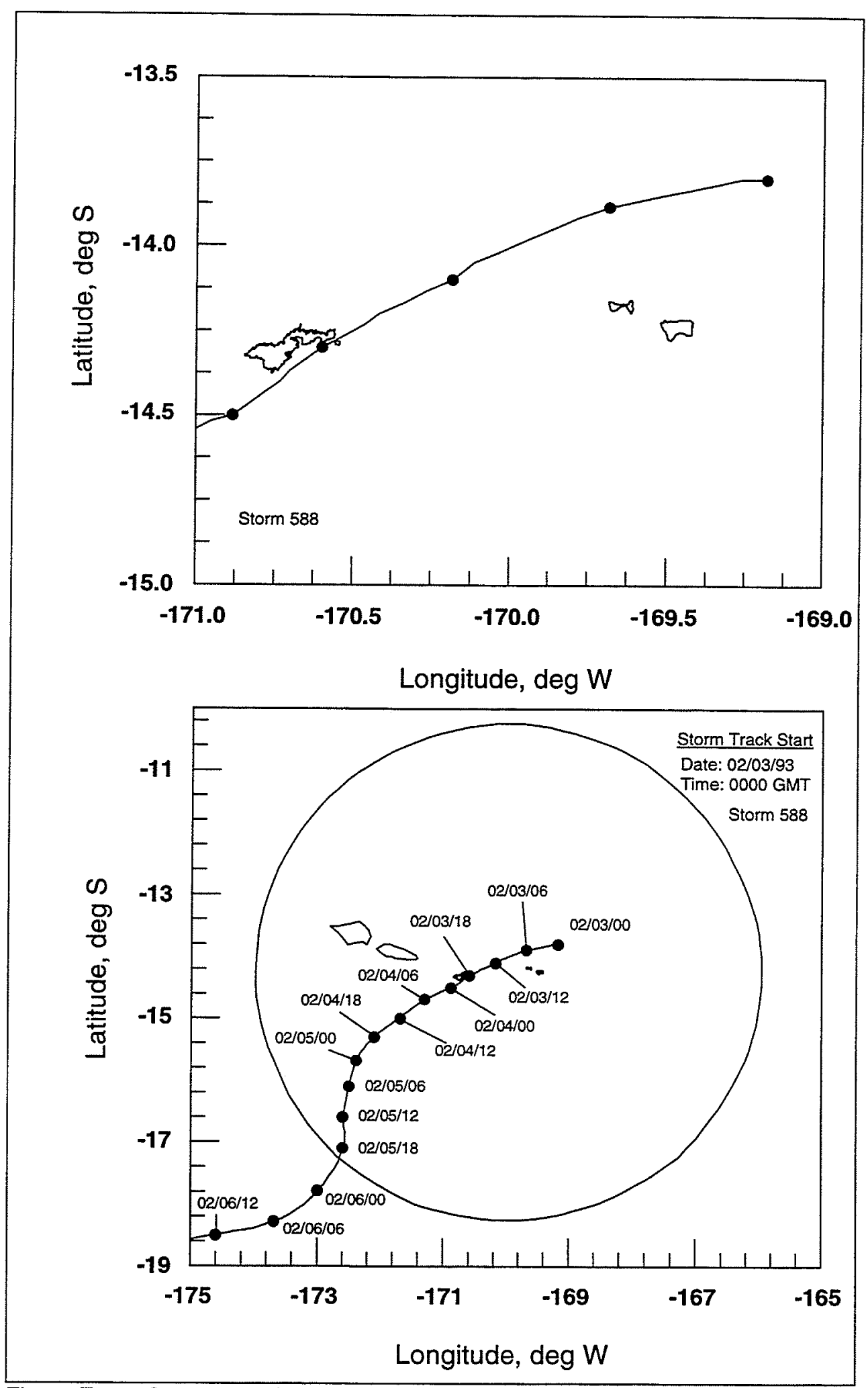

Figure E.31. Storm track for storm number 588 


\section{Appendix F Mathematical Notation}

$\begin{array}{ll}a_{1}, a_{2} & \text { Empirical ponding water-level coefficients } \\ C_{f} & \text { Bottom friction coefficient } \\ d & \text { Water depth } \\ D & \text { Total water depth for long-wave model } \\ f & \text { Coriolis parameter } \\ F(n) & \text { Simulated cumulative probability of occurrence } \\ F_{x}(x) & \text { Cumulative probability density function } \\ \hat{F}_{X}\left(X_{(r)}\right) & \text { Empirical estimate of cumulative probability density function } \\ g & \text { Acceleration due to gravity } \\ h & \text { Ambient water depth } \\ h_{b} & \text { Water depth at breaking } \\ H & \text { Wave height } \\ H_{b} & \text { Height of breaking wave } \\ H_{0} & \text { Deep-water significant wave height } \\ n & \text { Number of random vectors; number of years } \\ p_{i} & \text { Probability of a specific event } \\ P_{a} & \text { Ambient atmospheric pressure } \\ P_{C} & \text { Central pressure } \\ P_{S} & \text { Atmospheric pressure at water surface } \\ \text { Pr } & \text { Probability } \\ r & \text { Rank } \\ r_{i} & \text { Response parameters } \\ r & \text { Response vector } \\ R & \text { Radius of the Earth } \\ R^{2} & \text { Coefficient of determination } \\ s_{j} & \text { Probability segment } \\ S_{x x} & \text { Cross-shore component of the cross-shore directed radiation } \\ T & \text { stress } \\ U & \text { Wave period } \\ & \text { Component of current parallel to the East-West axis } \\ & \\ & \end{array}$




$\begin{array}{ll}v_{i} & \text { Input parameters } \\ \underline{v} & \text { Input vector } \\ V & \text { Component of the current parallel to the North-South axis } \\ W & \text { Maximum wind speed } \\ x & \text { Cross-shore distance } \\ x_{i} & \text { Response vectors corresponding to event } X \\ X_{i} & \text { Random event vectors } \\ \alpha & \text { Empirical coefficient } \\ \beta & \text { Empirical coefficient } \\ \gamma_{b} & \text { Breaking depth index } \\ \zeta & \text { Free-surface water elevation relative to the geoid } \\ \xi & \text { Effective Newtonian equilibrium tide potential } \\ \eta & \text { Ponding water level } \\ \bar{\eta} & \text { Mean still-water level } \\ \lambda & \text { Number of events per year } \\ \phi & \text { Latitude } \\ \varphi & \text { Longitude } \\ \rho & \text { Water density } \\ \Omega & \text { Angular speed of the Earth's rotation } \\ \tau_{S \phi}, \tau_{S \varphi} & \text { Surface stresses } \\ \tau_{*} & \text { Bottom stress } \\ \Re^{d_{v}} & \text { dimensional space }\end{array}$




\section{REPORT DOCUMENTATION PAGE}

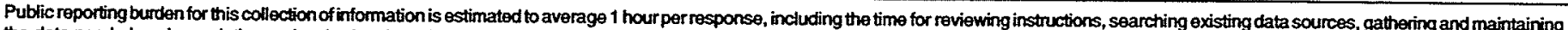

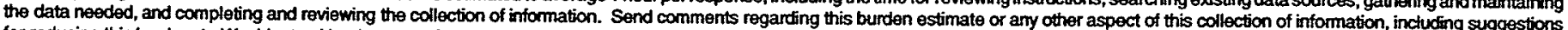

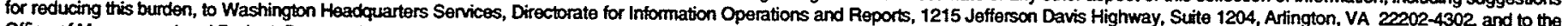
Office of Management and Budget, Paperwork Reduction Project (0704-0188), Washington, DC 20503.
1. AGENCY USE ONLY (Leave blank) 2. REPORT DATE
December 1998
3. REPORT TYPE AND DATES COVERED
Final report

4. TITLE AND SUBTTILE

\section{Hurricane-Induced Stage-Frequency Relationships for the Territory of} American Samoa

6. AUTHOR(S)

Adele Militello, Norman W. Scheffner

\section{PERFORMING ORGANIZATION NAME(S) AND ADDRESS(ES)}

U.S. Army Engineer Waterways Experiment Station 3909 Halls Ferry Road

Vicksburg, MS 39180-6199

8. PERFORMING ORGANIZATION REPORT NUMBER

Technical Report CHL-98-33
9. SPONSORINGMONITORING AGENCY NAME(S) AND ADDRESS(ES)

U.S. Army Engineer Division, Pacific Ocean

Ft. Shafter, HI 96858-5440
5. FUNDING NUMBERS

\section{SUPPLEMENTARY NOTES}

Available from National Technical Information Service, 5285 Port Royal Road, Springfield, VA 22161.

12a. DISTRIBUTION/AVAILABILITY STATEMENT

Approved for public release; distribution is unlimited. 12b. DISTRIBUTION CODE

13. ABSTRACT (Maximum 200 words)

This report describes the methodology for computing storm-surge surface elevations versus frequency of occurrence relationships for the U.S. Territory of American Samoa. Maximum inundation levels were computed from combination of representative tidal elevations, storm surge, wave-induced setup, ponding level on fringing reefs, and wave runup. The statistical approach taken to calculated frequency-of-occurrence relationships was the Empirical Simulation Technique, which applies historical wave information and a nearest neighbor technique for simulating random storm events. Each phase of the study is described in detail and study results are presented in graphics and tables.

\section{SUBJECT TERMS}

Empirical simulation technique

Finite element modeling

Hurricane modeling

Storm surge
Wave runup

Wave setup

Wind wave modeling
15. NUMBER OF PAGES

286

16. PRICE CODE
17. SECURITY CLASSIFICATION OF REPORT

UNCLASSIFIED
18. SECURITY CLASSIFICATION OF THIS PAGE

UNCLASSIFIED
19. SECURITY CLASSIFICATION OF ABSTRACT
20. LIMITATION OF ABSTRACT 
